\title{
Enantioselective Synthesis Muqubilin and Negombatoperoxides B and C/D
}

Xiao-Tao Wang and Yikang $\mathrm{Wu}^{*}$

State Key Laboratory of Bioorganic and Natural Products Chemistry, Center for Excellence in Molecular Synthesis, Shanghai Institute of Organic Chemistry and the University of Chinese Academy of Sciences, Chinese Academy of Sciences, 345 Lingling Road, Shanghai 200032 China.

E-mail: yikangwu@sioc.ac.cn

\section{Table of Contents}

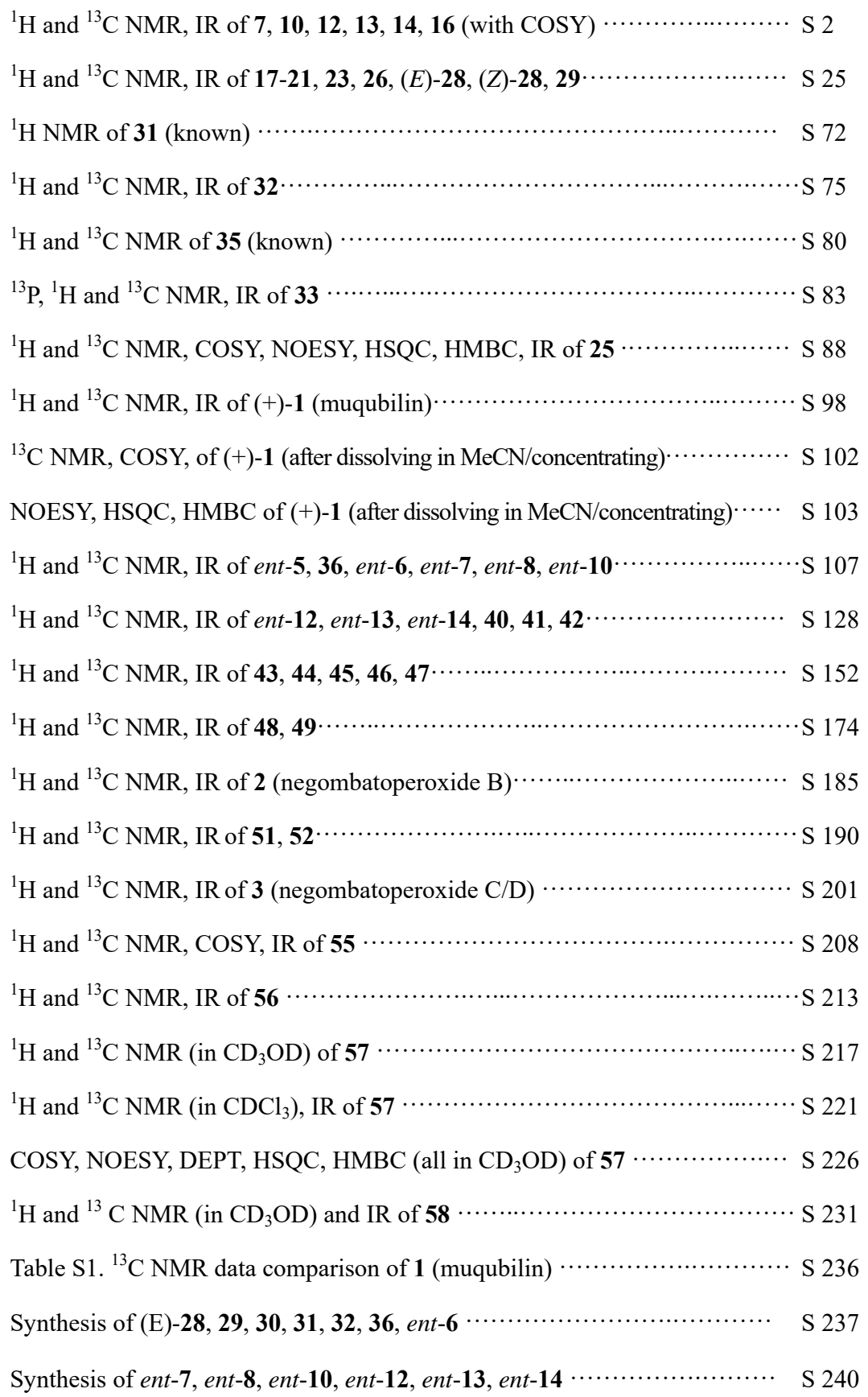




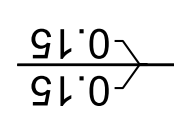

$\varepsilon$

50

90.1

EL ${ }^{\circ}$
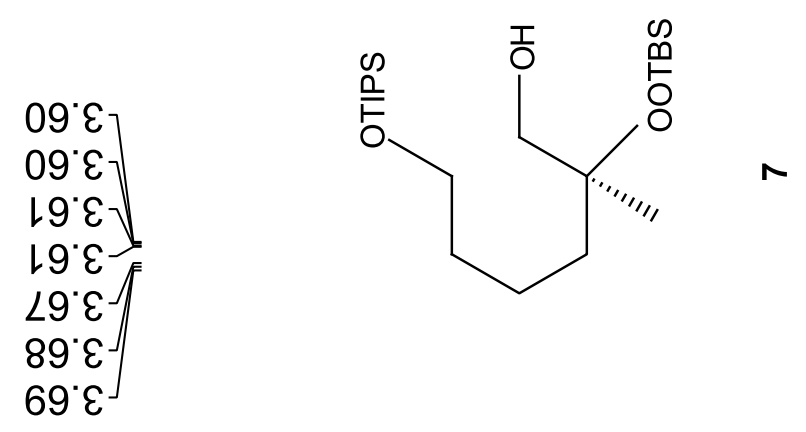

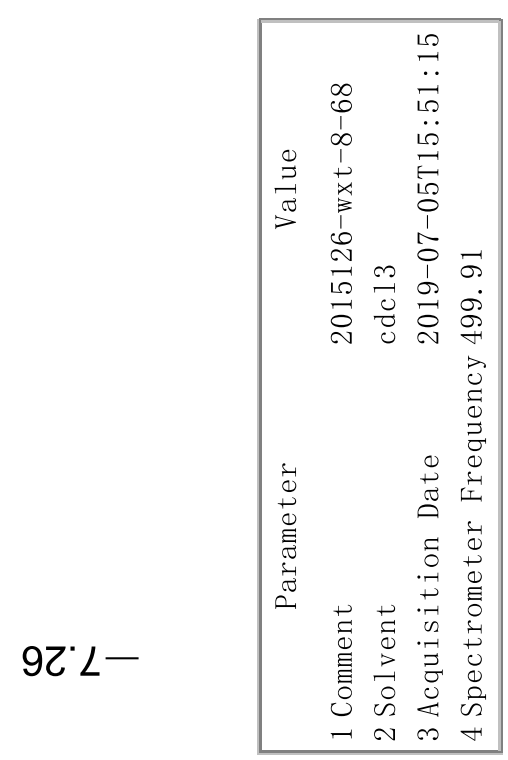


69.9-

89.

66ルー

10.81

$6 L^{\circ} 8 \mathrm{l}$

$\angle \varepsilon^{\circ} 8 \mathrm{~L}$

28 $6 \mathrm{l}$

งเ9Z-

$\left.6{ }^{*} \varepsilon \varepsilon\right\urcorner$

$00^{\circ} \nabla \varepsilon^{-}$

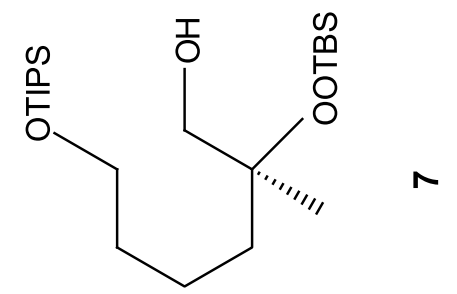

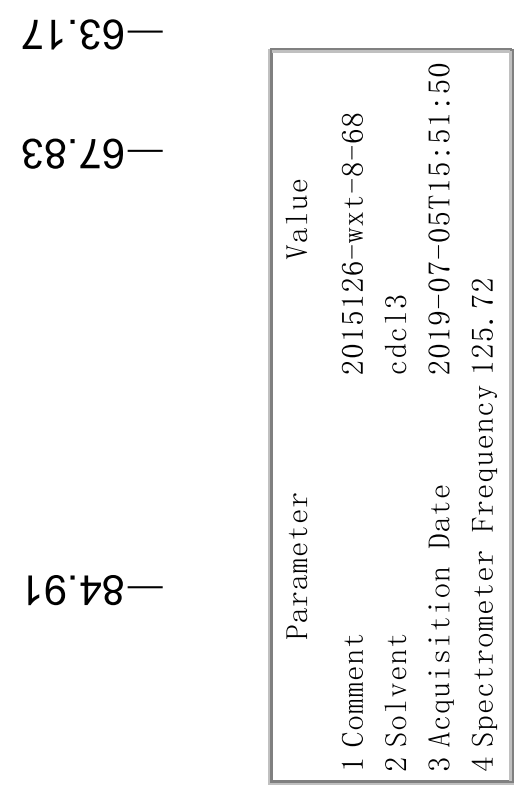




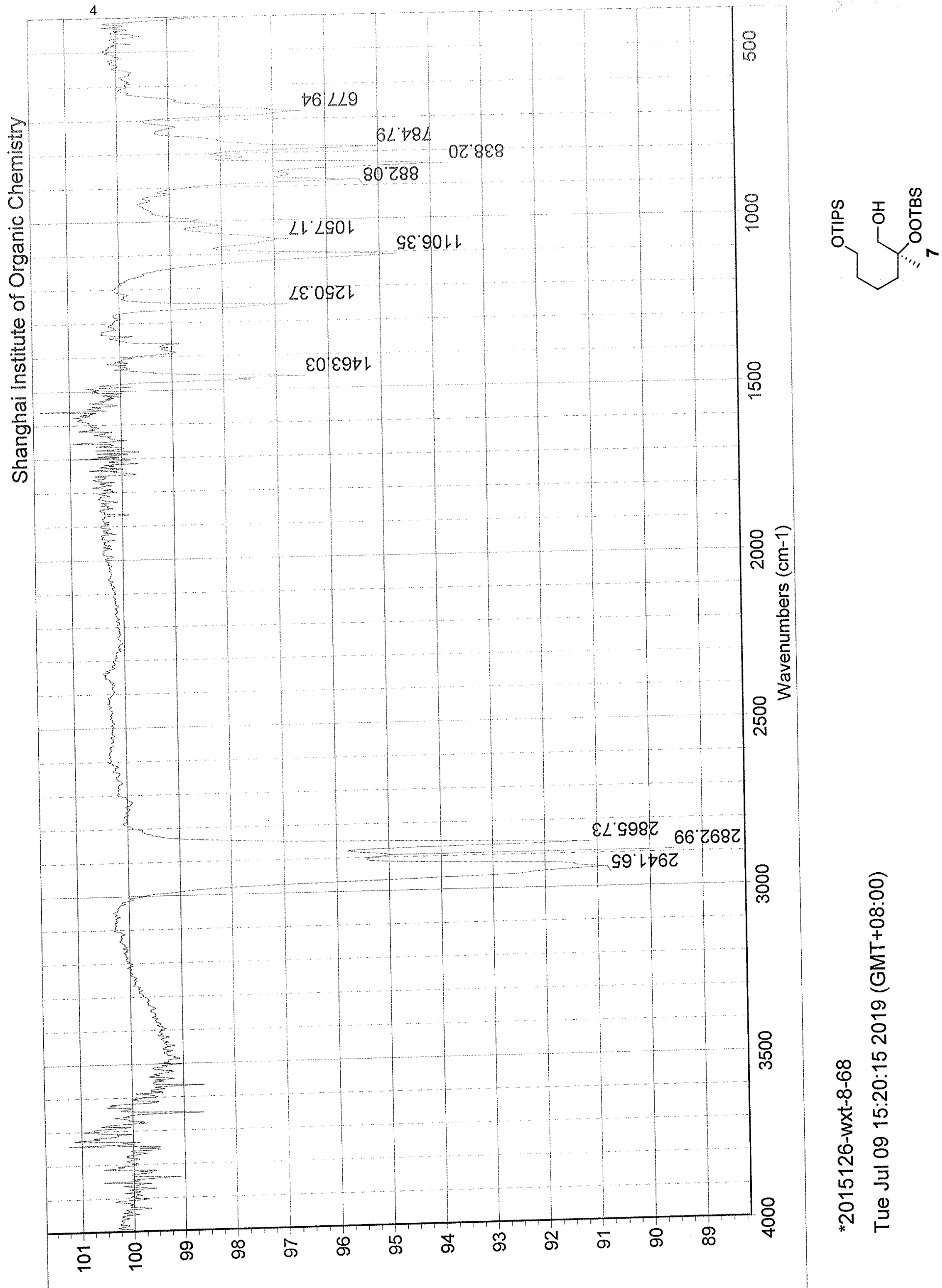


$\left.\begin{array}{l}\varepsilon \cdot 0 \\ \varepsilon \vdash \cdot 0\end{array}\right\}$

EL 0

$\left.\begin{array}{l}79^{\circ} \varepsilon \\ 99^{\circ} \varepsilon \\ \angle 9^{\circ} \varepsilon \\ \nabla L^{\circ} \varepsilon\end{array}\right]$

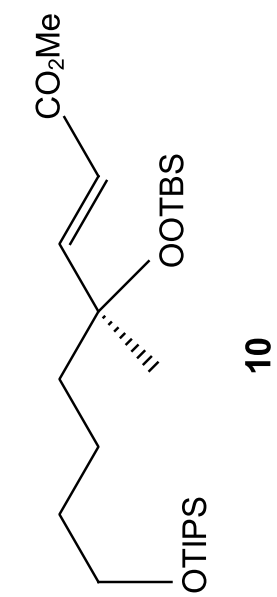

96.9-

$66^{\circ} 9^{\circ}$

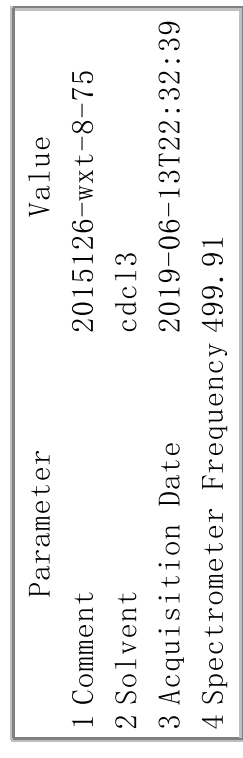

$9 Z^{\circ} L-$ 


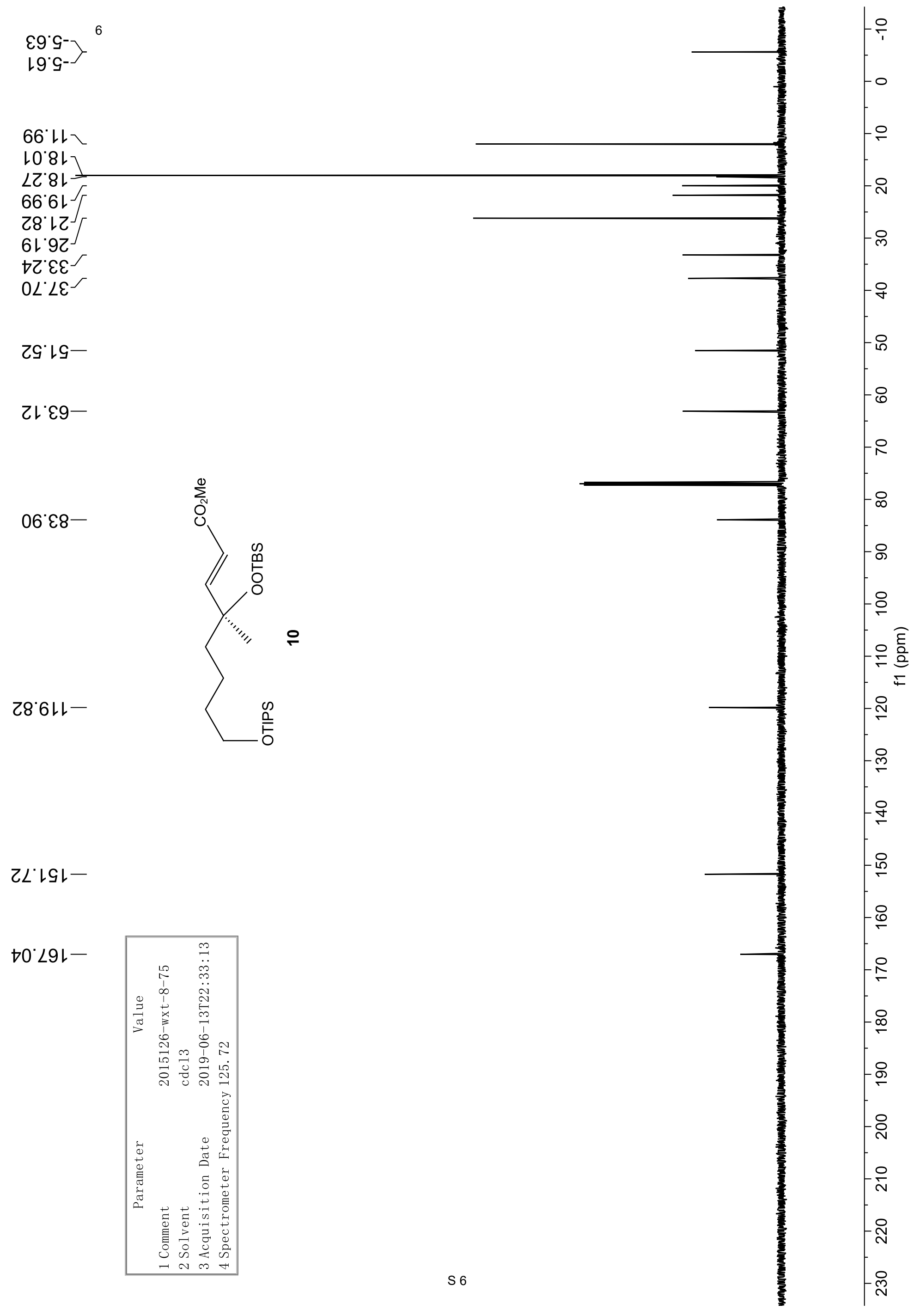




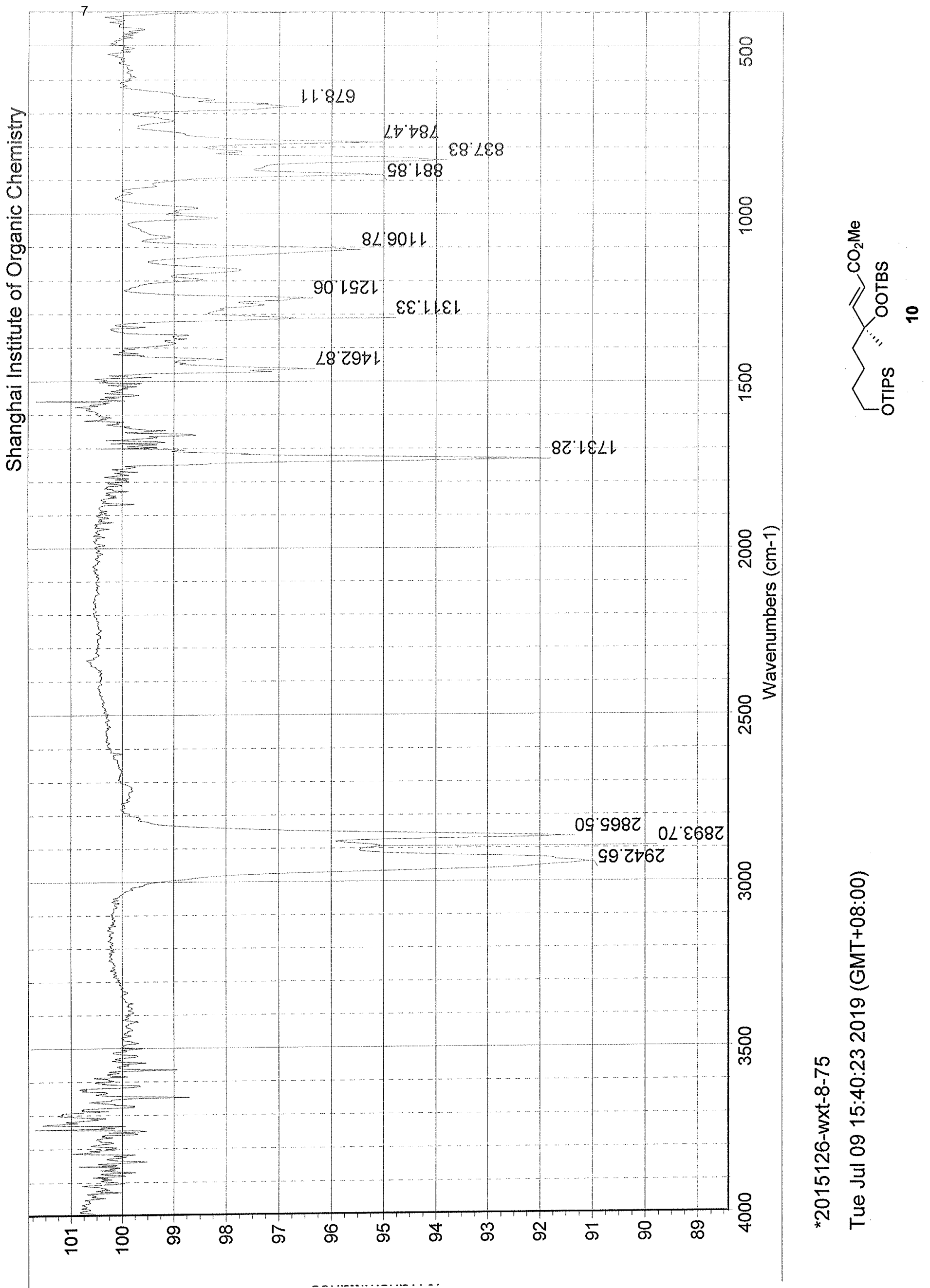


$\frac{16^{\circ} 0}{7017}$ Zl.

28.

$\nabla \varepsilon$

$\nabla \varepsilon^{\prime} 乙$

ง $\iota^{\circ}$

$\varsigma \varepsilon^{\prime} 乙$

$9 \varepsilon^{\prime \prime} 乙$

$\angle \varepsilon^{\prime} Z^{-}$

$8 \varepsilon^{\circ} \tau^{\circ}$

$99^{\circ} \varepsilon$
$29^{*} \varepsilon$
$89^{*} \varepsilon^{\top}$

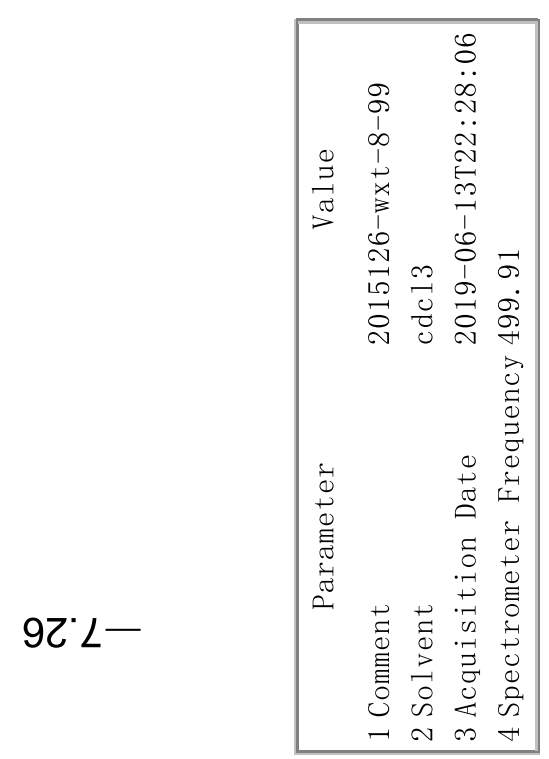

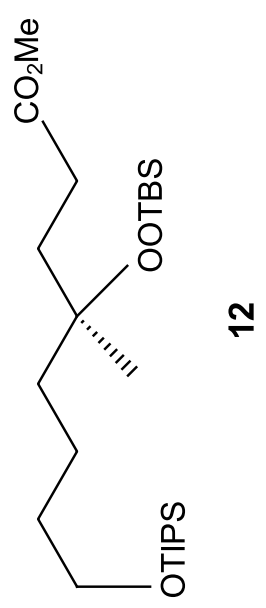
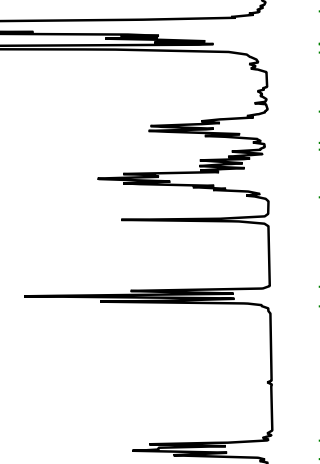

T 00

- $\varepsilon 6$.

in 
$79^{\prime} \mathrm{s}-$

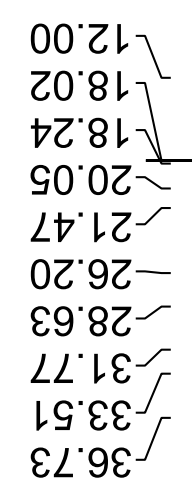

6ฑเงー

$\downarrow 乙 \cdot \varepsilon 9-$

$8 \varepsilon \cdot \varepsilon 8-$
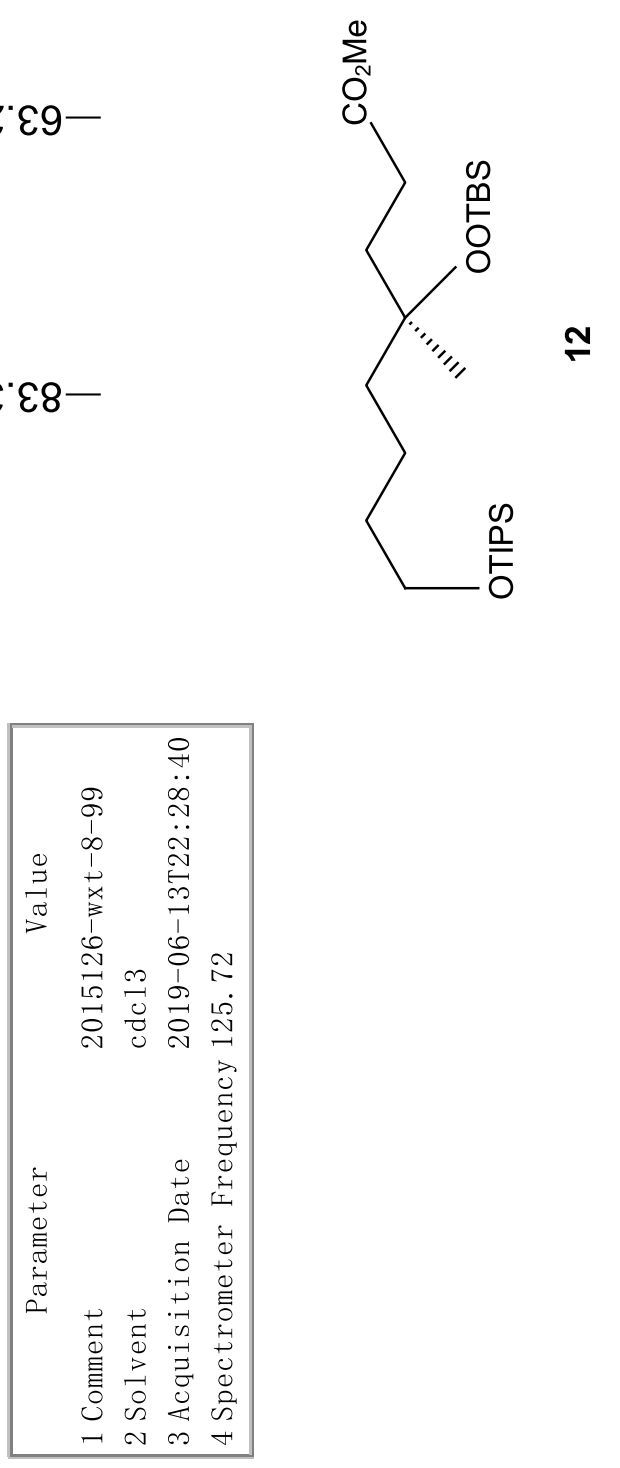


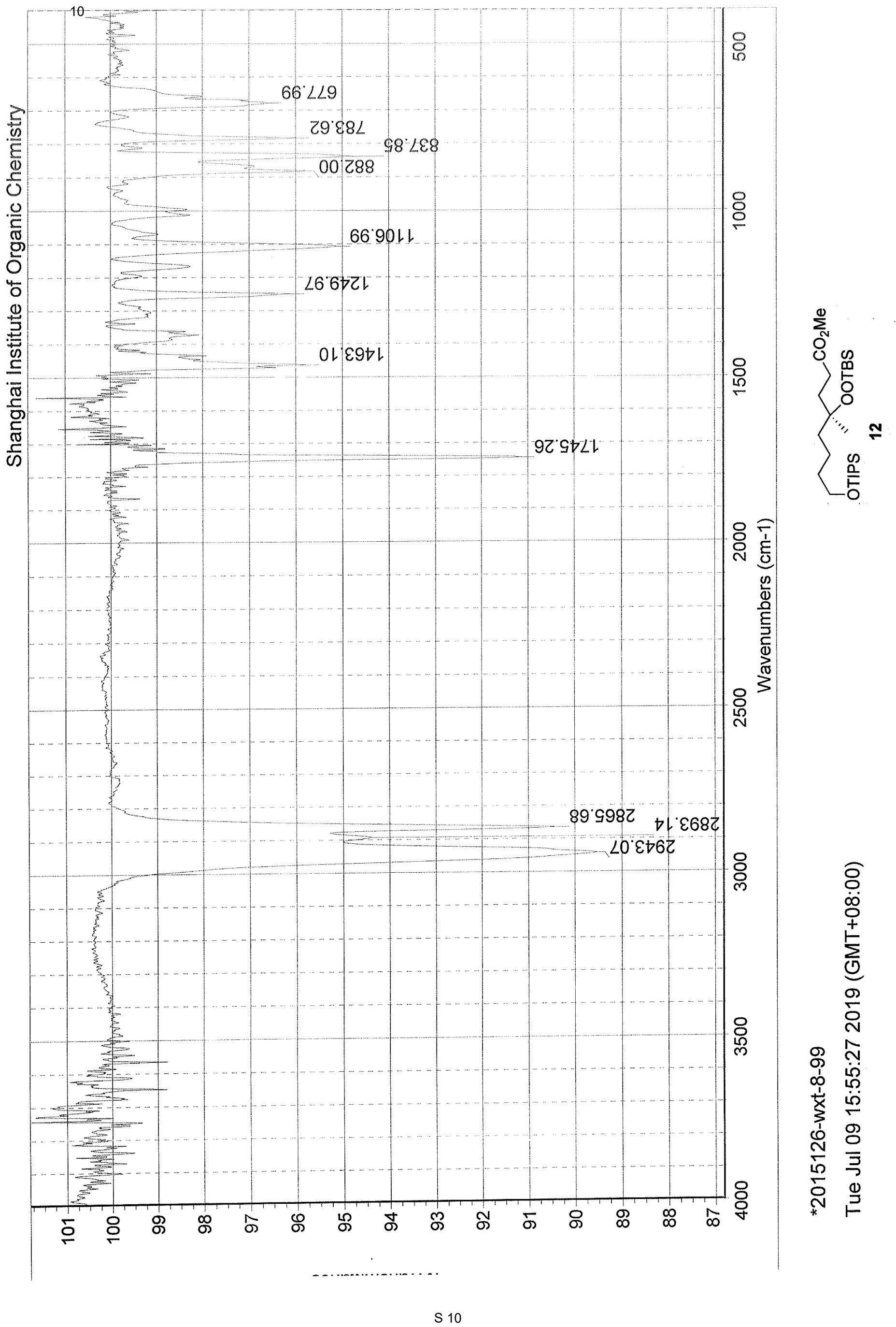




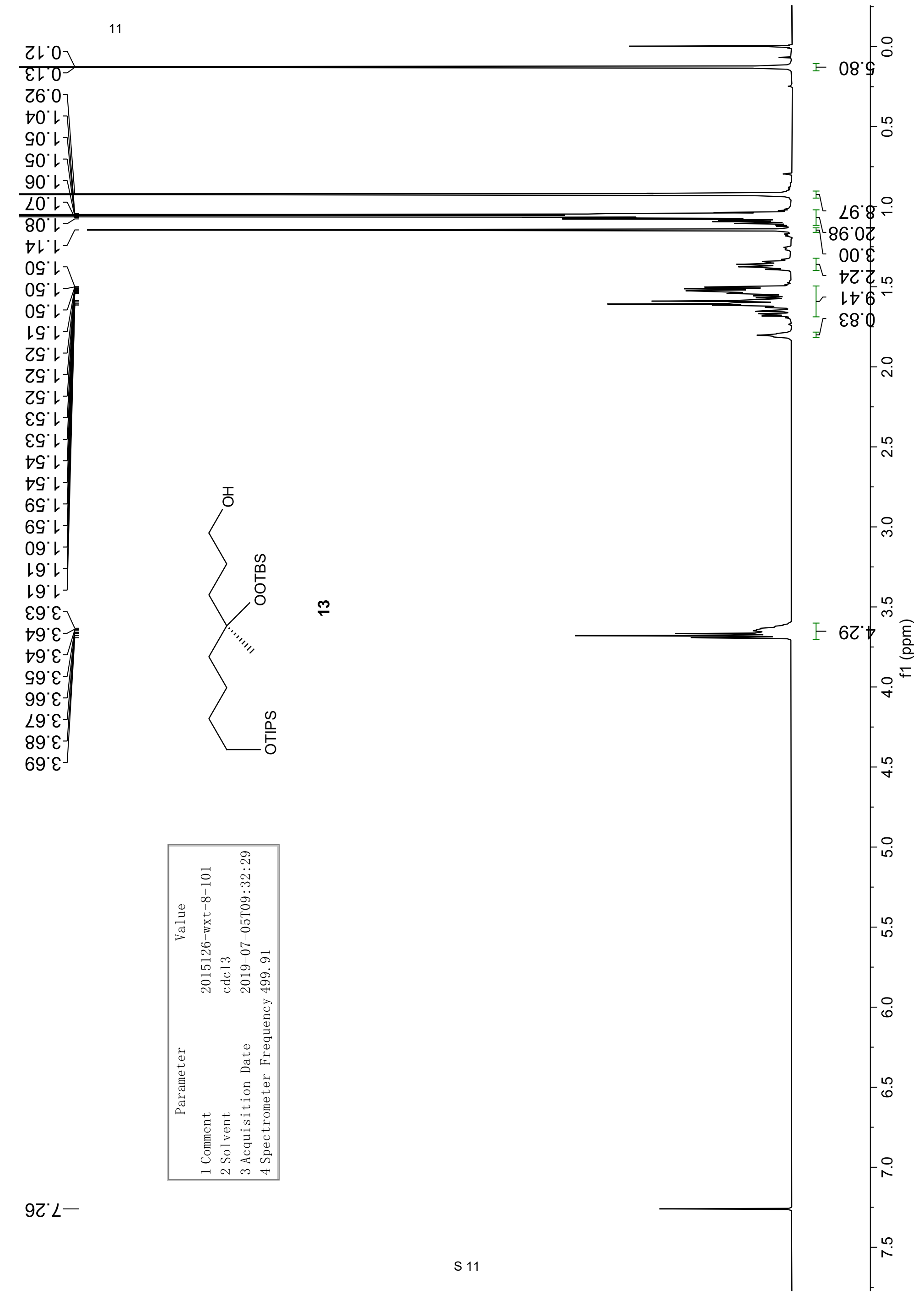


レ゚てレー

80.81
92.81

$9 \mathrm{~L}^{\circ} 0 \mathrm{Z}^{-}$

†0 $\mathrm{ZZ}^{5}$

レ゙9乙

$86^{\circ} 92^{-}$

$\angle Z 冗 \varepsilon-$

$\angle G^{\prime} \varepsilon \varepsilon^{-}$

$\varepsilon 9.9 \varepsilon-$

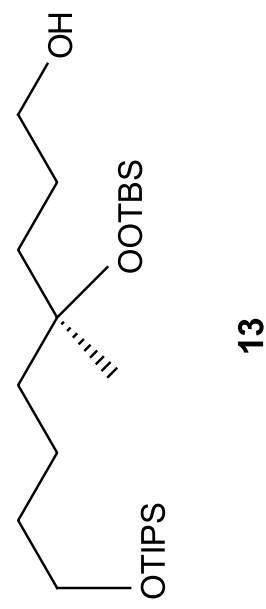

乙๐๕9

$\angle Z^{\circ} \varepsilon 9^{\top}$
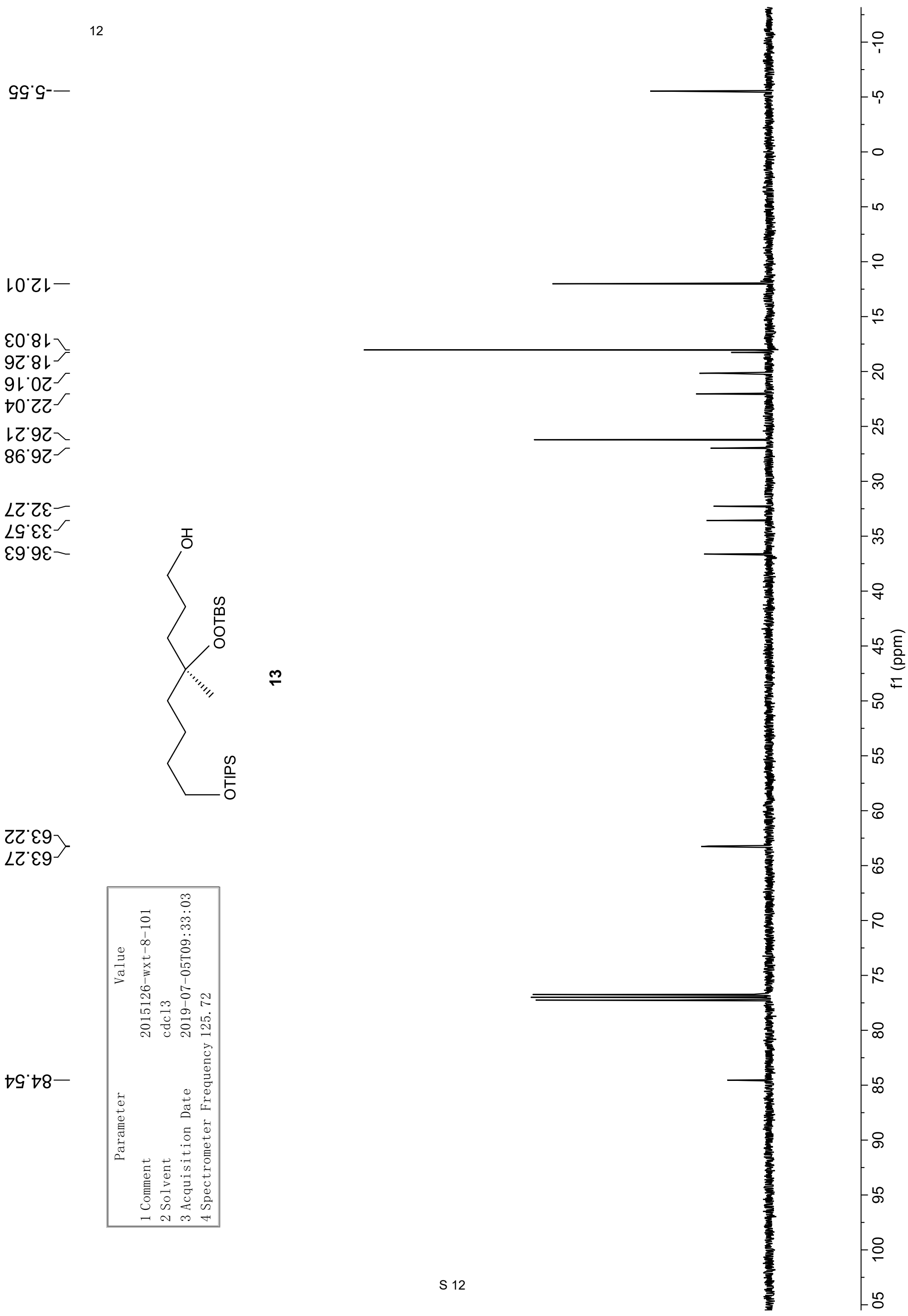

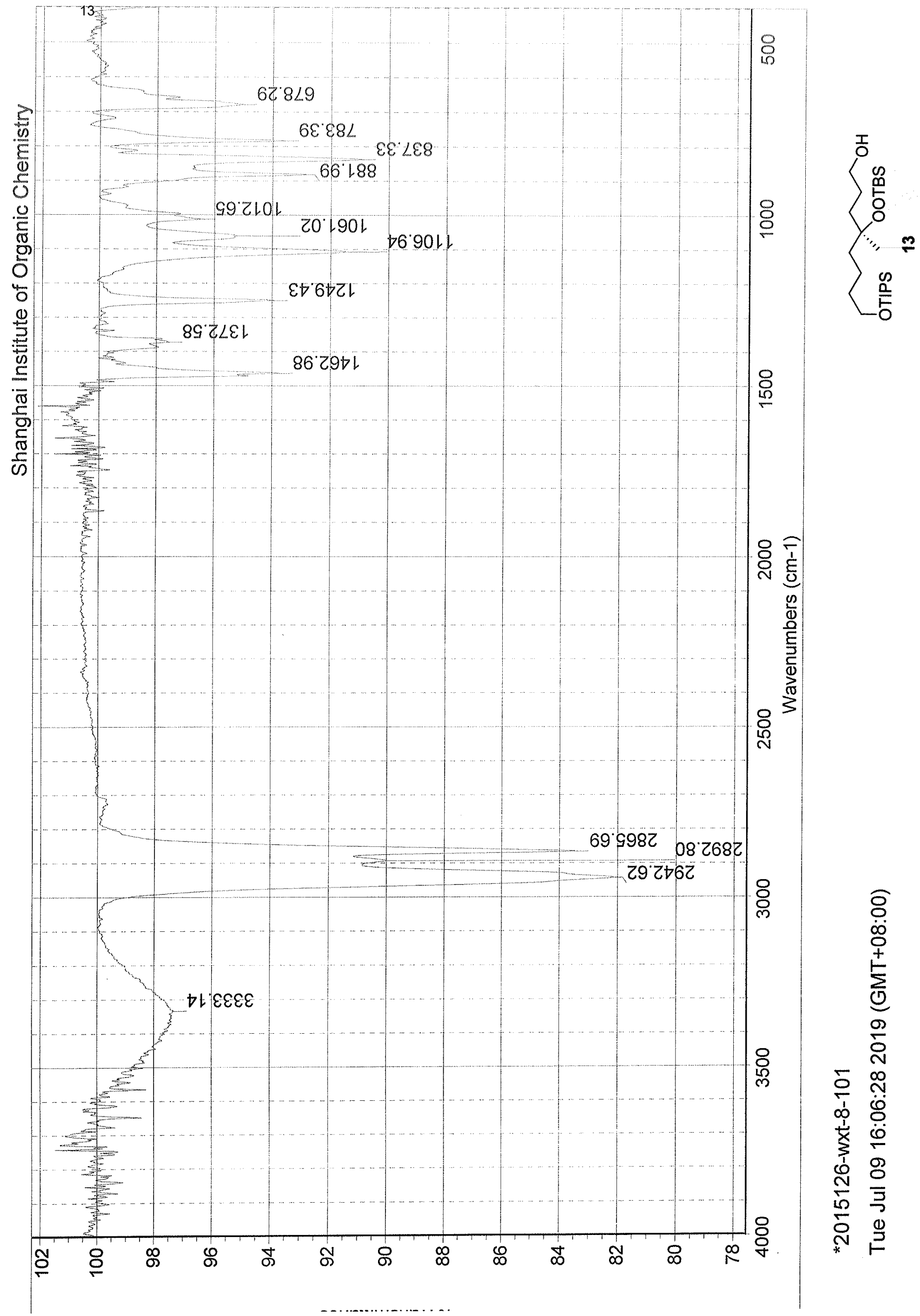


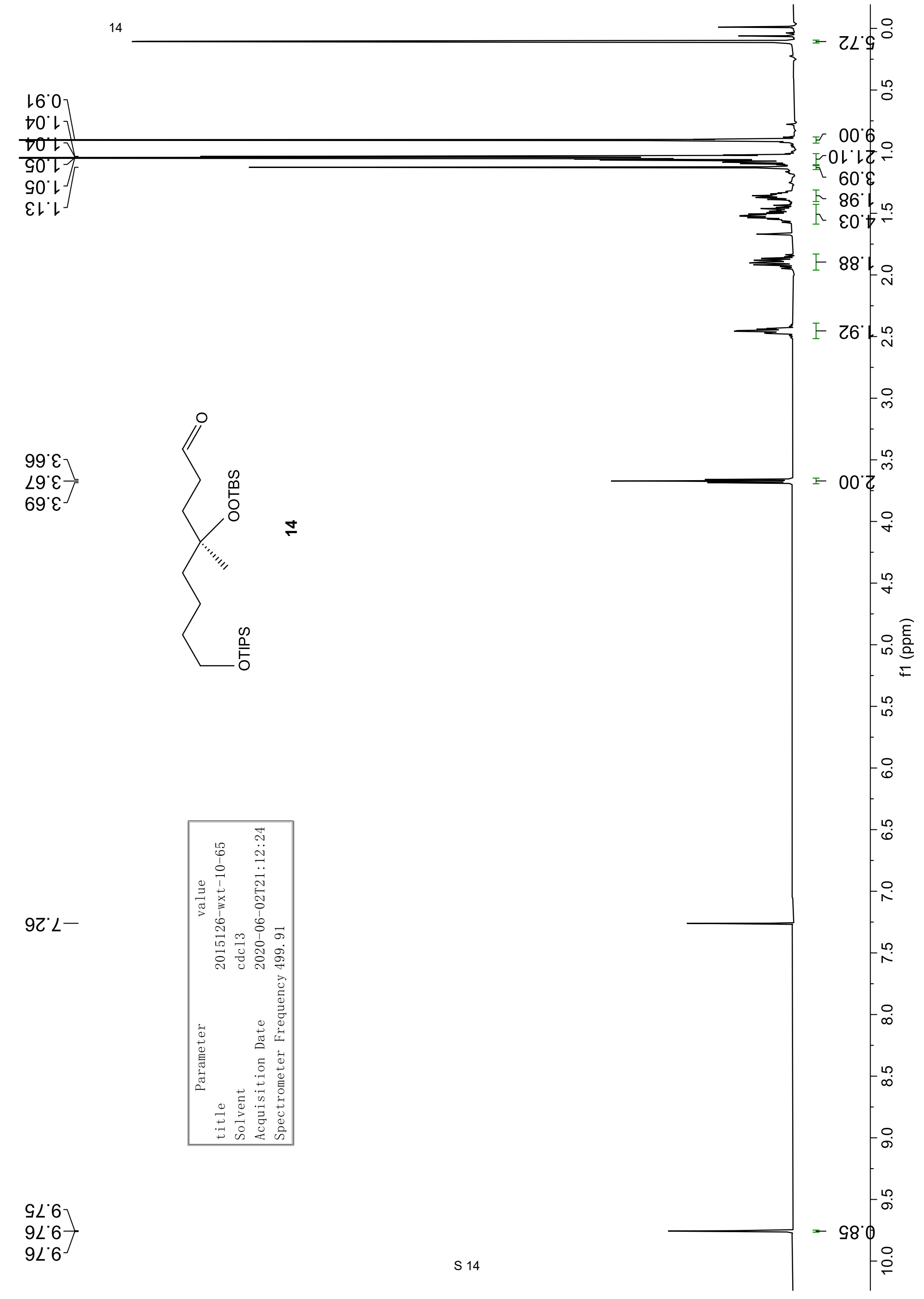



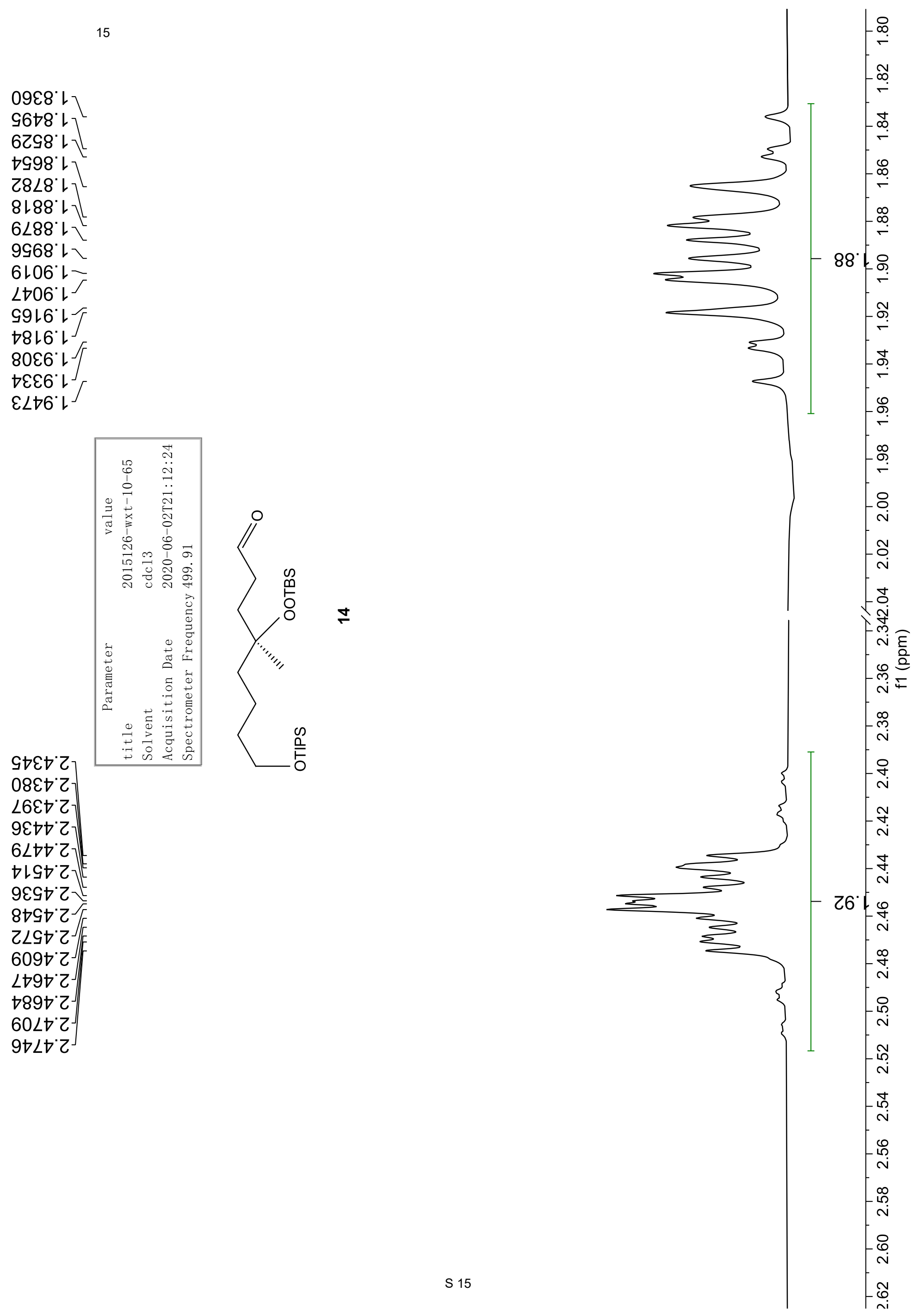
$\mathrm{G} 9^{\circ} \mathrm{G}^{-}$
$79^{\circ} \mathrm{G}^{-}$ $\mathrm{b}^{16}$

$\angle 6$ เレ

$108 \mathrm{~L}$

$02.8 \mathrm{~L}$

OL'OZ

69'l

$9 \mathbf{L}^{\circ} 9 \mathrm{Z}^{-}$

$88^{\circ} 8 \tau^{\top}$

$9 t^{\circ} \varepsilon \varepsilon^{\top}$

乙L' $9 \varepsilon$ 广

$0 \angle 8 \varepsilon$

9เะ9-

งซ๋ $\varepsilon 8-$

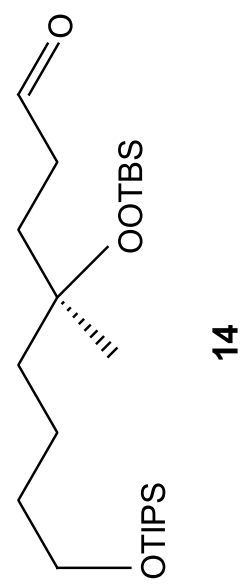

乙०

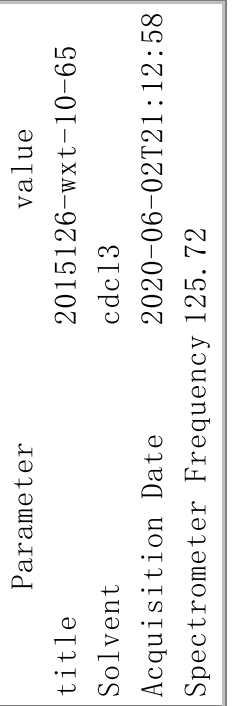




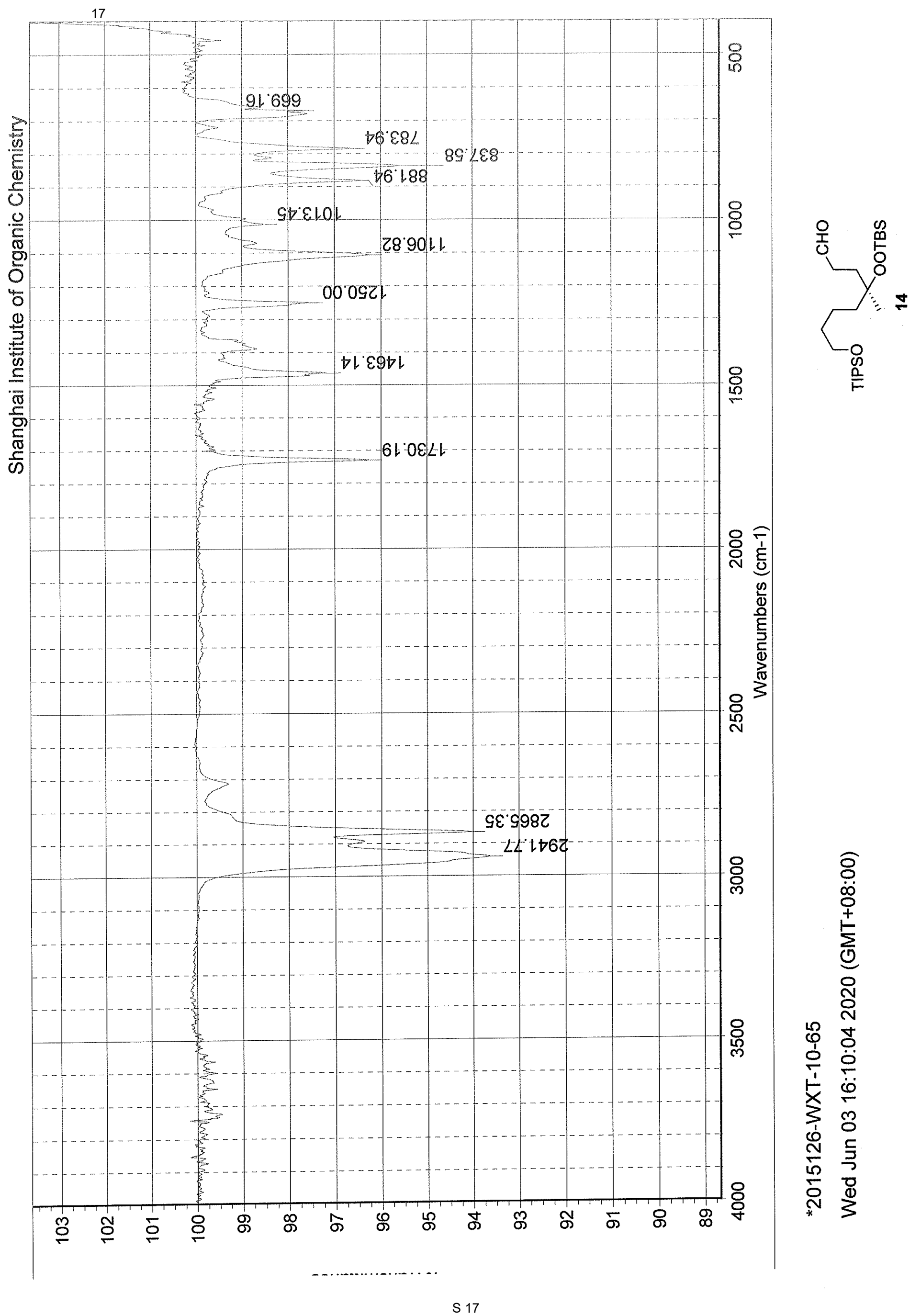


$-10.9^{\circ}$

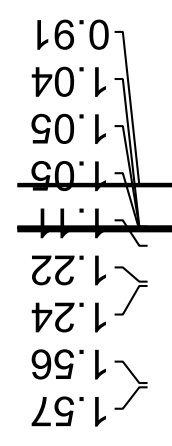

$98^{\circ}$ 乙
$28^{\circ}$
$68^{\circ} 乙$
$06^{\circ}$ 乙

$06^{\circ}{ }^{-}$
Z6

$99^{\circ} \varepsilon$

$\angle 9^{\circ} \varepsilon$

$89^{\circ} \varepsilon$

$8 L^{\circ} \varepsilon-$

$8 L^{\circ} \varepsilon$

$6 L^{\circ} \varepsilon$

$6 L^{\circ} \varepsilon$

$08^{\circ} \varepsilon$

เฉ $\varepsilon^{\circ}$

$18^{\circ} \varepsilon$

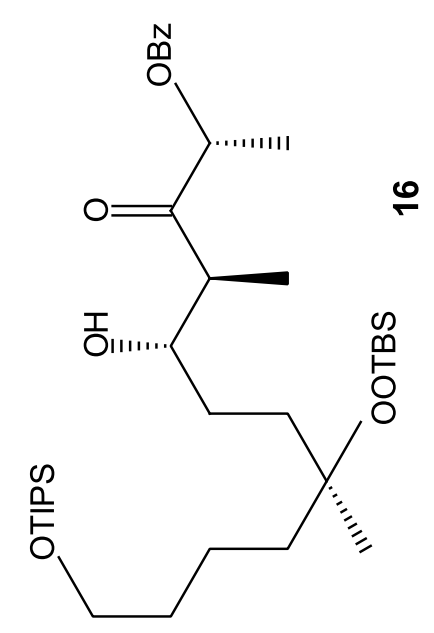

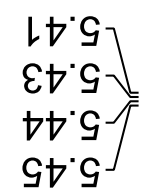

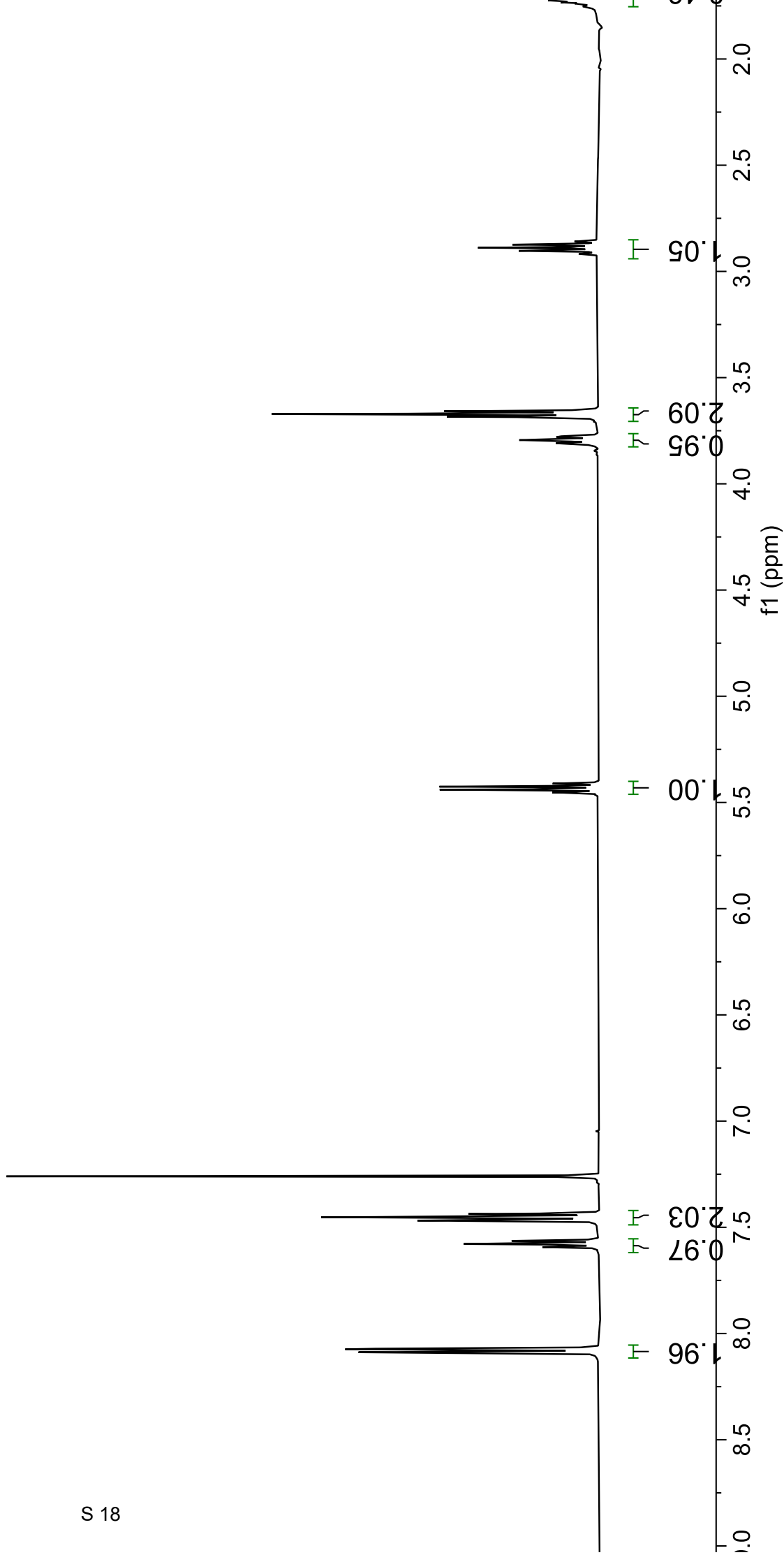




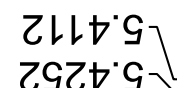

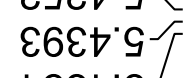
$\bullet \varepsilon S t^{\circ} \mathrm{s}$

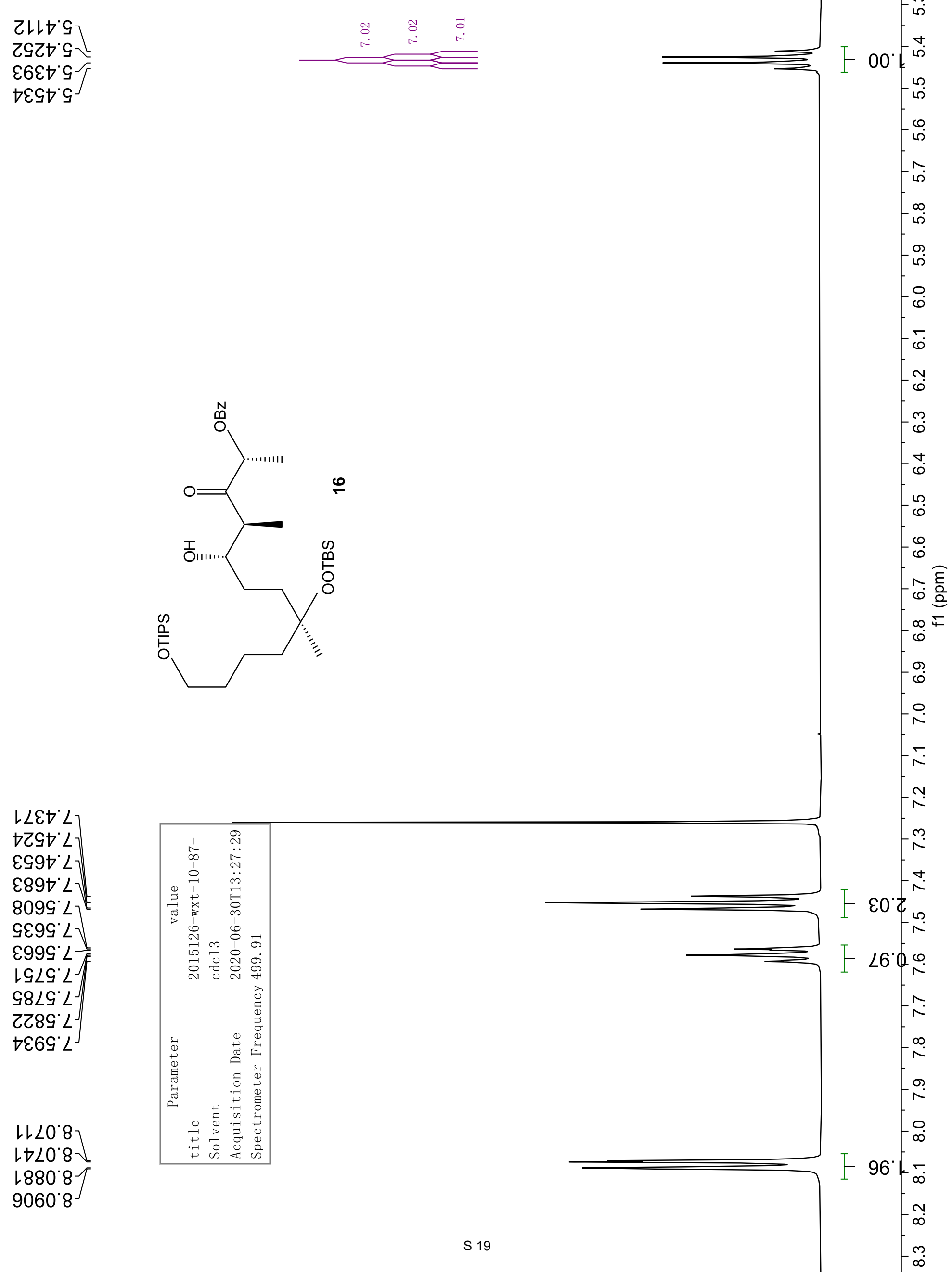


9698 $2 久$ 0† $\angle 8^{\circ} Z 2$ 9888 $2-$ ยع06 $乙^{\circ}$ $9 \angle 16 \mathrm{C}^{\top}$

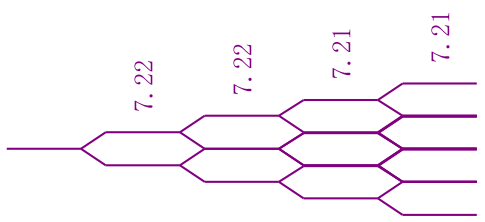

$\mid$
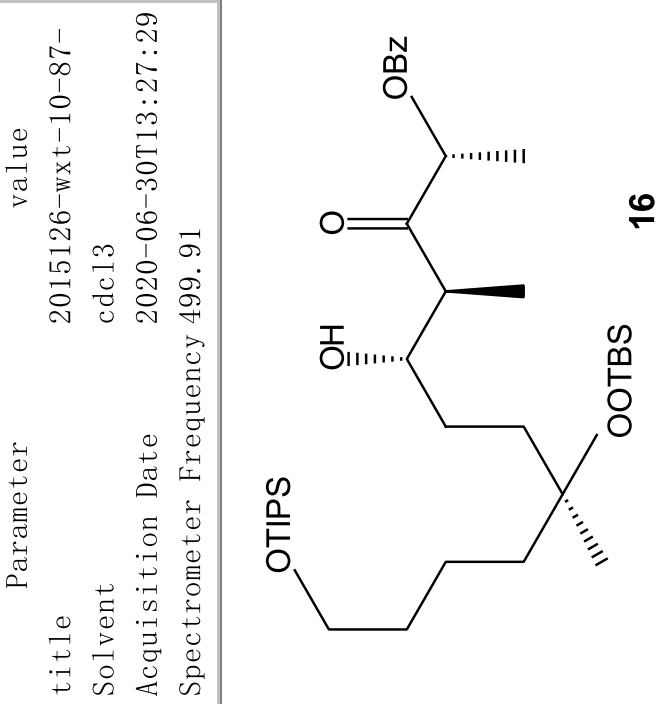

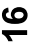

$\varepsilon 899^{\circ} \varepsilon-$

Zᄂ $\angle 9^{\circ} \varepsilon-$ 乙†89. $\varepsilon^{-}$

$\nabla G \angle L \cdot \varepsilon$ $66 \angle L^{\circ} \varepsilon$ 006 L' $\varepsilon$ $0 \triangleright 6 L^{\circ} \varepsilon-$ $\varepsilon 86 L^{\prime} \varepsilon$ $6808^{\circ} \varepsilon$
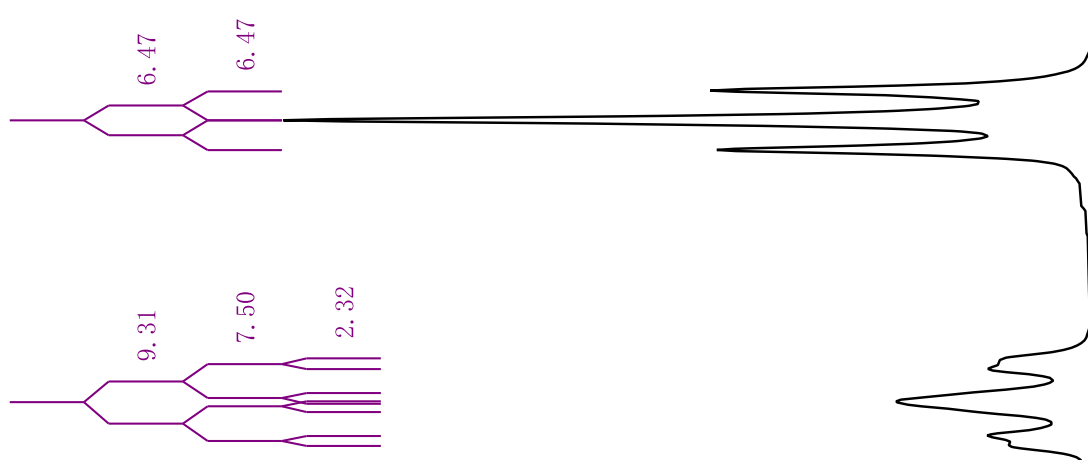
$\angle \varepsilon+8^{\circ} \varepsilon$

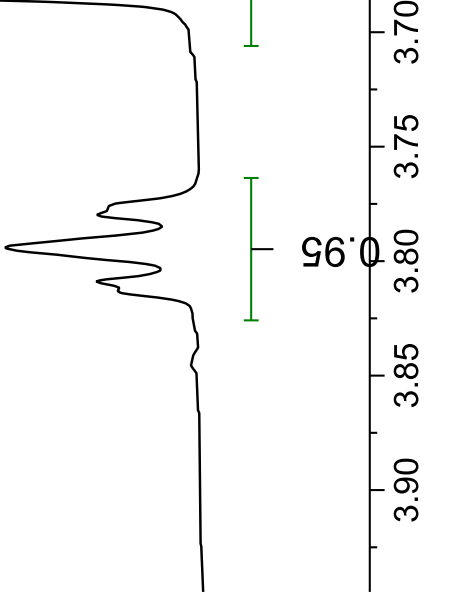



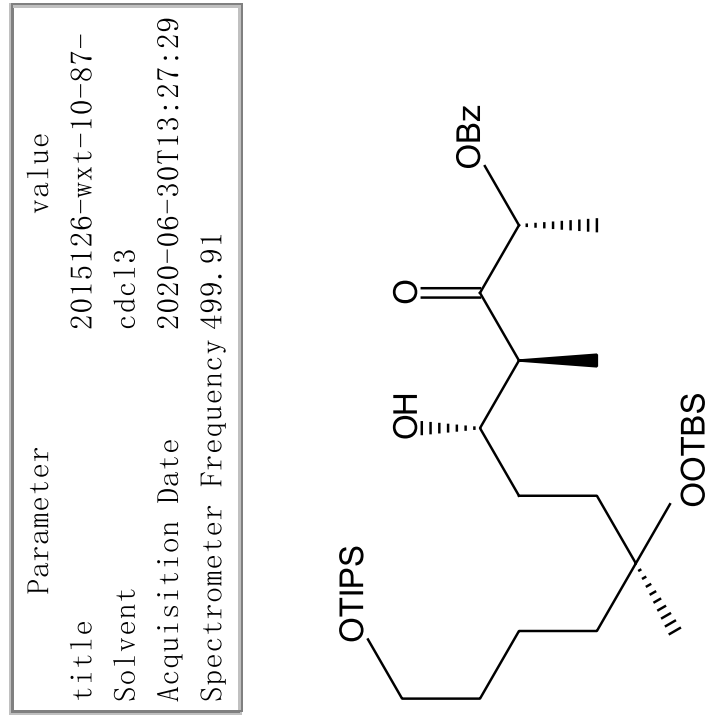

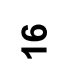

ELO6'O

\section{$\angle \varepsilon \neg 0^{\circ}$}

StSOL

990 'L

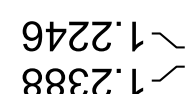

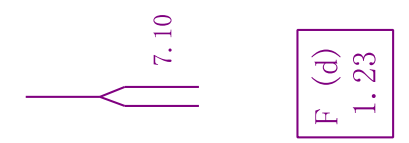

0899' ᄂ

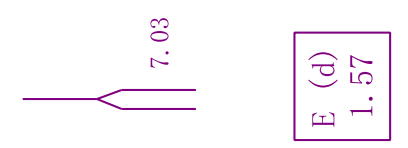

OZLG'

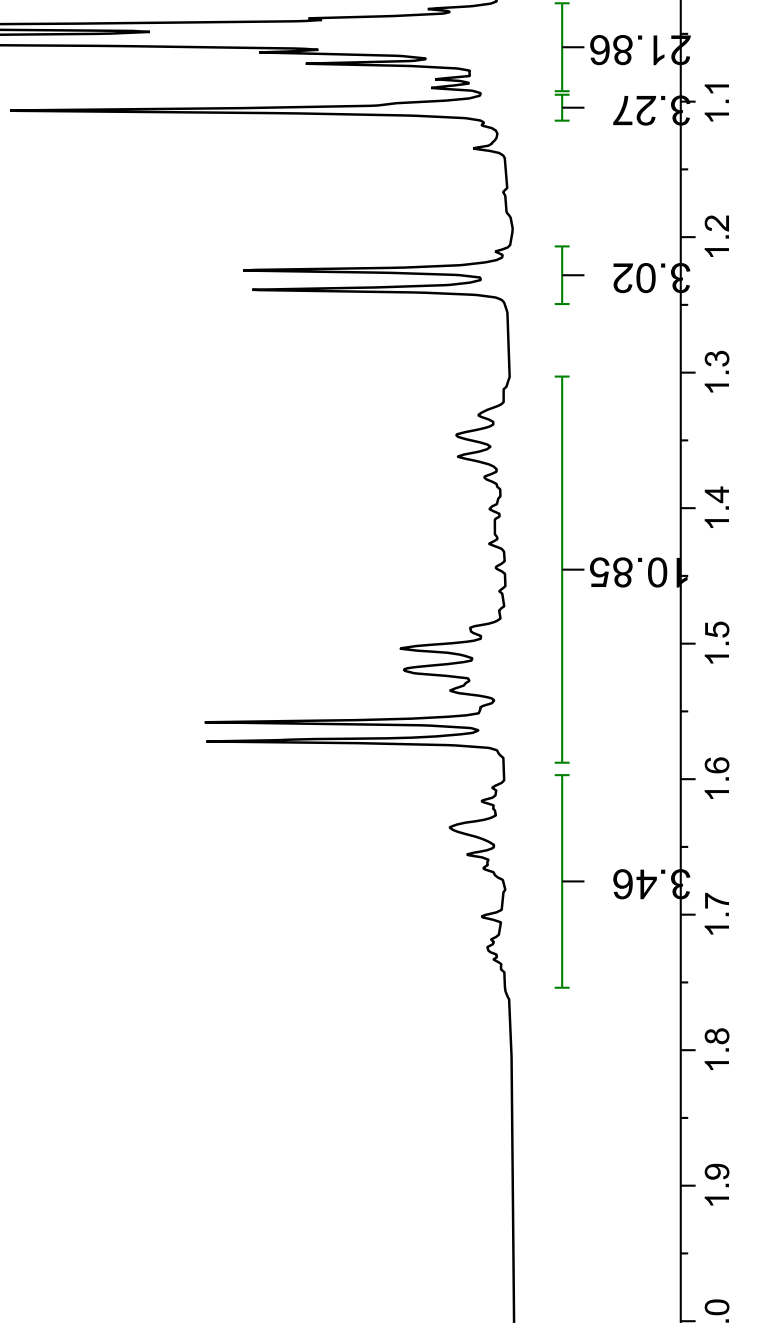



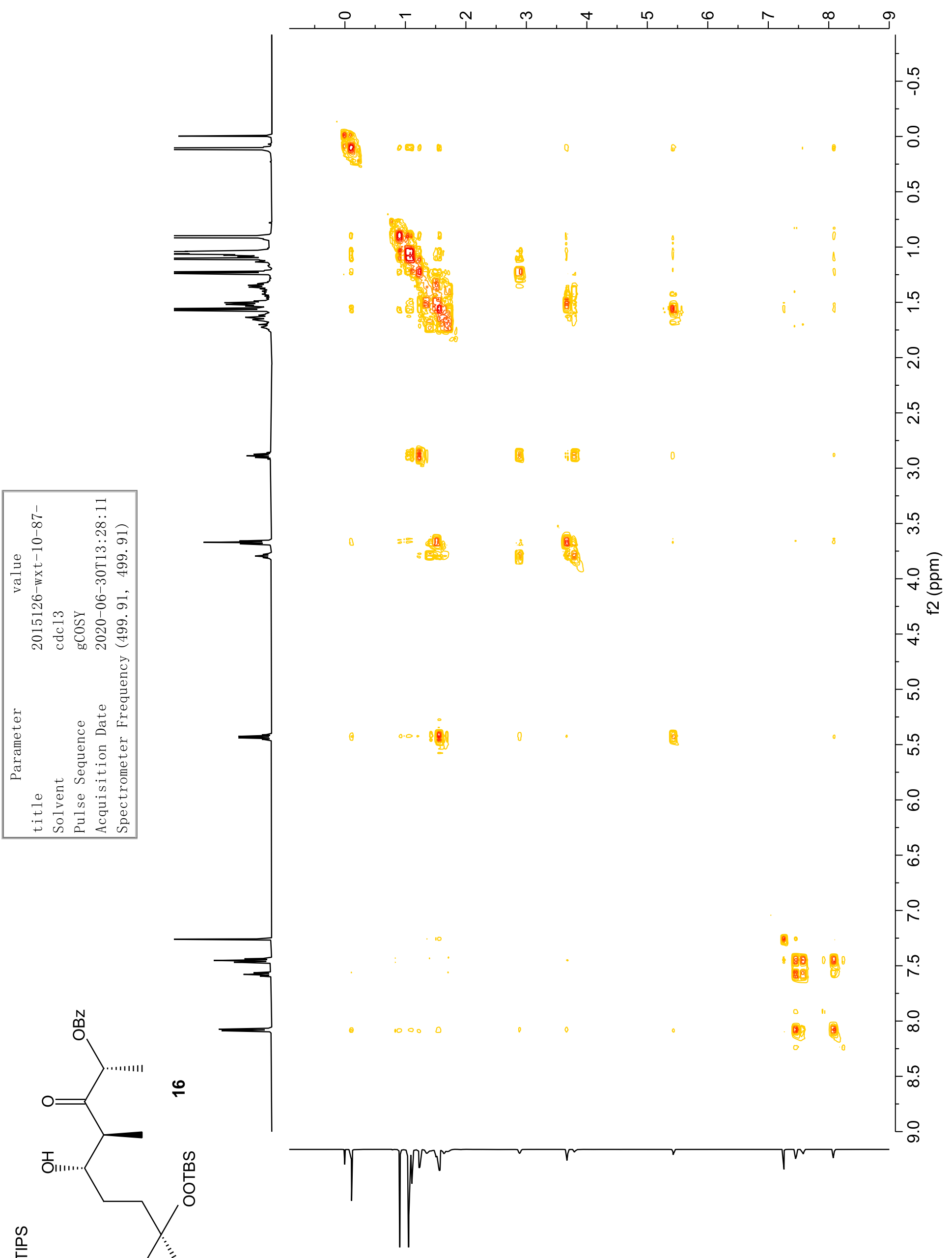

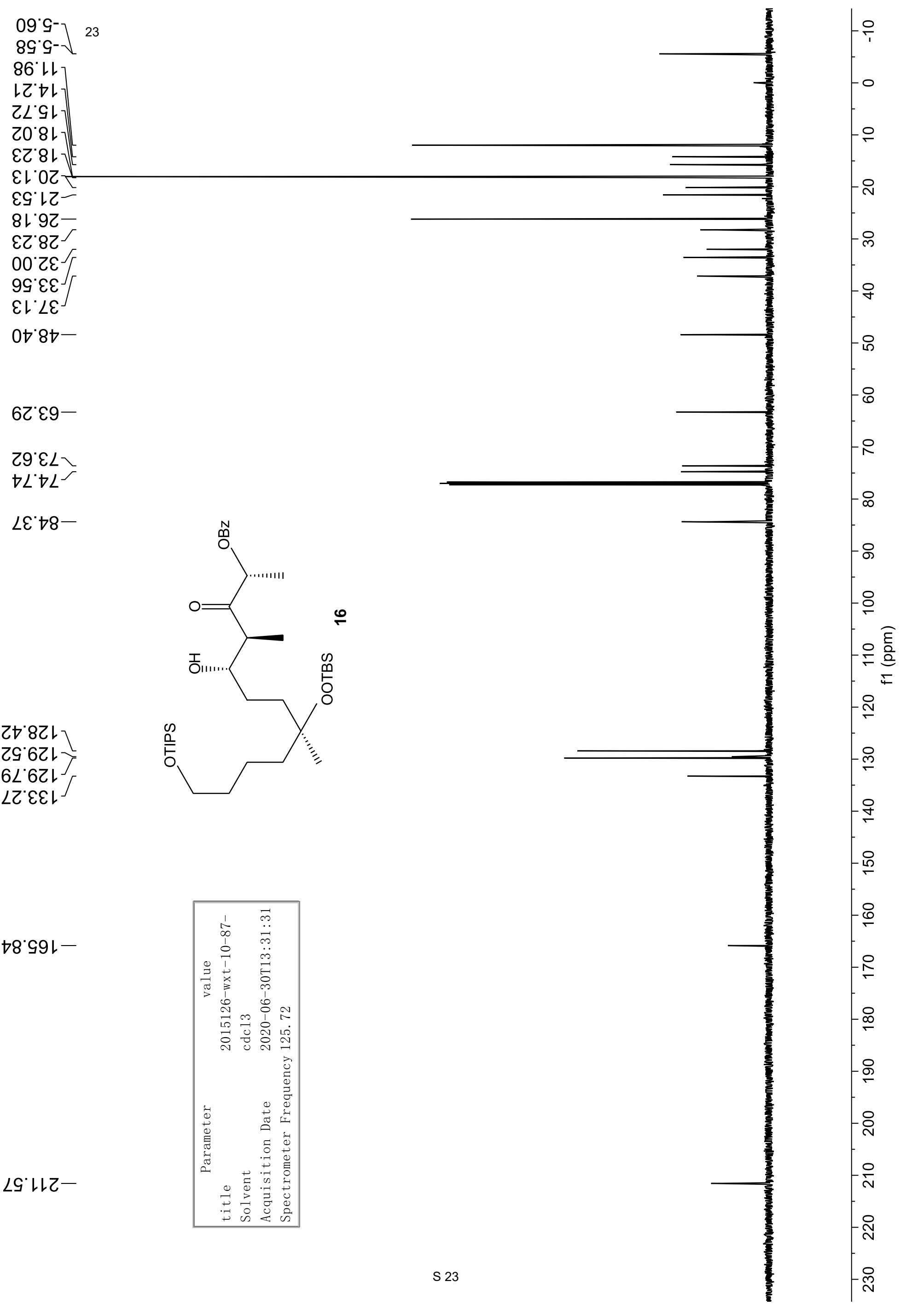

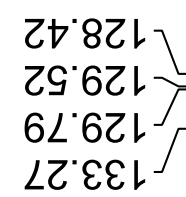

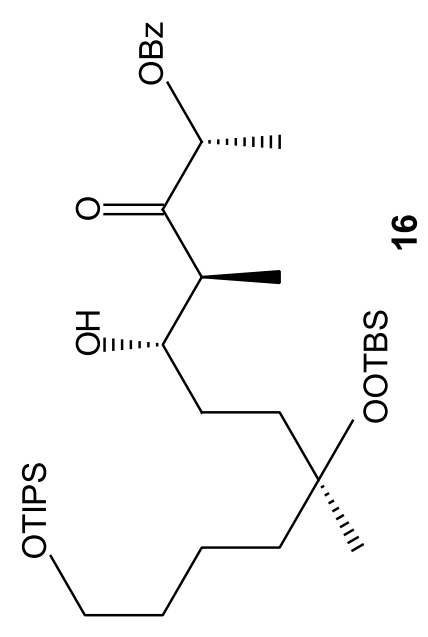

เ8.991-

LGレスー

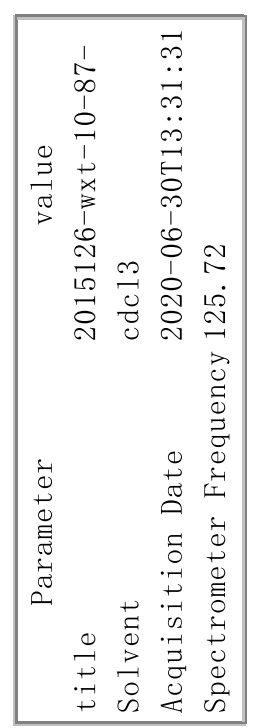




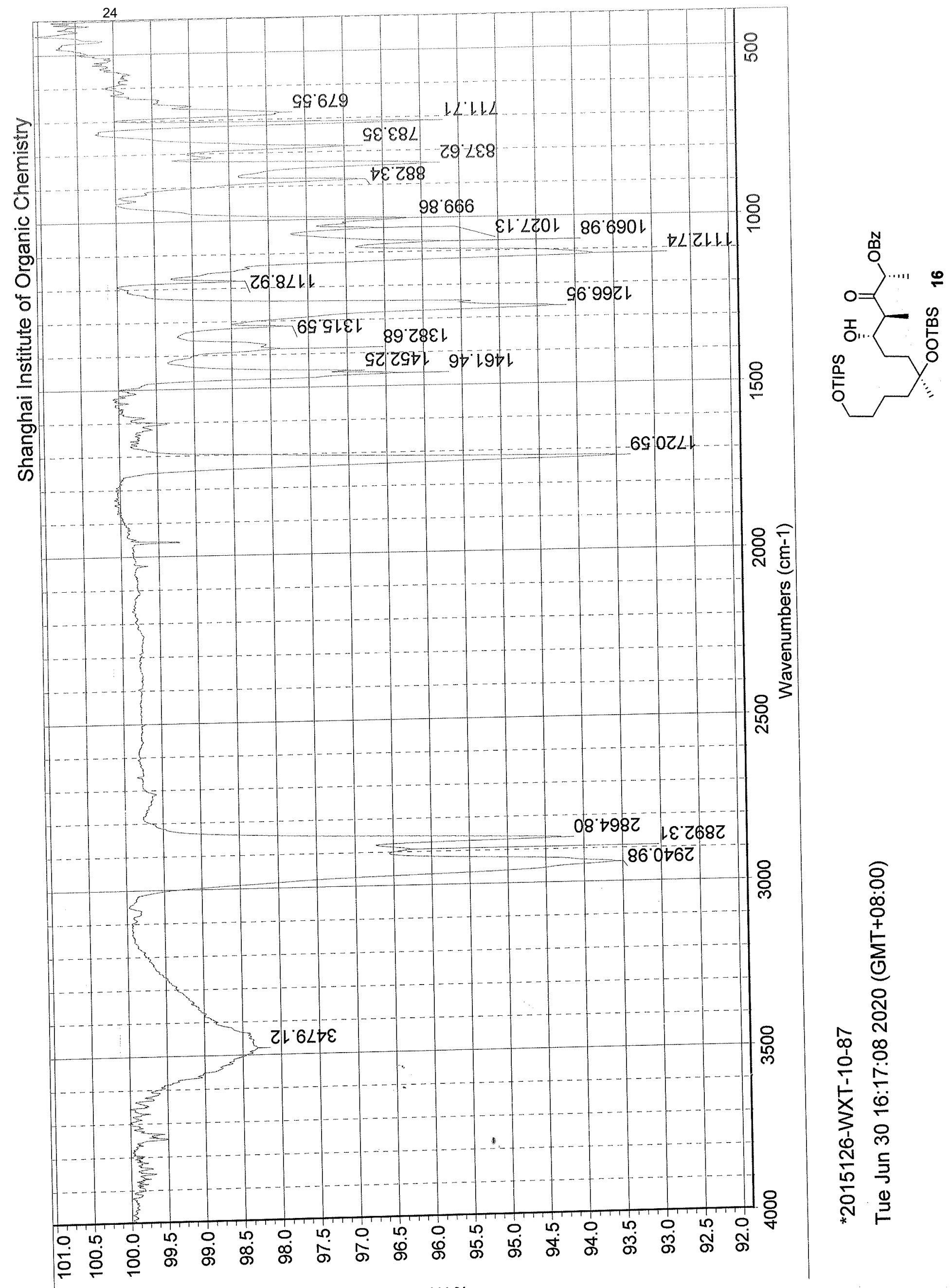




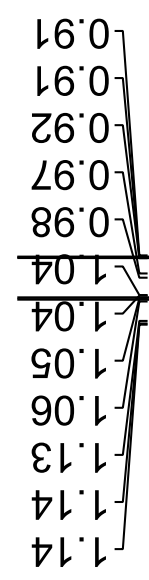

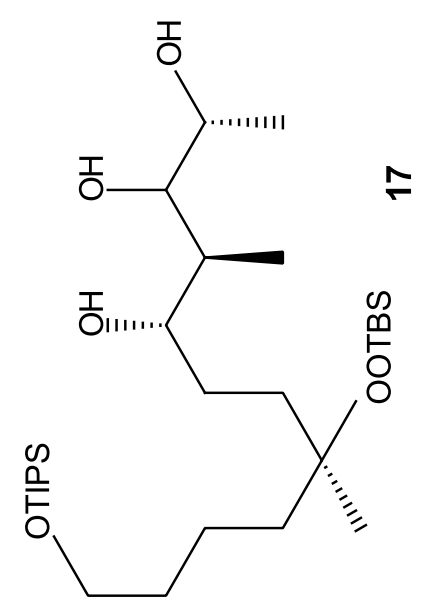

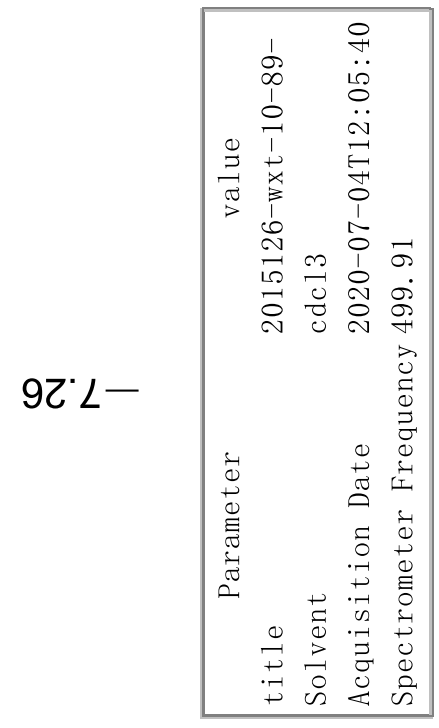

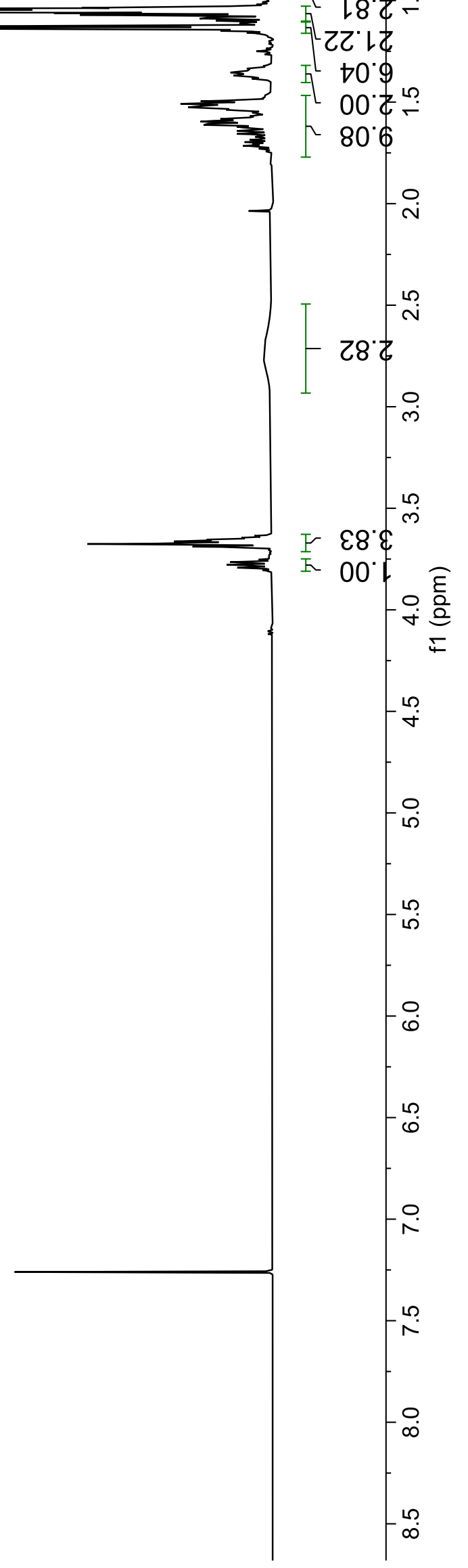



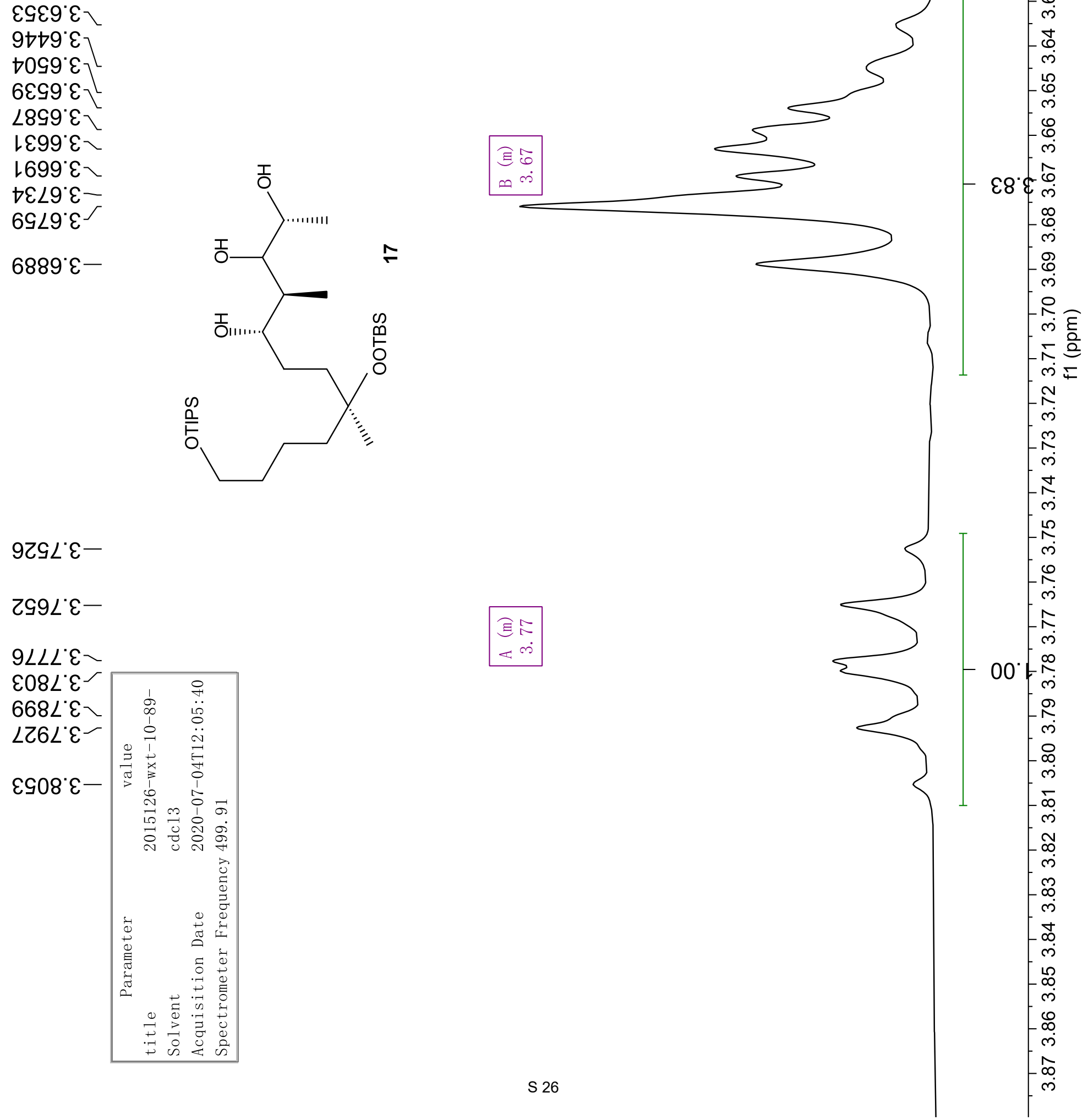
29ルレ L6ルレ $10.8 \mathrm{~L}$ 七乙.81 98.8L

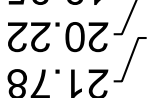
$9 \mathrm{l}^{\circ} 9 \mathrm{Z}^{\Gamma}$ $\angle \varepsilon^{\prime} 6 乙^{-}$ レナてと乙 เG'EE$16.9 \varepsilon$ $\left.\varepsilon 6^{\circ} 8 \varepsilon\right]$

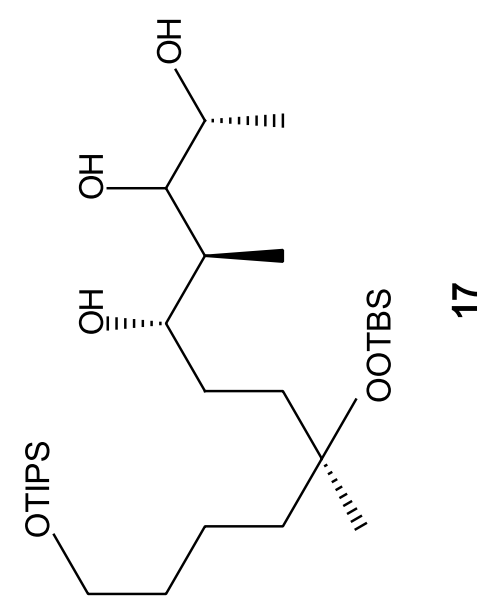

LL'89-

Oオ'9L
LS'9L

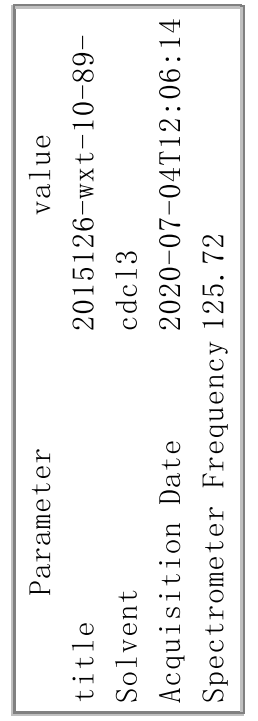

$9\llcorner " \nabla 8-$ 


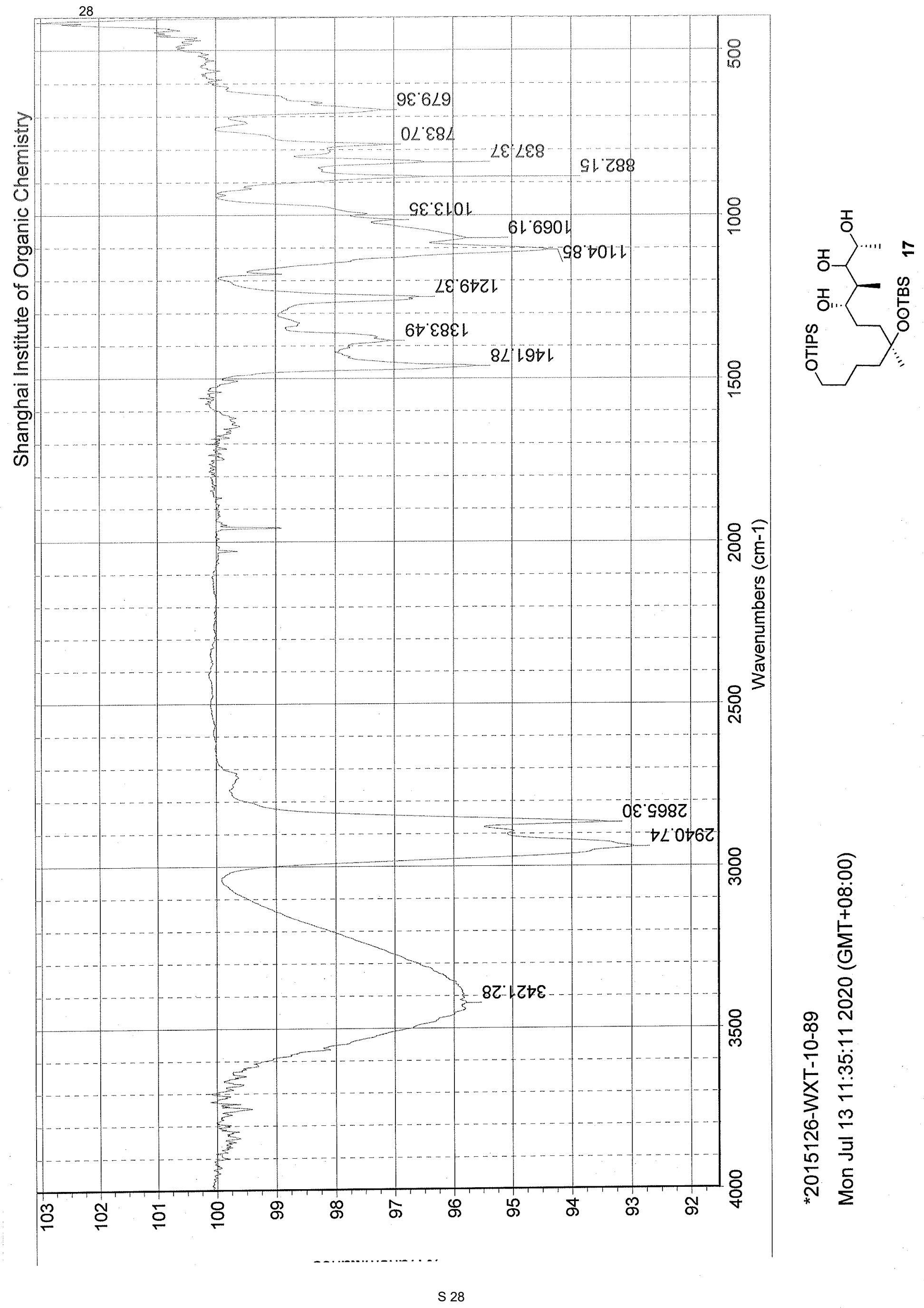



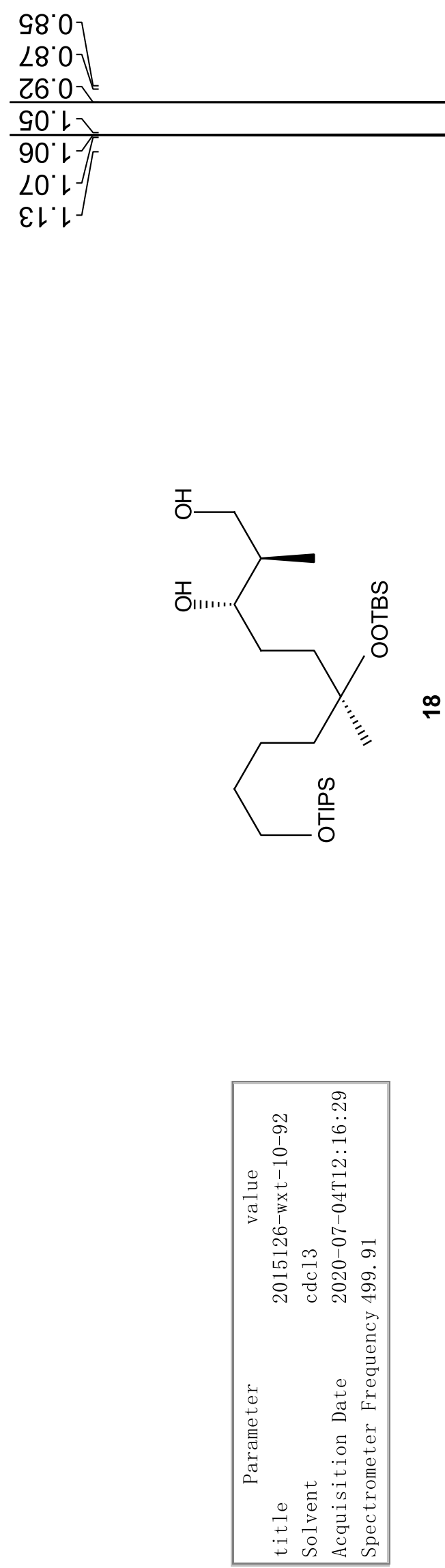


$$
\begin{aligned}
& 00 \angle G^{\circ} \varepsilon- \\
& 9 \varepsilon \angle G^{\circ} \varepsilon^{\digamma} \\
& { }^{\circ} 89^{\circ} \varepsilon \backslash \\
& \text { 乙Z69 }{ }^{\circ} \varepsilon- \\
& \varepsilon \varepsilon 09^{\circ} \varepsilon \backslash \\
& { }_{8909}{ }^{\circ} \varepsilon- \\
& 0 Z 79^{\circ} \varepsilon- \\
& \text { ५8ح9' } \varepsilon- \\
& \text { 8\&†9 } \varepsilon- \\
& 1999^{\circ} \varepsilon- \\
& 68 \angle 9^{\circ} \varepsilon \backslash \\
& \neg 289^{\circ} \varepsilon- \\
& \downarrow 689^{\circ} \varepsilon- \\
& 6\left\llcorner 69^{\circ} \varepsilon^{\top}\right. \\
& \text { ᄂ } 0 L^{\circ} \varepsilon- \\
& \text { ルレLとー }
\end{aligned}
$$
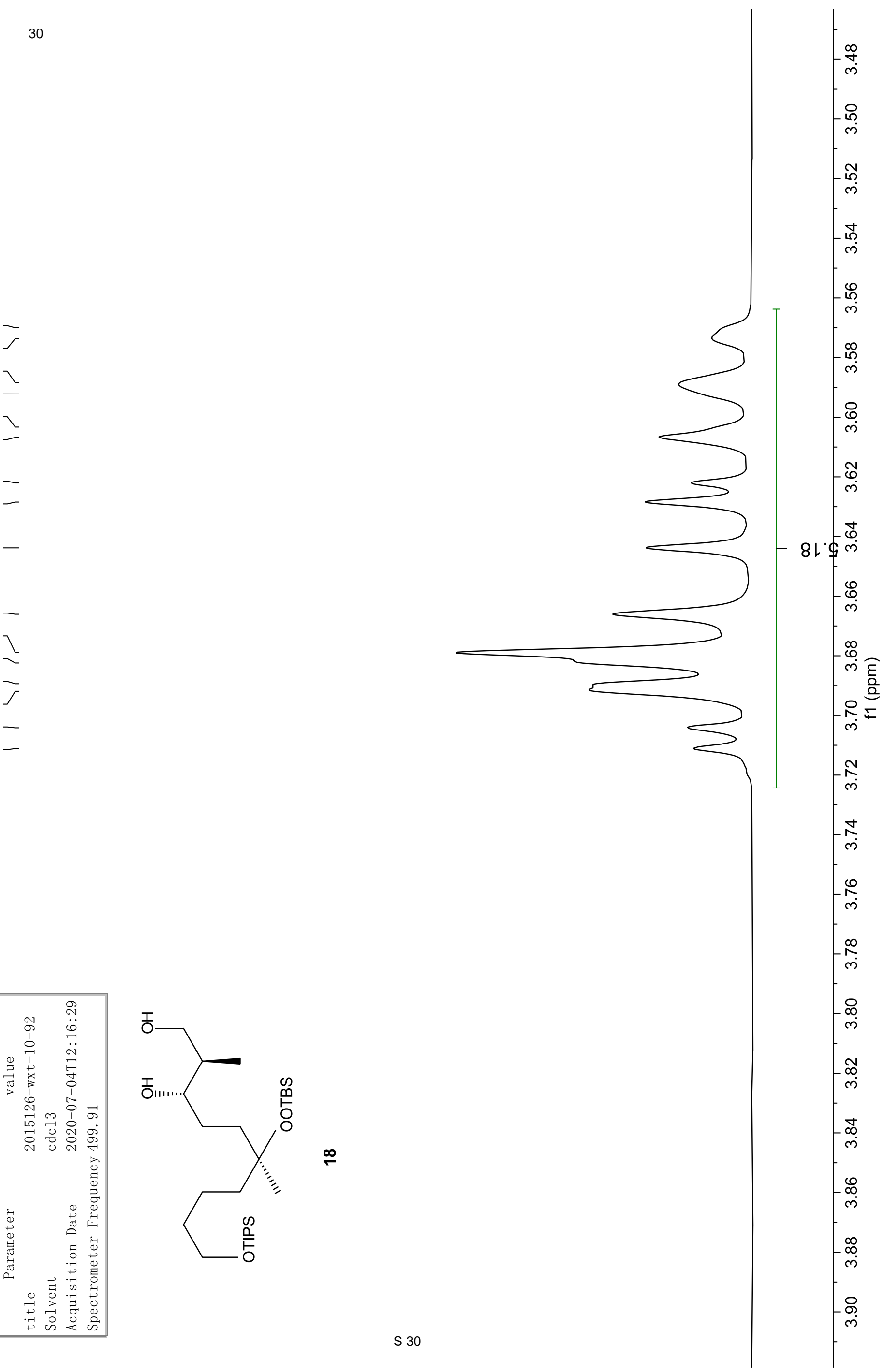
$\angle G^{\prime} \mathcal{G}^{-}$
$9 \mathcal{G}^{\prime} \mathrm{G}^{-}$

86ルー

ต6"ยน-

$20.8 \mathrm{~L}$

$7 Z^{\circ} 8 \mathrm{~L}$

$8 \mathrm{Z}^{\circ} 0 \mathrm{Z}^{-1}$

69"レて
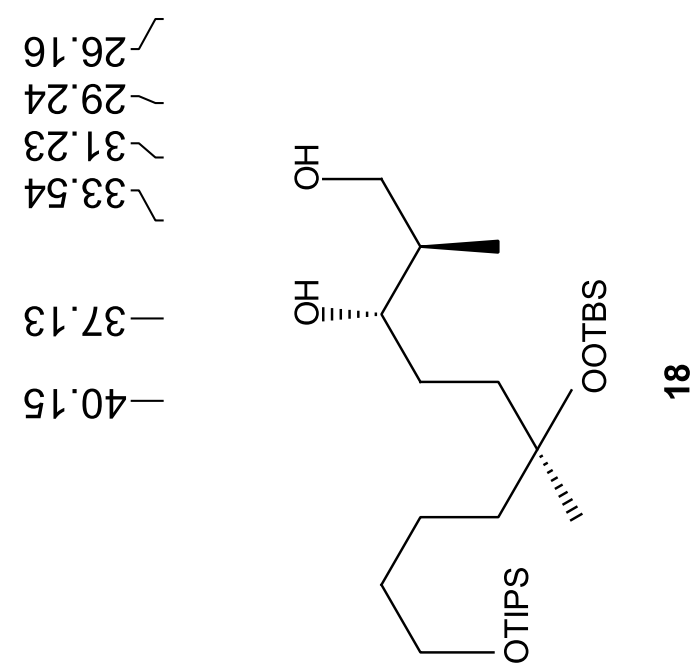

Lてะ9-

0ะ'89-

$\angle 6^{\circ} \angle L-$

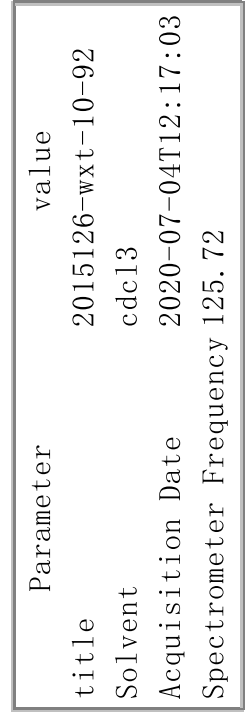

$\stackrel{\infty}{\circ}$

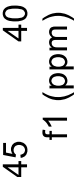

요

一뉴

8

눙

웅

$\stackrel{2}{\sim}$

$\infty$

$\stackrel{\infty}{\infty}$

乙6" ฤ8一 


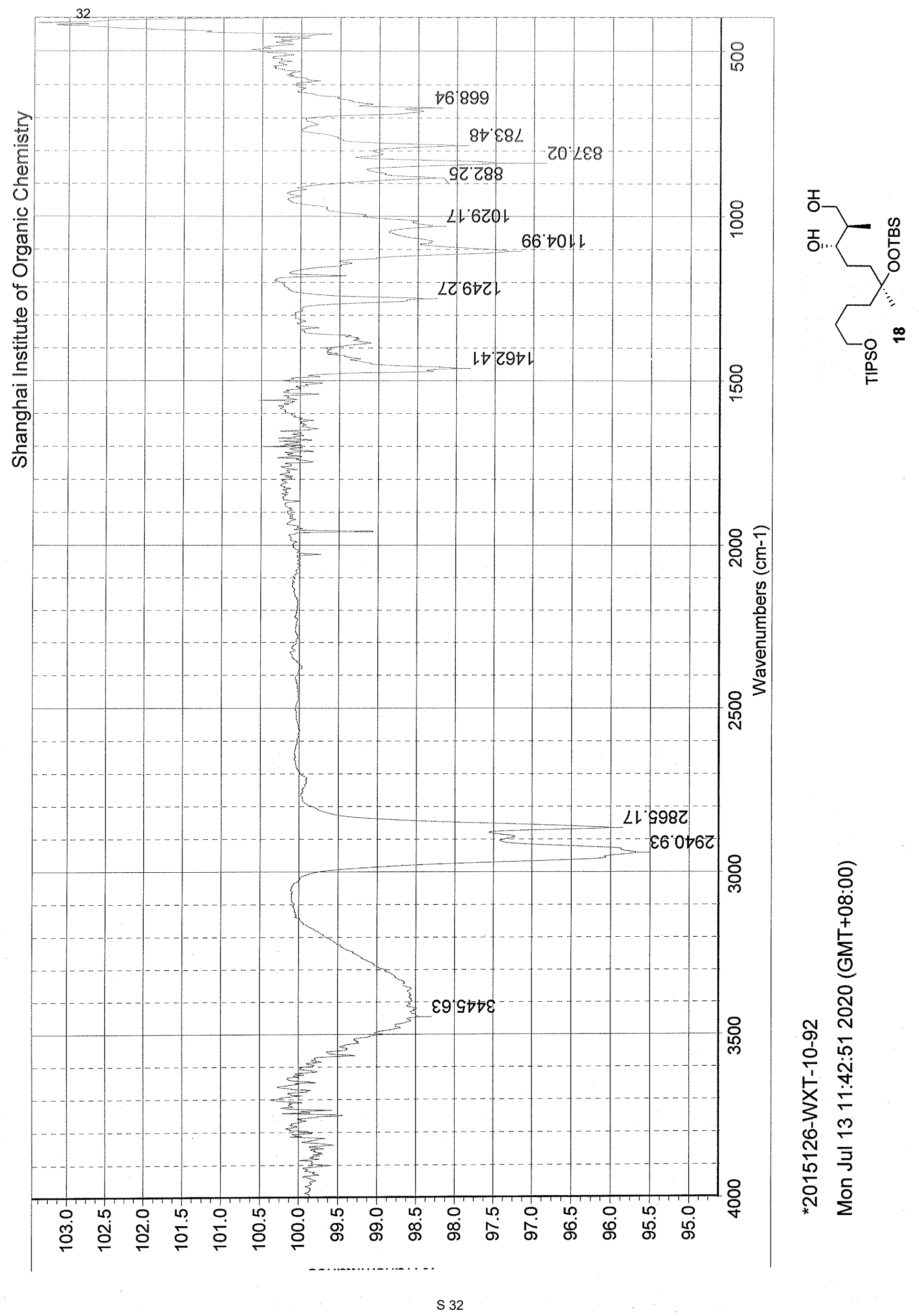


$\left.\begin{array}{l}0 L^{\circ} 0 \\ L\end{array}\right\}$

$16^{\circ} 0$

$\angle 6^{\circ} 0$

$86^{\circ} 0$

G0.

प्रण1.

$80 \%$

EL"

L8'乙-
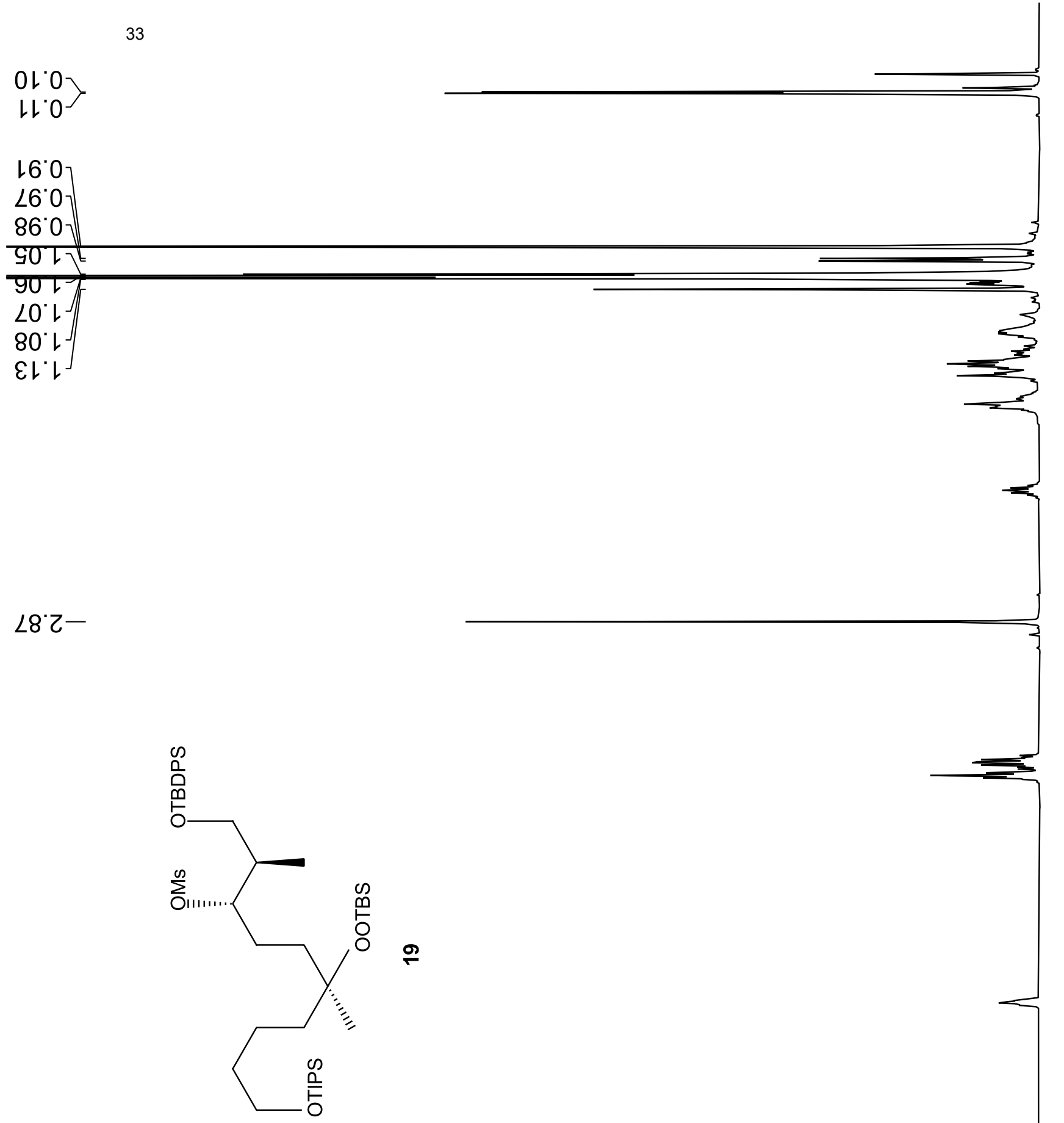

F 00

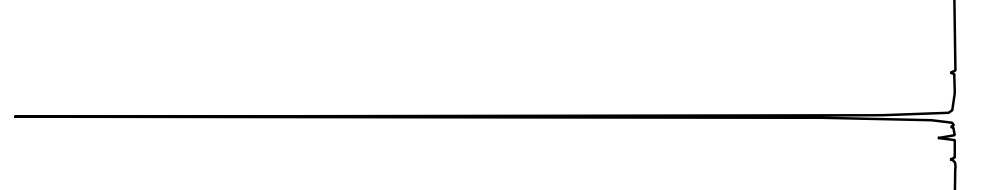

工 60

\section{$\angle \varepsilon$}

$\angle E^{\circ} L$

$8 \varepsilon^{\circ} L$

$6 \varepsilon^{\circ} L$

$6 \varepsilon^{\circ} L$

$0 \nabla^{\circ} L$

Ot $\angle$

$\sqcup \nabla^{\circ} L$

Z

$\varepsilon \nabla^{*} L$

$\varepsilon 9^{\circ} L$

$\nabla 9^{\circ}<\frac{1}{1}$

S9. $\angle$

$99^{\circ} \mathrm{L}$

$99^{\circ} L$

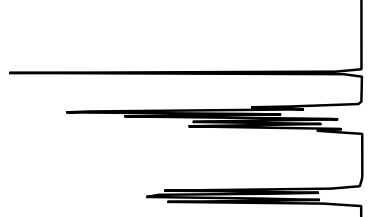



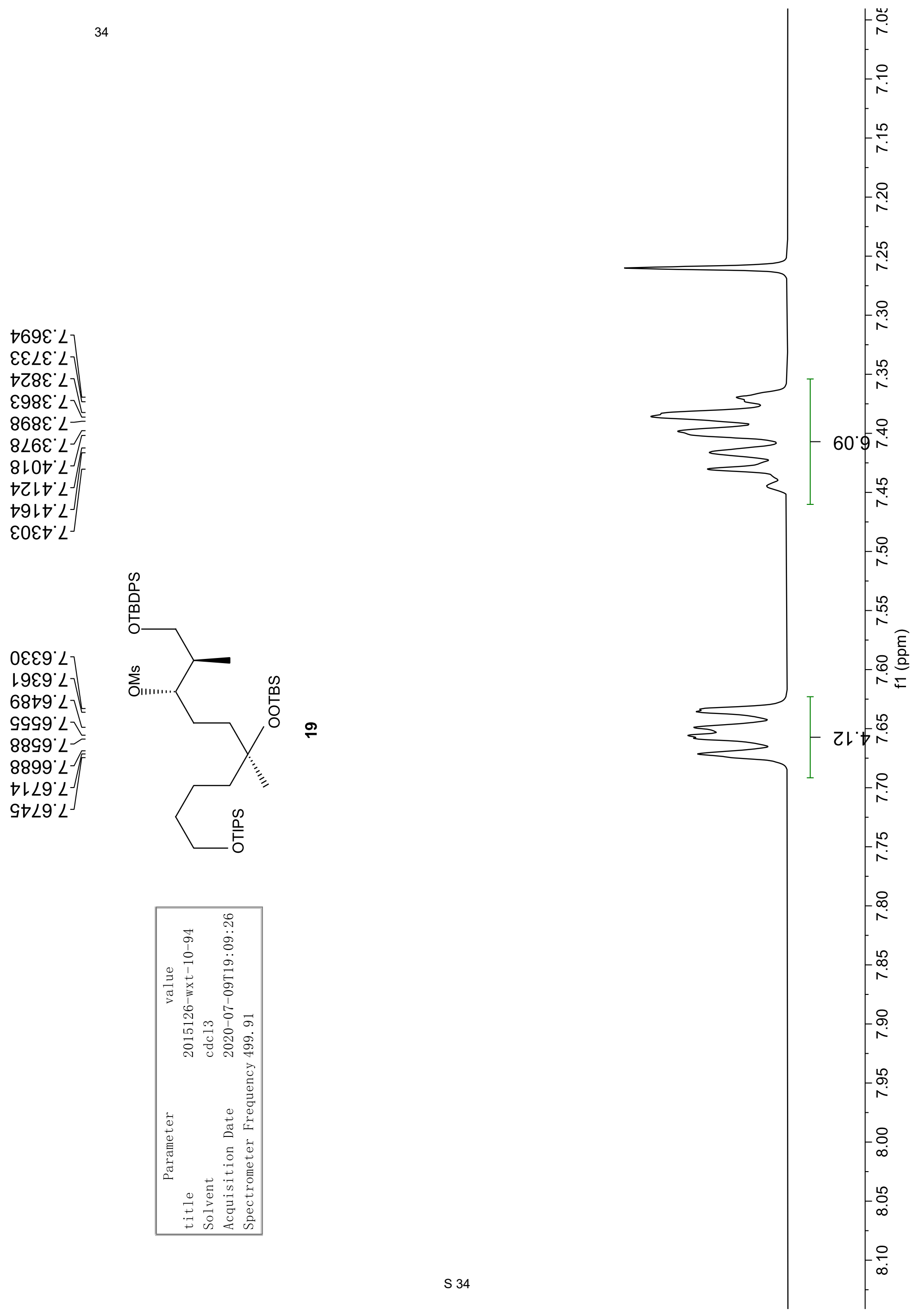
$\angle \angle 9 G^{\circ} \varepsilon \backslash$ G6 $\angle G^{\prime} \varepsilon \backslash$ $2889^{\circ} \varepsilon \backslash$ 9009 ${ }^{\circ}$ SS09 $\mathcal{~} \mathcal{-}$ $\angle \angle I 9^{\circ} \varepsilon \backslash$ $\mathrm{S}^{\circ}{ }^{\circ} \varepsilon>$ ह8ะ9 $\varepsilon \backslash$ $\angle \angle G 9^{\circ} \varepsilon$ $\left.\varepsilon_{099} \varepsilon\right]$ $80 \angle 9^{\circ} \varepsilon$ $\nabla \varepsilon \angle 9^{\circ} \varepsilon-$ $6 \varepsilon 89^{\circ} \varepsilon$ $\downarrow 989^{\circ} \varepsilon^{-}$
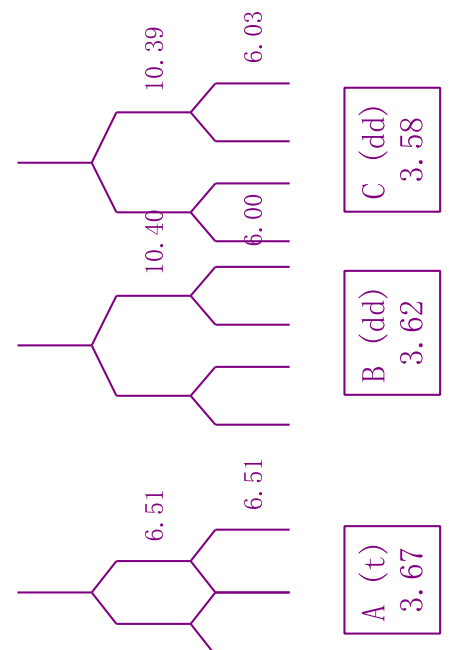
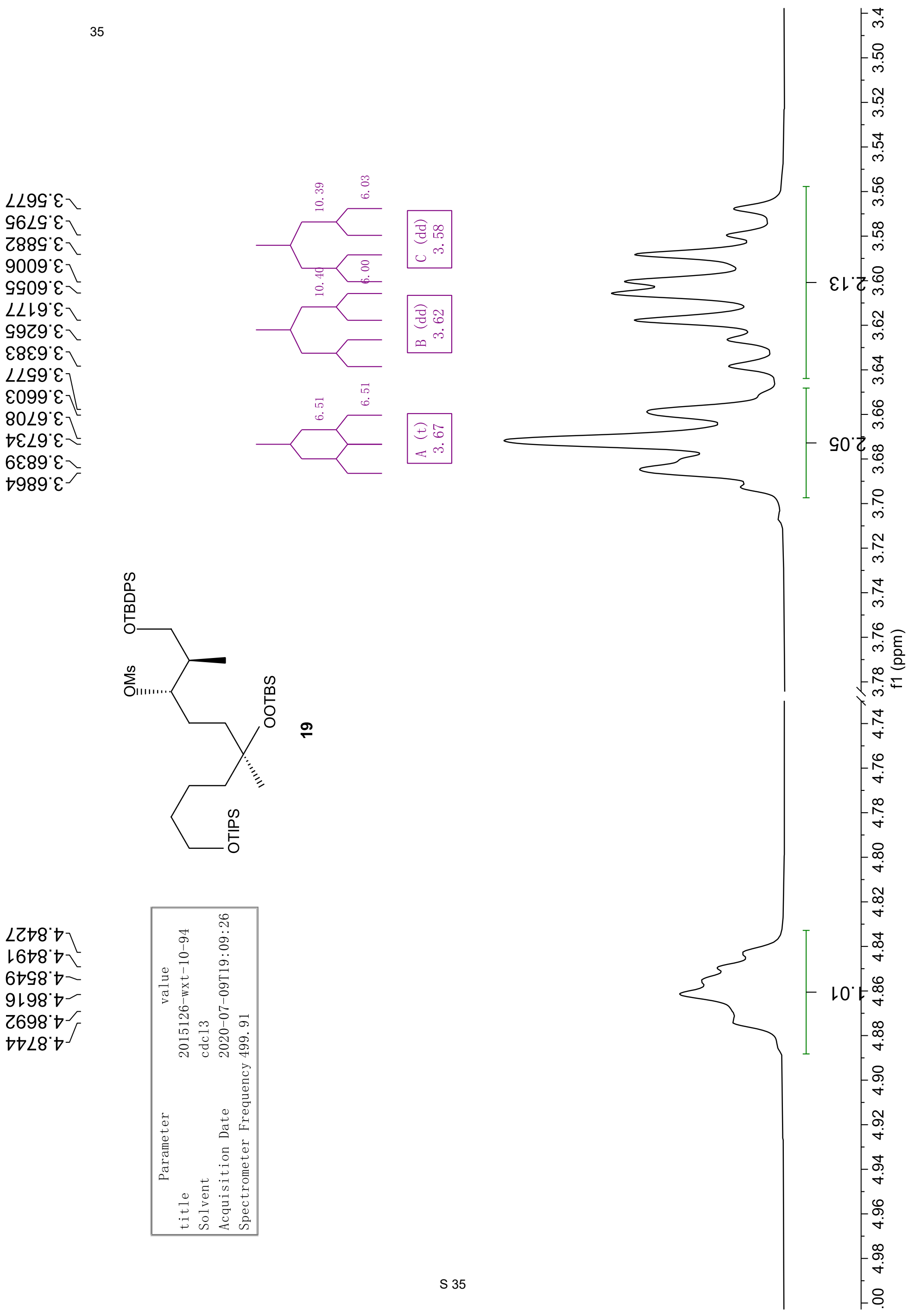
$\left.\begin{array}{l}09 \cdot 9^{-} \\ 89^{-9}\end{array}\right\}$

66・レ

E乙 $2 L$

$\varepsilon 0{ }^{\circ} 8 \mathrm{~L}$

हट'8ᄂ

乙2.6L

$70^{\circ} 0 \mathrm{Z}^{\circ}$

$98^{\circ} \iota^{\top / F}$

$89^{\circ} \nabla \tau^{\prime}$

乙乙'9乙

98.9乙

Z6'

$\angle \mathcal{G}^{\circ} \varepsilon \varepsilon^{\circ}$

$99^{\circ} 9 \varepsilon$

$0 \nabla^{\circ} 8 \varepsilon$

$\varepsilon \tau^{\prime} 6 \varepsilon^{\circ}$

$\varepsilon \varepsilon^{\prime} \varepsilon 9-$

乙レ'99-

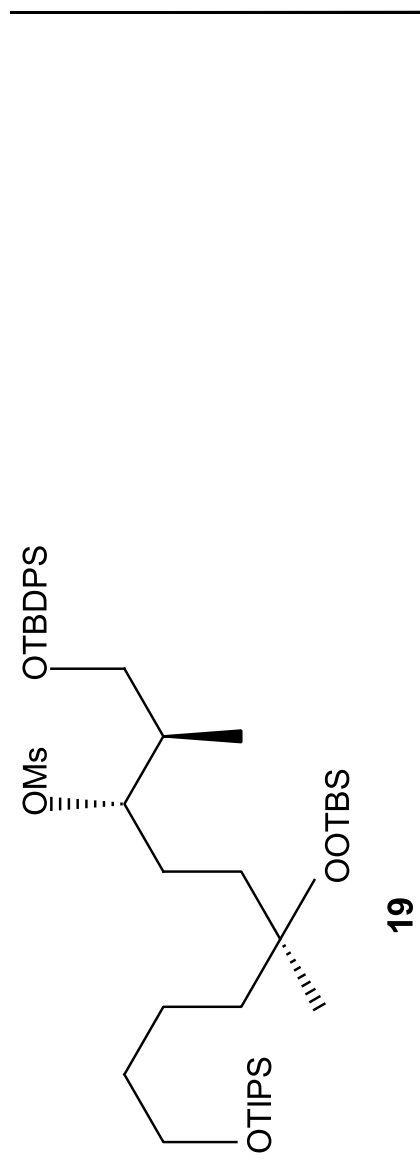

†L' $\varepsilon 8$

$\angle \forall 98-$
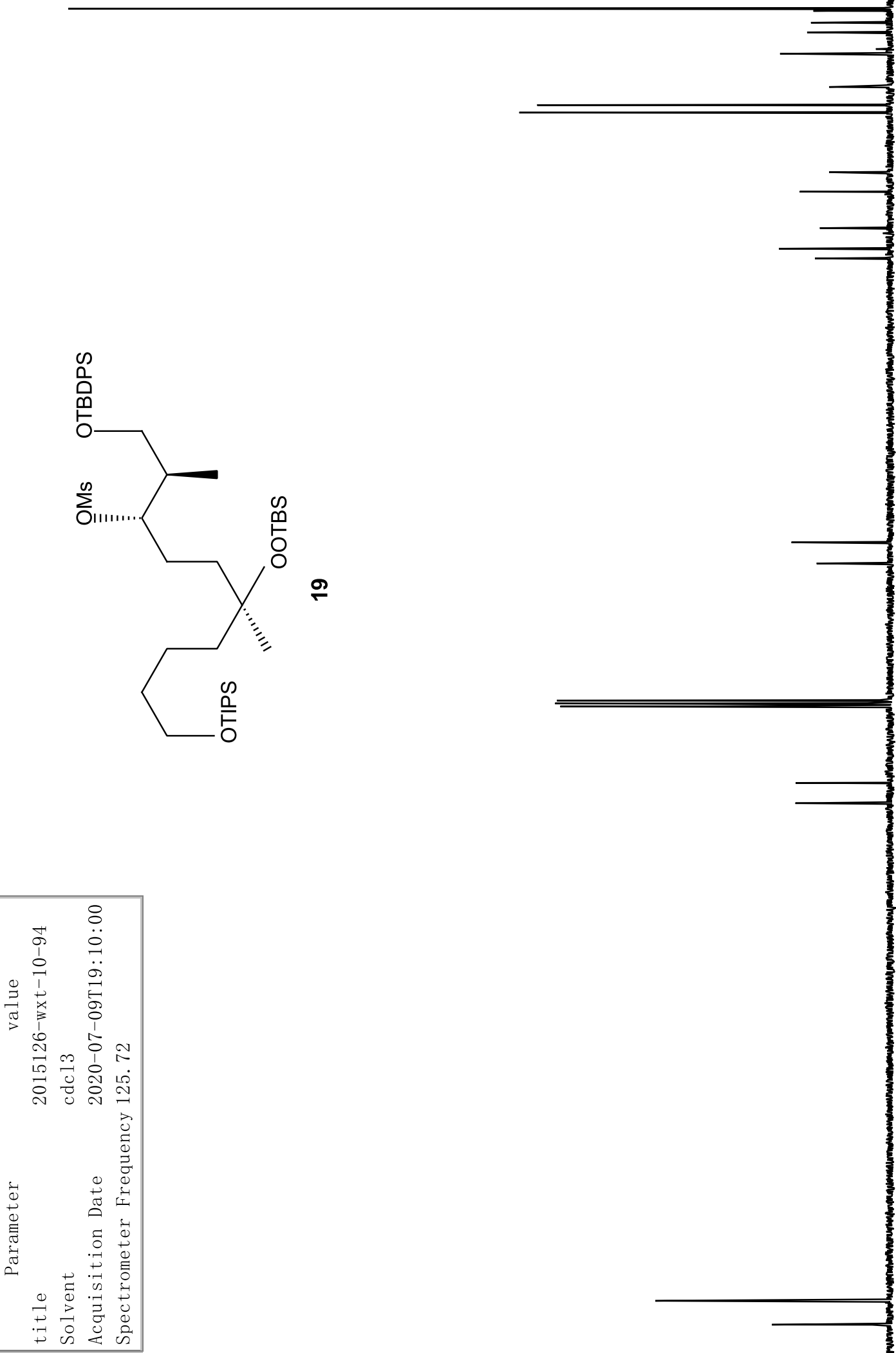

L6ZL
$2 L 62 !$

$\angle \varepsilon^{*} \varepsilon \varepsilon\llcorner-$ 


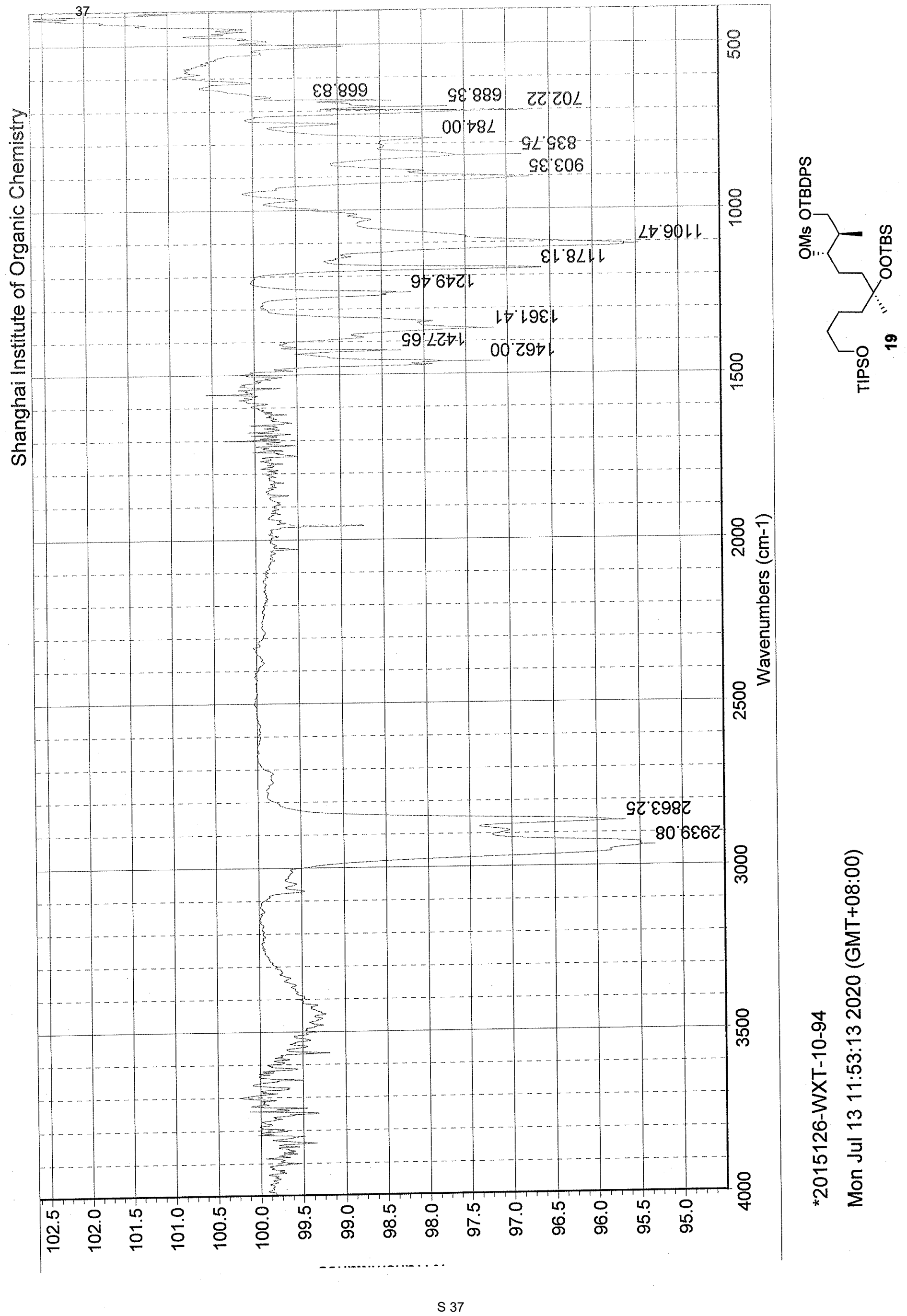


$\angle 6^{\circ} 0$

$66^{\circ} 0$

2015

$\mathrm{Ol}^{\circ} \mathrm{L}$

98: Z-

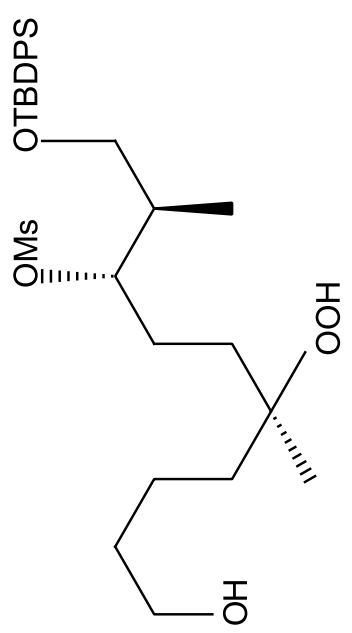

กิ
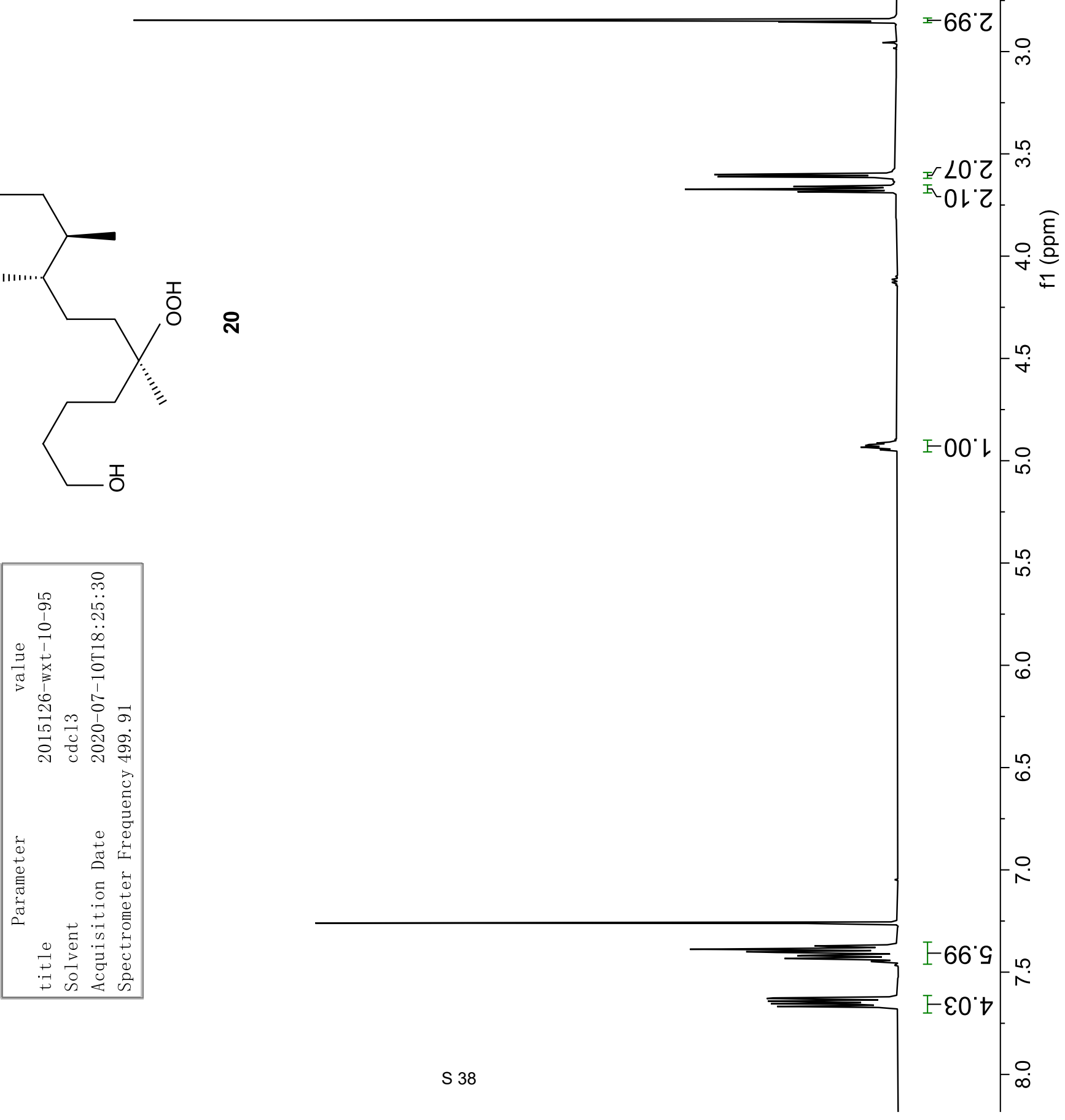


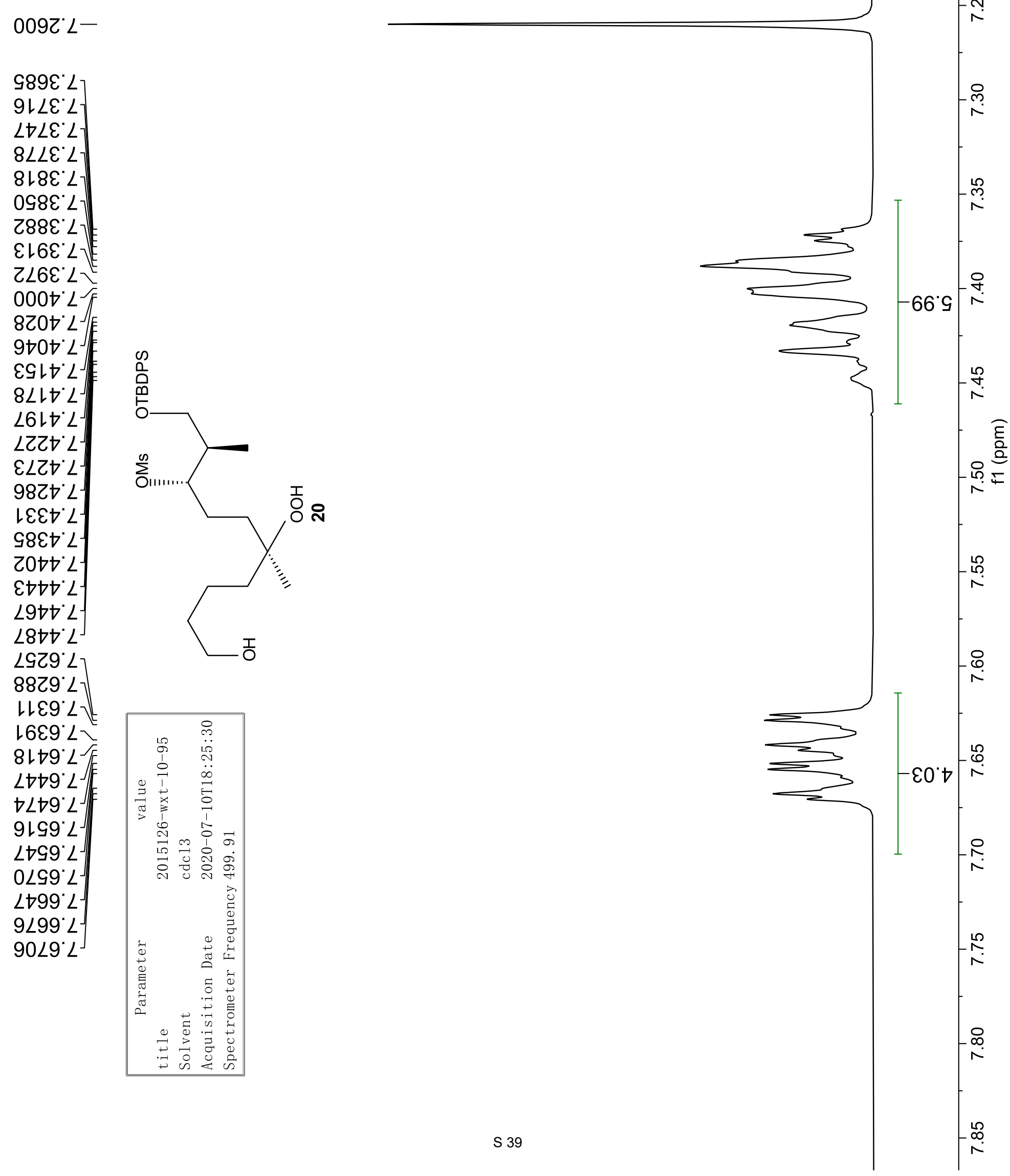



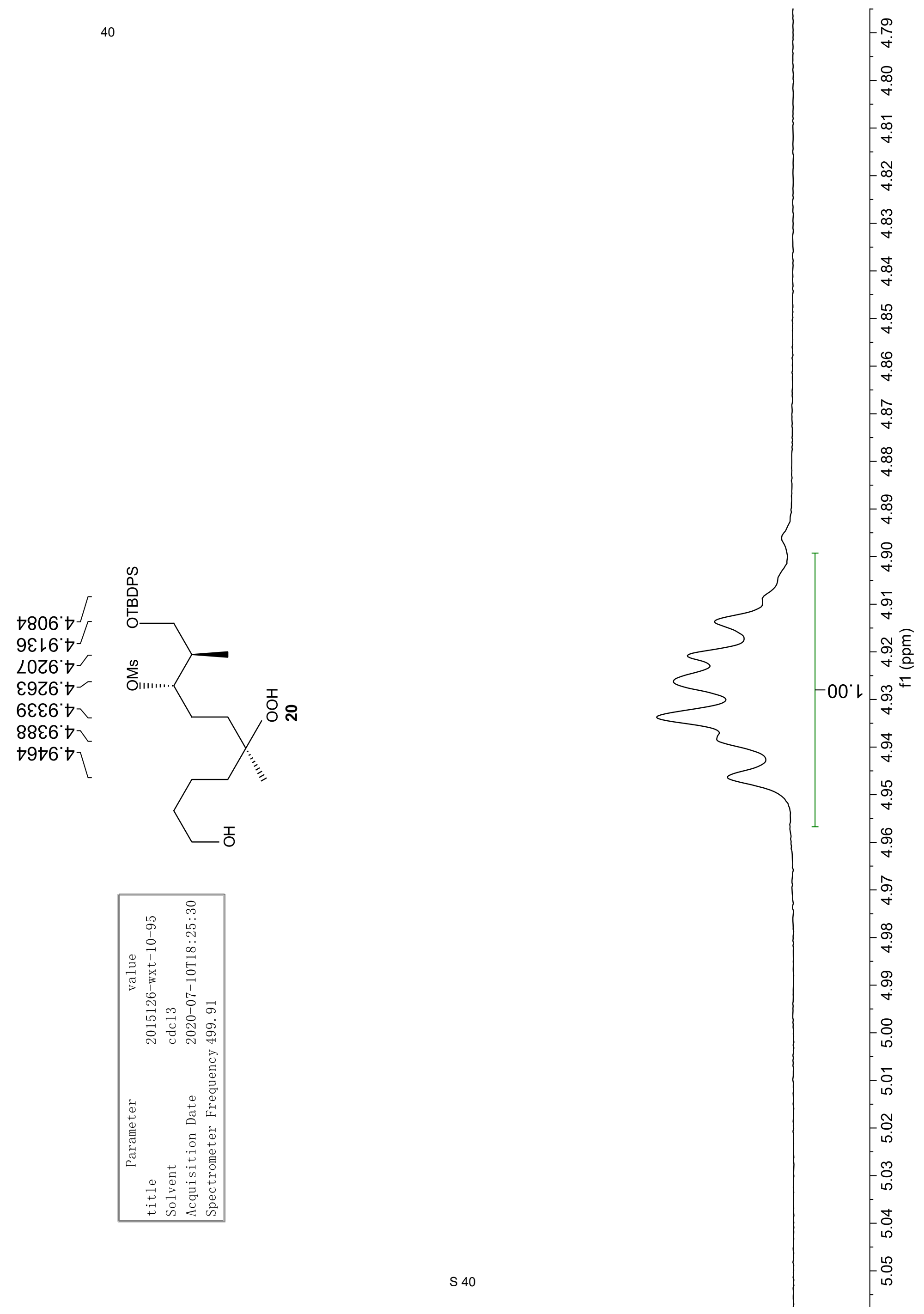
9とててレー

ᄂ 6 L

$\nabla \varepsilon^{*} 6$ ᄂ

$\angle L^{\circ} \mathrm{OZ}$

$09^{\circ} \nabla Z^{-}$

$\downarrow 8.9 Z^{-}$

99.0 -

† 乙E-

8เ' $9 \varepsilon>$

† $8 \varepsilon=$

$9 \angle 8 \varepsilon^{\circ}$

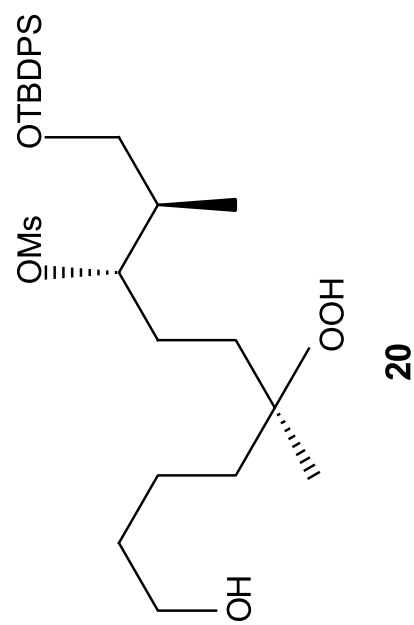

St29-

$\nabla 6.79$

$69^{\circ} \varepsilon 8-$

$66^{\prime} \nabla 8$

LLCZL

$\nabla L$ LZL

LL'6ZL

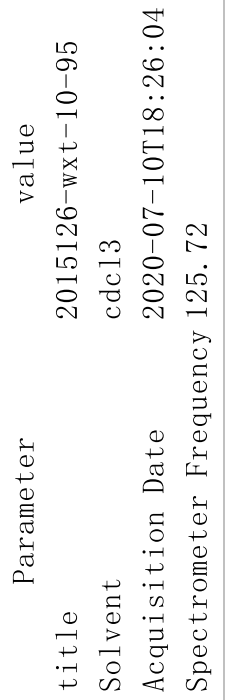

8L"6ZL

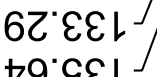

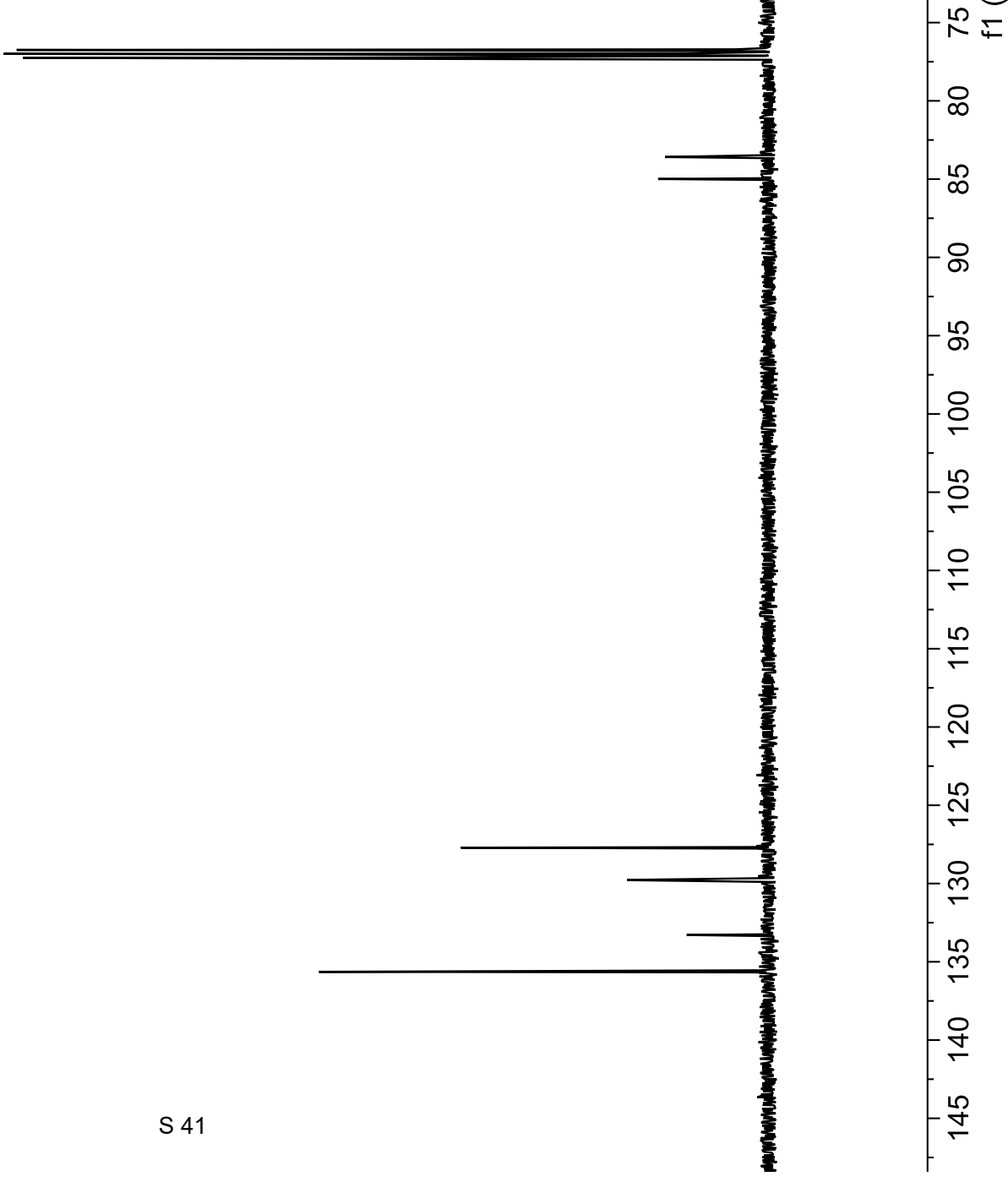




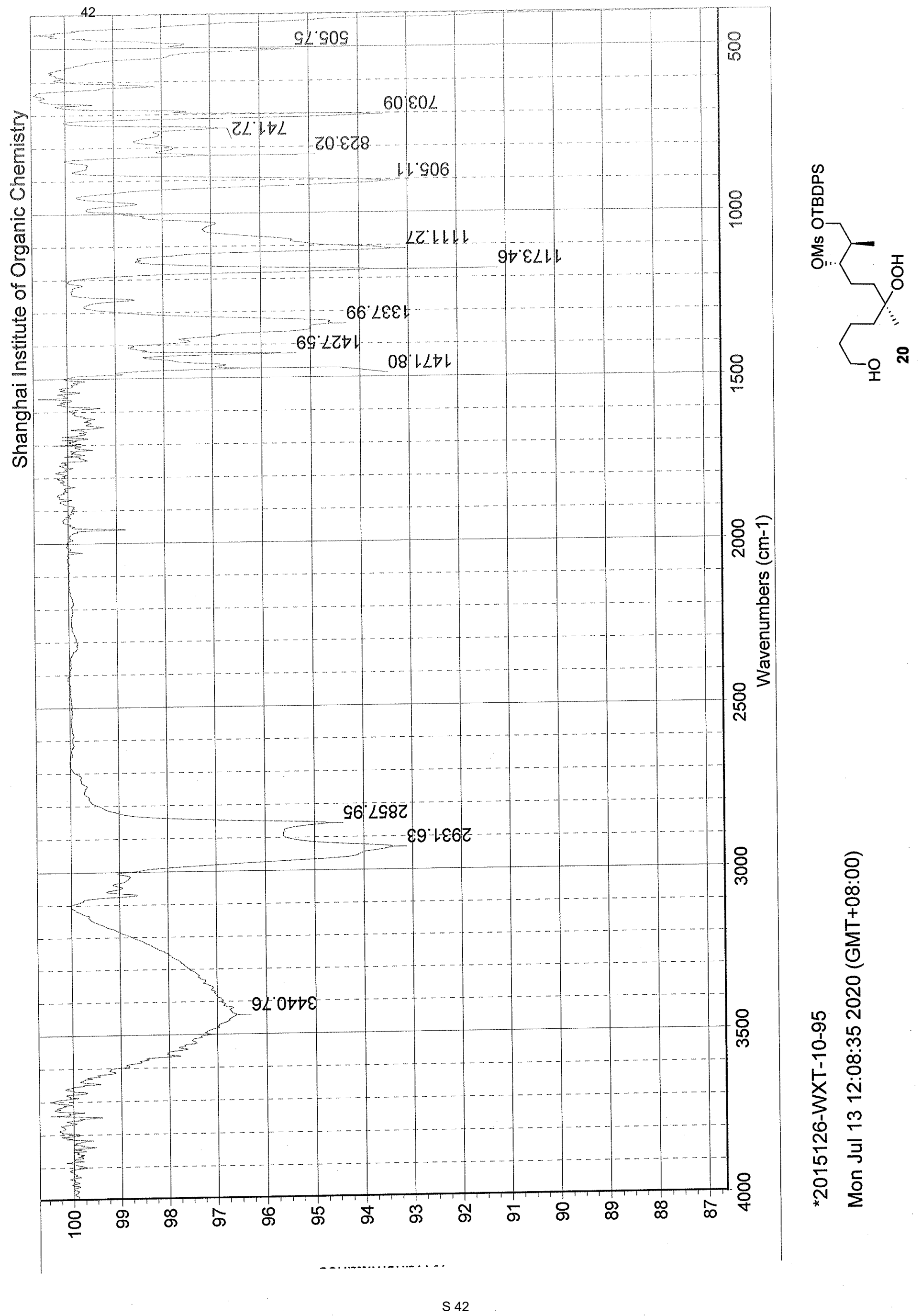




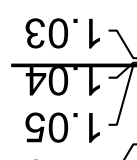

62 เ

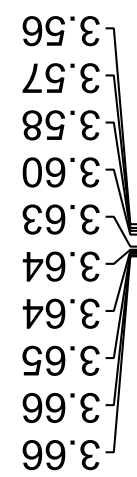

$\infty$
0
0
0
0

$\bar{N}$
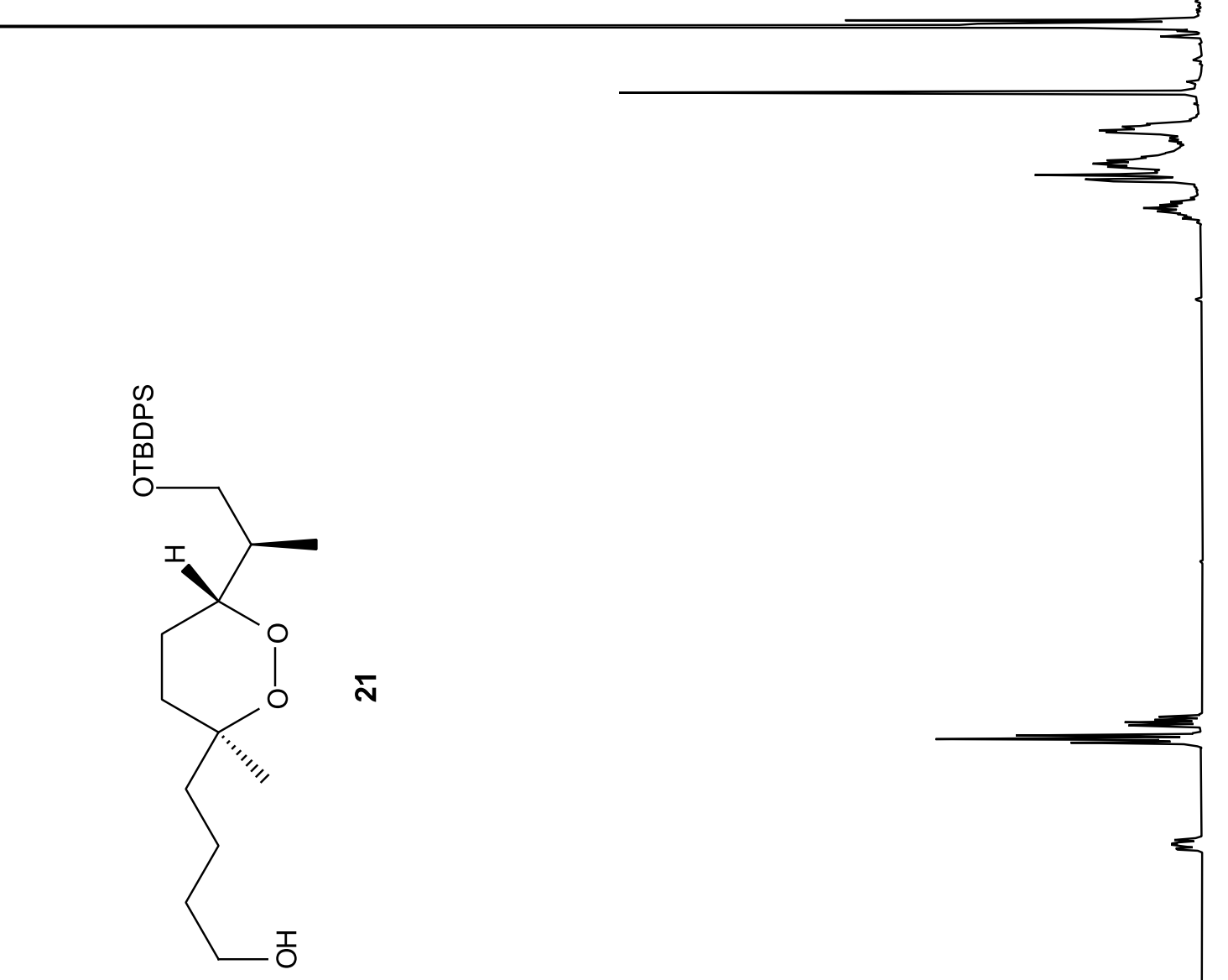

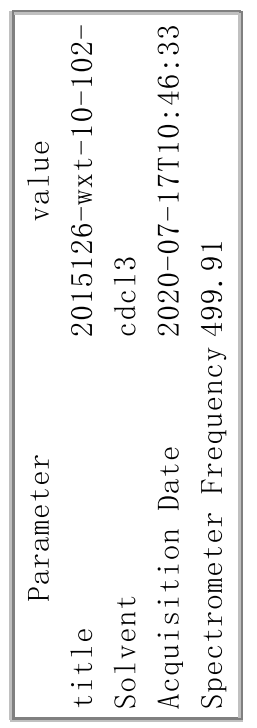

凡 672

ऽ $18 \%$

I $6 \varepsilon^{\prime} \&$

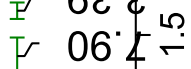

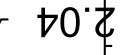


0092 L-

8SSE $L$

$\angle 89 \varepsilon^{\circ} \angle$

9เ9\& $L$

$9 \succ 9 \varepsilon$ L

เ69\& $L$

$\varepsilon Z \angle \varepsilon^{\circ} \angle$

ZSLE $\angle]$

18LE' $\angle$

$\nabla \nabla 8 \varepsilon<2$

$0 \angle 8 \varepsilon \angle$ T

Z68E $L$

ZLGE $L$

$6 Z 0 t^{\circ} L$

290t L

$\varepsilon 60 t<$

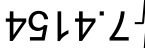

90Zt $L$

89Zt L

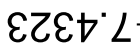

ZSE† L
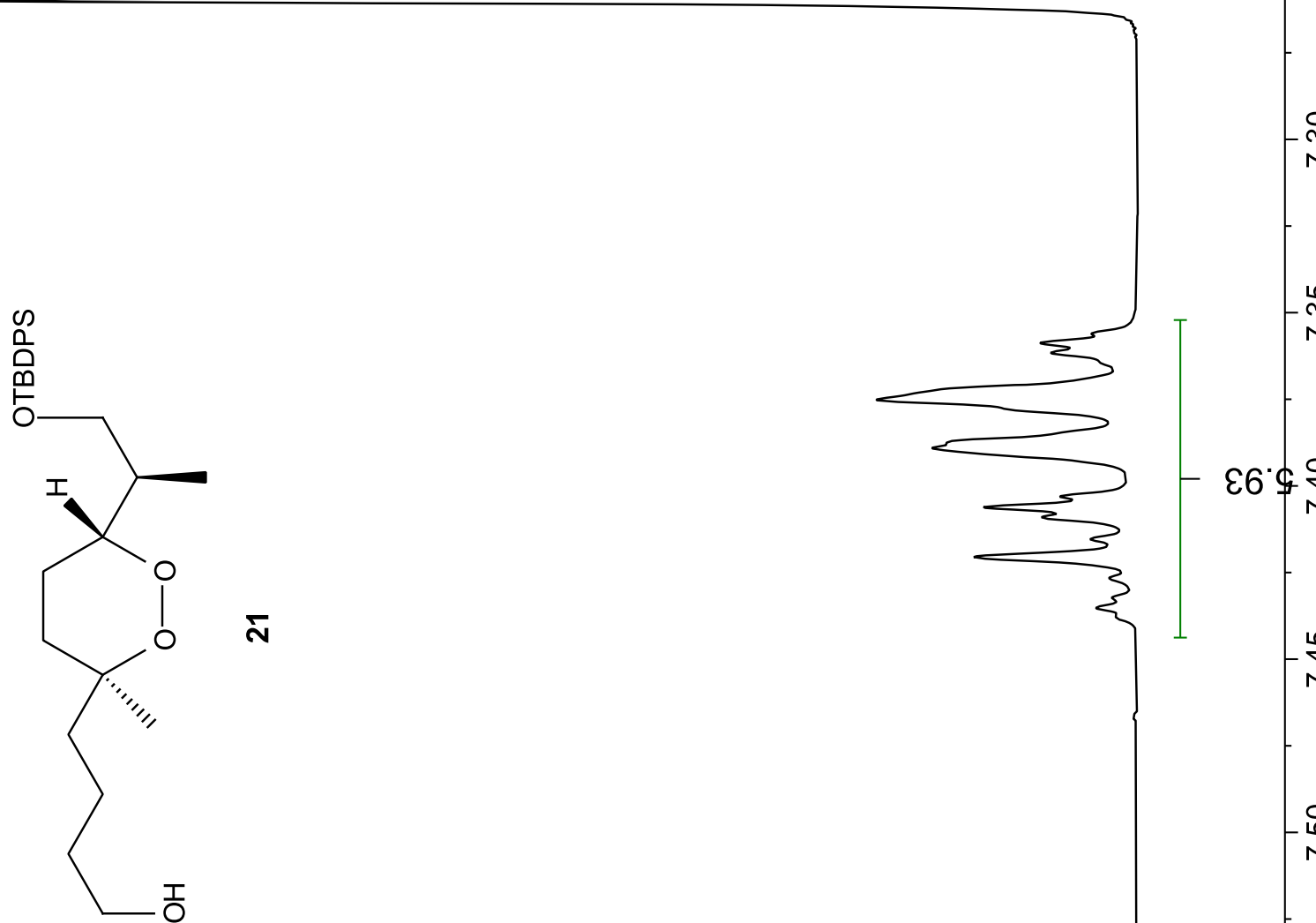

$\bar{N}$

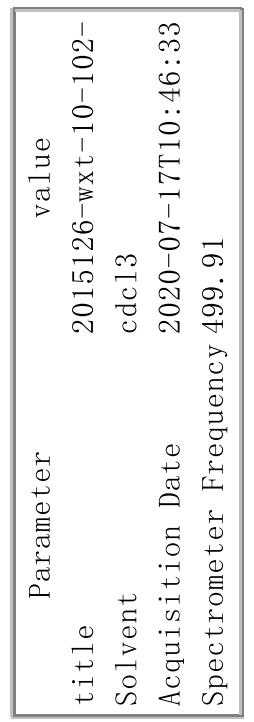


†ะ99' $\varepsilon-$

$8 \nabla \angle G^{\circ} \varepsilon-$

9ะ89 ${ }^{\circ}-$

0S69 $\varepsilon$ -

$\angle L 9^{\circ} \varepsilon$

$\varepsilon เ \nabla 9^{\circ} \varepsilon$

$6 \succsim t 9^{\circ} \varepsilon$

† $199^{\circ} \varepsilon-$

$6 \angle 99^{\circ} \varepsilon^{-}$

9เ99.
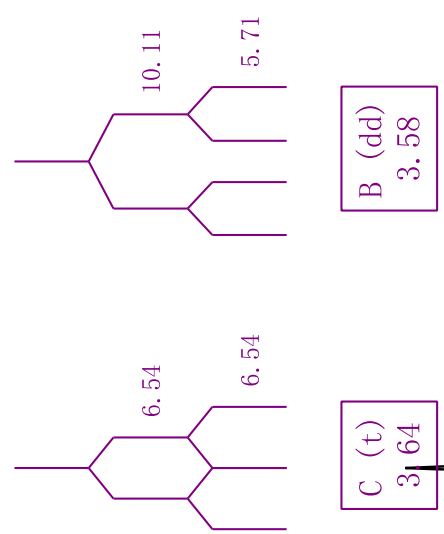

\section{ङ}
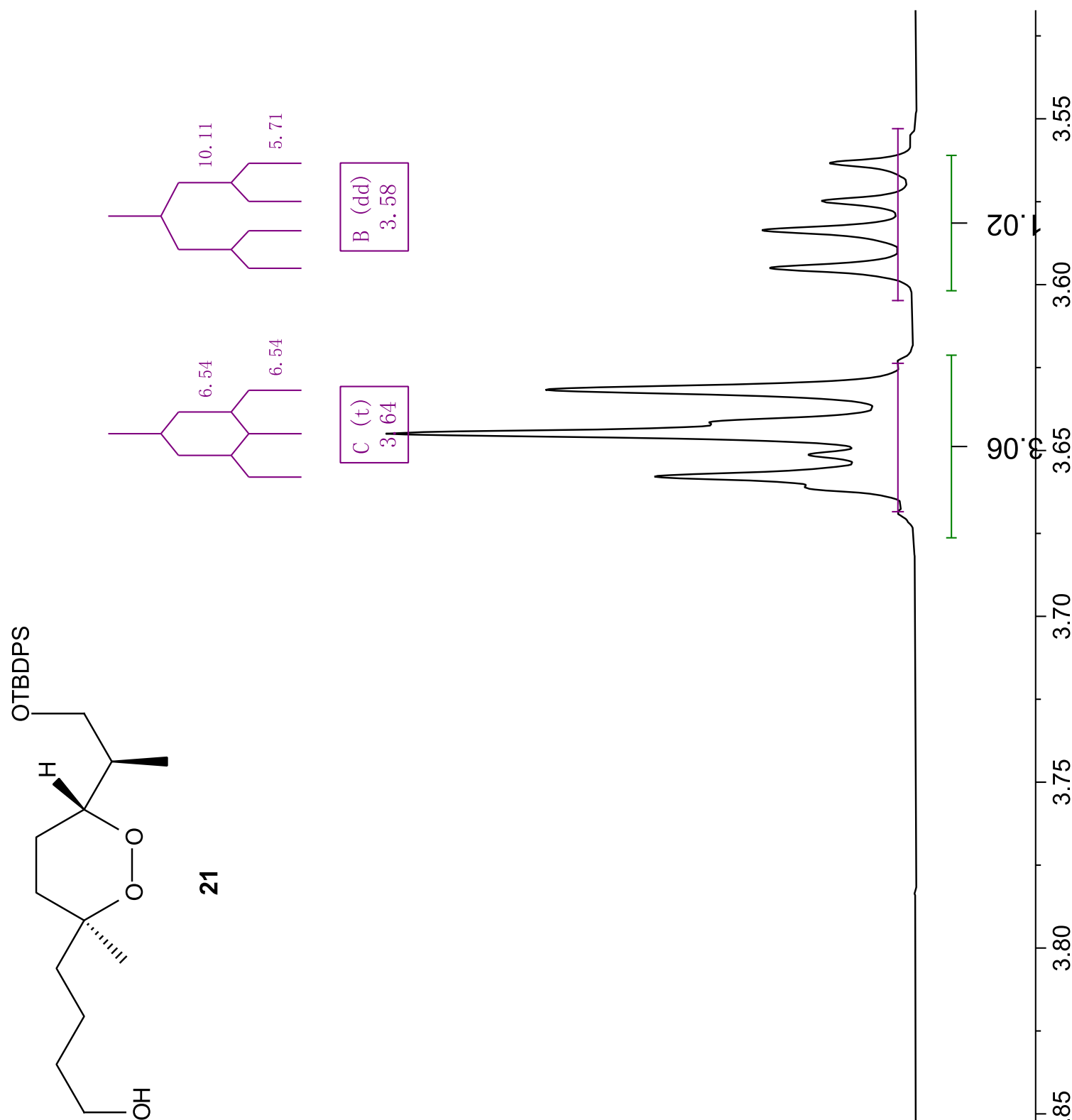

암

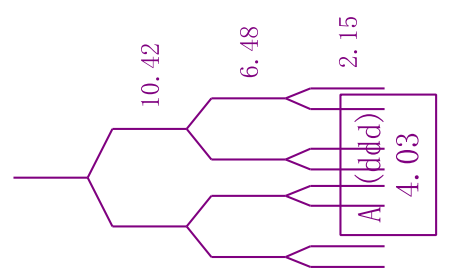


$20^{\circ} 0^{--}$

9よてレー

$0 \varepsilon^{\circ} 6$ -

$9 Z^{\circ} 0 Z^{\nearrow}$

89. \& $^{-}$

98.92-

$6 L$ '乙E

8เ $\varepsilon \varepsilon^{\prime}$

$\angle \varepsilon^{\prime} 6 \varepsilon>$

$\varepsilon 乙^{\circ} 0 \nabla^{\top}$

$n$
0
0
0
0

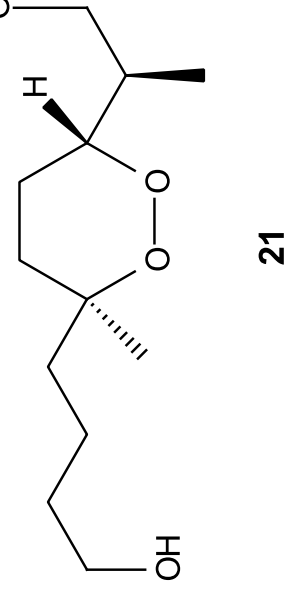

$\bar{N}$

LL'Z9-

89'99-

98'6L-

81'28-

Z9 $\angle Z L$ 69.6Zレ †9 $\varepsilon \varepsilon\llcorner$

GL'EEL】 6G"GELT
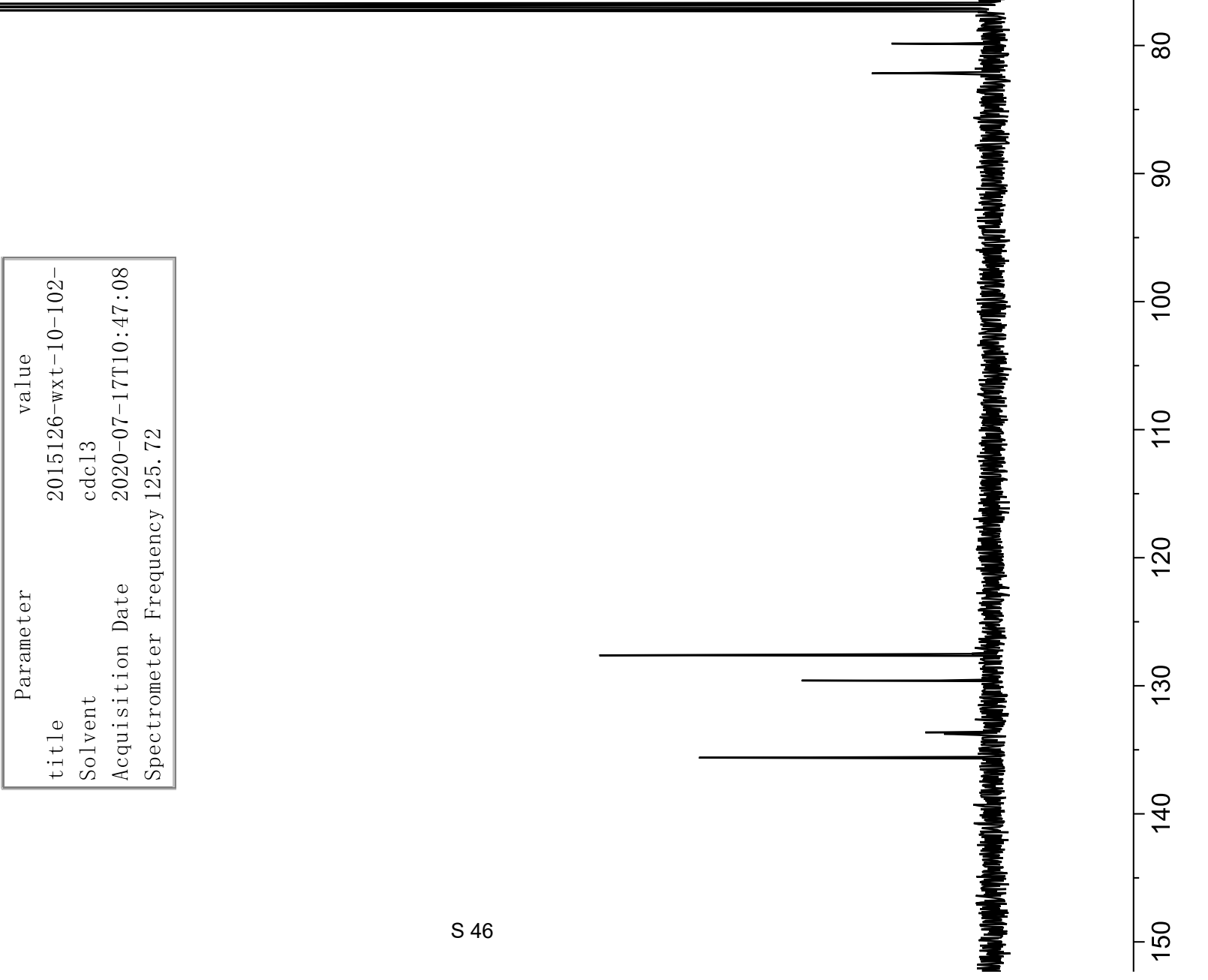


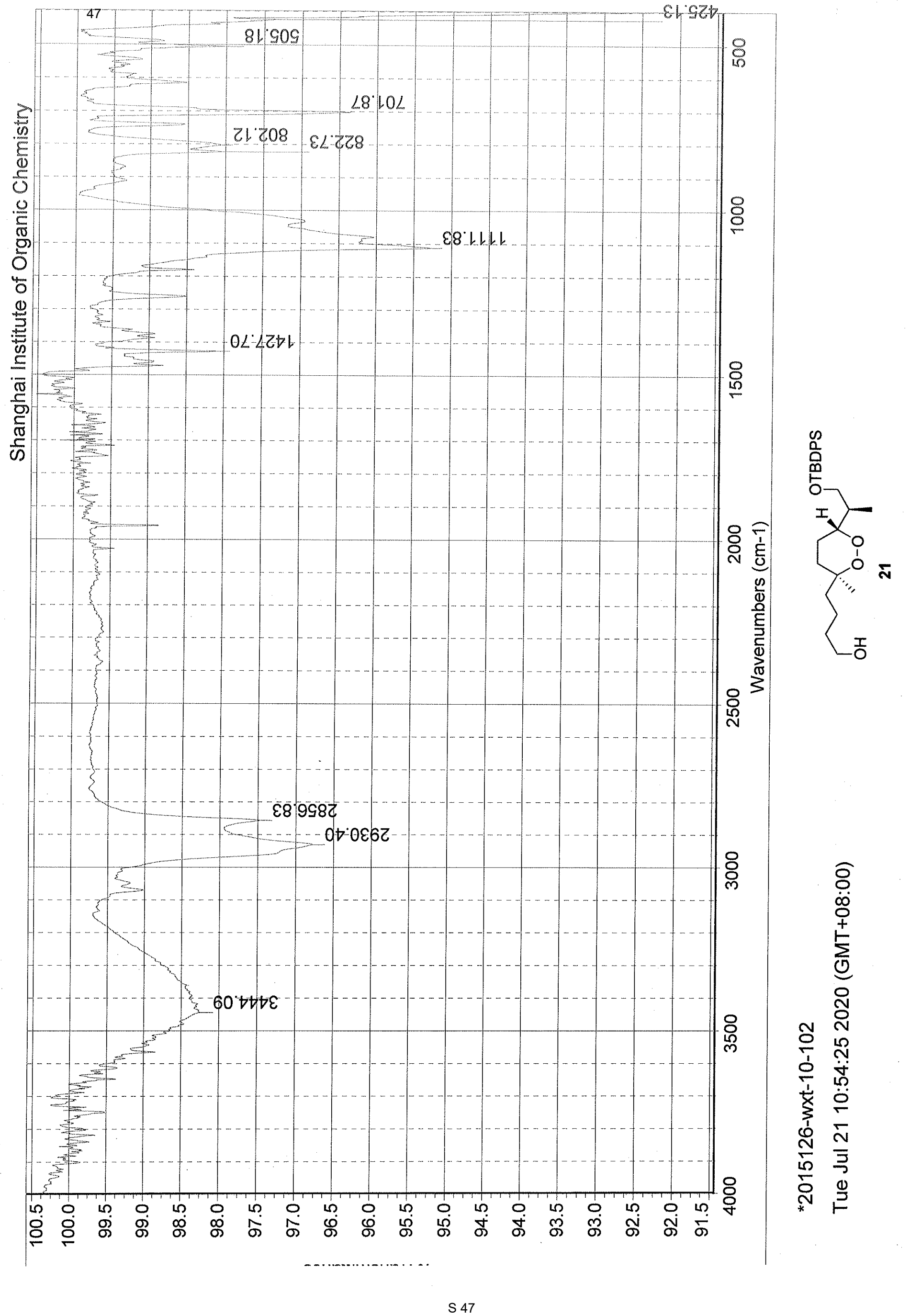


70レา 90. เE้

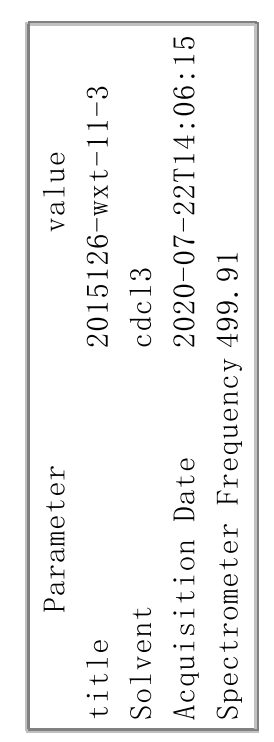

$79^{\circ} \mathrm{L}$ $\nabla 9^{\circ} L$ $\nabla 9^{\circ}<$ $99^{\circ}<$ $99^{\circ} \mathrm{L}$ $99^{\circ} \mathrm{L}$ 99. $L J$ $99^{\circ} L$ $99^{\circ} \mathrm{L}$
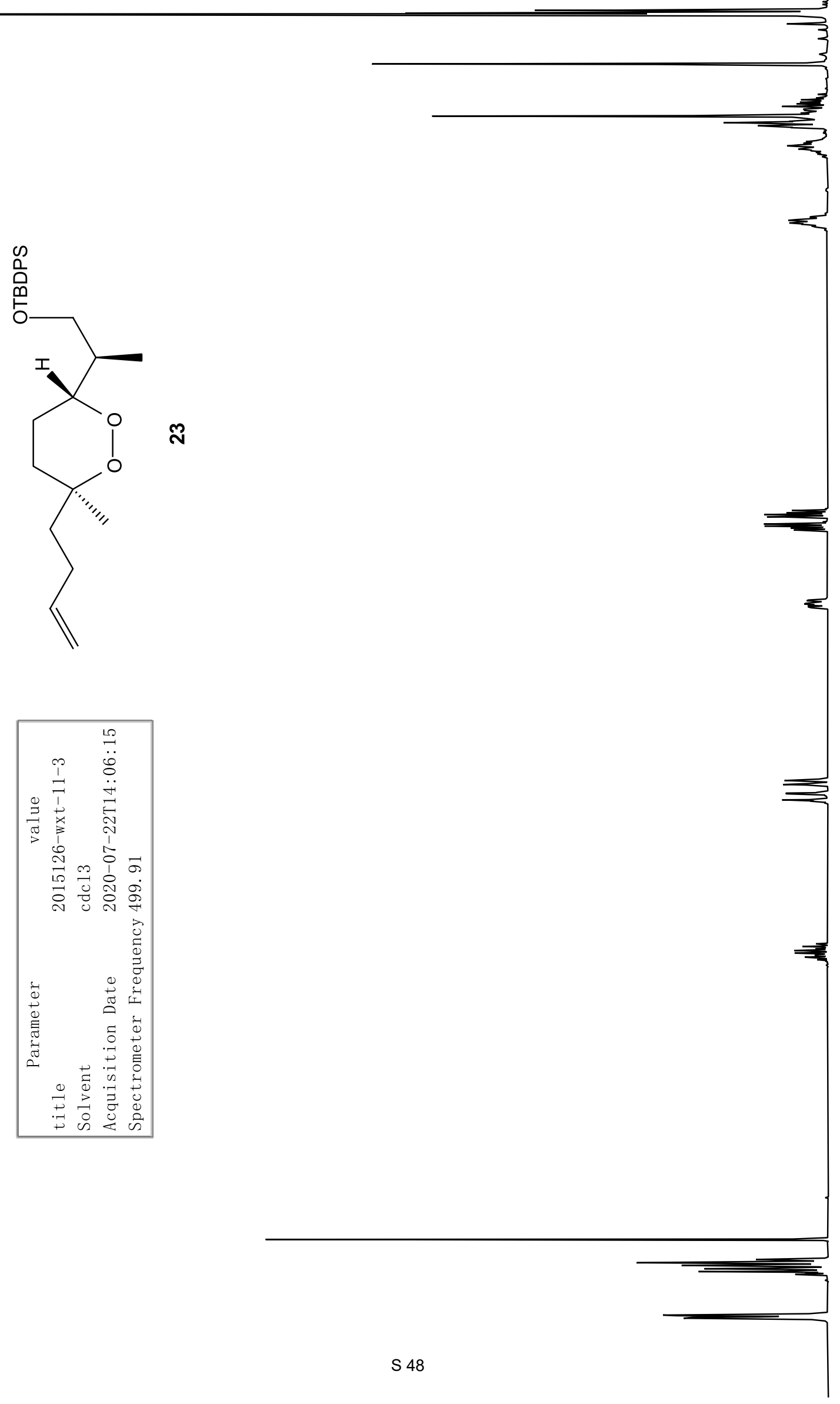


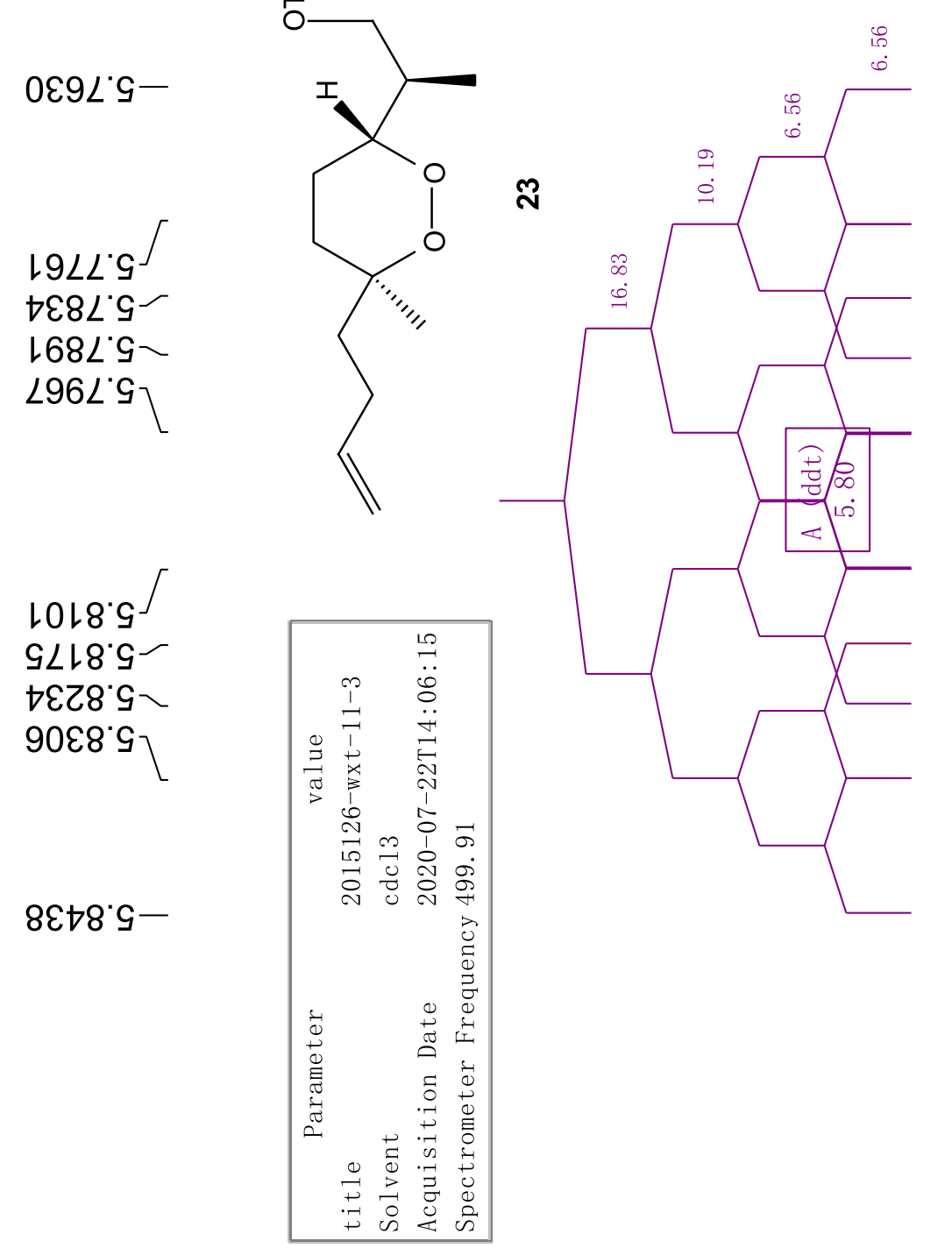

c

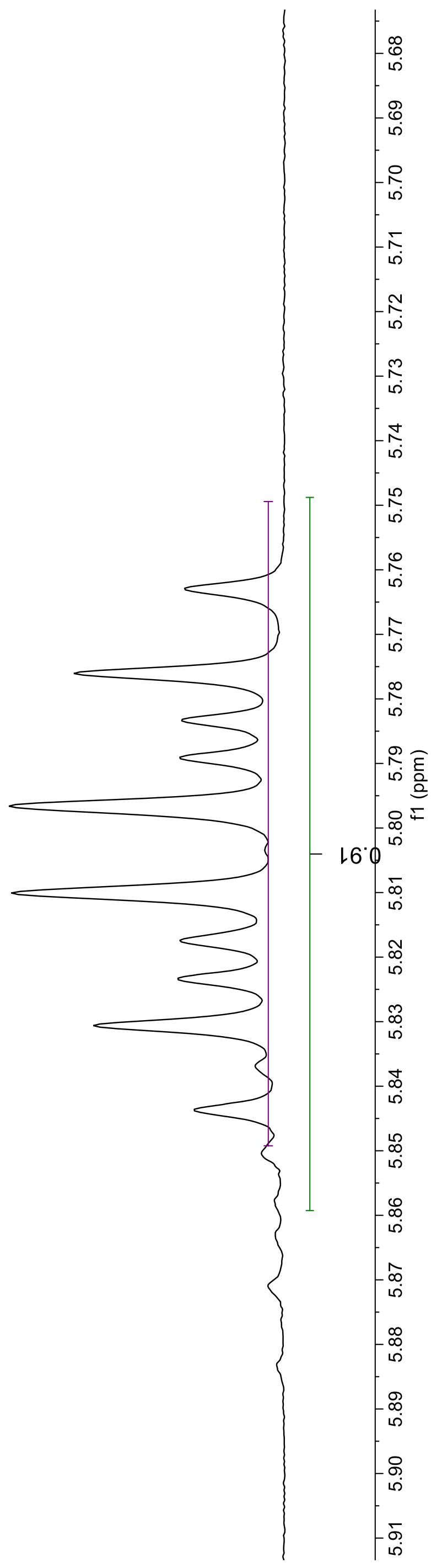


$\angle 626^{\circ} \circ-$ $8 Z \varepsilon 6^{\circ} \downarrow-$ $09 \varepsilon 6^{\circ} \downarrow-$ $98 \varepsilon 6 \bullet-$

$1096.7-$ 6296 ○$\varepsilon 996^{\circ} b-$ 0696 $\bullet-$

$\varepsilon 9666^{\circ}{ }^{\circ}$ 9666 ○一 Zع00 9 $9900^{\circ} \mathrm{S}>$

รOع० $9^{-}$ 6\&\&0 $9-$ $\nabla \angle E O^{\circ} S_{-}$ 60t0'
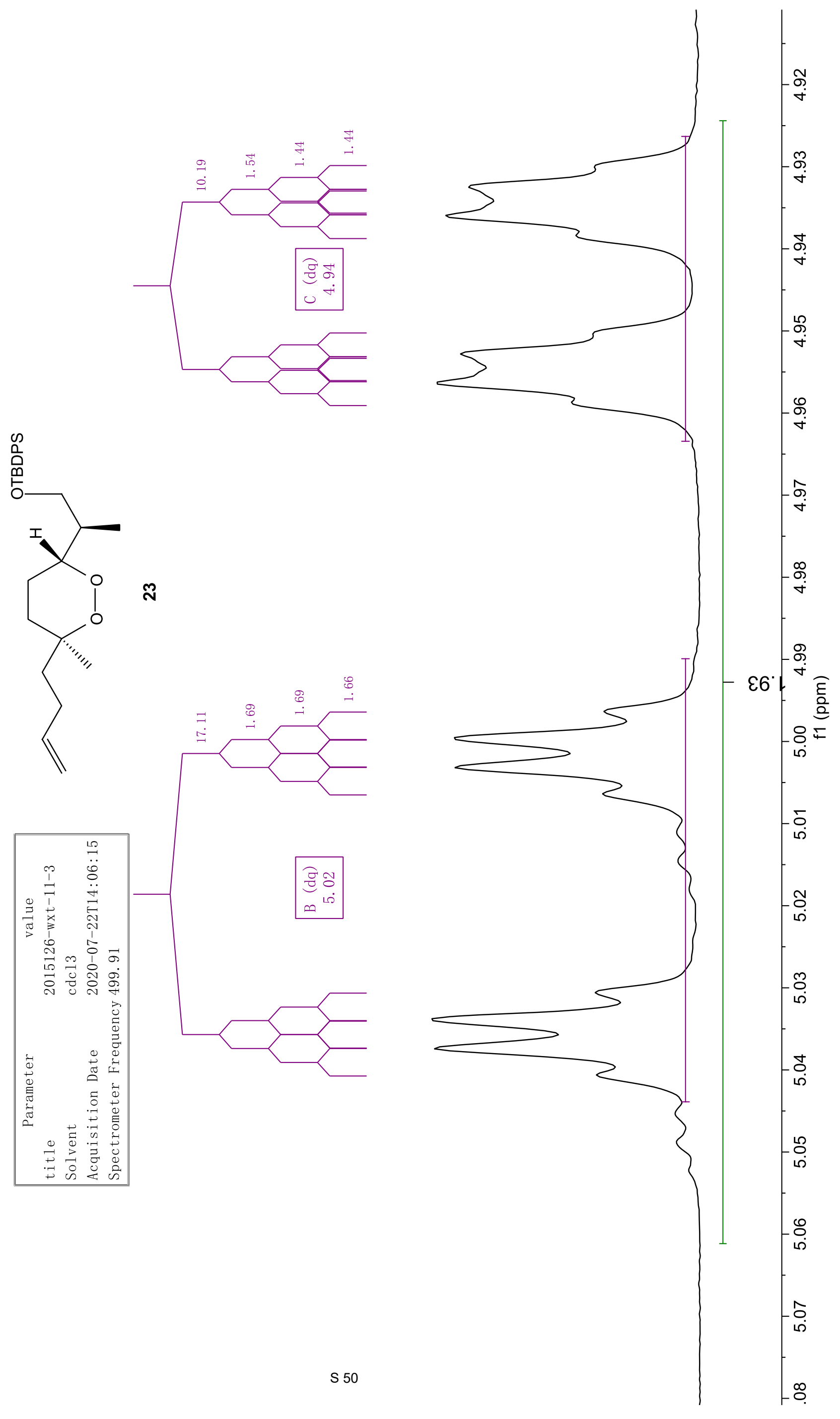
$8899^{\circ} \varepsilon-$ 2089' $\varepsilon-$ $0689^{\circ} \varepsilon-$ ${ } 009^{\circ} \varepsilon-$

$0 \angle \varepsilon 9^{\circ} \varepsilon-$ 乙L† ${ }^{\circ} \varepsilon-$ $\varepsilon \angle G 99^{\circ} \varepsilon-$ S $\angle 99^{\circ} \varepsilon-$
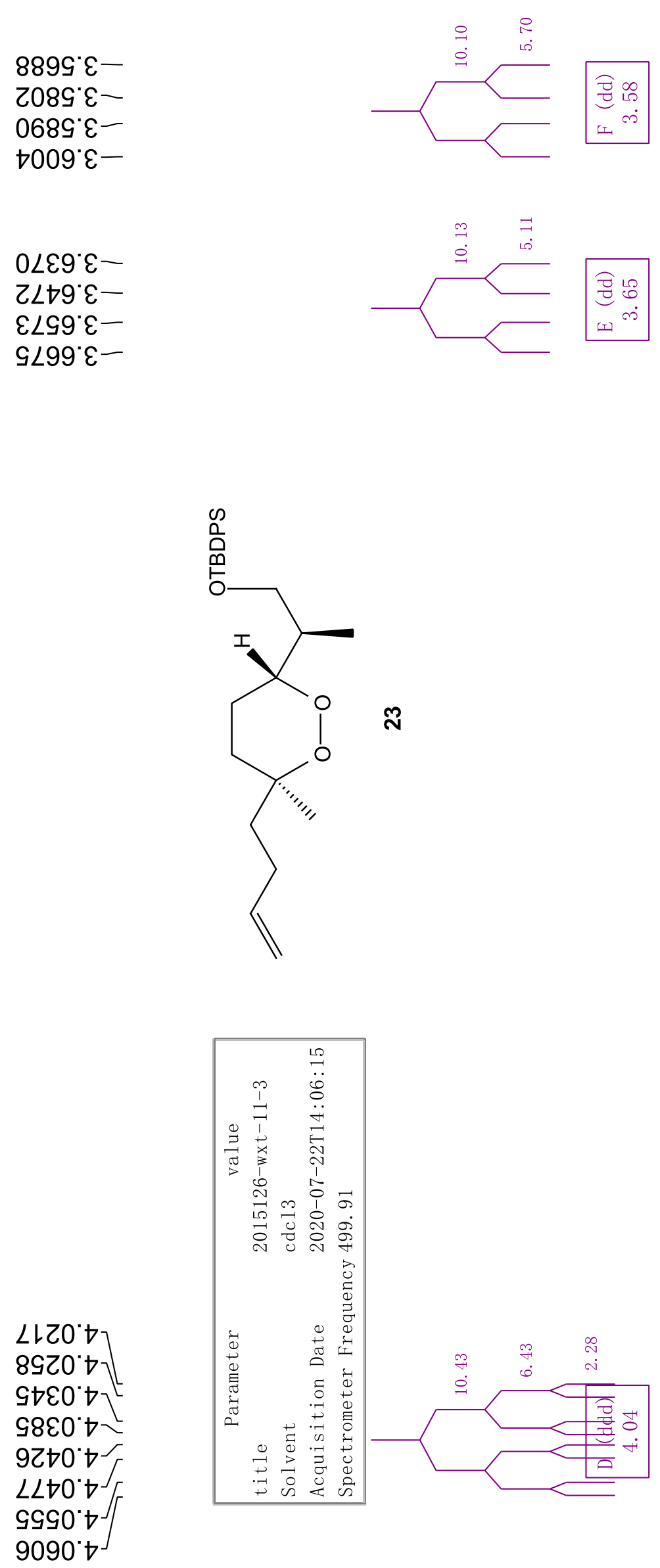

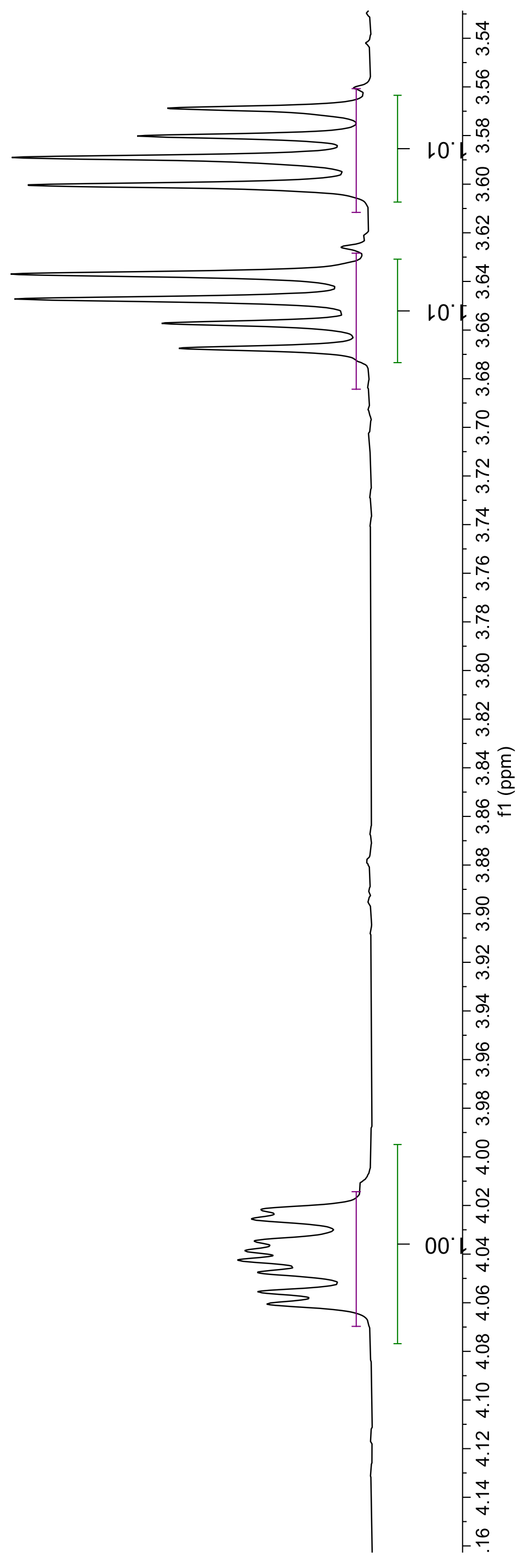


†レてレー

0ย'6ᄂ

9乙.0乙一

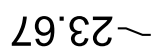

$98^{\circ} 9 Z$

$\angle \mathcal{E}^{\prime} \angle 乙^{\top}$

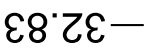

$8 \varepsilon^{\circ} 6 \varepsilon$

$79^{\circ} 6 \varepsilon^{\top}$

69.99-

99.6L-

9L'Z8-
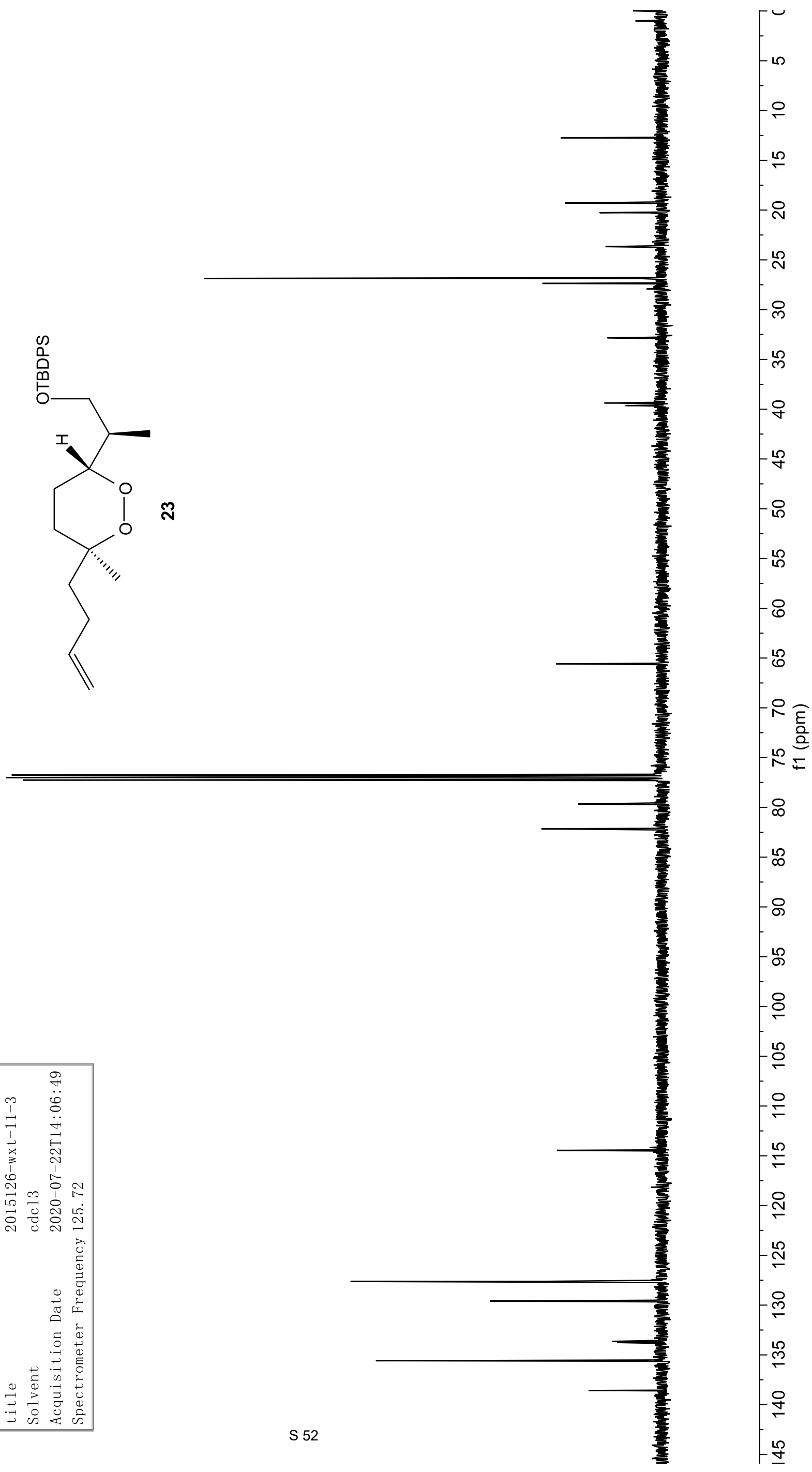


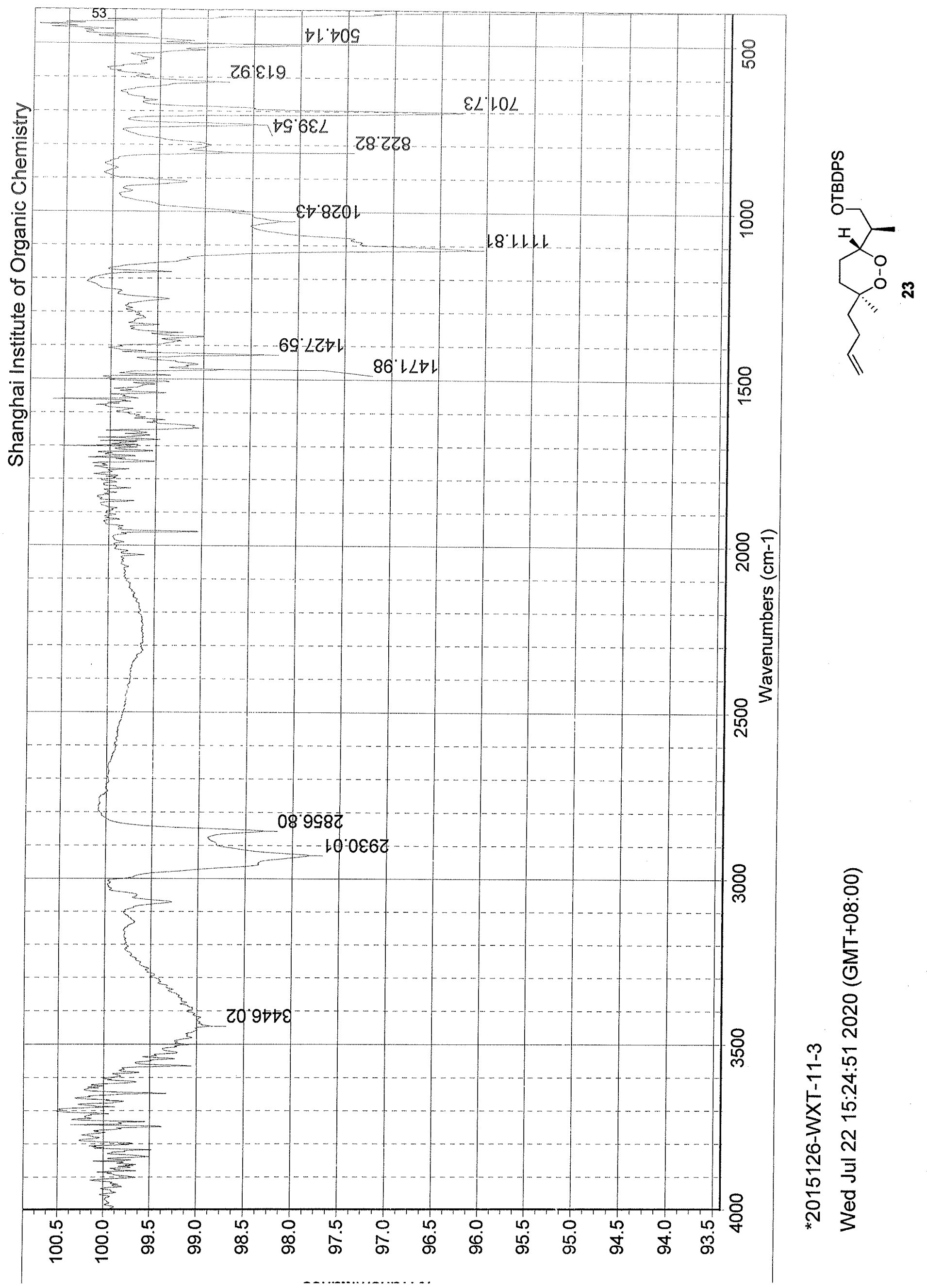



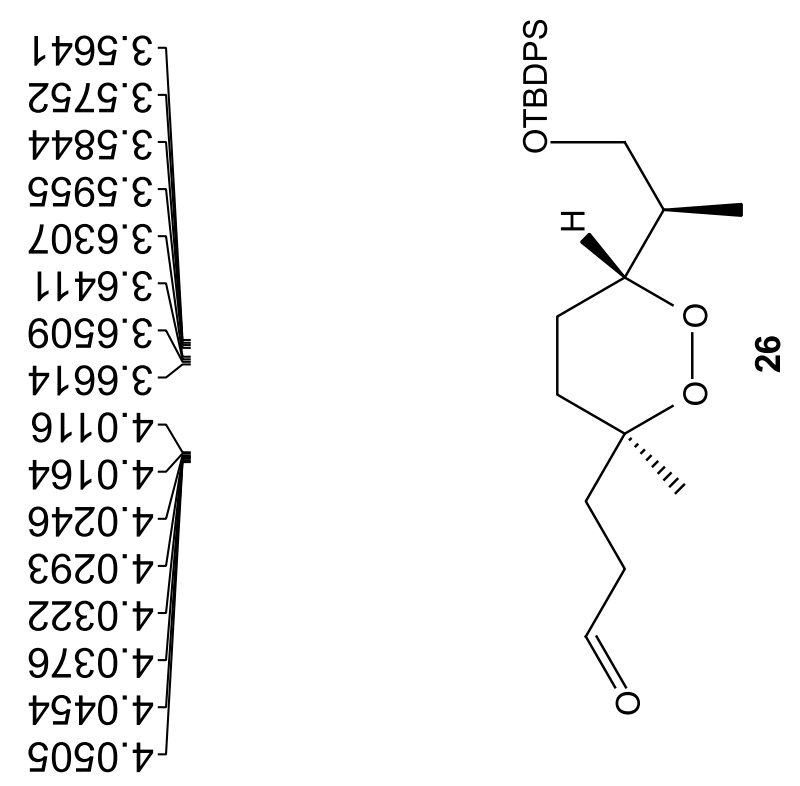

\section{요}

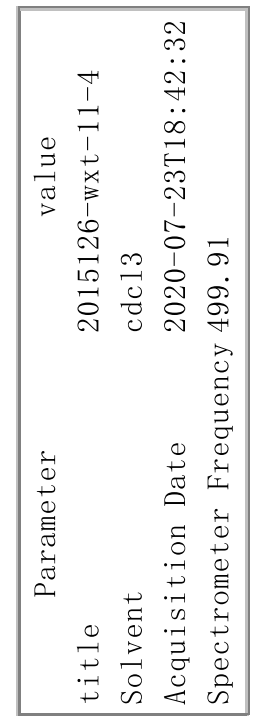

8DLL'6

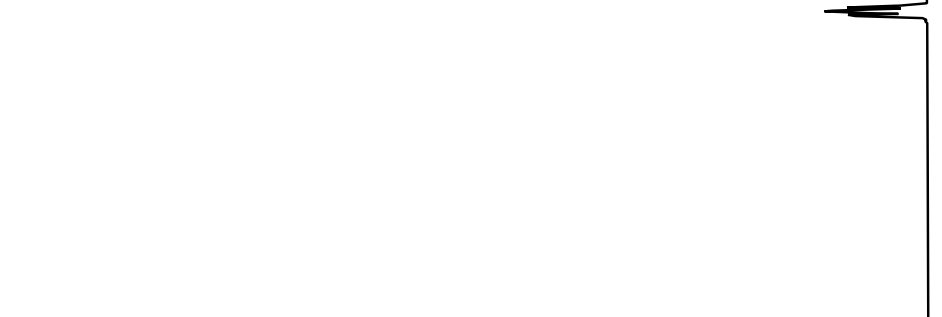


$\vdash \rightarrow 99^{\circ} \varepsilon-$ 乙S $\angle \mathcal{G}^{\circ} \varepsilon-$ $\nabla 789^{\circ} \varepsilon-$ S969' $\varepsilon-$

$\angle 0 \varepsilon 9^{\circ} \varepsilon-$ เレ๋9' 6099.

†เ99 $\varepsilon-$

$940^{\circ}$ $7910^{\circ} t$ $9 \forall Z 0^{\circ} t$ घ620" ZZEO० $9 \angle \varepsilon 0^{\circ} \nabla$ $\nabla S t 0^{\circ} t$ SOSO $\Rightarrow$
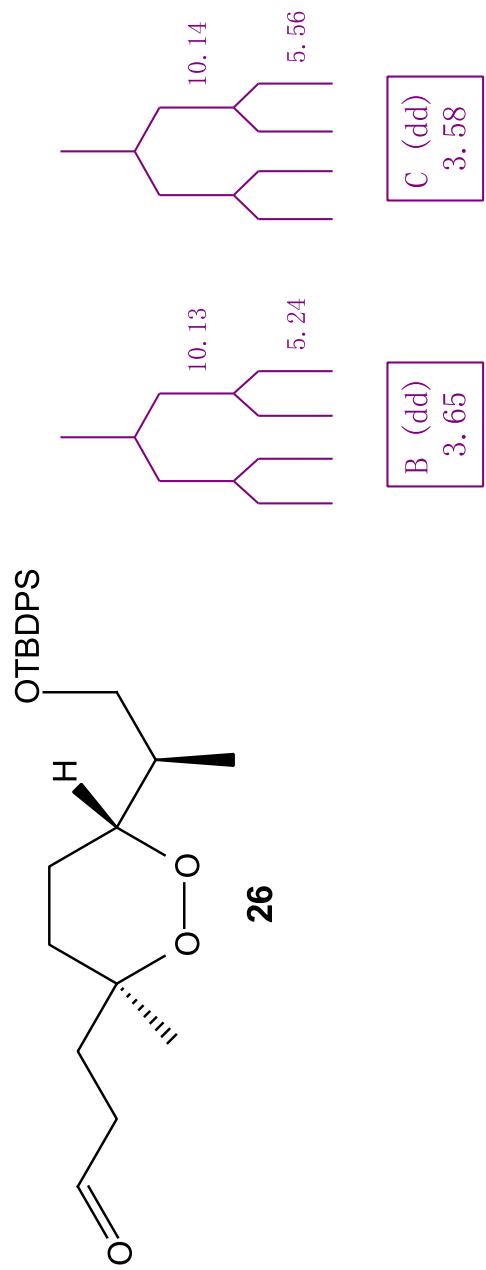
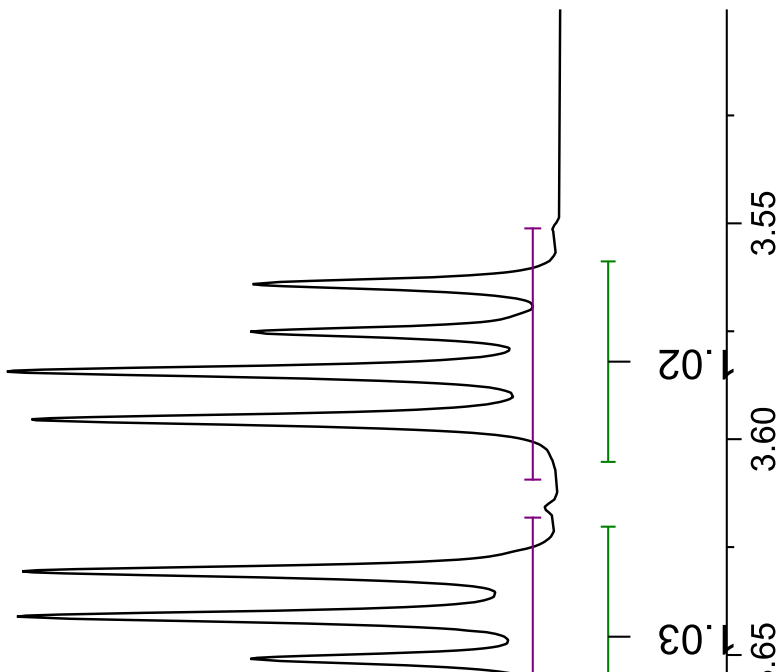

लं

is

馆

$\infty$

हิㅡㄹ

in

$\infty$

8

m

오ำ

तथ क

亏े 웜
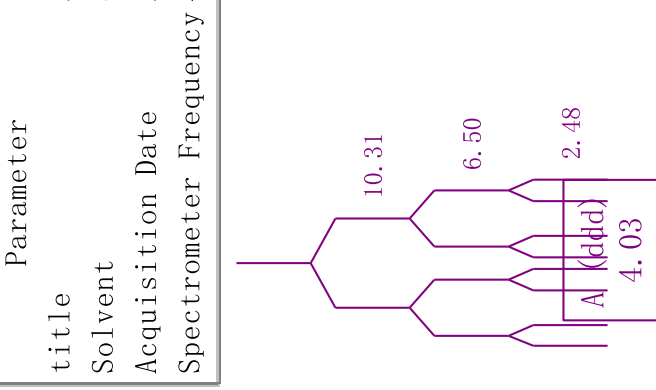
29'99-

Z8. 8 L $70.28-$
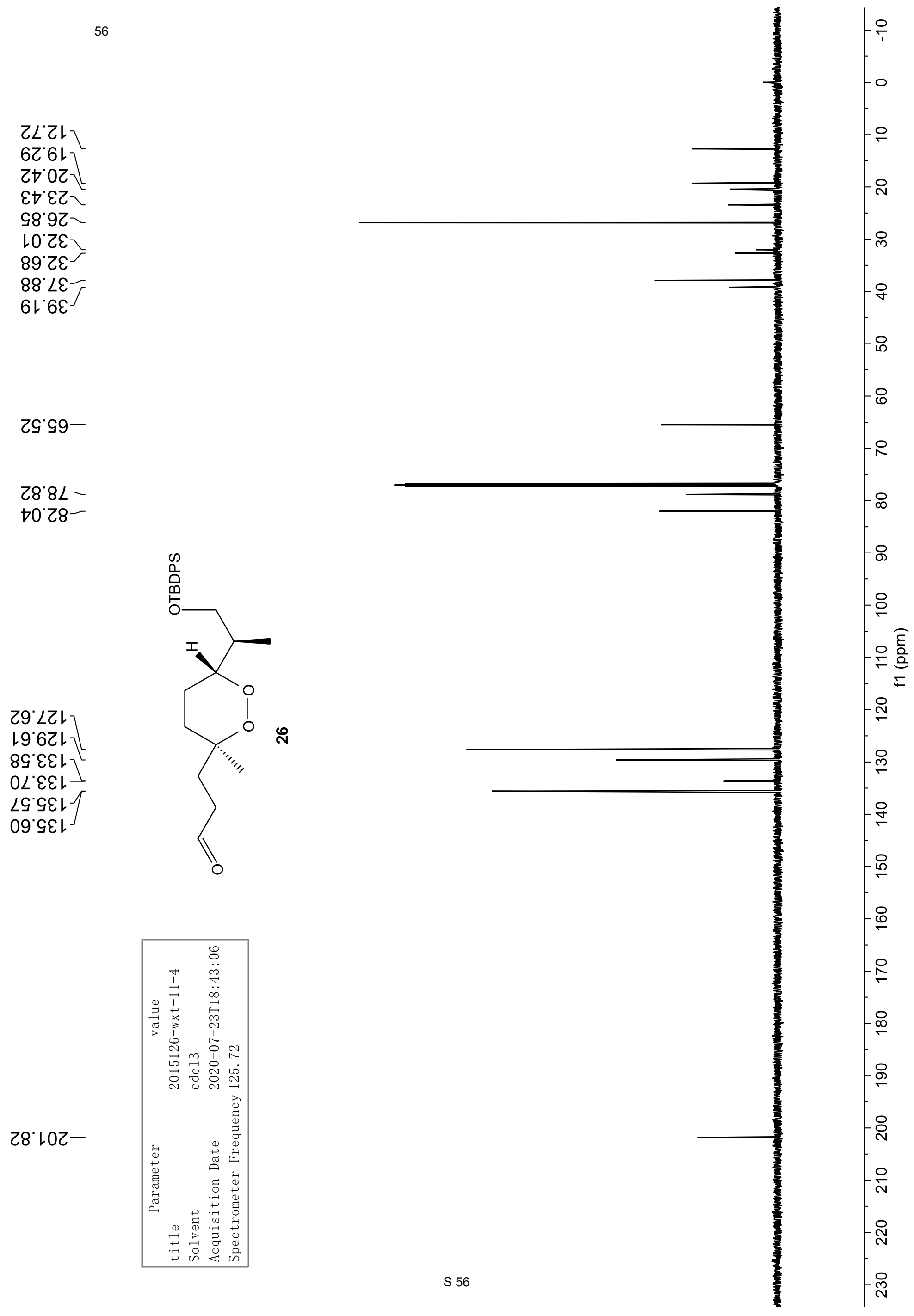


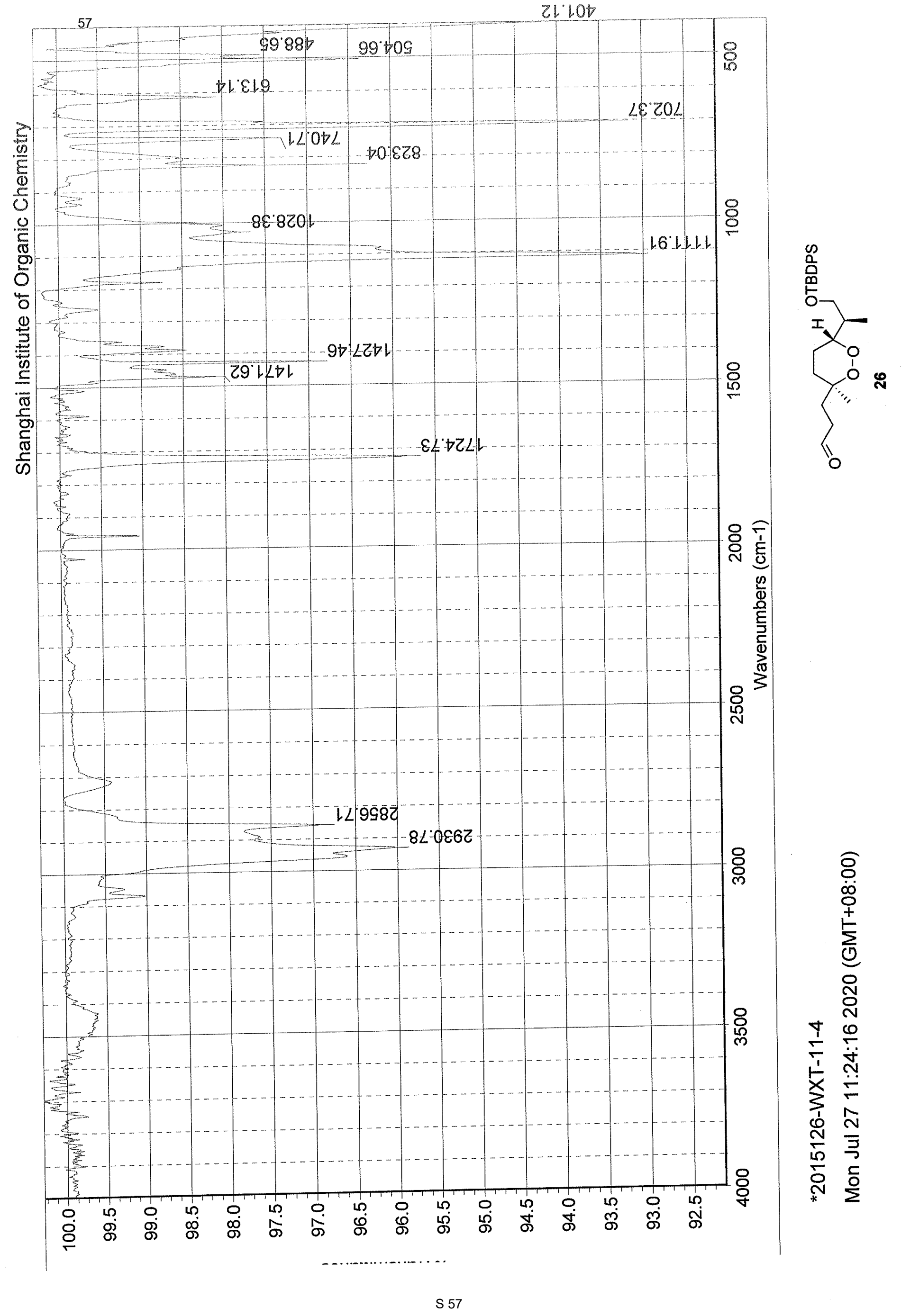




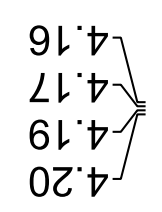

$79^{\circ} L$ $\nabla 9^{\circ}<$ $99^{\circ}<$ $99^{\circ}<-1$ $99^{\circ} \mathrm{L}$ $99^{\circ} \mathrm{L}$
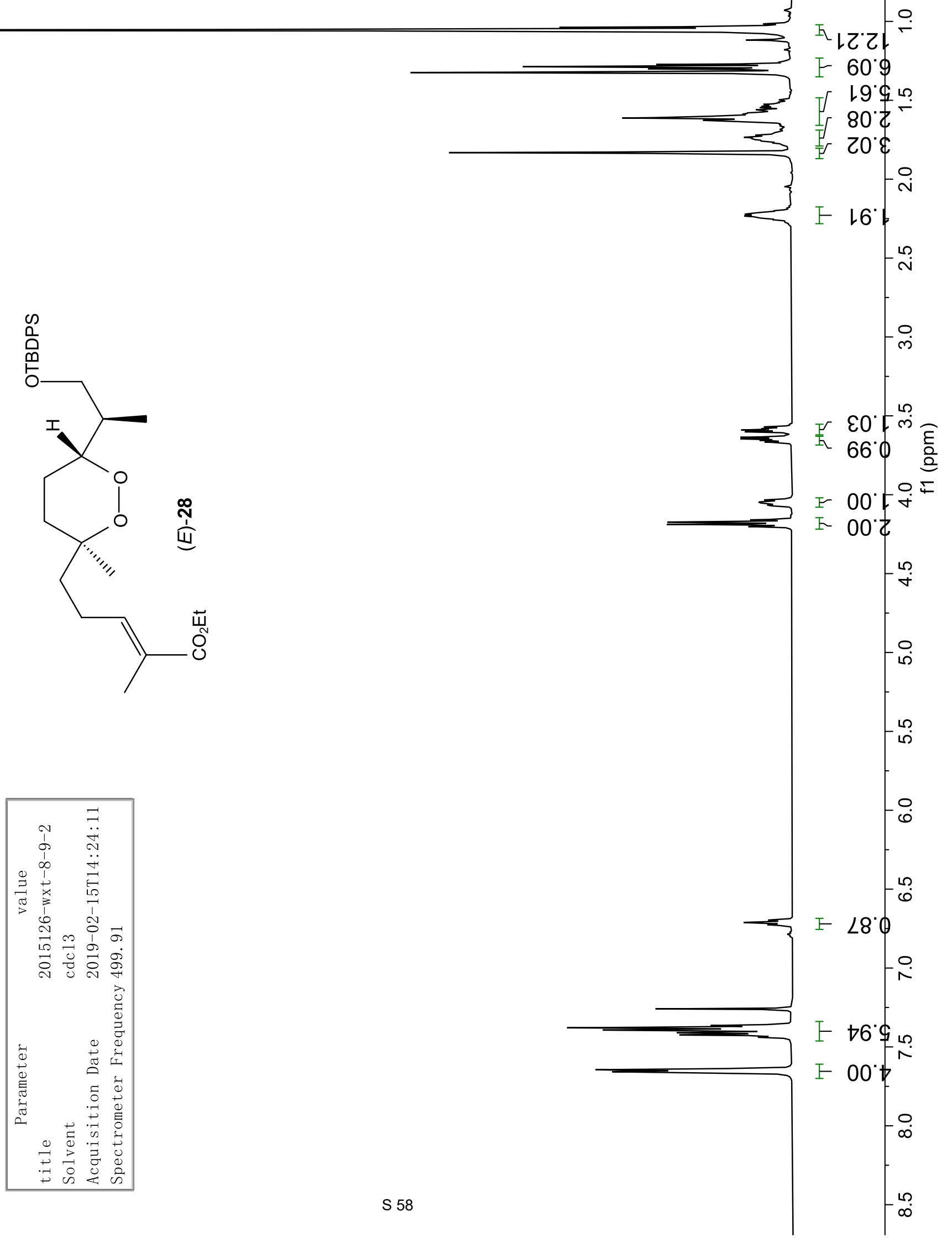
ع969'9 ר

ルレL'98SZL' 9 $\angle \varepsilon 9 \varepsilon^{\prime} \angle$ $\downarrow \angle 9 \varepsilon^{\circ} \angle$ $\angle 8 \angle E^{\circ} \angle L$ LE6E $L-$ カOレ゙ オレレ゙ $\angle \nabla 乙 \nabla^{\circ}$ 06Et $L$

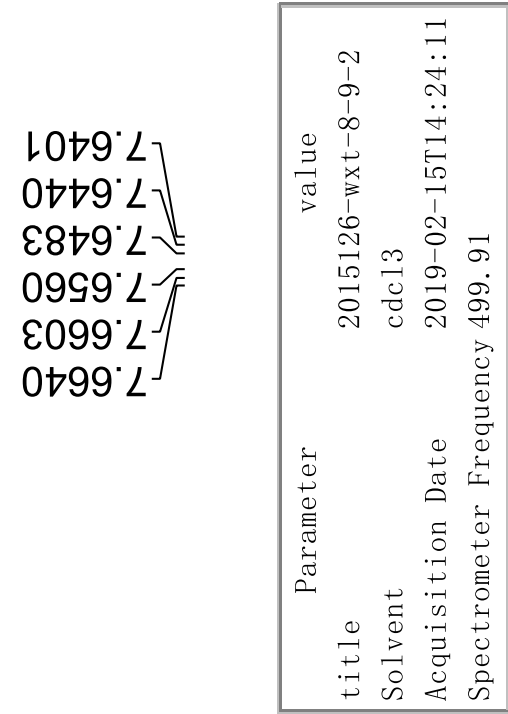
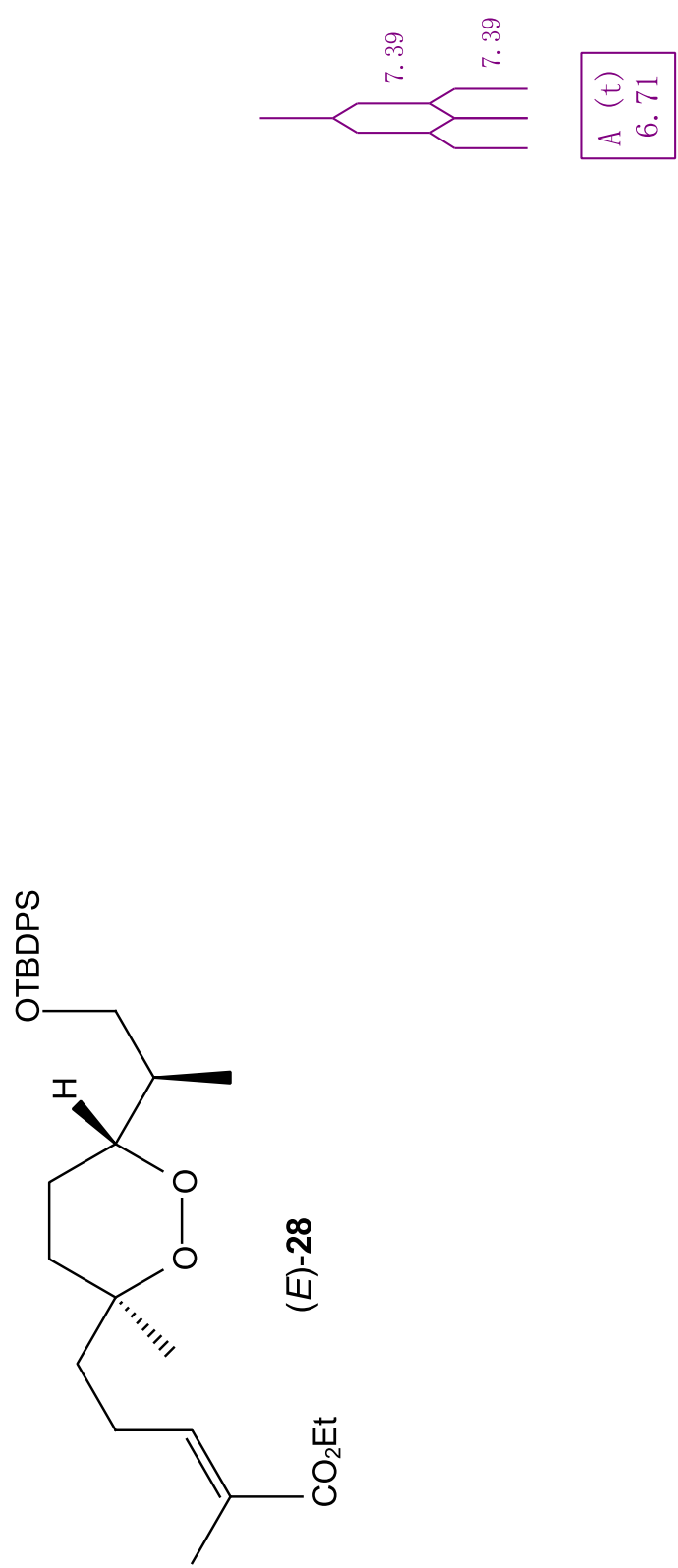
$00 \angle G^{\circ} \varepsilon \sim$ $\varepsilon 189^{\circ} \varepsilon-$ Z069' $\varepsilon-$ Sl09' $\varepsilon^{-}$ $6989^{\circ} \varepsilon-$ $29+9^{\circ} \varepsilon-$ Z999 $\varepsilon-$ $9999^{\circ} \varepsilon^{-}$
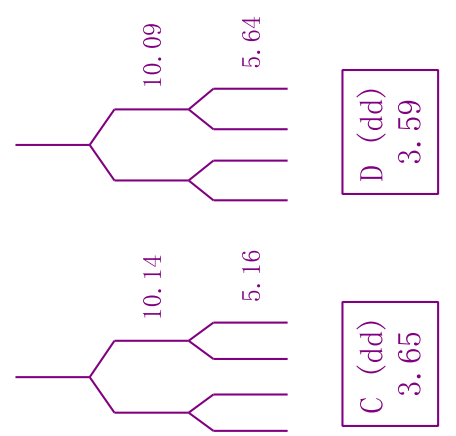

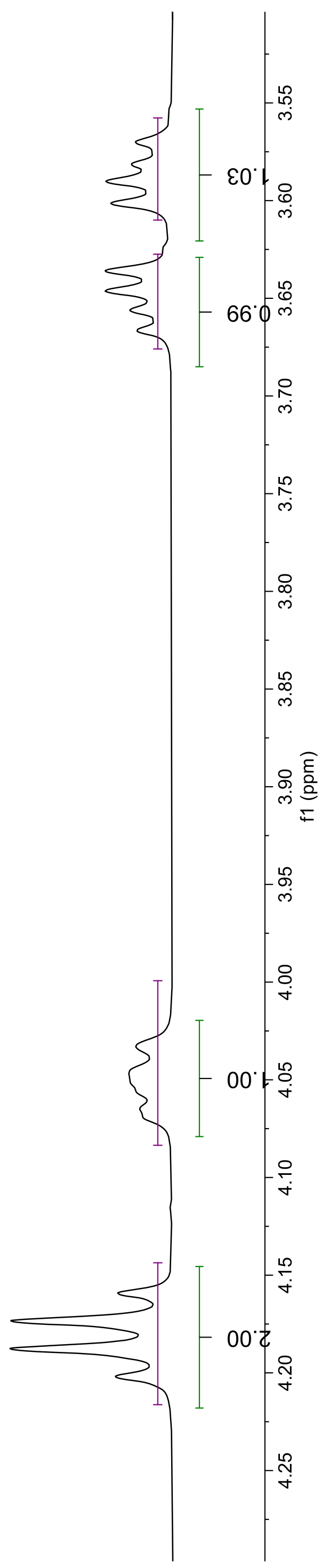

E6Gレー $9 \varepsilon \angle L{ }^{\circ} \nabla-$ 8L8L' เZOZ゙

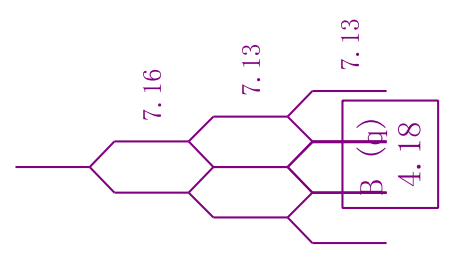




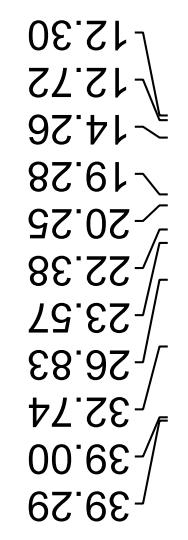

$$
\begin{aligned}
& \text { 乙†.09- } \\
& \text { २९.99- }
\end{aligned}
$$

$8 \varepsilon^{\circ} 6 L-$
$80^{\circ} \mathrm{Z8}$

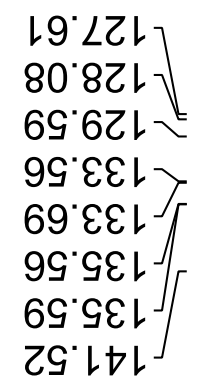

$\infty$
0
0
0
0

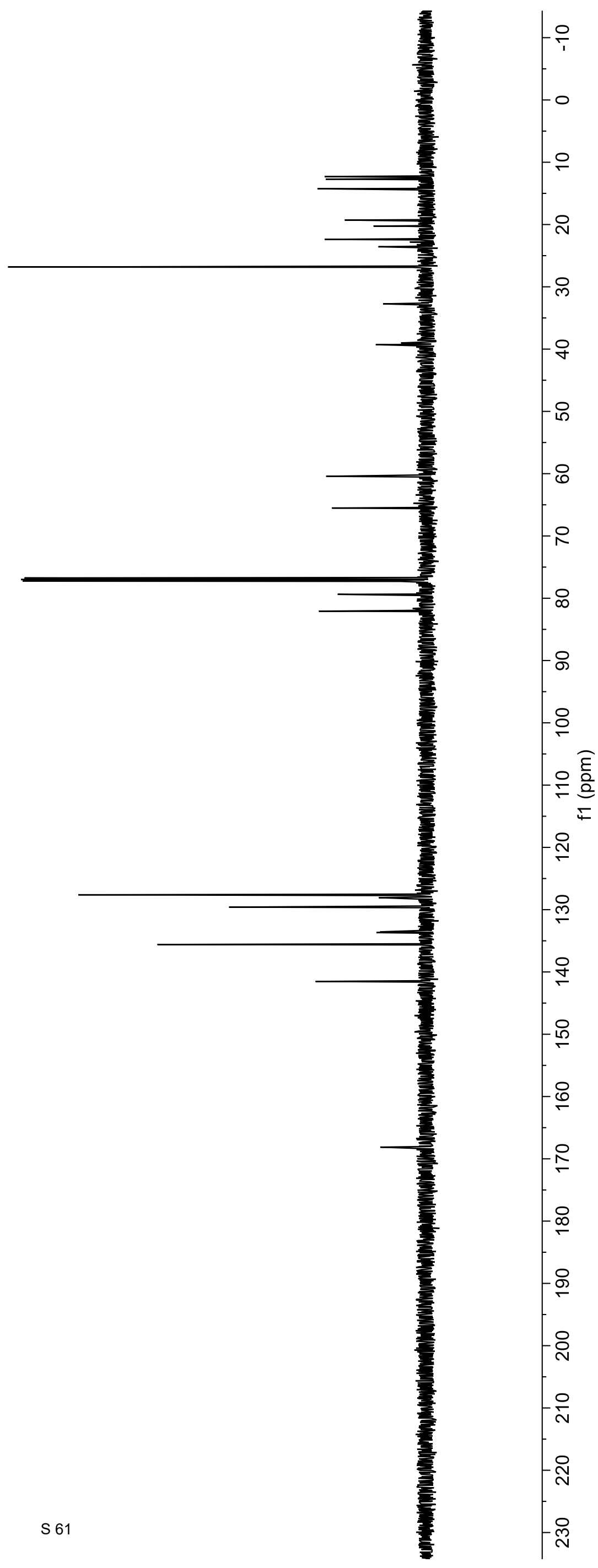




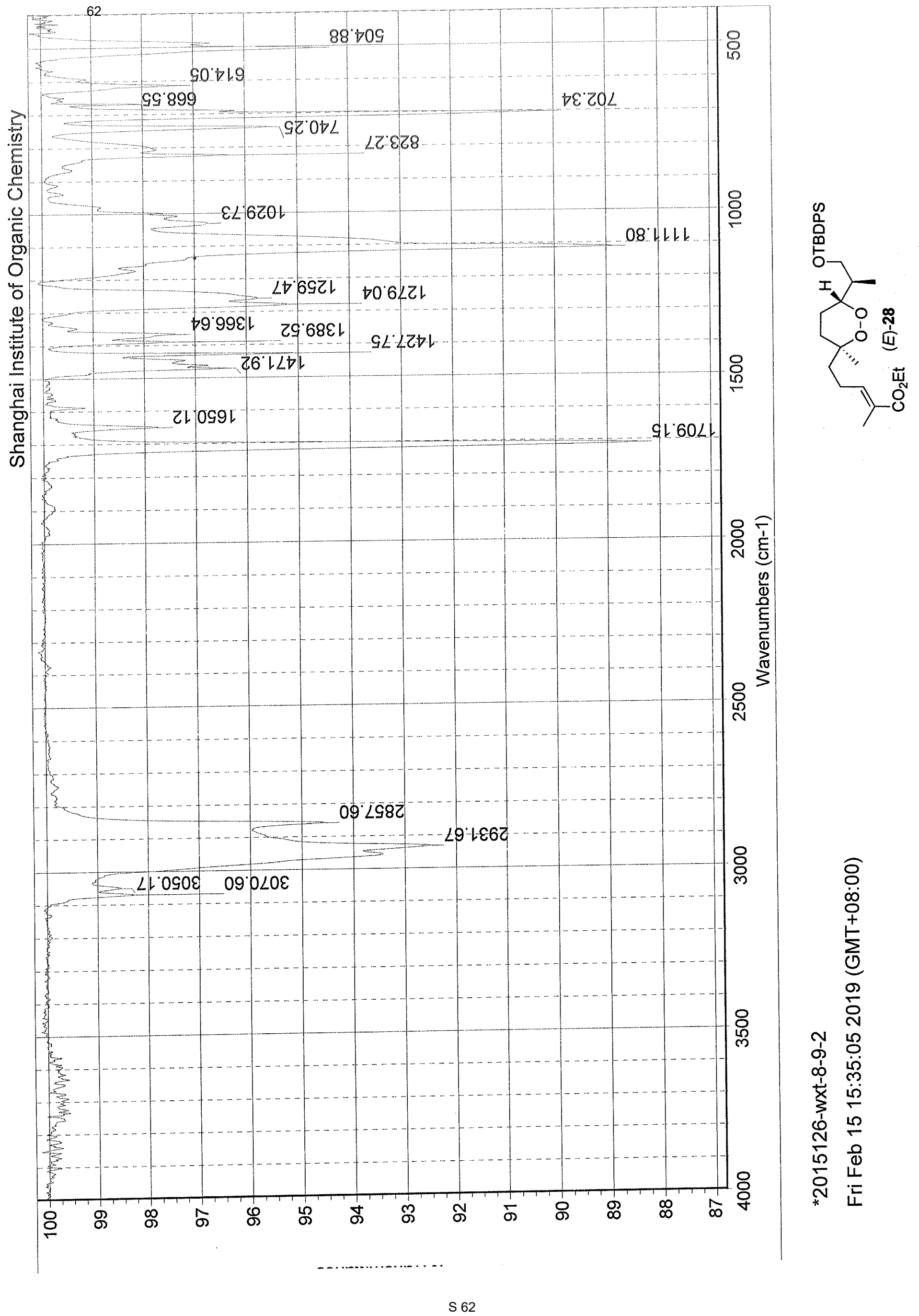




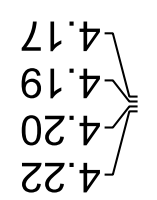

$\nabla 9^{\circ} L$ $\nabla 9^{\circ}<$ $\nabla 9^{\circ} \mathrm{L}$ $79^{\circ}<$ $99^{\circ}<$ $59^{\circ}<$ $99^{\circ} \angle$ $99^{\circ} \mathrm{L}$
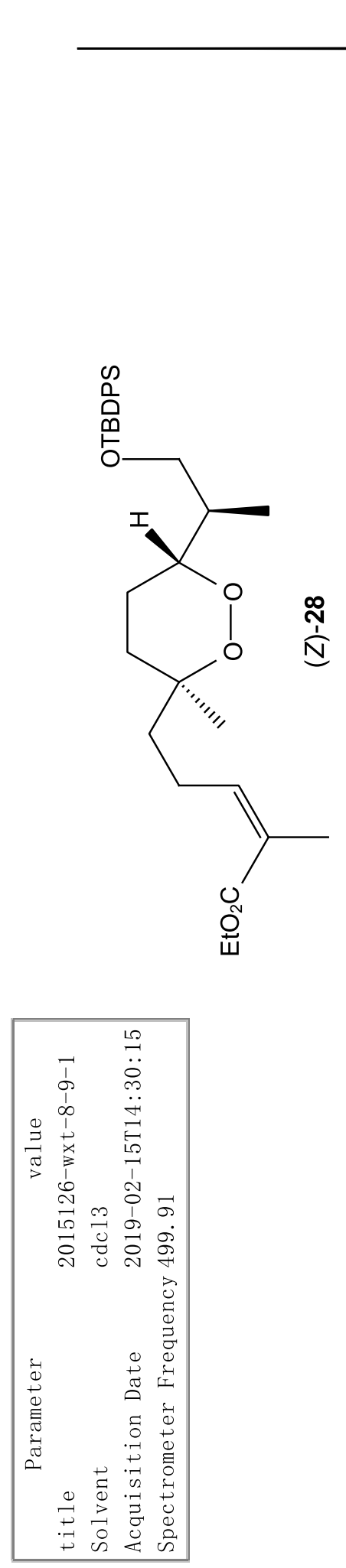

$\underbrace{}_{90} 2$

ᄃ $2 L S$

T) $\varepsilon 0.9$ 움

If $\varepsilon L \cdot z$

टा. .

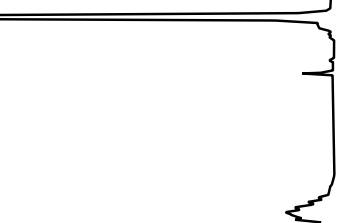

$1 \quad$

F 66.0 吹

F $10.2 \stackrel{\circ}{\mathrm{N}}$

울

을

要 $00^{\circ}$

$\Longrightarrow$ F $00 . F$

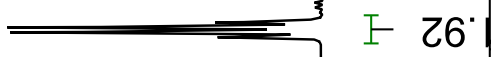

I 26.

F 68
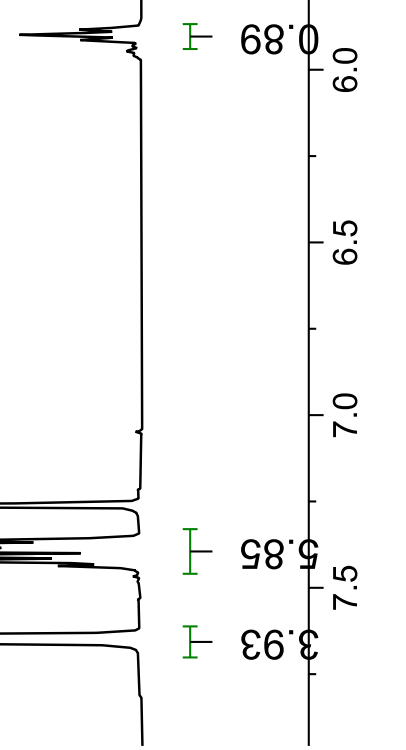
2เ88'

9†88 9

$2968^{\circ} \mathrm{G}$

$\angle 668^{\circ} 9^{-}$

9เL6

8ㄴ6.9
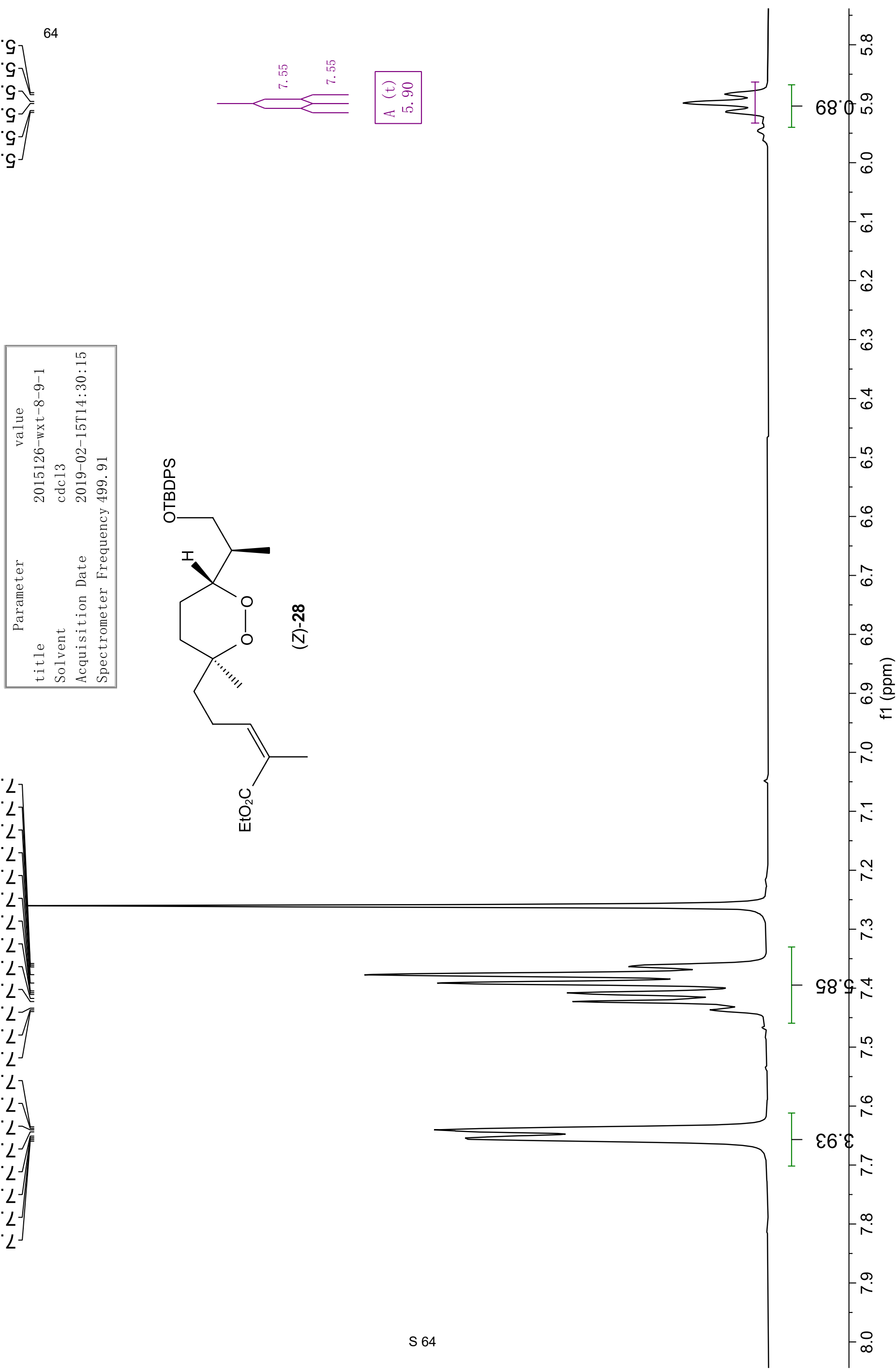

Z8SE 'L

เI9E $L$

$0 † 9 \varepsilon^{\circ} \angle$

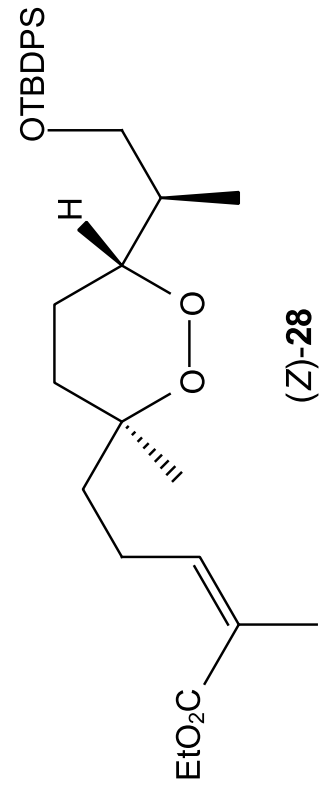

LLLE' $\angle$

EL6E $L$

$\angle \supset O \nabla^{\circ} \angle$

8LOt $L]$

80Lt L

GLレV

L乙てヤ' L

O†Eヤ" $L T$

89Et $L$

L6Et $\angle$

乙SE9 $L$

08E9 $\angle$

$\neg 0 \nabla 9^{\circ} \angle$

$\angle E \nabla 9^{\circ} \angle$

OLS9 L

$8 \varepsilon 99^{\circ} L$

S9S9 $L$

$\checkmark 699^{\circ} \mathrm{L}$ 
9199' $\varepsilon$ $0 \varepsilon \angle G^{\circ} \varepsilon-$ 8เ89' $\varepsilon-$ 乙ย6९ 'ย$6829^{\circ} \varepsilon \sim$ เ6ع9 $\varepsilon-$ 26+9. $\varepsilon-$ $\varepsilon 6 \varsigma 9^{\circ} \varepsilon^{-}$
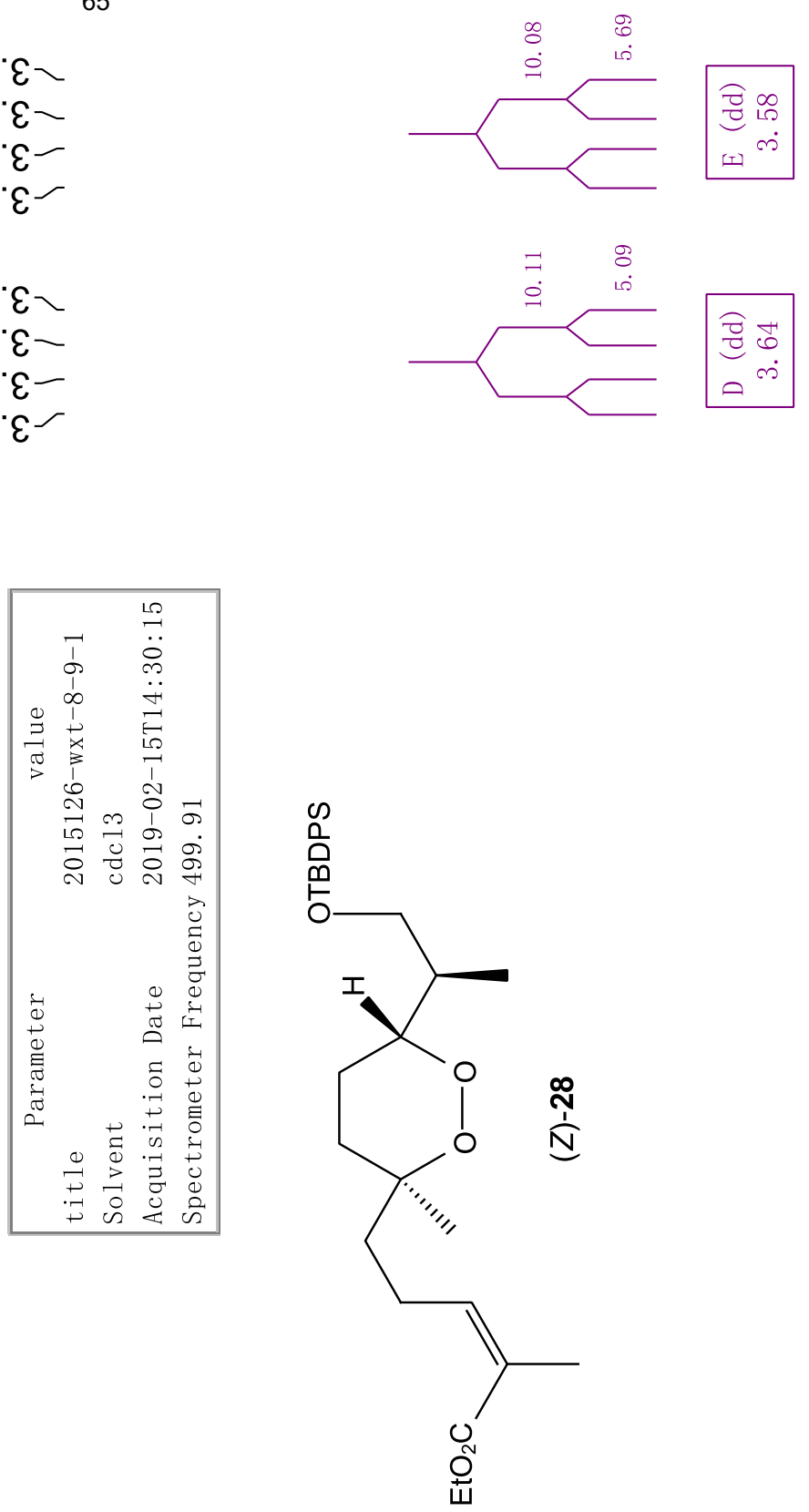

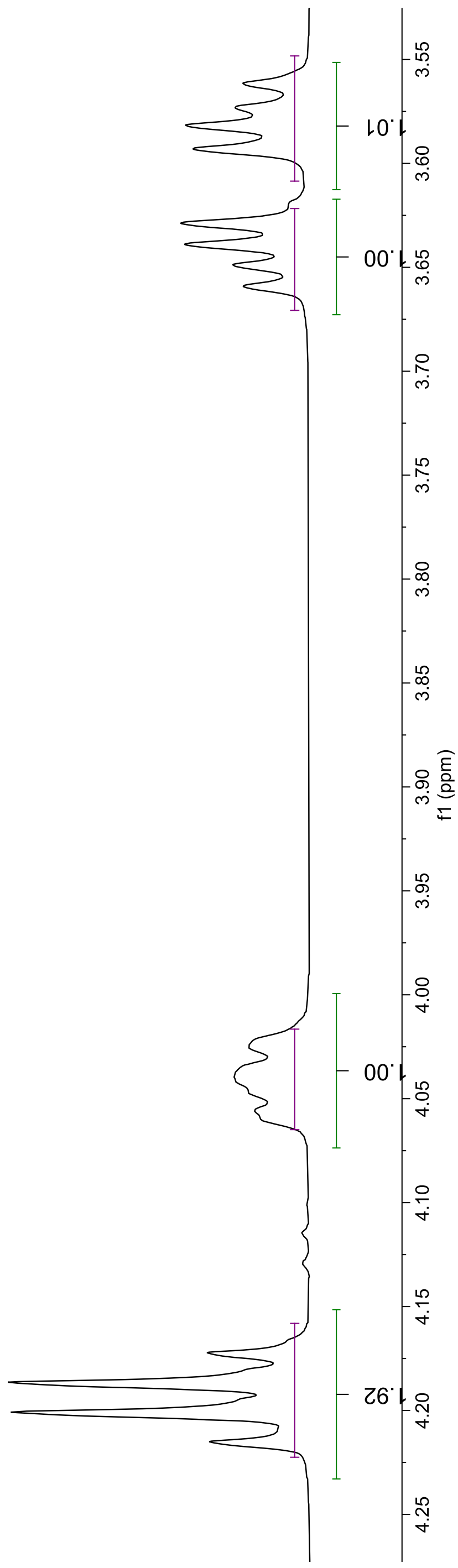

लं

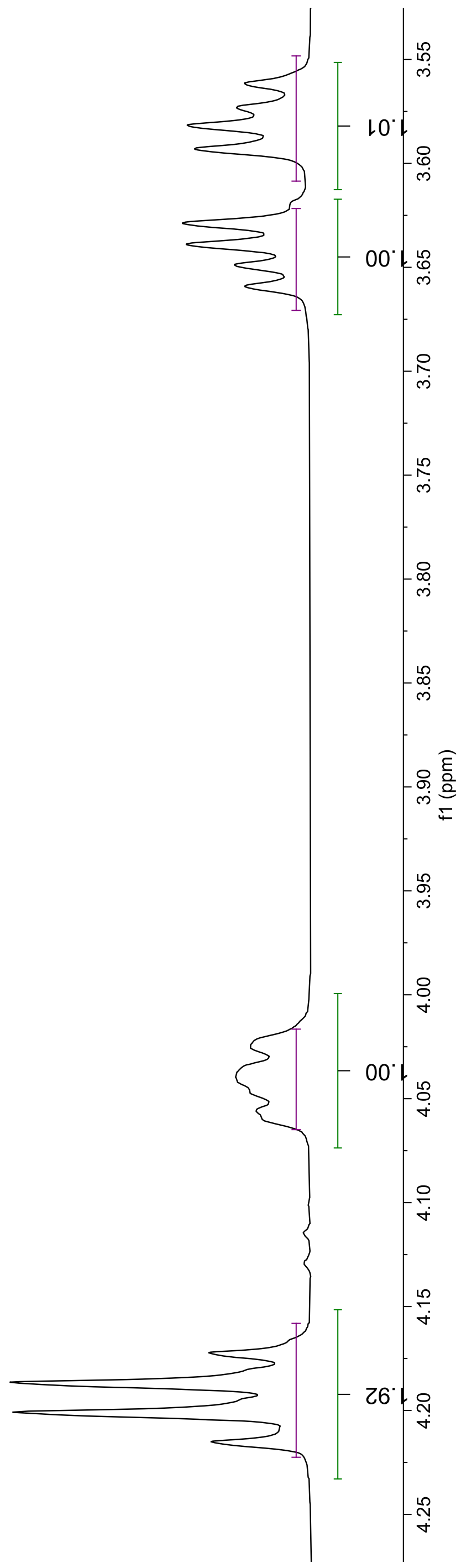

9ZLLレー 6981'bてレーでー †Sでヤ

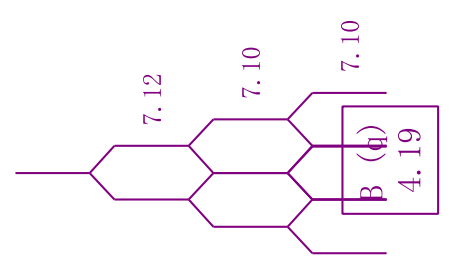


$\left.\begin{array}{l}\nabla L^{\circ} Z L \\ 6 Z^{\circ} \nabla L \\ 6 Z^{\circ} 6 L \\ \varepsilon Z^{\circ} 0 Z \\ 99^{\circ} 0 Z\end{array}\right]$

$\nabla \mathcal{G}^{\circ} \varepsilon 乙$

$\neg 9^{\circ} \varepsilon{ }^{\top}$

$\varepsilon\llcorner\nabla 乙$

$\neg 8 \mathrm{C}^{\circ}$

$19.2 \varepsilon$

ง $\varepsilon^{\circ} 6 \varepsilon-$

$$
\begin{aligned}
& \text { OL.09- } \\
& \text { E9'99- }
\end{aligned}
$$

$\varepsilon L^{\circ} 6 L-$

乙レ'Z8-
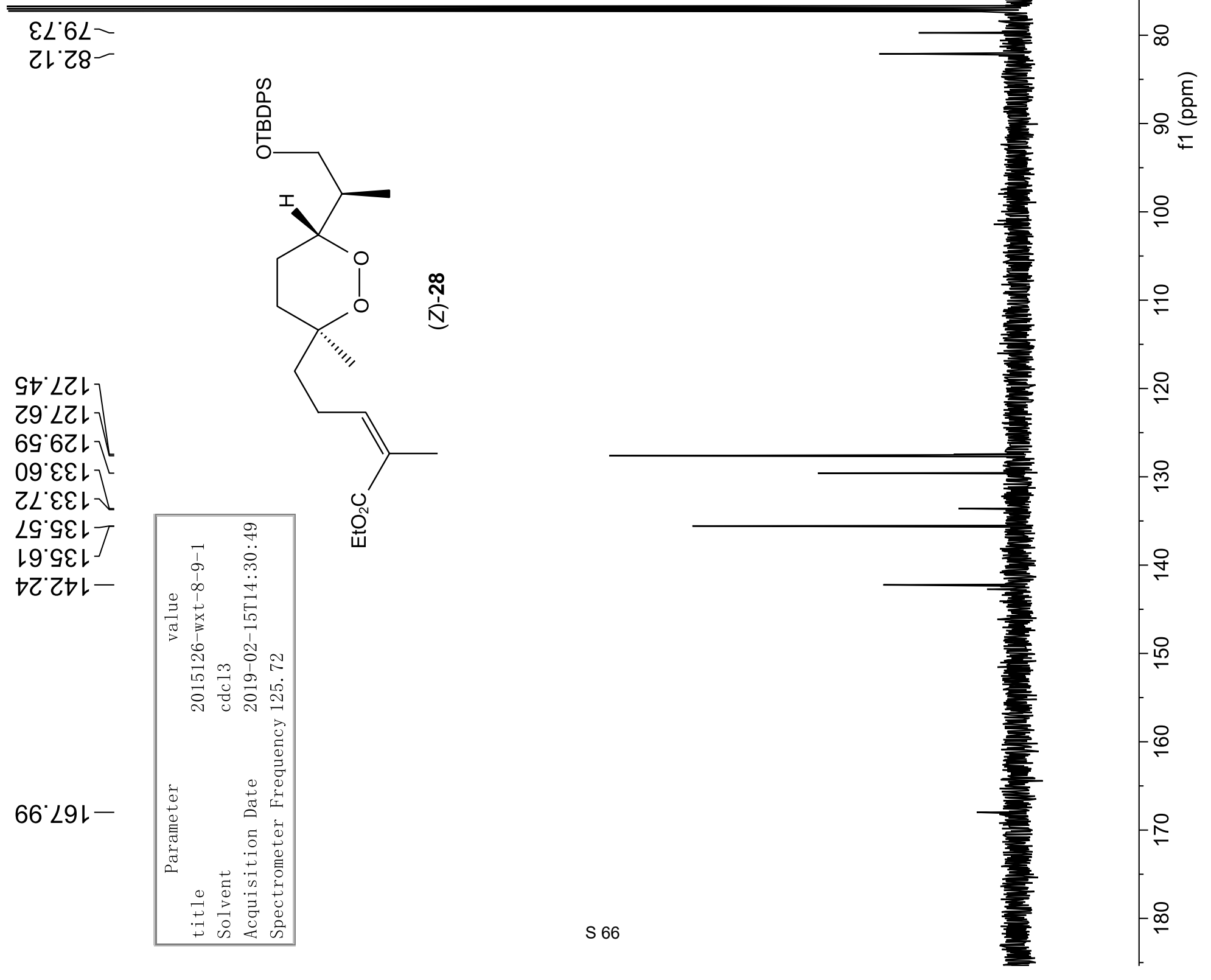


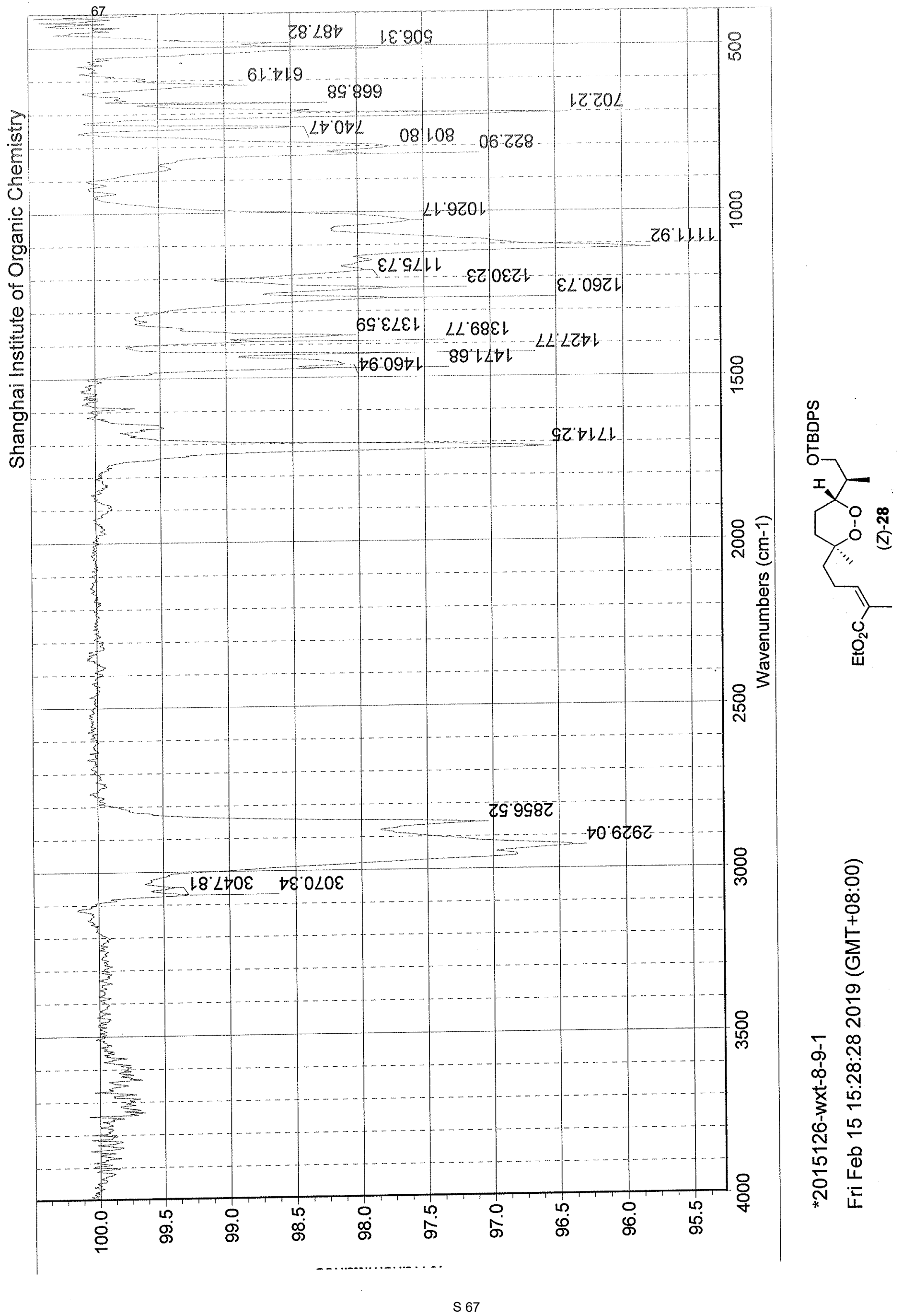




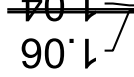

乙ย๋レ-

L9レ-

$66^{\circ} \varepsilon-$

S9. $L$

$99^{\circ} L$

$99^{\circ} L J$
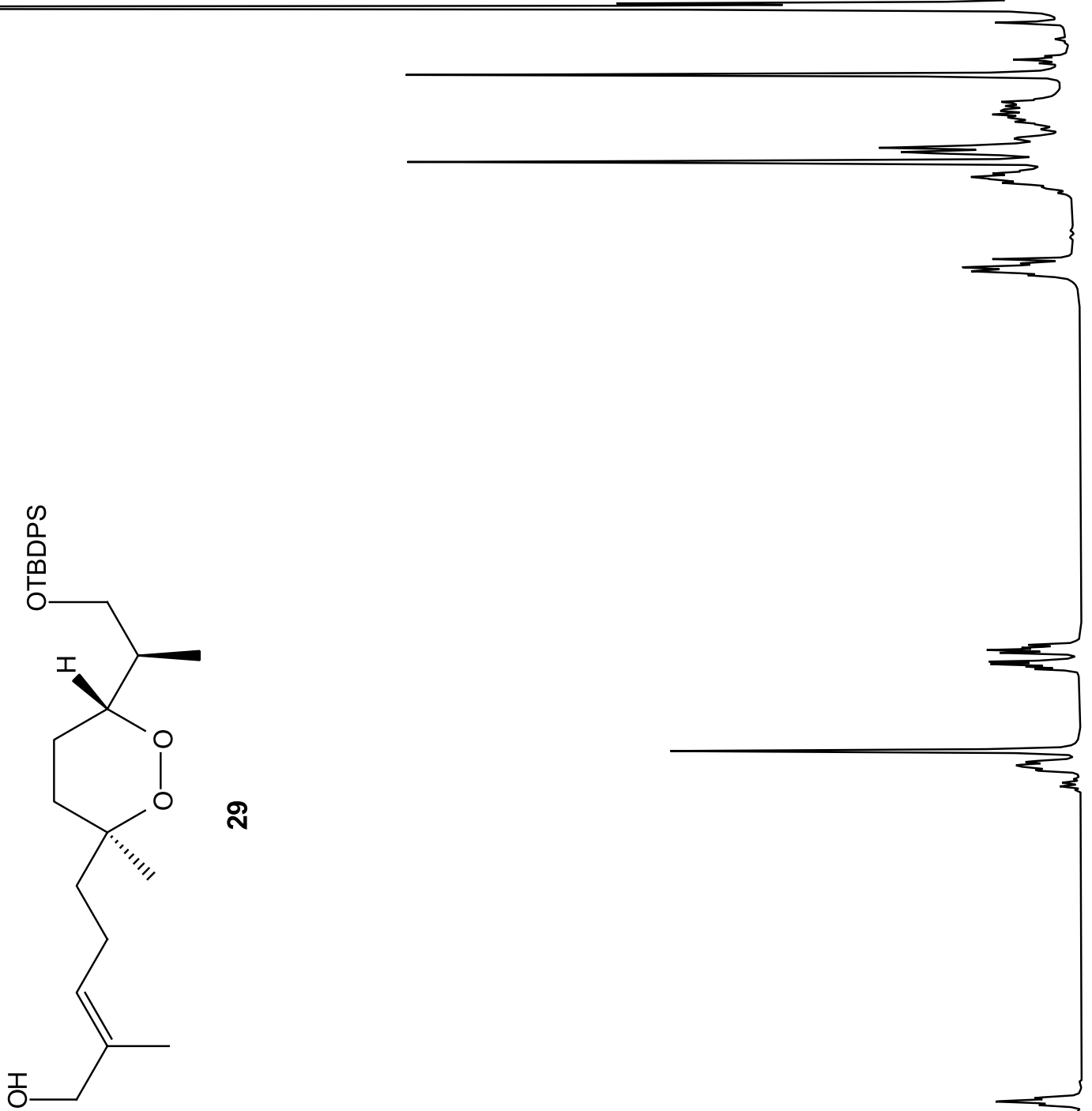

$\AA_{9121} \stackrel{\circ}{-}$

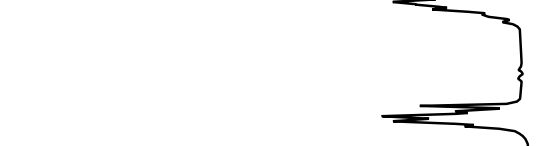

న

F $12: \% \stackrel{\mathrm{N}}{\mathrm{N}}$

ก

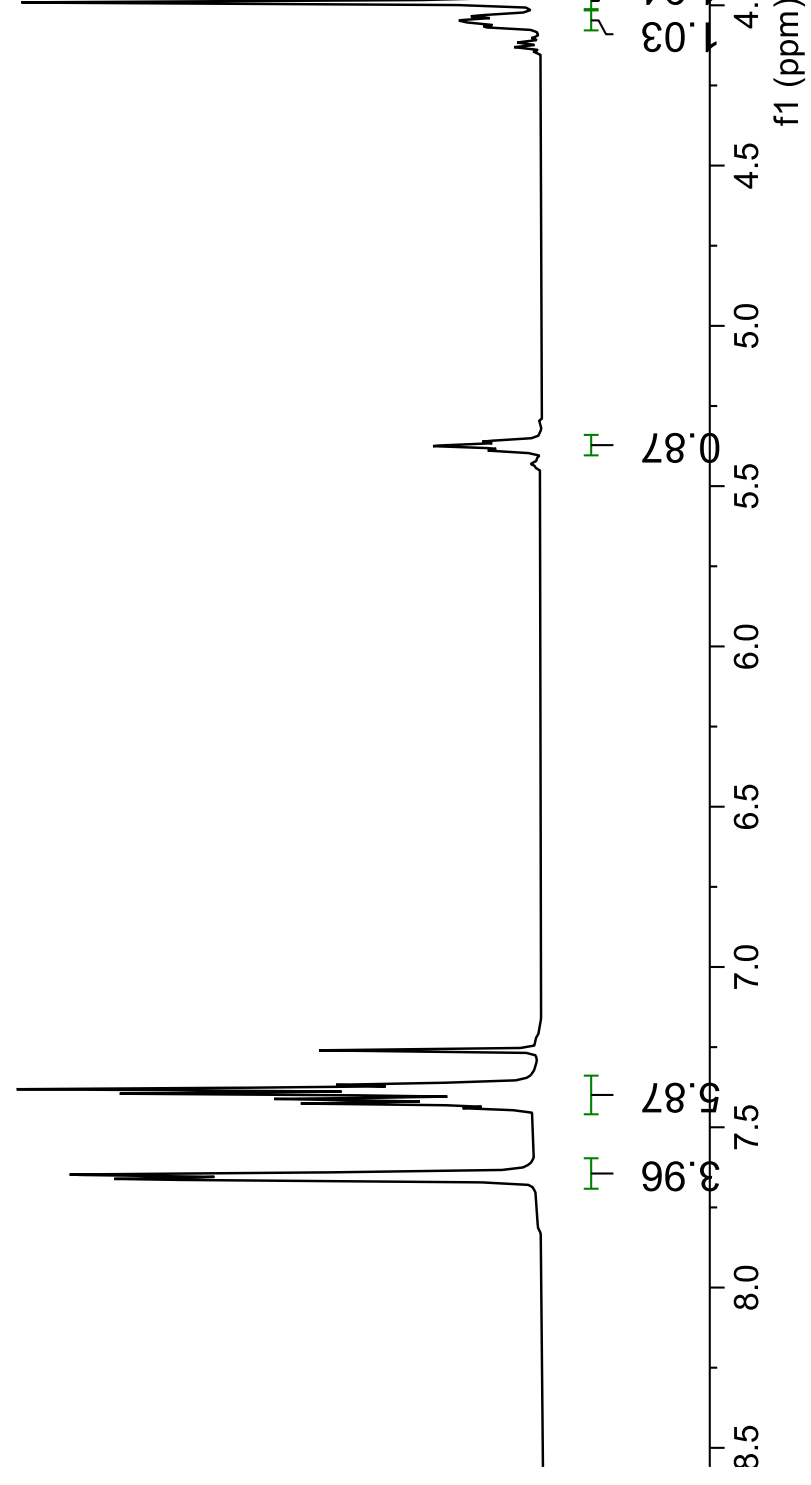



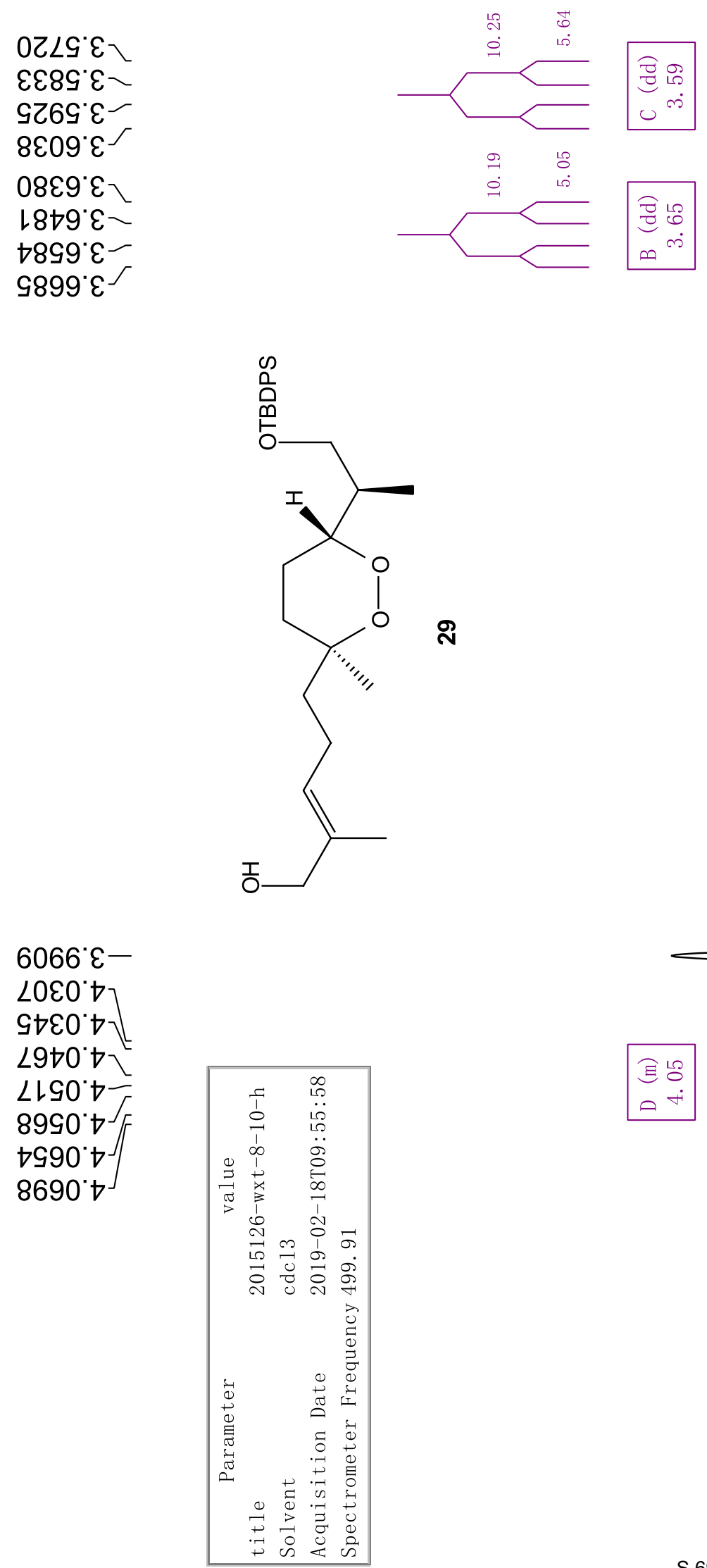

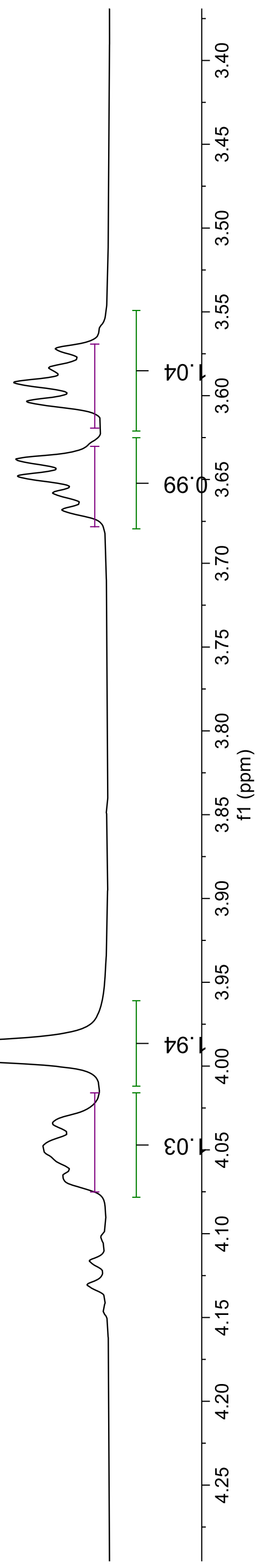




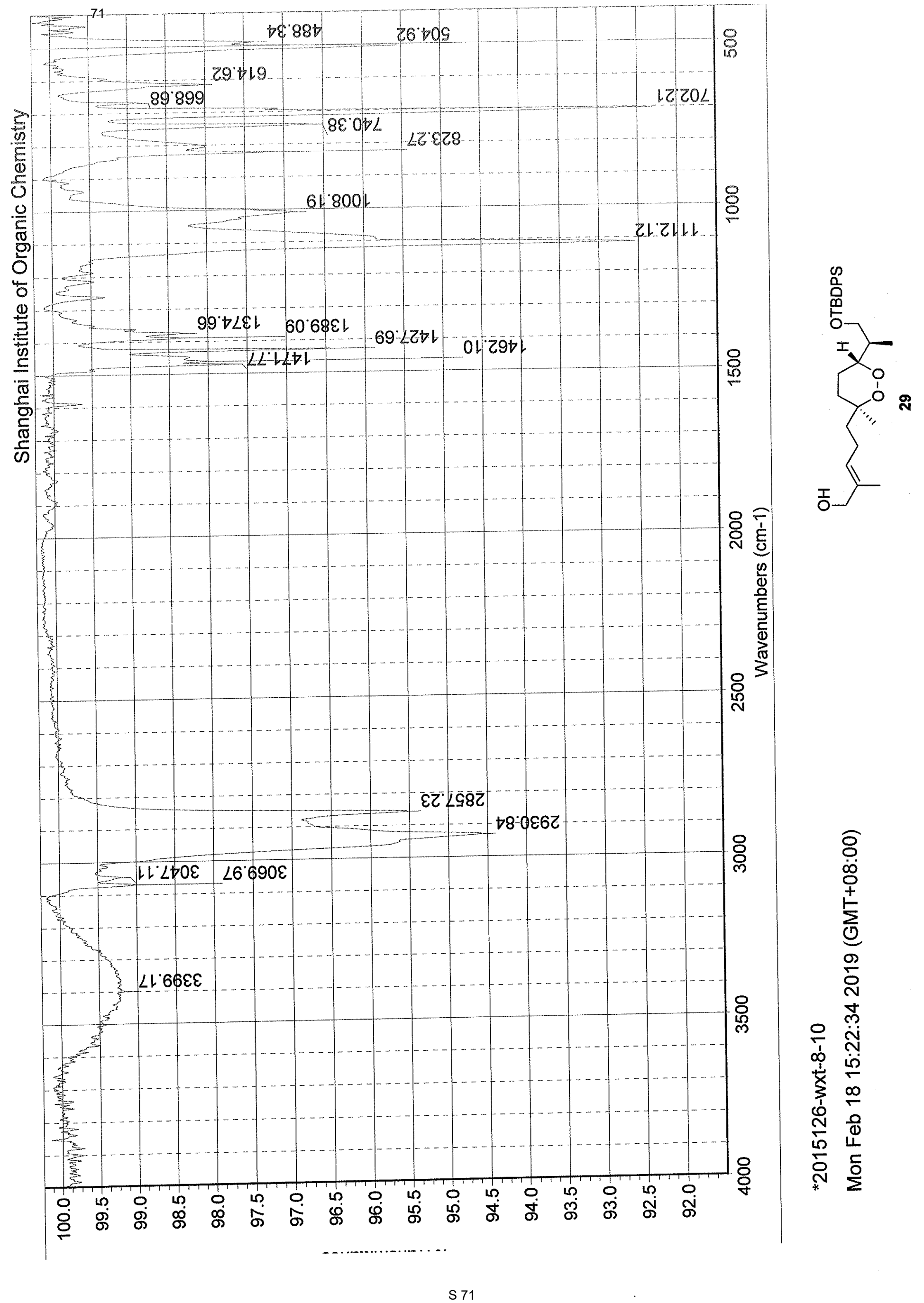




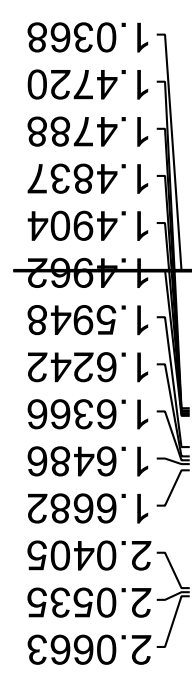

$6 เ 96^{\circ} \varepsilon-$

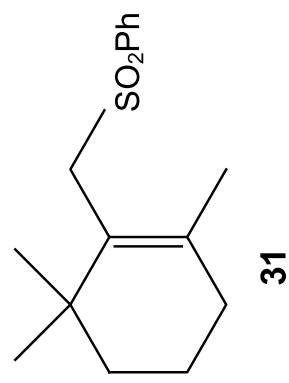

069Z $L$

06EG $\angle$

SOGG $L$

EtG $G^{\circ} L$

ZLGS $L$

L $\left.\angle 99^{\circ} \angle\right]$

ZOLG $L$

$8819^{\circ} L$

$\bar{m}$

$9616^{\circ} \mathrm{L}$

9Z26 $L-$

99Z6 L

8E\&6 $\angle$

$\angle 9 \varepsilon 6^{\circ} \mathrm{L}$

$68 \varepsilon 6^{\circ} \mathrm{L}$
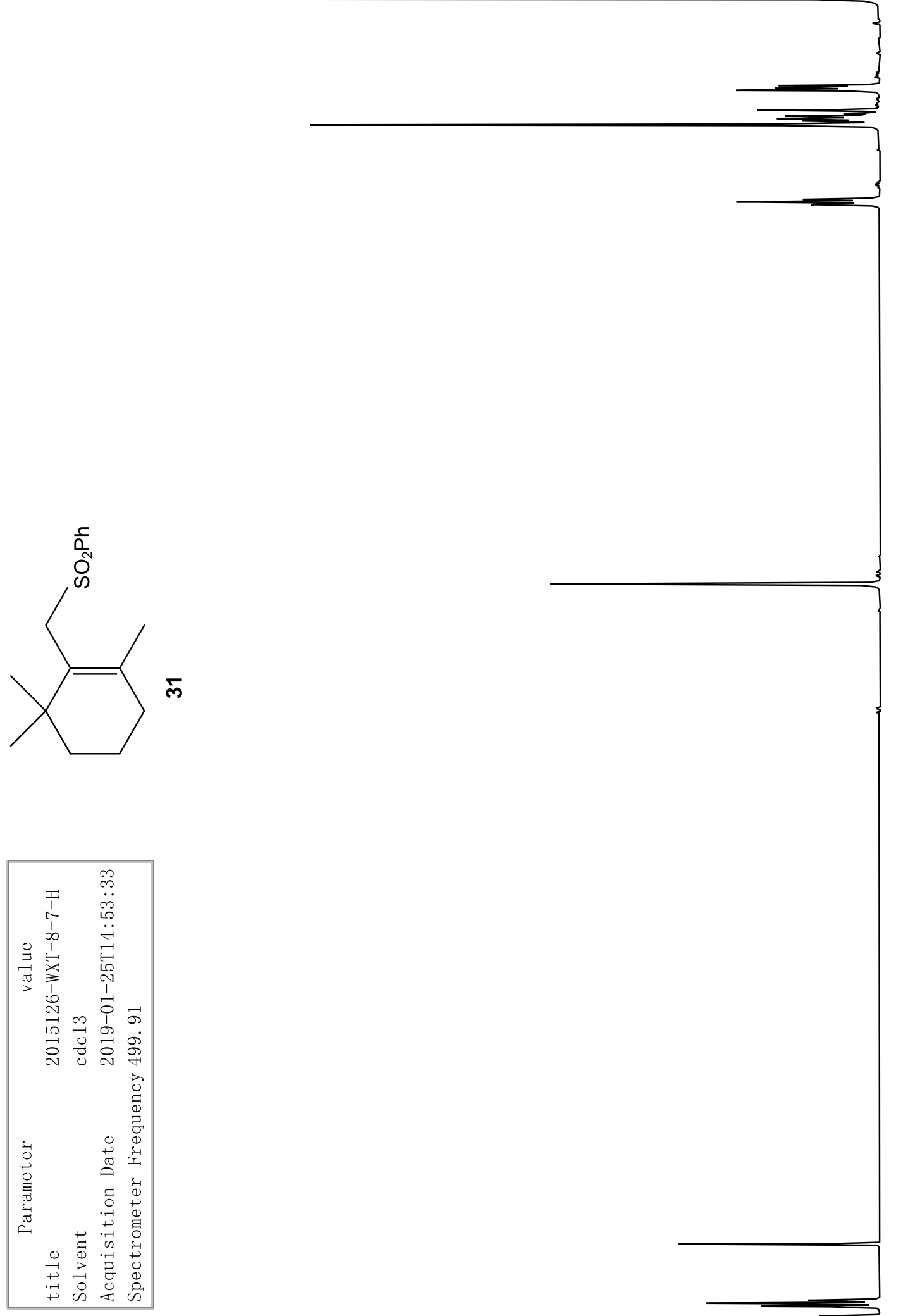

FEน $9 \stackrel{\circ}{-}$

ז $90^{\circ} 乙$

๘レ乙

一七 $\supset 0^{\circ} \varepsilon$

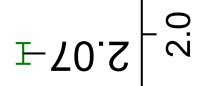

$=00^{\circ}$ 
069Z L-

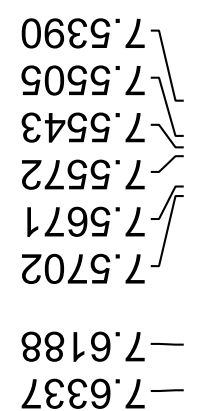

96L6' $\mathrm{L}$ 9ZZ6 L 99Z6 L L ${ }^{8 \varepsilon \varepsilon 6^{\circ} L} \bar{F}$ $\angle 9 \varepsilon 6^{\circ} \angle$ 68E6 ${ }^{\circ} \mathrm{L}$
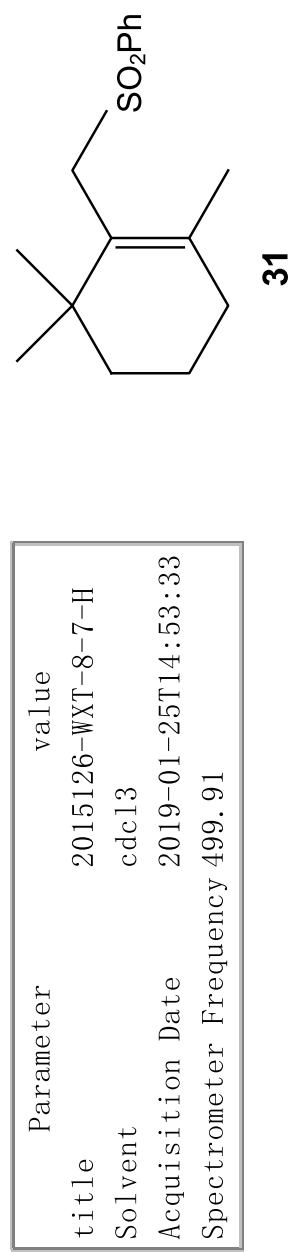

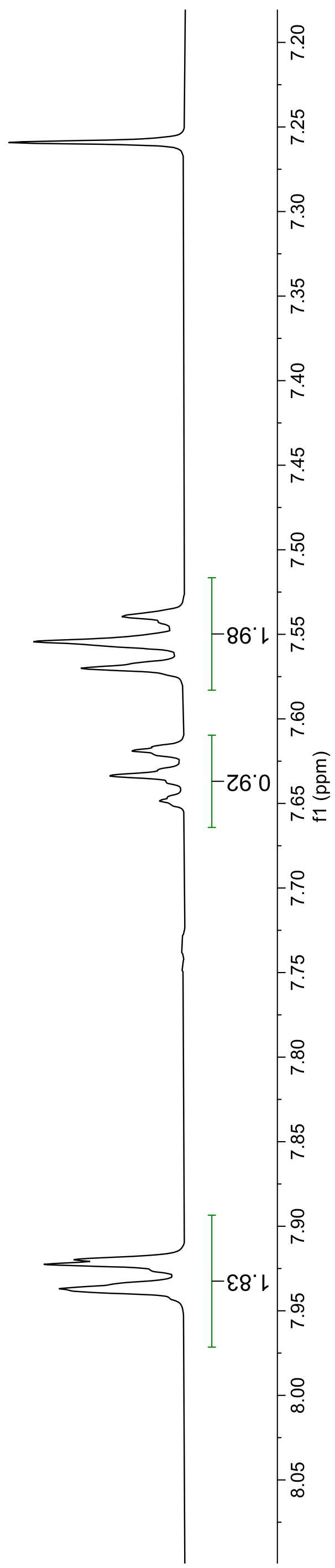




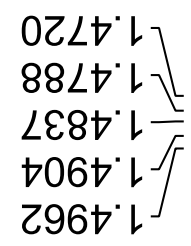

8†69' ᄂ

Z७Z9 9989 ᄂ 9879' ᄂ Z899.

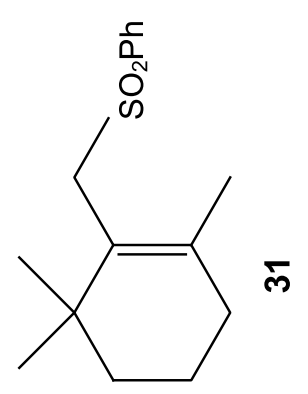

$\bar{m}$

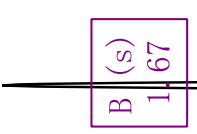


0
0
0
0
0

న్ల

$\varepsilon$ है $^{\circ}$
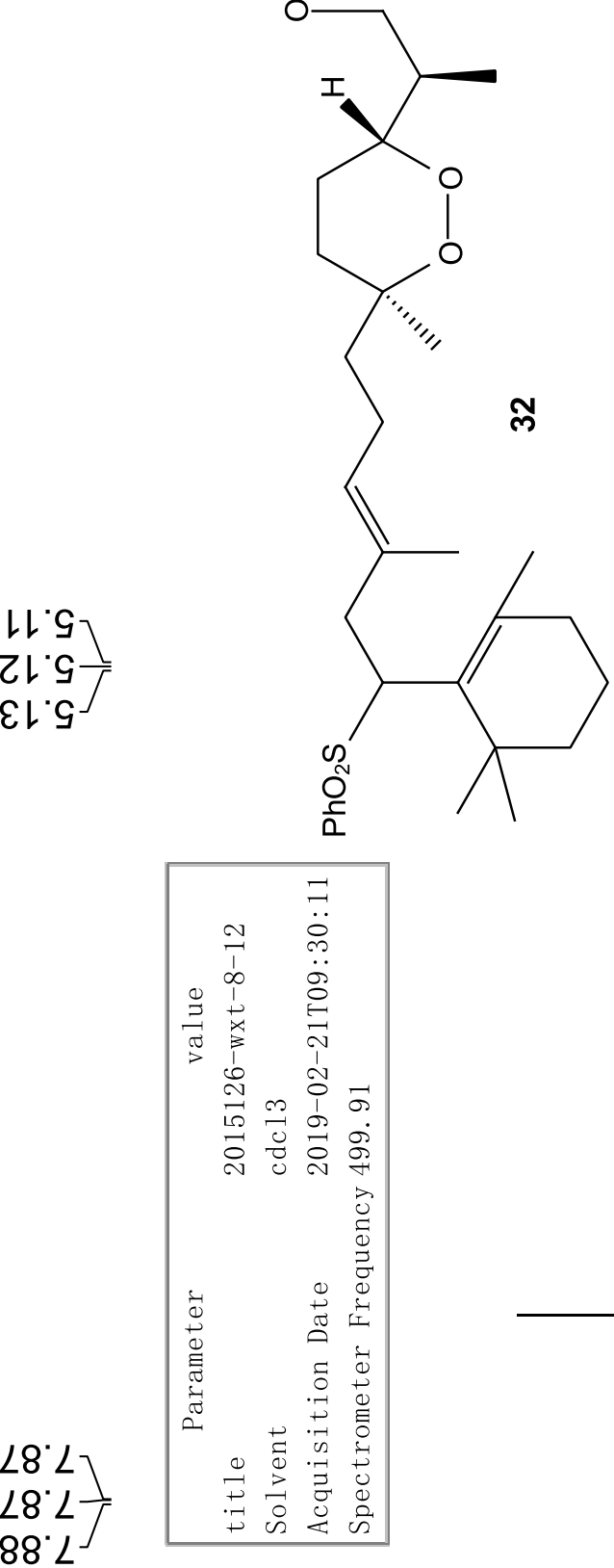

$0<\%$

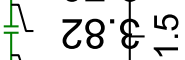

$92 \%$

h $9 t^{\circ}$

瓦 $\varepsilon 6^{\circ}=$

瓦 $\angle 0^{\circ} \circ$ 엇

t 1 .

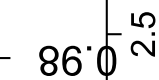

$=86^{\circ} 0^{\circ}$

$\Rightarrow$ F 960 .

$=560^{\circ}$

$00 \%$

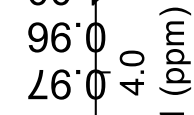

F $\angle 6 . \% \stackrel{\circ}{\circ}$

$+16.0^{\circ}$

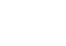


\&8SE ' L

OZ9\& $\angle$

LELE' $\angle$

$G \angle 8 \varepsilon^{\circ} \angle$

乙E0t $\angle]$ L

G90t L]

$\left.\angle 60 \nabla^{\circ} \angle\right]$

†Gレ゙ $L$

EOZち L

レLZヤ L

G†Et"L广

LE6t $L$

$0867^{\circ} L$

$0009^{\circ} \mathrm{L}$

$\nabla 809^{\circ} \mathrm{L}$

E†LG $L$

L9LG'L

$\nabla \nabla 2 G^{\circ} L$

66Z9 ${ }^{\circ}$

LZE9 $L$

ESE9 $L$

L8E9 $\angle$

$\angle O t 9^{\circ} \angle \mathbb{E}$

$\angle E \nabla 9^{\circ} \angle$

G8t9 ${ }^{\circ}$

†LS9 $L$

$0799^{\circ} \mathrm{L}$

$\angle 999^{\circ} \mathrm{L}$ $n$
0
0
0

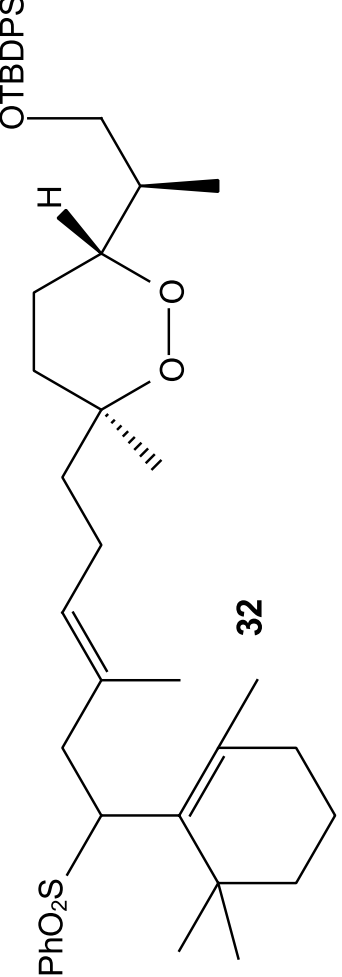

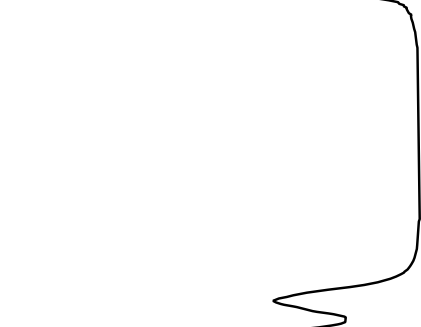
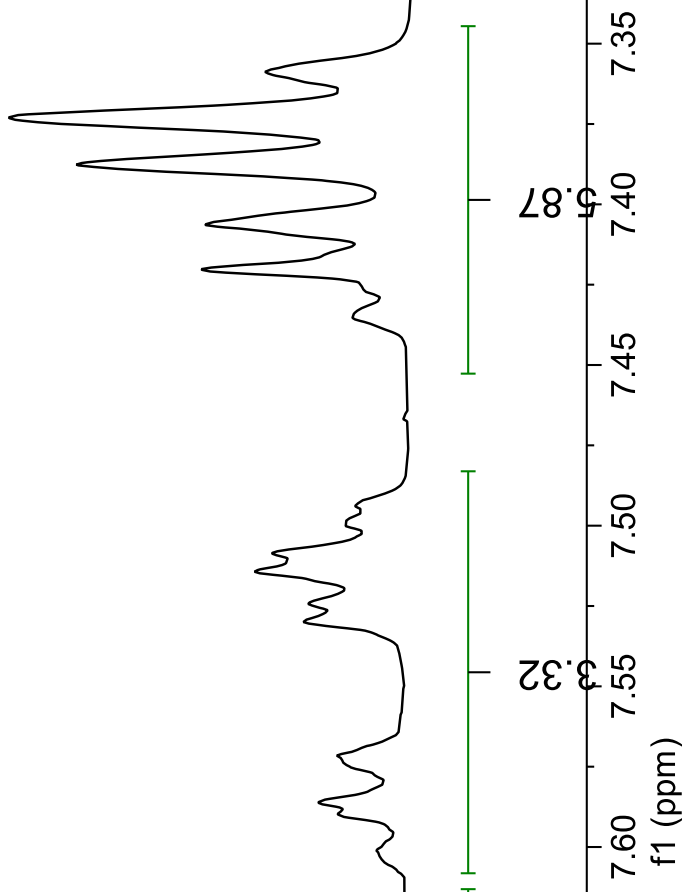

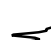
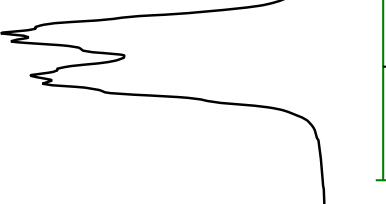

$-26 \& \stackrel{\circ}{\circ}$

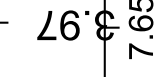
$\angle 698^{\circ} L^{\prime}$ $6888^{\circ} L^{-}$ 
$\angle E \nabla G^{\circ} Z \backslash$ L9G9'Z8ZLG'Z$\angle 989^{\circ} \mathrm{Z}^{\top}$

¿St6 Z E696' $\angle \forall \angle 6^{\circ} Z^{-}$ $2886^{\circ} \mathrm{Z}^{\top}$

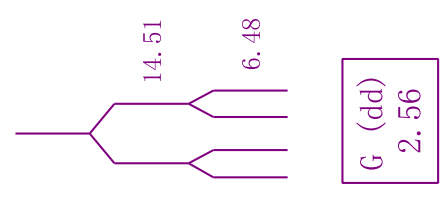

c
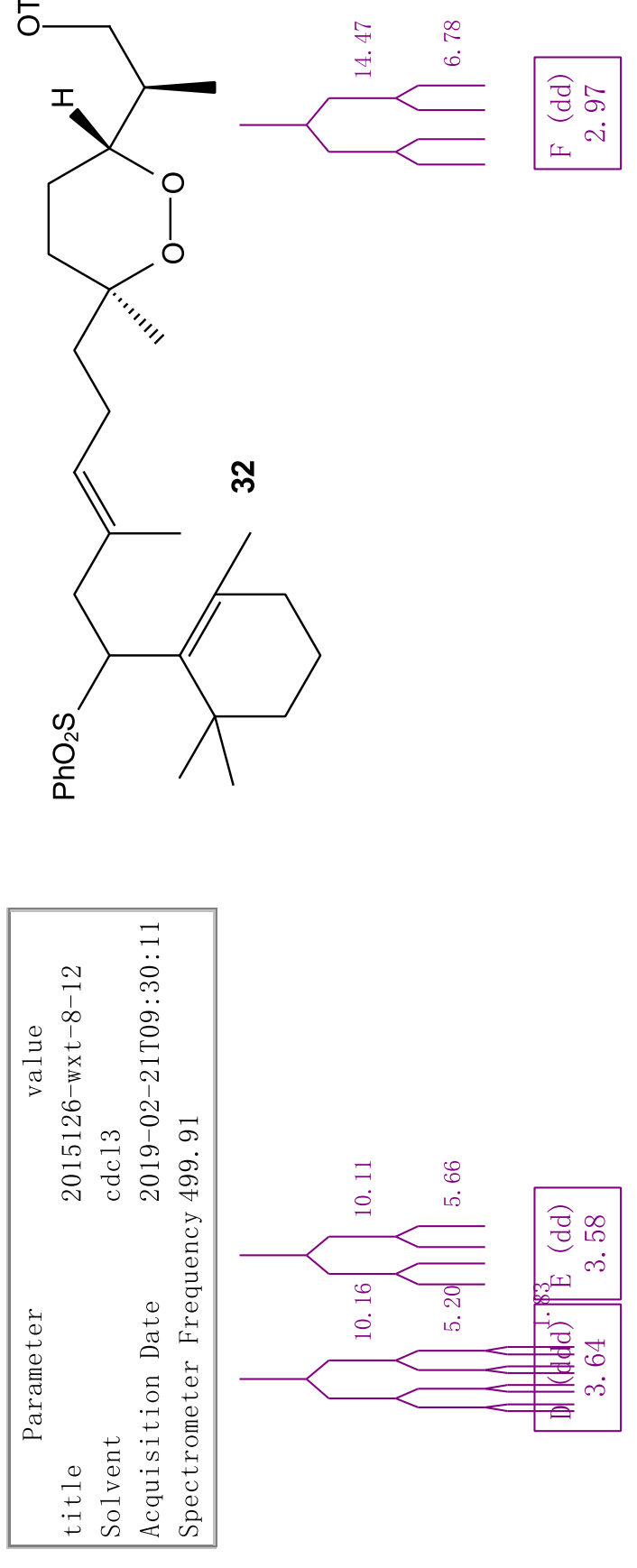

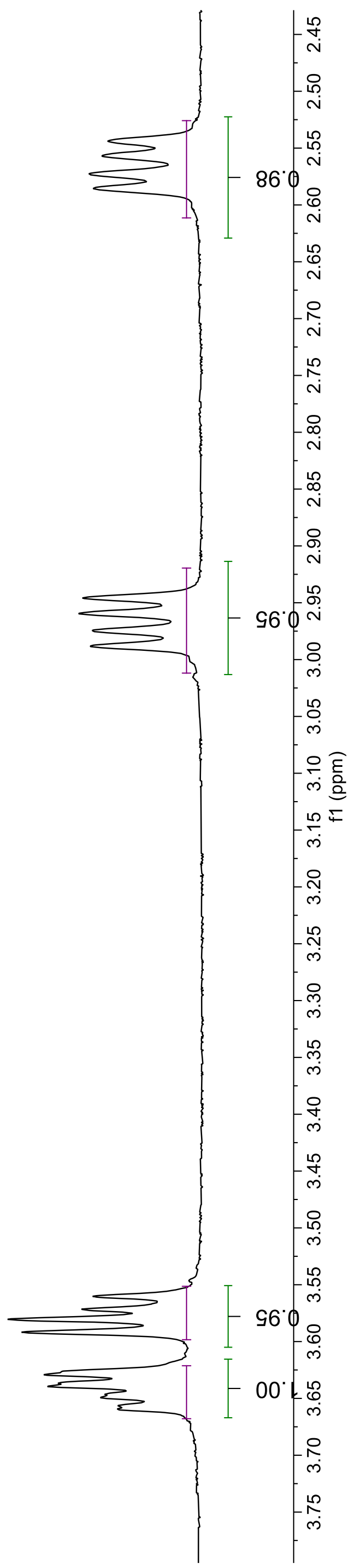


乙Lてレ OE'GL $206 \mathrm{~L}$ เ 6 เ $\left.6 L^{\circ} 0 Z\right]$ 99.乙 $\left.\neg \varepsilon^{\prime} \varepsilon 乙\right] E$ 99. $\varepsilon 乙-$ $98 \cdot 92$ 乙 LE. $8 Z-$ $6 L^{\circ} 62^{5}$ $\nabla \mathcal{G}^{\circ} \nabla \varepsilon \backslash$ $\varepsilon 0.9 \varepsilon-$ $\angle \varepsilon^{\circ} 6 \varepsilon$ $\varepsilon L 6 \varepsilon^{\top}$ $81 \cdot{ }^{\circ}$

L799 99.99

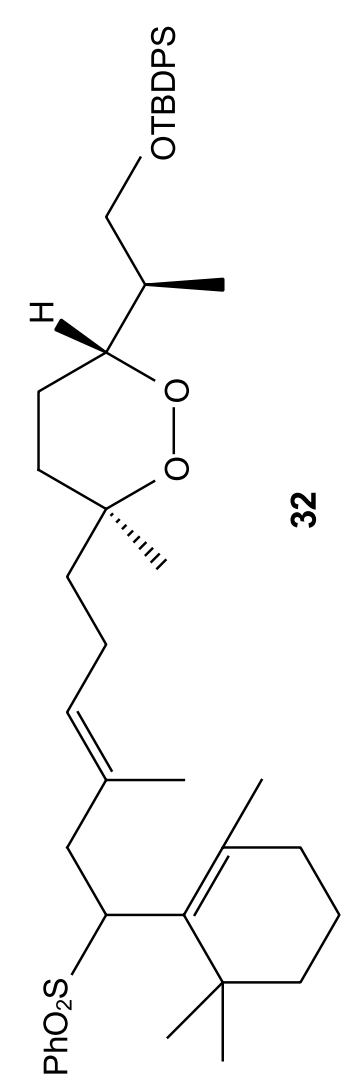

99.6Lר $60^{\circ} \mathrm{Z8}$ แ28
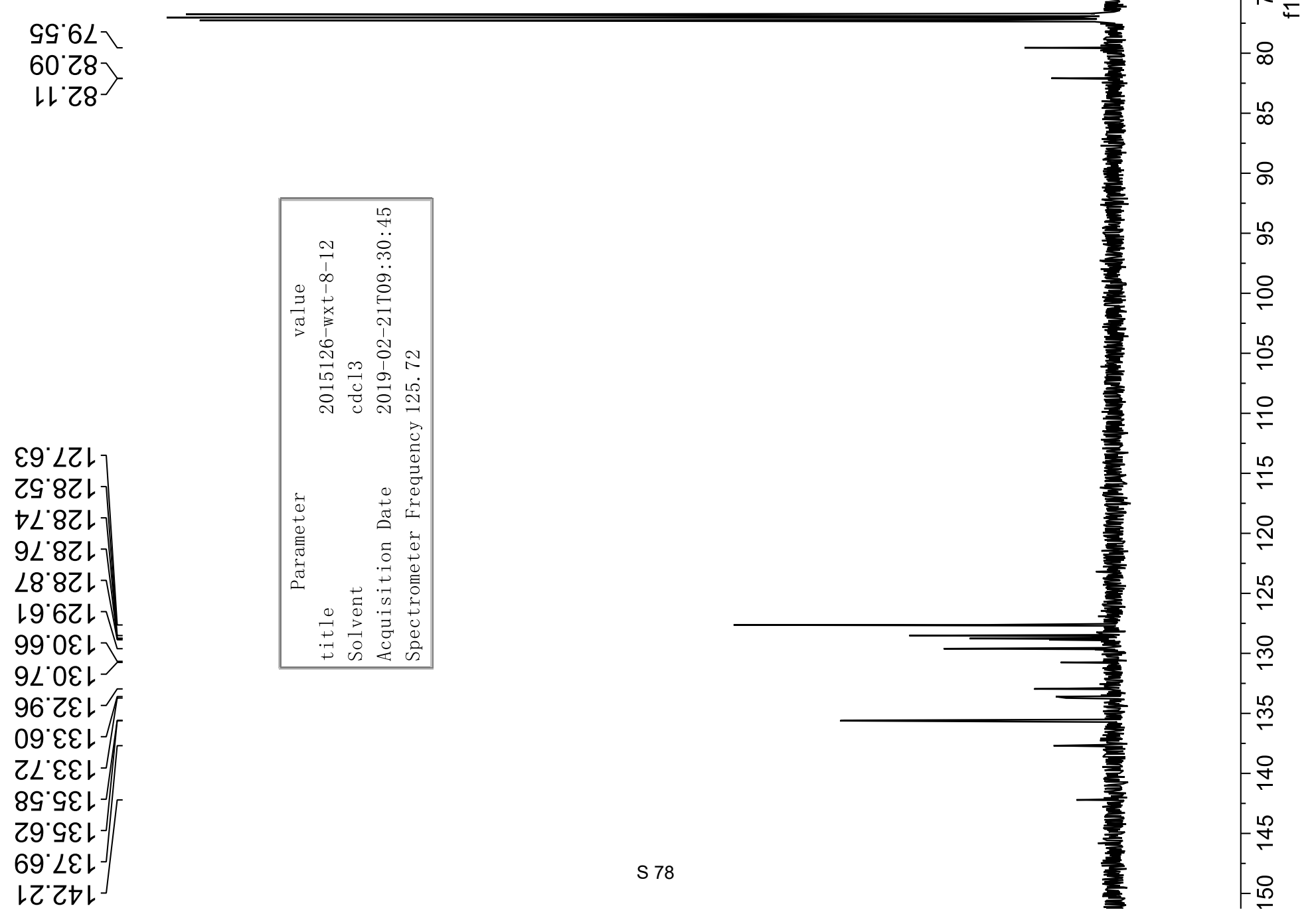


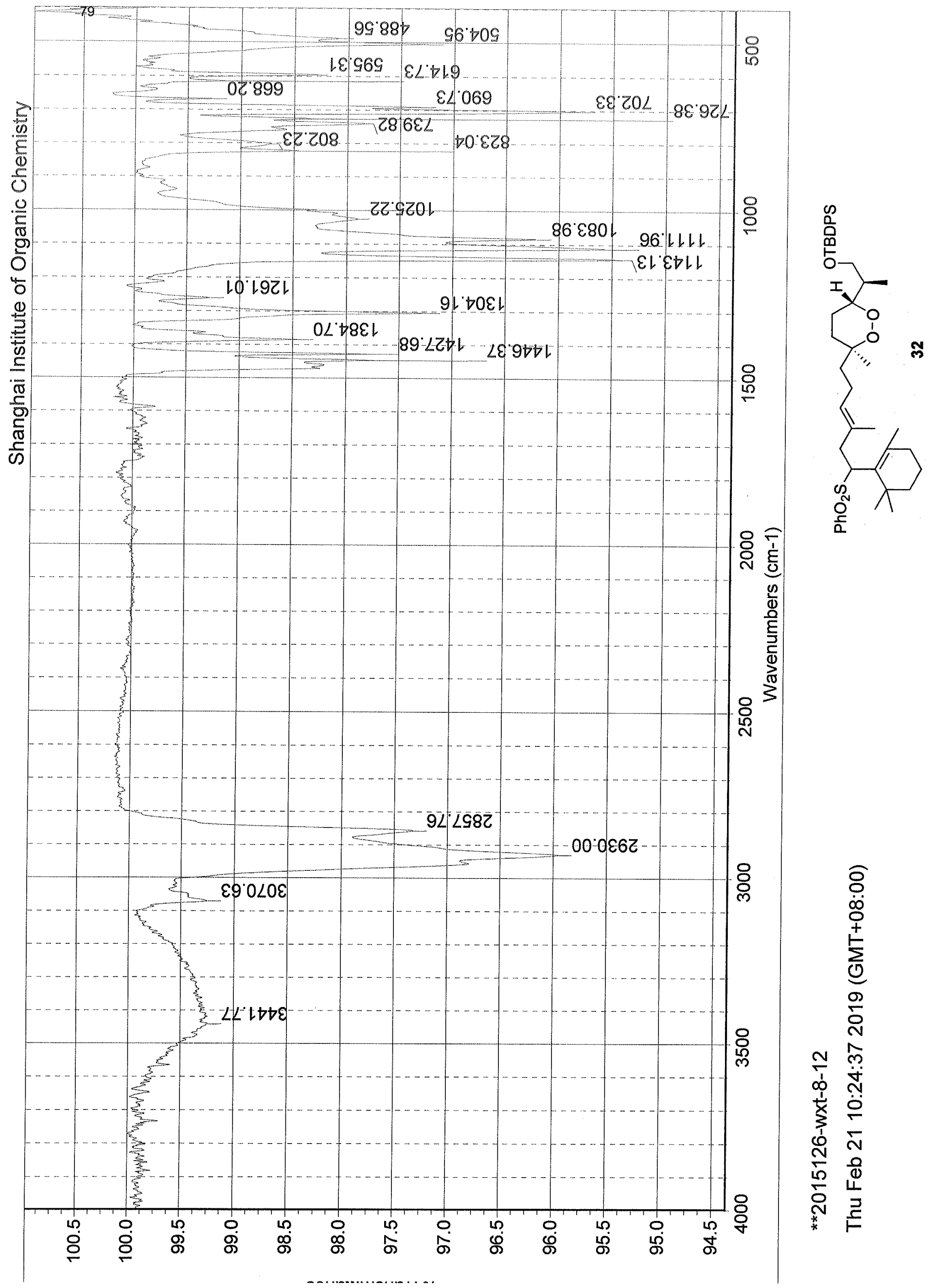


$\frac{66^{\circ} 0}{66^{\circ} 0^{-}}$
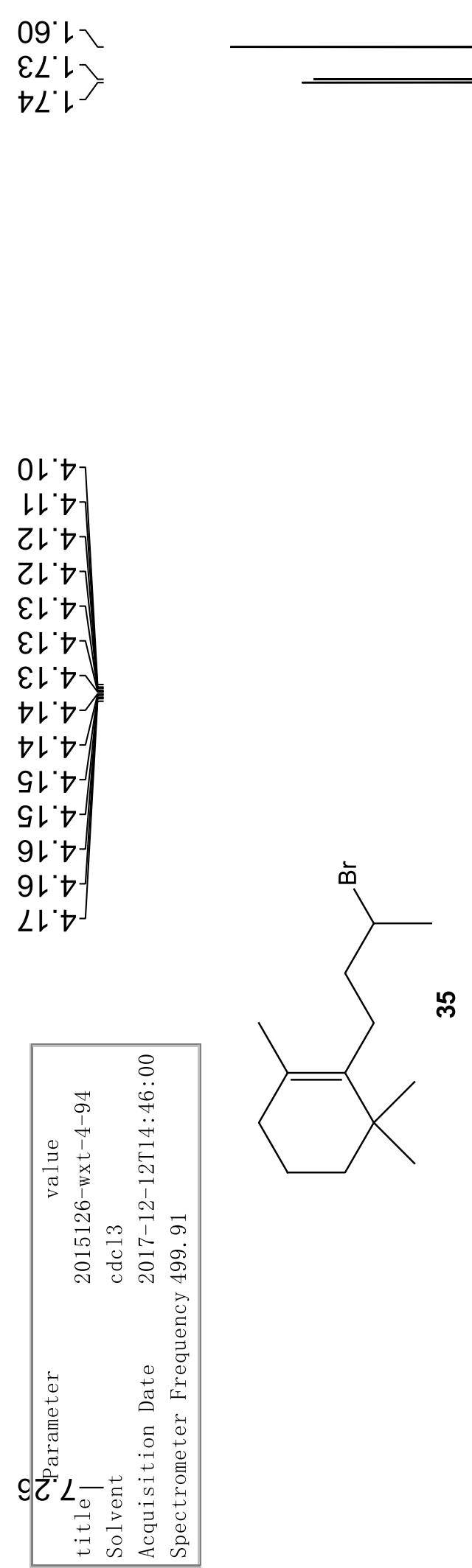
ยャ0เっ

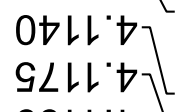
66ル・マ GLZL'わ LOEL $\nabla$ เEEL $\nabla$ LOtL'

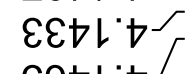
G9tレ' 乙†Gレ'万 G99l. ك'

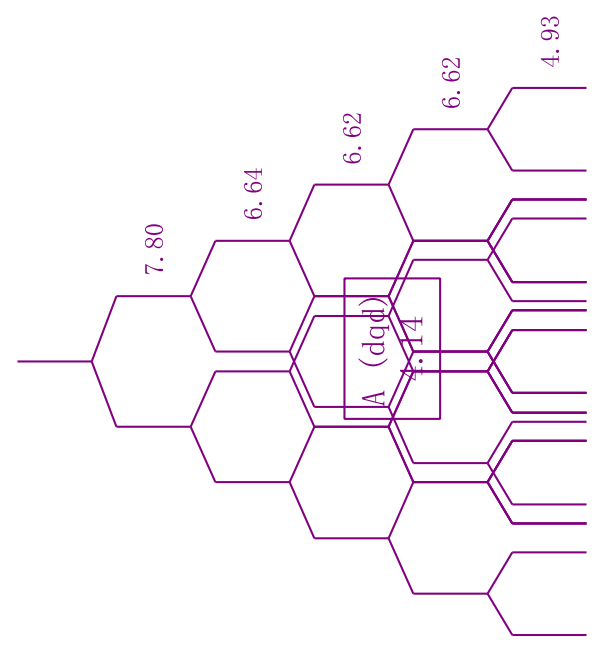

乮

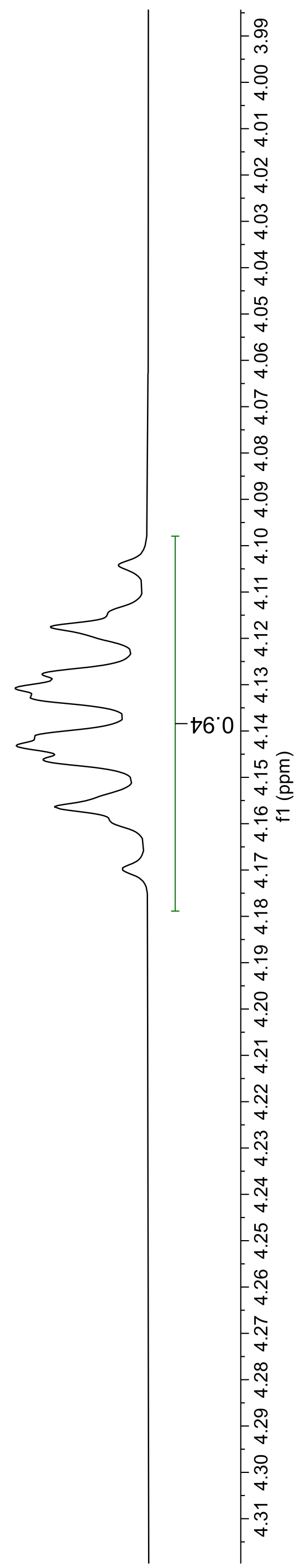


$8 t^{\circ} 6$ L

986 ᄂ

$\varepsilon \varepsilon^{\prime} 9 乙_{-}$

เ6.9乙

89.8Z

GL $2 \varepsilon^{\prime}$

10.9

$08^{\circ} 6 \varepsilon-$

$\angle \varepsilon^{\prime} レ \nabla^{\prime}$

IG'ZS-
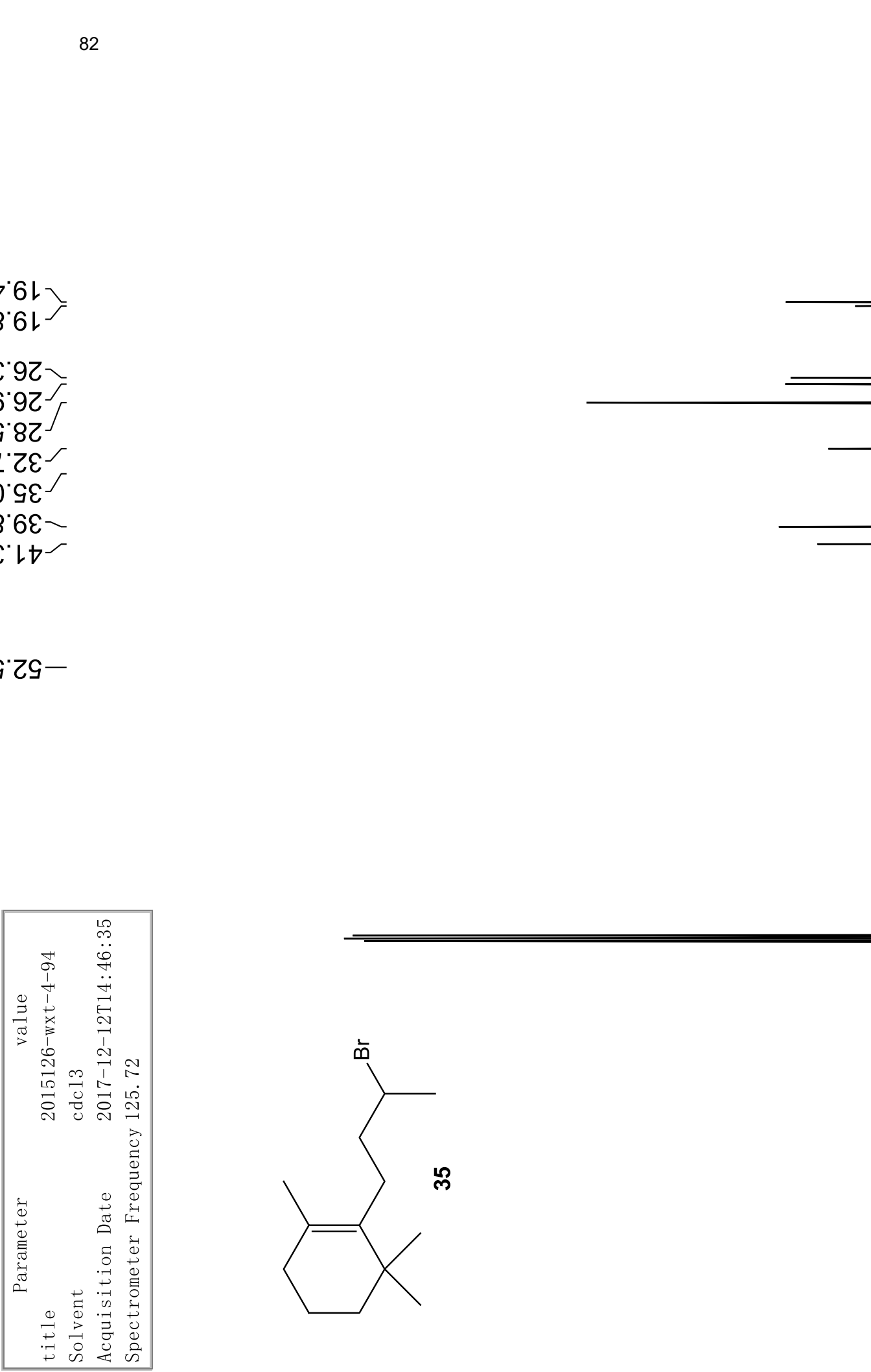


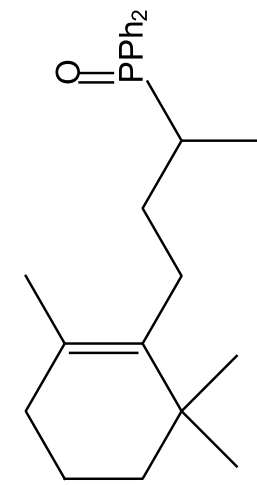

m

GL $\angle E-$

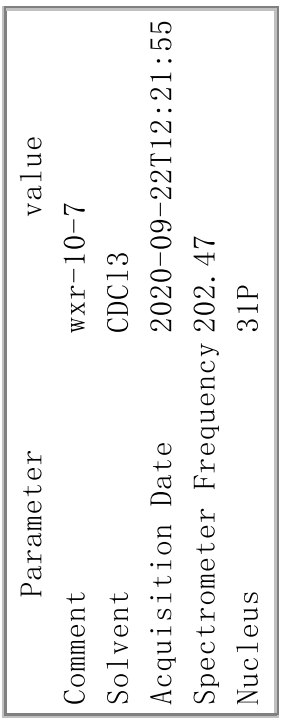


6L'0 88.0 LC' $\varepsilon Z^{\circ}$ $\nabla Z^{\circ}-$ $9 Z^{\circ}$ L $\angle \varepsilon^{\circ}$ $\varepsilon Z^{\prime} Z$

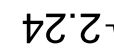
$9 Z^{\circ} Z$ $\angle Z Z^{\prime} Z$ $8 Z^{\circ} Z$ $8 Z^{\prime} Z$ $6 z Z$ $0 \varepsilon^{\circ} Z$ $0 \varepsilon$

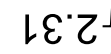
$1 \varepsilon^{\circ} Z$ 乙ะ乙 $\varepsilon \varepsilon^{\circ} 乙$ $\varepsilon \varepsilon$ ' $\nabla \varepsilon ' Z$ $\varsigma \varepsilon^{\prime} 乙$

$\angle L ' L$ $\angle L ' L$ $8 L^{\circ} L$ $8 L^{\circ} L$ $6 L^{\circ} L$ $6 L ' L$ $6 L ' L$ $08^{\circ} \mathrm{L}$ $08^{\circ} \mathrm{L}$ $18 \mathrm{~L}$ $18<$ 18.2 $28^{\circ}<$ $28 \mathrm{~L}$ $28^{\circ} \mathrm{L}$ $78^{\circ} \mathrm{L}$ $78^{\circ} \mathrm{L}$

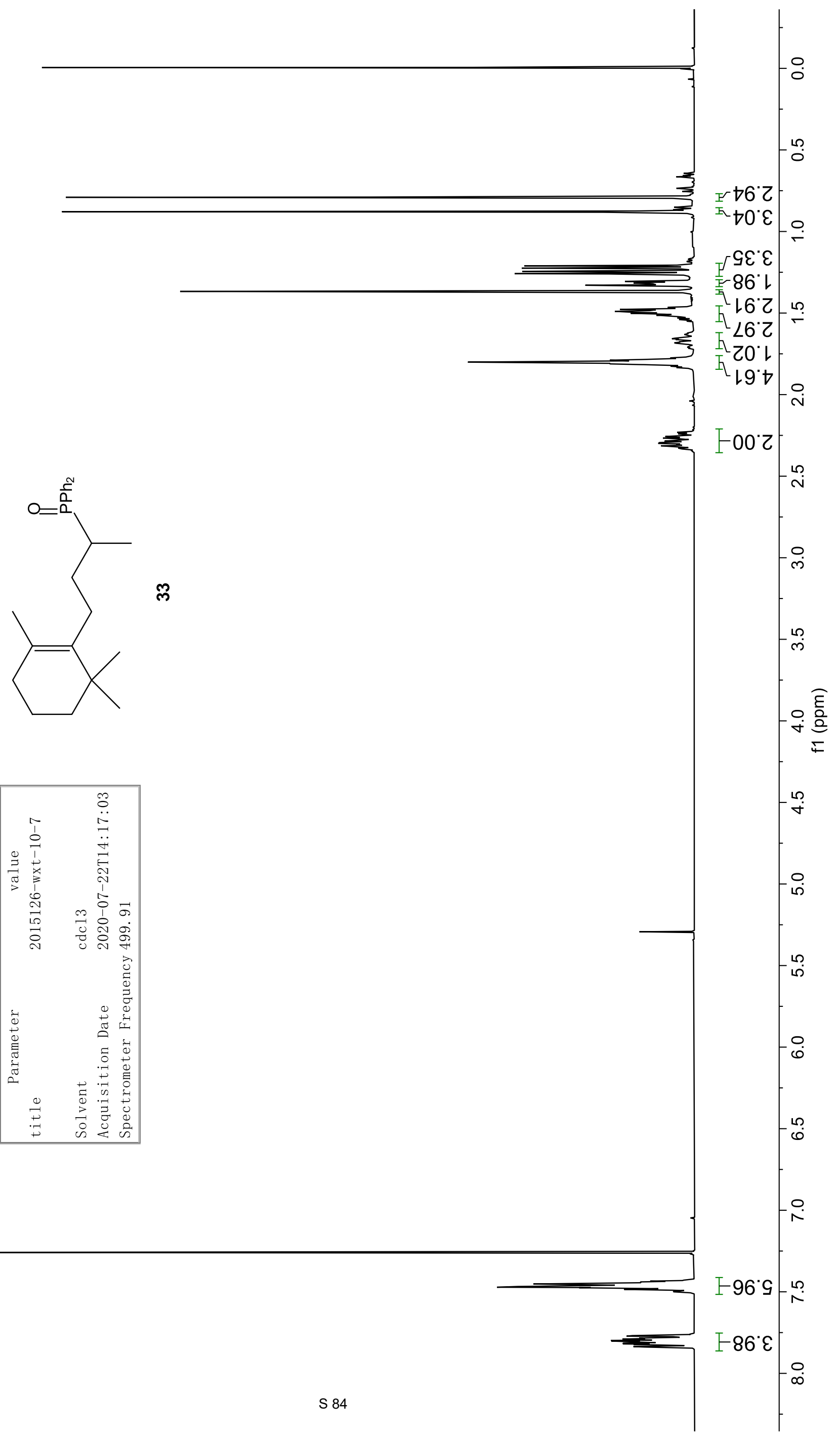


S06L'0-

$98 \angle 8^{\circ} 0-$

60レてレ
09ててレ

9カ七てレ

8892 ᄂ

$9 \angle 9 \varepsilon^{\circ}\llcorner-$

$8879^{\circ}$

GE99.

$\nabla \angle S 9^{\circ} \mathrm{L}$

8299.

GSL9.

†เ89

七๑8 ᄂ

$8689^{\circ}$ L

StLL'

GL8LL

U08.

2เL8. F

Sㄴ.

E9Z8

OSE8 L

0เย乙 乙

ルレてて

0LGZ乙

ᄂ $\angle 9 Z Z$

G08乙 乙

$6 \sqcup 8 乙$ 乙

6E6乙乙

G66乙'乙

О९०८乙

เ80ย $乙$

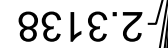

ย6เ $\varepsilon^{\circ} 乙$

乙৪乙ย乙

ธยहE 乙

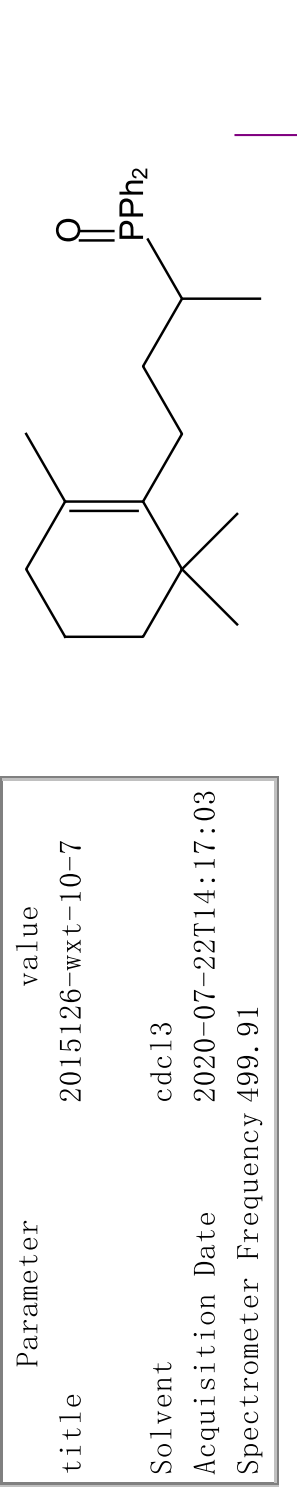

m్ల
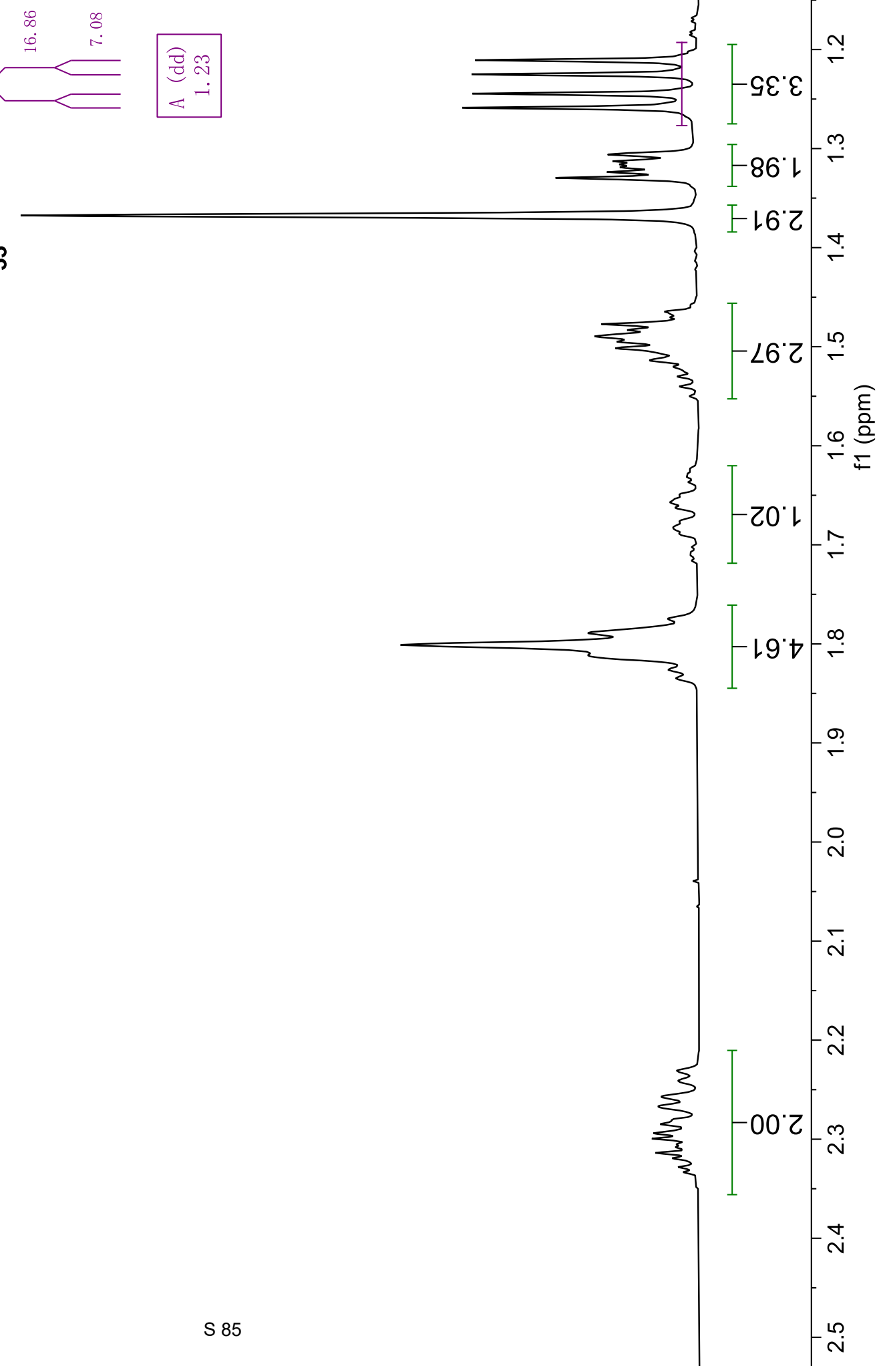


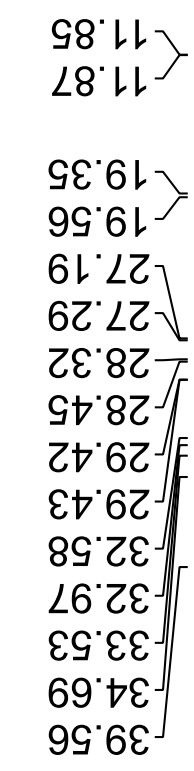

เG'LZL 七๐ $8 Z L$ 9ヤ゙ 8乙レ E9"8ZL SG:8ZL L8.0ع $88^{\circ} 0 \varepsilon L$ $06^{\circ} 0 \varepsilon$ L $\angle 6^{\circ} 0 \varepsilon L$ 乙E'LEL $G \varepsilon^{\circ} L \varepsilon L$ $6 \varepsilon^{\circ} L \varepsilon L$ เナ゙レレ S6. $L E L$. G乙'乙ع LL'ZEL $00^{\circ} \varepsilon \varepsilon\llcorner-$ $\diamond Z^{\prime} 9 \varepsilon \downarrow$
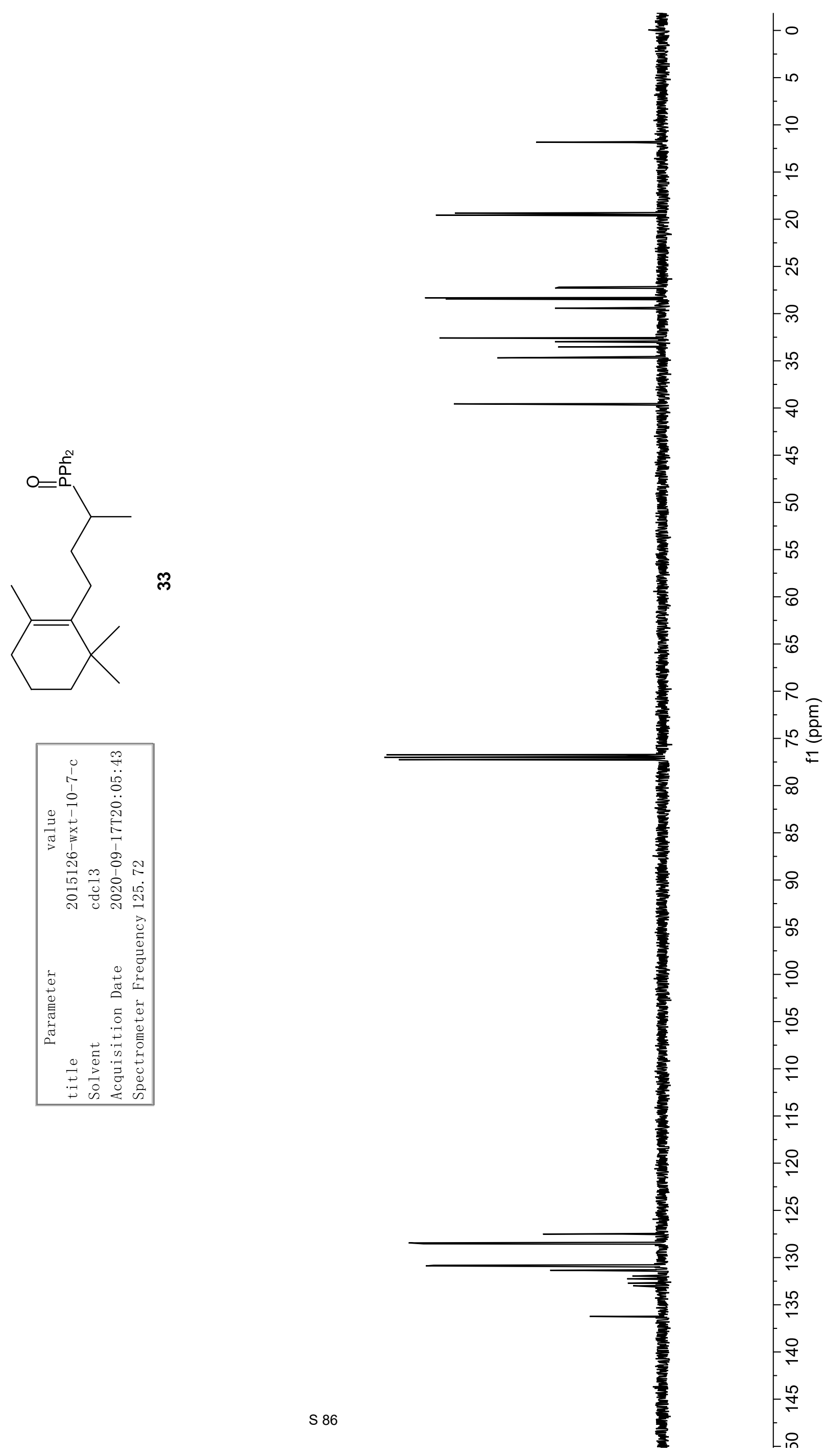


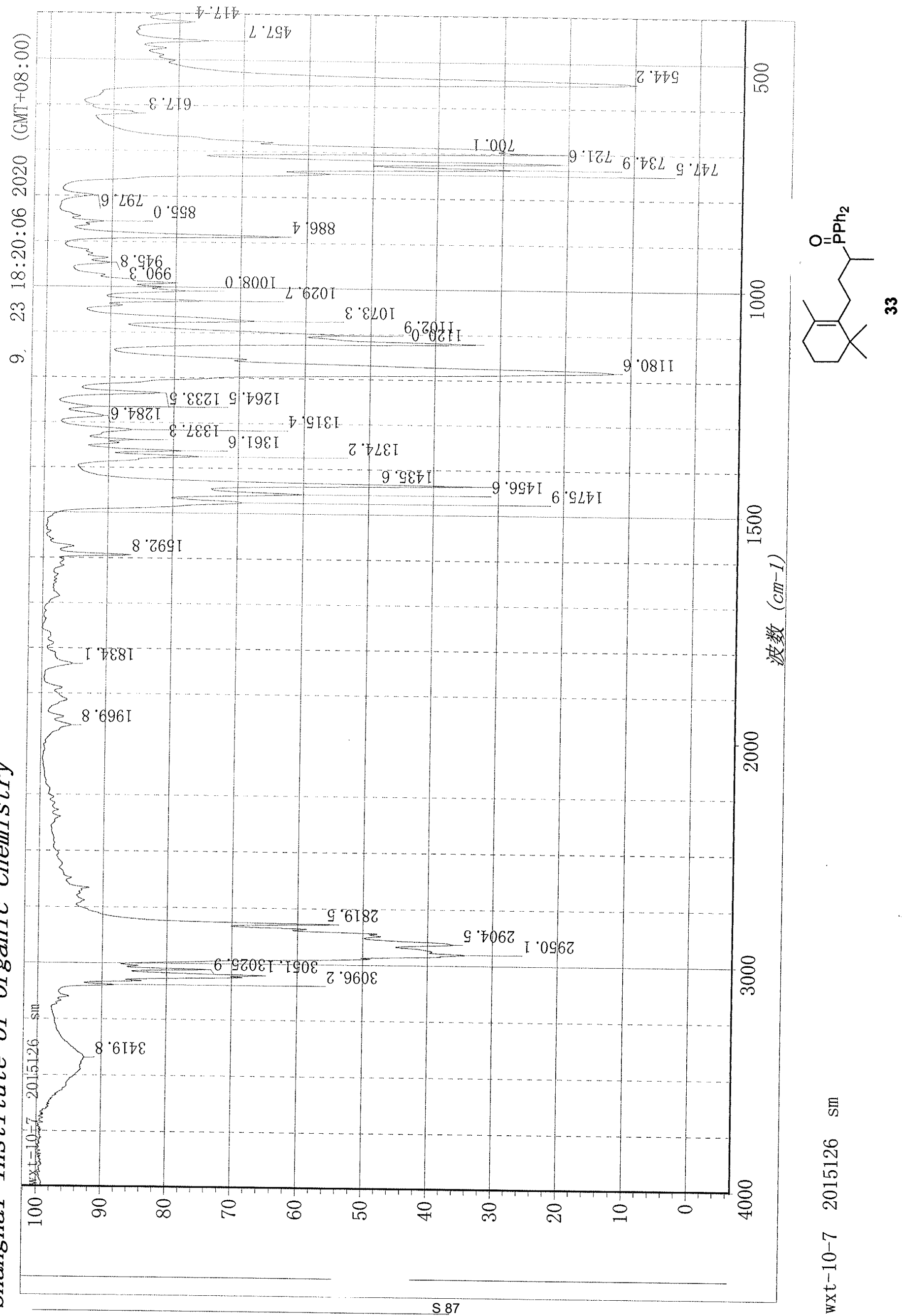



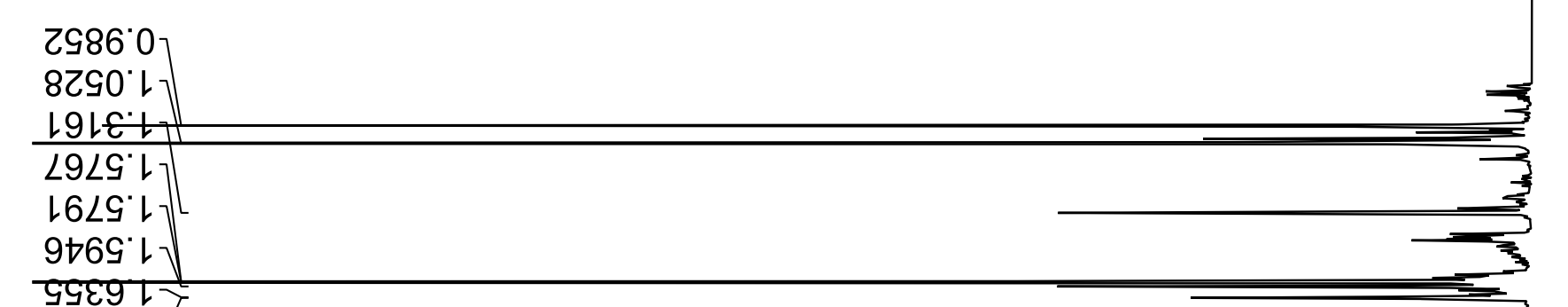

द589ᄂ

Z988.レ
Z668.L
ZZL6.

$\angle 999^{\circ} \varepsilon$

$\varepsilon 8 \angle G^{\circ} \varepsilon$

6989 $\varepsilon$

${ }^{\circ} \mathrm{CG}^{\circ} \mathrm{\varepsilon}$

6५ร9. $\varepsilon$

09t9 $\varepsilon$

เ999 $\varepsilon$

$\varepsilon 999^{\circ} \varepsilon$

$\varepsilon \angle Z 0^{\circ} \nabla$

9LE0 $\nabla$

乙ot0 $\nabla$

$\varepsilon \triangleright \nabla 0^{\circ} \nabla$

S\&७0 $\bullet$

$\nabla \varepsilon 90^{\circ} \nabla$

Z190"

$1990{ }^{\circ} \textrm{ }$

$7980^{\circ} \mathrm{s}^{-1}$

900 ' $\mathrm{L}$

9レレ9
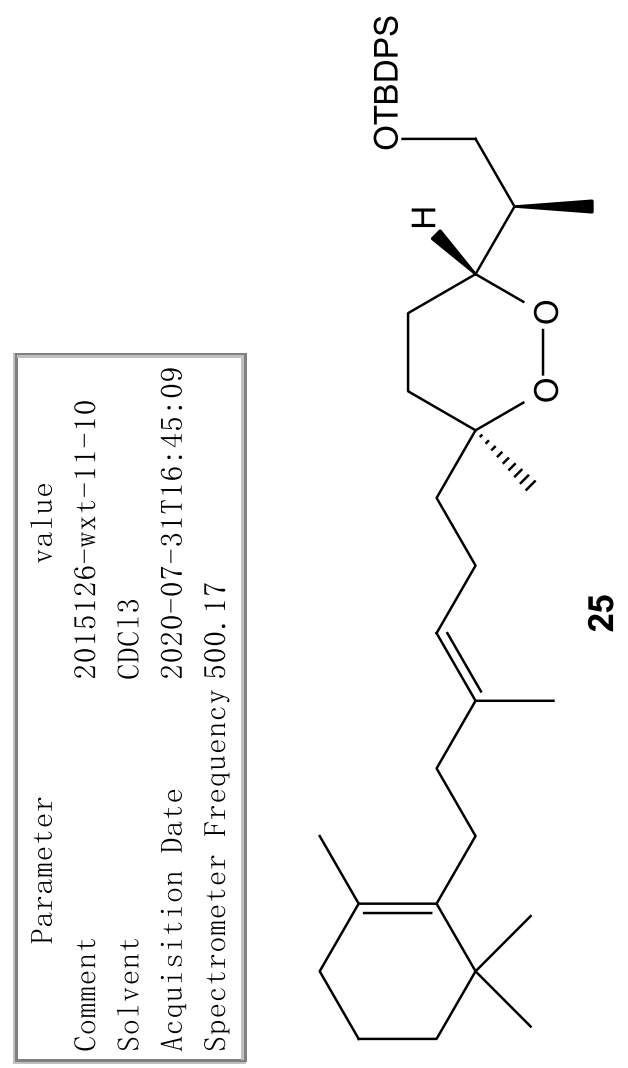

ผ

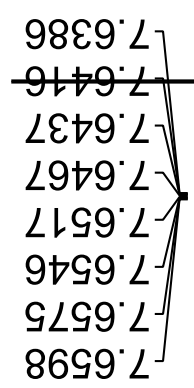



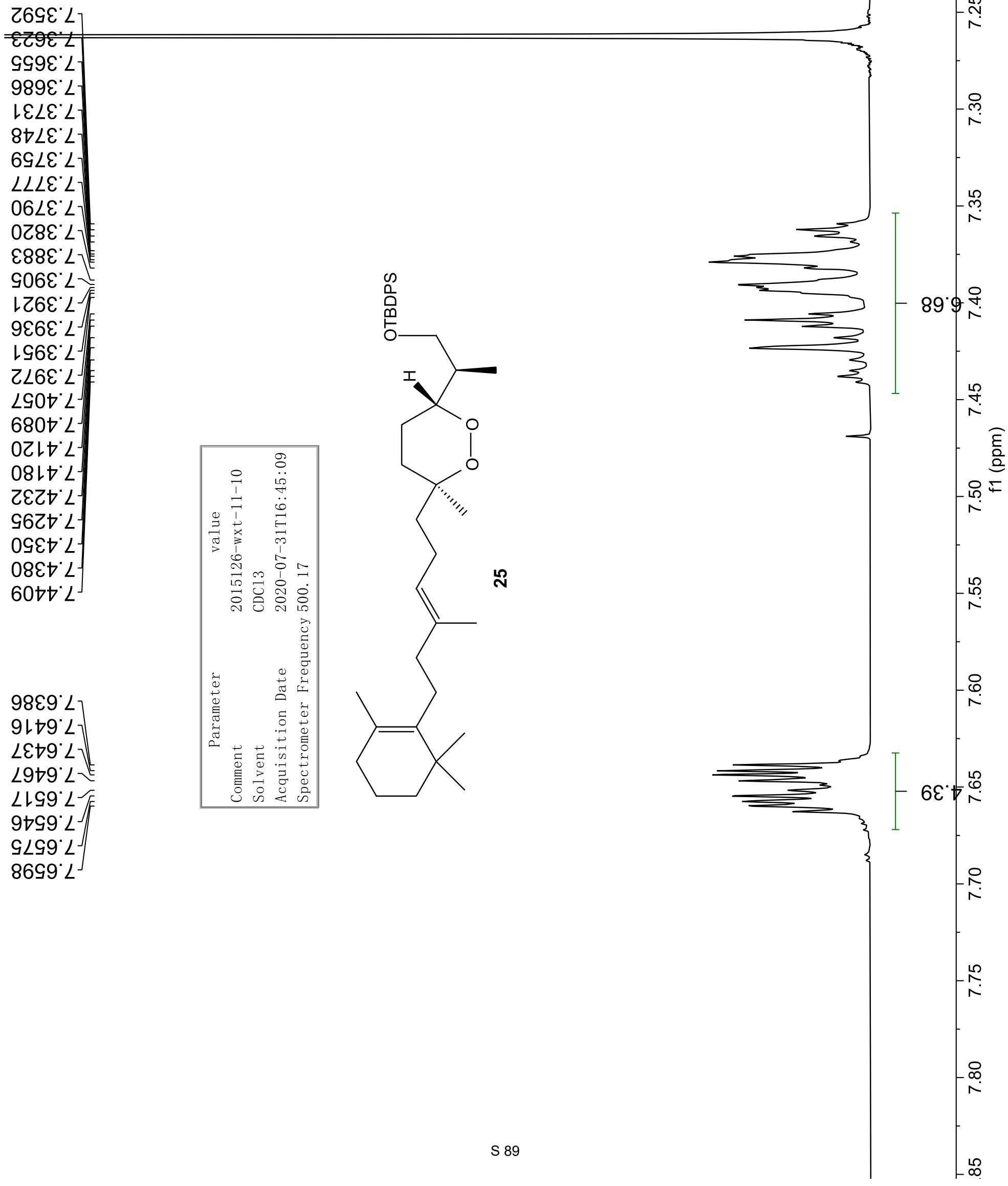
\&LZO॰ 9LEO" $\Rightarrow$ 乙0†0 $\bullet$ $\varepsilon \triangleright \nabla 0^{\circ} \nabla$

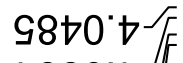
๑๕S0॰ 乙190' $1990^{\circ} \nabla^{\circ}$
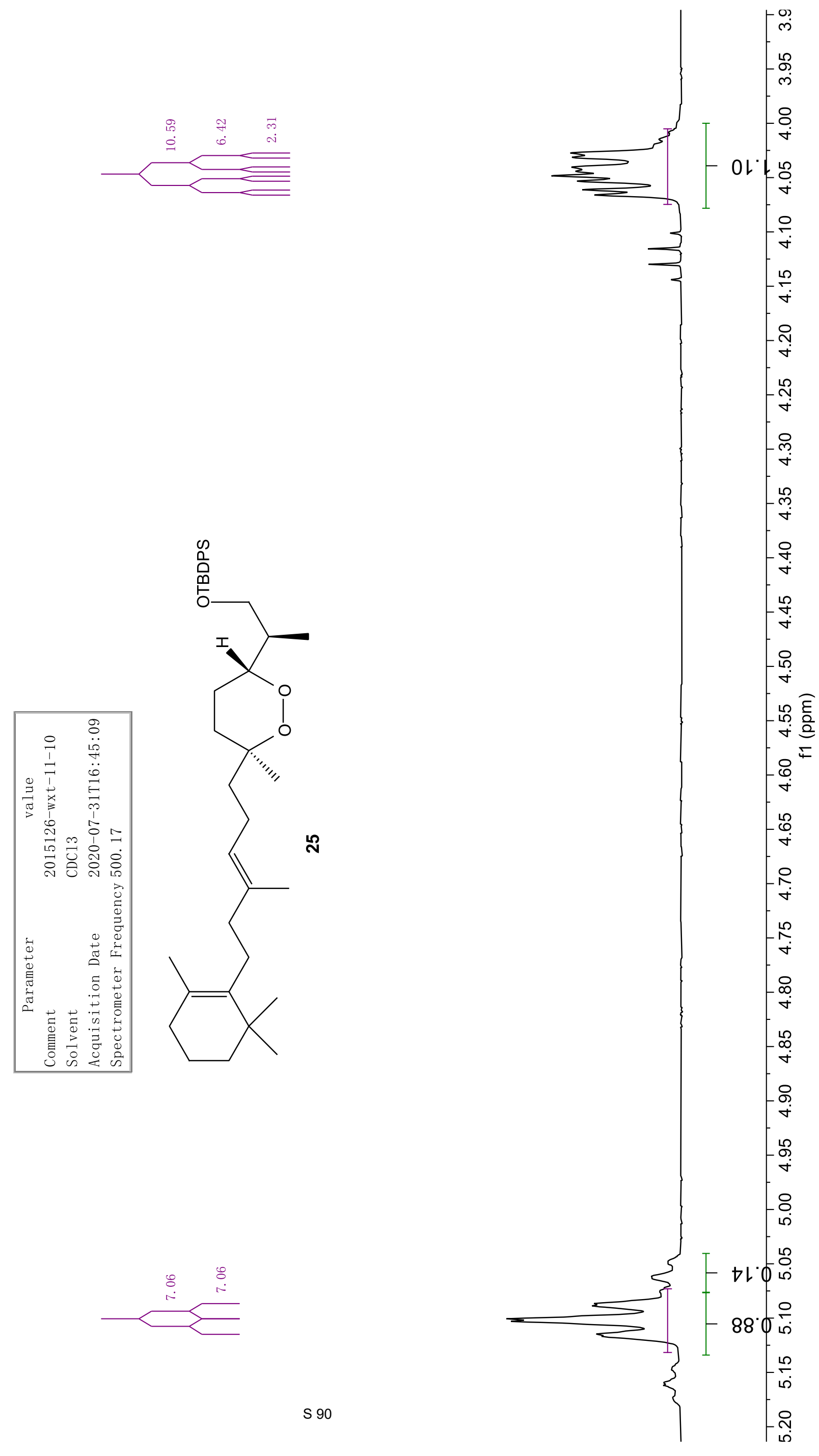

$7980^{\circ} \mathrm{c}$

900l'G-

9เレは
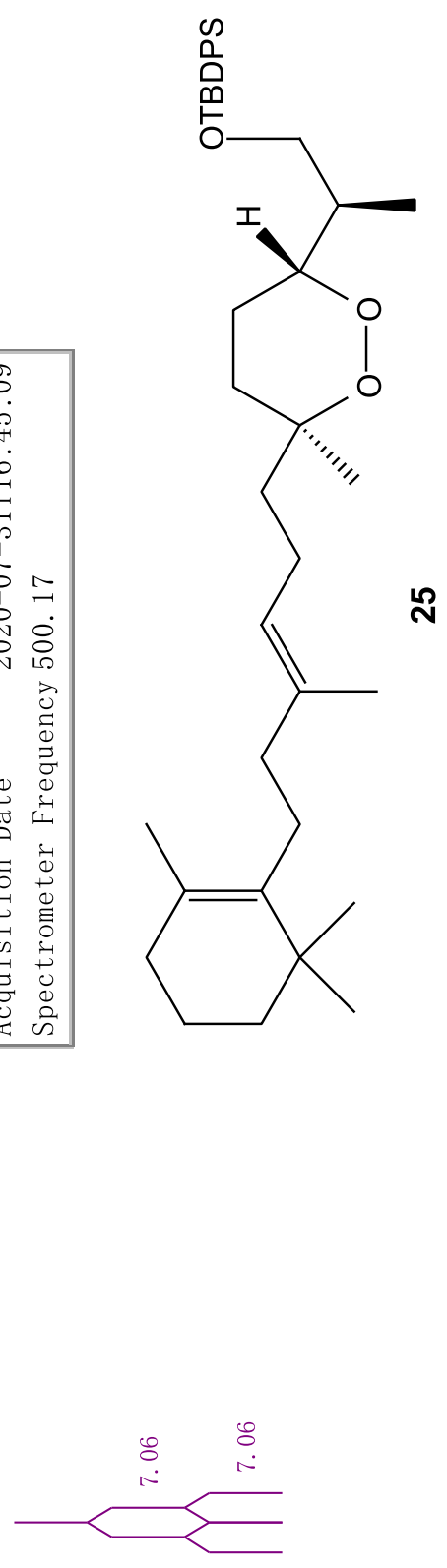
$\angle 999^{\circ} \varepsilon-$

$\varepsilon 8 \angle G^{\prime} \varepsilon-$ $6989^{\circ} \varepsilon-$ $\downarrow 86 \mathcal{G}^{\circ} \varepsilon-$

6S\&9 $\varepsilon-$

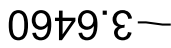
1999 $\varepsilon-$ ع999.
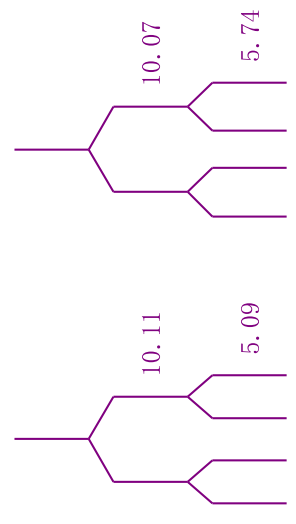

$\infty$
0
0
0
0
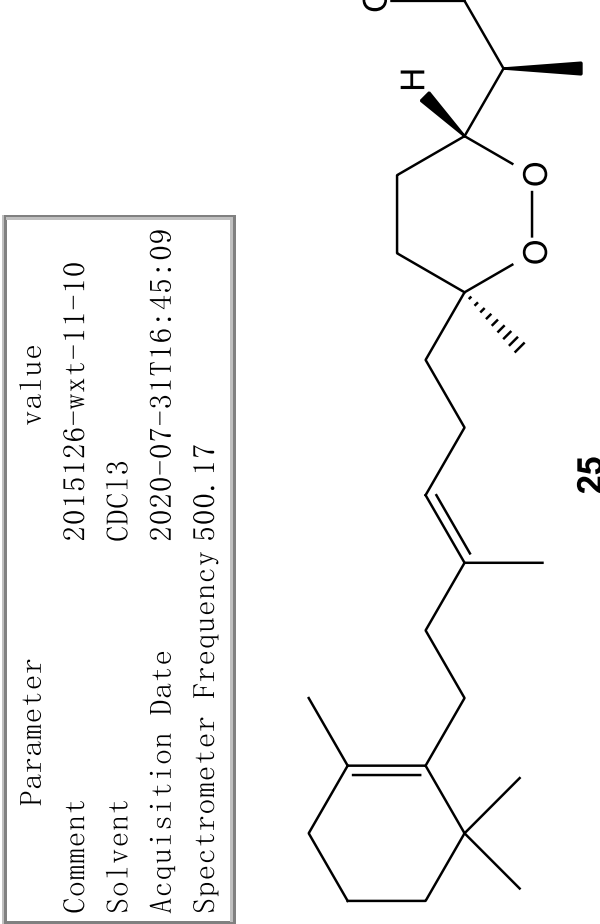

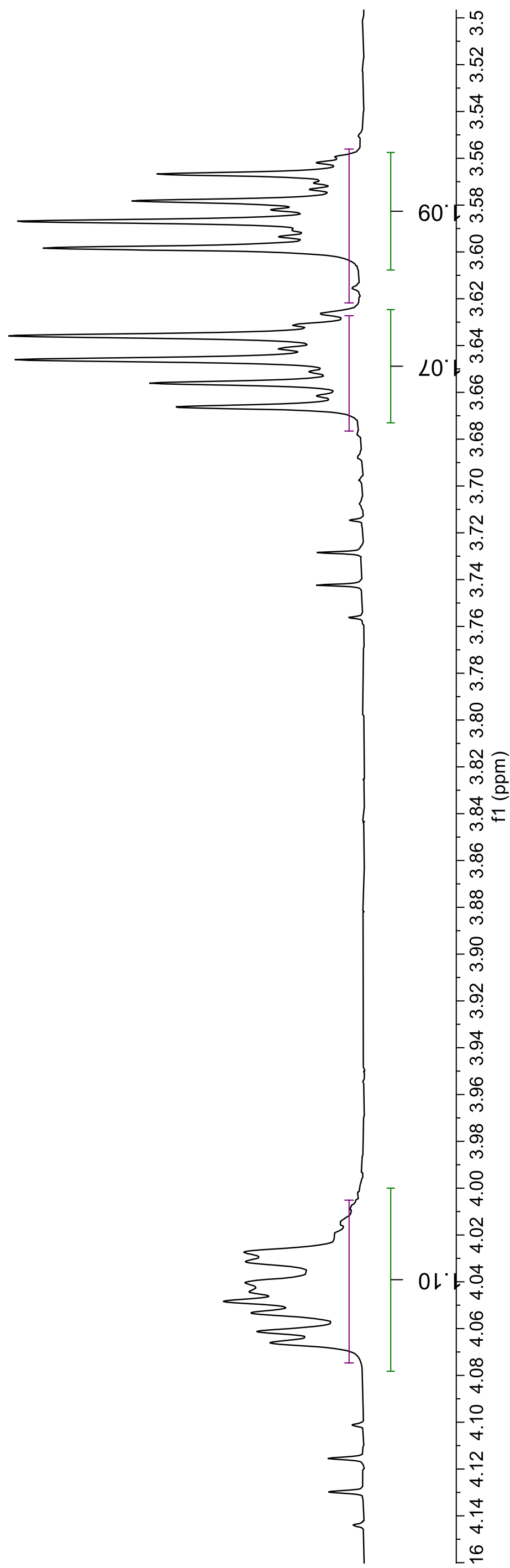


$Z L Z L$

$86^{\circ} \mathrm{sL}$

$6 Z^{\prime} 6 \mathrm{~L}$

$\varepsilon 9^{\prime} 6 \mathrm{~L}$

08.6 L

$\mathrm{ZZ} 0 \mathrm{Z}$

9G'L

$0<\varepsilon Z \backslash$

$78^{\circ} 92$

18.22

69.8Z

ZL ZE-

$08^{\circ} Z \varepsilon$

$96 \circ \varepsilon$

$6 \varepsilon^{\circ} 6 \varepsilon$

$18.6 \varepsilon$

$0 Z^{\circ} 0 t$

$190 t^{\circ}$

99.99-

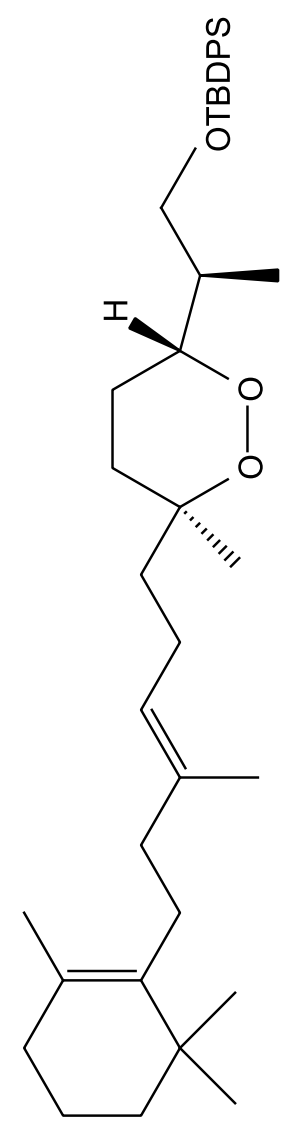

น

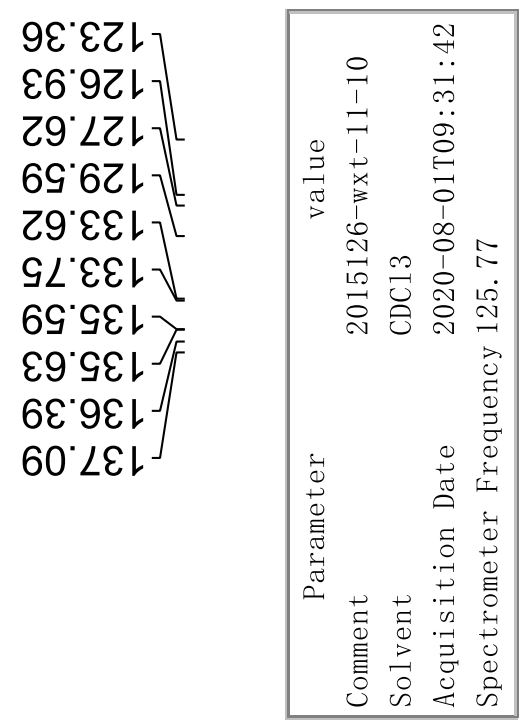




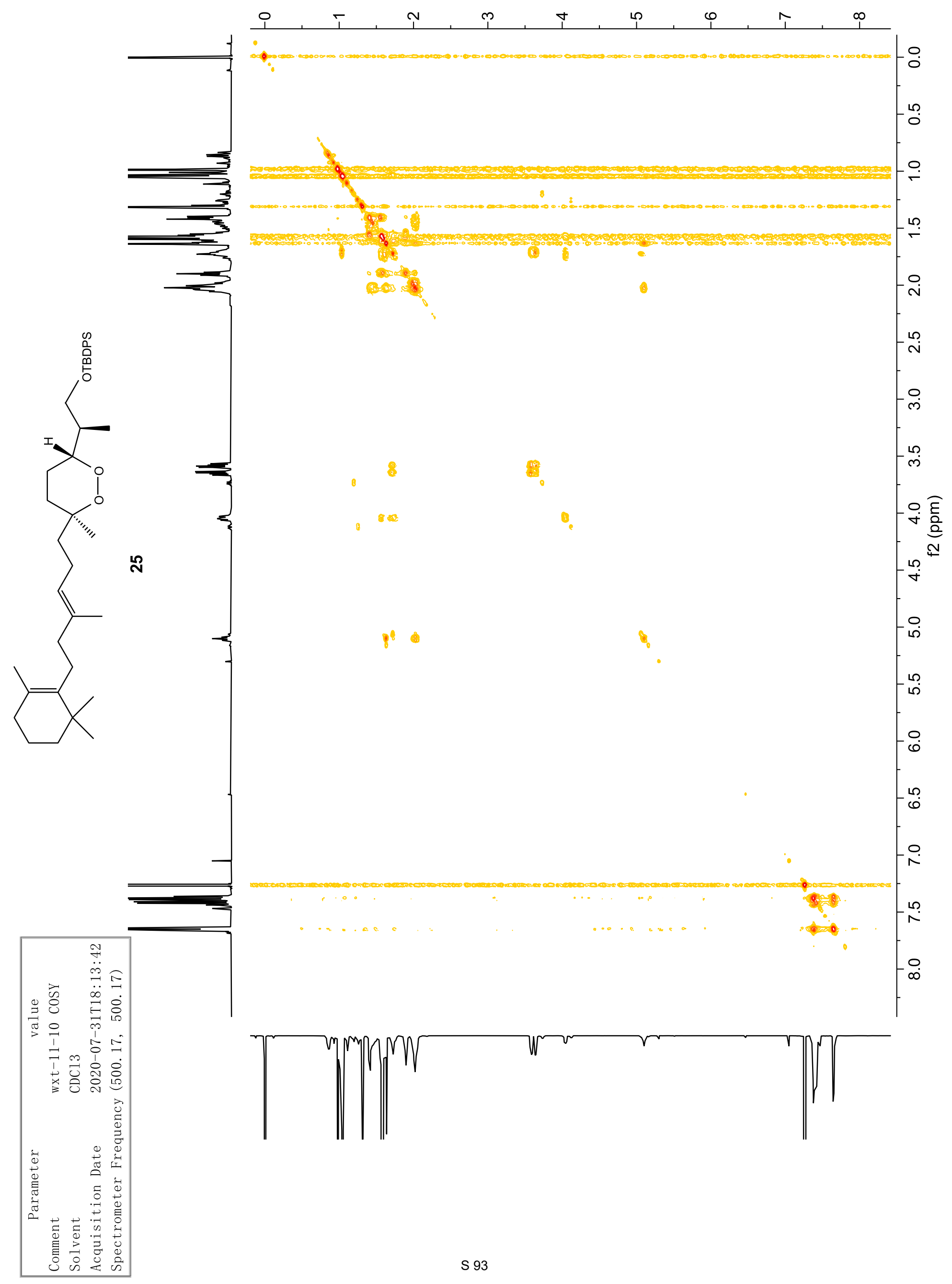




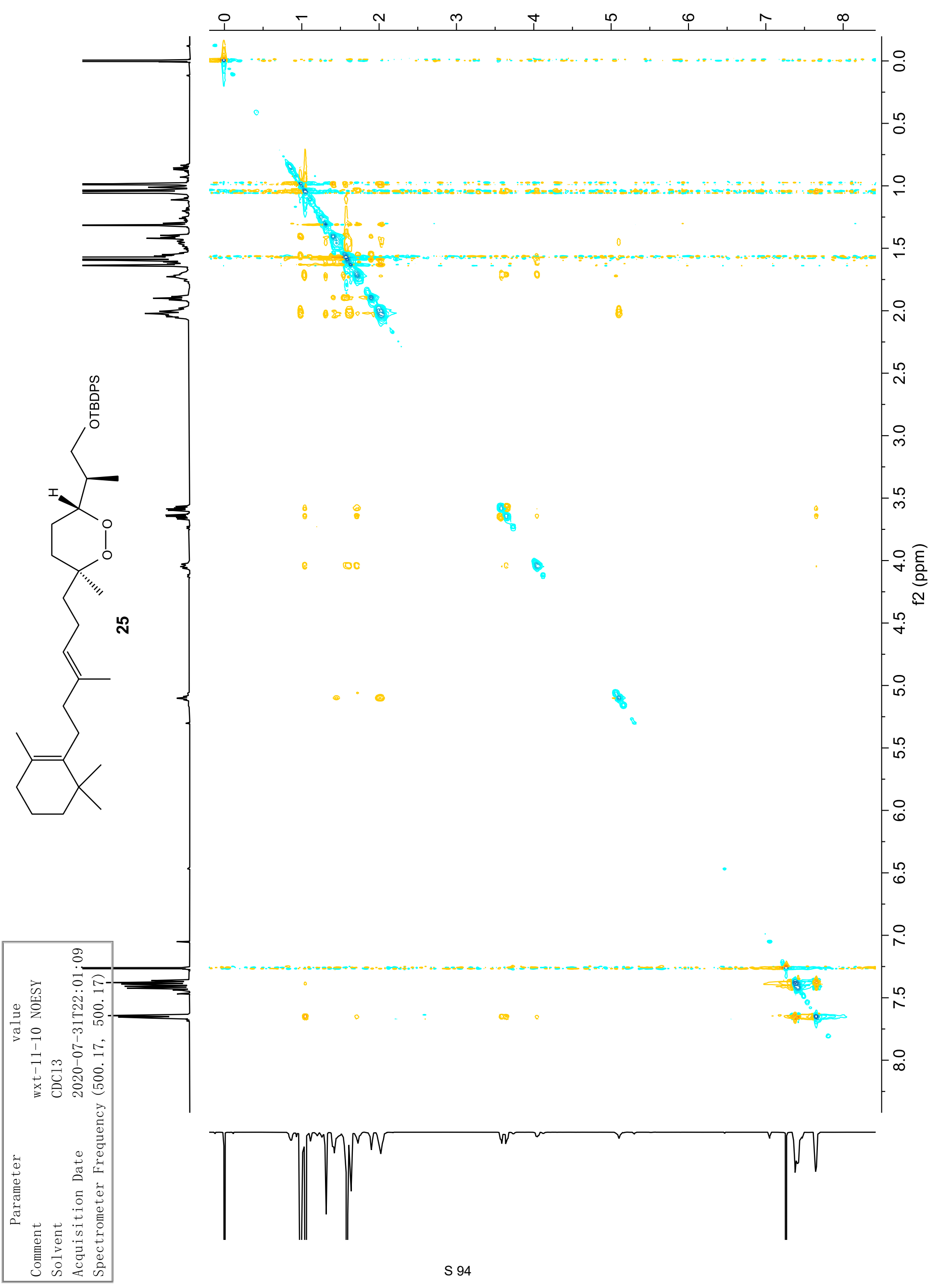




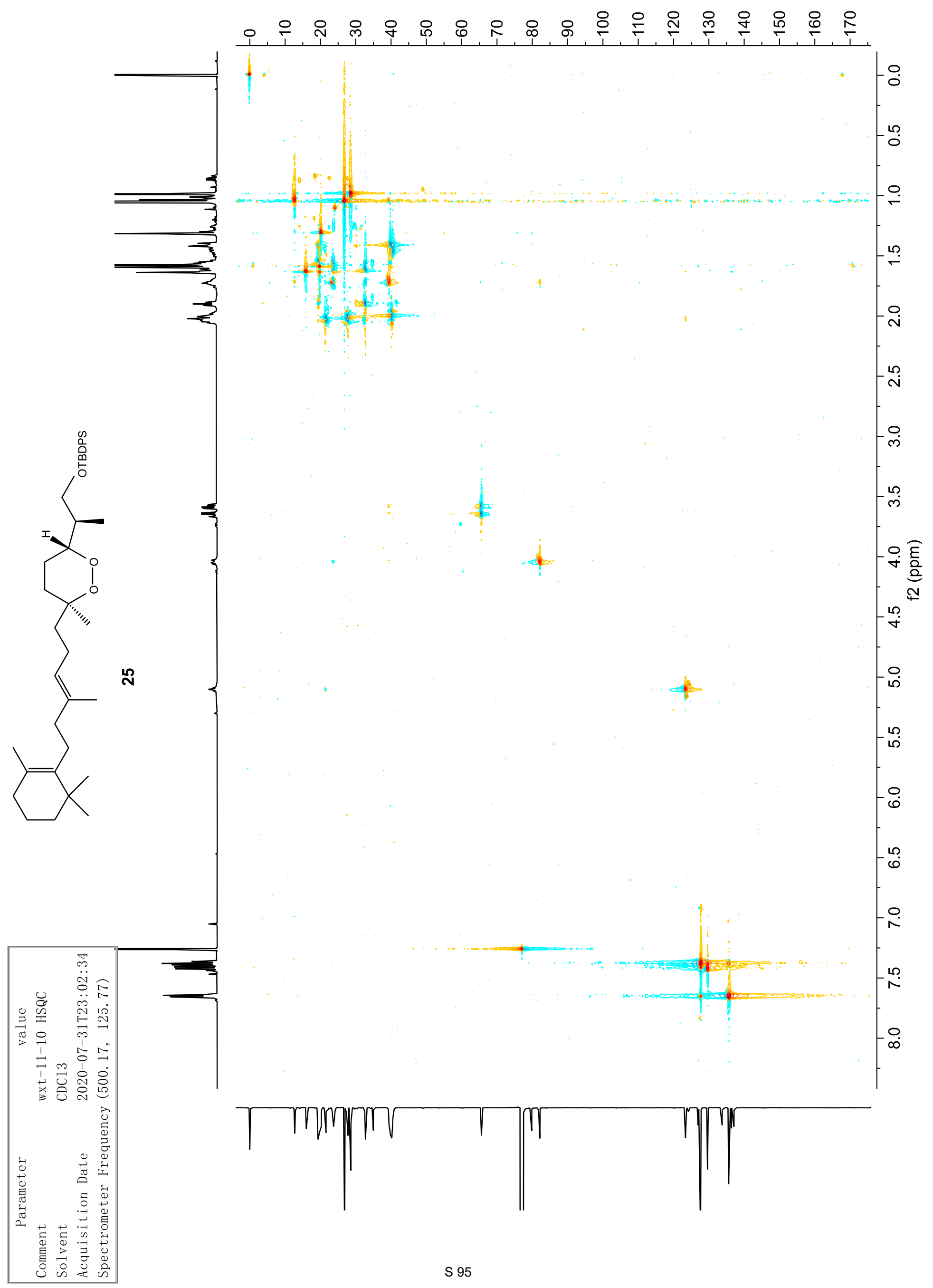




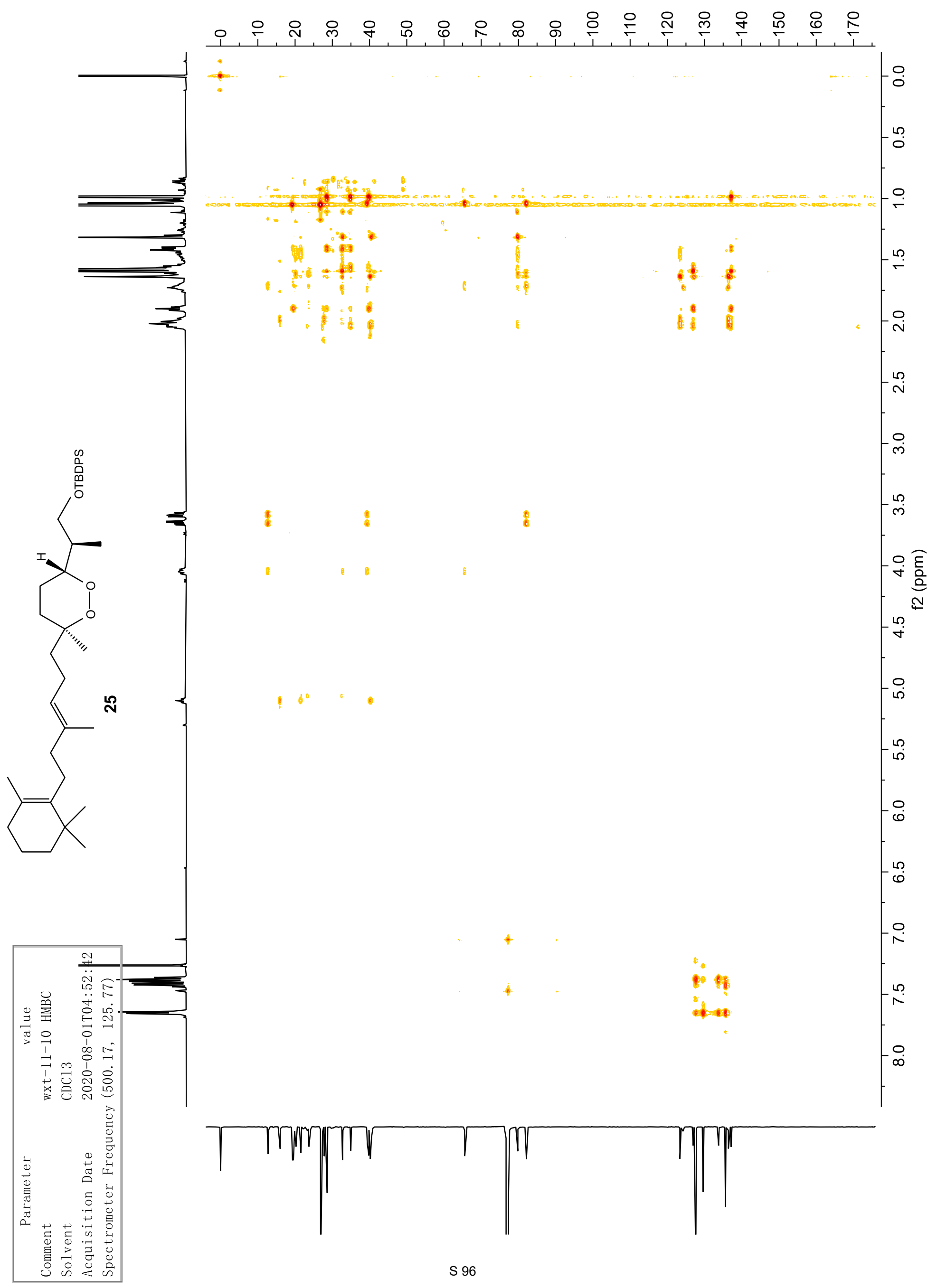




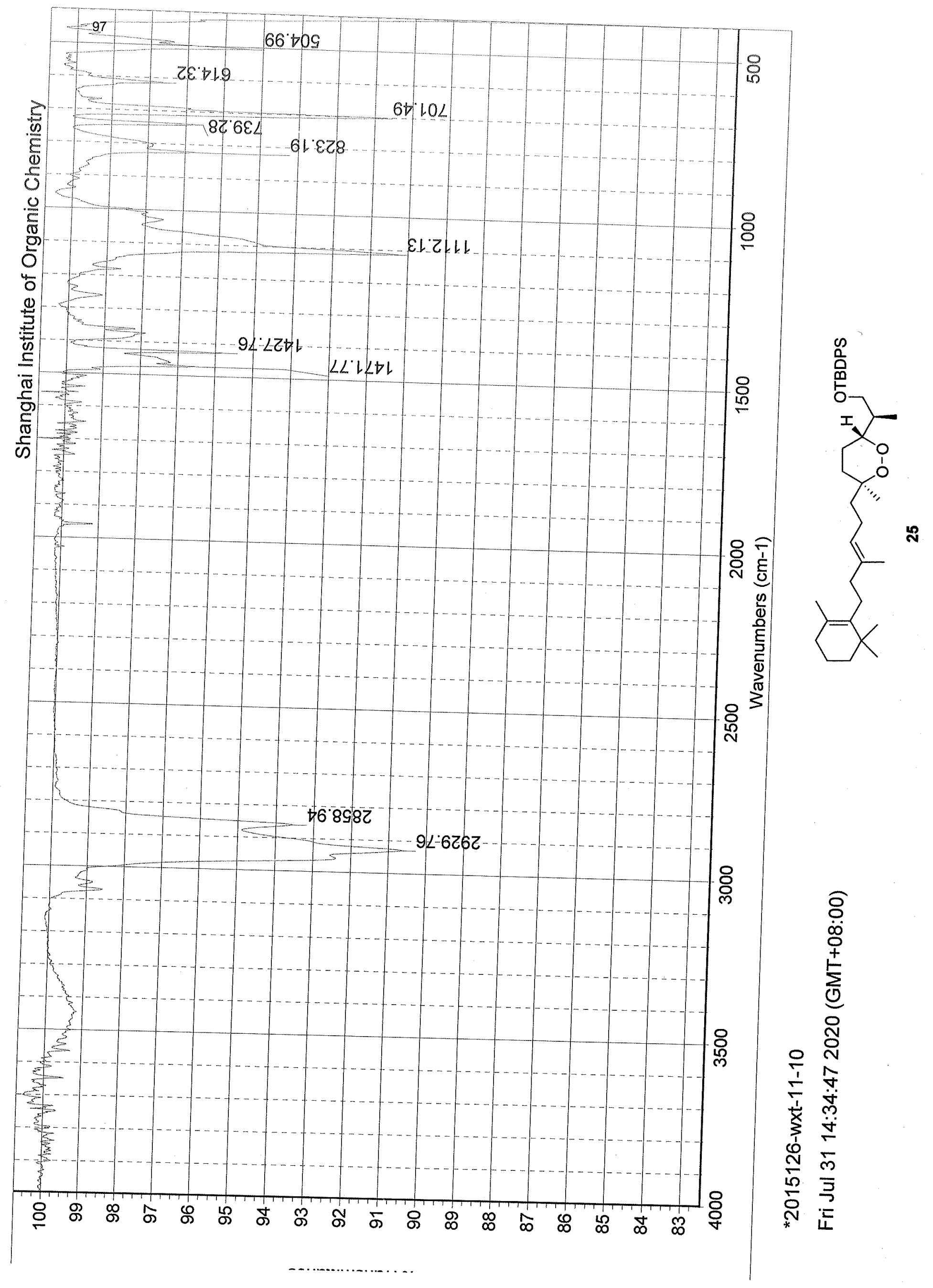


$86^{\circ} 0$
$82^{\circ} \downarrow$

$0 \varepsilon^{\circ} \downarrow$

เE

$69^{\circ}-$
$\varepsilon 9^{\circ}-$
$\forall 9^{\circ}$.

$\left.\begin{array}{l}89^{\circ} Z \\ 69^{\circ} z \\ 69^{\circ} z \\ 0 L^{\circ} z^{-}\end{array}\right]$

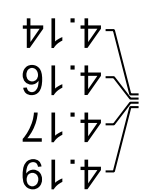

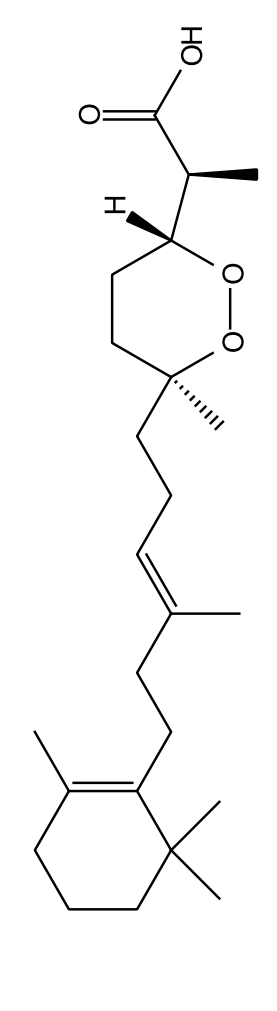

들
$\frac{\overline{0}}{5}$
$\frac{0}{2}$
$\frac{1}{ \pm}$
$\frac{1}{ \pm}$
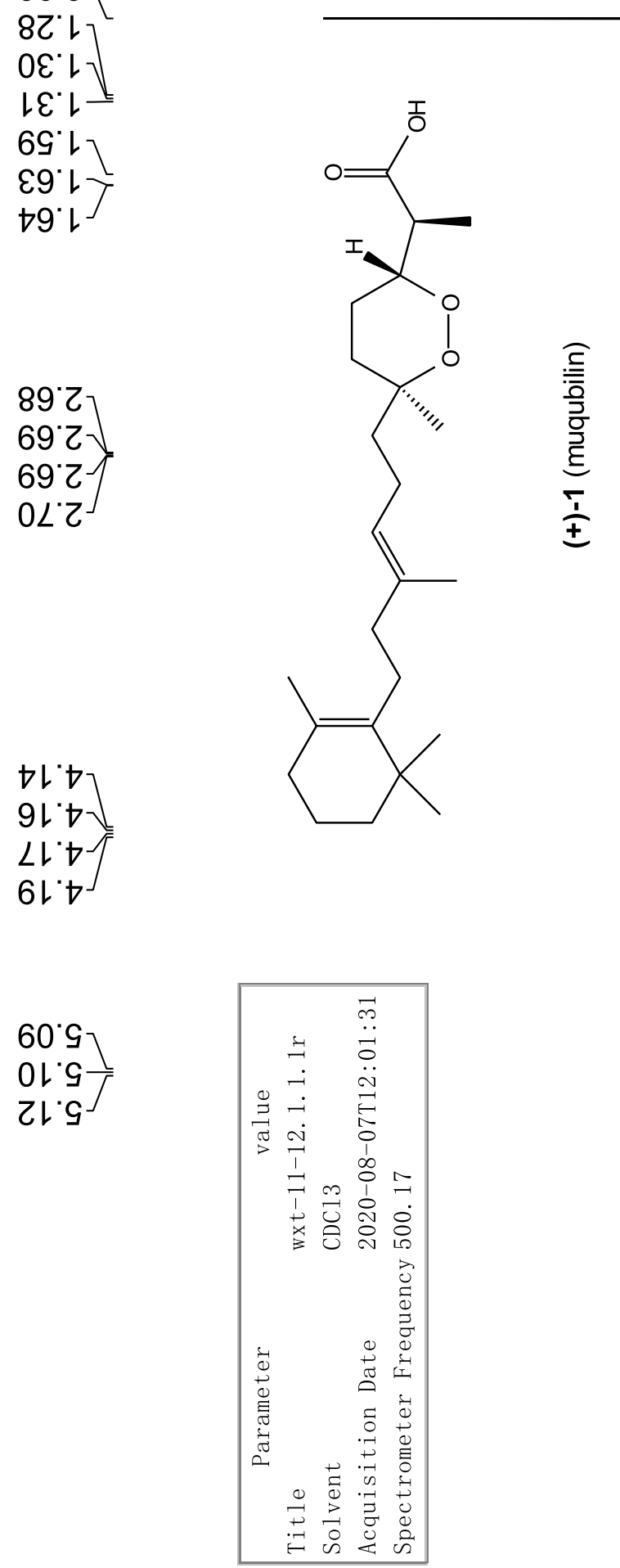

I $\varepsilon 8 . q^{\circ}$

$-0 \varepsilon^{\circ}<$

त $99^{\circ}$ \&

Tl9 EL-

$99^{\circ} z$

- $8 \varepsilon$ \%

I Gl:

3

in

$+20$

I 00

i

\{ 
カカカレ゙レ

6LGLDー てレレレー Z981'
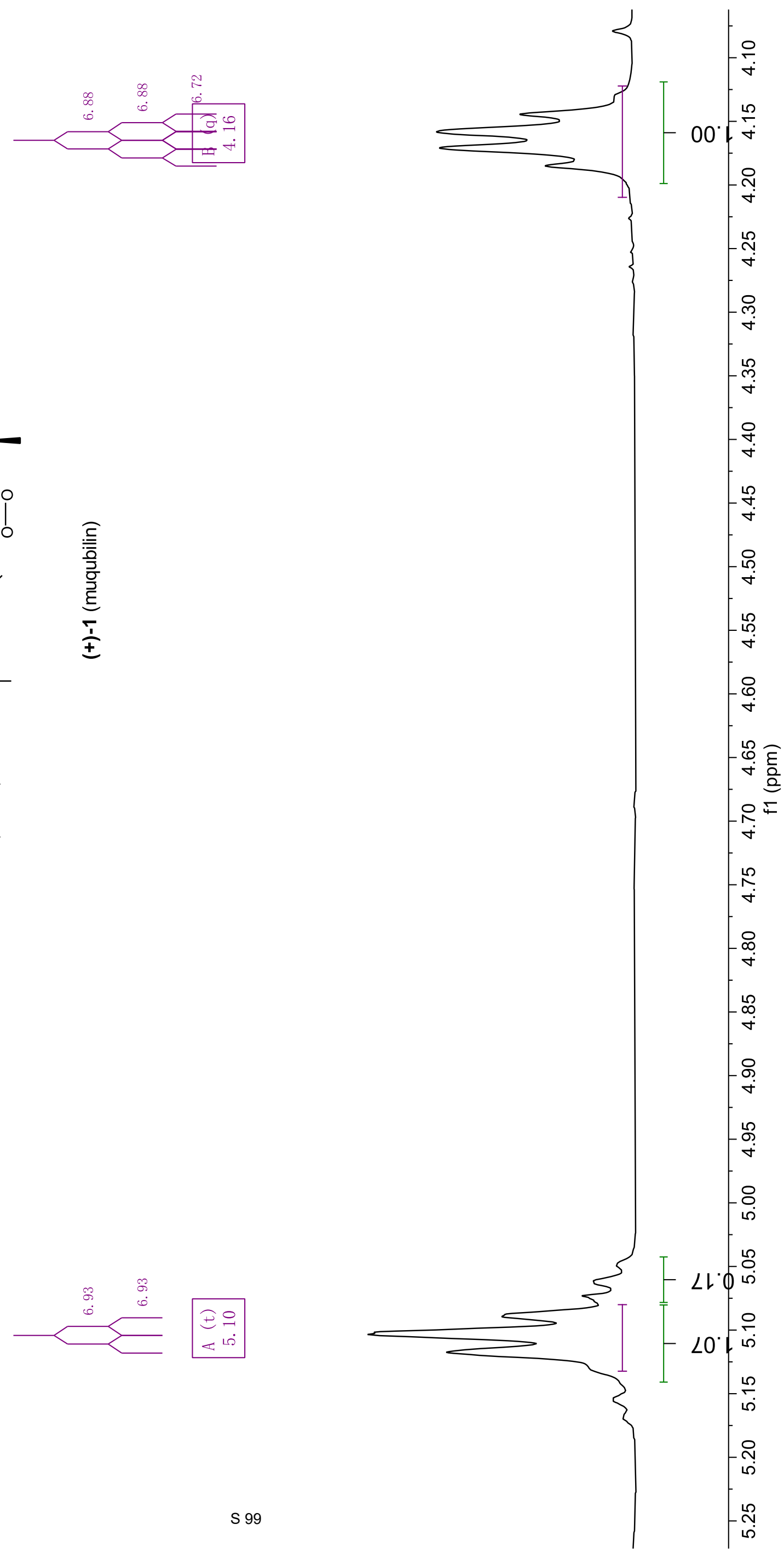


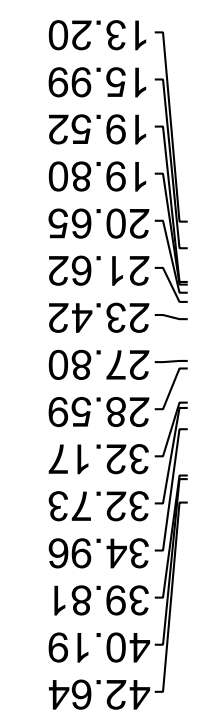

\begin{tabular}{l}
\hline 208 \\
00.18
\end{tabular}

6レ`ย乙レ 96.9ZLL9.9L $\angle O^{\circ} \angle E L$
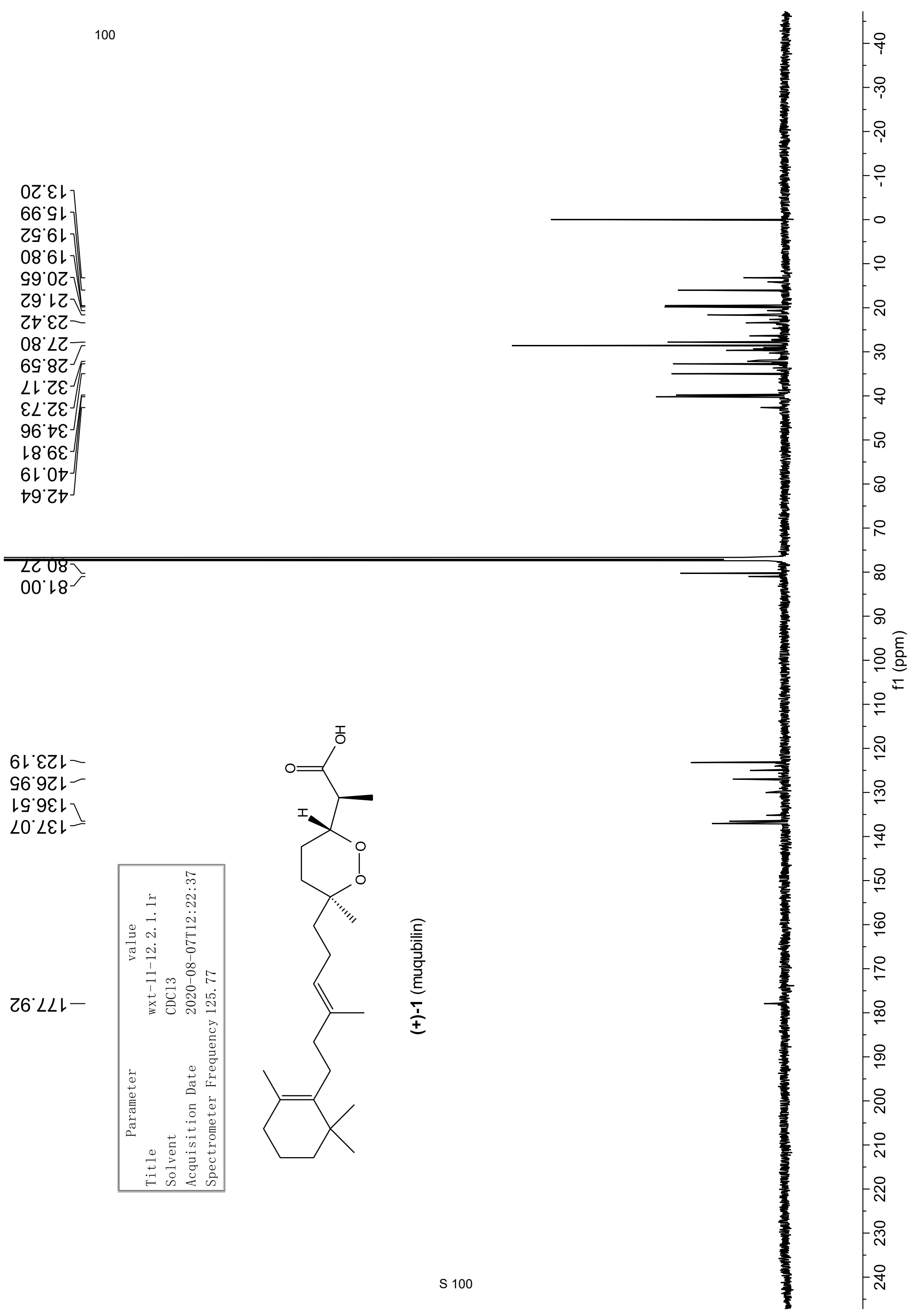


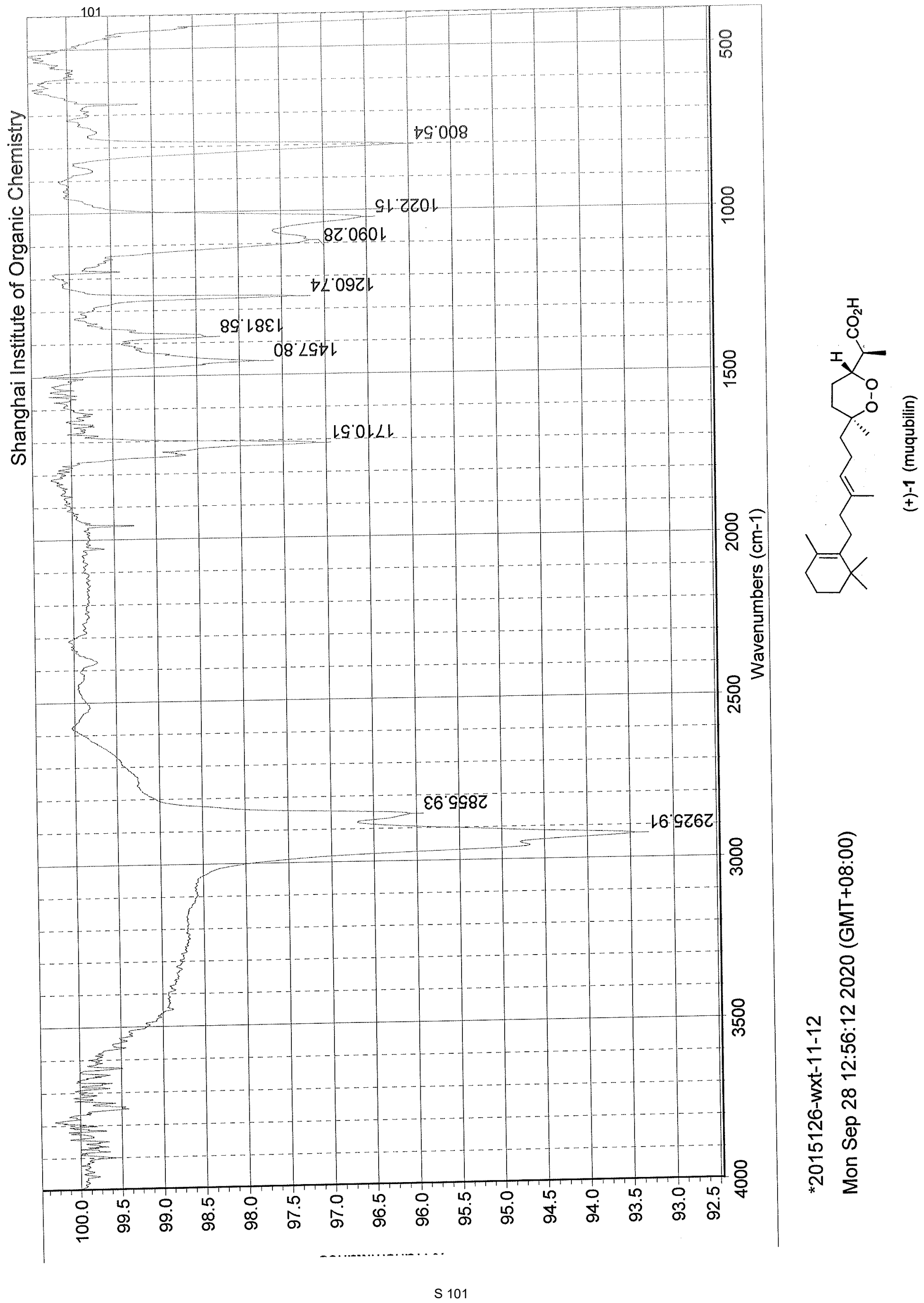




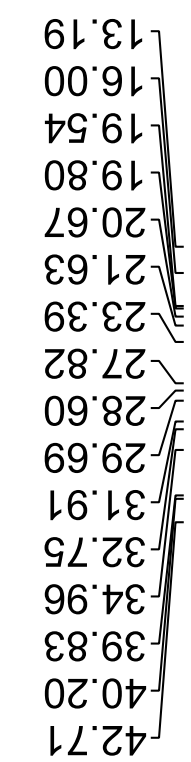

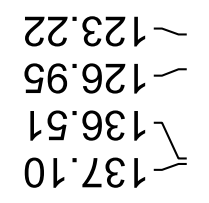

9608
86.08
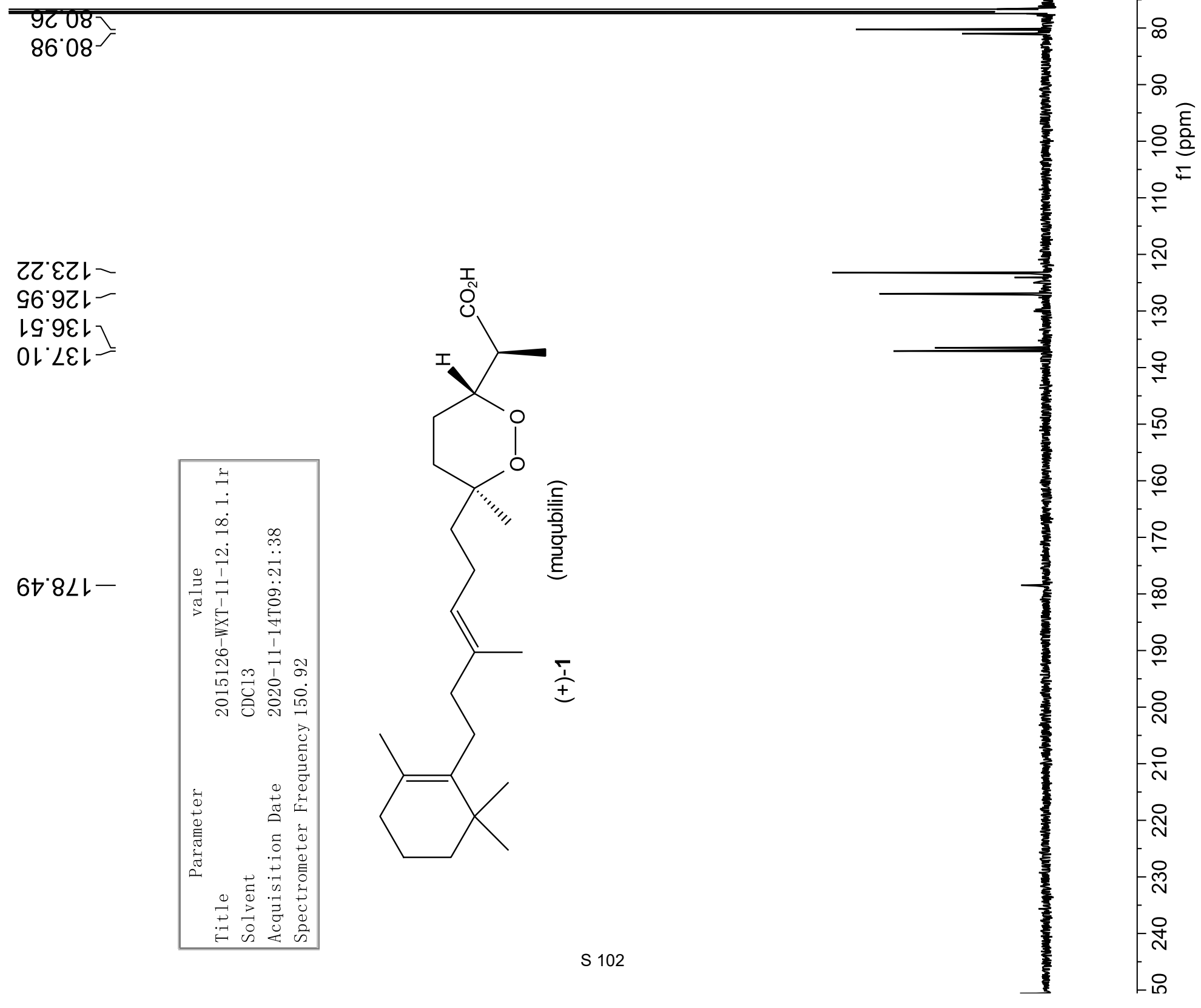


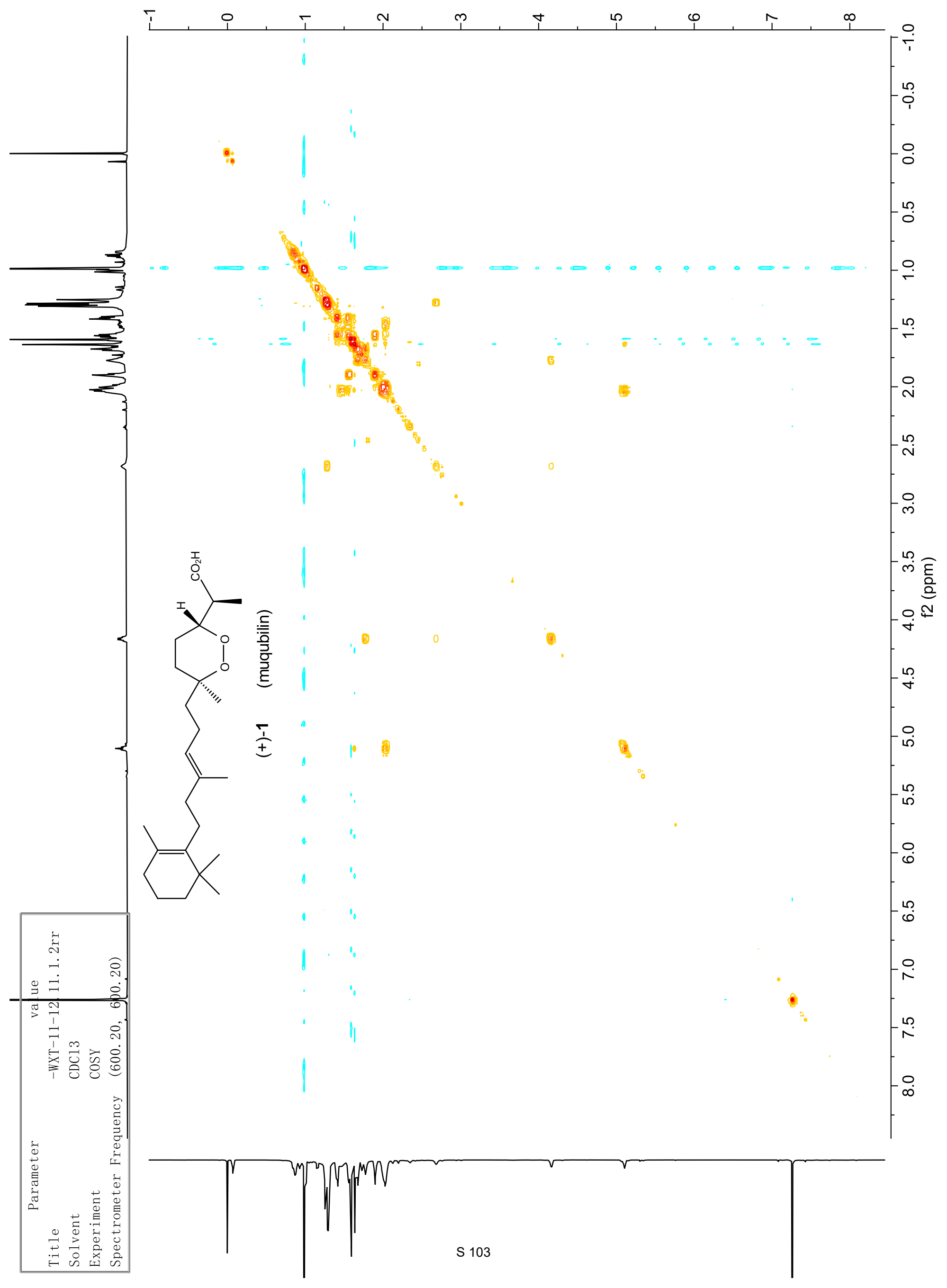




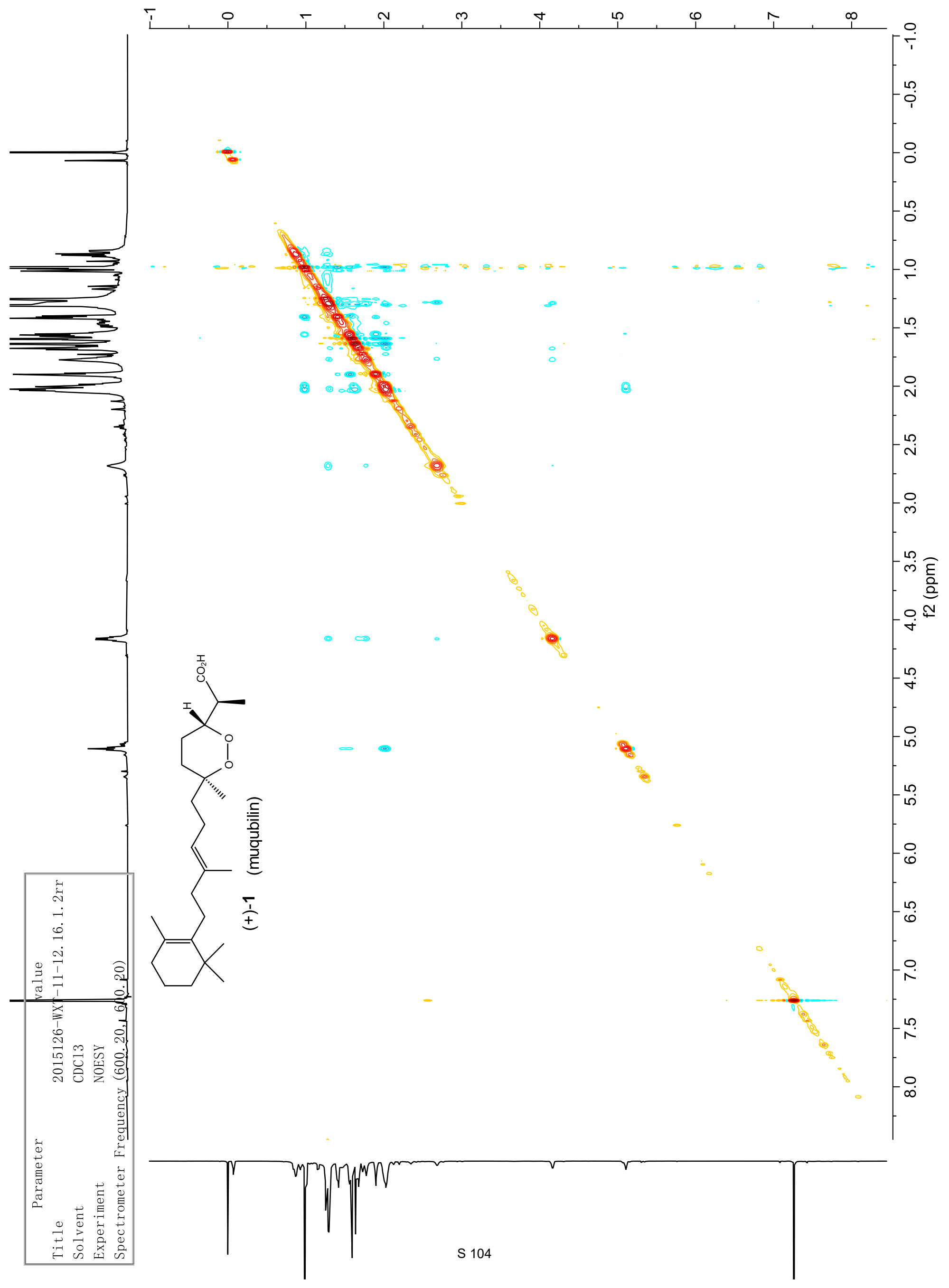




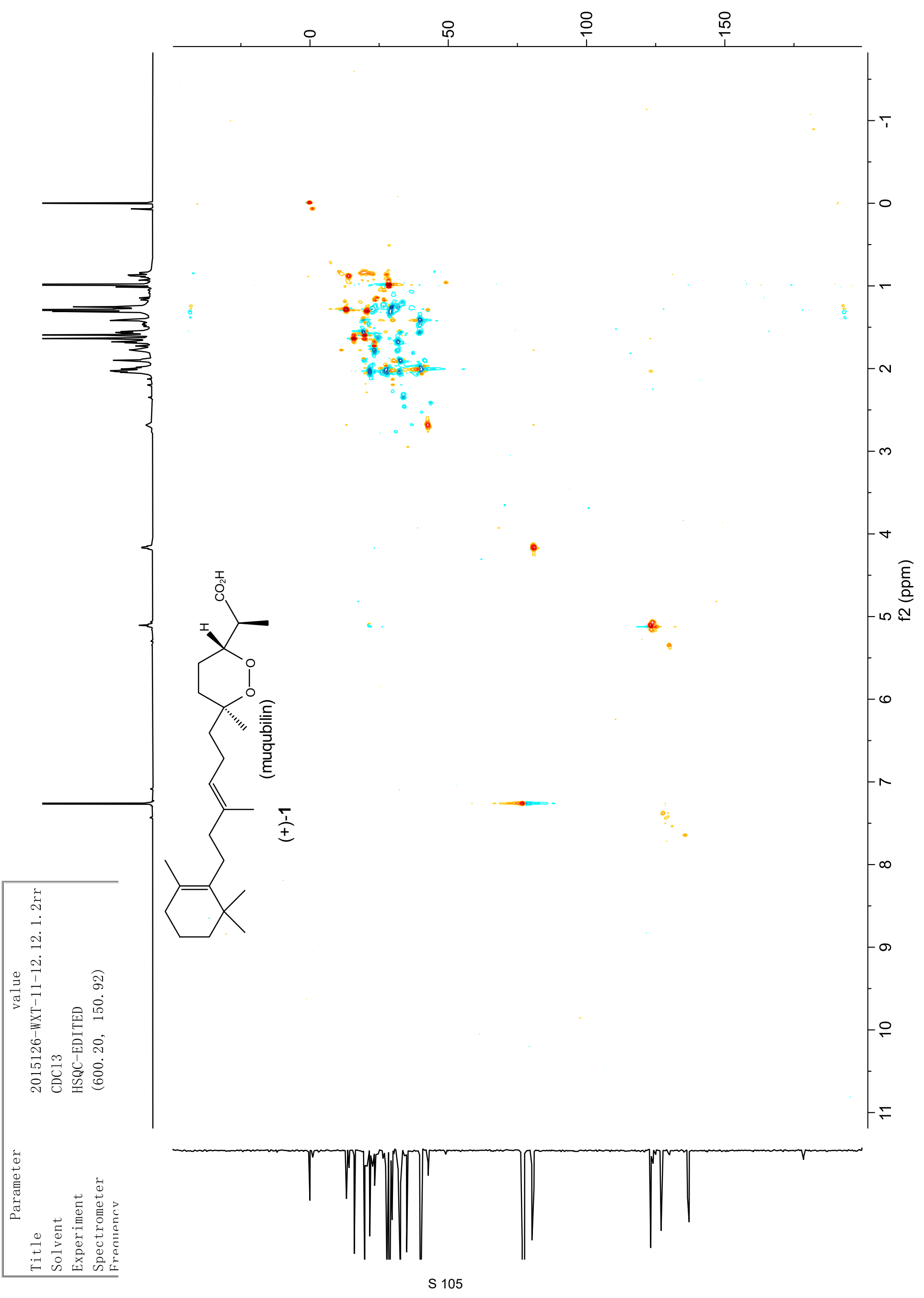


(udd) If

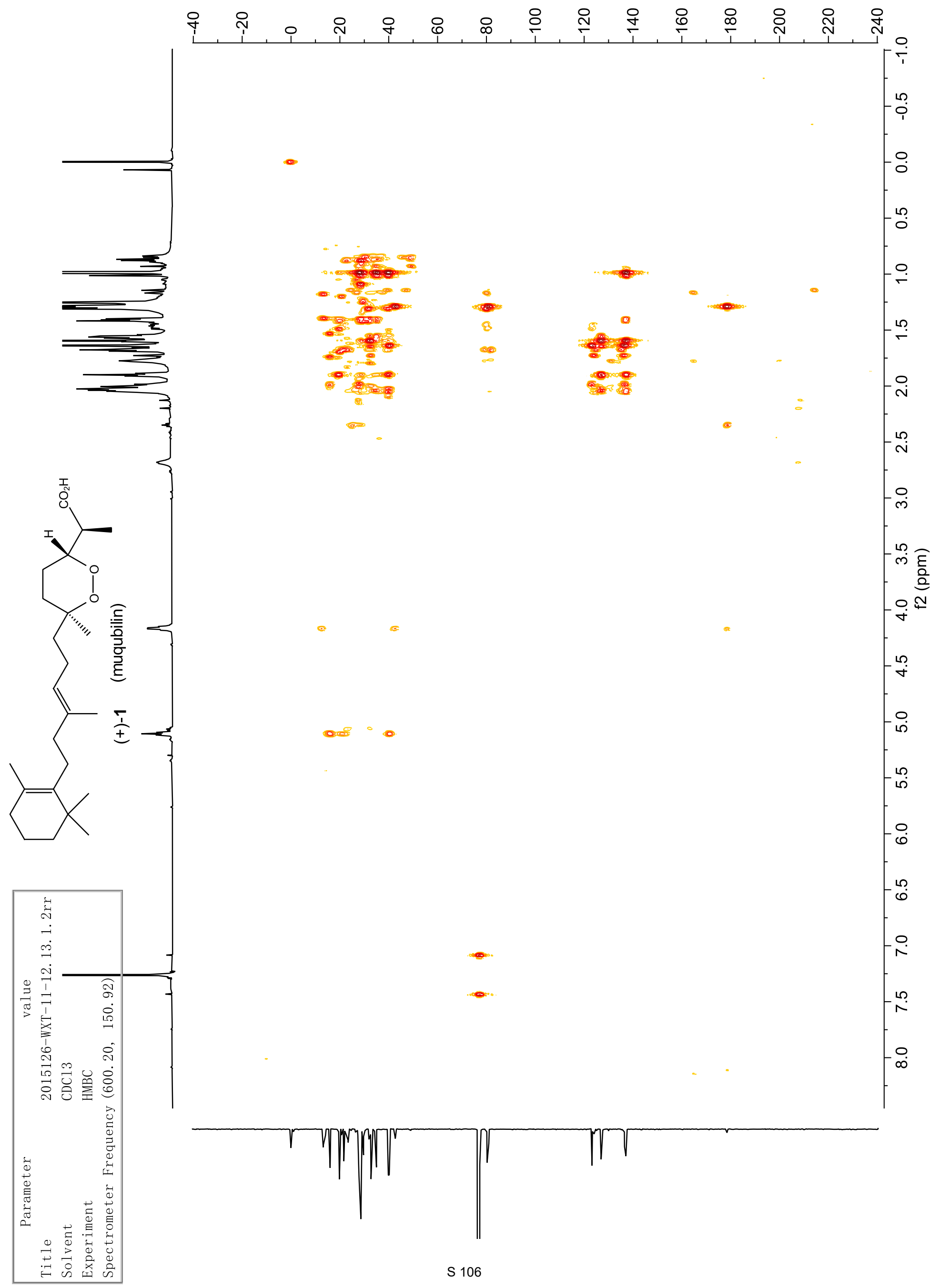



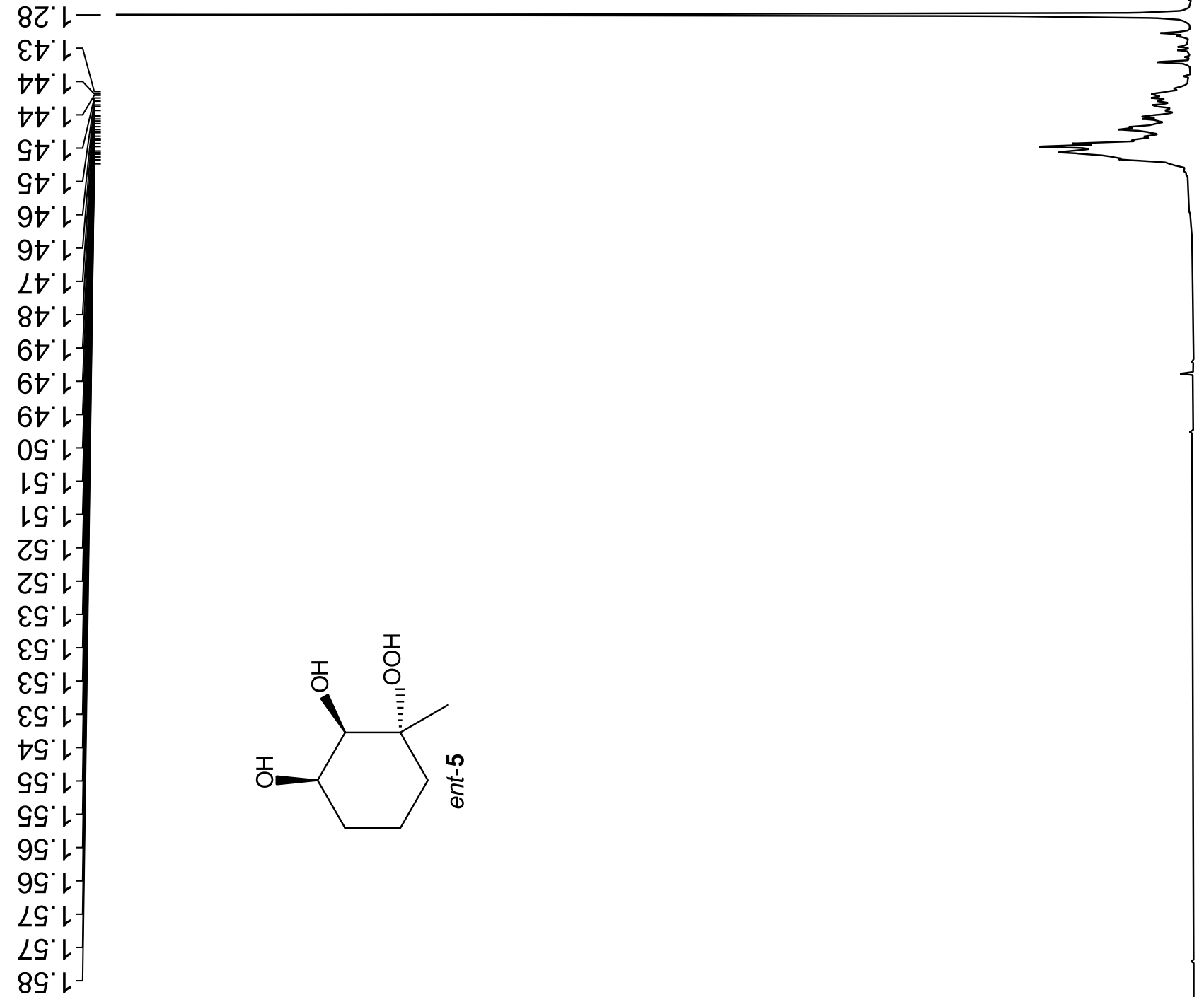

Gีเ

$9 \nabla^{\circ}$

$\angle \nabla^{\circ}$

$6 \nabla$

$6 \nabla^{\circ}$

IG'

IG'L

ZG'

EG"

$\varepsilon G^{*} L$

हs.

$\nabla S^{\prime} L$

.

99.

$99^{\prime \prime}$

$\angle G^{\circ} L$

$89^{\circ} L$

$\nabla L^{\circ} \varepsilon$

$G L^{\circ} \varepsilon$

$\varepsilon 8^{\circ} \varepsilon$

$\left.\varepsilon 8^{\circ} \varepsilon\right]$

$\succ 8^{\circ} \varepsilon$

$98^{\circ} \varepsilon-$

98. $\varepsilon^{\prime}$

$98^{\circ} \varepsilon$

$98^{\circ} \varepsilon$
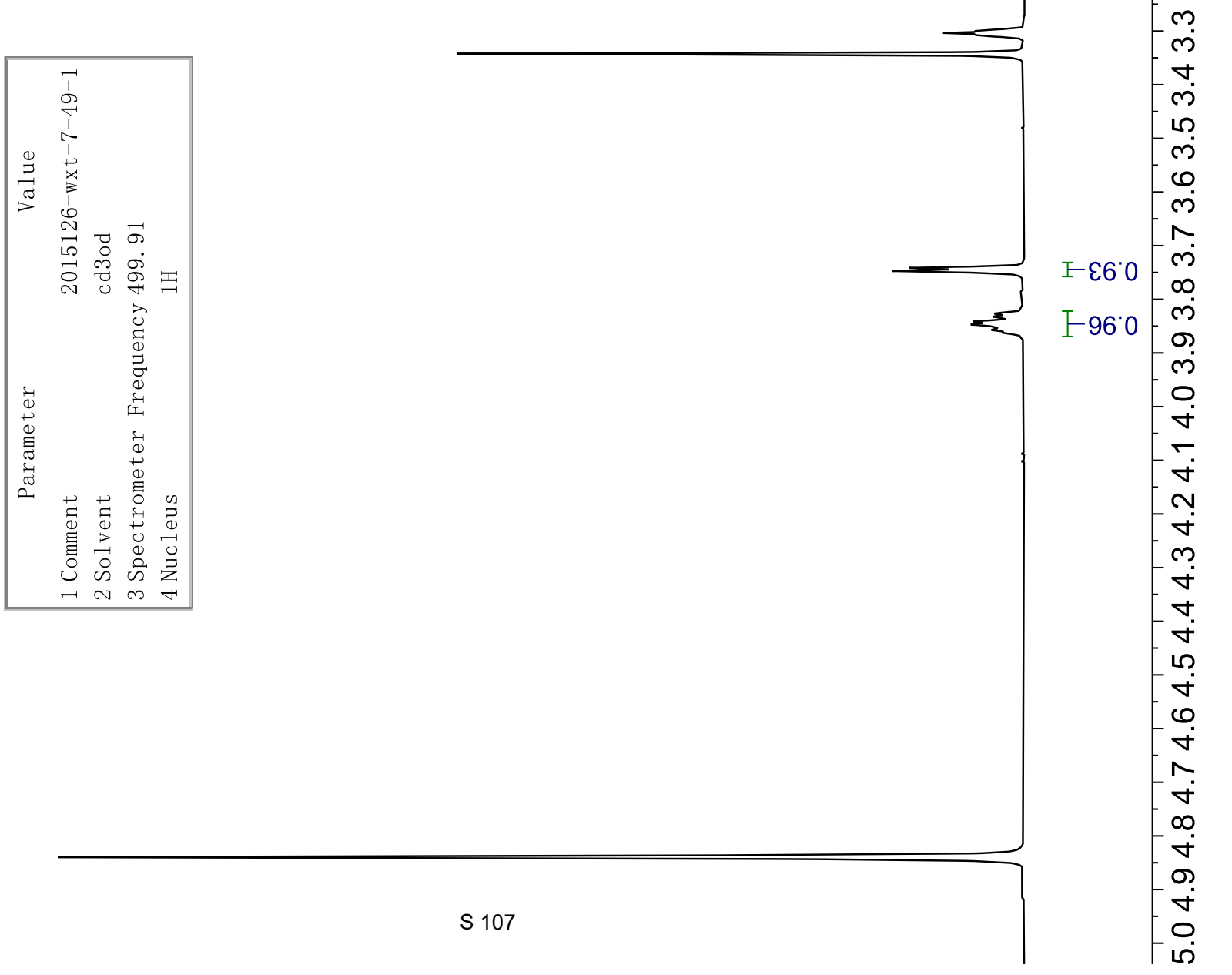
$89.81-$

s9.0Z-

$\varepsilon L \cdot \angle Z-$ $8062-$
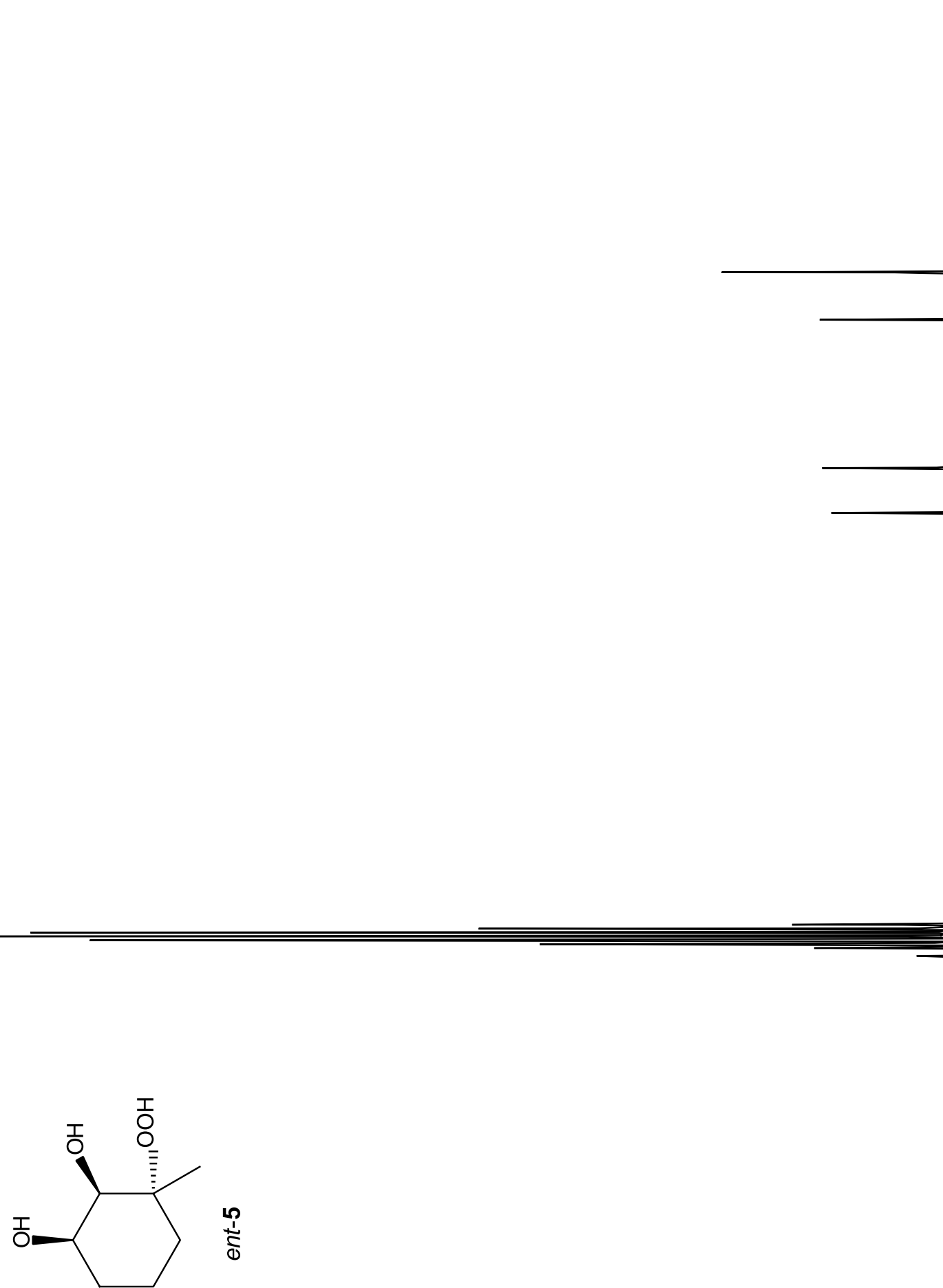

99'89-

$\angle 6^{\circ} L L-$

$6 L^{\circ}+8-$
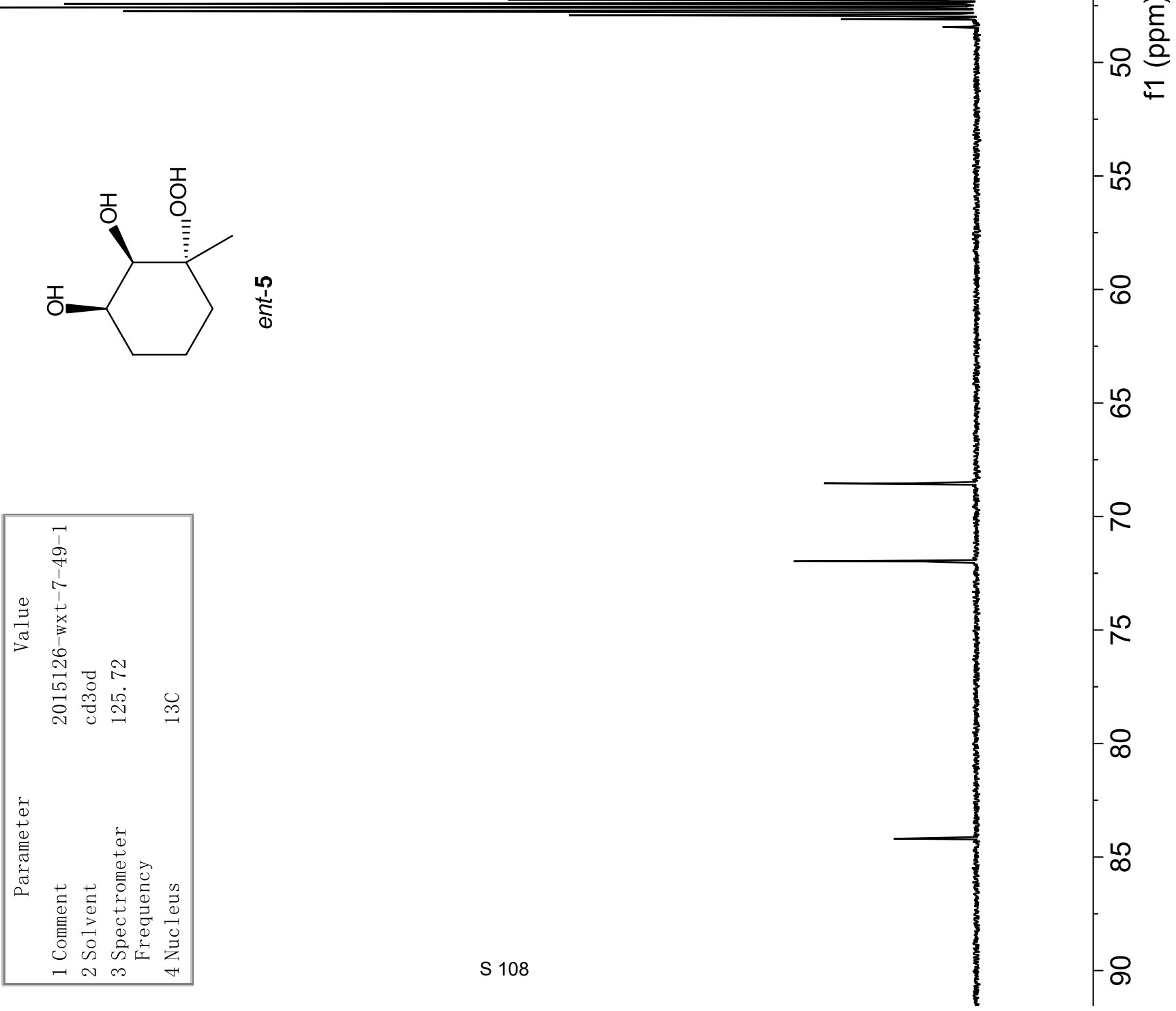


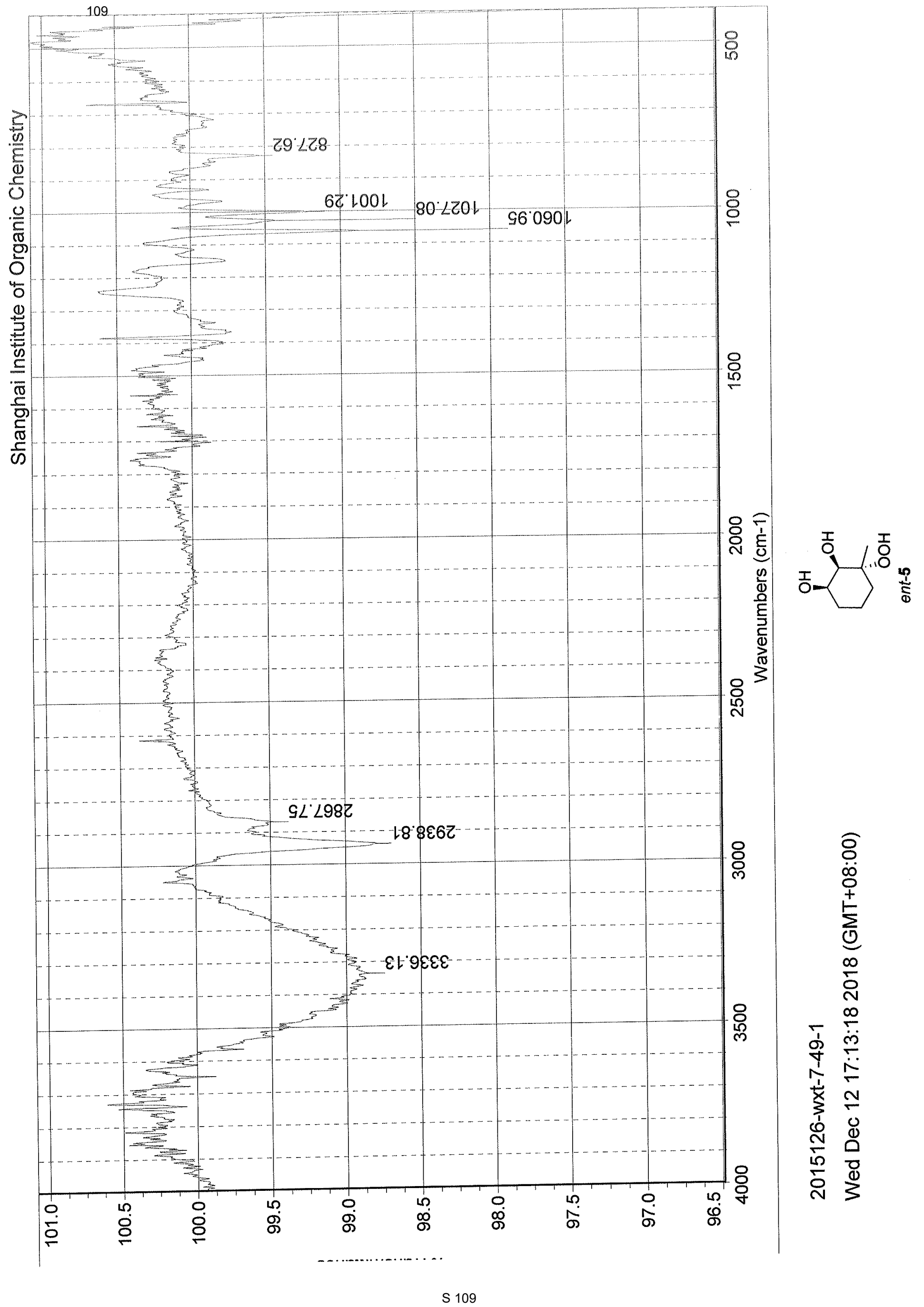


$\varepsilon L^{\circ} 0$
$\downarrow\llcorner$
$\circ$

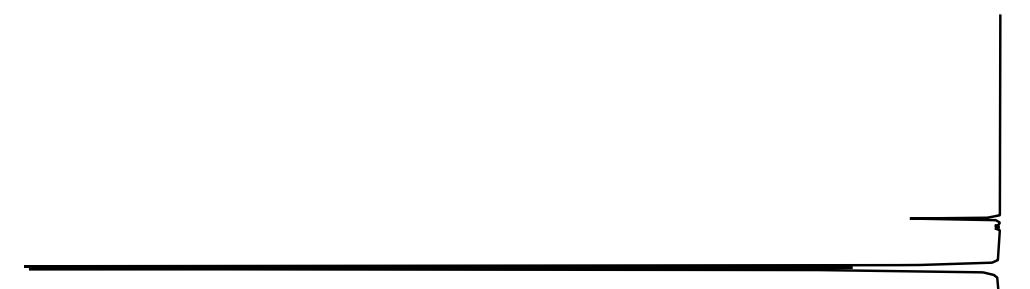

$\mid \begin{gathered}n \\ 0 \\ 0 \\ 0 \\ 0 \\ 0\end{gathered}$

-88'

そ๐०O

$9 \varepsilon^{*} レ-$

$8 Z^{\circ} 乙$

$8 Z^{\circ} 乙^{\zeta}$

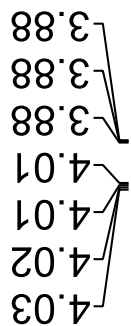

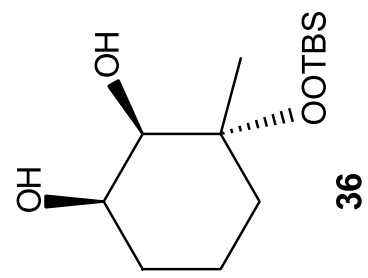

요
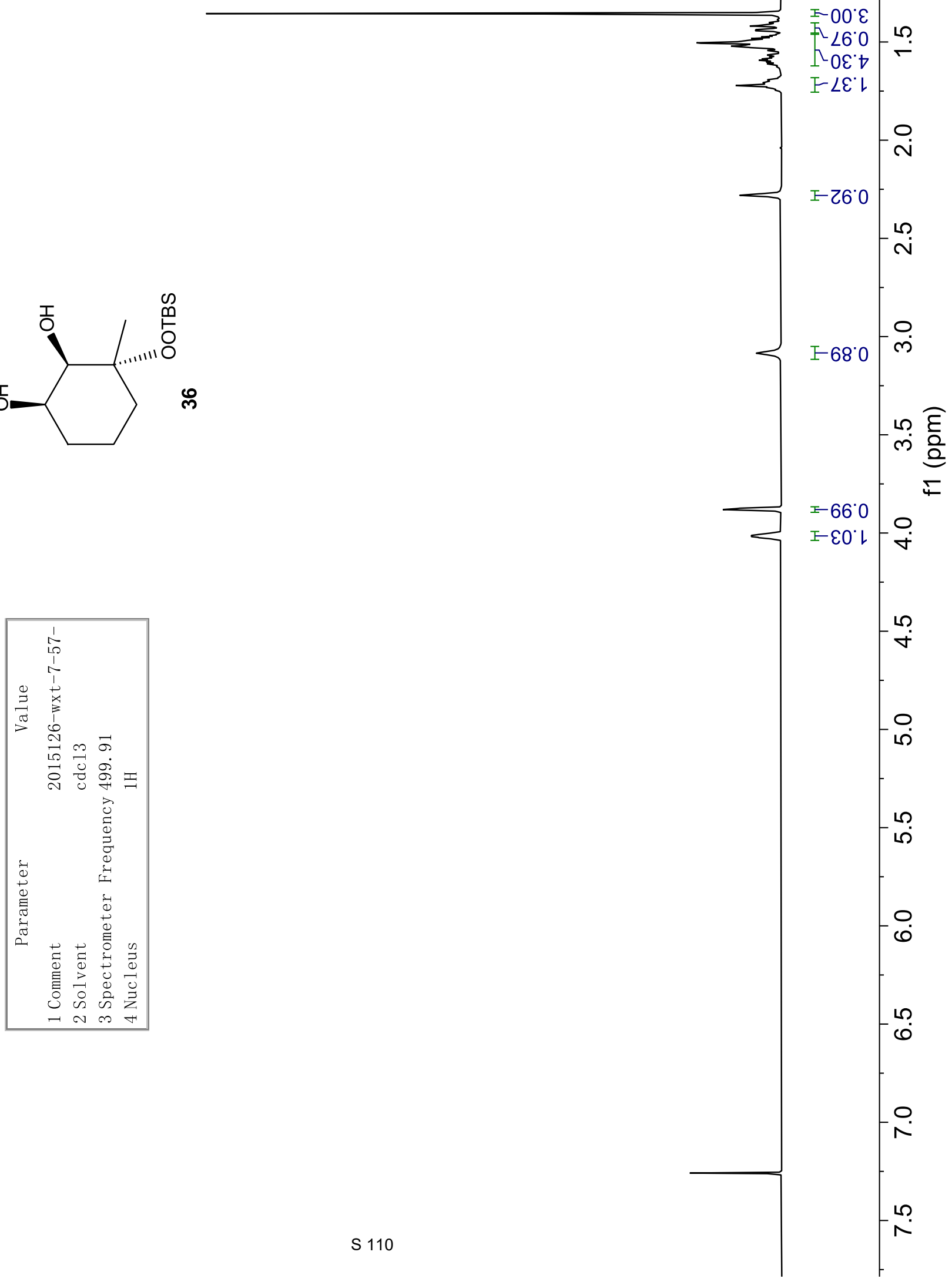
$79^{\circ} \mathrm{c}^{-}$

\&9. $9^{-}$

$9 L 8 L$
$7{ }^{\circ} 8 L$
$9 L^{\circ} 6 L$

$0 乙^{\circ} 9 乙^{\digamma}$

SL'8Z-

9L'L乙

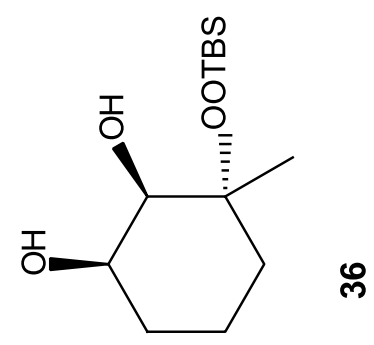

$\angle 8^{\circ} \nabla 8-$
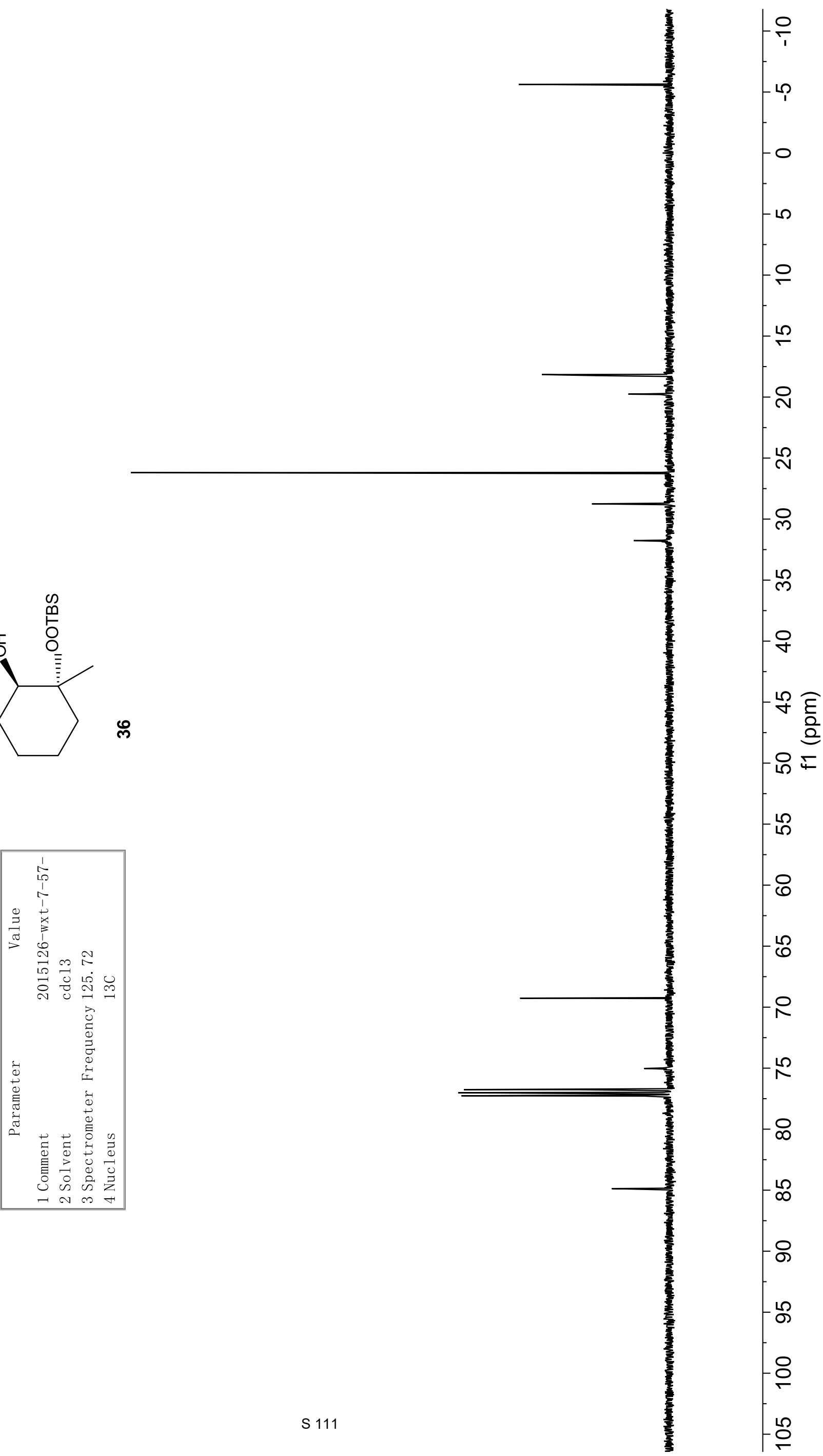


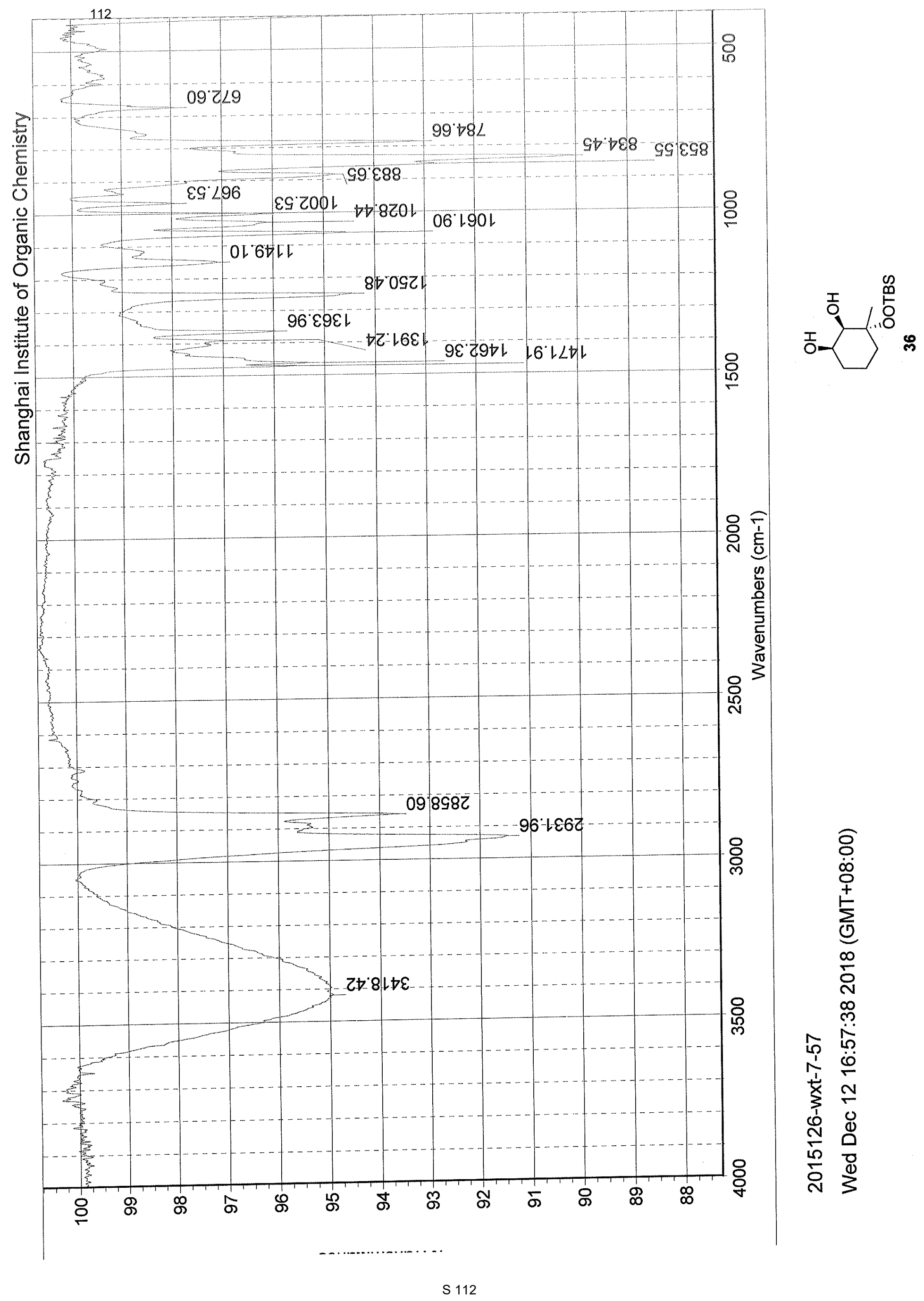


$\frac{\nabla L^{\circ} 0}{\Delta L^{\circ}}$ 
ง8Eち乙 乙 してGヤてカヤ9๑てて

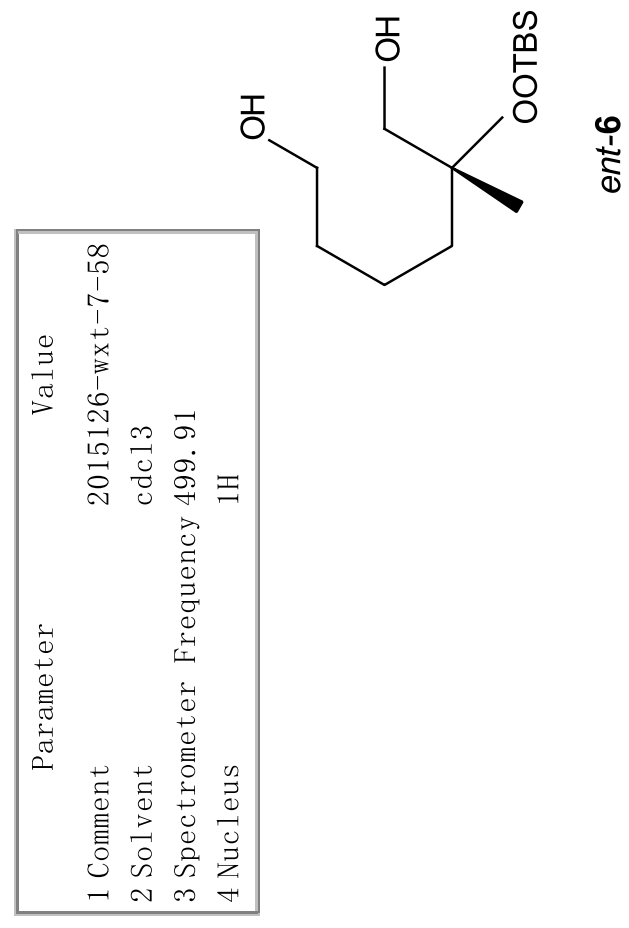

6งtG' 6699 $\varepsilon$ $\neg 699^{\circ} \varepsilon$ ] $\downarrow \varepsilon 89^{\circ} \varepsilon-$ $\varepsilon 019^{\circ} \varepsilon \backslash$ 8L乙9 $\varepsilon-$

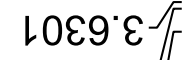

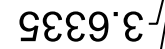
เS†9 I $^{\circ}$
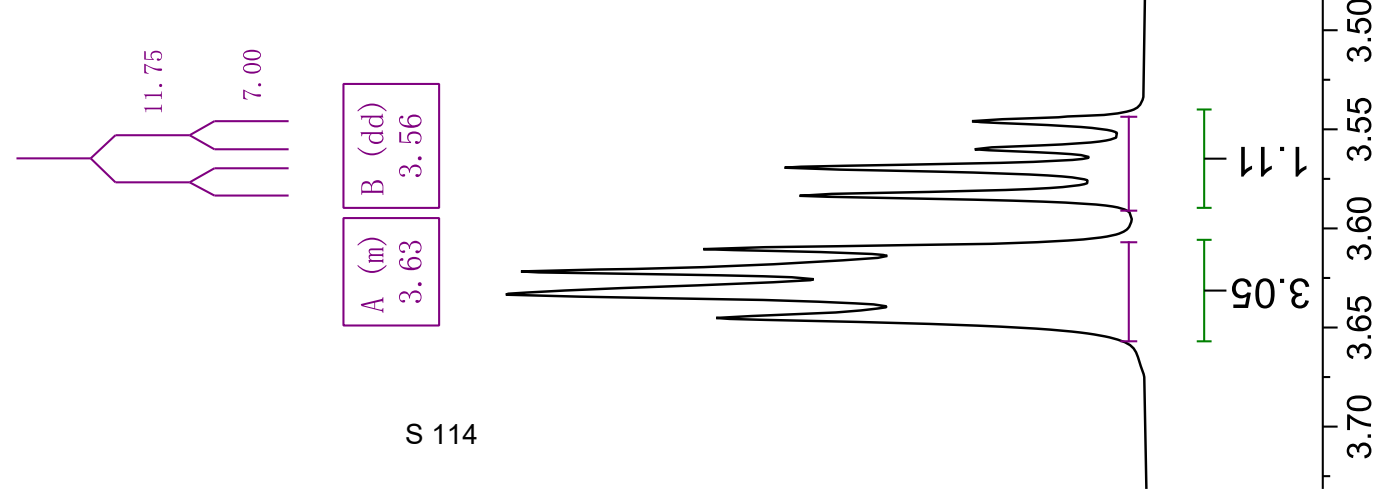

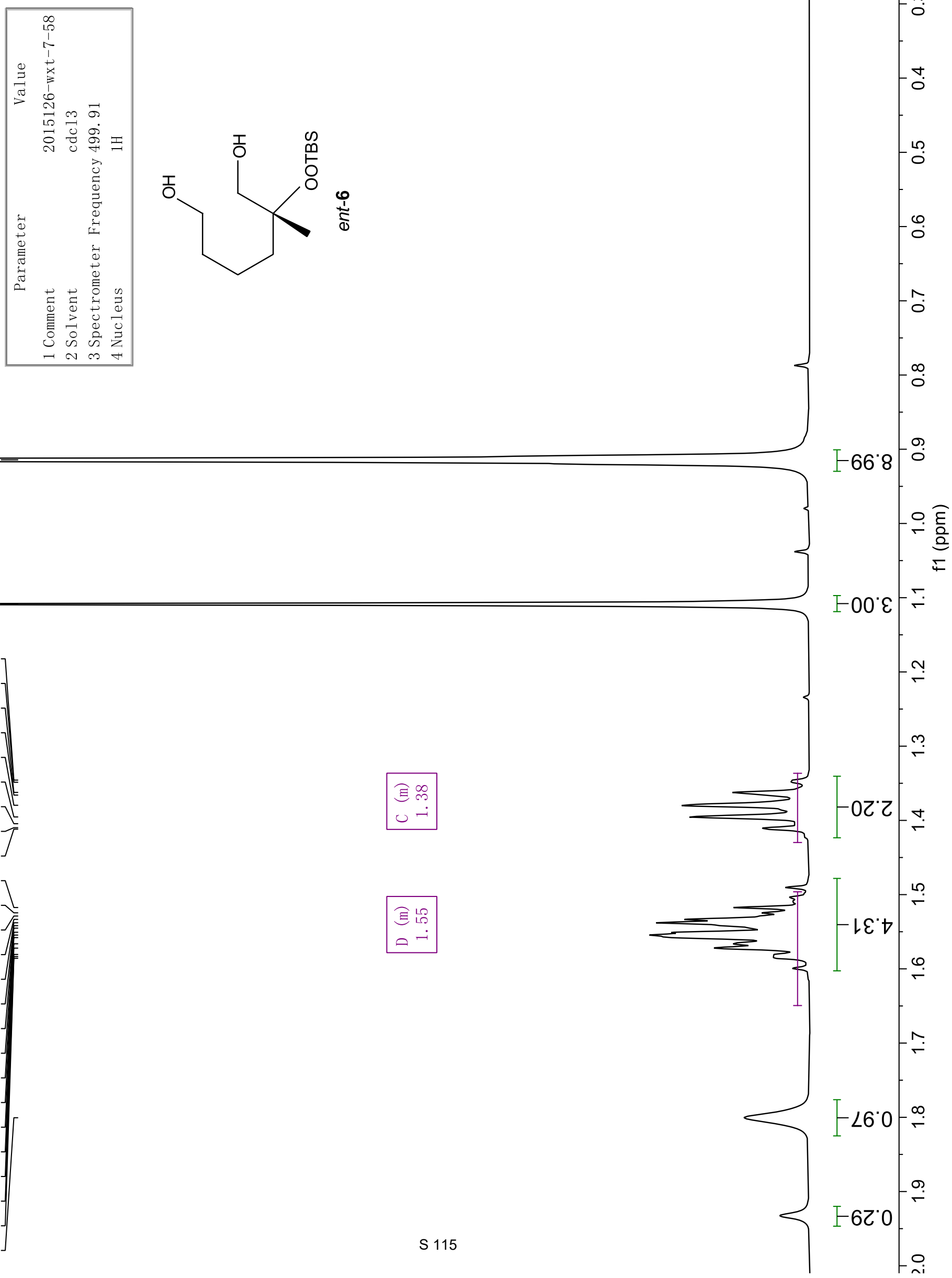
$6 L^{\circ} 8 L$
$87^{\circ} 81$

696l

จレ9乙

$90^{\circ} \varepsilon \varepsilon>$

$\angle \mathcal{G}^{\circ} \varepsilon \mathcal{E}^{-}$

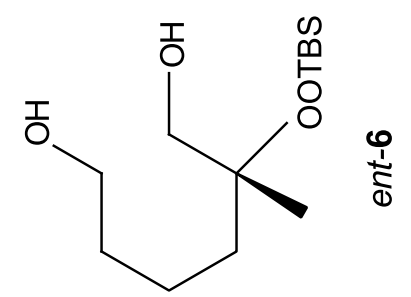

09'29-

IS $29-$
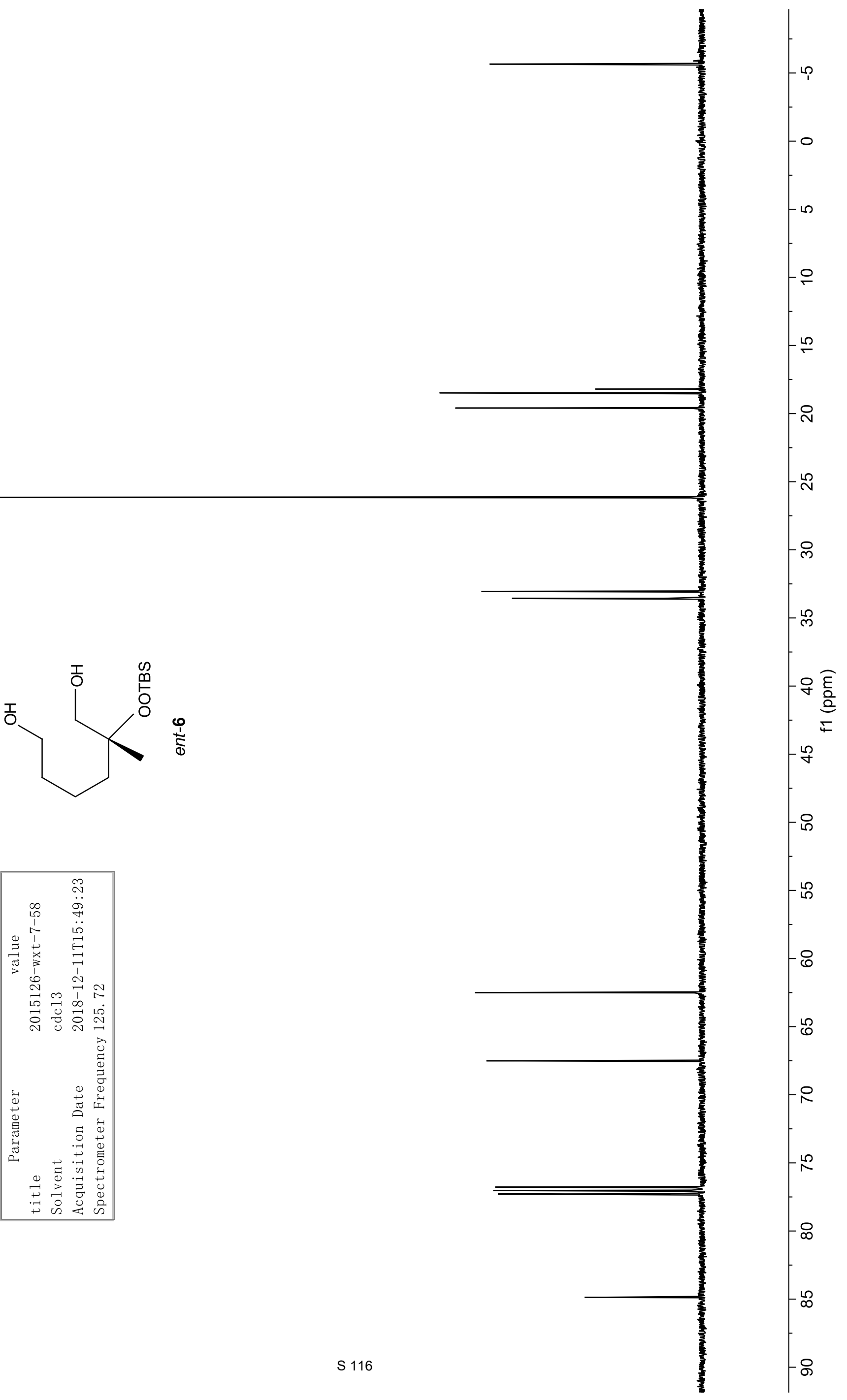


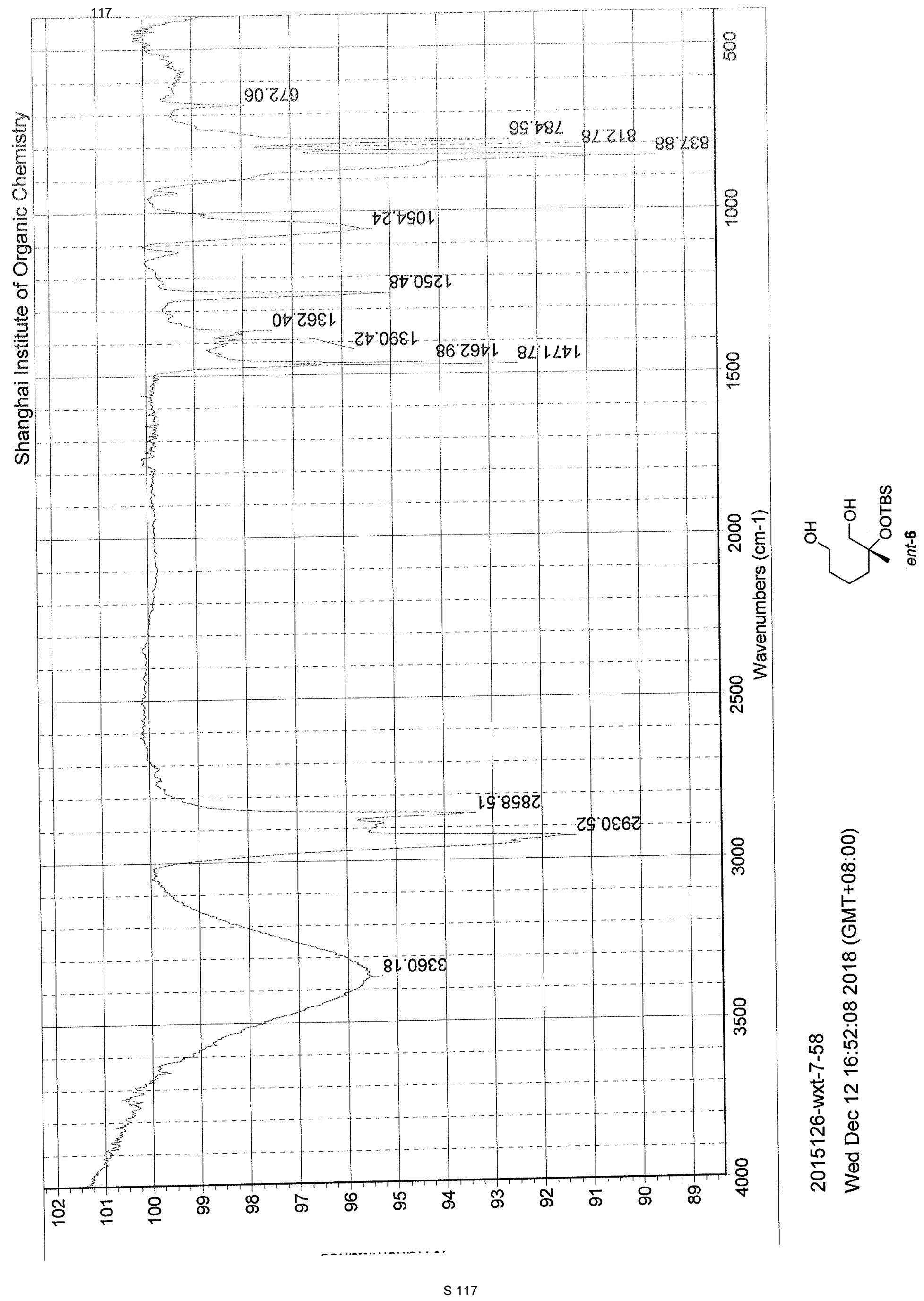


s.0

$\mathrm{Sl}^{\circ} \mathrm{O}^{-}$

$26^{\circ} 0$

$70 \mathrm{~L}$

हl.

$9 \varepsilon^{\prime} Z$
$9 \varepsilon^{\prime} z$
$8 \varepsilon^{\prime} \tau^{\top}$

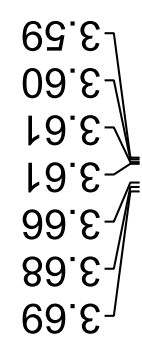

$9 Z^{\prime}\llcorner-$
กิ

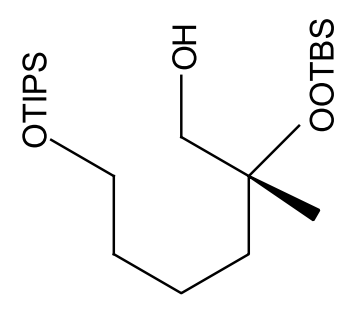

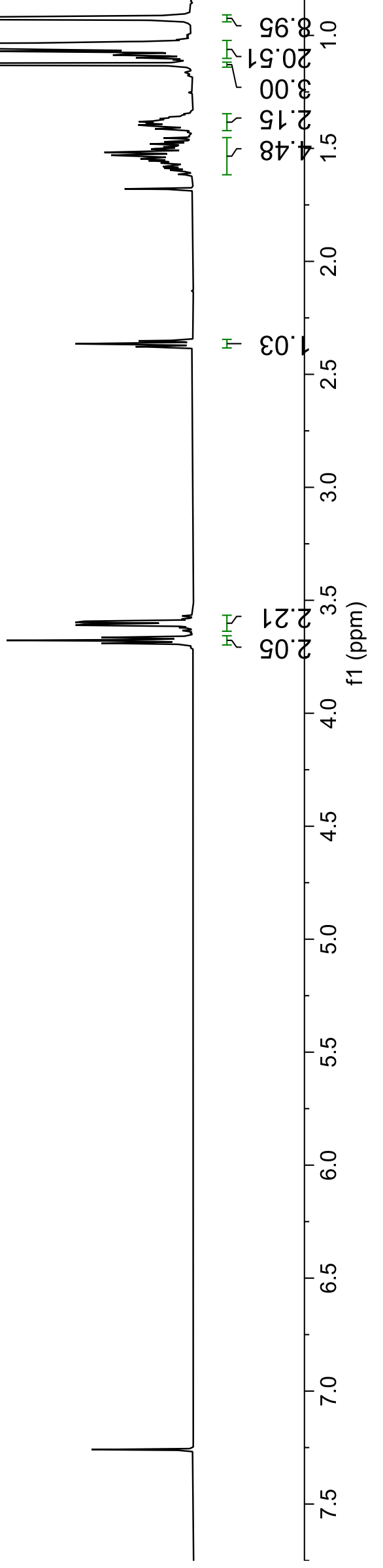


${ }{ }^{\circ} 9^{\circ} \varepsilon-$

ع乙8૬'

LZ6G' $\varepsilon^{\varsigma}$

$\angle L 69^{\circ} \varepsilon^{-}$

$\angle S 09^{\circ} \varepsilon \backslash$

G०เ9'

ル乙9

6єع9 ${ }^{\circ} \varepsilon-$

$\angle \succ 99^{\circ} \varepsilon-$

$G \angle \angle 9^{\circ} \varepsilon-$

$\varepsilon^{8069^{\circ} \varepsilon-}$

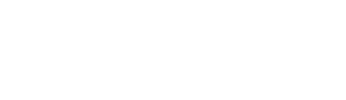

กิำ<smiles>CC([O])([O])CCCCO[O-]</smiles>
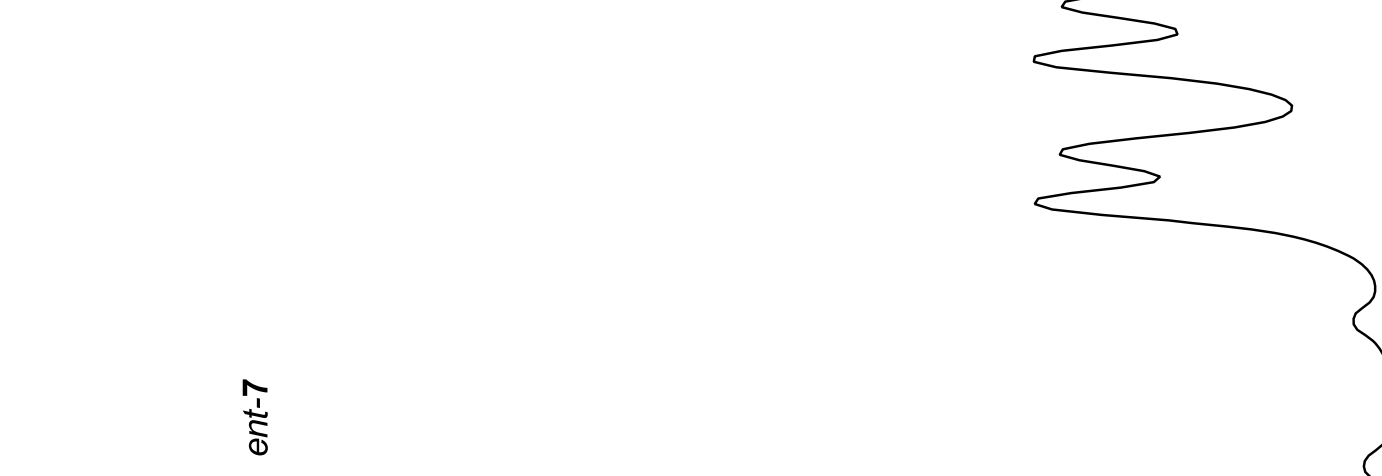

ङ
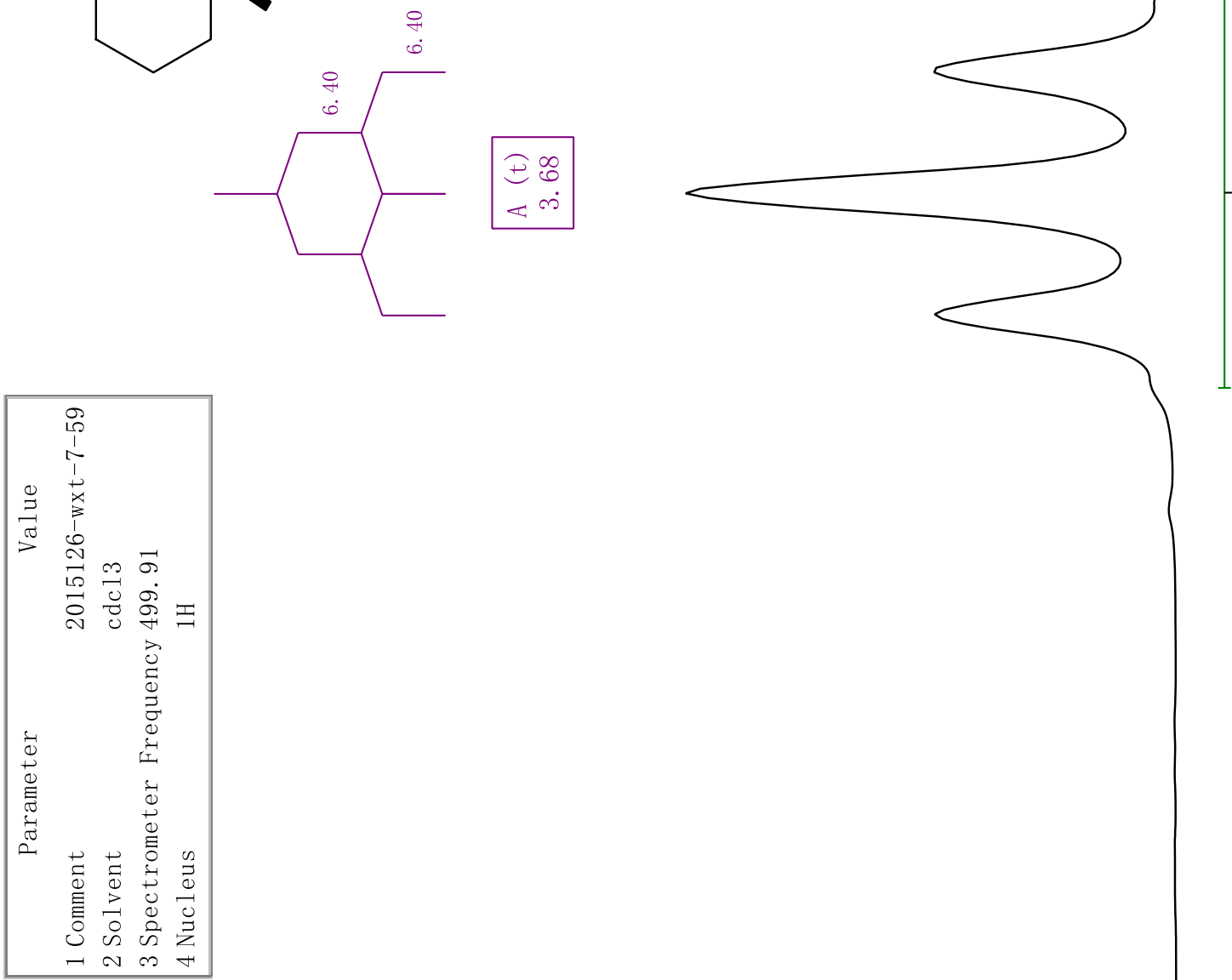

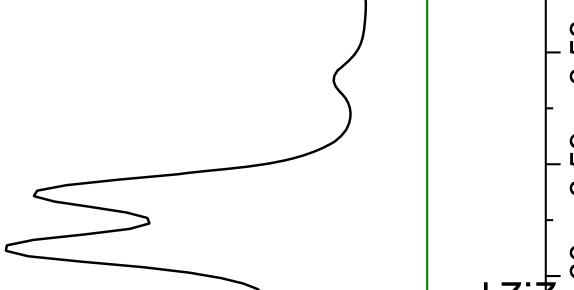


$\left.\begin{array}{l}89^{\circ} G^{-} \\ \left\langle 9^{\circ} G^{-}\right.\end{array}\right\rangle{ }^{120}$

L6ルー

$20 \% 8 \mathrm{~L}$

$6 \mathrm{~L} 8 \mathrm{~L}$

$\angle \varepsilon^{\circ} 8 \mathrm{~L}$

$28 \% \mathrm{~L}$

9レ'9Z-

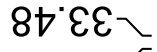

$\angle 6^{\circ} \varepsilon \varepsilon^{-}$

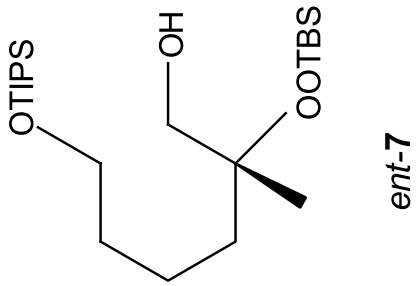

9.'ع9-

$\neg 8^{\circ} \angle 9-$

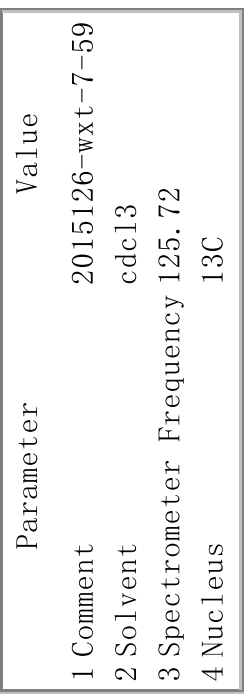

$06 * \nabla$ 


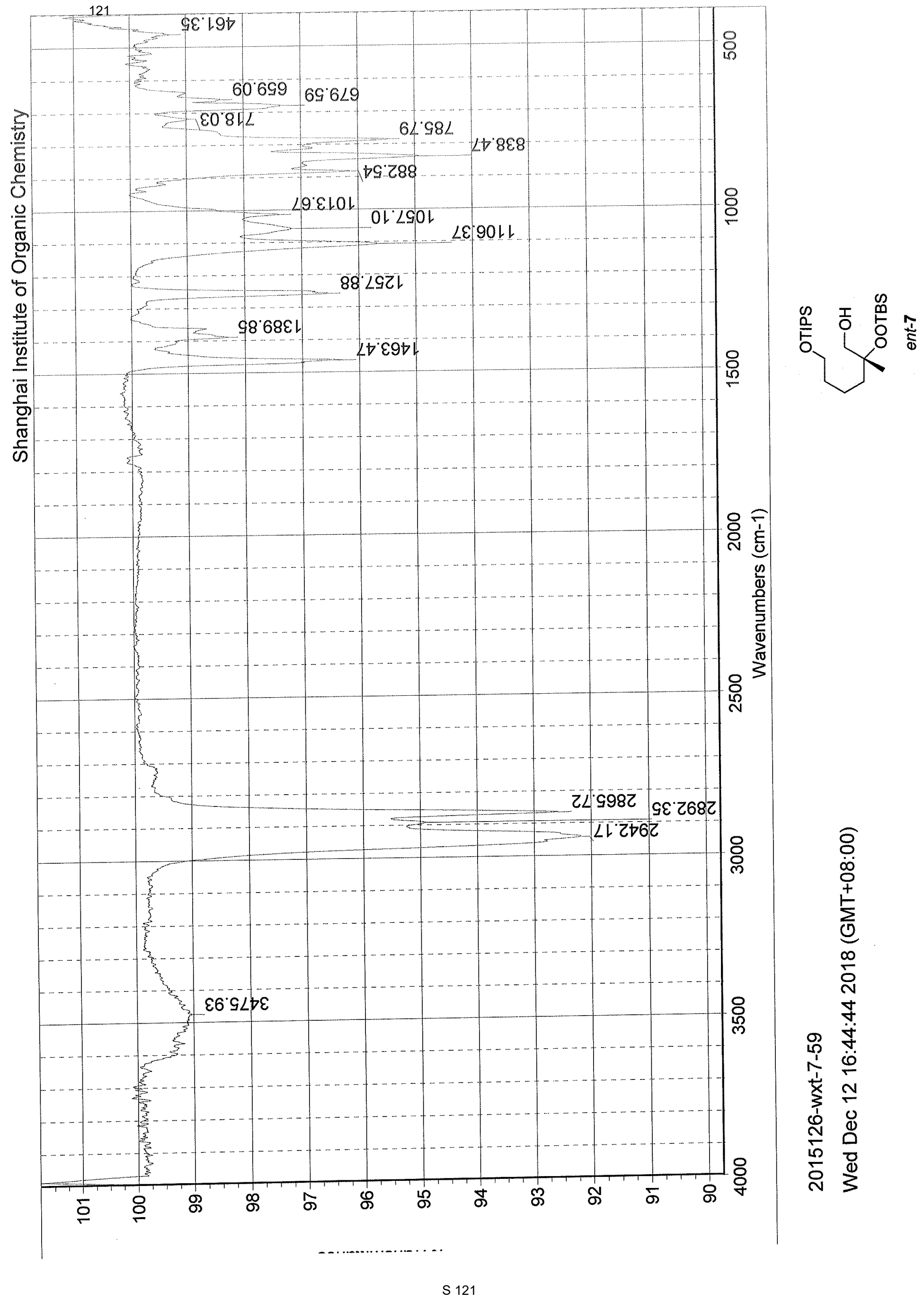




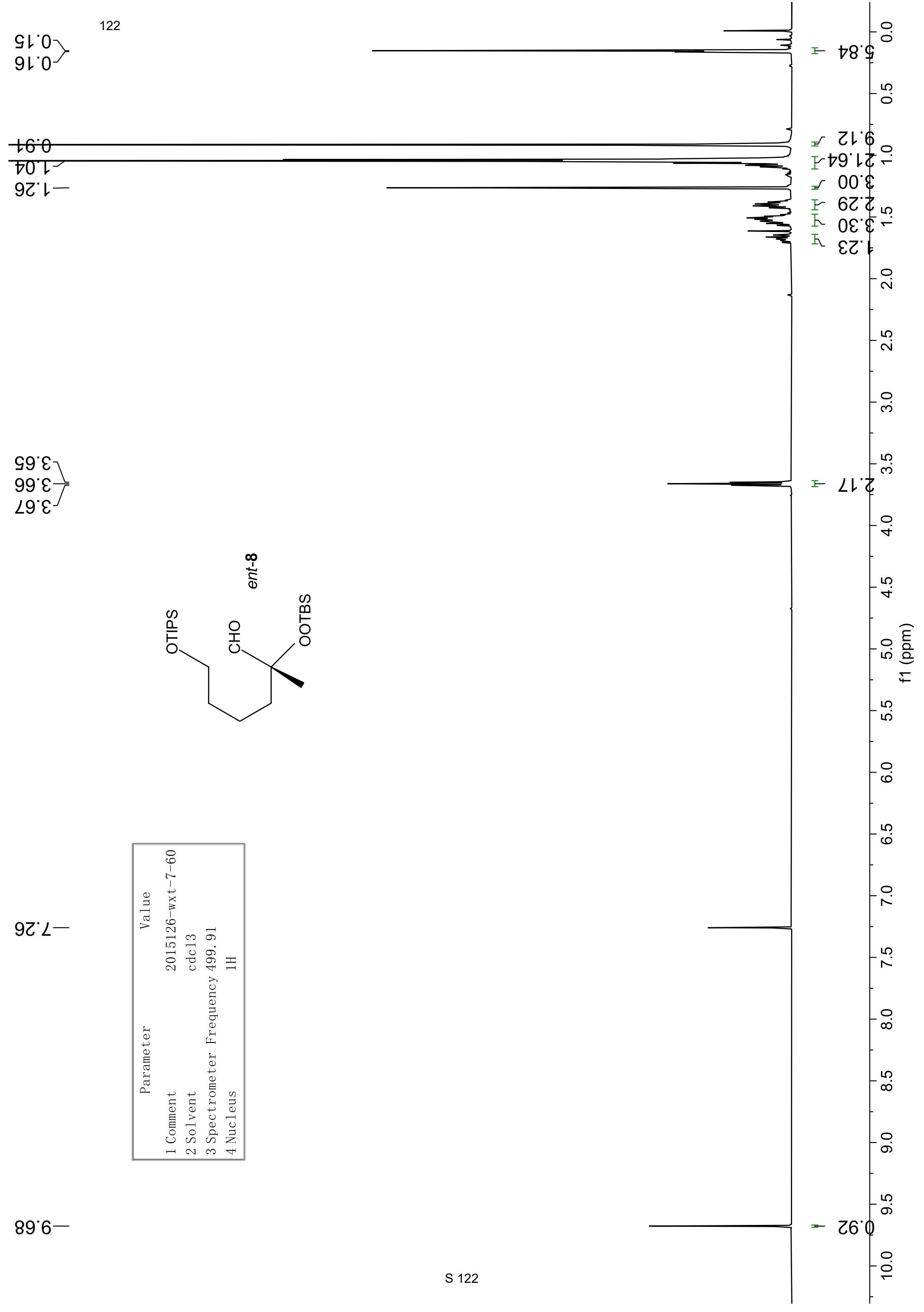


$\left.\begin{array}{l}G L G^{-} \\ \nabla L G^{-}\end{array}\right\rangle^{123}$

ย6เレ

$19.9 \mathrm{l}$

$108 \mathrm{~L}$

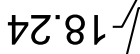

$026 \mathrm{l}$

$20.92^{\varsigma}$

$66^{\circ}$ 乙E

O乙' $\varepsilon \varepsilon^{-}$

$76.29-$

$\varepsilon L ' 88-$
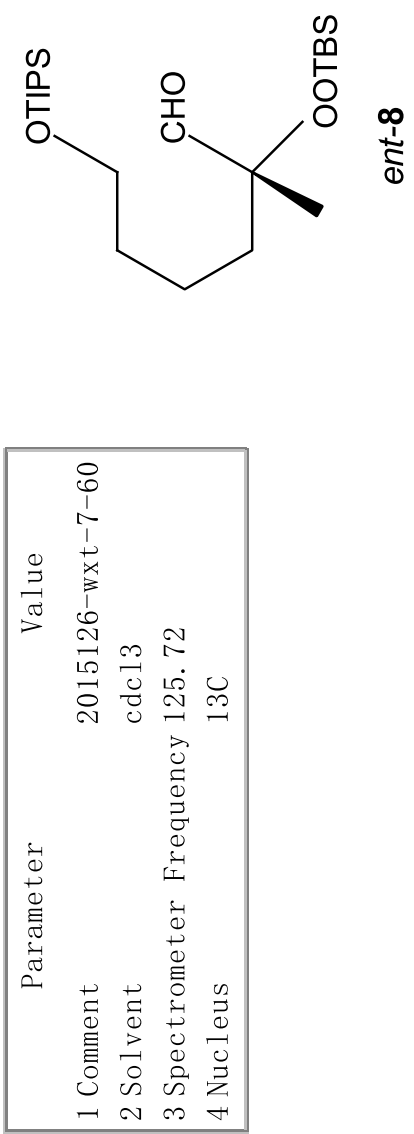


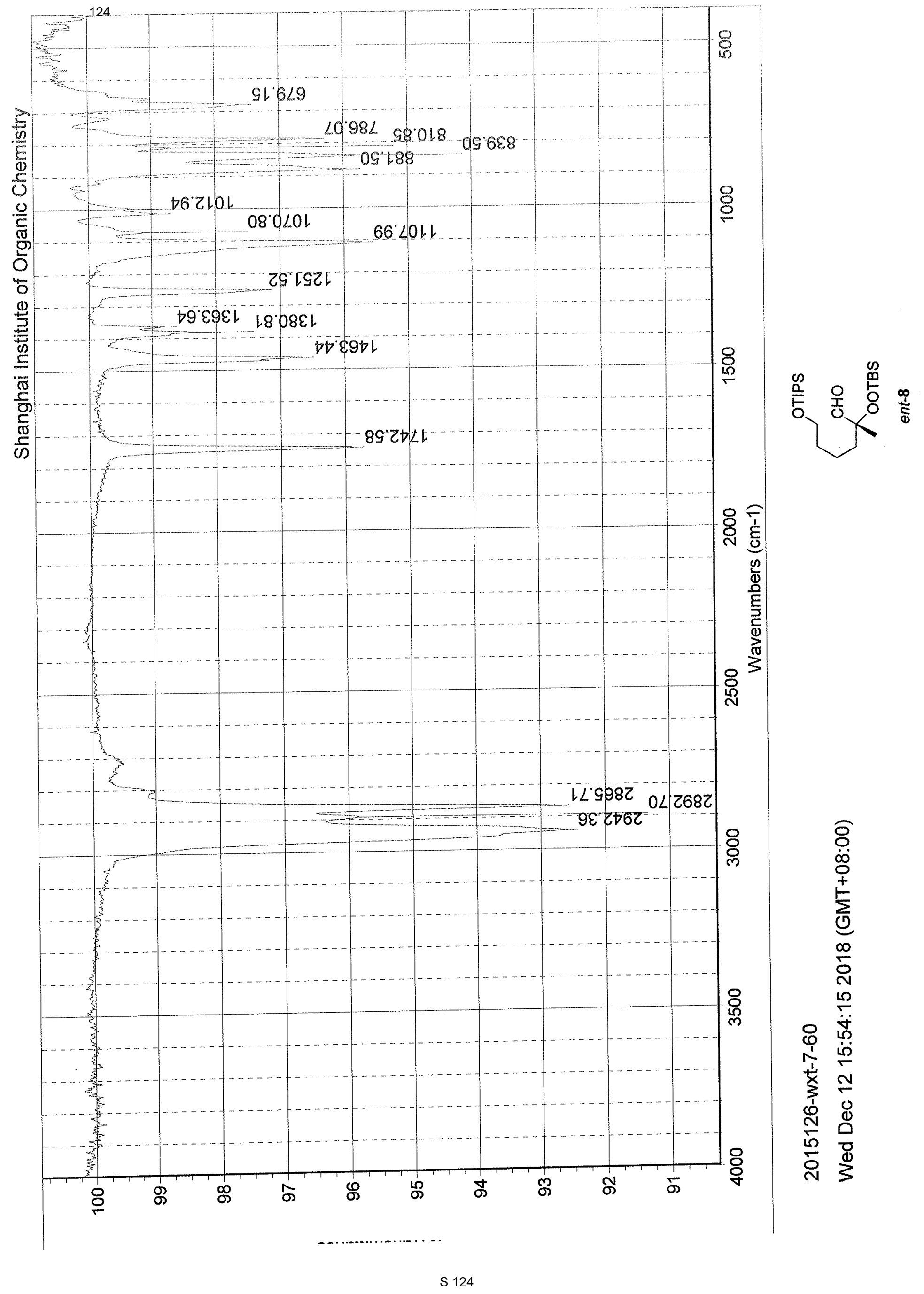


$\varepsilon L^{\circ} 0$
$\varepsilon L^{\circ} 0$

$20^{\circ} 0$

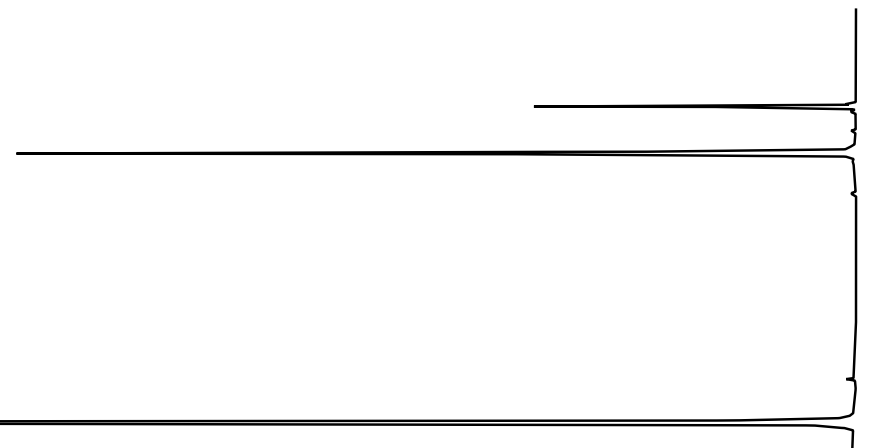

to

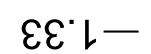

$\not 9^{\circ} \varepsilon$
$99^{\circ} \varepsilon$
$\angle 9^{\circ} \varepsilon^{\prime}$
$\dagger L^{\circ} \varepsilon$

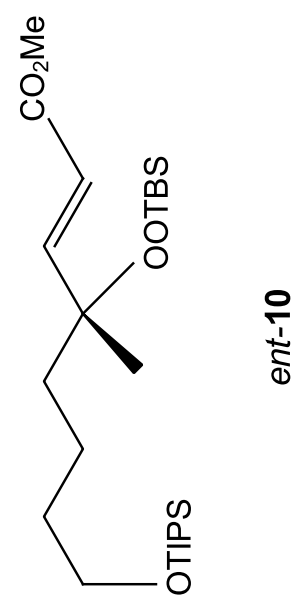

28.9

$06^{\circ} 9^{-}$

96.9

$66^{\circ} 9$

$9 Z^{\circ} \mathrm{L}$

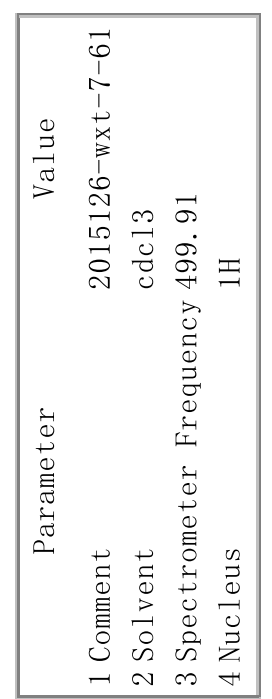


Z9.9- $>126$

96 ルレ
1081
$28^{\circ} 81-$
$\angle 6.6 L$ $08 \mathrm{~L}^{5}$

6เ.92-

乙乙 $\varepsilon \varepsilon-$

$89^{\circ} \angle \mathcal{E}-$

99'IS-

ル๕89-

68' $88-$

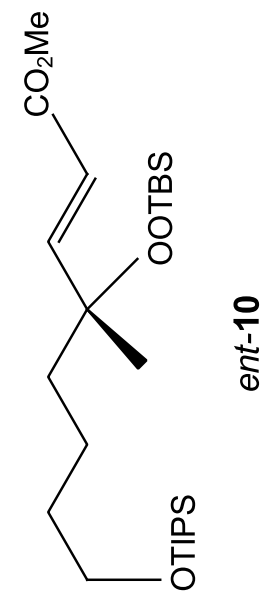

086ルー
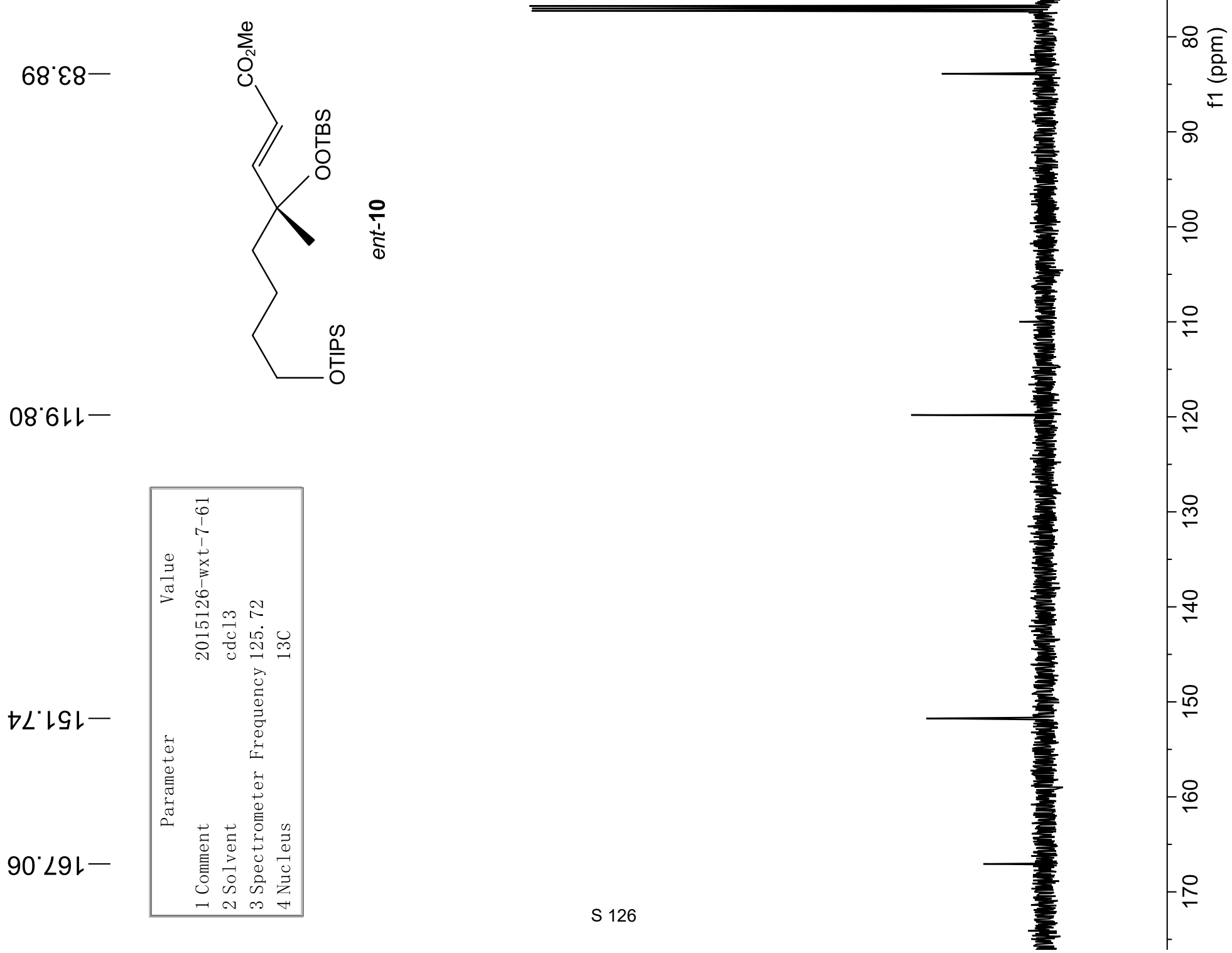


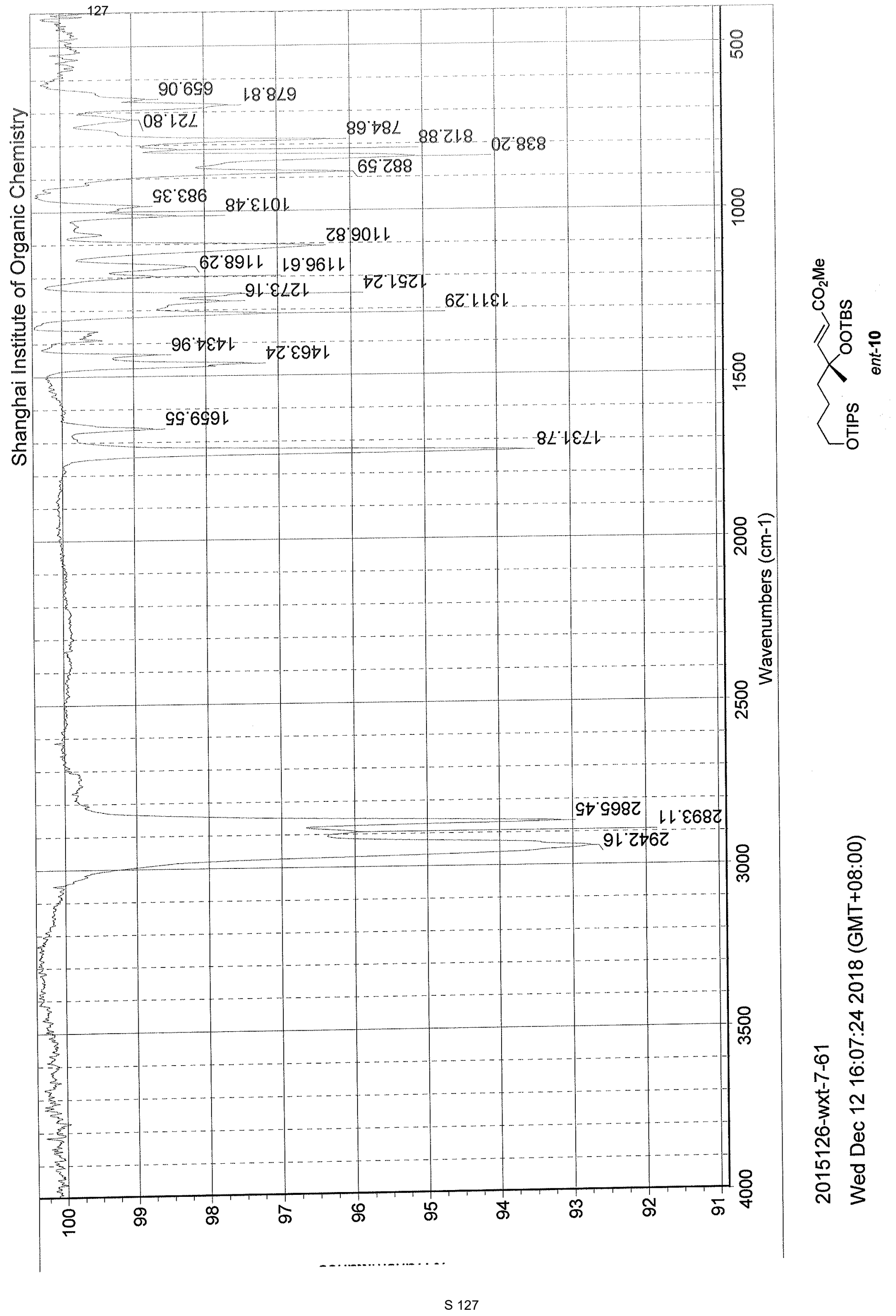




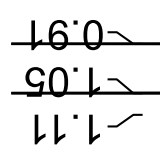

$$
\begin{aligned}
& \text { L8 } \\
& 06^{\circ} \text { เ } \\
& \nabla \varepsilon^{\prime} 乙 \\
& \nabla \varepsilon ' 乙 \\
& \text { GE'乙- } \\
& 9 \varepsilon^{\prime} 乙 \\
& 9 \varepsilon^{\circ} 乙 \\
& 9 \varepsilon " 乙 \\
& \angle \varepsilon^{\circ} 乙 \\
& 8 \varepsilon^{\circ} \tau^{\circ}
\end{aligned}
$$$$
\left.\begin{array}{l}
99^{\circ} \varepsilon \\
99^{\circ} \varepsilon \\
\angle 9^{\circ} \varepsilon \\
89^{\circ} \varepsilon
\end{array}\right]
$$

$89 \varepsilon$
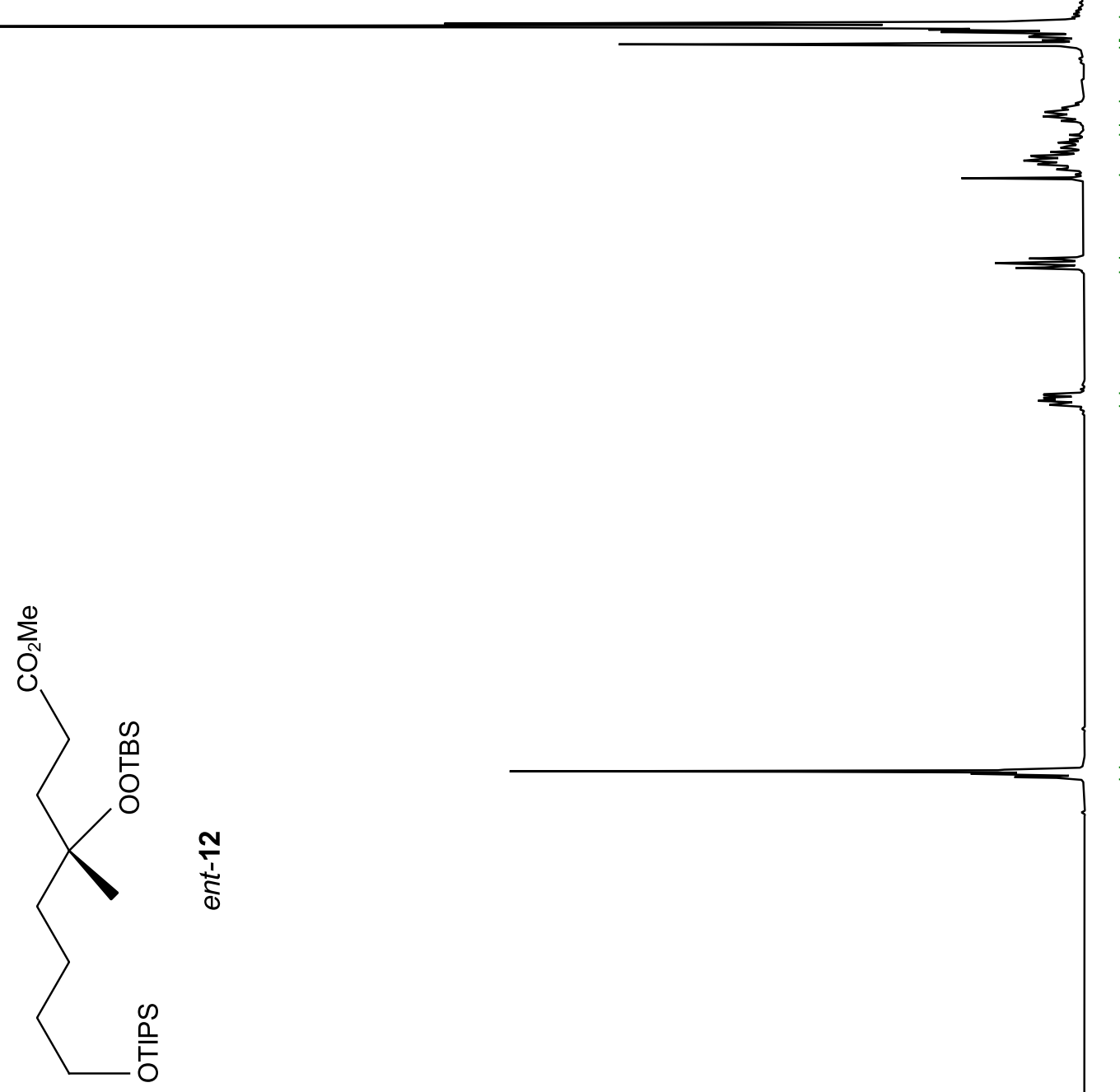

- $62 \% 0$

下o.0を U⿺ - $0 \varepsilon$ $09+\stackrel{n}{\circ}$

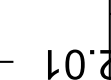

i

I 76

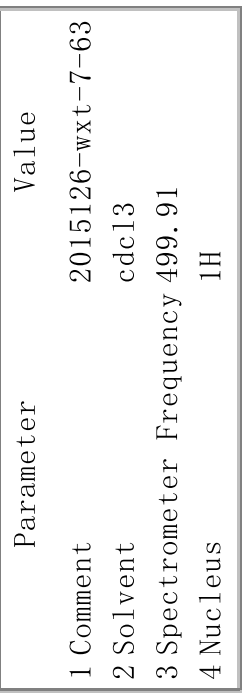

웅 
Z998'

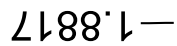
†868'
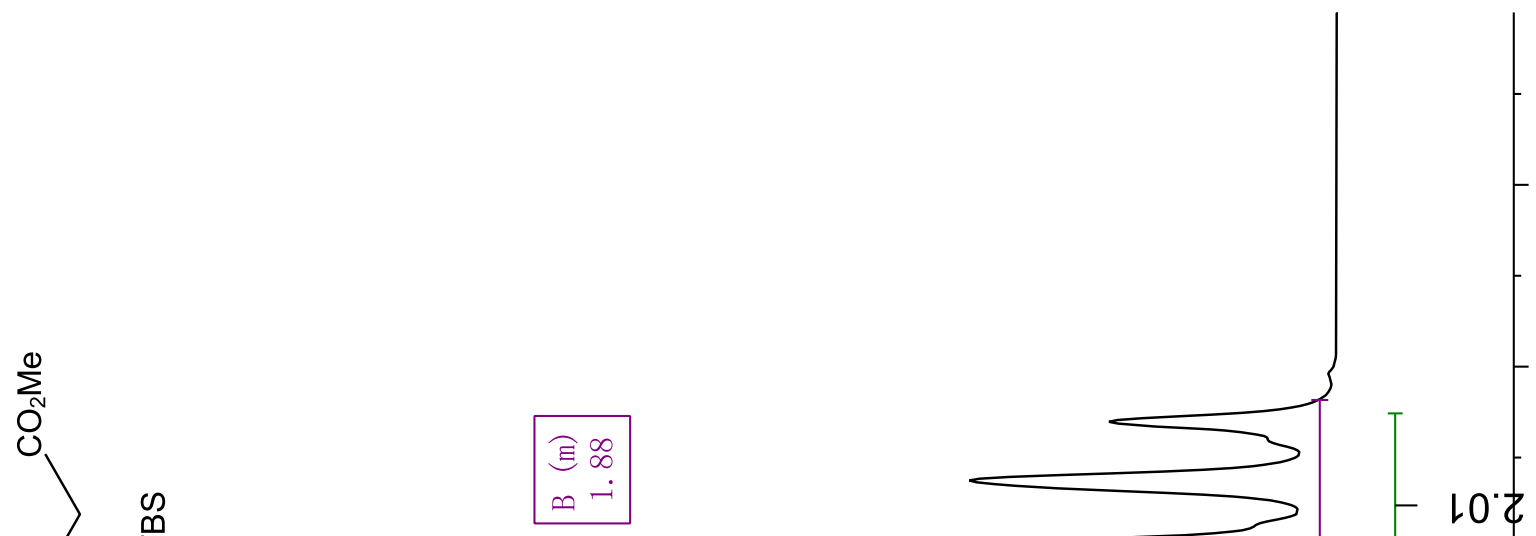

$\stackrel{\infty}{-}$

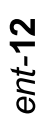

$\stackrel{\infty}{\stackrel{\infty}{+}}$

8

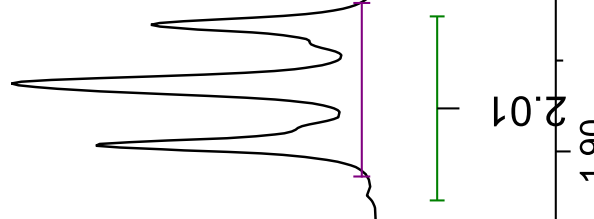

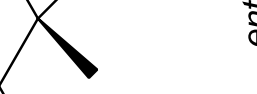

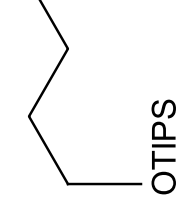

$\frac{0}{\infty}$

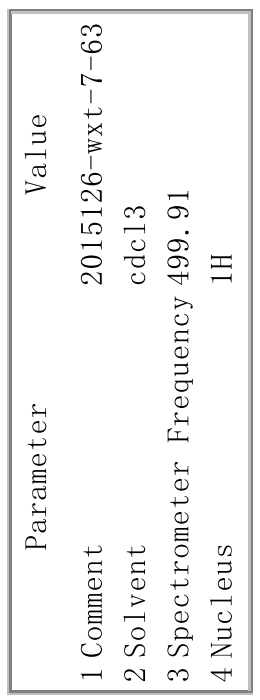

$90 \downarrow \varepsilon 乙$

$9 \nabla \downarrow \varepsilon ' 乙$

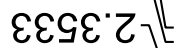

乙LGE乙 乙

$\angle L 9 \varepsilon^{\circ} Z^{-}$

9†9ع'乙]

$\downarrow \varepsilon \angle \varepsilon^{\prime}{ }^{\prime}$

$\begin{array}{ll}E & \infty \\ \infty & - \\ & -1\end{array}$

政

욤

乙8L $\mathcal{E}^{\prime}$

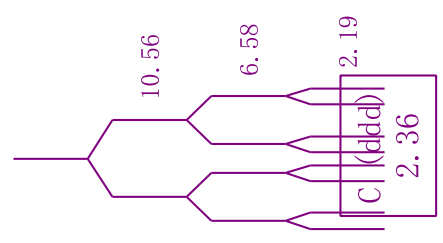


L6・レ

$\varepsilon 0.81$
$92.81]$

$70^{\circ} 0 \mathrm{Z}-$

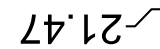

l乙.9Z-

$29^{\circ} 8 \mathrm{Z}^{-}$

乙L'L

OS $\varepsilon \varepsilon^{\circ}$

$69^{\circ} 9 \varepsilon^{\prime}$

SG'LS-

$\nabla{ }^{\prime} \varepsilon 9-$

૬ع'๕8-
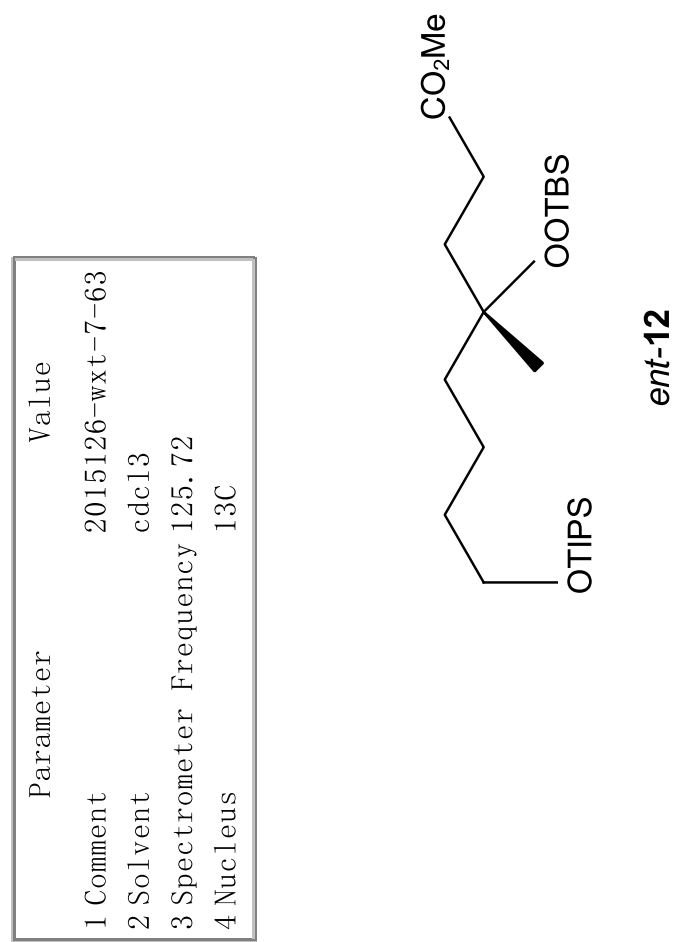


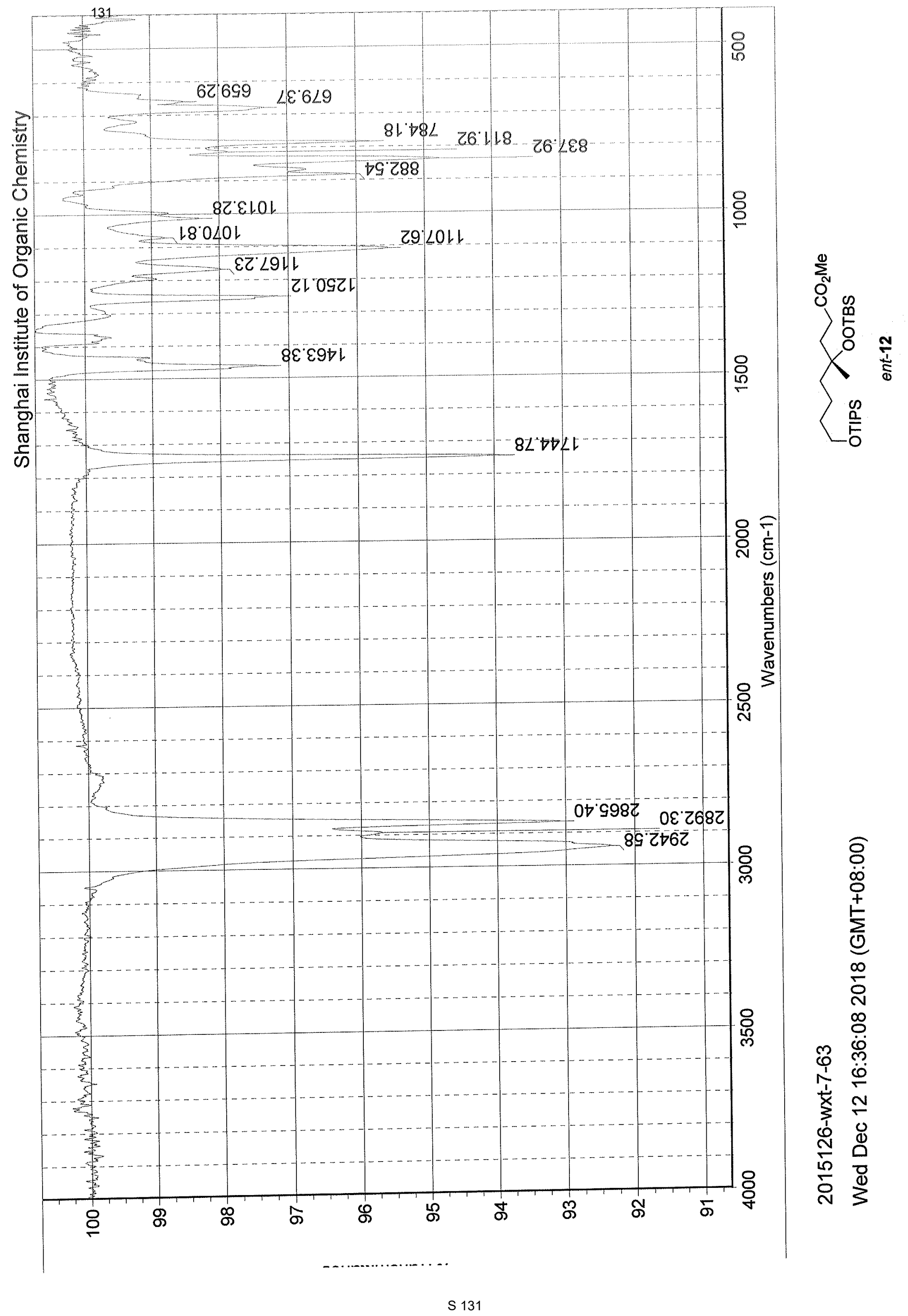


$2 L^{\circ} 0-$

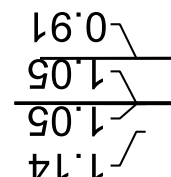

90.1
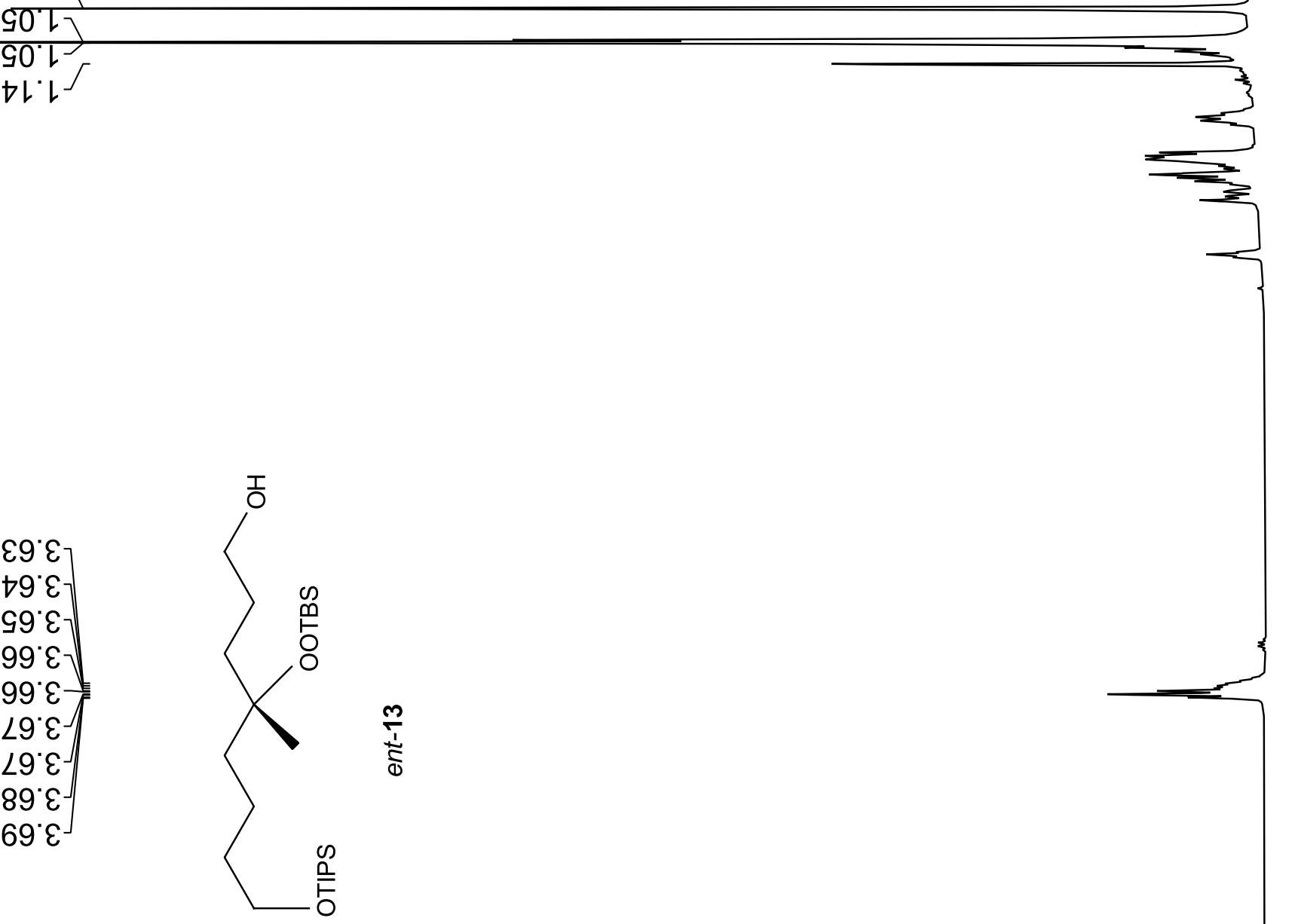

瓦 $86 \%$

$8 \varepsilon 6$

$00 \%$

10

91.6

$$
2
$$


$\nabla \mathrm{G}^{\prime} \mathrm{G}-$

26レー

ع 8 L

92 $8 \mathrm{~L}$

9L"OZ

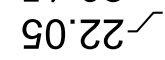

0乙 9 乙

96.9Z

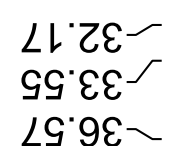

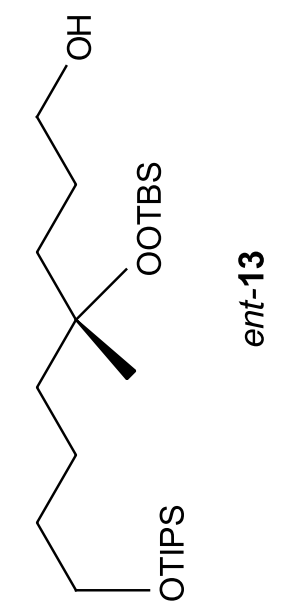

$\left.\begin{array}{l}2.89 \\ 97.89\end{array}\right\}$

ธน" $\varepsilon 9^{-}$
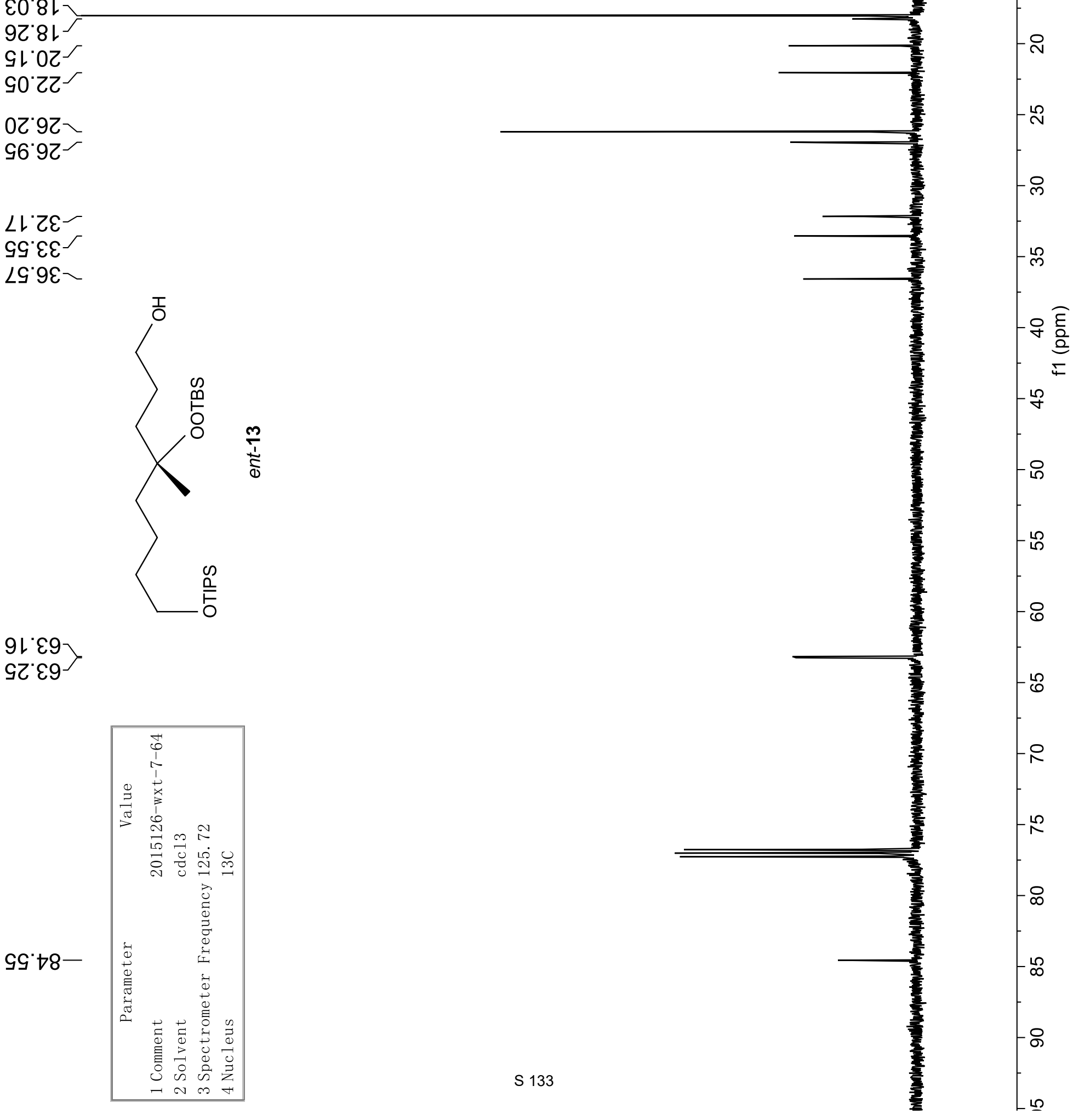


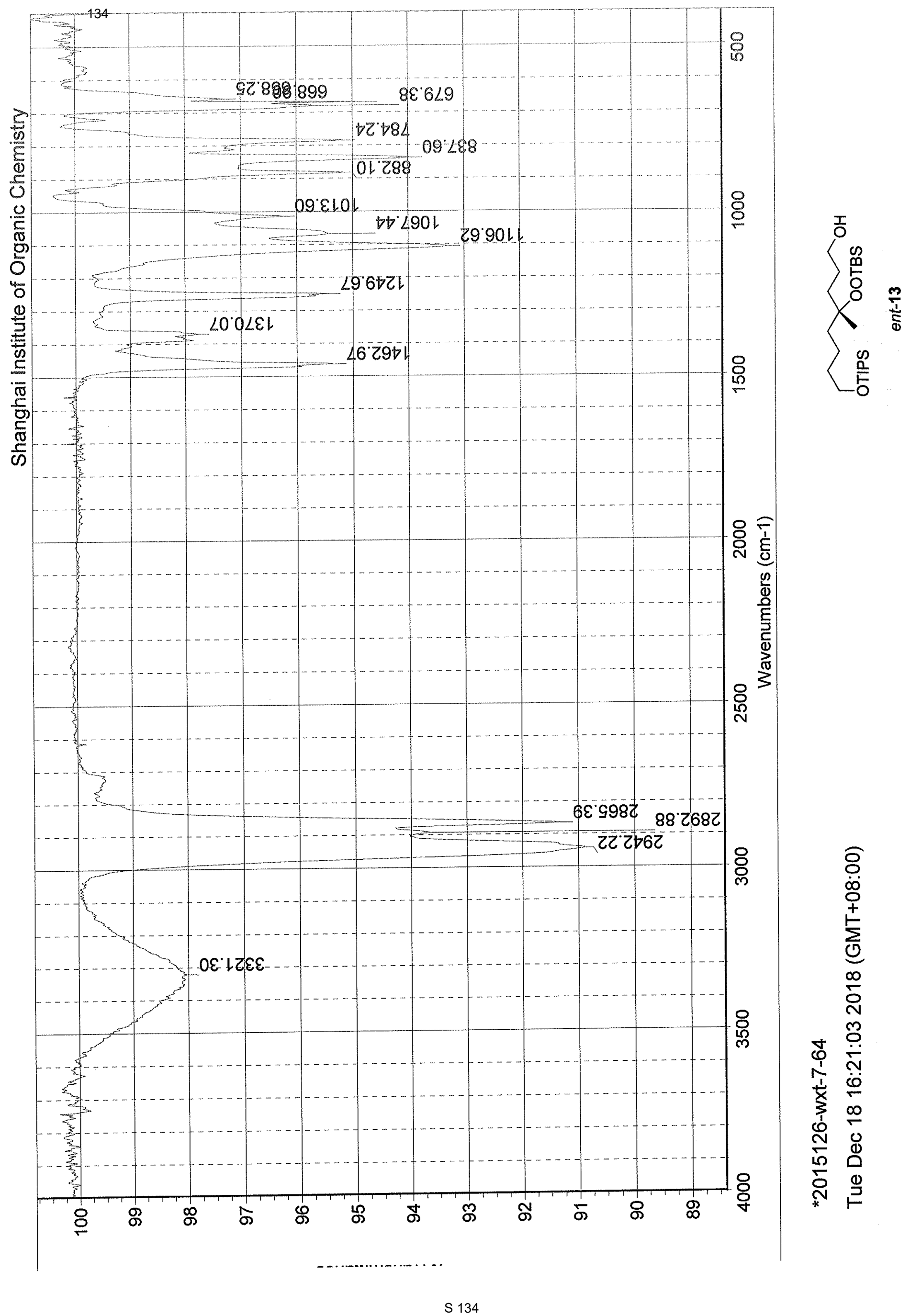




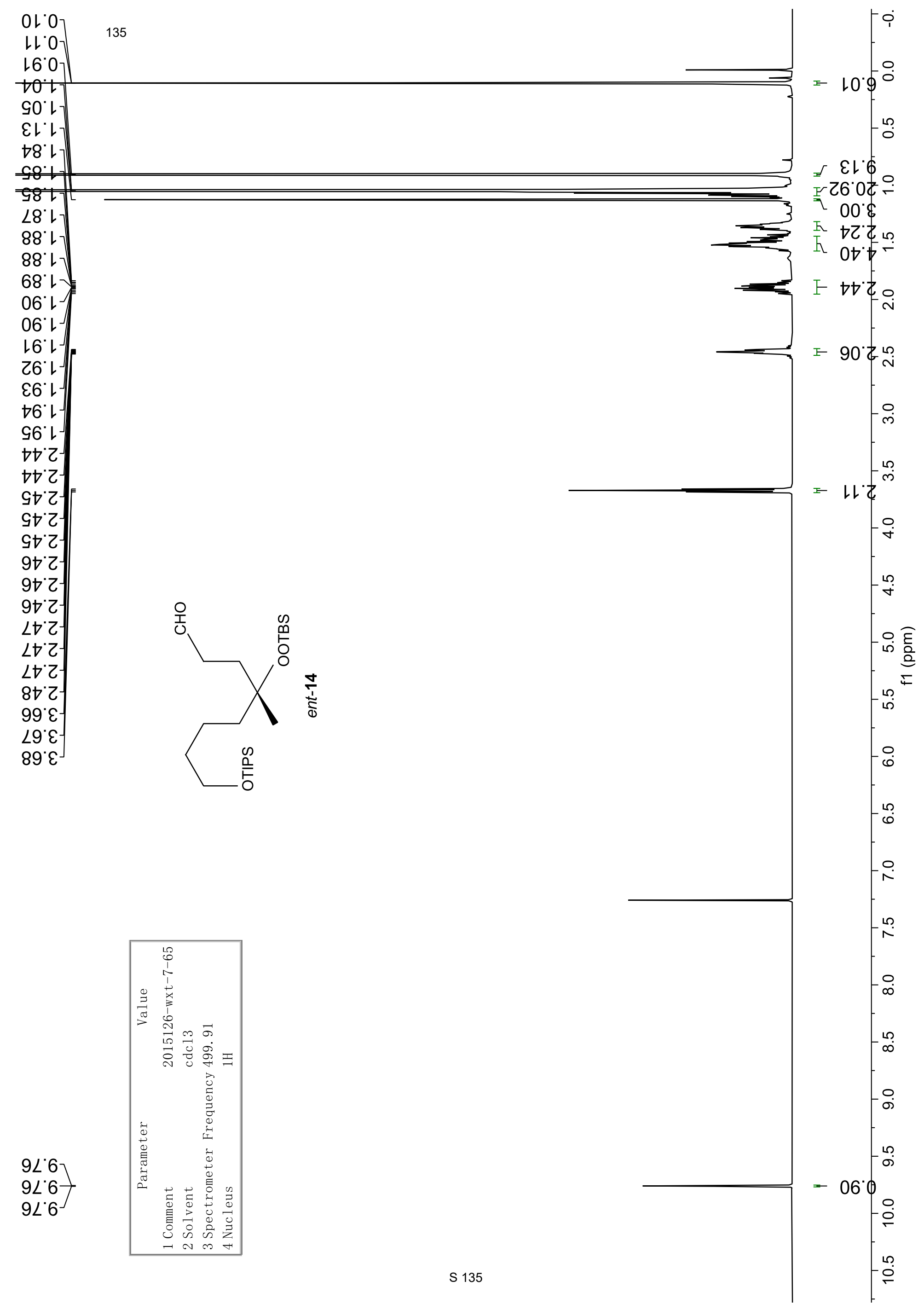



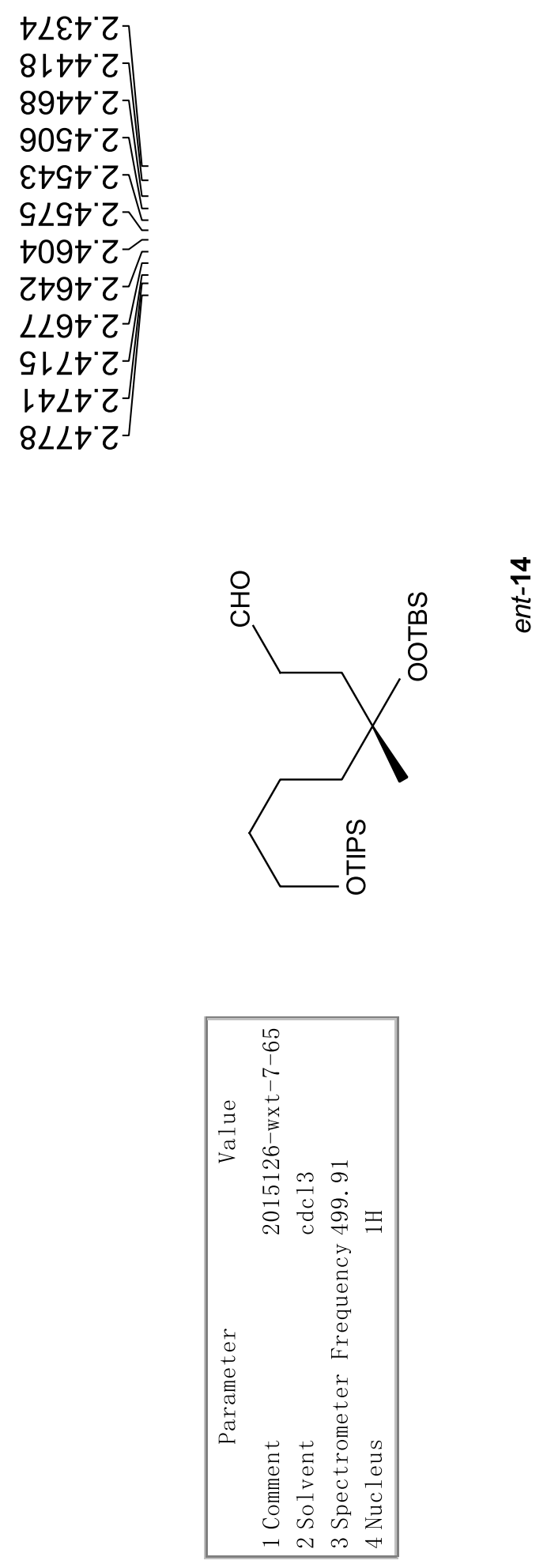

$0699^{\circ} \varepsilon-$

$6 เ \angle 9^{\circ} \varepsilon-$ $\angle \succ 89^{\circ} \varepsilon-$
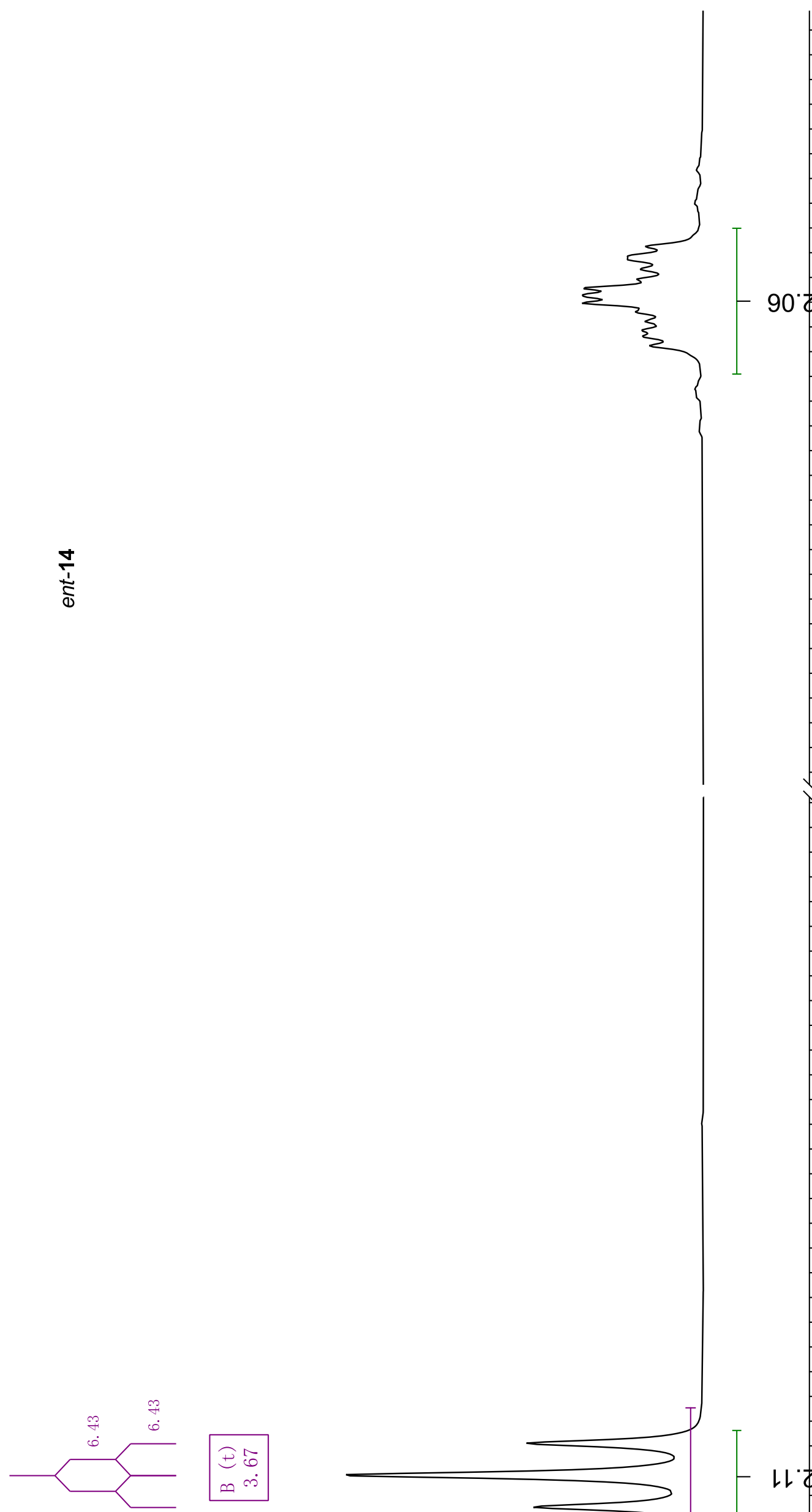
$\left.\begin{array}{l}\text { Z9.9-} \\ \text { l9.9- }\end{array}\right\}^{137}$

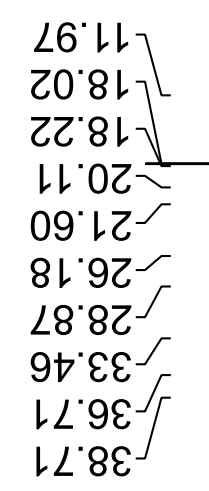

9.89-

ฤฤะ8-
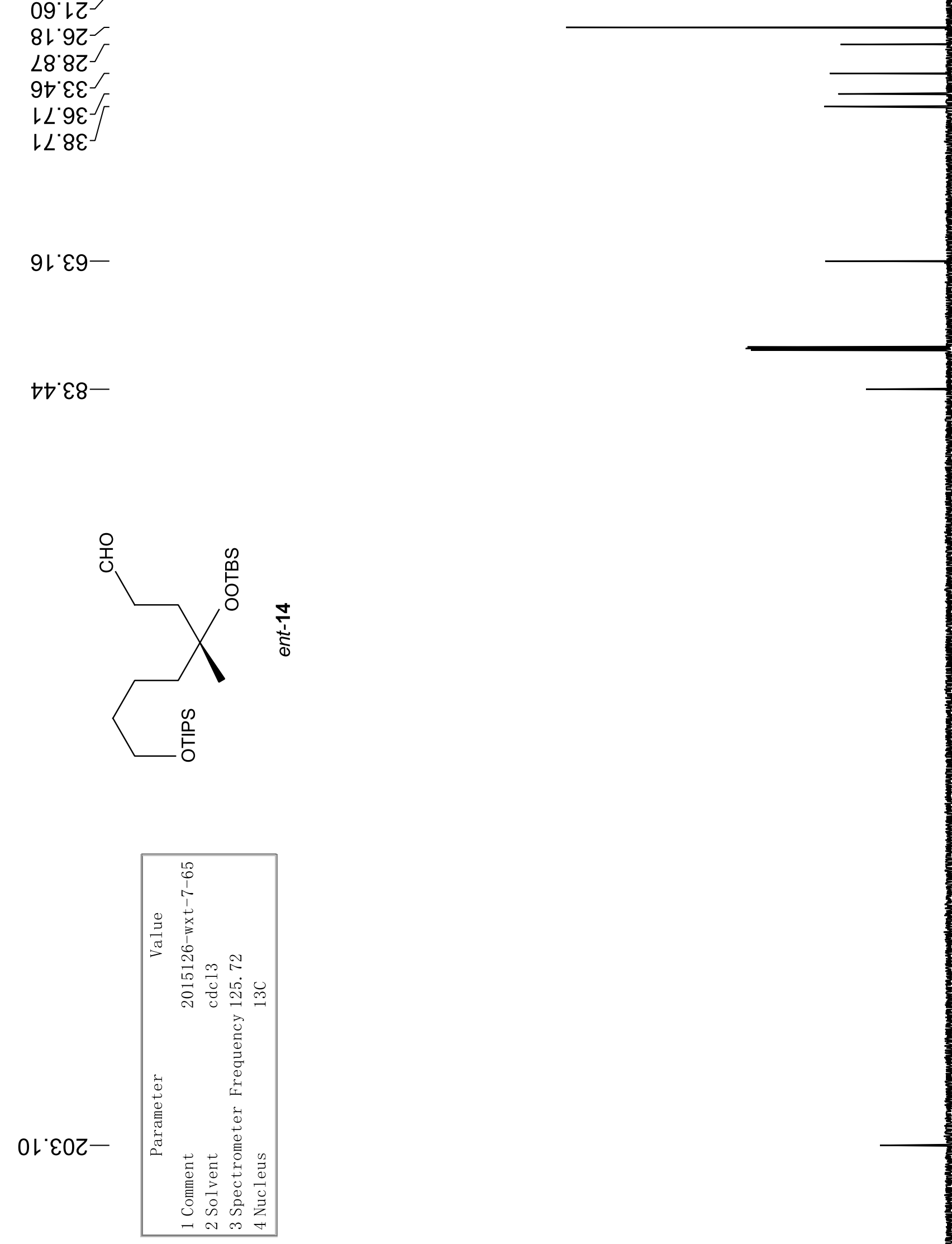


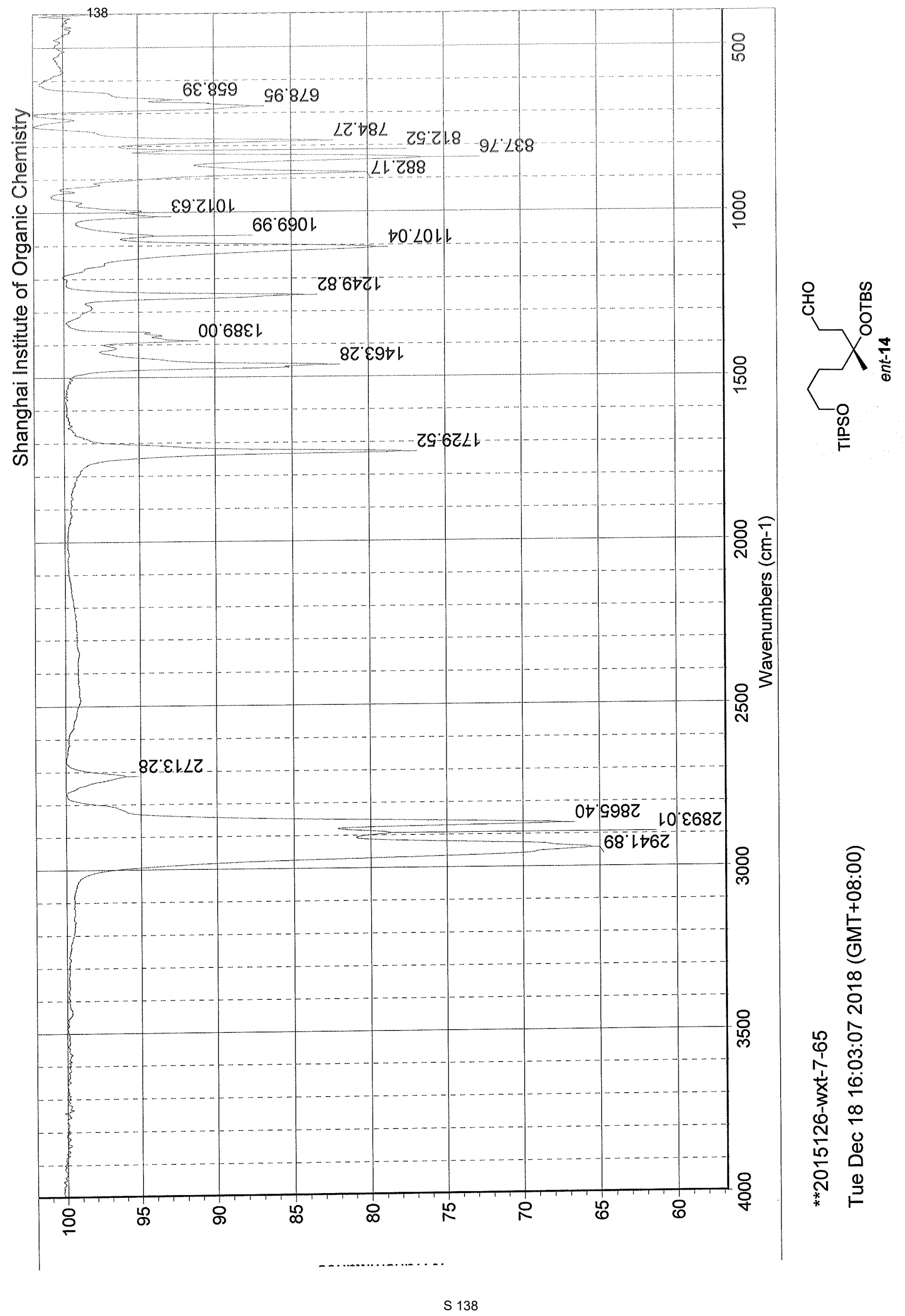




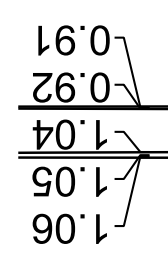

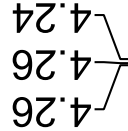

$$
\begin{aligned}
& \angle 9^{\circ} \nabla \\
& 69.5
\end{aligned}
$$

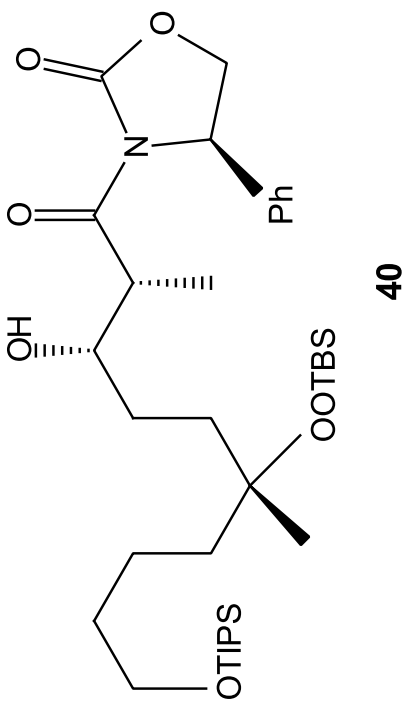

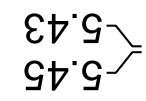
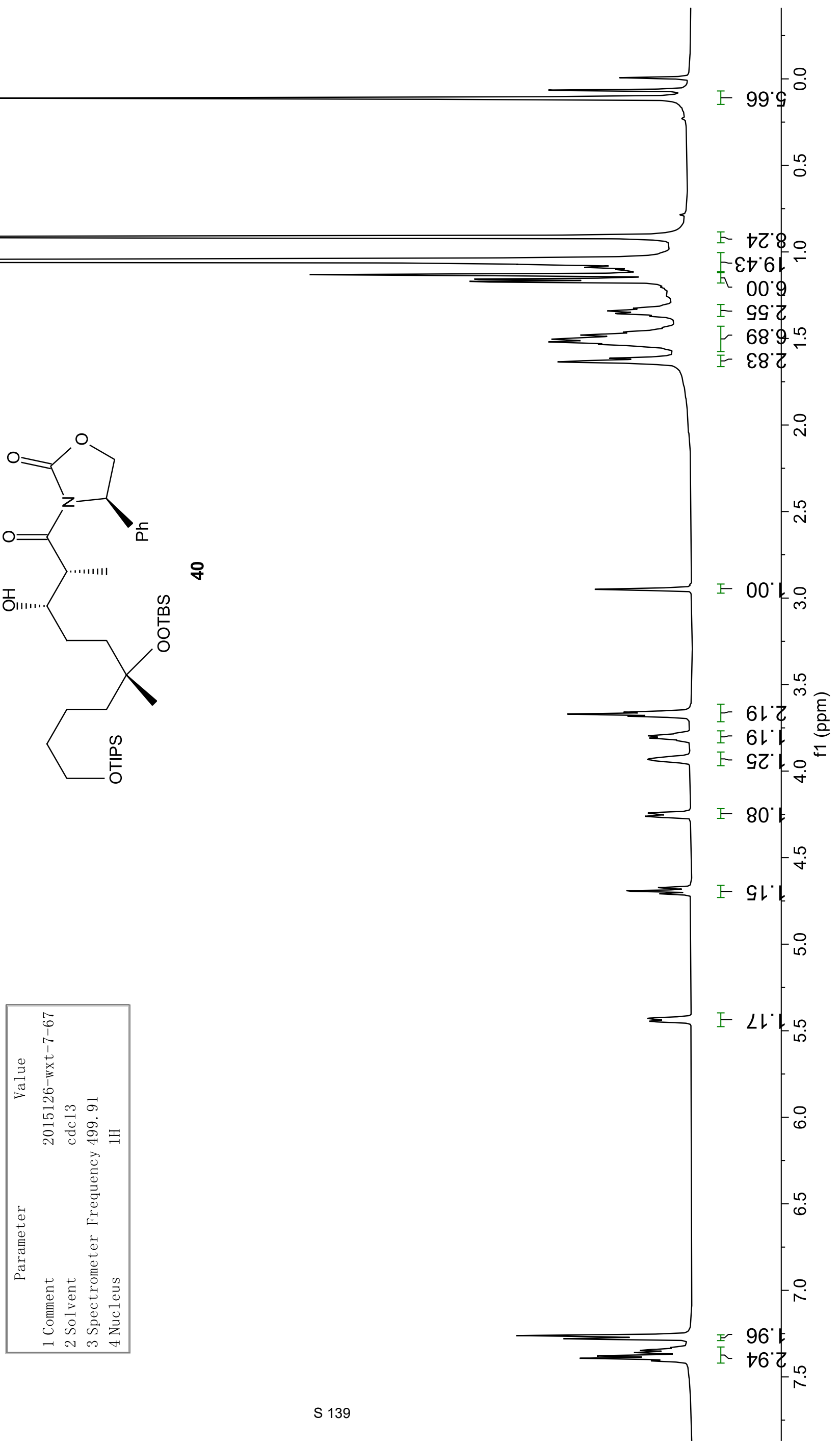


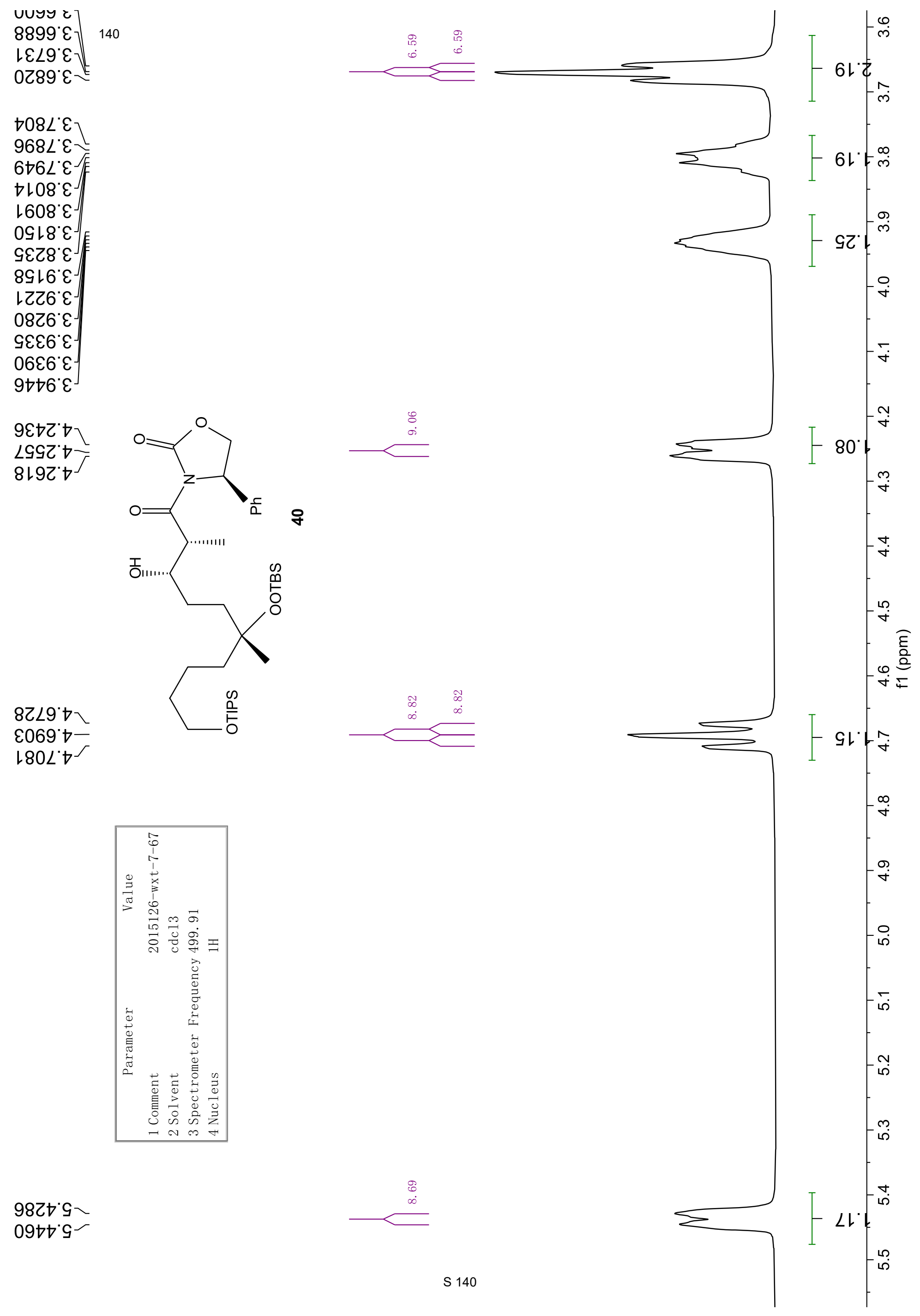


GG:G-— ${ }^{141}$

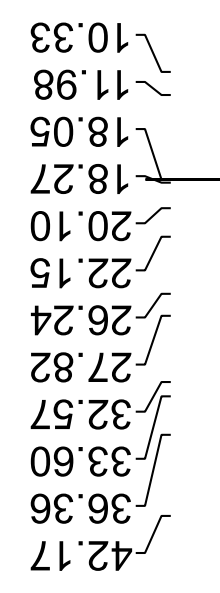

$99^{\circ} \angle \mathcal{G}^{\top}$
$\nabla \varepsilon^{\circ} \varepsilon 9-$
$98^{\circ} 69$
$09^{\circ} L \backslash \backslash$

$\angle Z^{\circ} \nabla 8-$

GG'GZレ

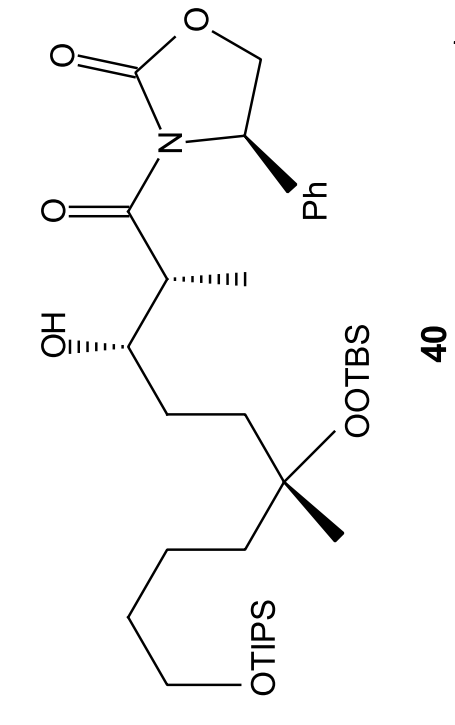

6L'8Zレ】
$0 \varepsilon^{\circ} 6$ ᄂ

$0 \varepsilon^{\circ} 6 L^{-}$

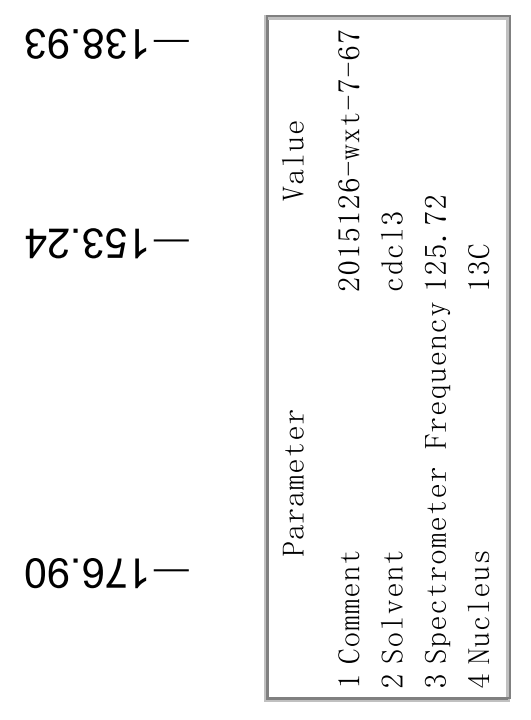




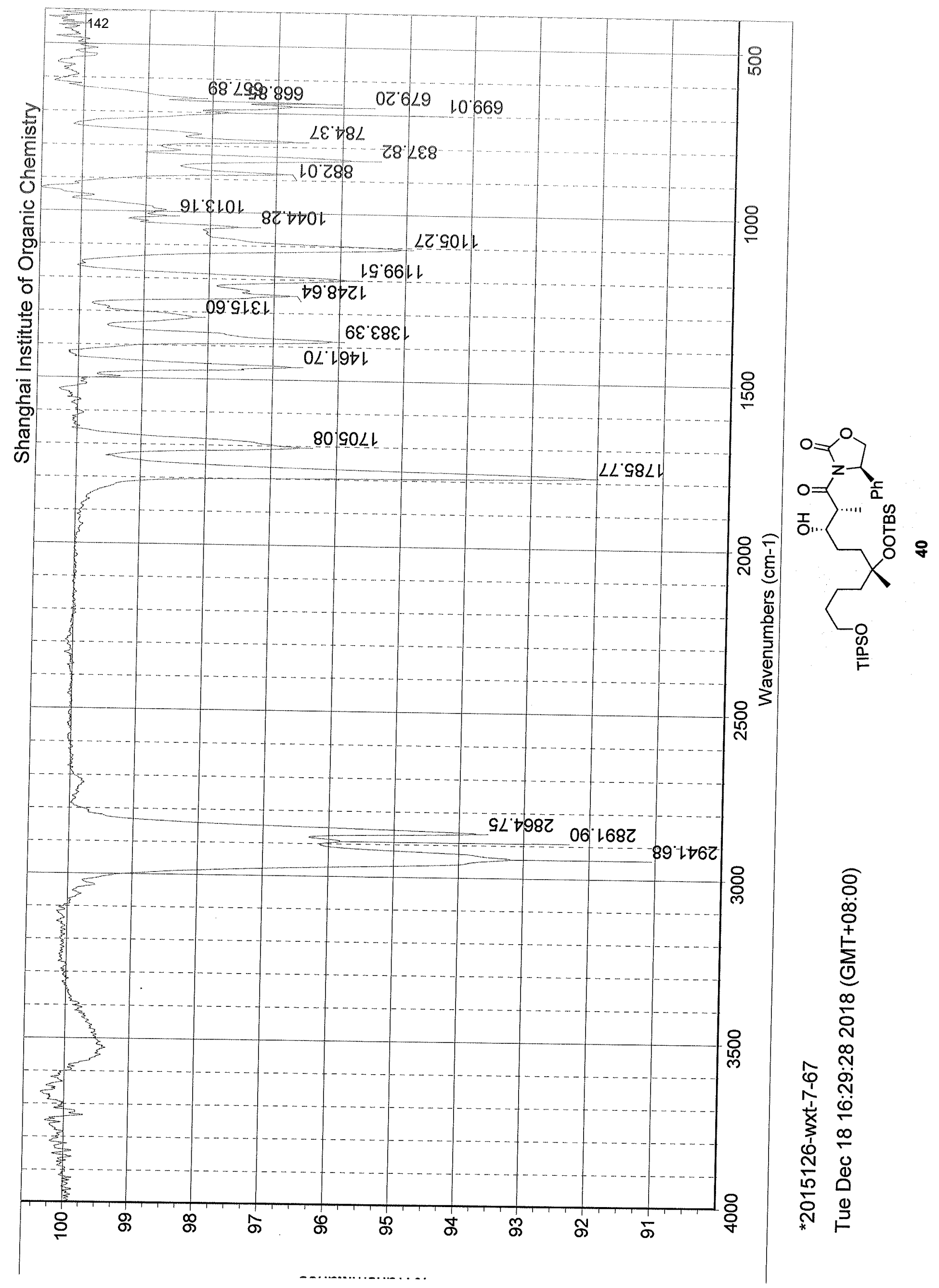


$26^{\circ} 0$

$70{ }^{\circ} \mathrm{L}$



$\angle 9^{\circ} Z-$

$98^{\circ} \mathrm{Z}-$

$99^{\circ} \varepsilon$

$89^{\circ} \varepsilon$

$69^{\circ} \varepsilon$

$0 L^{\circ} \varepsilon$

L $L \varepsilon^{\varepsilon}$
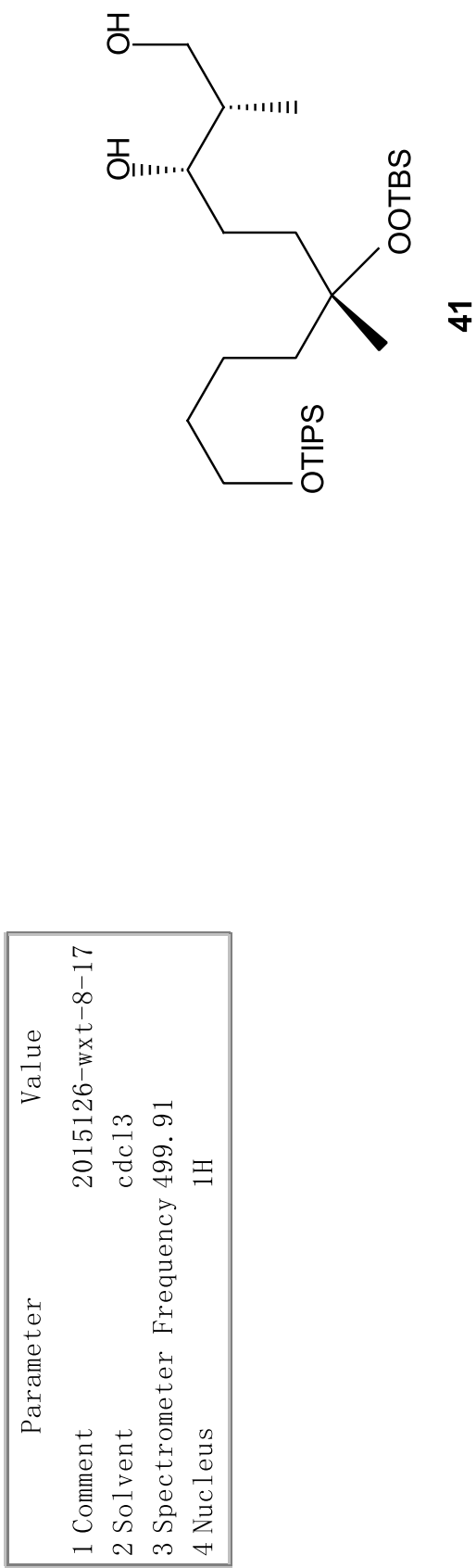


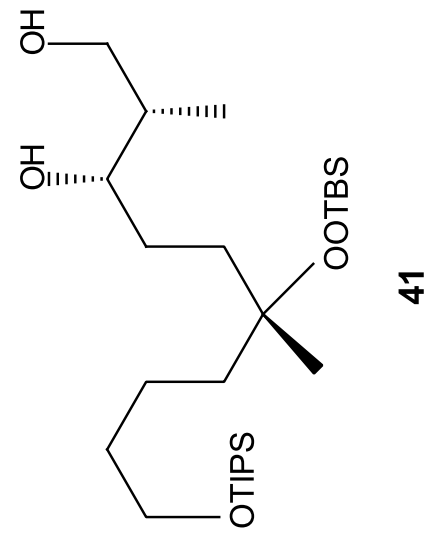

6Z99' $\varepsilon^{-}$

9S $\angle 9^{\circ} \varepsilon-$

$9889^{\circ} \varepsilon \sim$

G00 L $\mathcal{} \mathcal{}$ 乙

$660 L^{\prime} \varepsilon \sim$
$0 S \angle 8^{\circ} \varepsilon>$ $\angle L 88^{\circ} \varepsilon-$ 0เ68 8 $\neg 006^{\circ} \varepsilon-$ 乙L06. $\varepsilon^{-}$

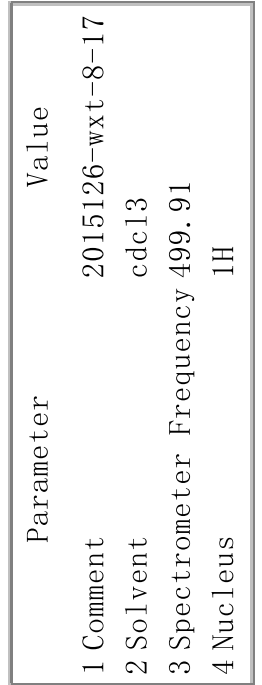


$20.8 \mathrm{~L}$

9278

$0 Z^{\circ} 0 Z^{-}$

เZZZ

$81.92-$

$\varepsilon 6^{\circ} L Z-$
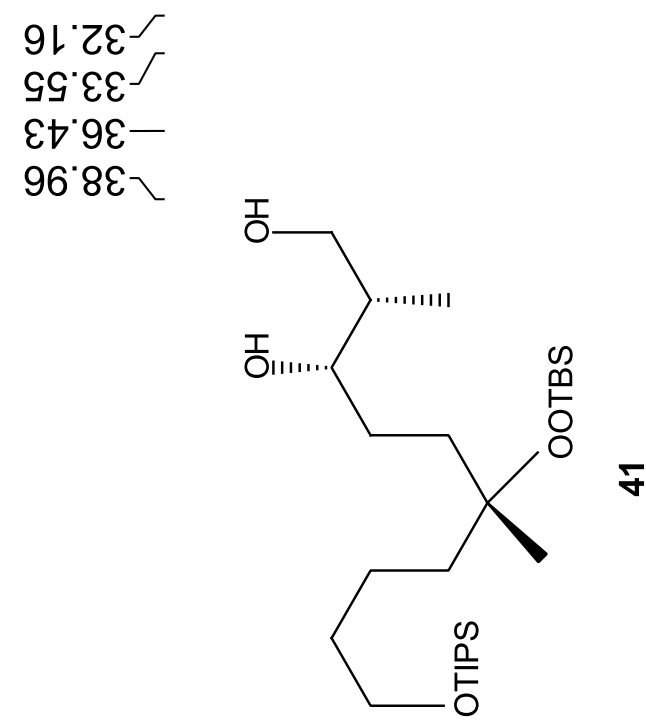

$\varepsilon Z^{*} \varepsilon 9-$
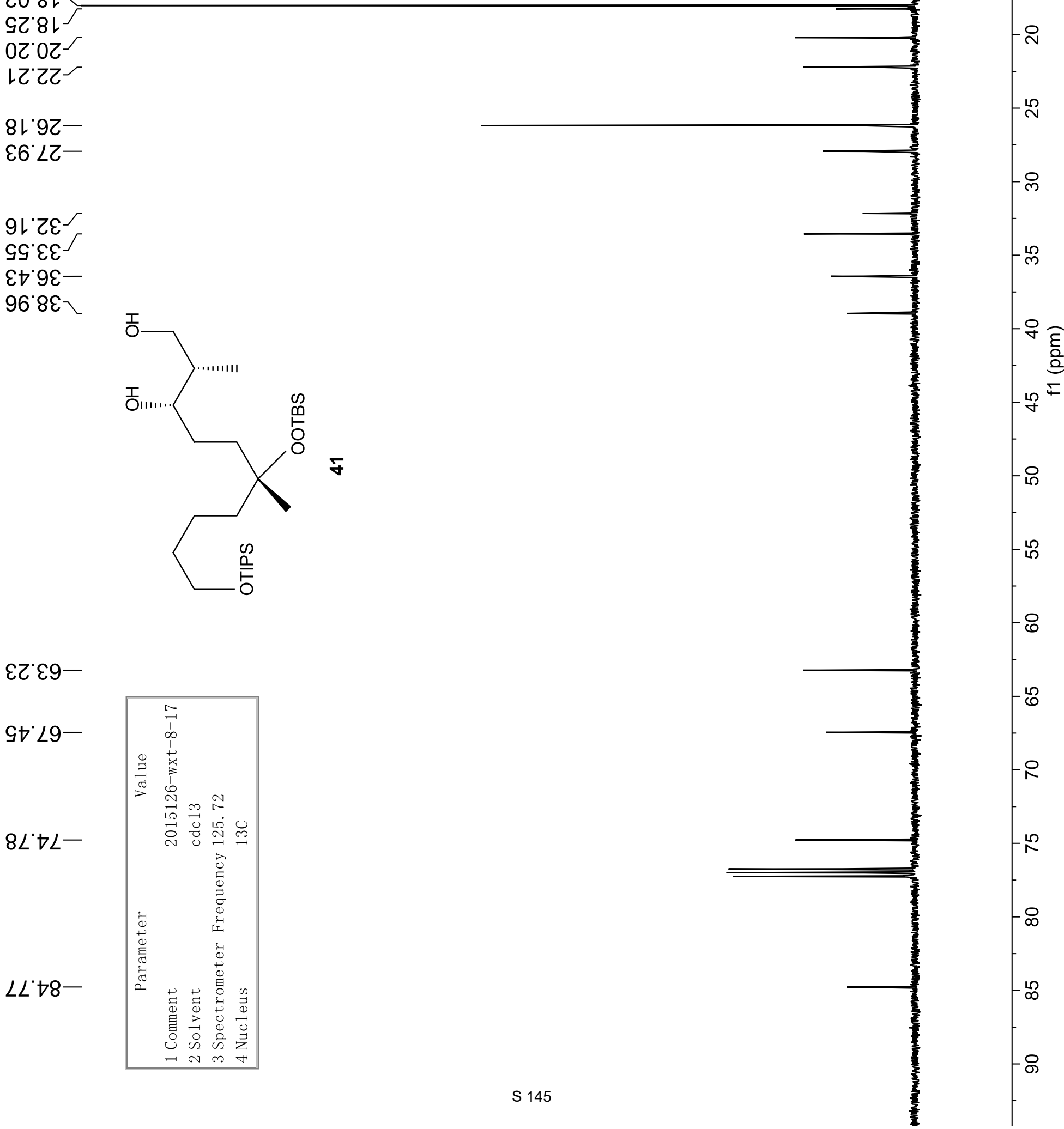

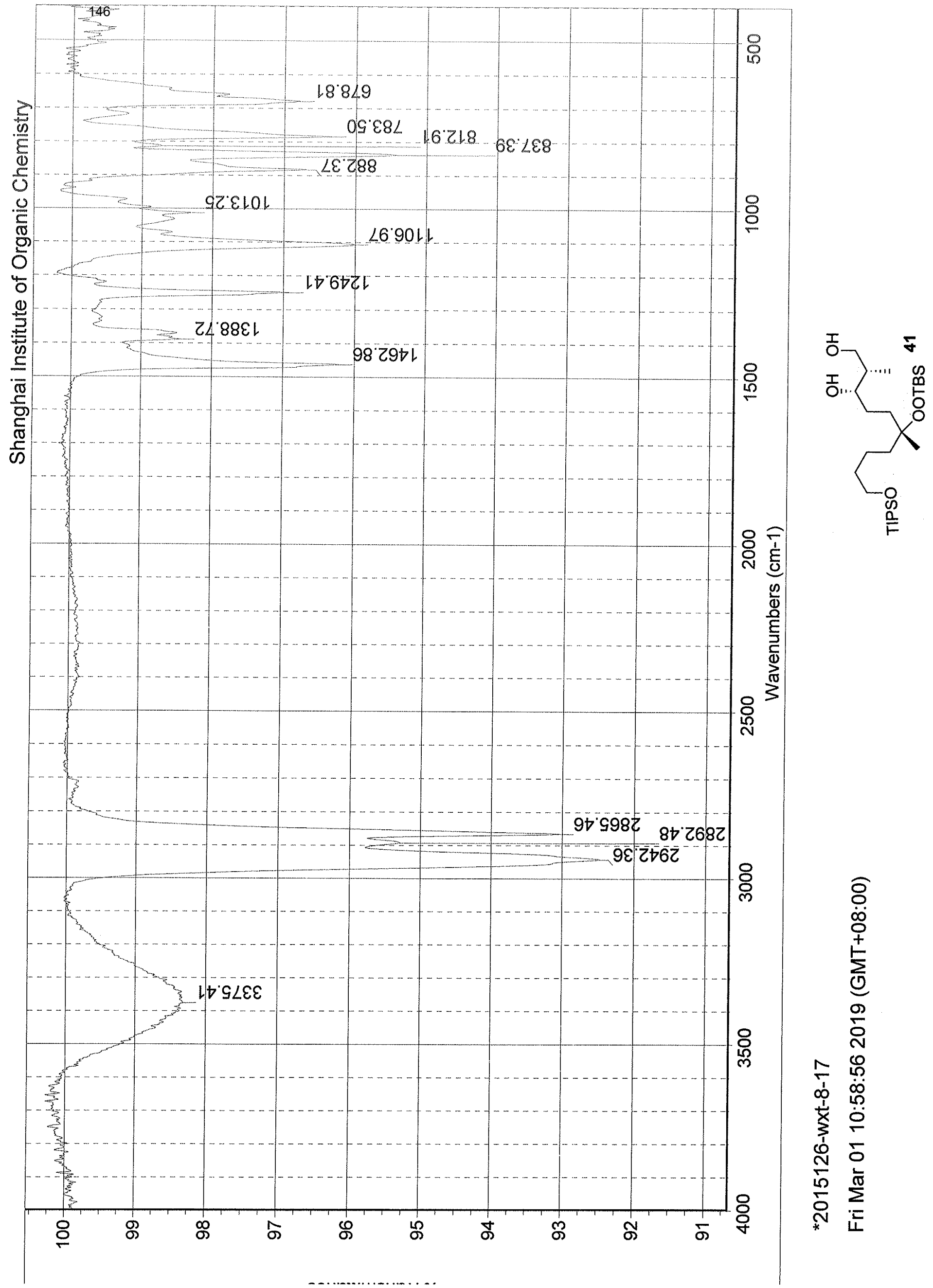

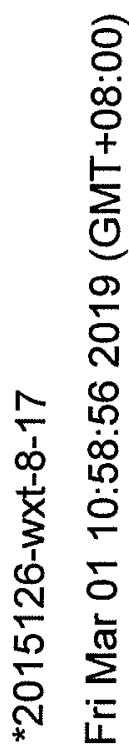



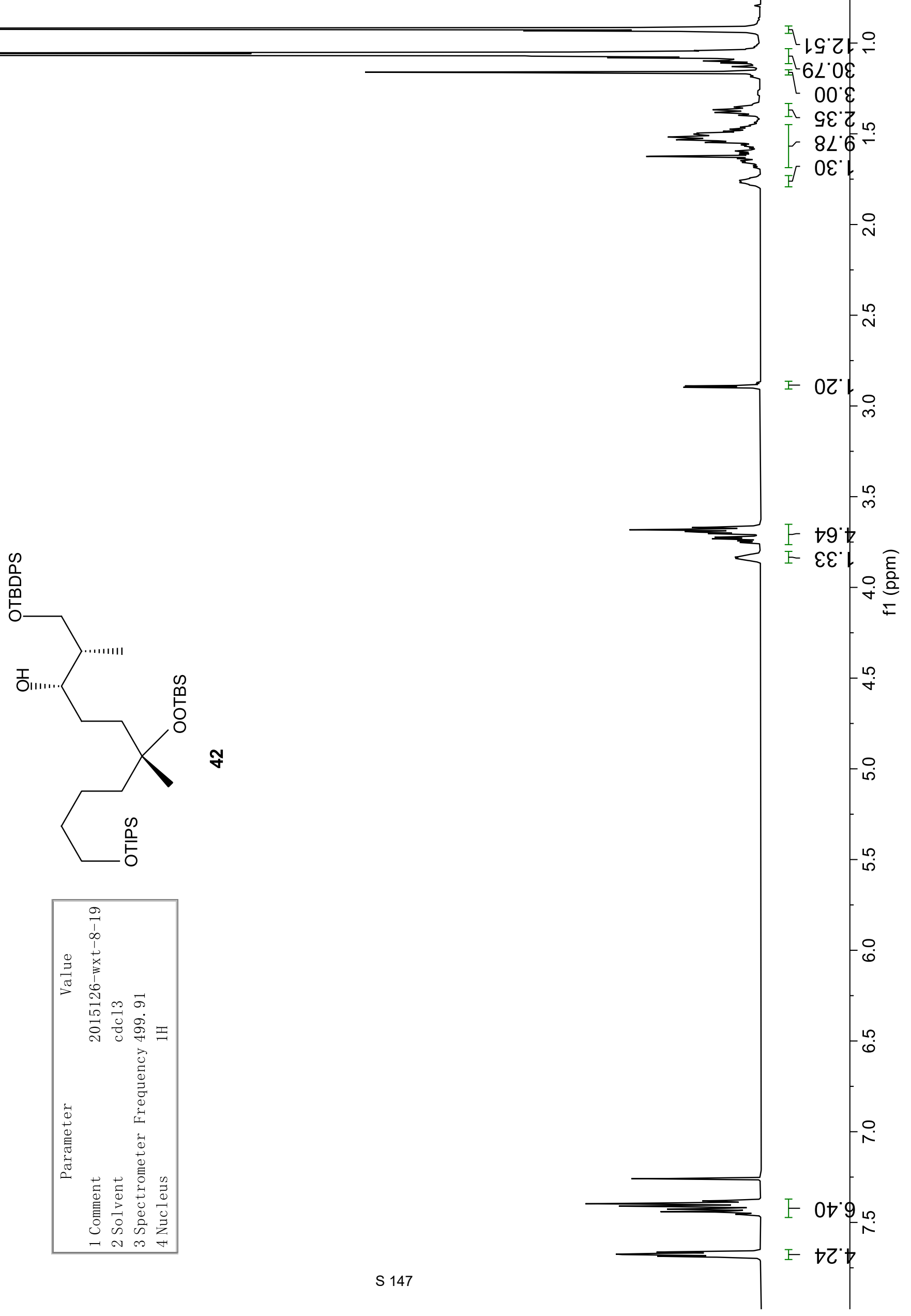

$99^{\circ} L$

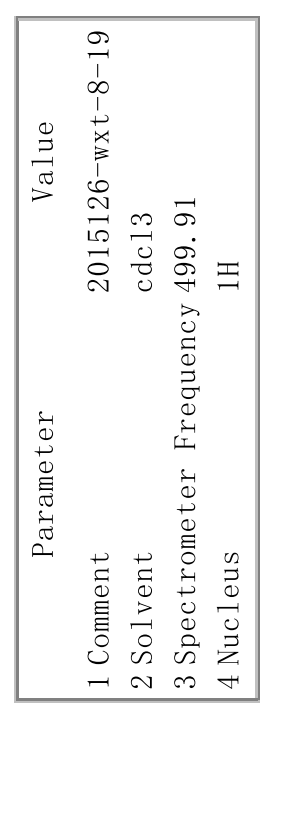




\section{ZLLE $\angle$ $\angle 08 \varepsilon^{\circ} \angle$ 乙७8 $\varepsilon^{\circ}\llcorner$ $6 \varepsilon 6 \varepsilon^{\circ}\llcorner$ $\left.\checkmark \angle 6 \varepsilon^{\circ} \angle\right]$ $680 t^{\circ} L$ 9レレ゙ 6Z2ち L ย9Zち L G6乙ち $\llcorner$ ¿ $\angle G E \nabla^{\circ} \angle$ 80t5 $L$ $\nabla \angle \nabla \nabla^{\circ} \angle$ 8LG L ESGt L}
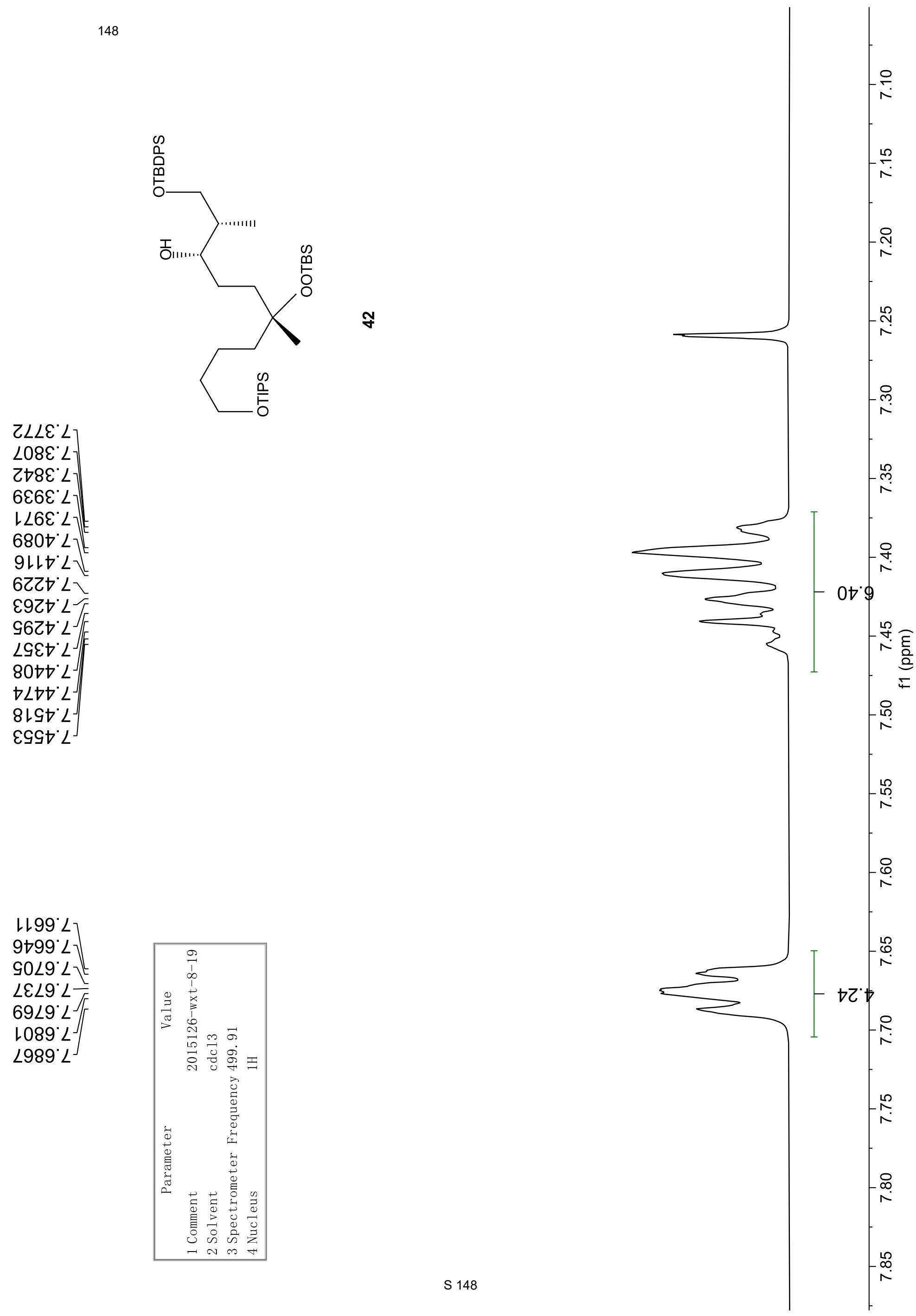
9688 乙

\&L68 乙
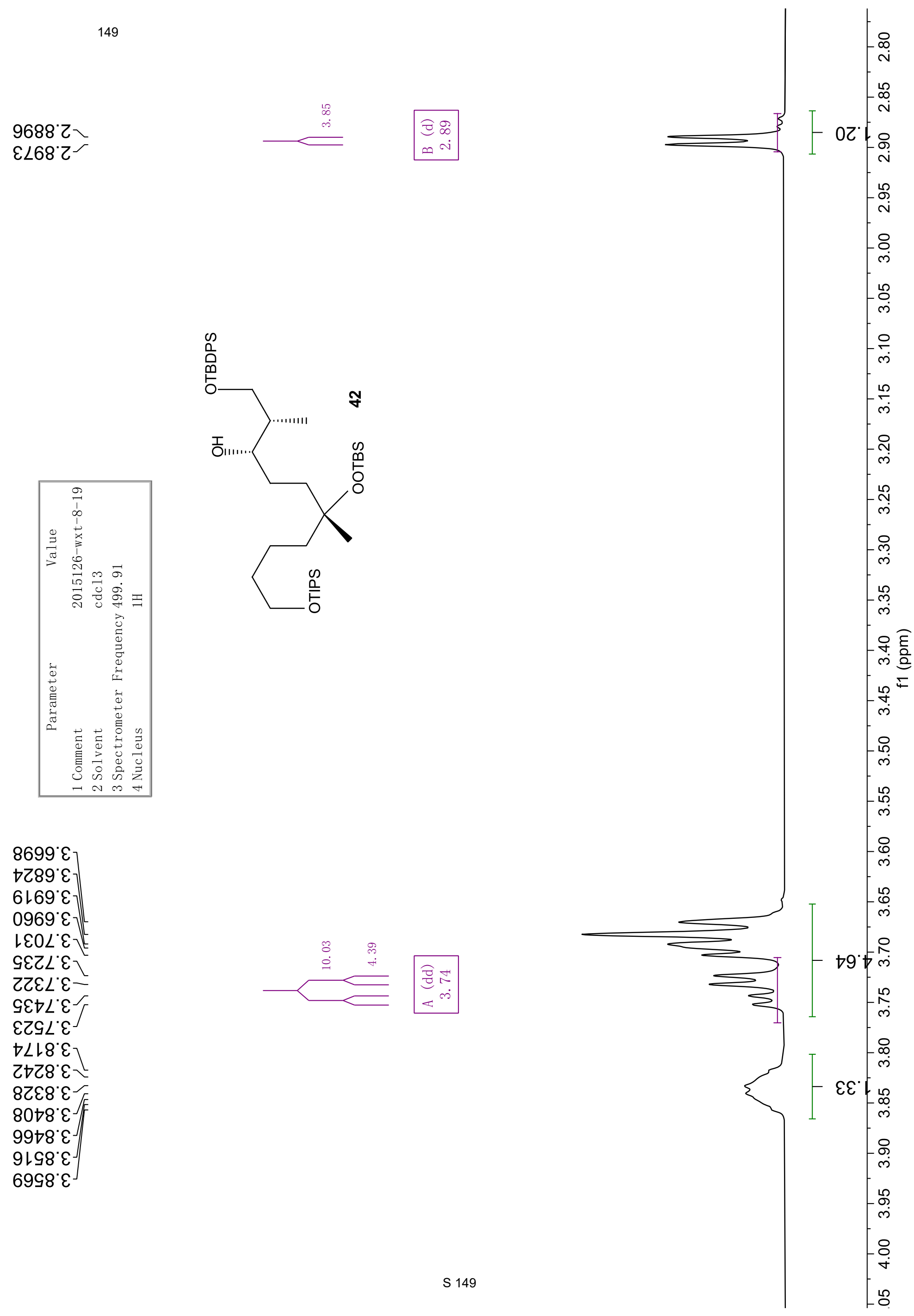
$\nabla G^{\prime} \mathrm{G}^{-}$

9Zㅇㄴ

10てレ-

$70.8 \mathrm{l}$

$\angle 28 \mathrm{~L}$

9L6L

ㄴ.0乙

6乙'乙乙

งて'9乙/[

$98 \cdot 92$

ᄂ乙 $8 乙^{\top}$

$\varepsilon 0^{\circ} \varepsilon \varepsilon^{\lrcorner}$

$\succ 9^{\circ} \varepsilon \varepsilon$

$\varsigma \varepsilon^{\prime} 9 \varepsilon$

Gเ $6 \varepsilon$
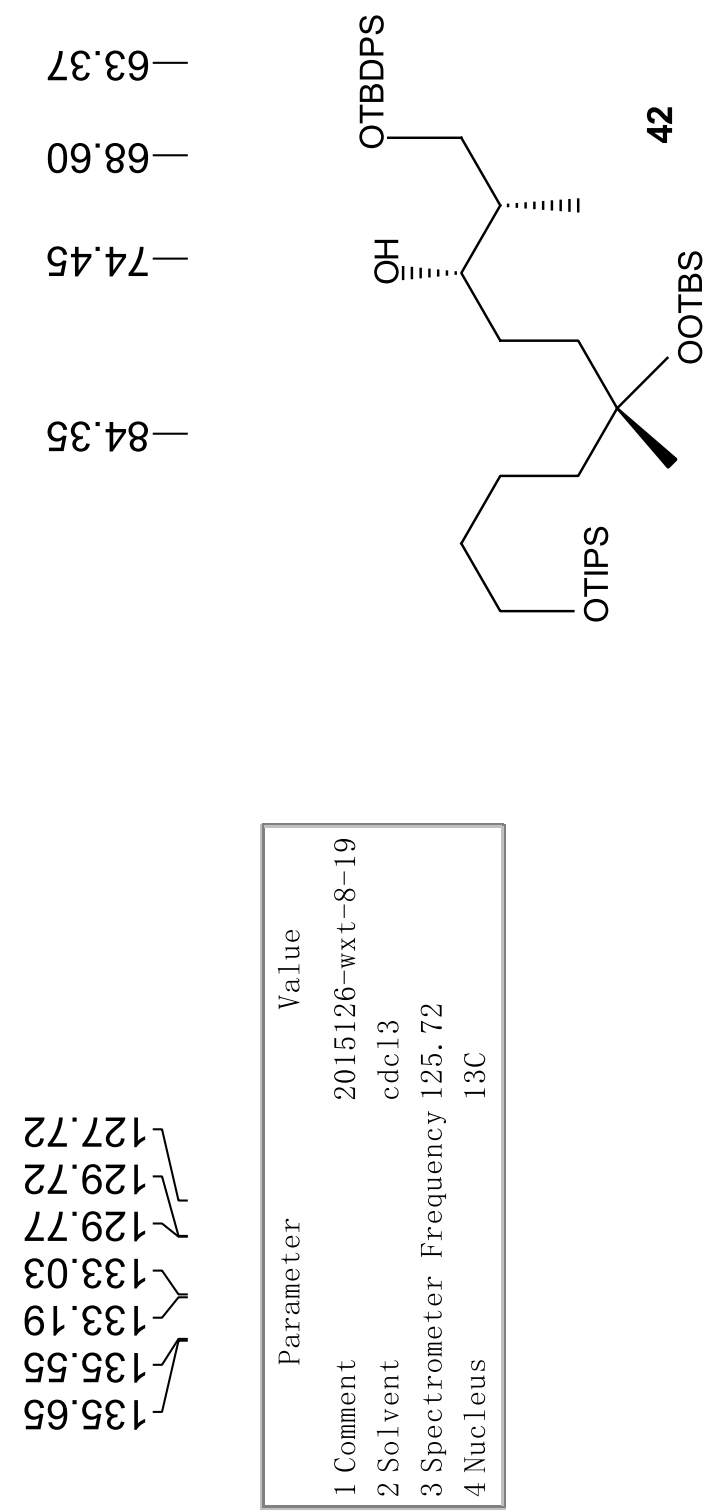


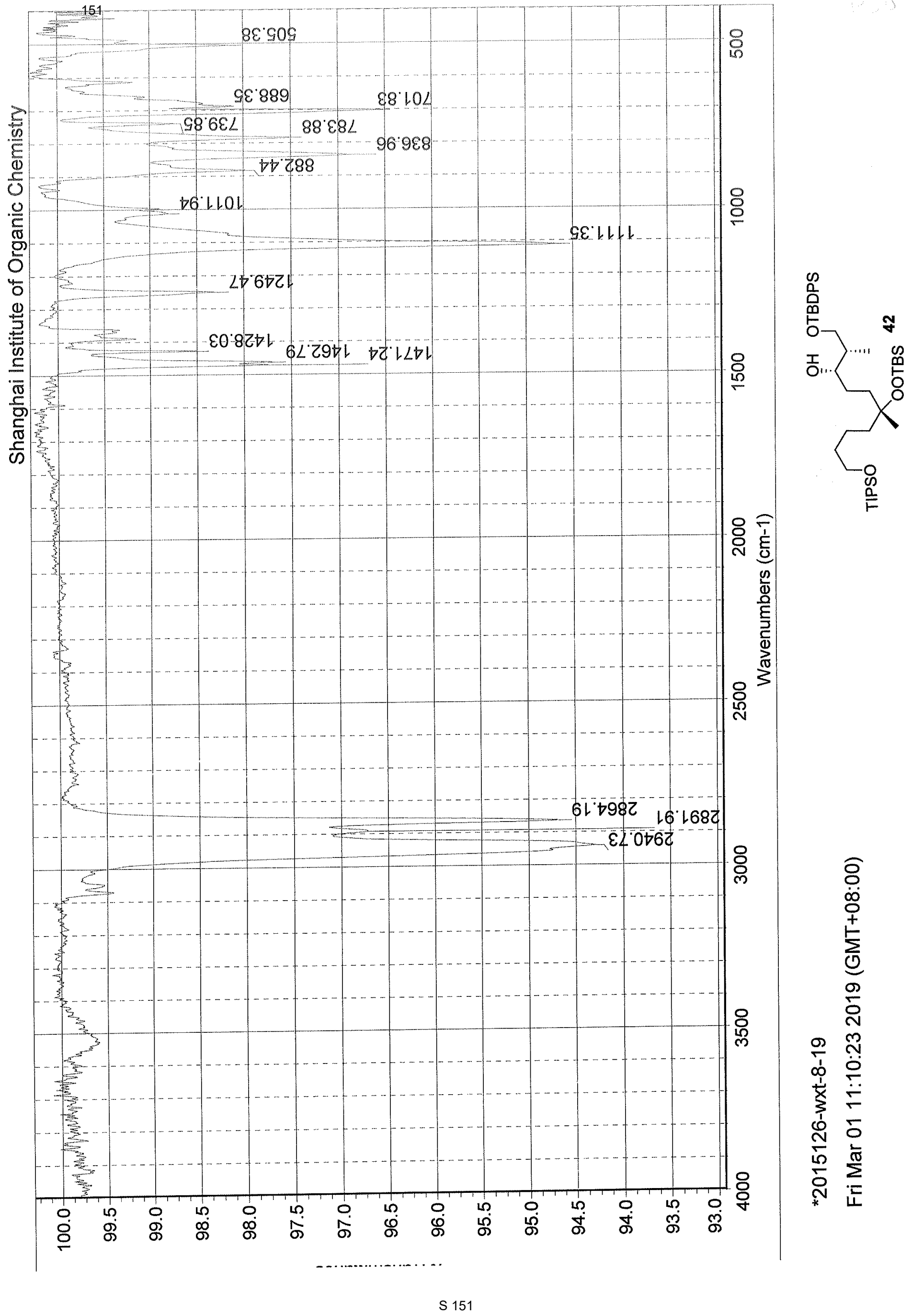




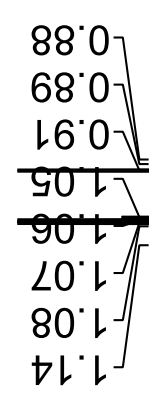

ع6"

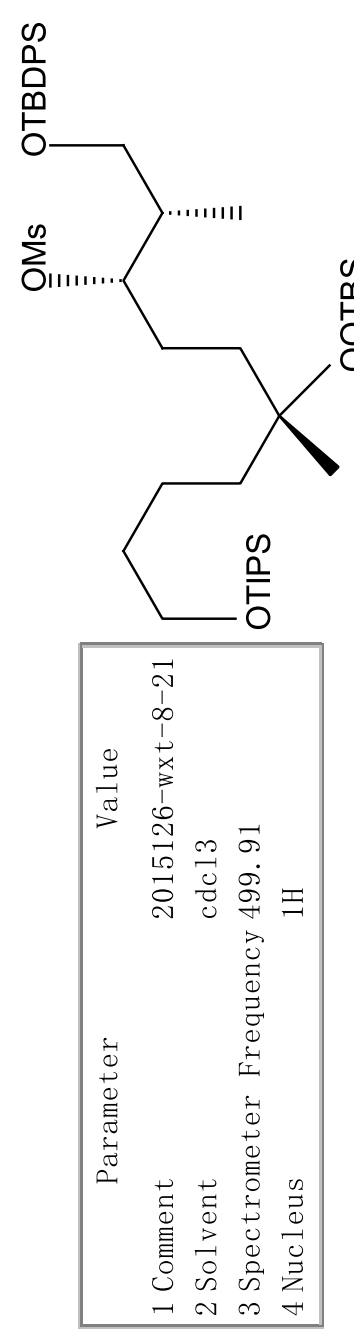

?

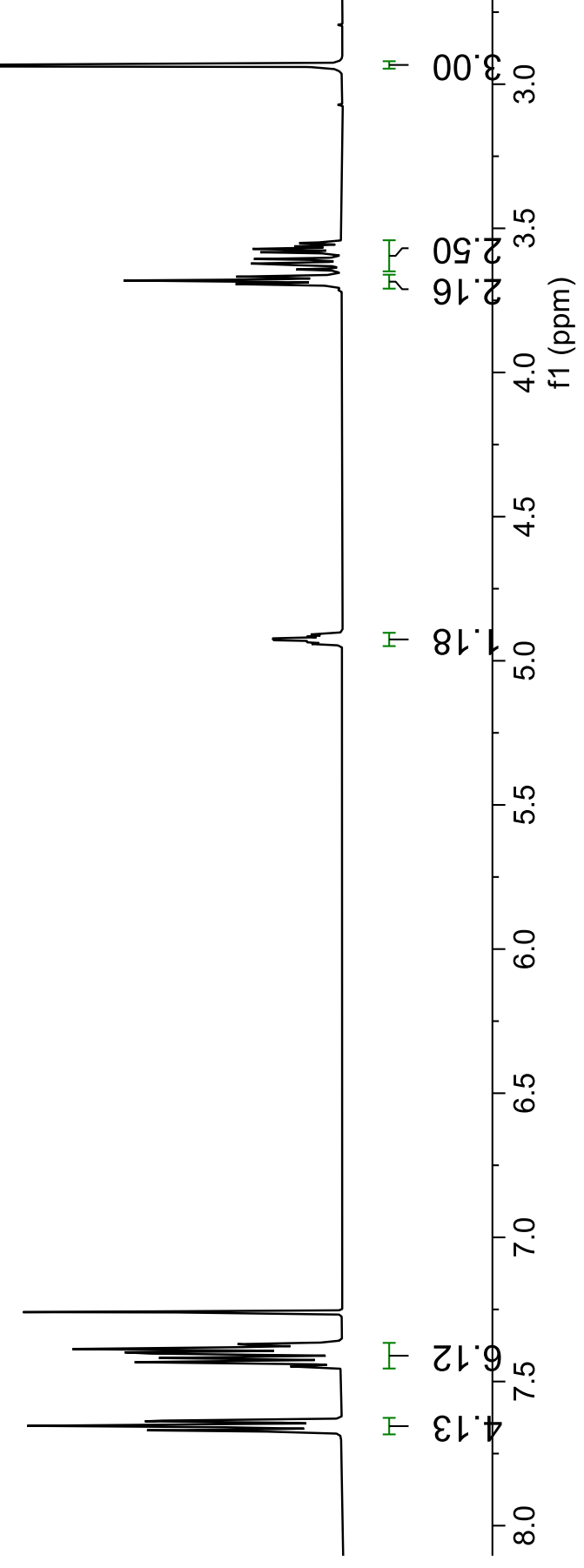



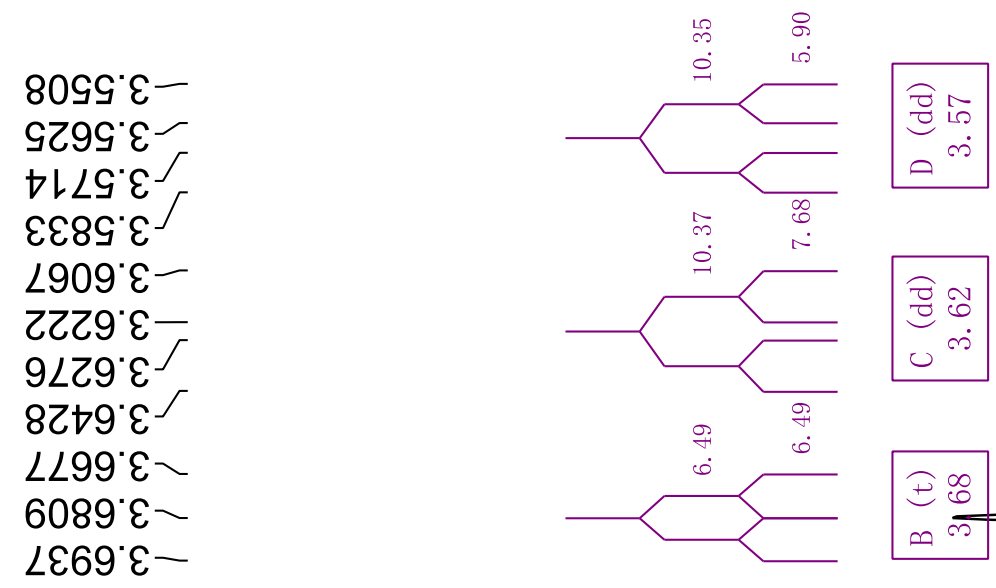

- 0sを。
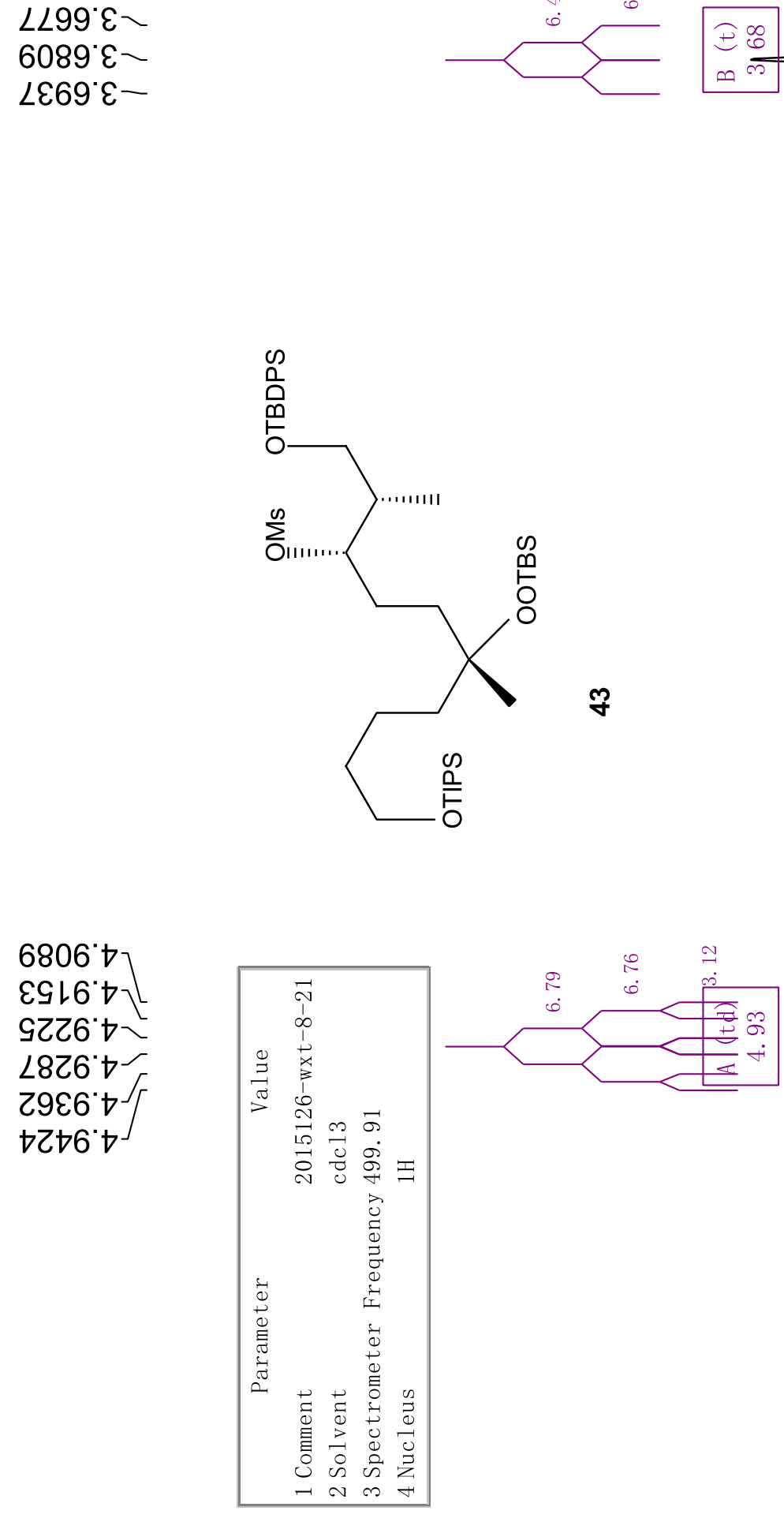
$6 G^{\circ} \mathrm{G}^{-}-154$

69.0L

00てレ-

†0.8L

हट.8L

6L'6L-5

90.0乙

E6 L乙

乙乙.9乙-

$\downarrow Z^{\prime} 9 z^{\prime}$

$98^{\circ} 9 \tau^{\circ}$

$\nabla G^{\prime} \mathrm{्}$

$89^{\prime} \varepsilon \varepsilon^{\top}\left[{ }_{F}\right.$

$\varepsilon G^{\prime} 9 \varepsilon$

$0 \varepsilon^{\circ} 8 \varepsilon$

IS' $8 \varepsilon^{\circ}$
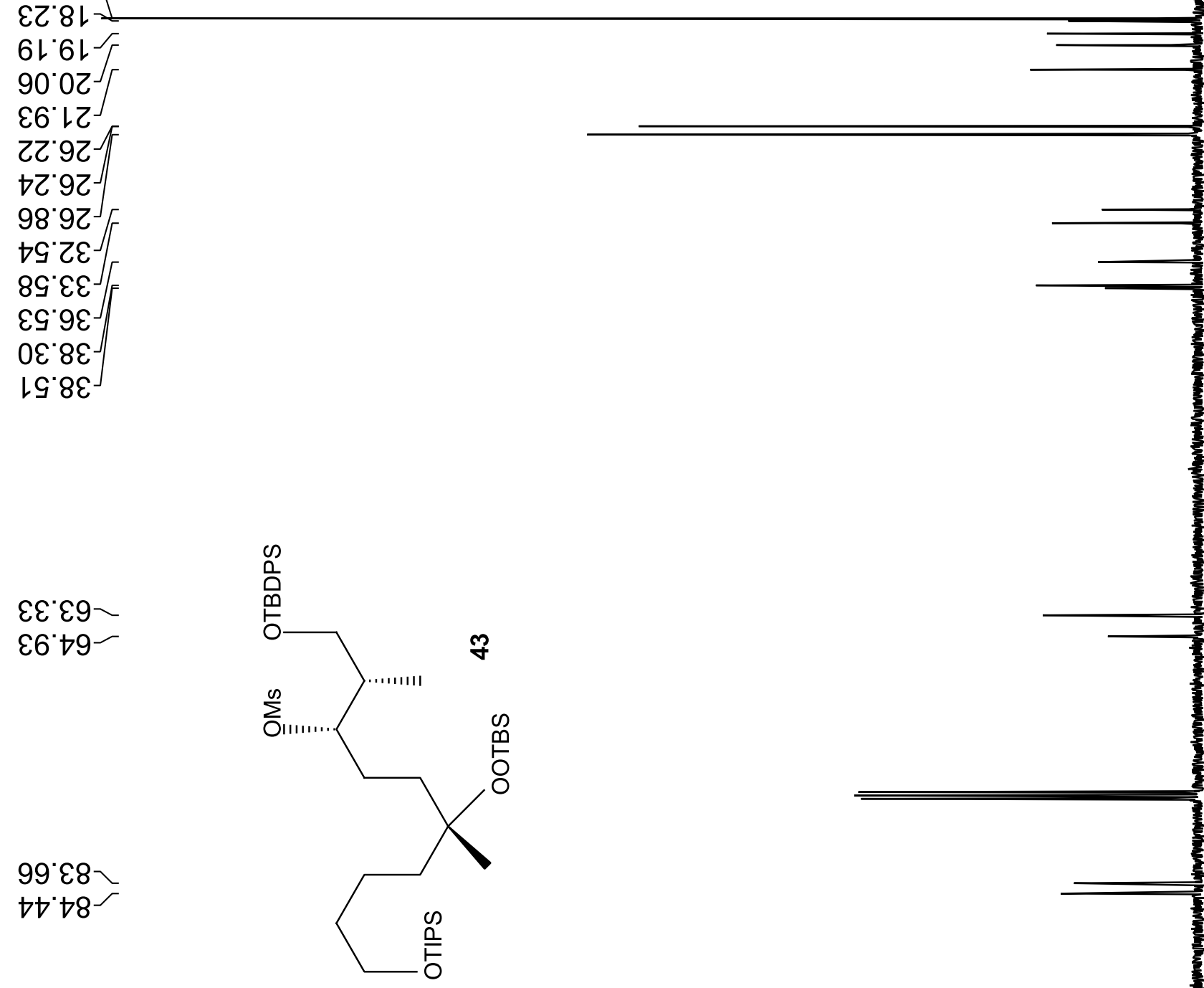


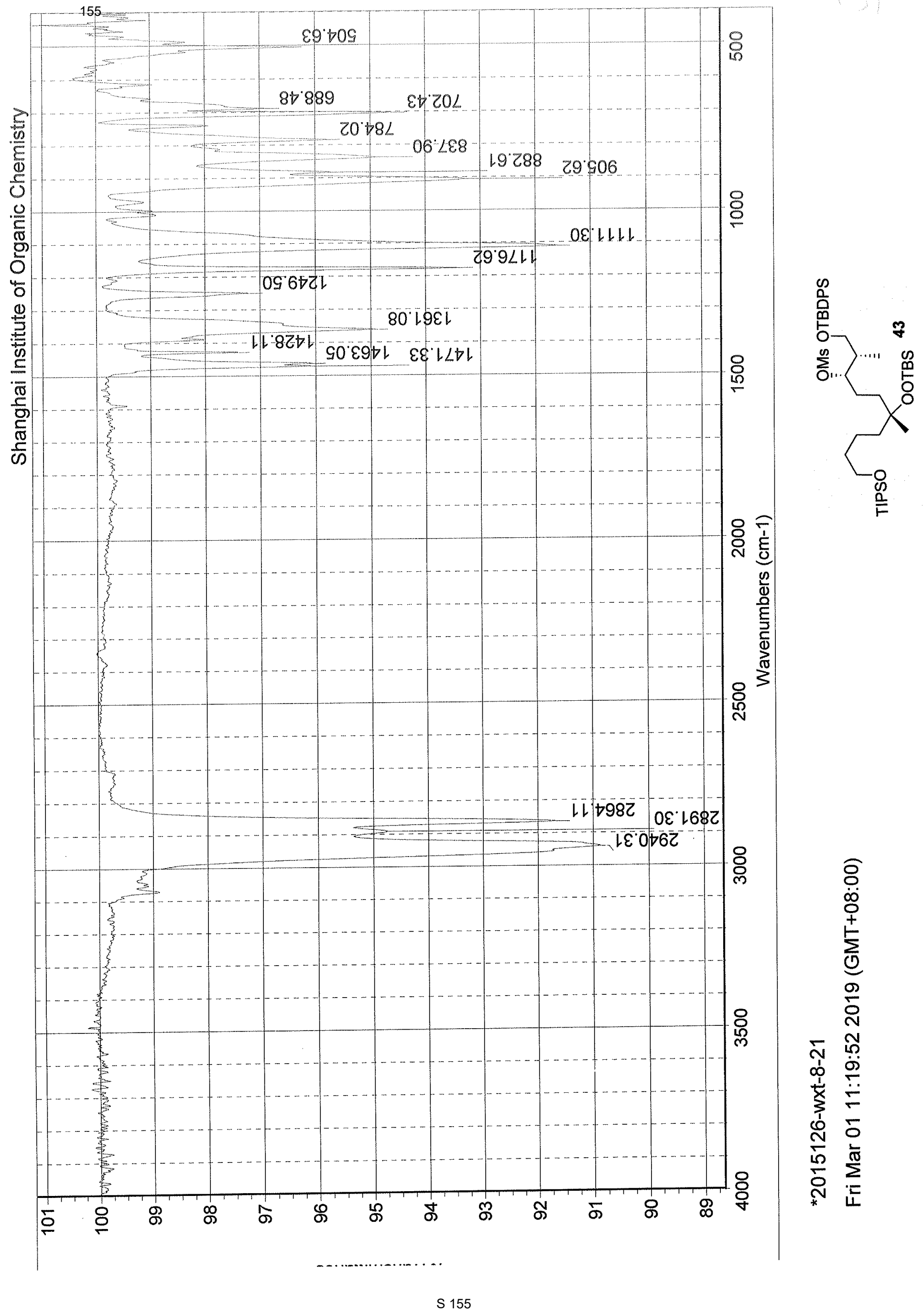


$260^{\circ}$

86.0 2015

96"Z-

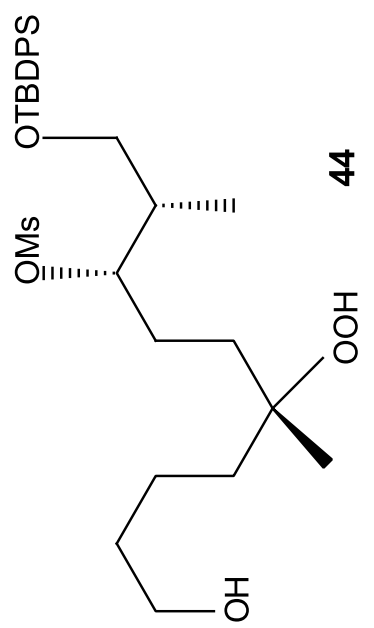

\&

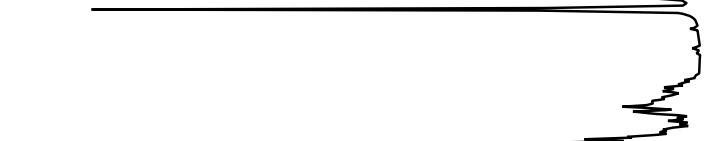

$r^{2} 6 L^{\circ} \varepsilon$

r $21.6-$

$10^{\circ} \varepsilon$

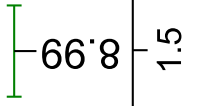

$-\subseteq \varepsilon \cdot Z$

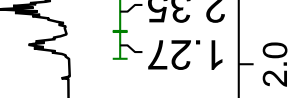

i

$=00 \varepsilon \stackrel{-}{\sim}$

(2)

(n)

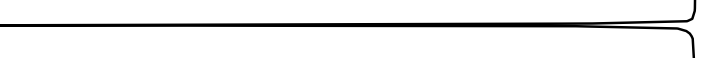

$00 \varepsilon$

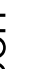

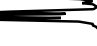




\section{$G \varepsilon<\varepsilon^{\circ}<\backslash$ 99LE'L- E68E L- SEOt L 60Zち L 6EZち L- 9LEt L 86t大 L厅} 6ZE9 L 09ع9 $L \backslash$ 0979 L $16+9.2$ E699. L 5 c)
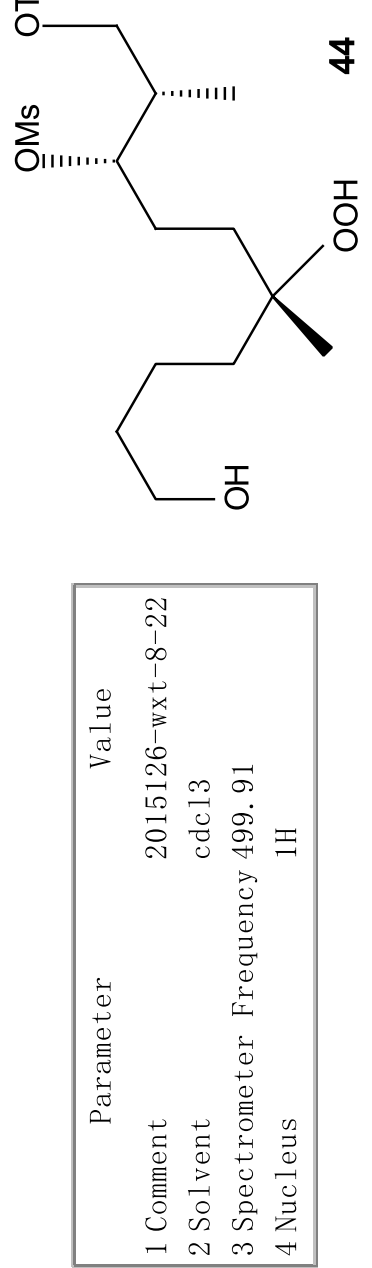

\&

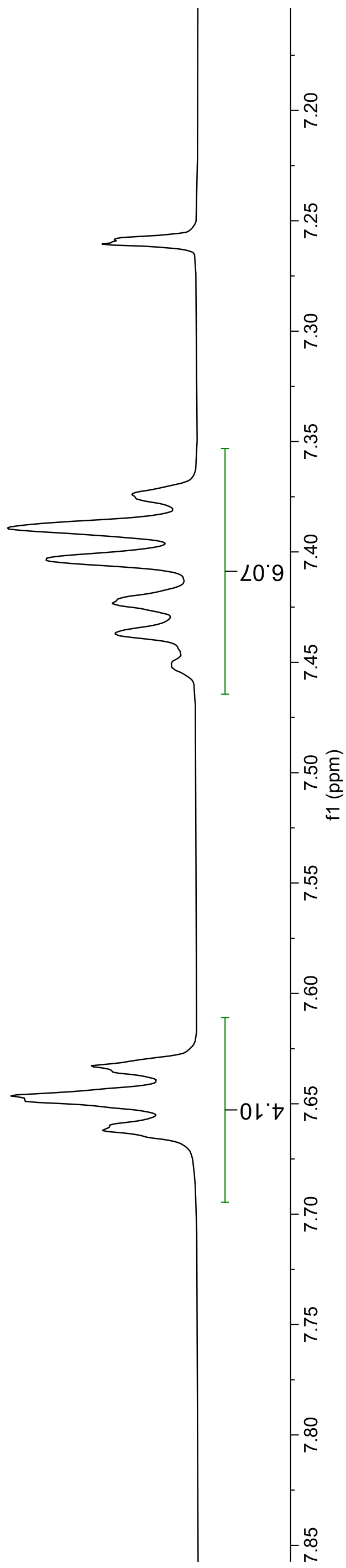



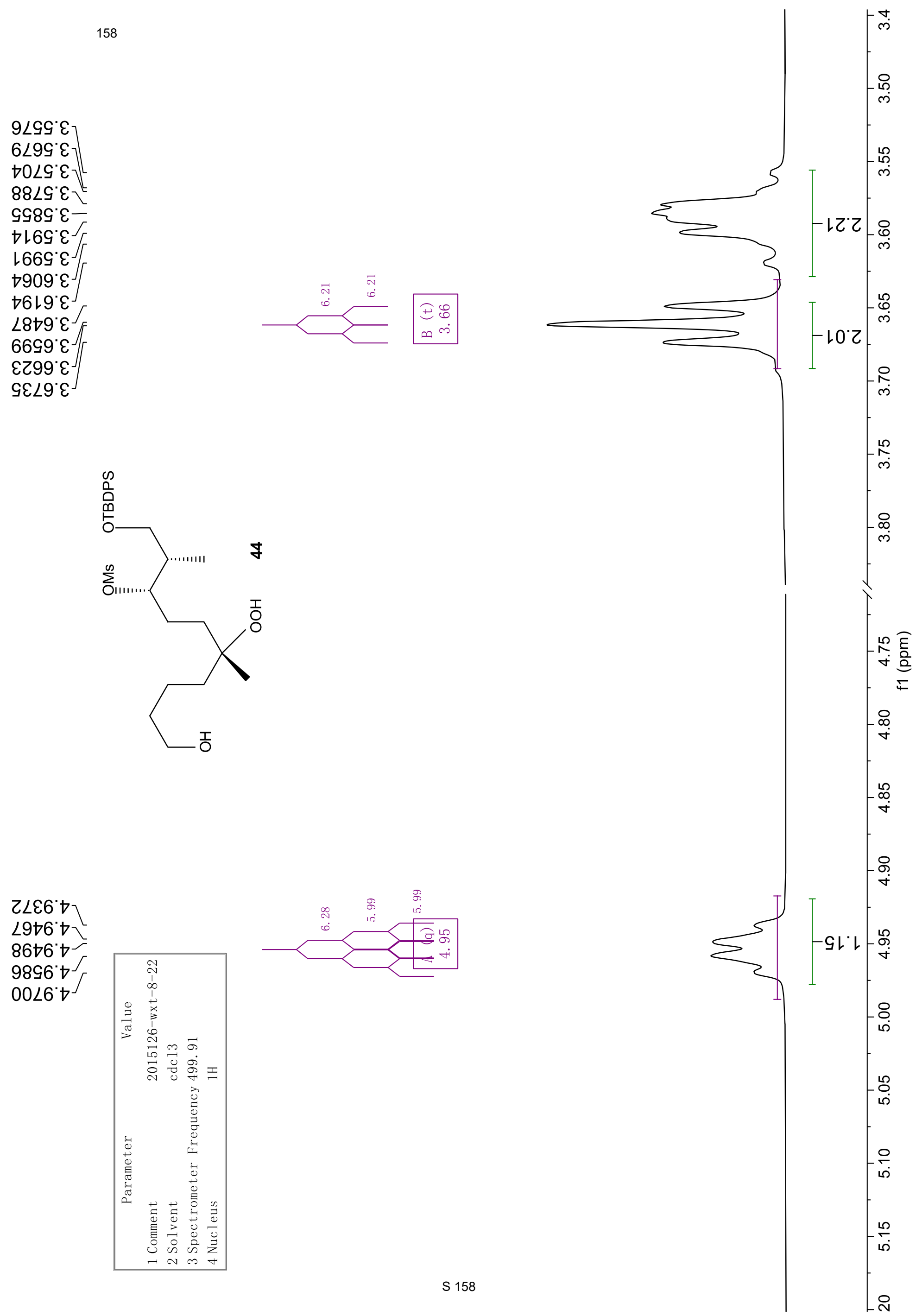
Lレレー

$0 Z^{\circ} 6 \mathrm{~L}$
$8 \varepsilon^{\prime} 6 \mathrm{~L}$

$0 Z \cdot Z^{\top}$

$9 \varepsilon^{\prime} 9 Z$

$\angle 8^{\circ} 9 Z^{-}$

$\angle 9 \cdot 1 \varepsilon \backslash$

$\nabla L G \varepsilon \backslash$

乙६. $8 \varepsilon$

IS' $8 \varepsilon^{\top}$

$\angle \varepsilon ' 29-$

$16+5$

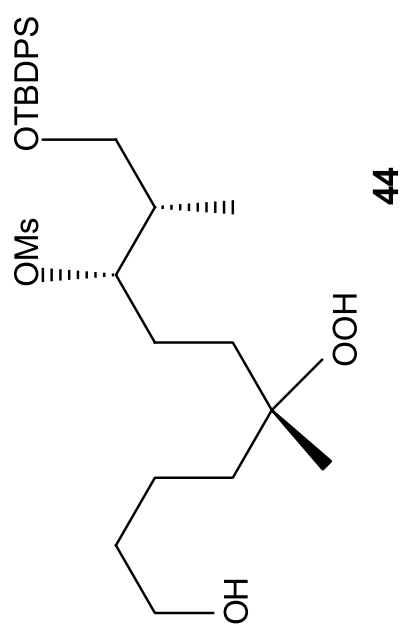

$\downarrow 9^{\circ} \varepsilon 8$ 久

6ฑ・ロ8 $9 \angle \angle Z L$ $08^{\circ} 6 \mathrm{ZL}$ Z8.6Z1 乙Z हEᄂ $8 \varepsilon^{\circ} \varepsilon \varepsilon \downarrow$

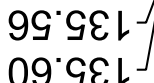

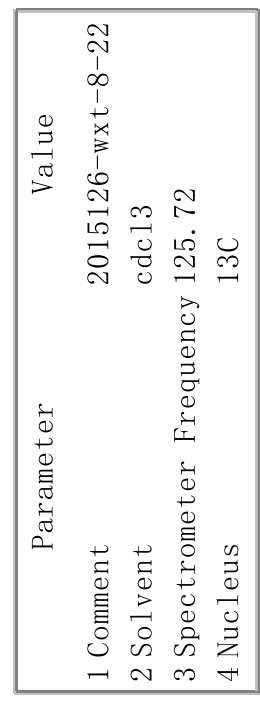




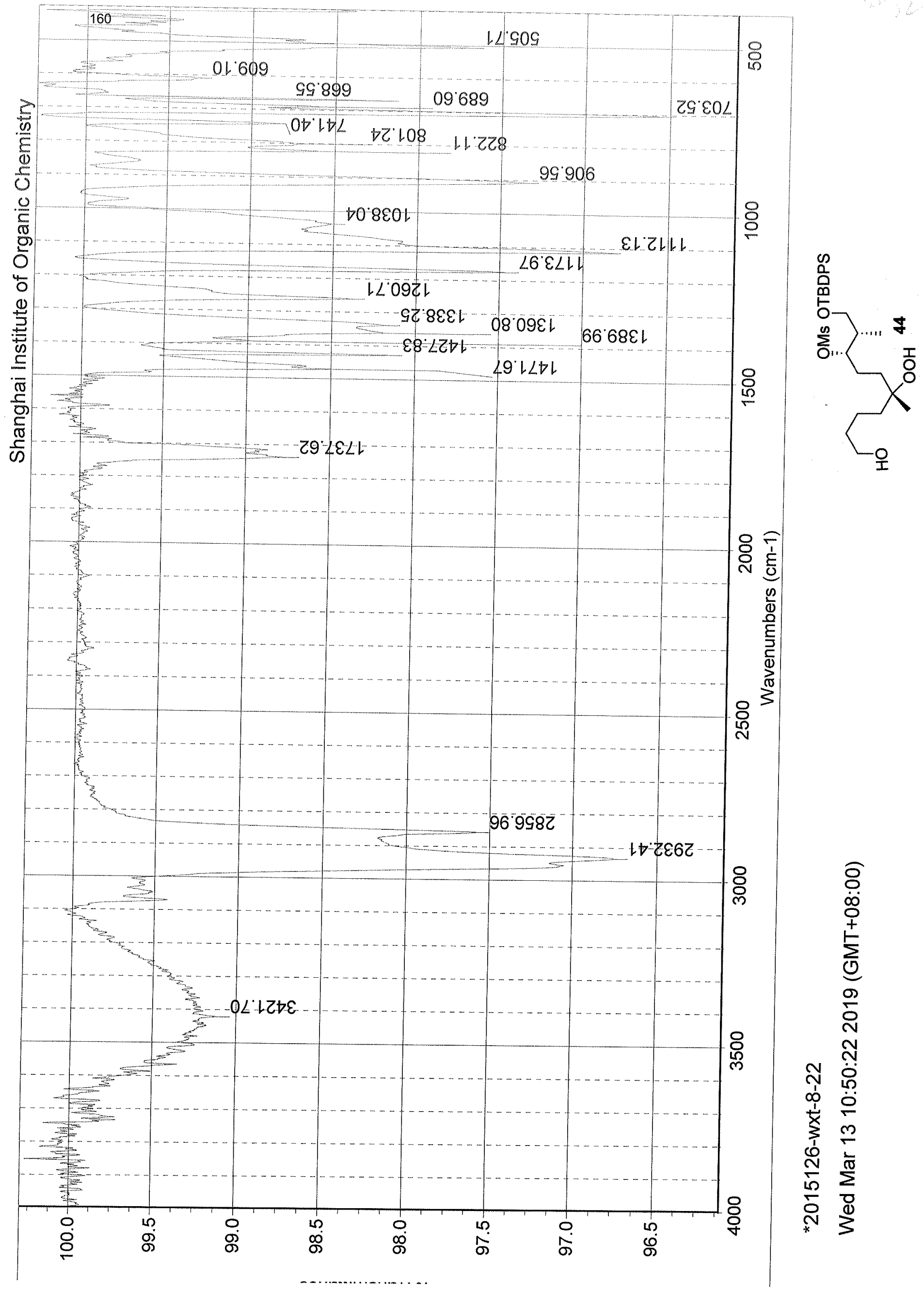



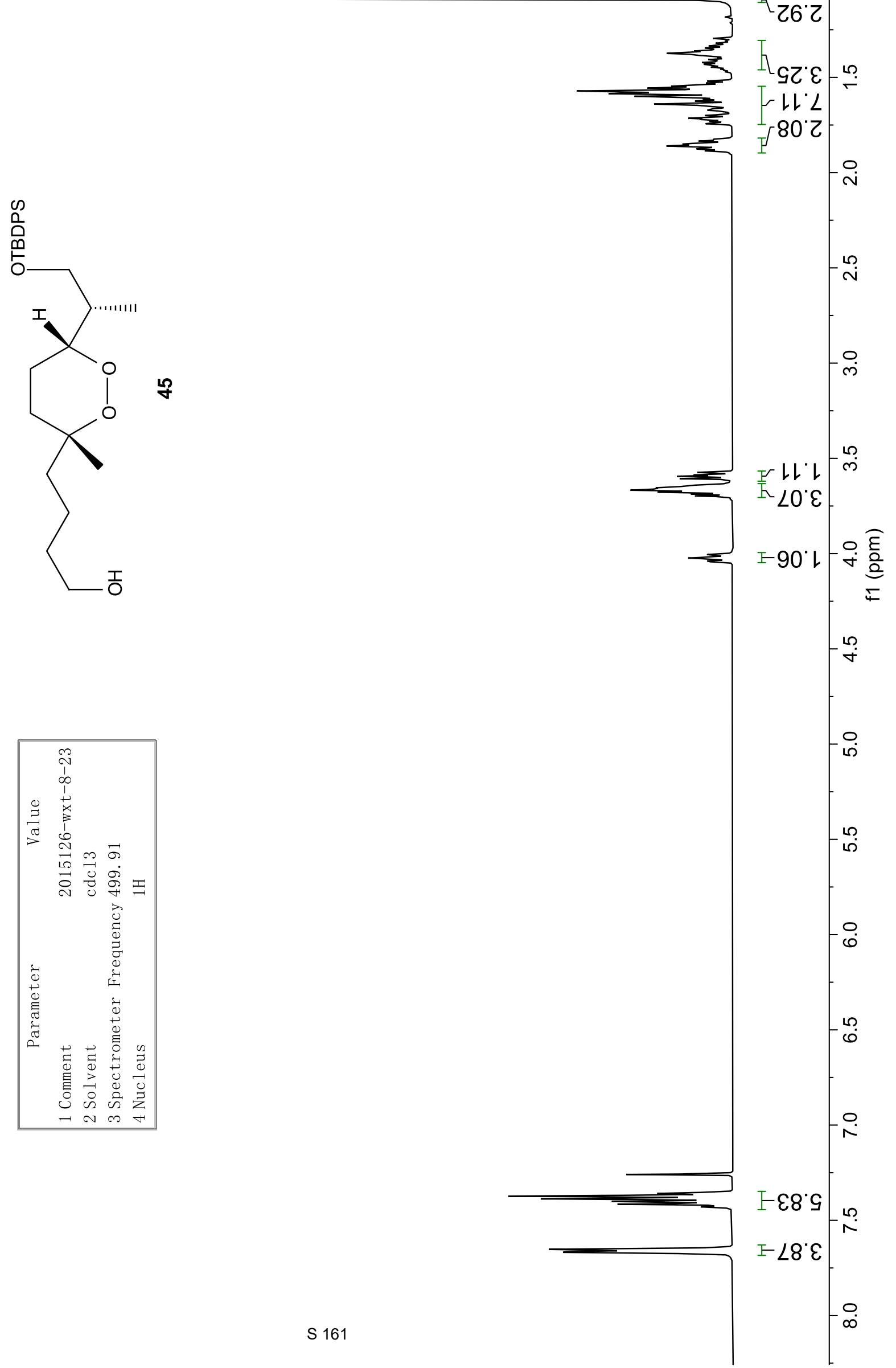


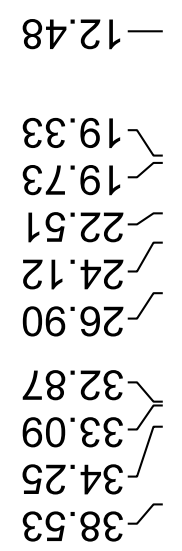

$28 \cdot 29-$
$82 \cdot 99-$

$99^{\circ} 6 L$

$6218-$
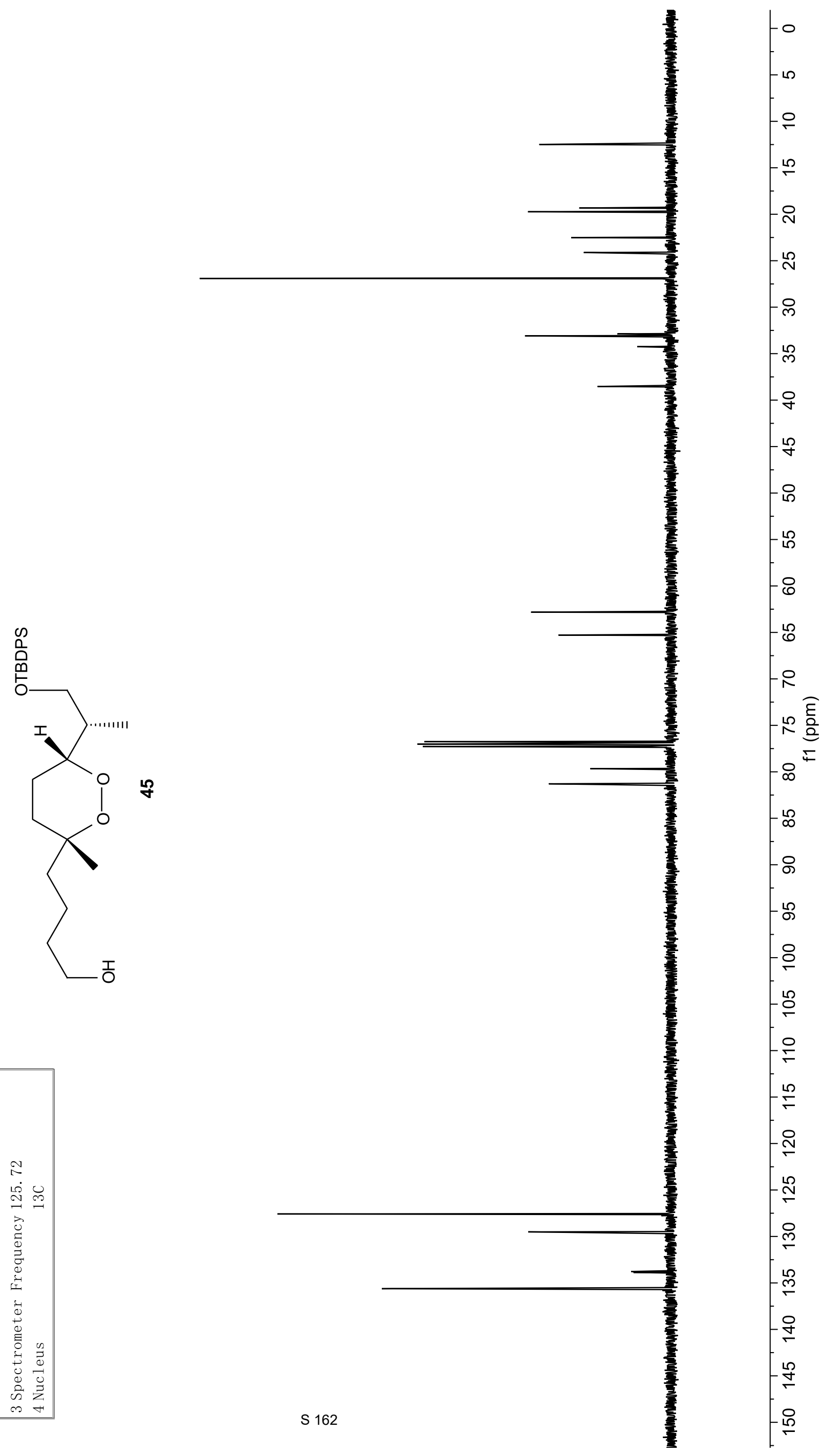


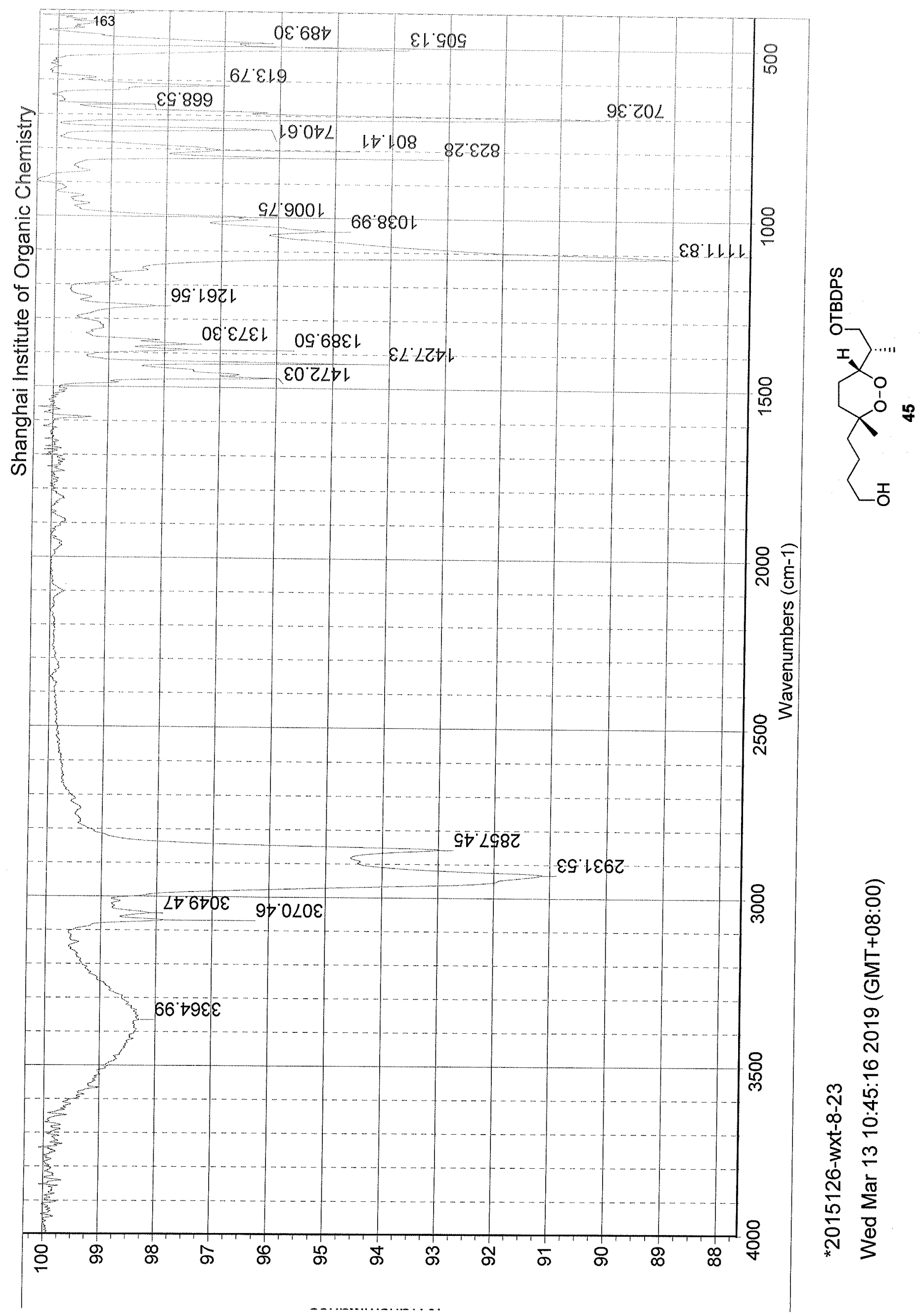



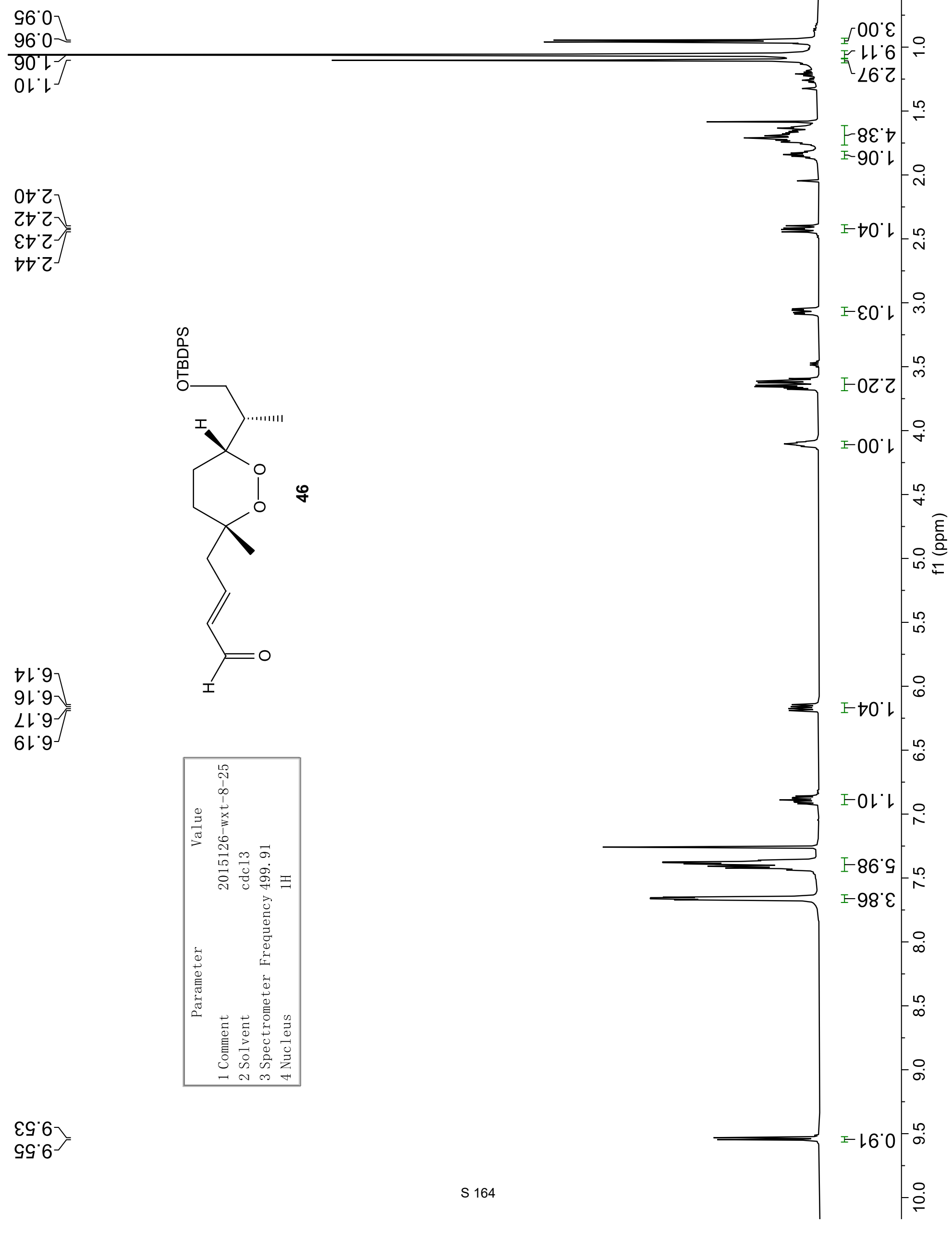
8Ztレ.9 889.9\&† LL'9Z06.'9

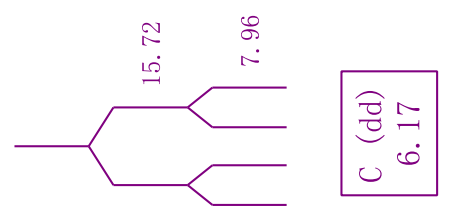

ㅇ
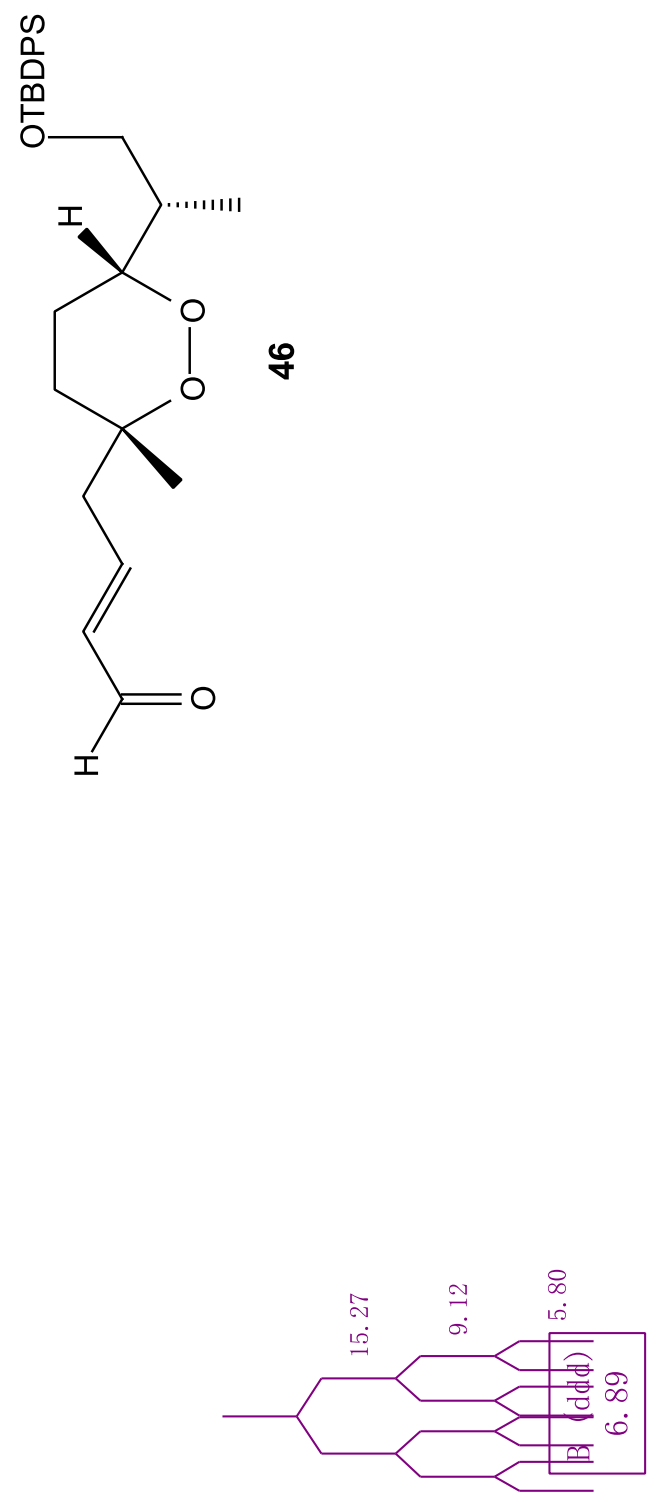

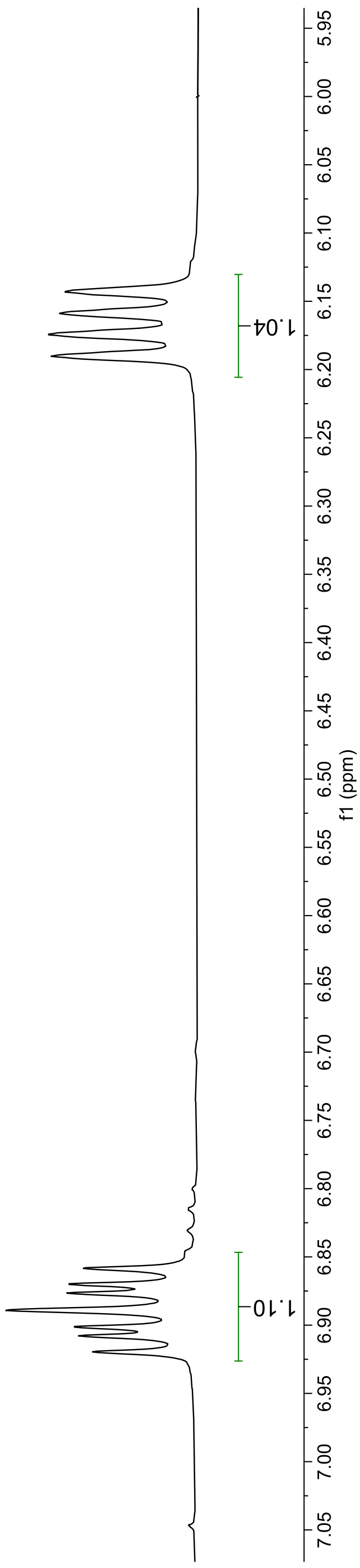


$6870^{\circ} \varepsilon \backslash$

$9690^{\circ} \varepsilon-$

$6 \angle \angle 0^{\circ} \varepsilon-$ $9680^{\circ} \varepsilon^{\gamma}$

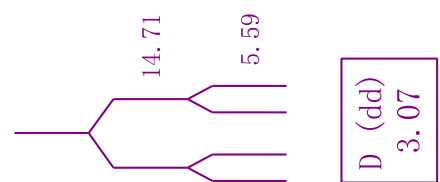

$\infty$
0
0
0

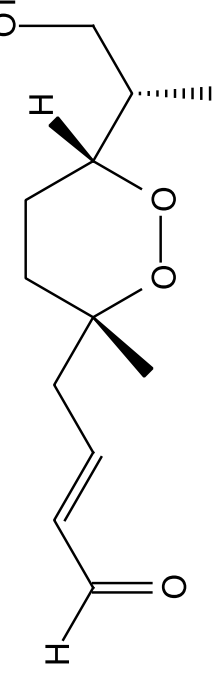

\&
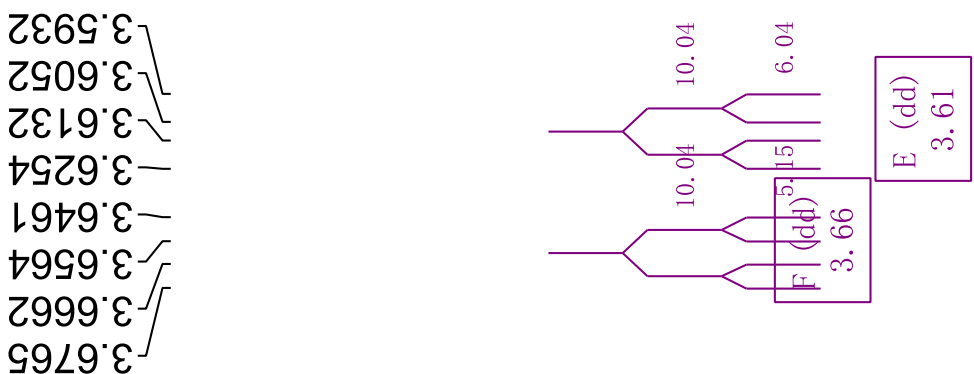

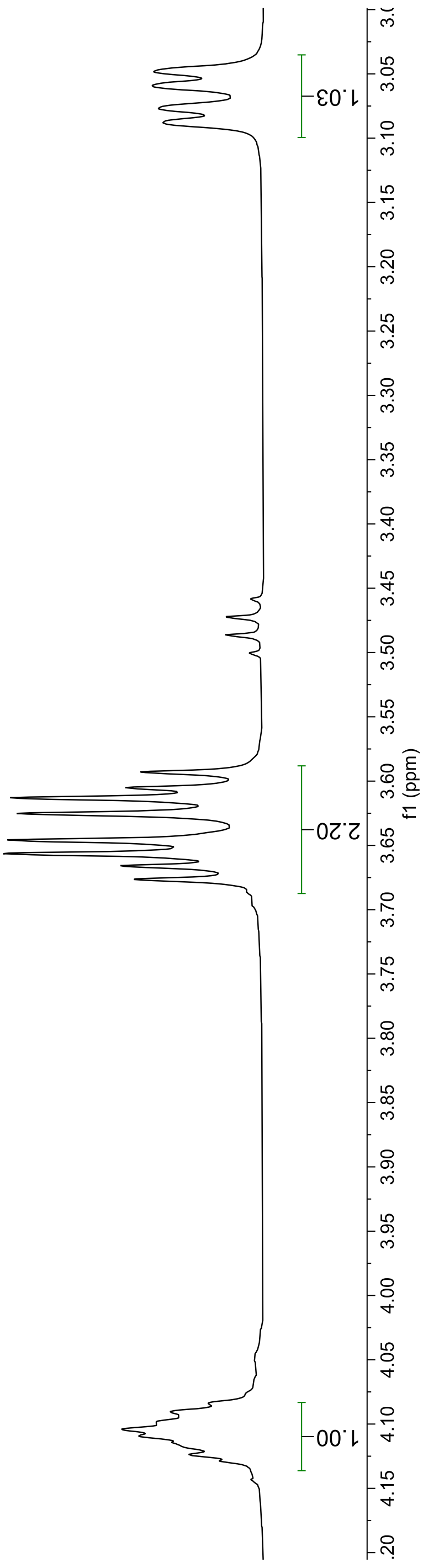

I EO. 
とカてレー 乙E'6卜 99.2Z $\varepsilon s^{\circ} \circ Z-$ 68.92乙๐ $\varepsilon \varepsilon>$ $\left.\angle 6^{\circ} \angle E\right]$ $99^{\circ} 8 \varepsilon-$

20.99-

8162 t5. 18

$19 \angle Z L$ $89^{\circ} 6 Z 1$ 69.6ZL] $\rightarrow 9^{\circ} \varepsilon \varepsilon \downarrow$ $\nabla L \varepsilon \varepsilon 1-$ $\angle \nabla$ SEL09. $9 \varepsilon$ I9 $\subseteq \varepsilon \downarrow$

$\infty$
0
0
0

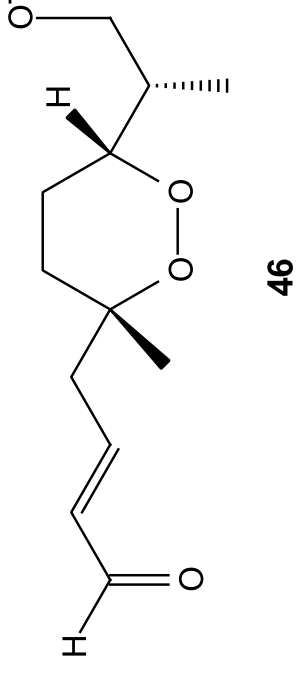

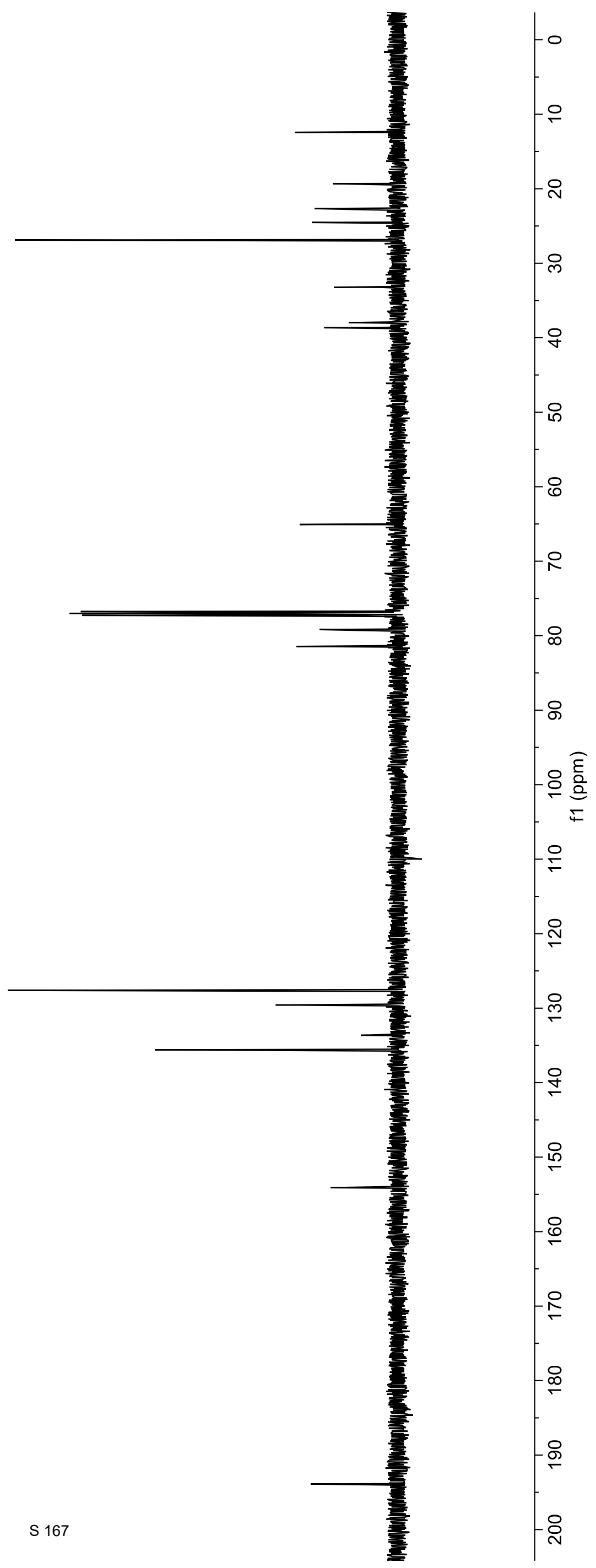



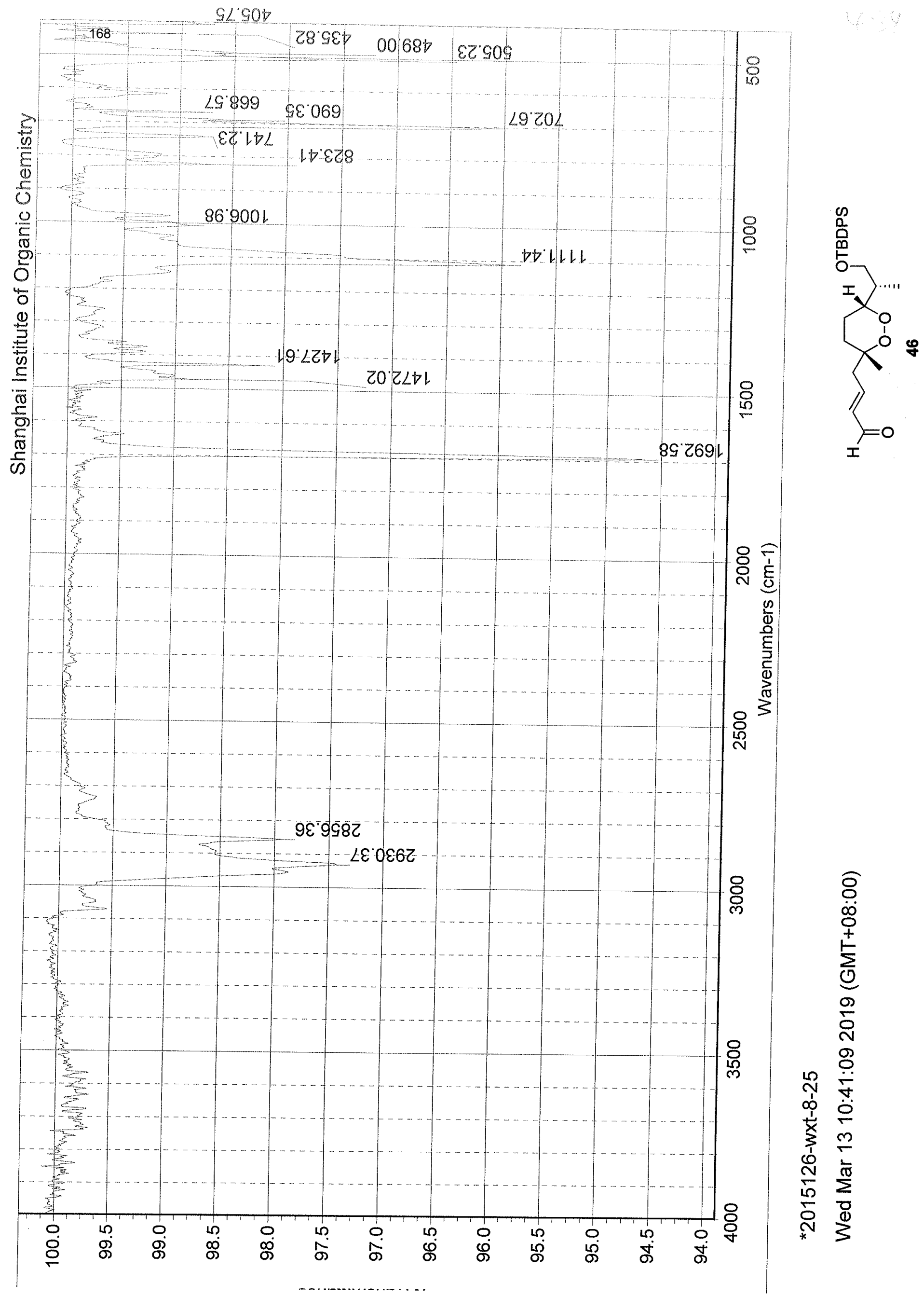
$\varepsilon 6^{\circ} 0$
$96^{\circ}-\gamma$

$9 Z^{\circ} \downarrow$

$\angle Z^{\circ}{ }^{\circ} \mathcal{}$ $\left.\begin{array}{l}\angle S^{*} \varepsilon \\ 89^{\varepsilon} \varepsilon \\ 69^{\circ} \varepsilon \\ 09^{\circ} \varepsilon \\ 99^{\circ} \varepsilon- \\ \angle 9^{\circ} \varepsilon \\ 89^{\circ} \varepsilon \\ 69^{\circ} \varepsilon\end{array}\right]$

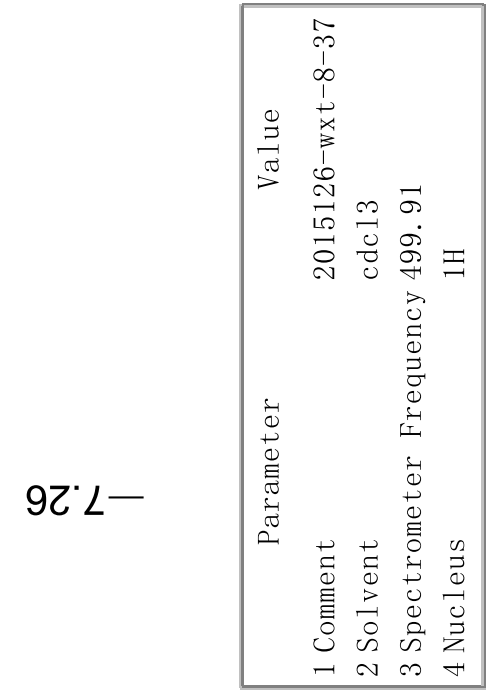
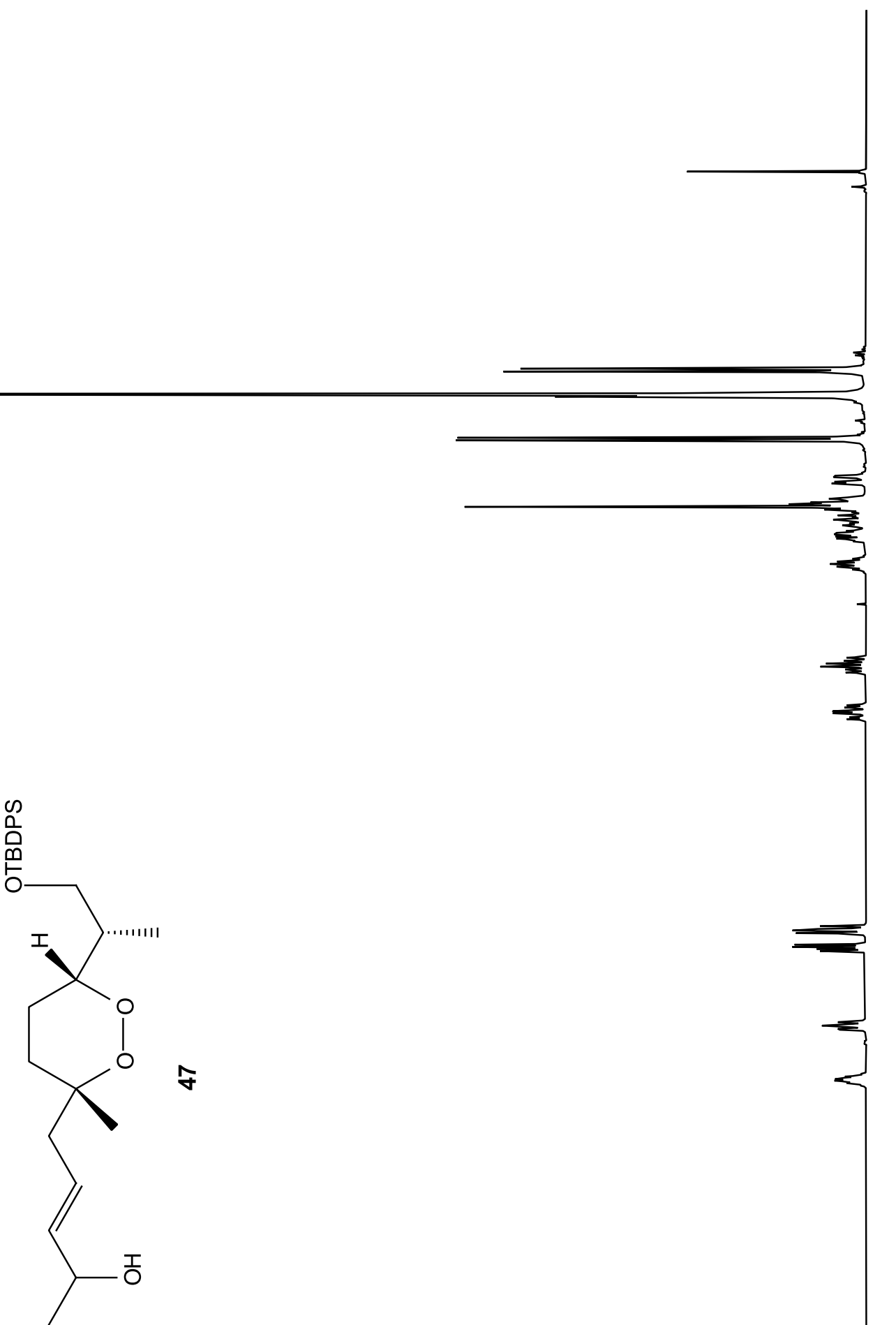

F $00 \notin 0$

ᄃ $87^{\circ} 11$

- $26^{\circ} \mathrm{C}$

< $88^{\circ} 0$ เ

= $09^{\circ}-$

r 99.2

Zl.

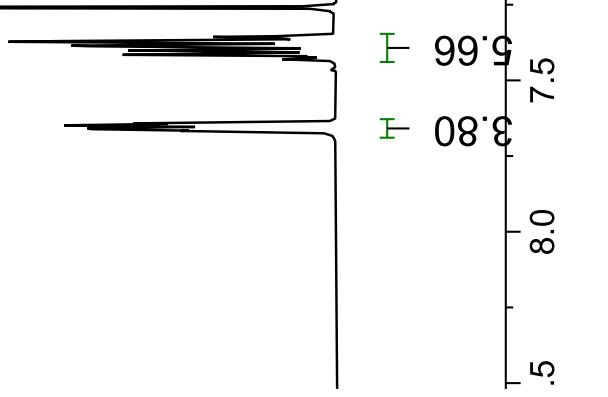


$\supset 0 \angle \mathcal{G}^{\circ} \varepsilon \backslash$ เE89' $\varepsilon$ $\supset 069^{\circ} \varepsilon^{-}$ เE09. $9 \angle 99^{\circ} \varepsilon \backslash$ $1899^{\circ} \varepsilon-$ 9LL9' $\varepsilon^{\prime}$ $6 \angle 89^{\circ} \varepsilon$
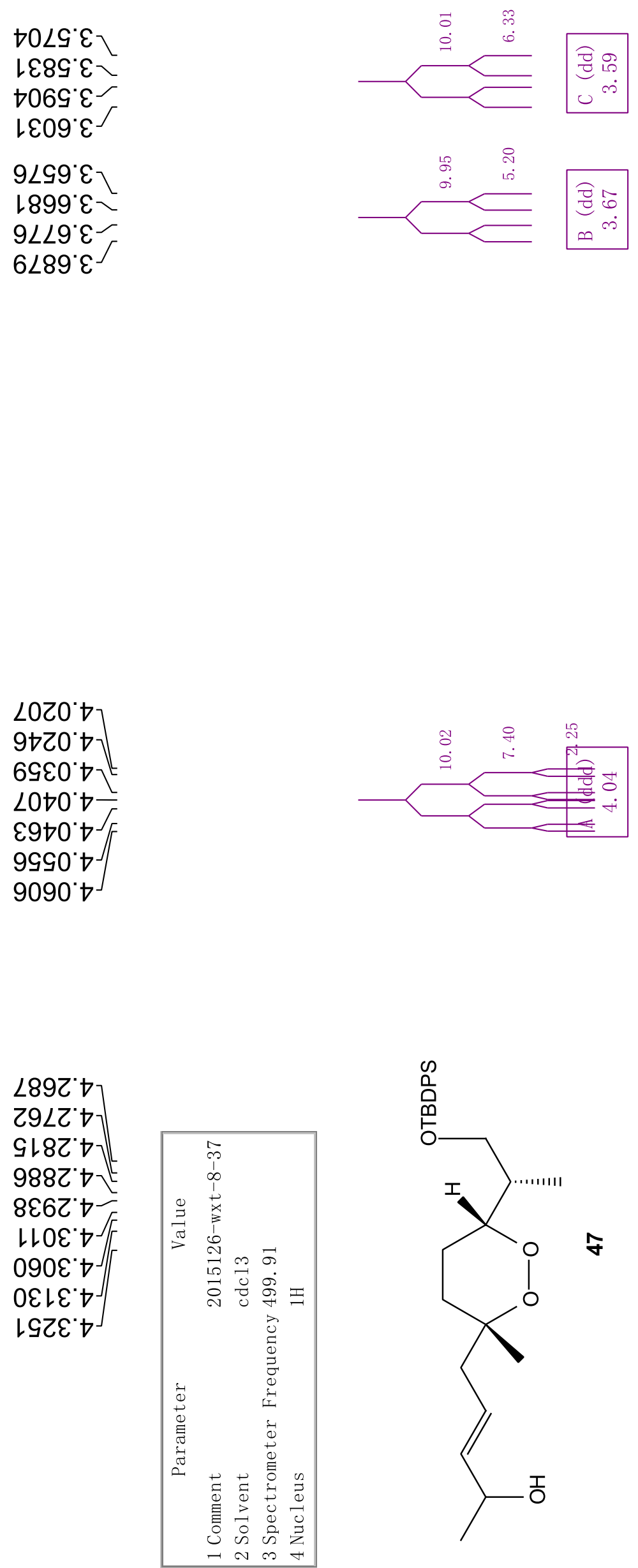

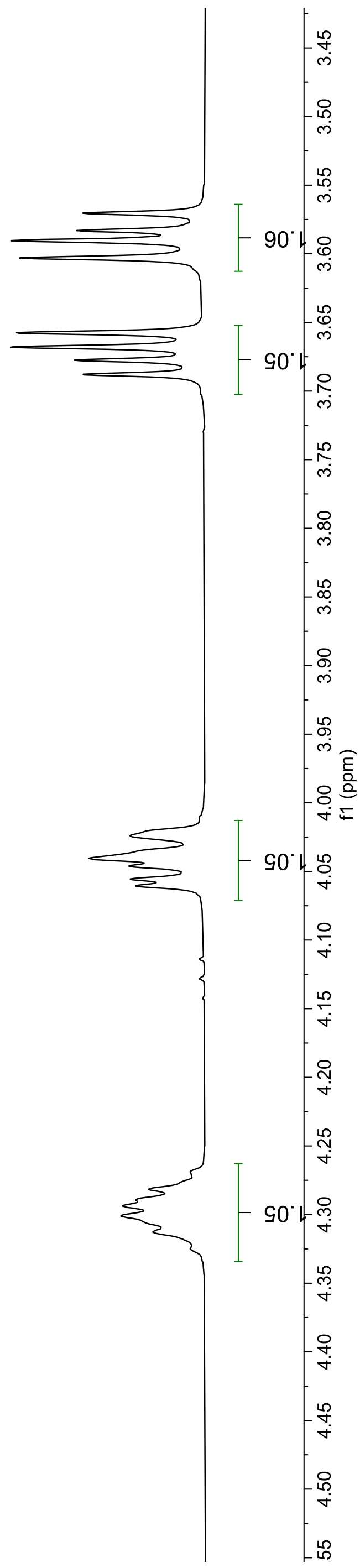


966乙てー

6ナレとてー

டL乙E'てー

レて๖どてー

૬GSE'て-

ย69ع 乙一

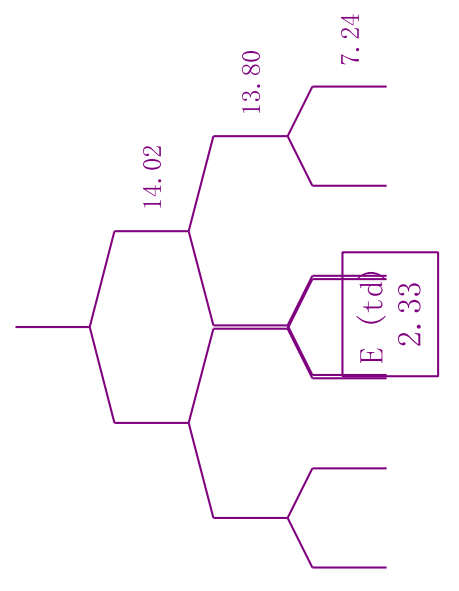

$n$
0
0
0
0
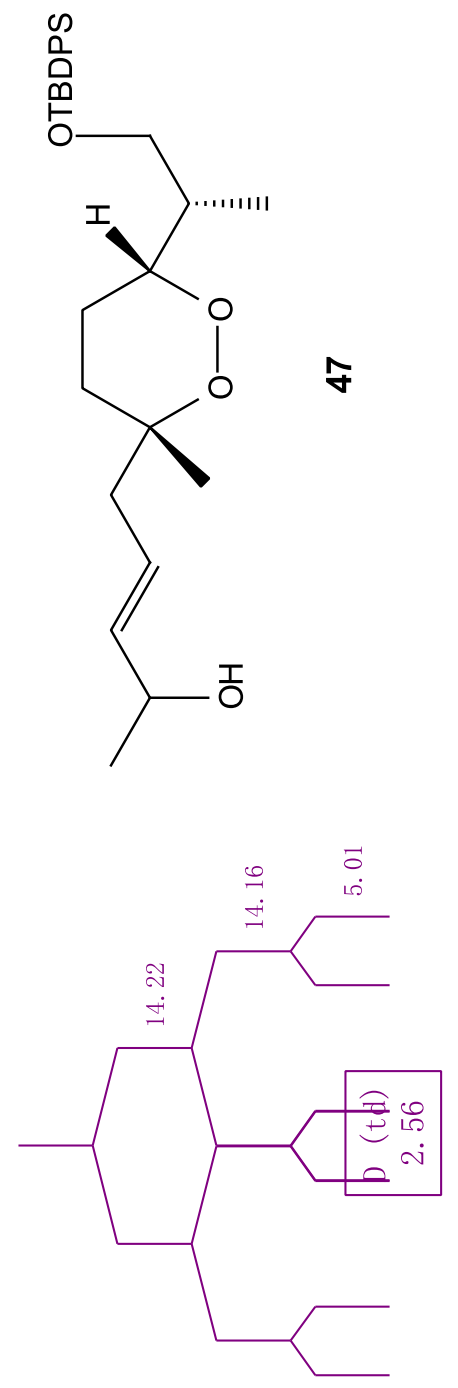

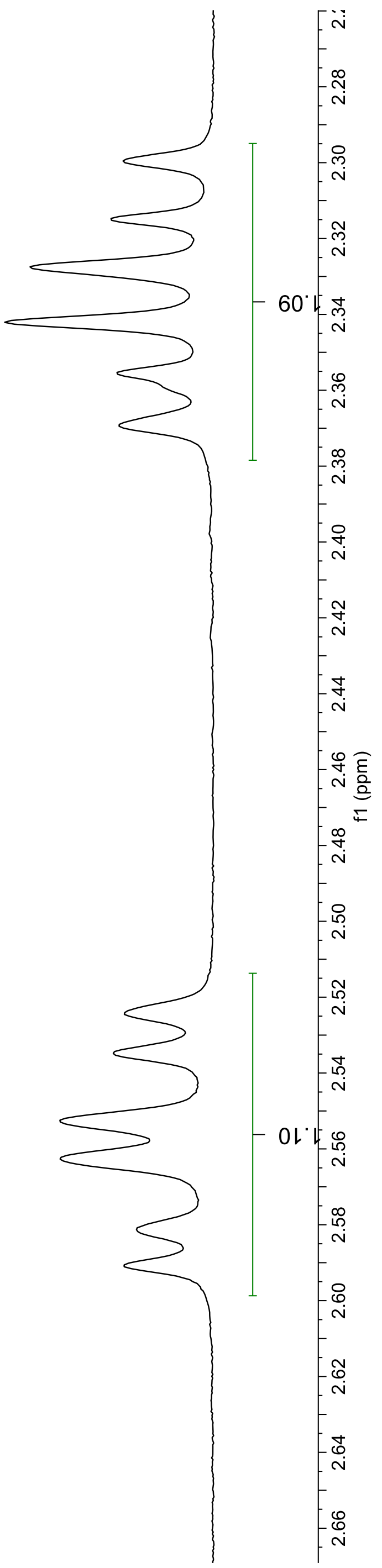




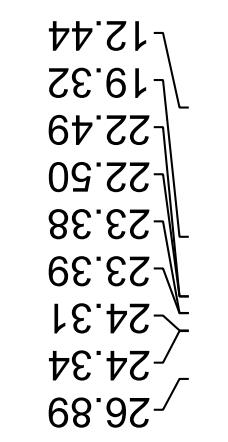

$\angle Z^{\circ} Z \varepsilon$
$Z \varepsilon^{\circ} Z \varepsilon^{-}$
$97^{\circ} \angle \mathcal{} \simeq$
$89^{\circ} 8 \varepsilon^{-}$

27.99

18.89

78.89

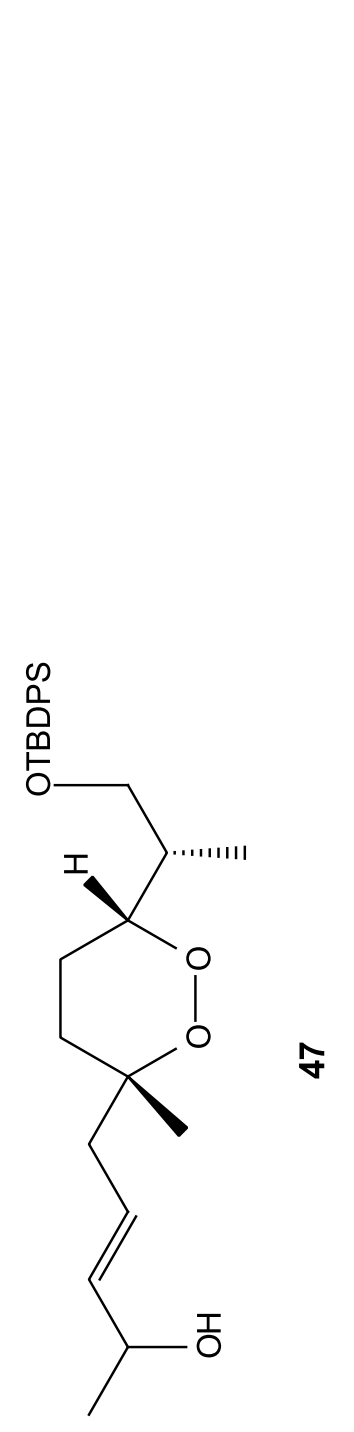

EG.6L-

GE'L -

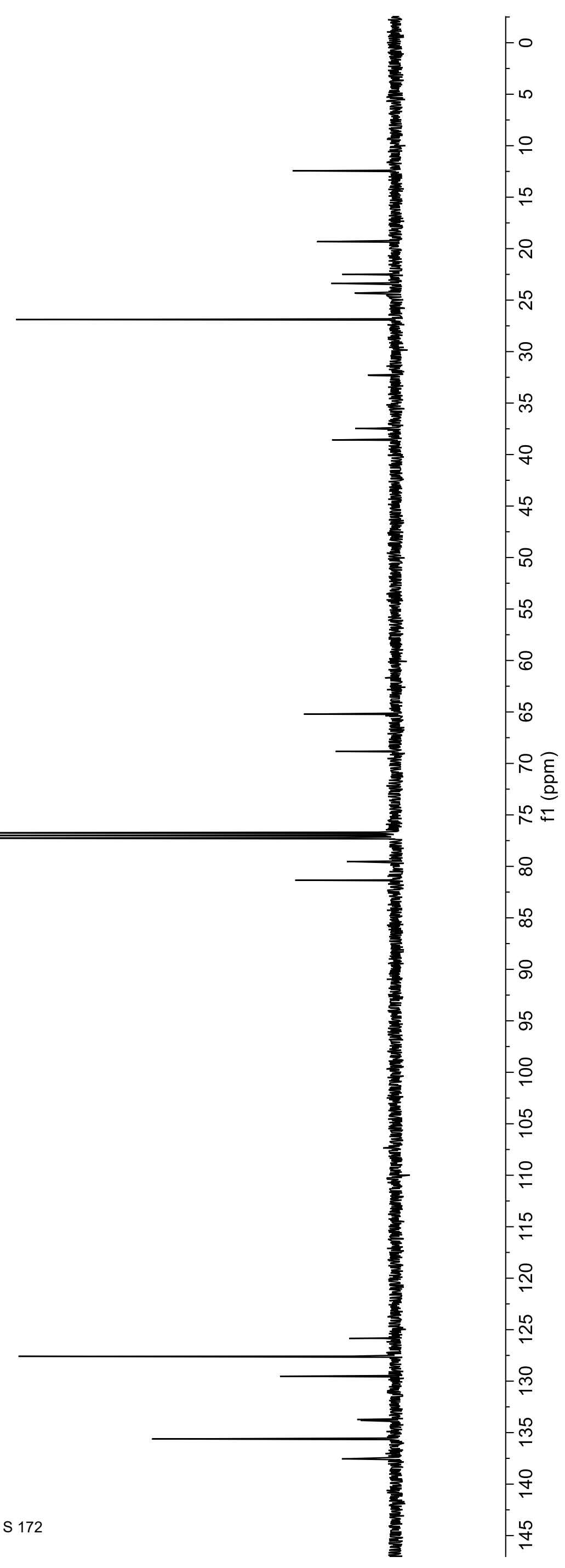




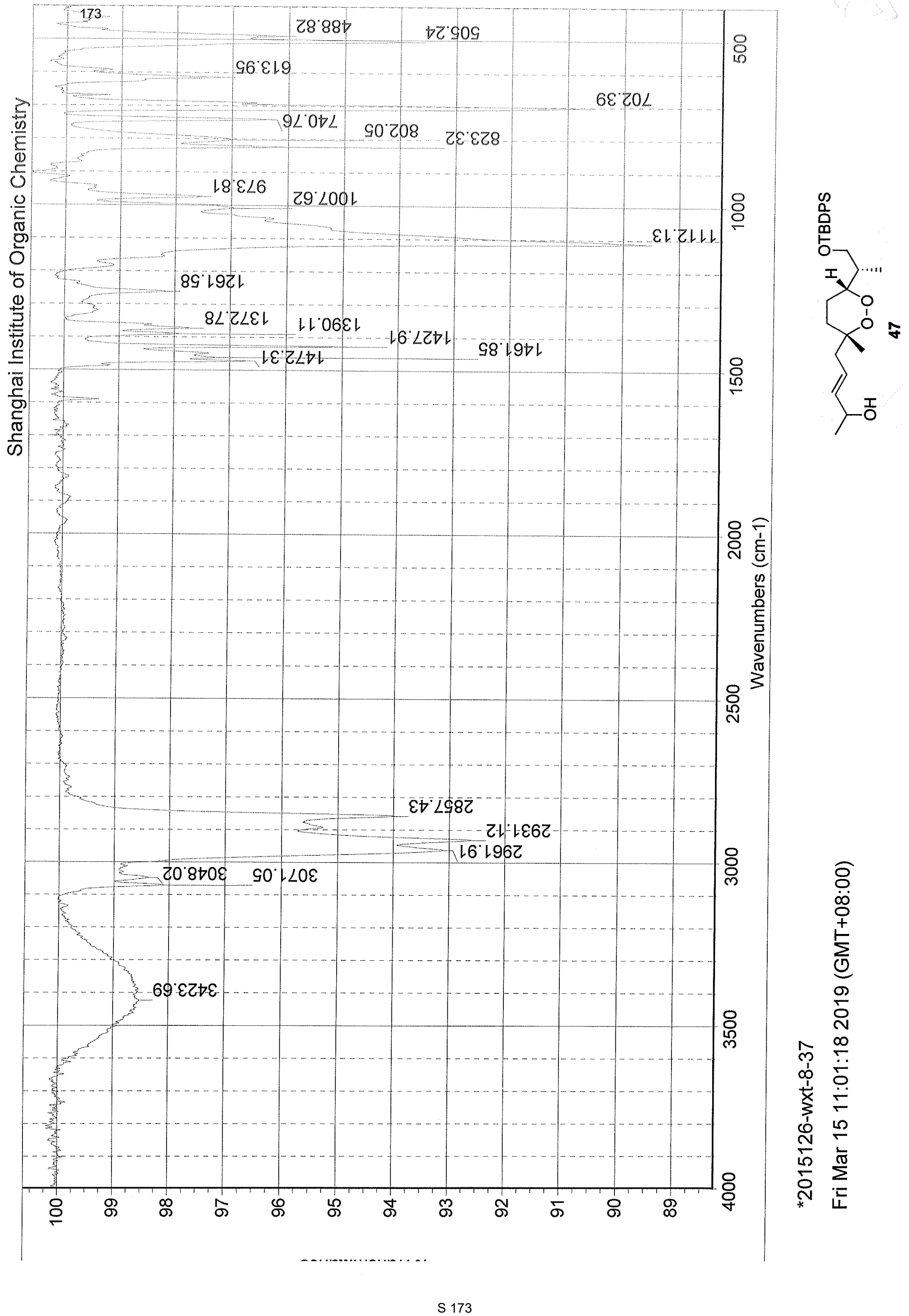


$76^{\circ} 0$

$96.0-$

9015

$60^{\circ}$ ᄂ

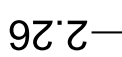

$\infty$
0
0
0
0

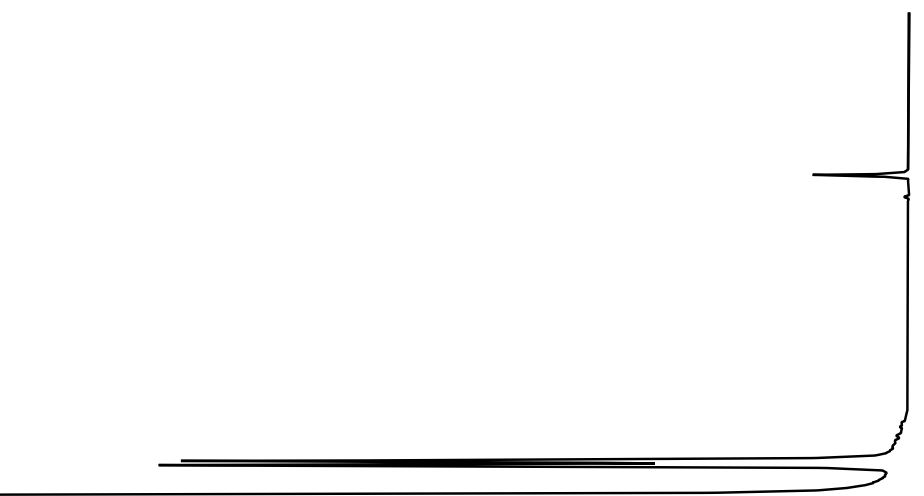

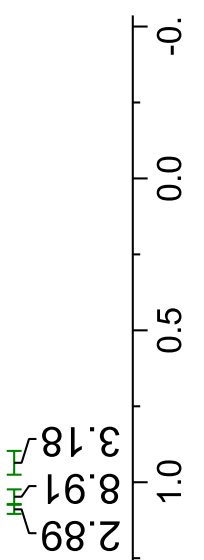

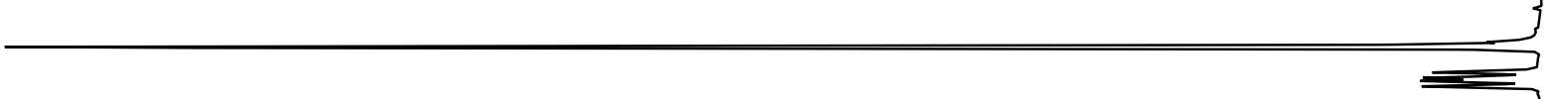

3

$9 \angle 乙$

$96^{\circ} 0$
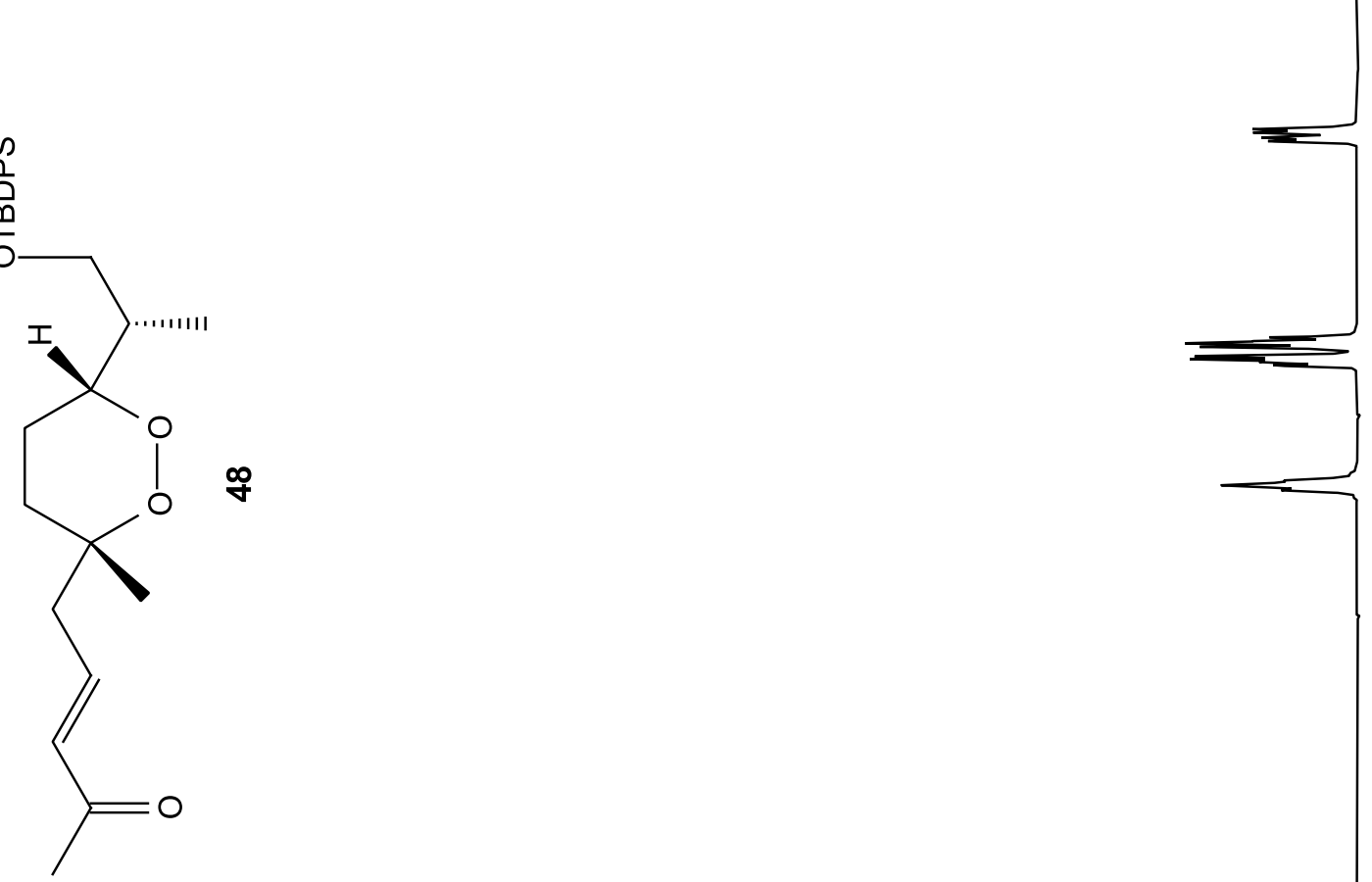

$60{ }^{\circ}$
乙レ'9

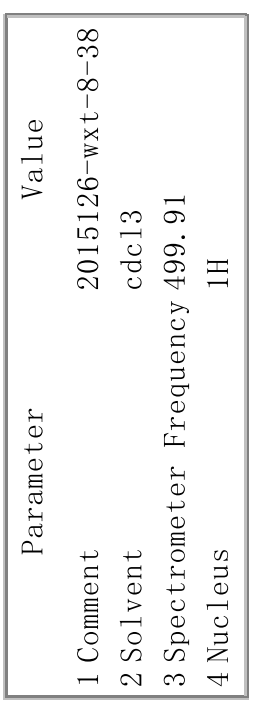

文

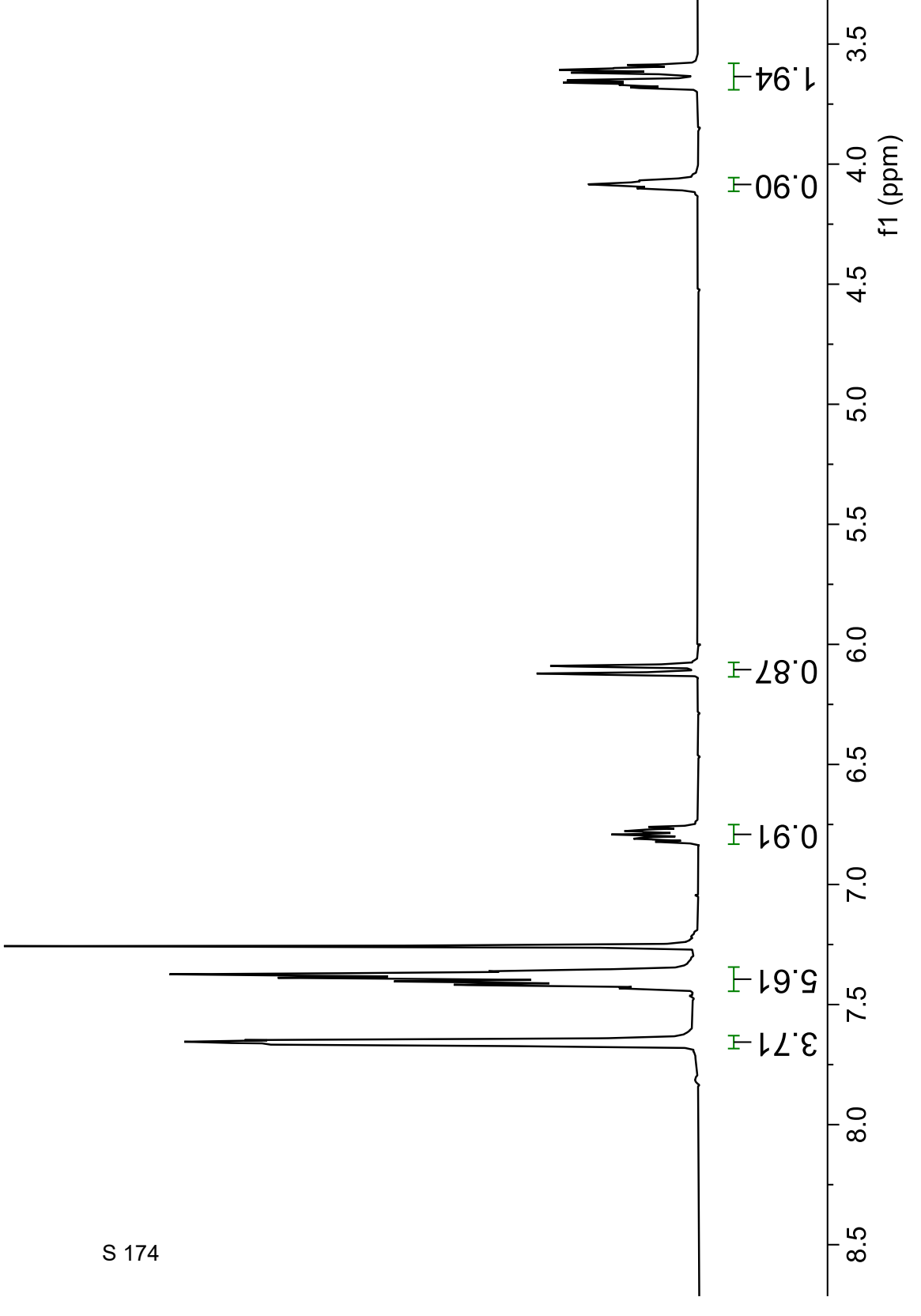




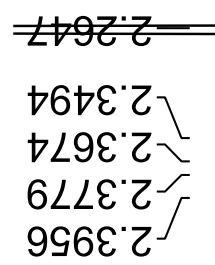

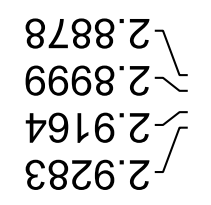

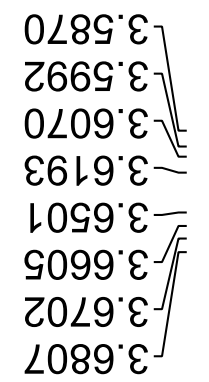

$\angle 089^{\circ} \varepsilon$
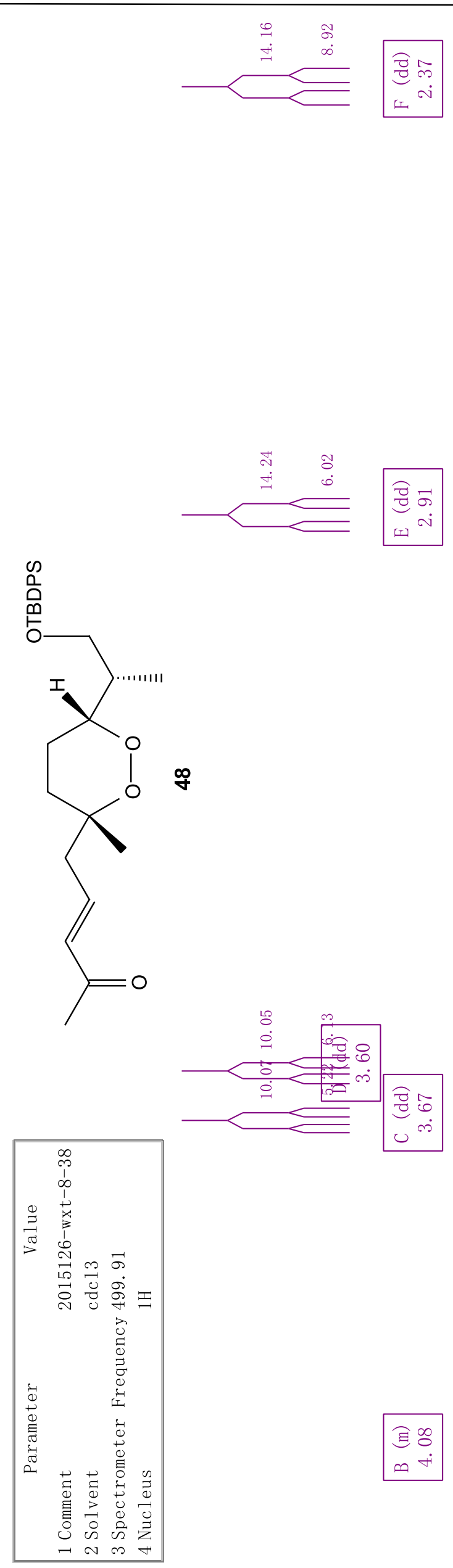
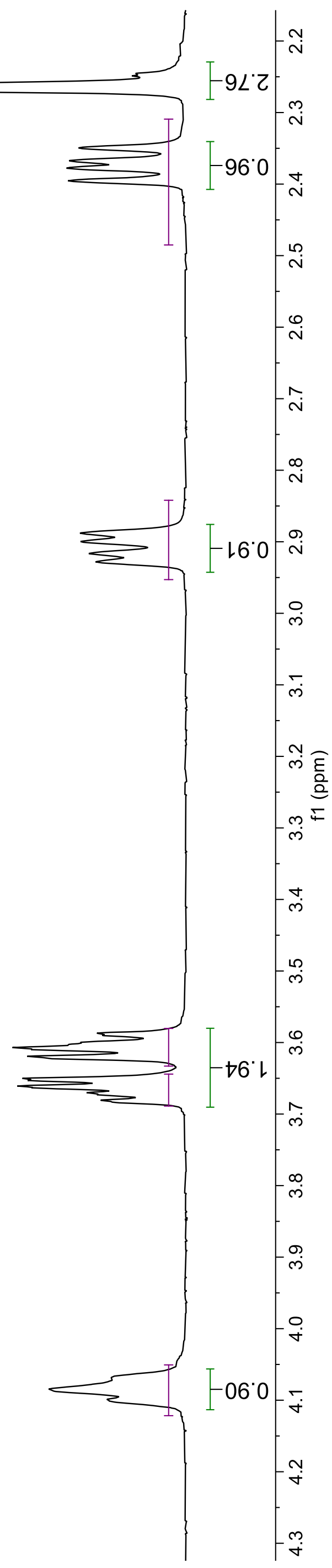
レナてレ

L.6L

19.Z乙

$\nabla 9{ }^{\circ} \nabla 2$

$\varepsilon L 9 Z \succ$

$88.9 乙^{\top}$

$\varepsilon 0^{\circ} \varepsilon \varepsilon-$

$\angle 8^{\circ} \angle E \backslash$

$79^{\circ} 8 \varepsilon^{-}$

Eเ:S9-

LE $6 L$

งt'18-
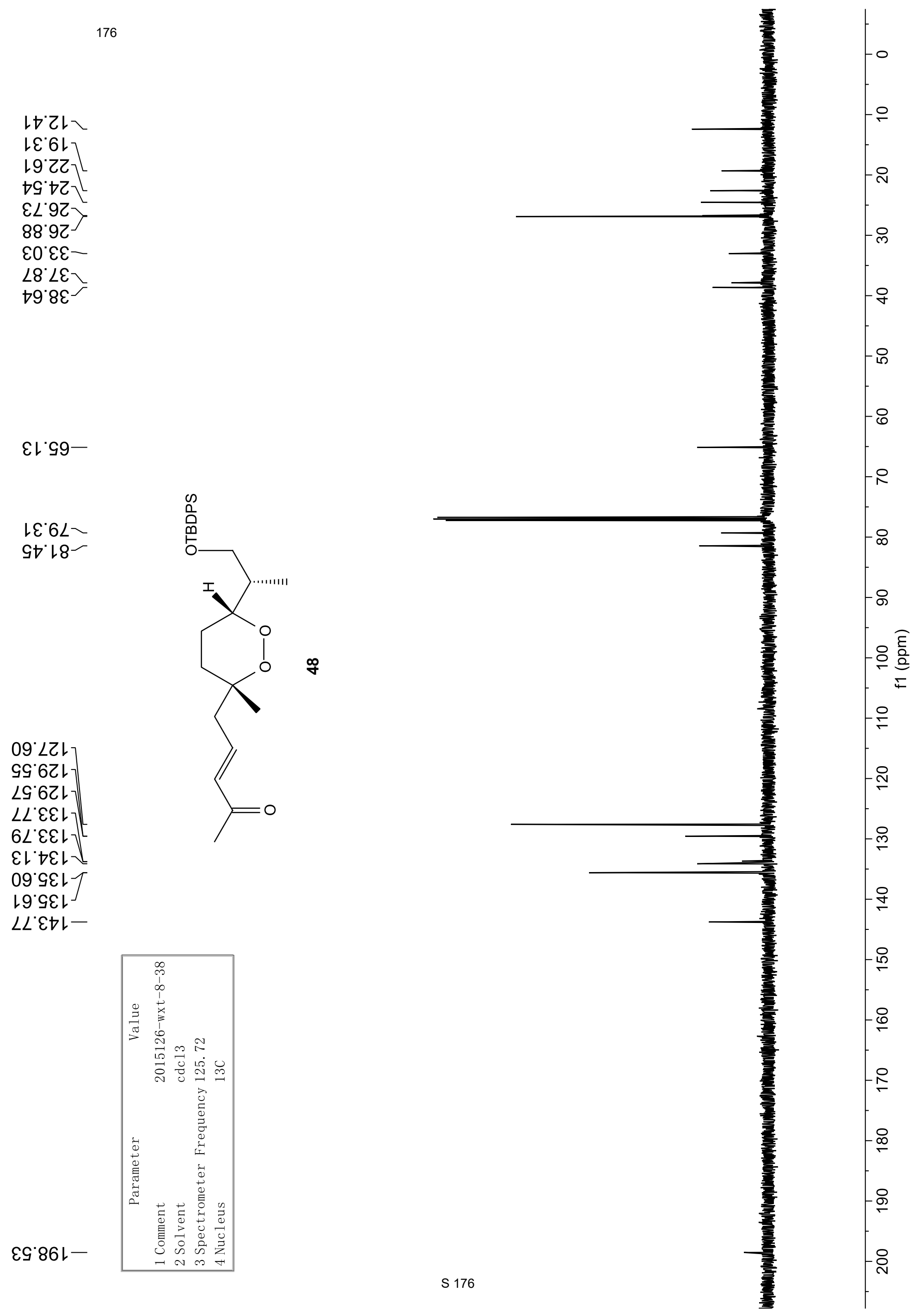


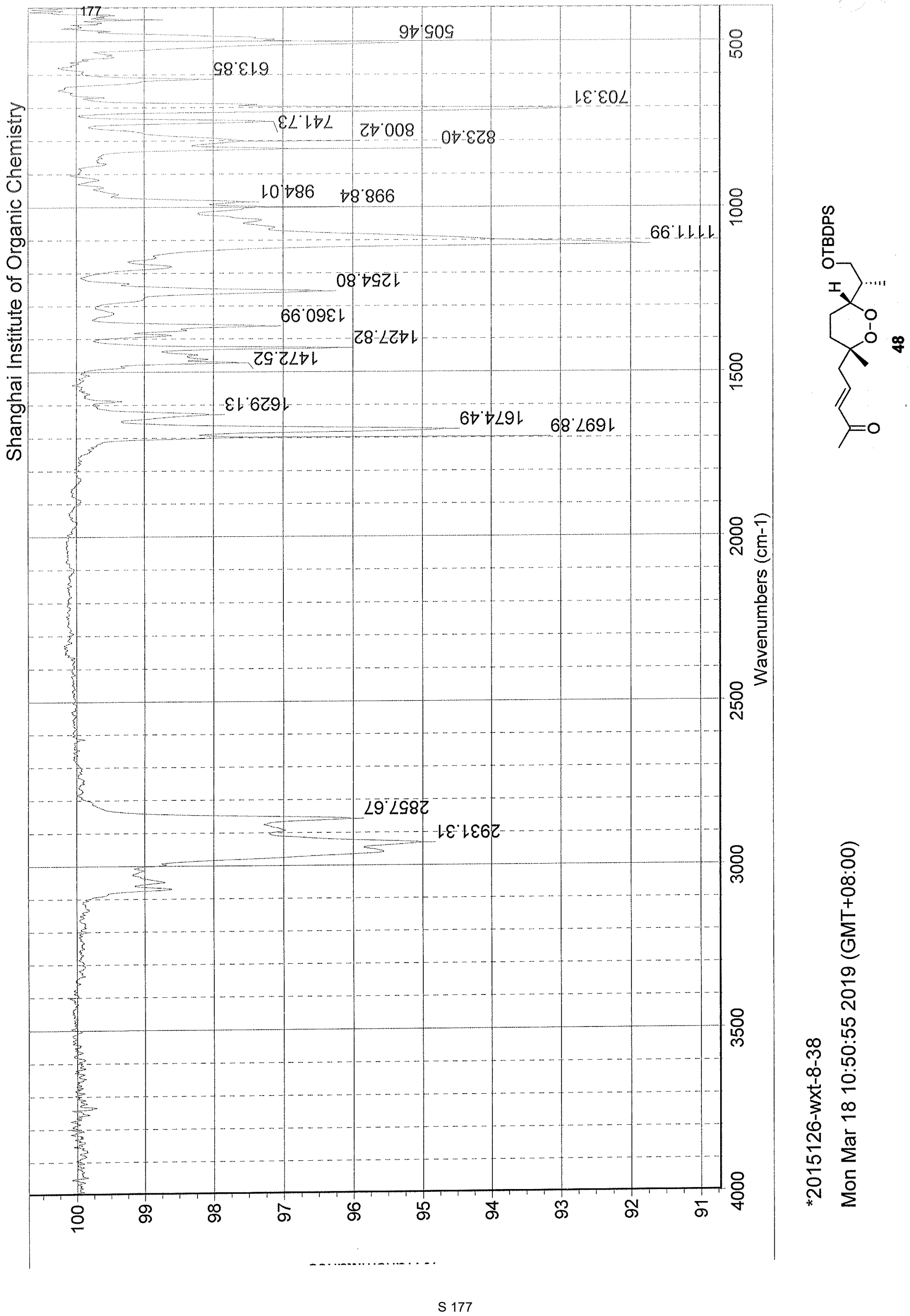


$26^{\circ} 0$

$+60^{\circ} 0^{-}$

09.レ-

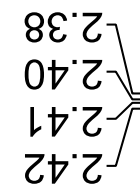

$26^{\circ} \mathrm{Z}$

$\varepsilon 6^{\circ} Z$

$\nabla 6{ }^{\circ}-1$

$96{ }^{\circ} \mathrm{Z}^{\circ}$

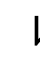

$\nabla L^{\circ} 9$

9L. 9

$\angle L \cdot 9$

$8 \angle 9$

6L'9

089

18.9

ट०. 9

$9 Z \angle-$

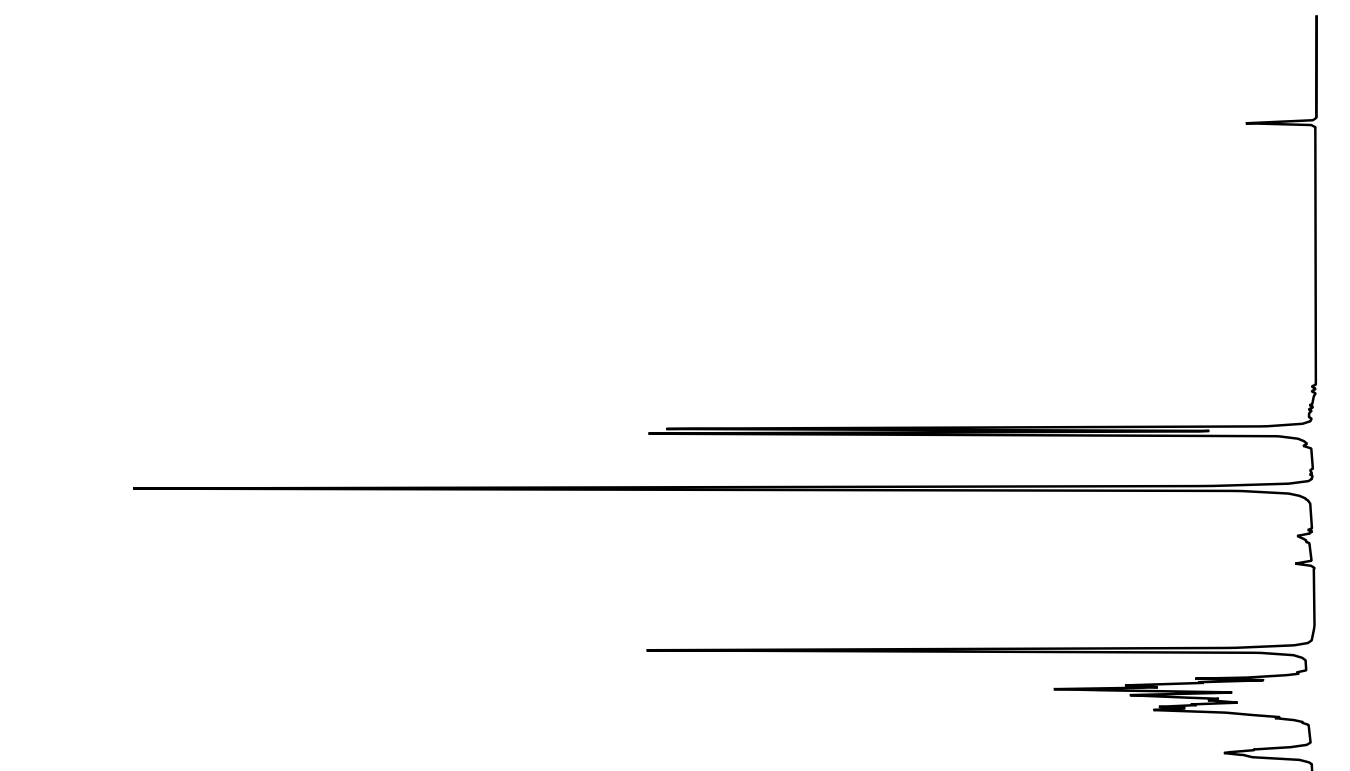

F $00^{\circ} \varepsilon$

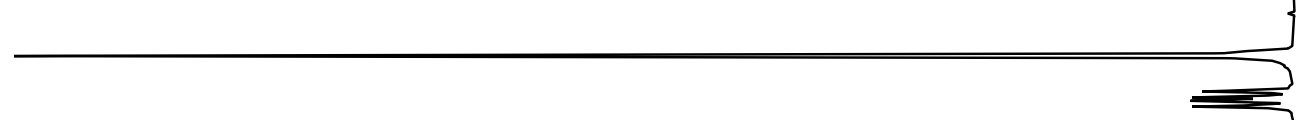

F $06^{\circ} \mathrm{Z}$

F 80. L

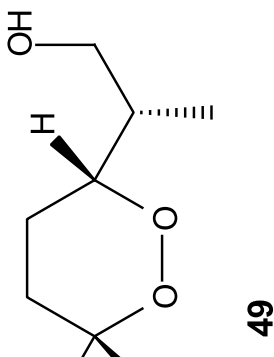

8

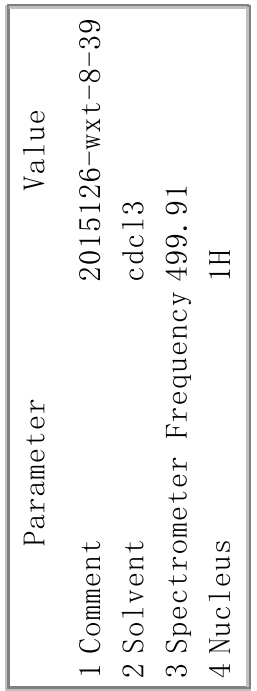


9เルレ9-

9عเレ9-
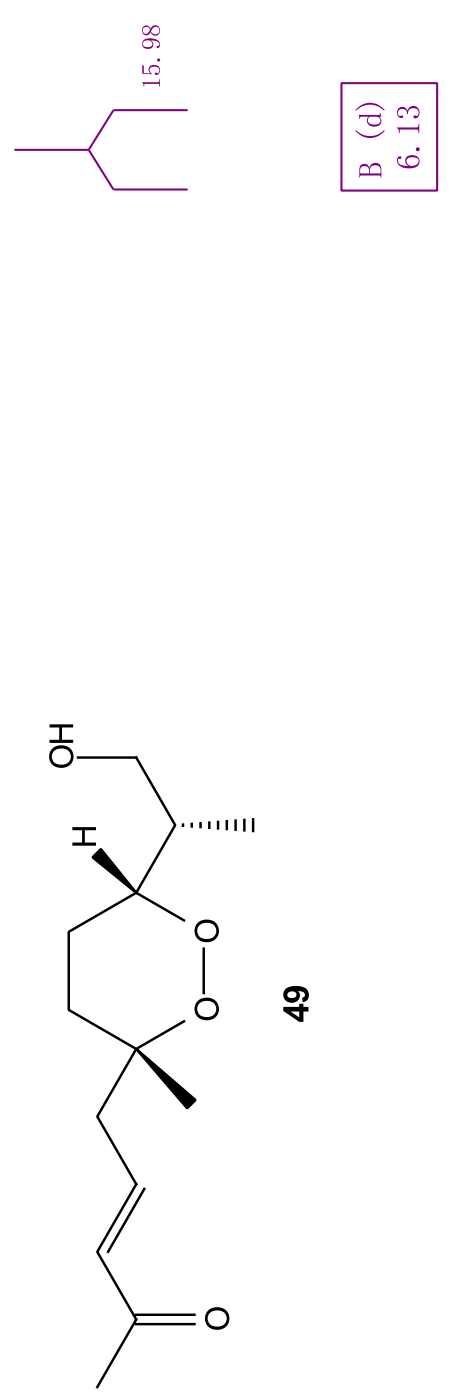

L6SL'9

8LLL'9

$\varepsilon \angle L L 9-1$

668L'9-

8ह08.9

E608.9

แLZ8.9

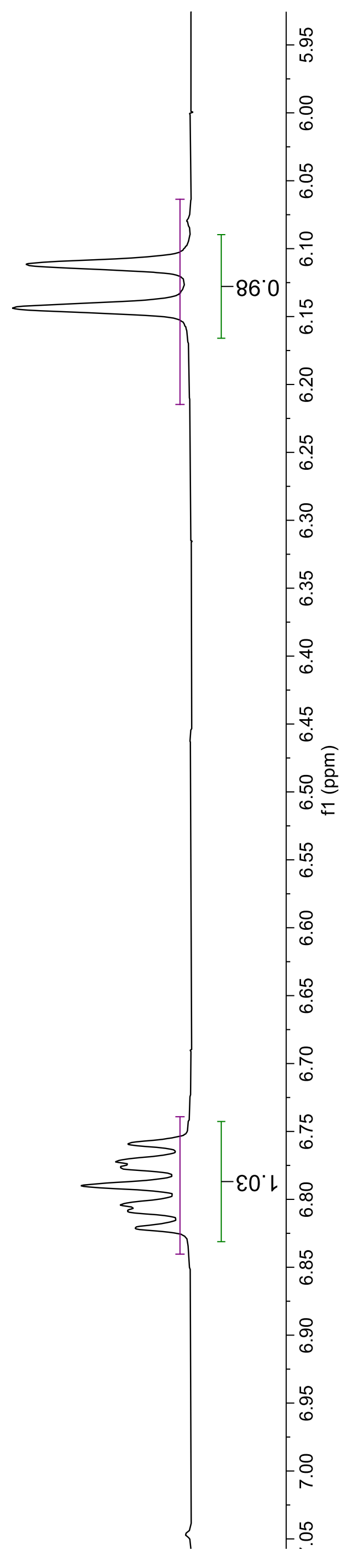


OSLG $\mathcal{C}^{\circ}$

$\nabla 789^{\circ} \varepsilon-$

GL8G $\varepsilon^{\top}$

SL6G' $\varepsilon^{\prime}$

$8809^{\circ} \varepsilon^{-}$

'0Z9. $\varepsilon^{-}$

レ乙ち9

ELS9. $\varepsilon-$

$\angle Z 99^{\circ} \varepsilon-$

$\varepsilon \neg 89^{\circ} \varepsilon-$
乙S $\angle 9^{\circ} \varepsilon-$
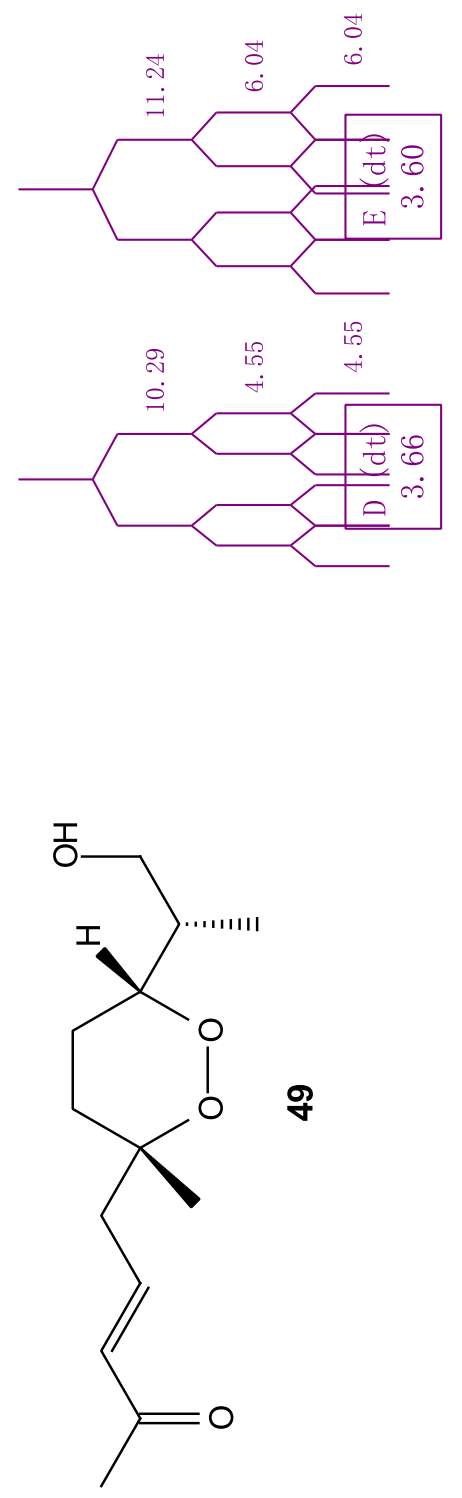

$6 \angle \angle 6^{\circ} \varepsilon>$

$6786^{\circ} \varepsilon-$

$\angle 966^{\circ} \mathcal{E}-$

9200

GELO"

8610

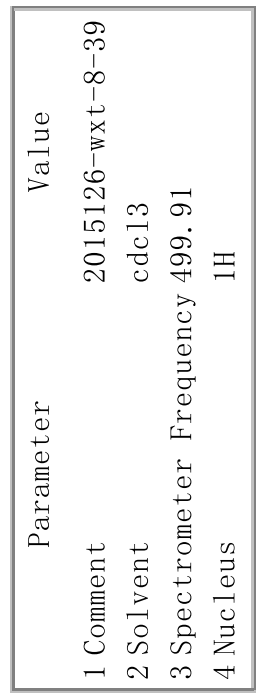

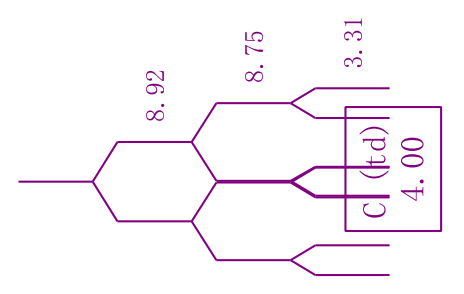

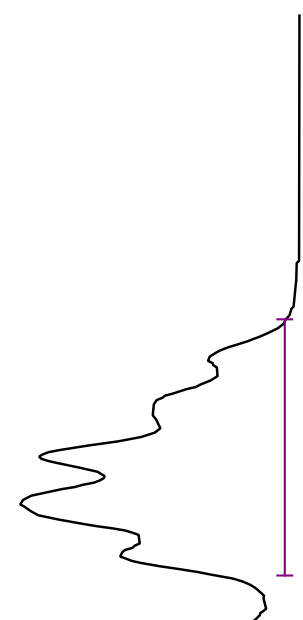


$\angle 8 \angle \varepsilon^{\prime} Z-$

$\angle 96 \varepsilon^{\circ} 乙-$

レレOオてー

0૬てヤてー

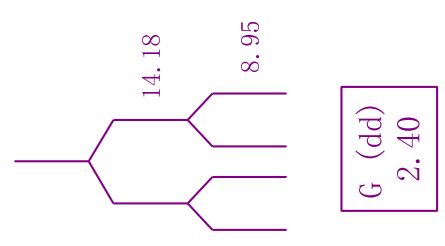

69เ6て

て8Z6 て-

カฤヤ6 てー

8996 こ一
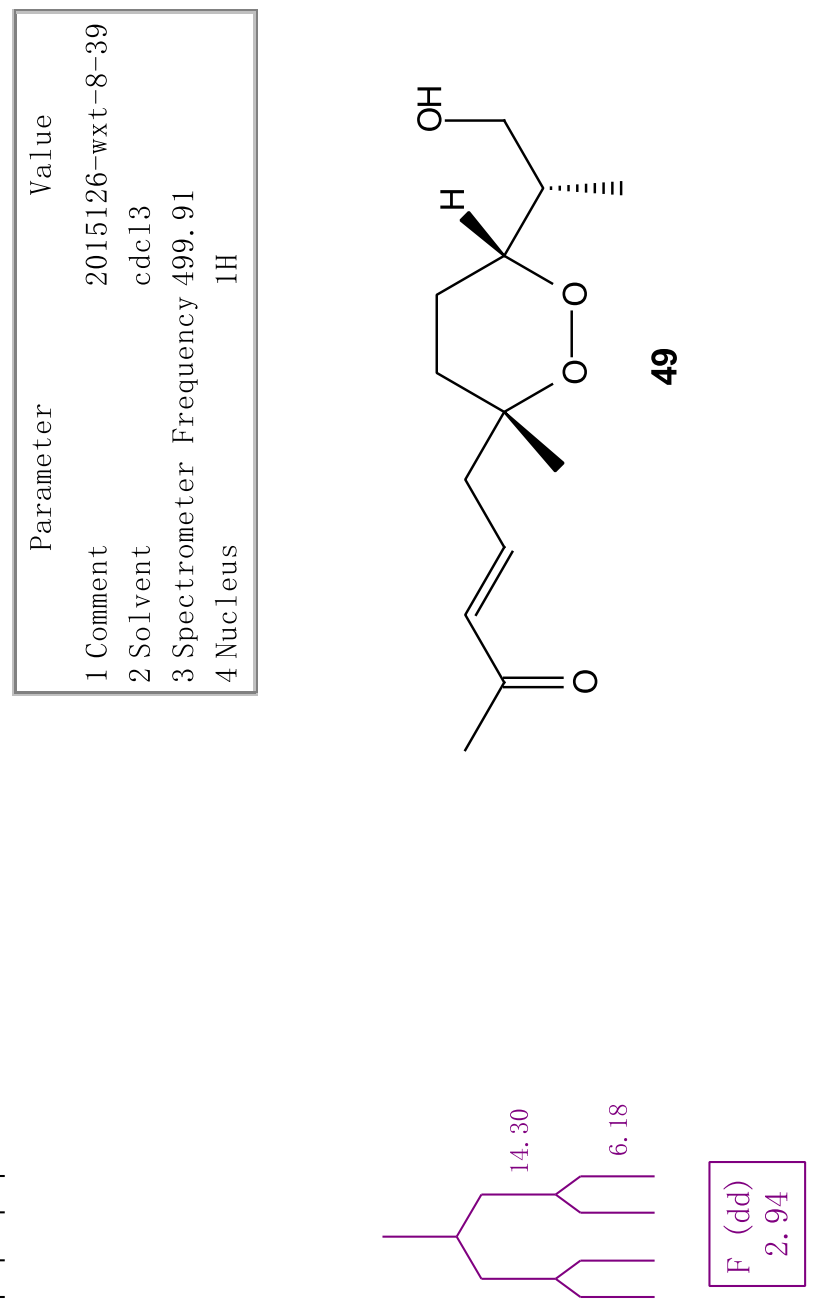

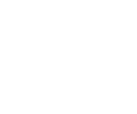<smiles>C=CCC</smiles> 
0 0七6 ${ }^{\circ} 0$

08E6 $0^{\circ}$

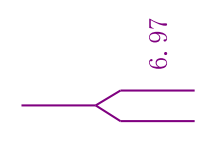

\section{요}

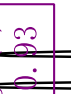

6๖0レレー

(으으

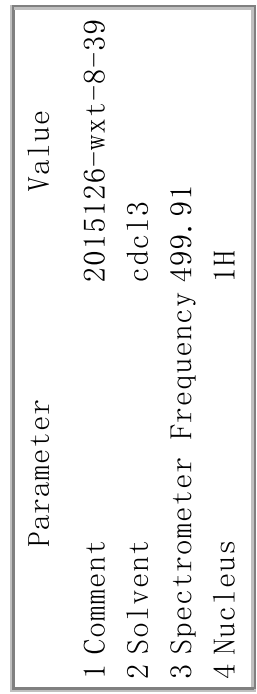

L969'レー

6乙89 ᄂ

$\angle E 69^{\circ}$ L

SE0L'L]

EGLL'L]

L乙EL'L]

$\varepsilon 8 \varepsilon L^{\circ}\llcorner$

86tL L -

แ $9 L^{\circ} L$

†89L'

G8LL'

SZ8L'

LZ6L'L

GL68' ᄂ

$7606^{\circ}$ -

ELZ6" ᄂ
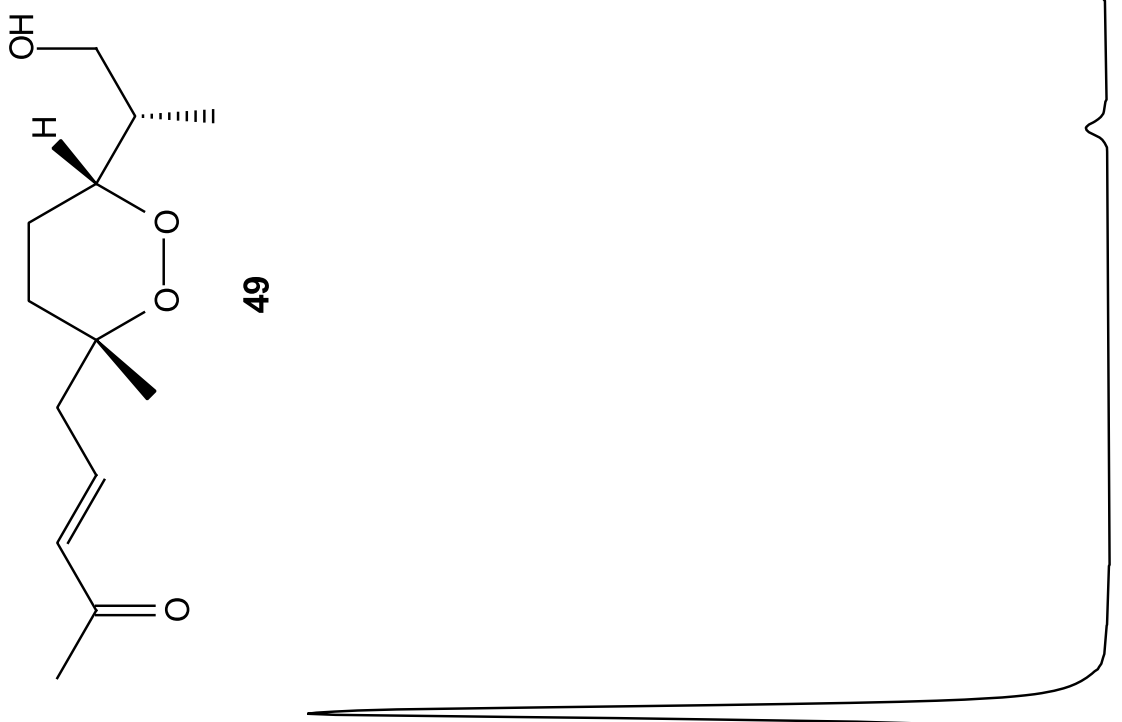

을

舟

움
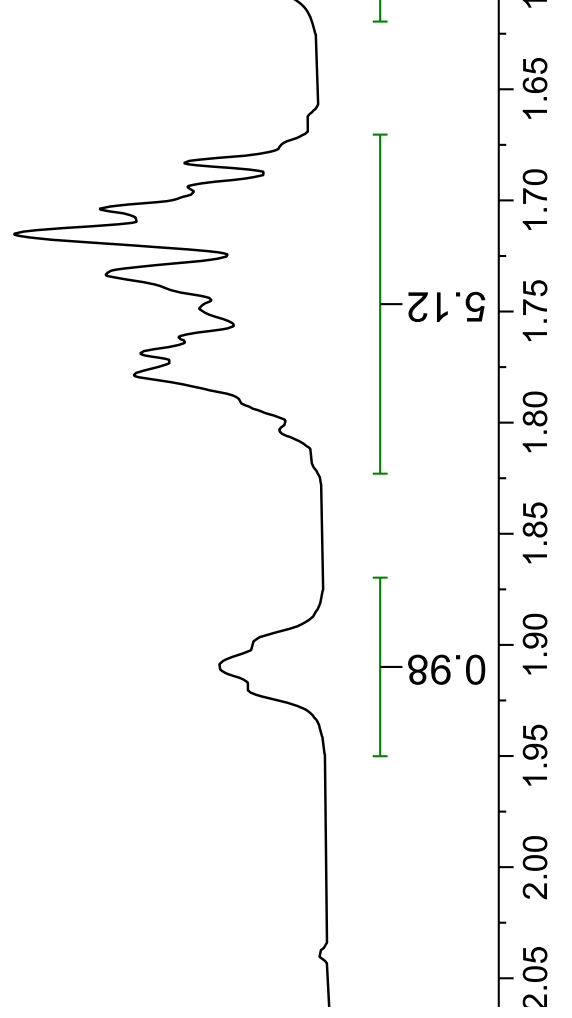
6Lててレー

$\angle 8^{\circ} \varepsilon Z$
$9 t^{\circ} \downarrow Z^{J}$

$28.9 z^{\top}$

$\succ 8 \cdot 2 \varepsilon-$

$\nabla 8^{\circ} \angle \varepsilon \sim$

$6 L^{\circ} 6 \varepsilon^{-1}$

†0'99-

E9 $6 L-$

$6 \nearrow^{\circ} \triangleright 8-$
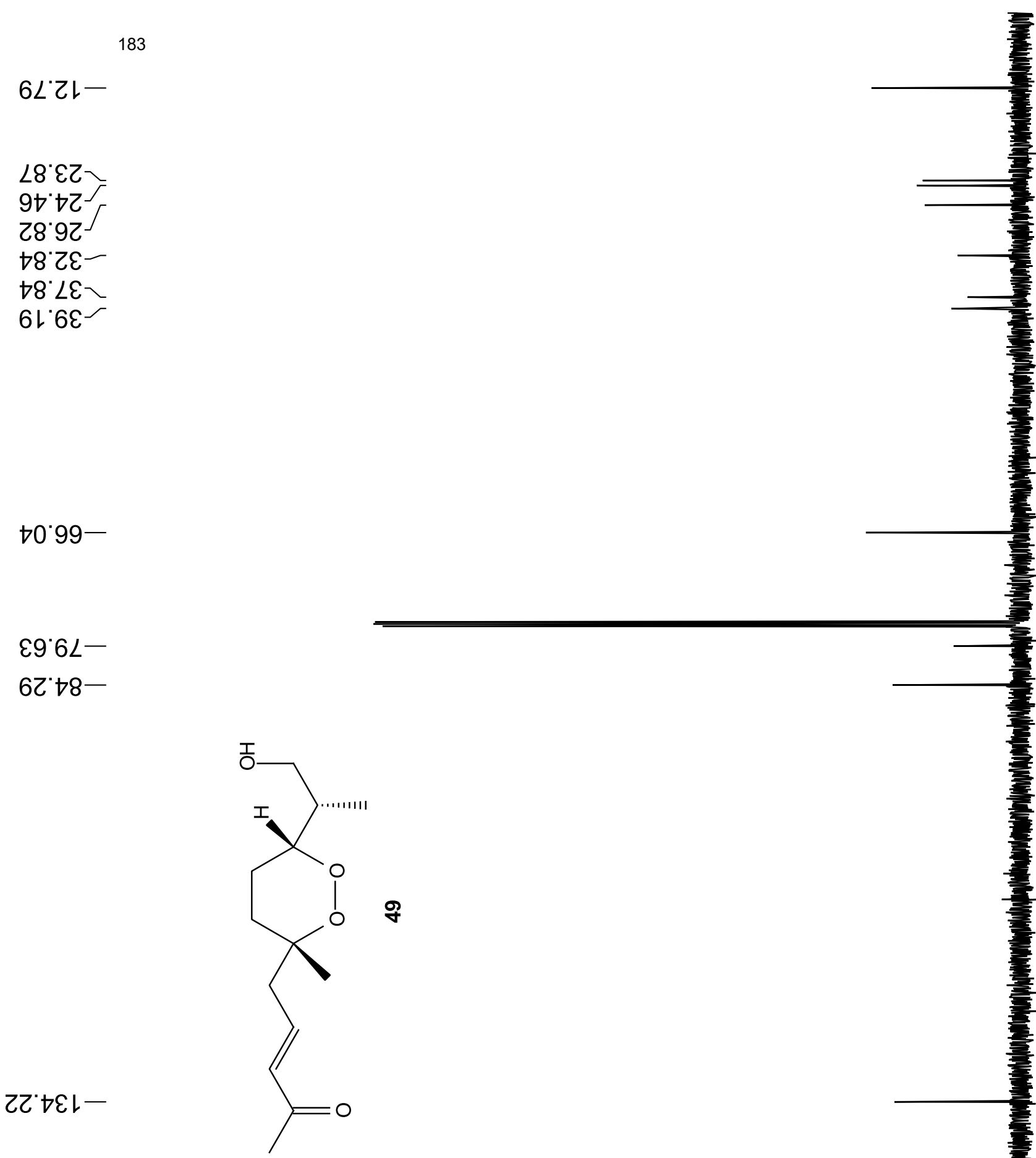

くでとヤレー

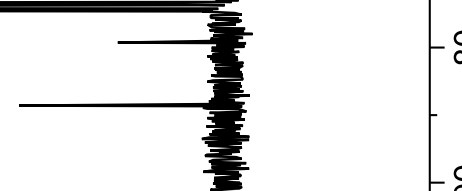

-8

음

हิ

은

$-\stackrel{ }{\sim}$

옴

옴

- 움

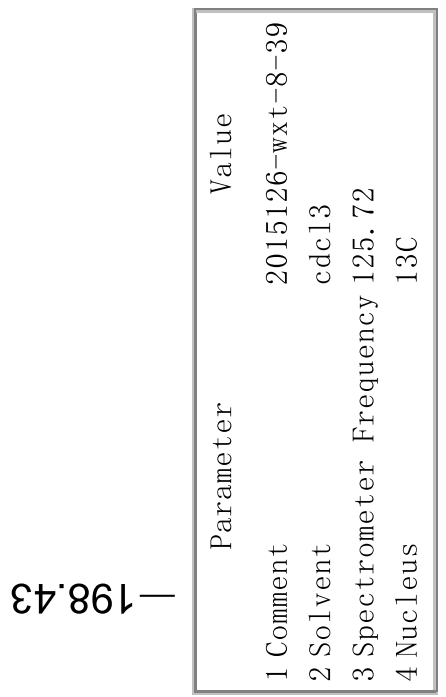




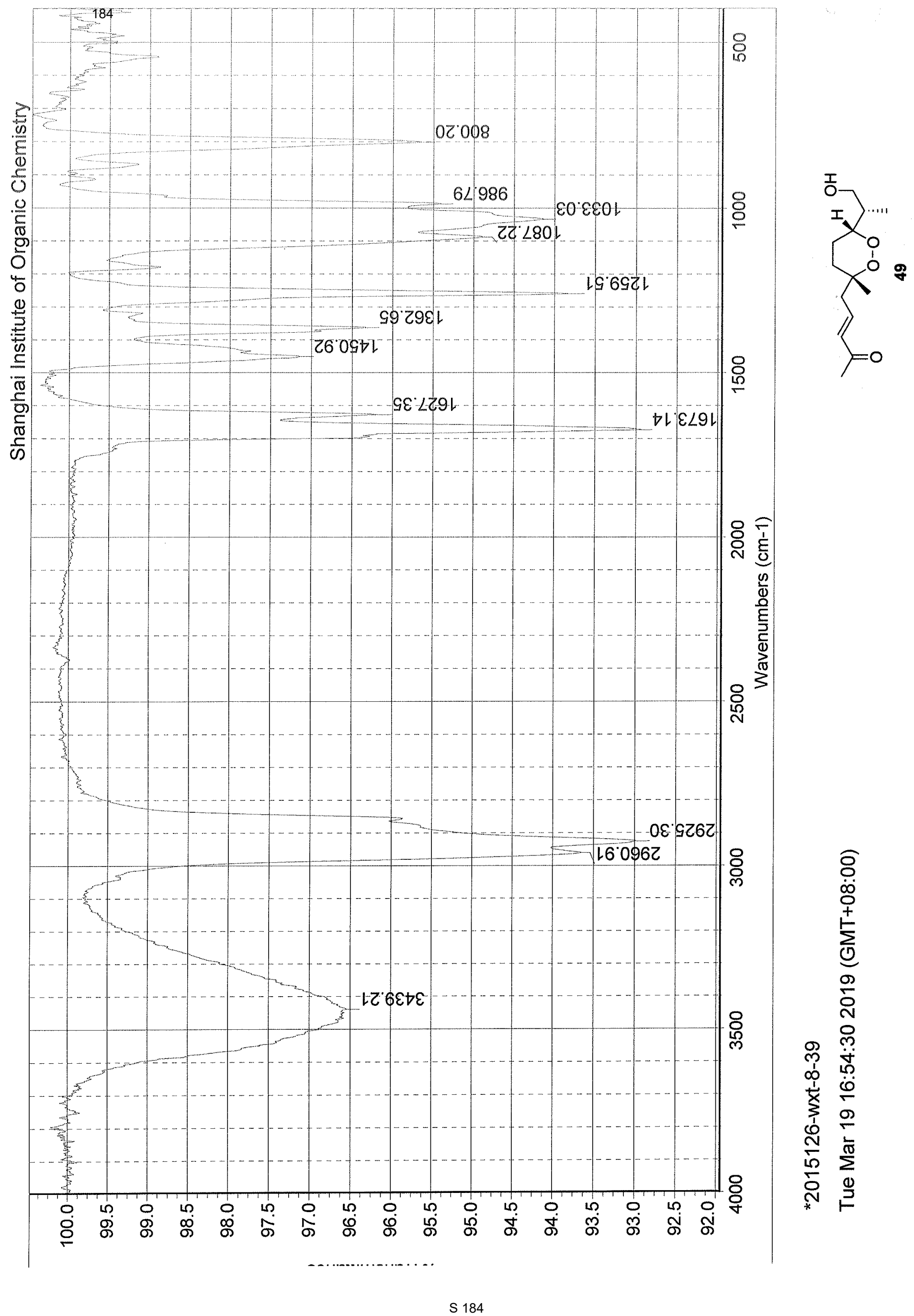



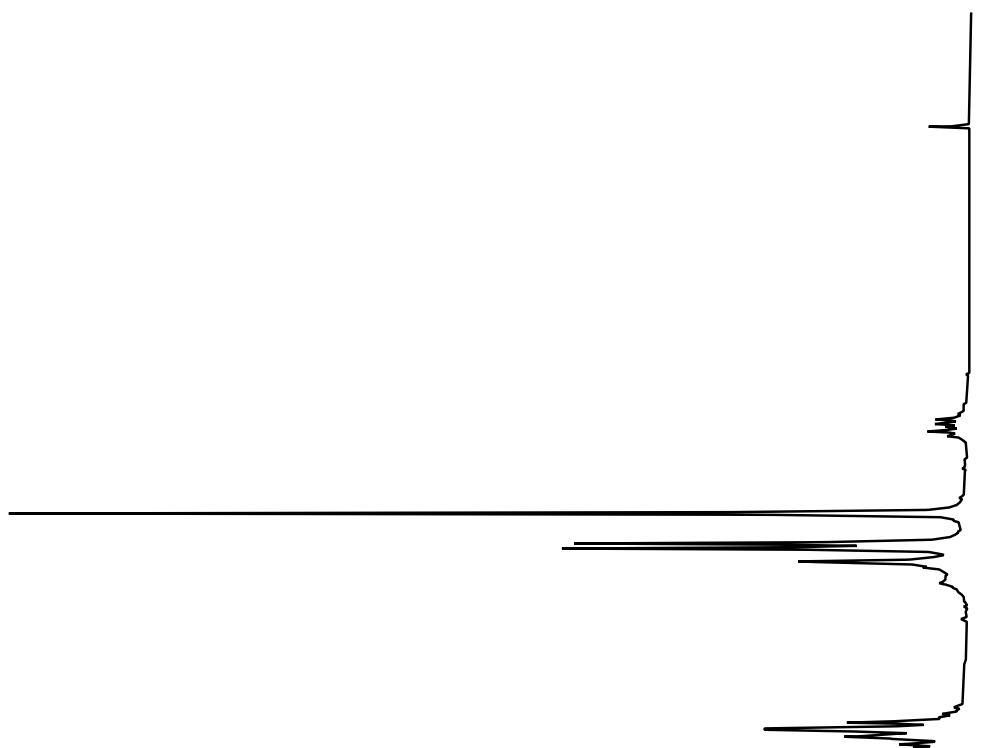

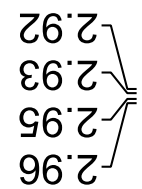
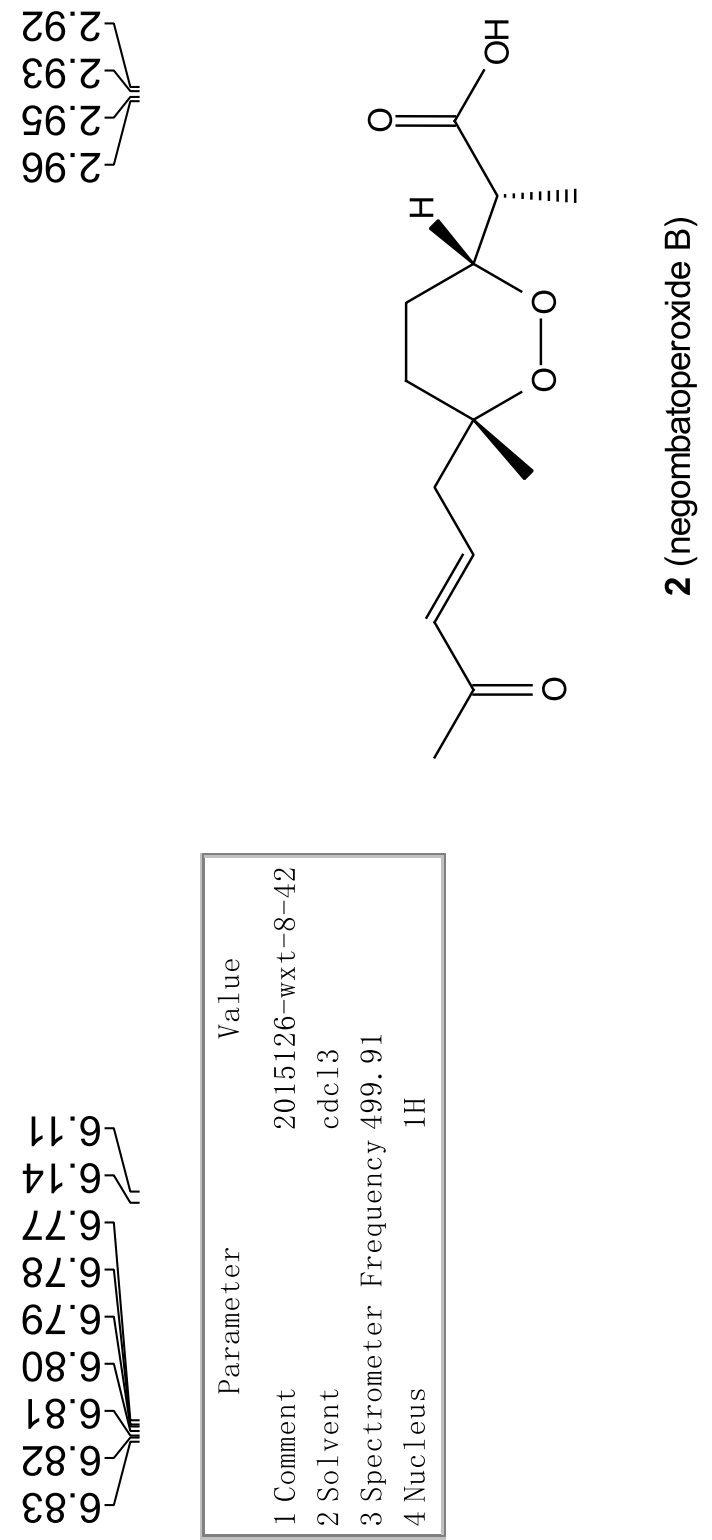

$9 Z^{\circ} L$

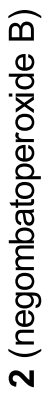

Fル・

$\stackrel{\circ}{\mathrm{i}}$ $88^{\circ} \mathrm{Z}$

К $61 . \mathrm{L}$ $\mathrm{F} 86^{\circ} 0^{\circ} \mathrm{N}$

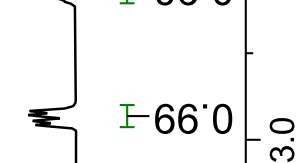

F $70^{\circ} \mathrm{L}$

?

$\widehat{\overline{\mathrm{g}}} \mathrm{a}$

$\circ$

F $20^{\circ}$

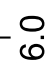
10

F $90^{\circ} \mathrm{L}$ 
0LOL'9-

ع6عเ'9-

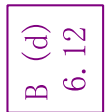

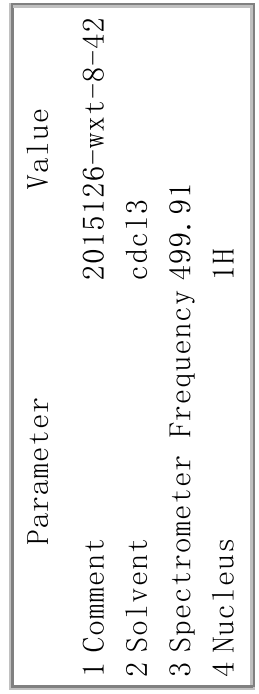

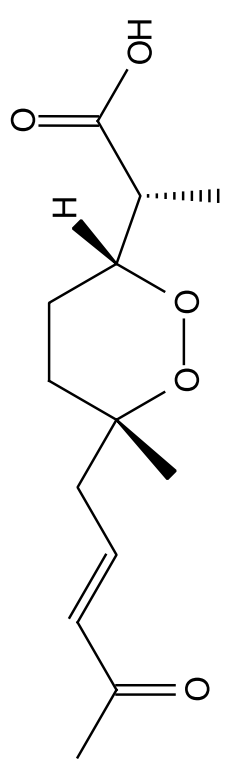

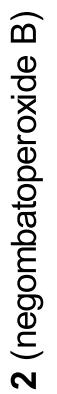

$\nabla \angle 9 \angle \cdot 9$

96LL'9]

$\checkmark G 8 L^{\prime} 9$

†86L'9-

ㄴ8ㅇ

ELL8'9

†628 9

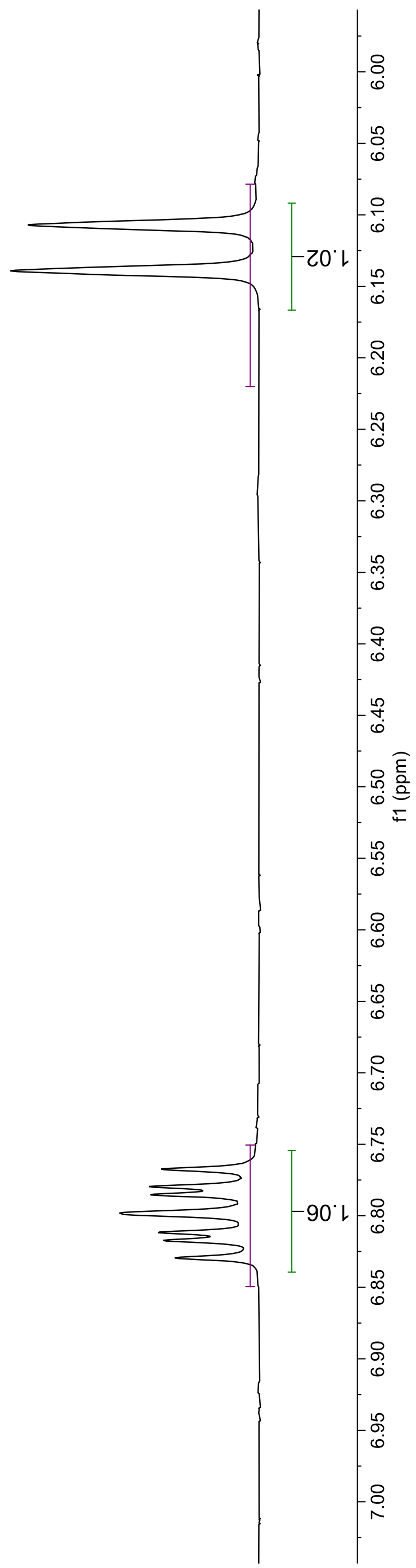




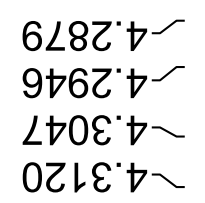

ง6てย๋ャー

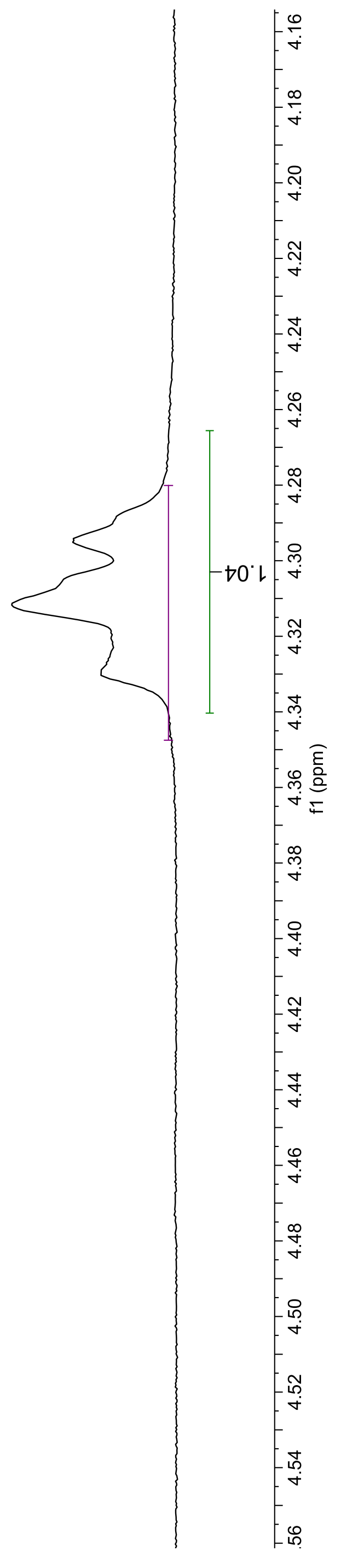

$\widehat{0}$
0
$\frac{1}{7}$
0
$\frac{0}{0}$
$\frac{0}{0}$
$\frac{0}{0}$
$\frac{0}{6}$
0
0
0
N 
SL'ZL-

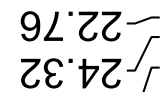

$$
\begin{aligned}
& 9 L \cdot 9 Z^{\prime} \\
& \text { } 7{ }^{\circ} z \varepsilon^{\top}
\end{aligned}
$$

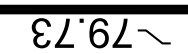

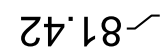

$\varepsilon \varepsilon^{*} \succsim \varepsilon\llcorner-$

ธ๕`๕ฑレ-
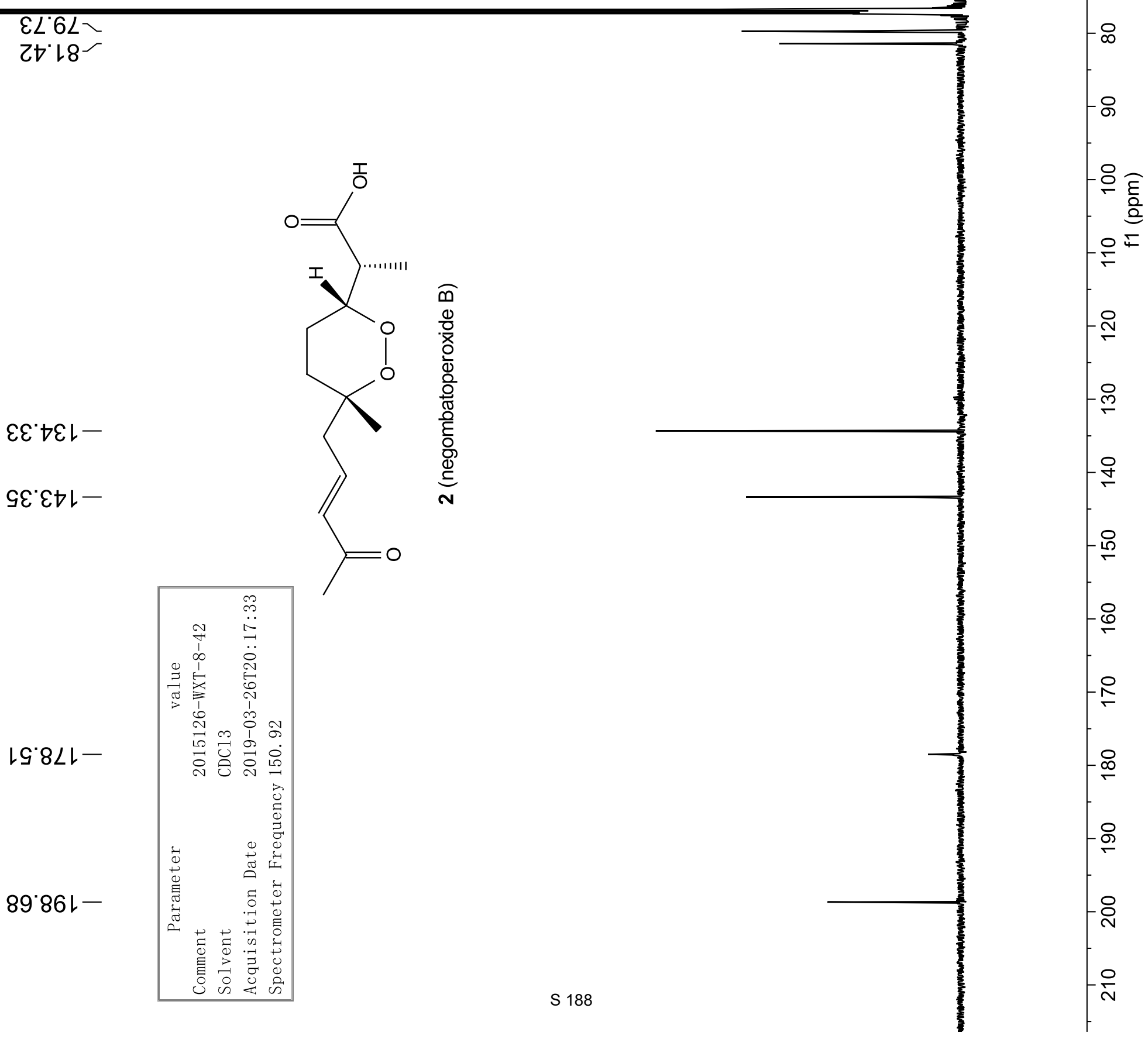


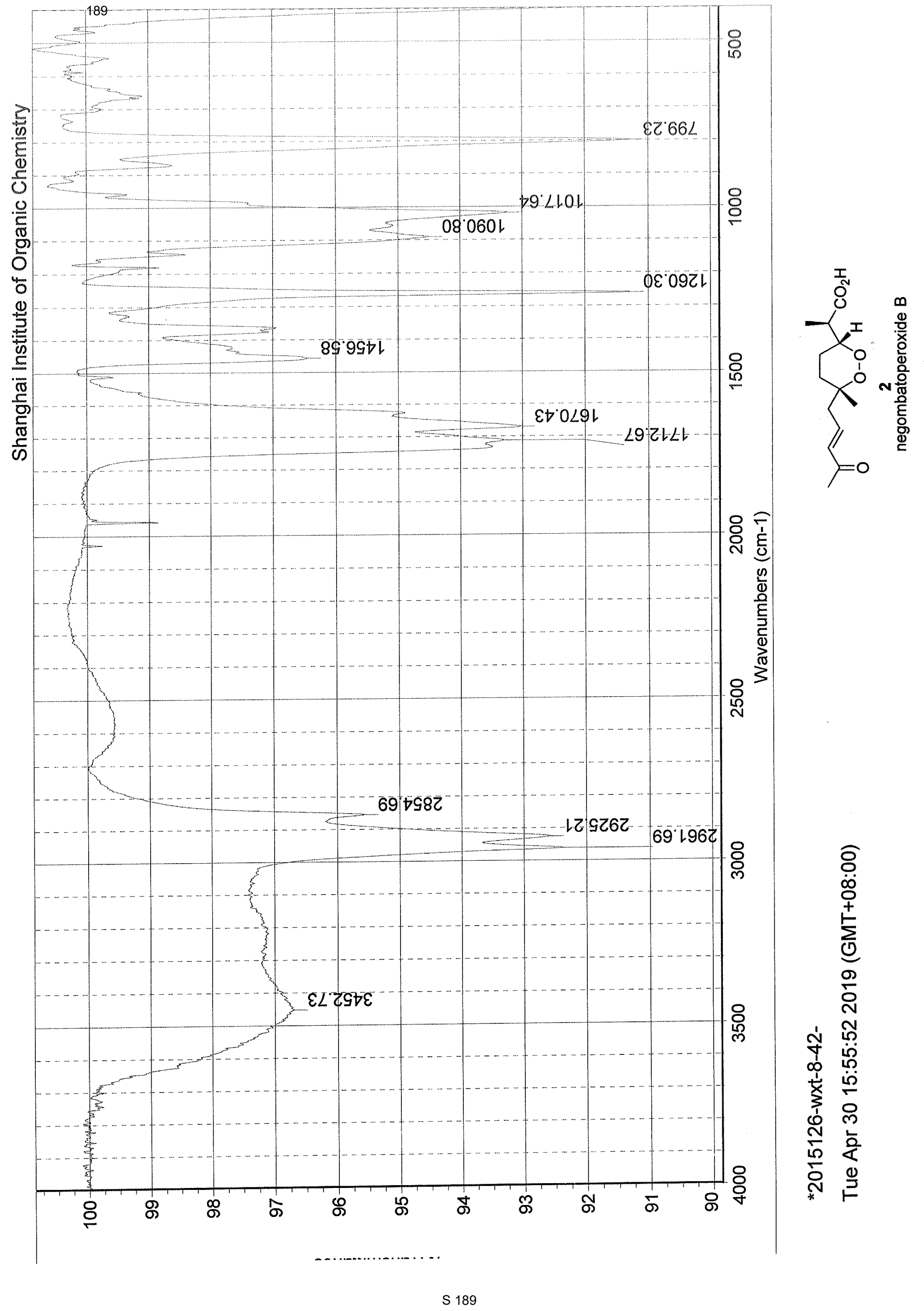


$\varepsilon 6^{\circ} 0$

$96.0-$

90.1

$90^{\circ} \mathrm{L}$

$8 \mathrm{C}^{\circ} \mathrm{I}$

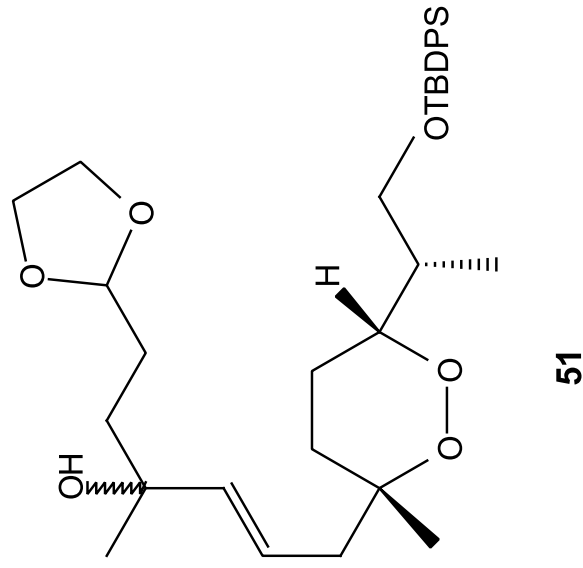

$99^{\circ} \mathrm{L}$

S9. $L$

$99^{\circ}<\sqrt{ }$

$99^{\circ} \angle J$

99. $L$

$99^{\circ} \mathrm{L}$

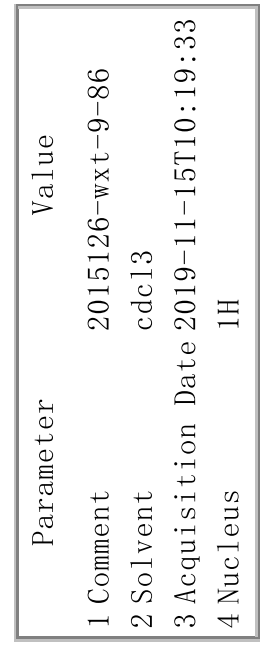

in
드 $00^{\circ} \&$ 웅

瓦L!

$\varepsilon 8$ है

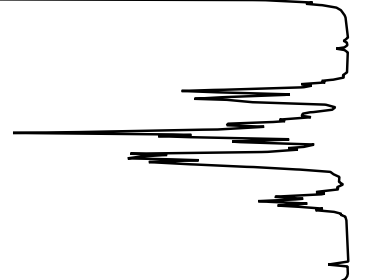

다 $\varepsilon 8$

L $89 \%$

त $\nabla Z^{\prime}$

$\angle 6.0 \stackrel{\circ}{\circ}$

$\Longrightarrow$

I 62द只

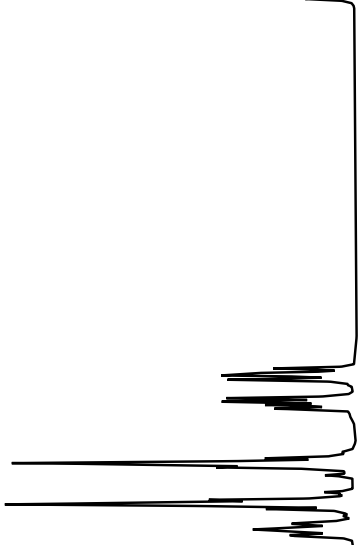

-

$12 b c$

厂片る

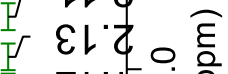

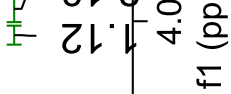

$\stackrel{4}{+\infty}$

웅

$\frac{\widehat{\varepsilon}}{\text { 을 }}$

$\stackrel{+}{+\infty}$

I $66^{\circ}$

잉

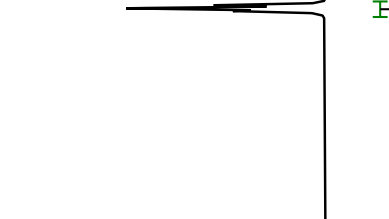

$=E \varepsilon \tau^{-\infty}$

10

움

I $\varepsilon 0^{\circ} \mathrm{g}$

$=t 0 . t$ 
6998 $\angle$

$609 \varepsilon \angle$

$\varepsilon Z \angle \varepsilon^{\circ} L \backslash$

$998 \varepsilon^{\circ} L$

$000 t^{\circ} \angle$

$9 \varepsilon 00^{\circ} L$

680t' L

0เレ๋ L

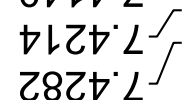
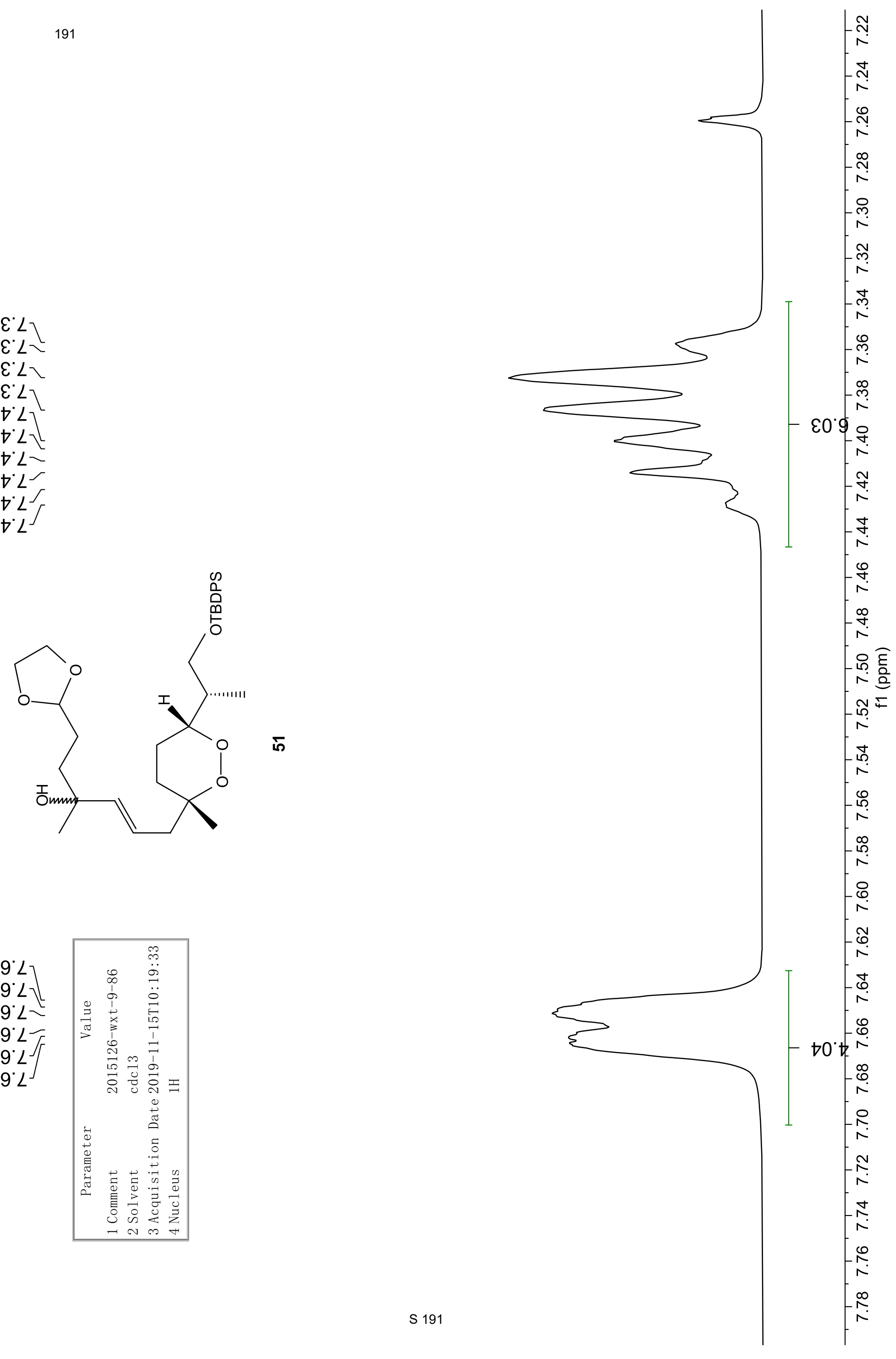
乙2 $\angle 9^{\circ} \varepsilon-$ $\angle 889^{\circ} \varepsilon-$ 1969' $\varepsilon$ $\angle 809^{\circ} \varepsilon-$

แ.99 $\varepsilon-$ SL $\angle 9^{\circ} \varepsilon-$ $0189^{\circ} \varepsilon-$ \&เ69' -
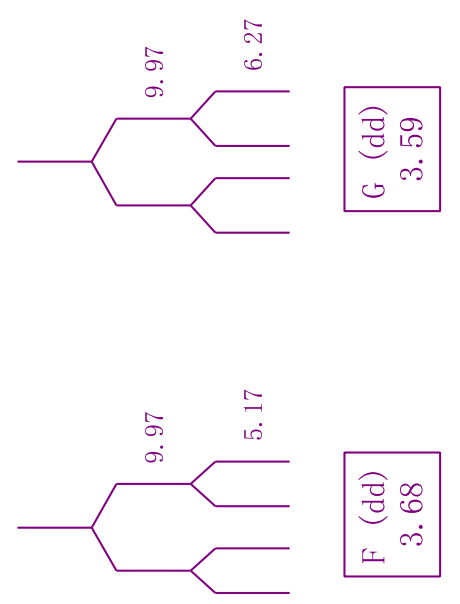

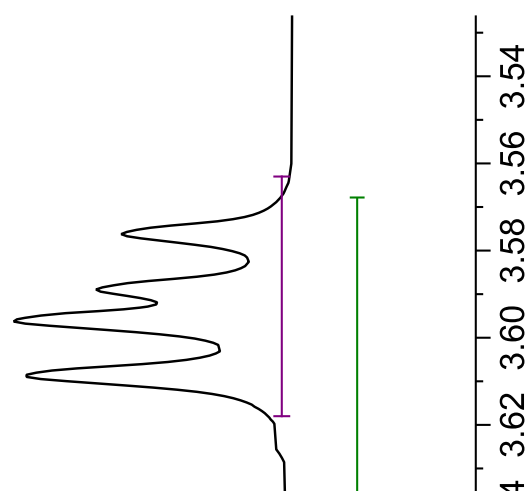

$\angle \forall$ द⿱

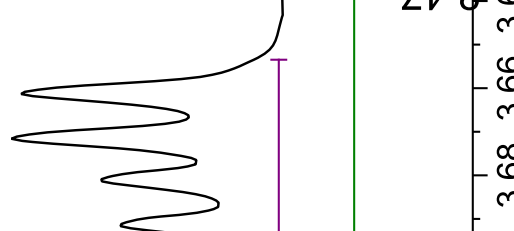

0
0
5
0

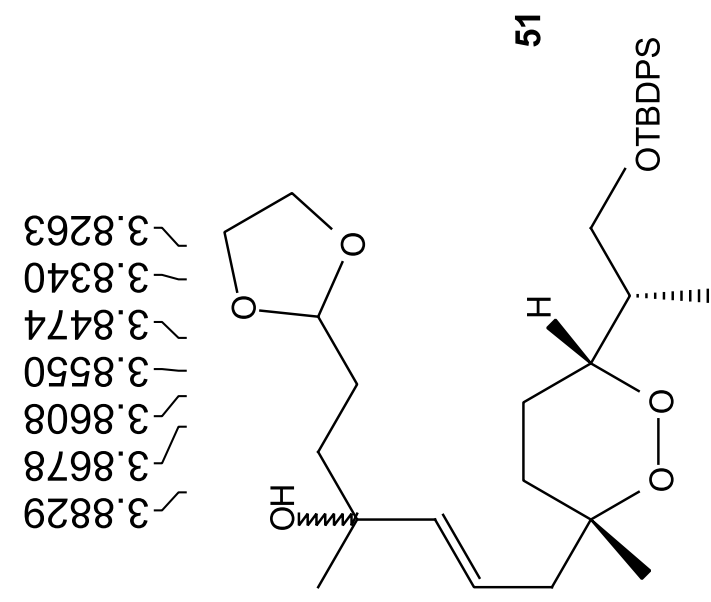

$\angle 0 \varepsilon 6^{\circ} \varepsilon \sim$

${ }^{\circ} 9 t 6^{\circ} \varepsilon \backslash$

$6296^{\circ} \varepsilon-$

$\varepsilon 696^{\circ} \varepsilon-$ $0996{ }^{\circ} \varepsilon^{\prime}$

$86 \angle 6^{\circ} \varepsilon^{-}$

$9<10^{\circ} \nabla$ ๑ZZ० $6 L E 0 \circ>$ 08E0 $\downarrow-$ LEt0 ${ }^{\circ}$ $0890^{\circ} \mathrm{t}$
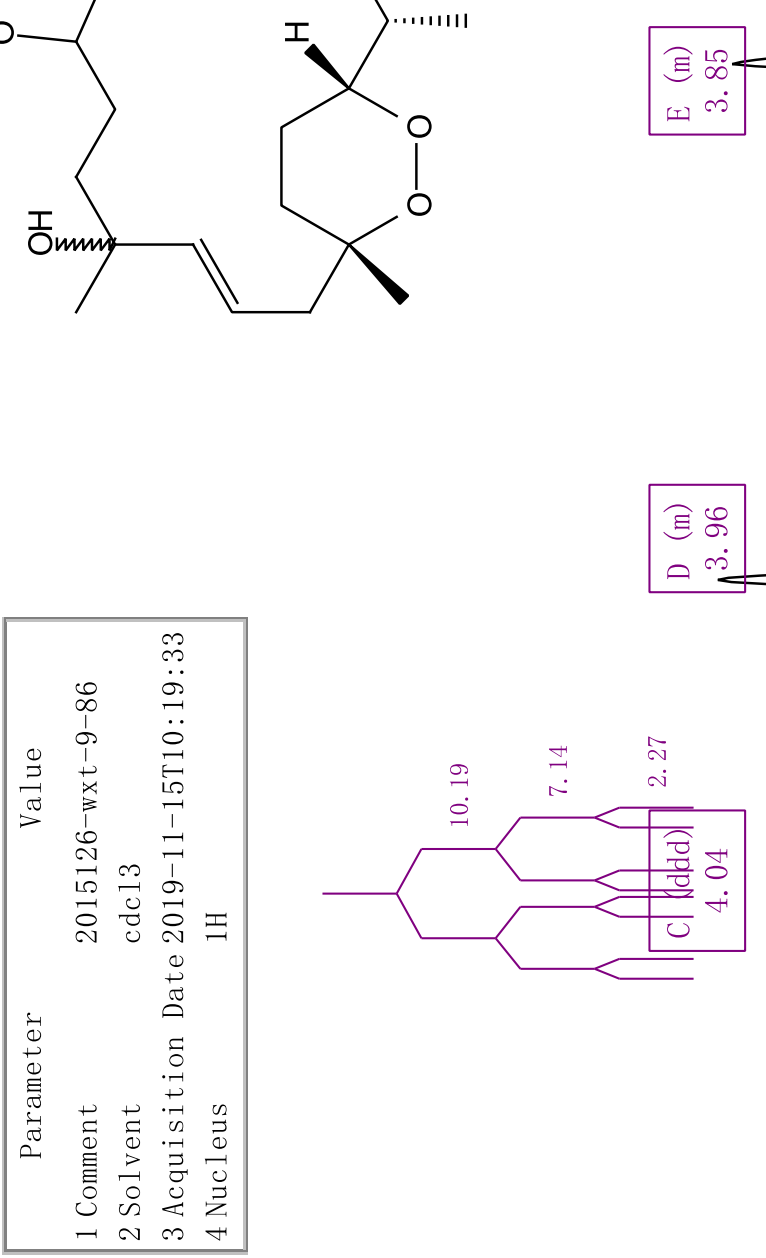

ह

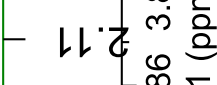
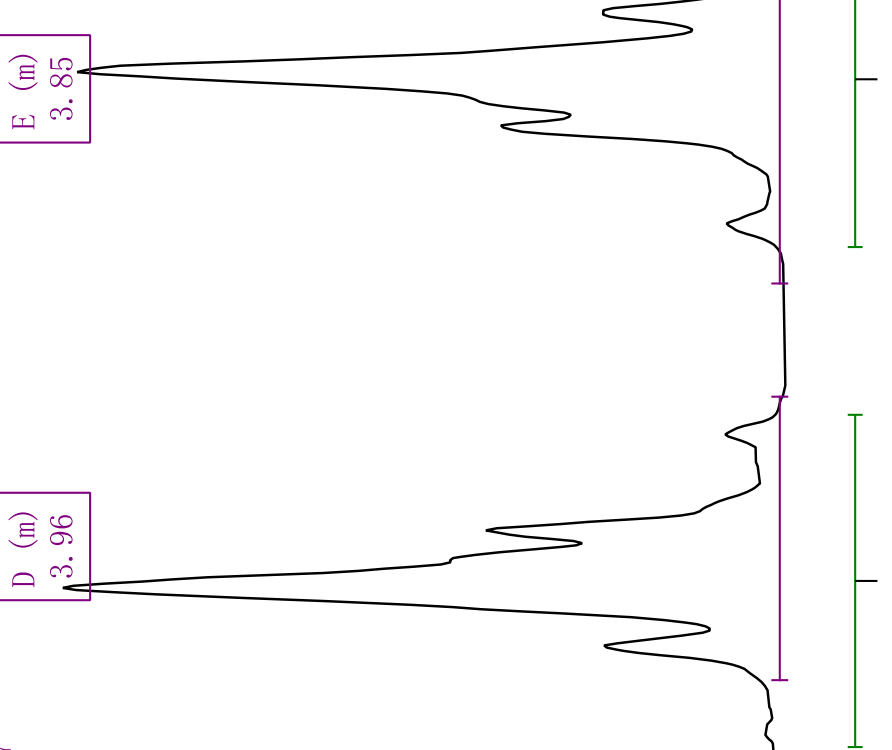

$\varepsilon \downarrow$ टे

$\dot{3}$

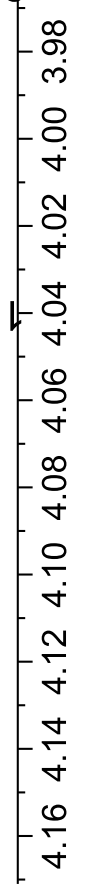


$\neg \downarrow \varepsilon 6^{\circ} 0$

乙8๑6 $0^{\circ}$
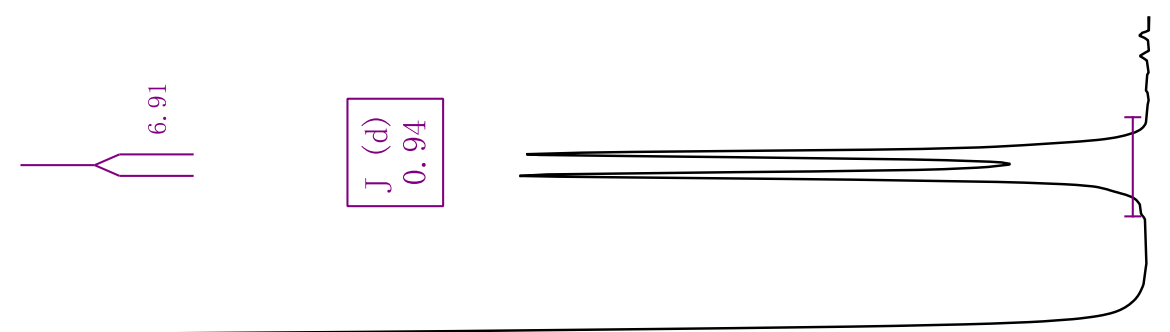

$\frac{1990^{\circ} 12}{9190^{\circ}-1}$

Z6LZ'

19tG'

EOSG' L

$\varepsilon \nabla 9 S^{\circ}:$

$\varepsilon 0 \angle S^{\circ}$.

$88 \angle 9^{\circ}$

E629.

†0t9

$19+9^{\circ}$

¿\&s9.

†099.

SL99.

$\angle L \angle 9^{\circ}$

$\angle Z 69^{\circ}$

692L'L

S†EL $L$

$\left.80 t L^{\circ} \cdot\right]$

OLSLL

L $\angle S L L-$

9Z9L' $\mathrm{L}$

$69 \varepsilon 8^{\circ} \mathrm{L}$

SOS8 L -

0E98 L

$19 \angle 8$.

$1688^{\circ}$
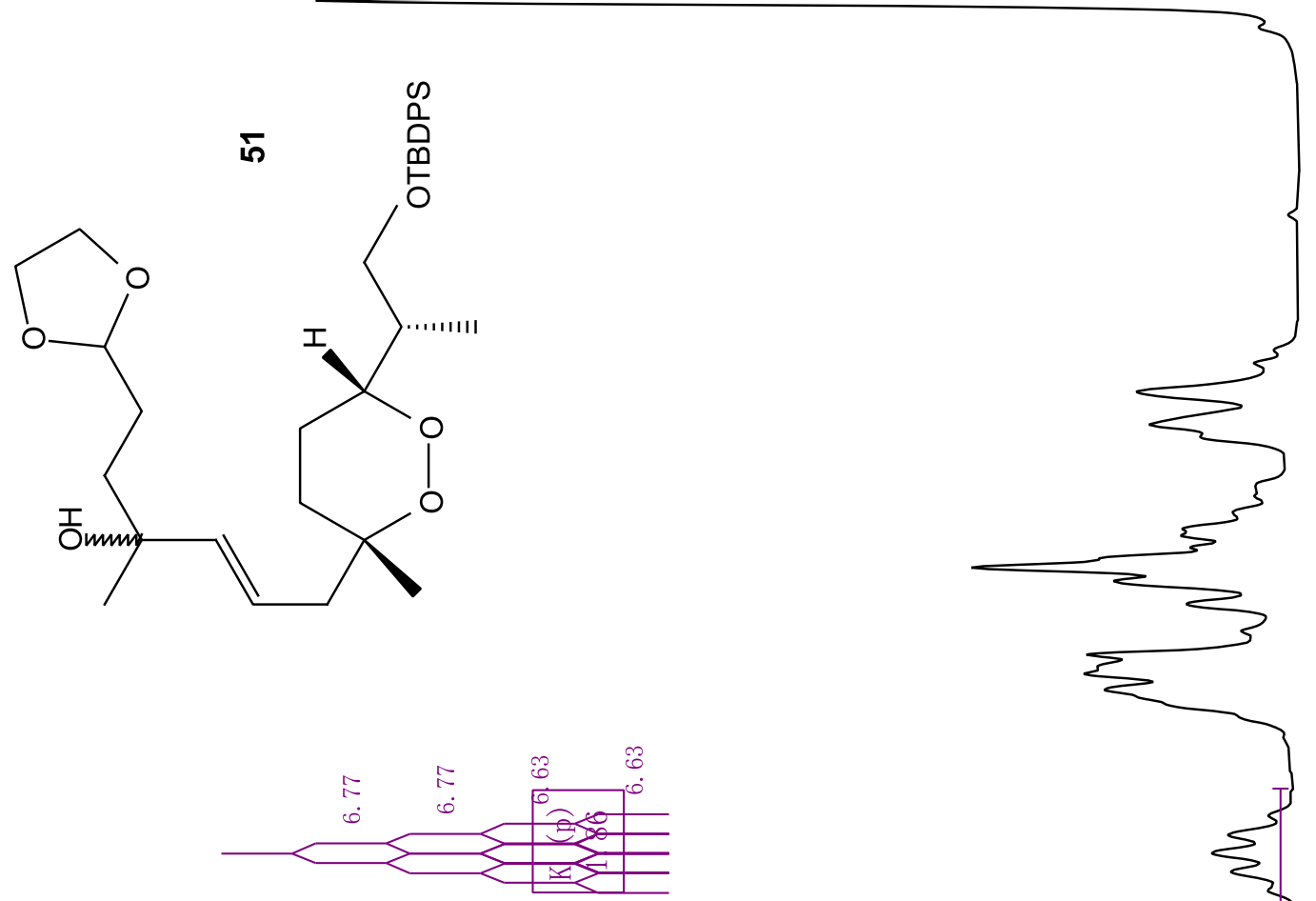

$F \varepsilon 8$

$-\varepsilon L \cdot$

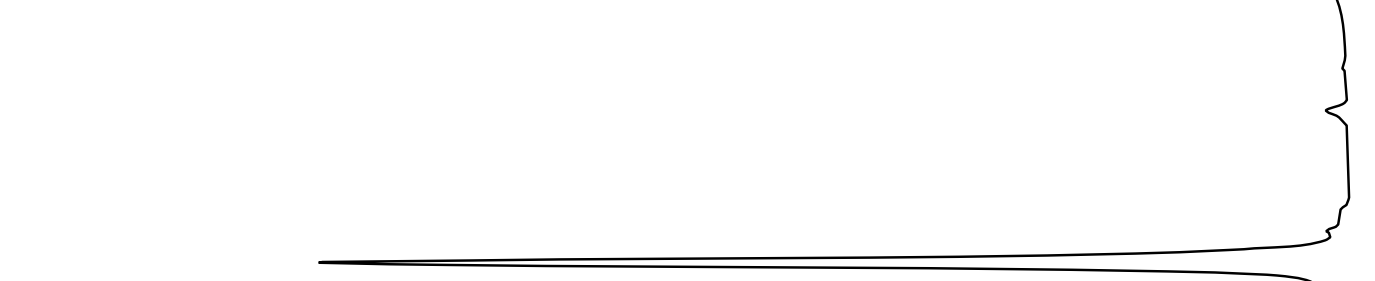

F $00 \%$

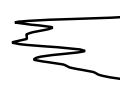

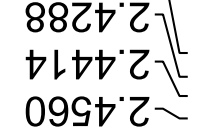
ELLV'乙G68t乙 GS09'乙 $\angle 9 L \mathcal{L}^{2} \mathrm{C}$

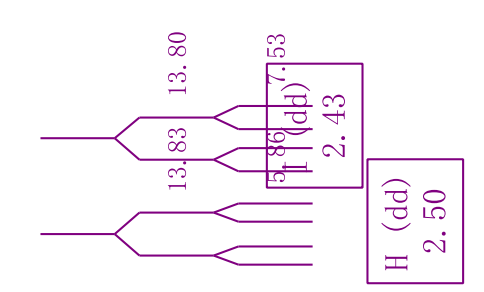




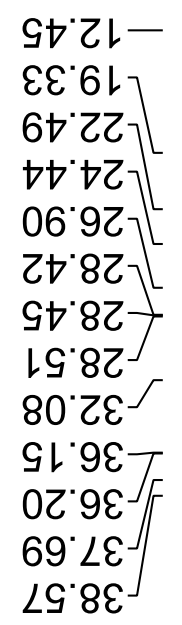

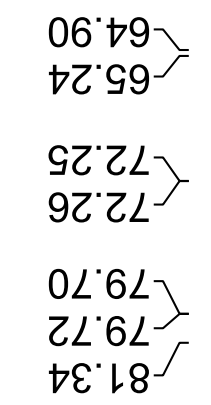

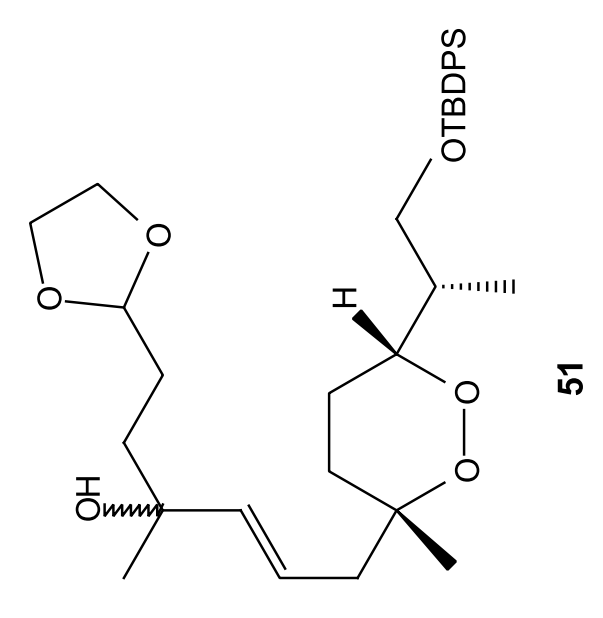

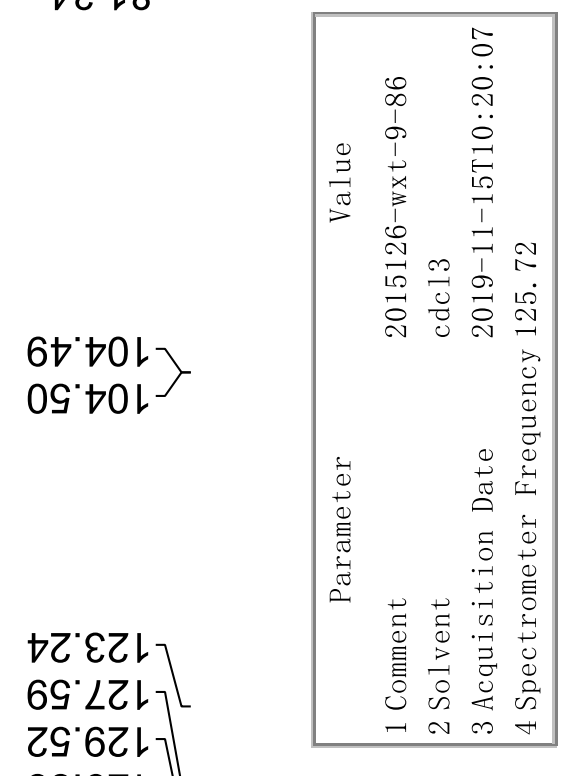
E9.6ZL】 $\left.\begin{array}{l}G L^{*} \varepsilon \varepsilon L \\ \varepsilon 8^{\circ} \varepsilon \varepsilon L\end{array}\right]$ 09.GEᄂ T Z9"GEL

60.0ㄴ-

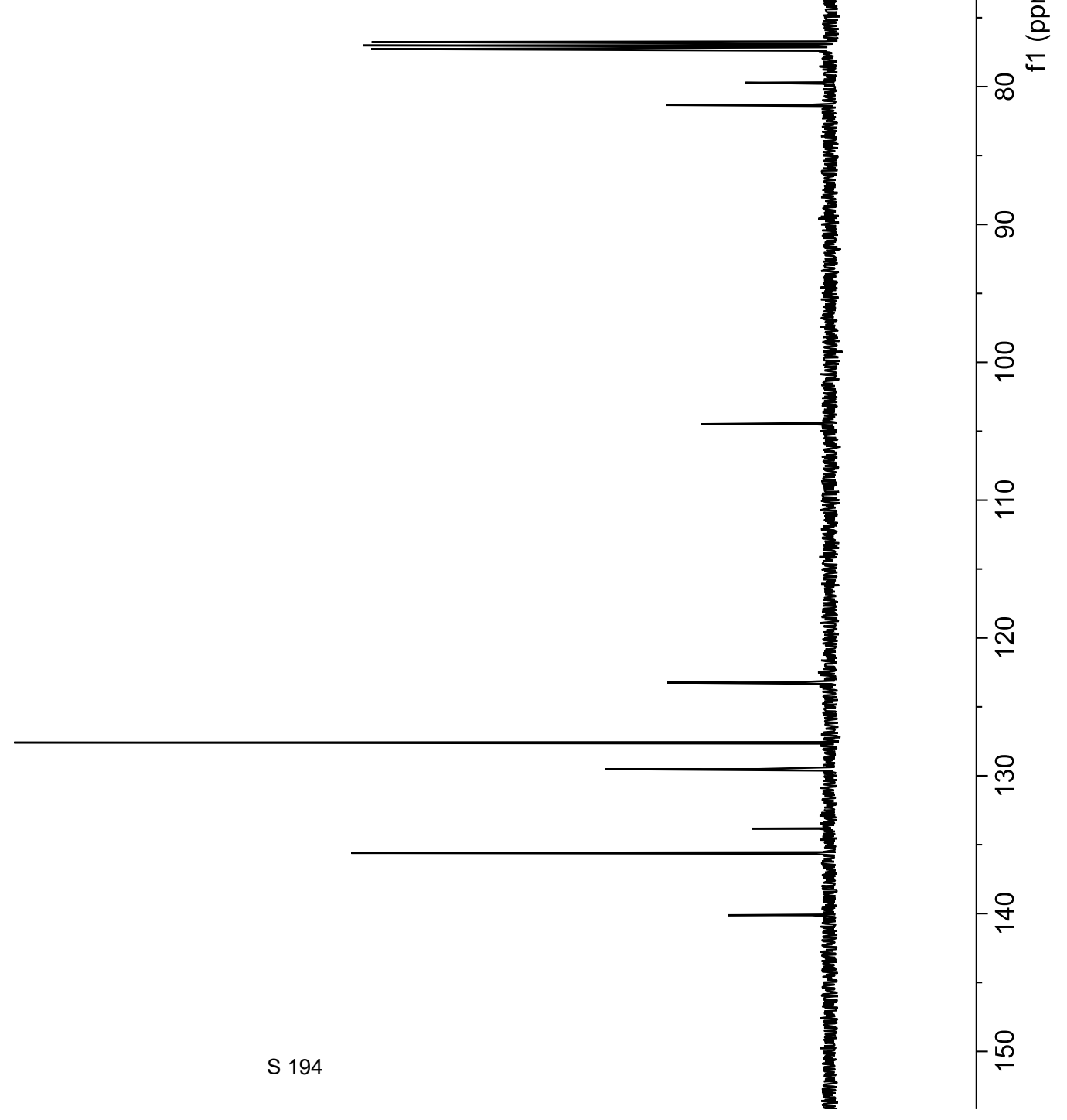




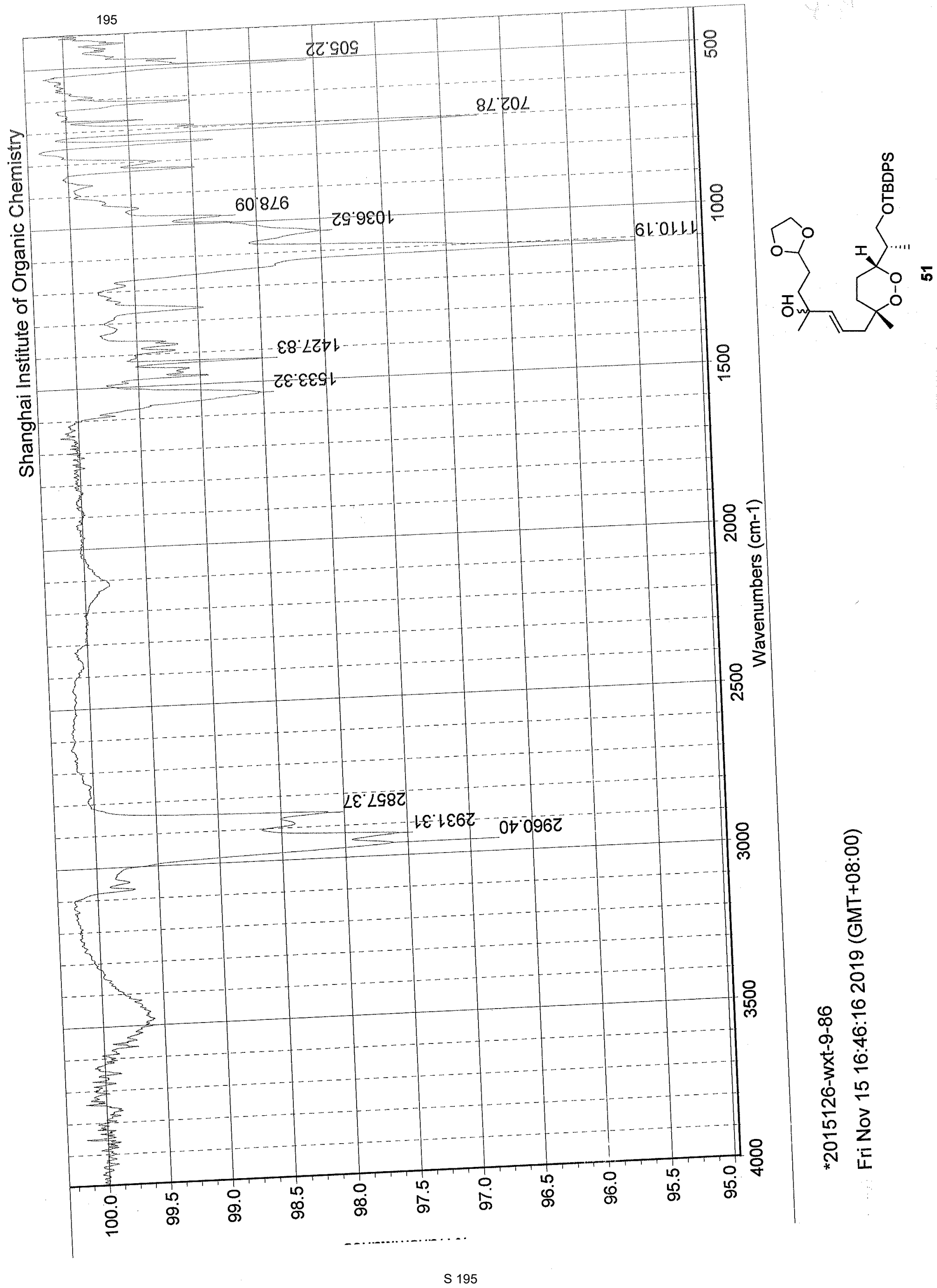


$\rightarrow 6^{\circ} 0$

96.0

90.1]

$0 S^{\circ}$

$0 S^{\circ} \downarrow$

$89^{\circ} \varepsilon$

$69^{\circ} \varepsilon$

$09^{\circ} \varepsilon$

$19^{\circ} \varepsilon$

$99^{\circ} \varepsilon-$

$\angle 9^{\circ} \varepsilon$

$89^{\circ} \varepsilon$

$69^{\circ} \varepsilon$
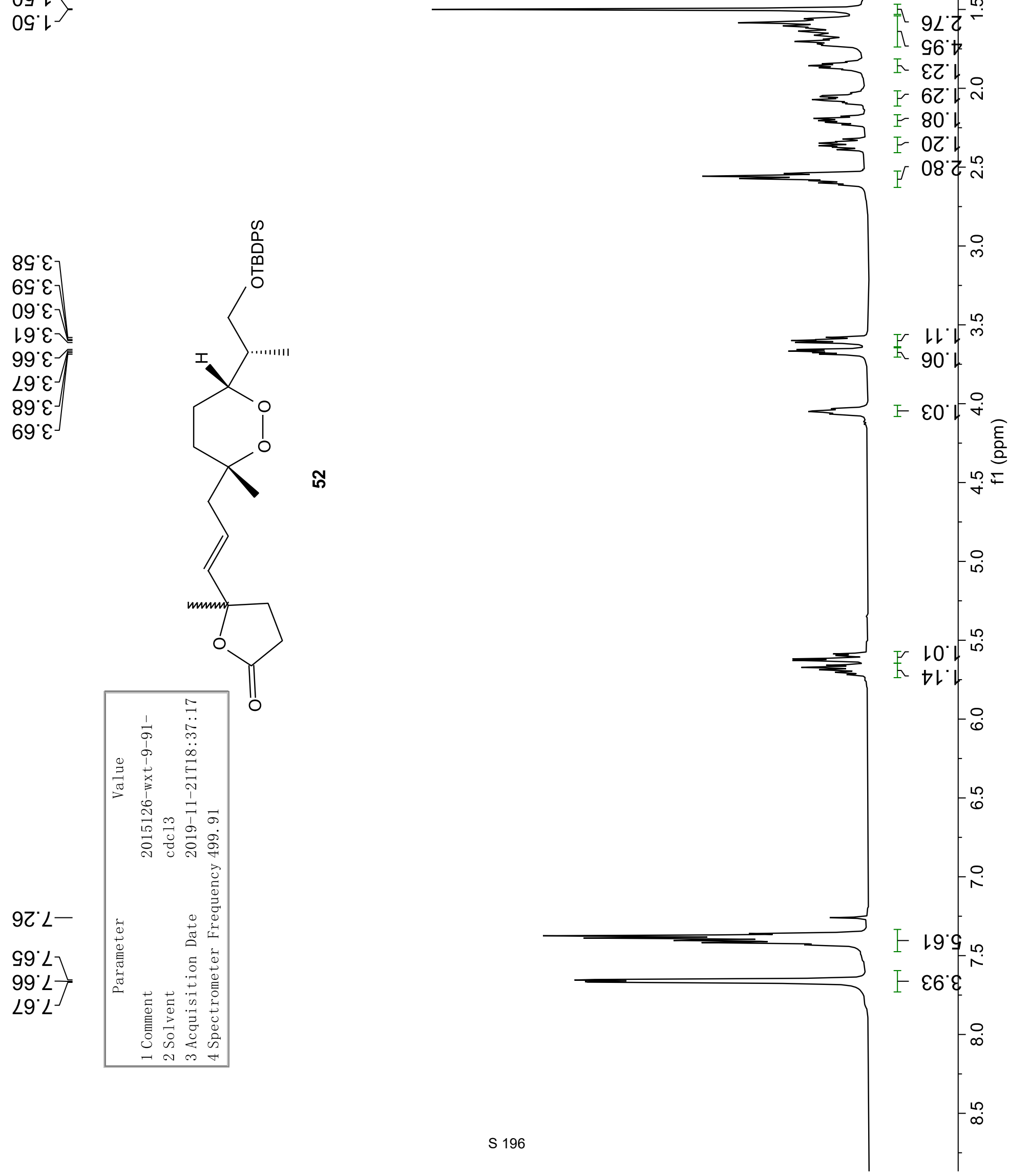
†989' 9 6969 9 9L19'9 2829'9 -

Z899.9 ZZL9'9 \&L89' 9 SEOL'G 08L'G
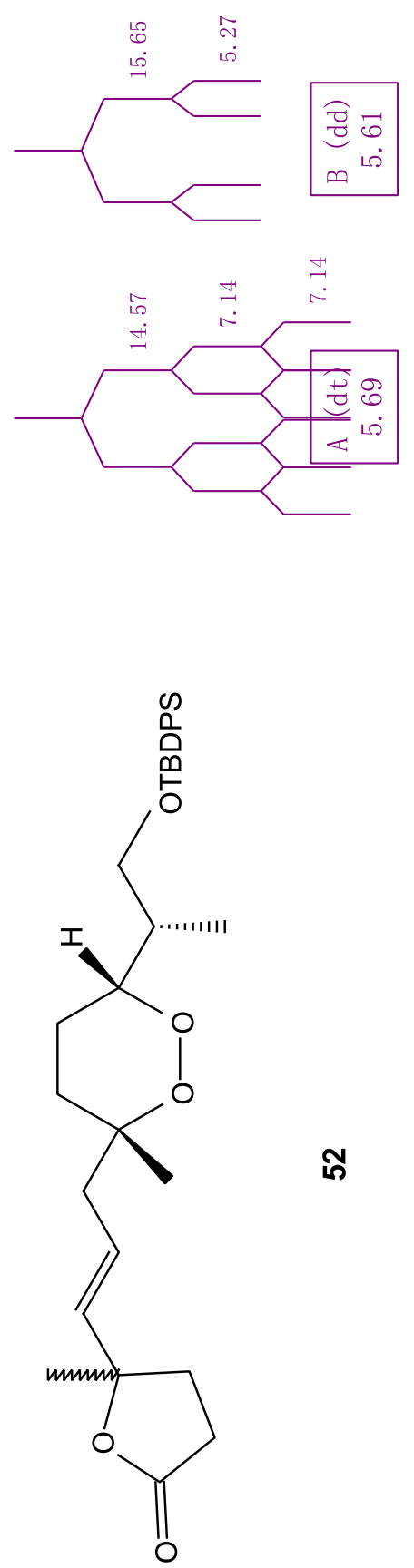

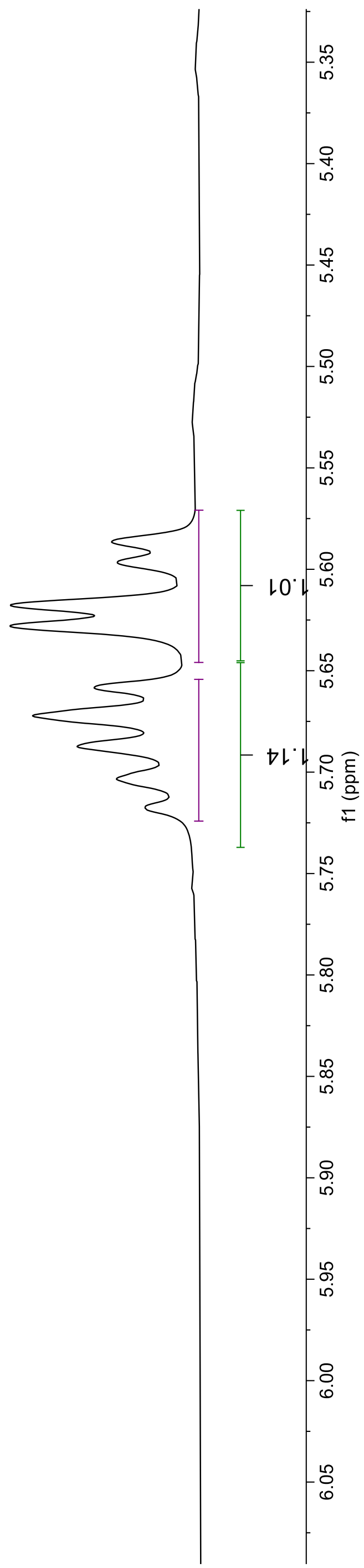


66L9' $\varepsilon$ $0269^{\circ} \varepsilon-$ $\angle 669^{\circ} \varepsilon^{-}$ †乙19 $\varepsilon^{-}$

$\angle G 99^{\circ} \mathcal{E}$ $0999^{\circ} \varepsilon-$ $99 \angle 9{ }^{\circ}-$ $1989^{\circ} \varepsilon^{-}$
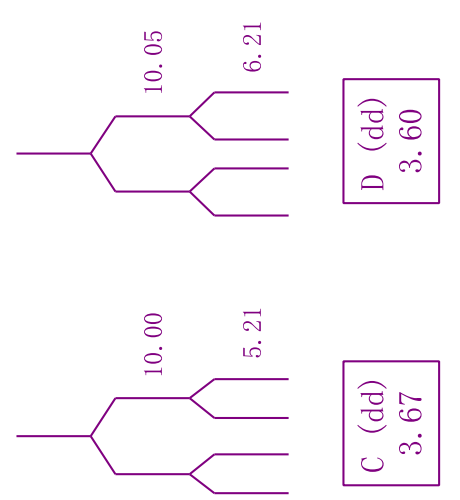

SIEO" -

ZSt0"

OLSO

Z990॰

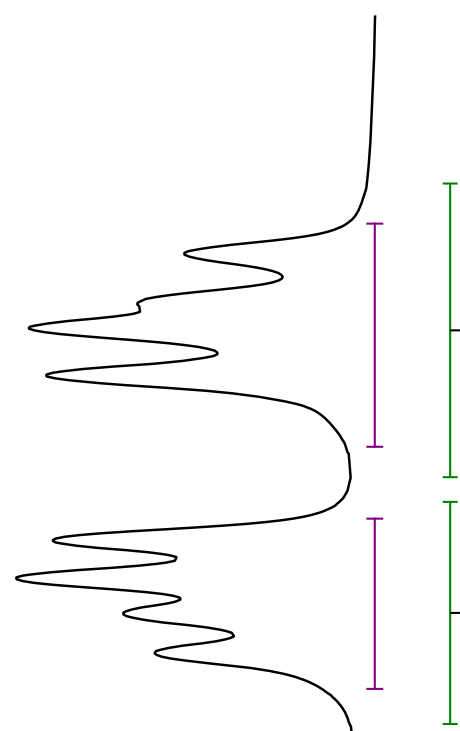

$\mid \begin{gathered}\mid \\ -\infty \\ 0\end{gathered}$

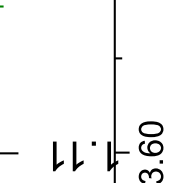

H H

กิ

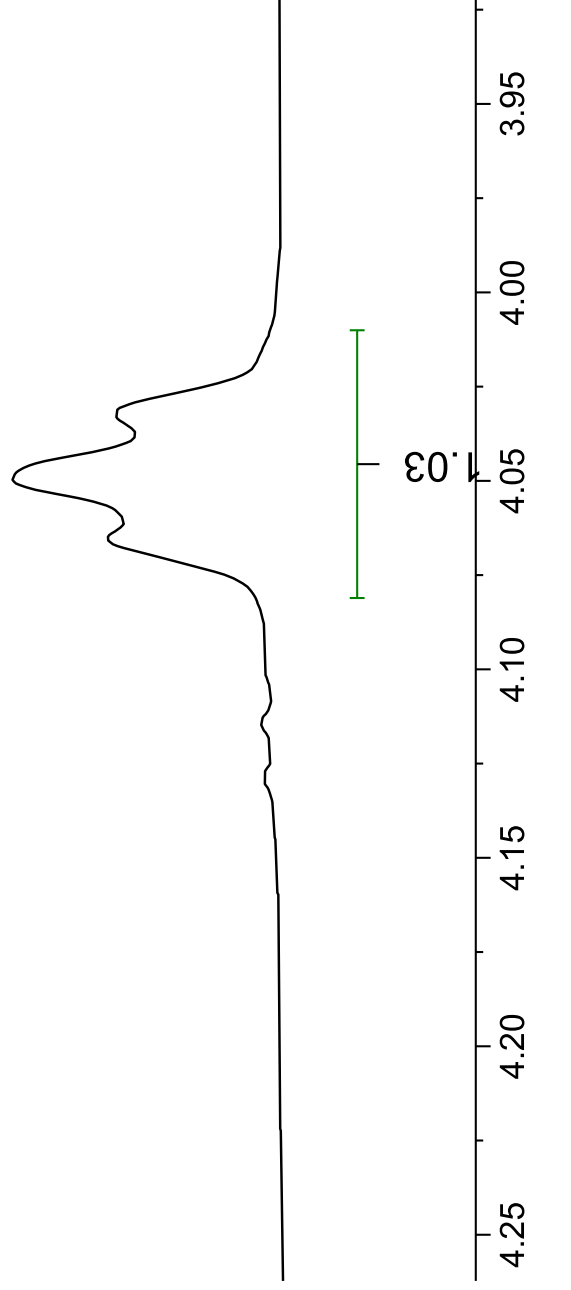




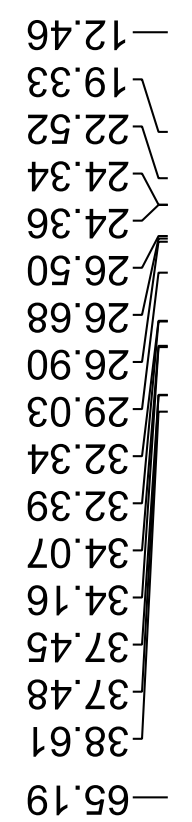

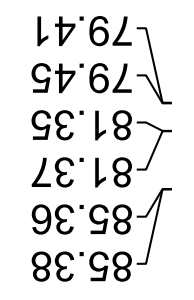

6乙"Gてレ

ย७゙Gてレ]

$09^{\circ} \angle Z L$

† 6 СL]

99.6乙レ ᄂ

$0 \angle$ $\varepsilon \varepsilon\llcorner$

$08 . \varepsilon \varepsilon\llcorner-1$

9乙 งEL

$\neg \varepsilon$ $ง \varepsilon l-$

09.GEL

I9.GEl

$\left.\begin{array}{l}0 \angle 9 L L \\ Z L \cdot 9 \angle L\end{array}\right\}$

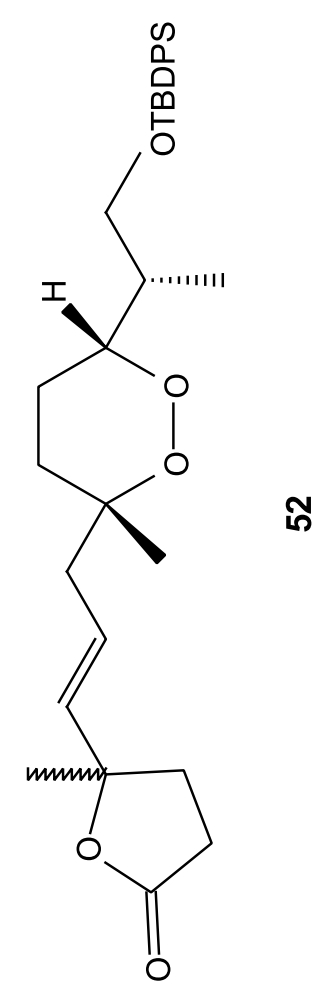

กิ

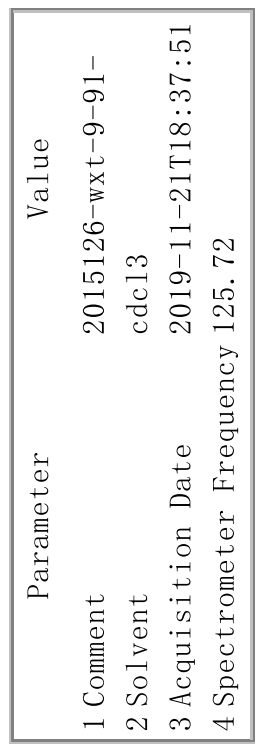




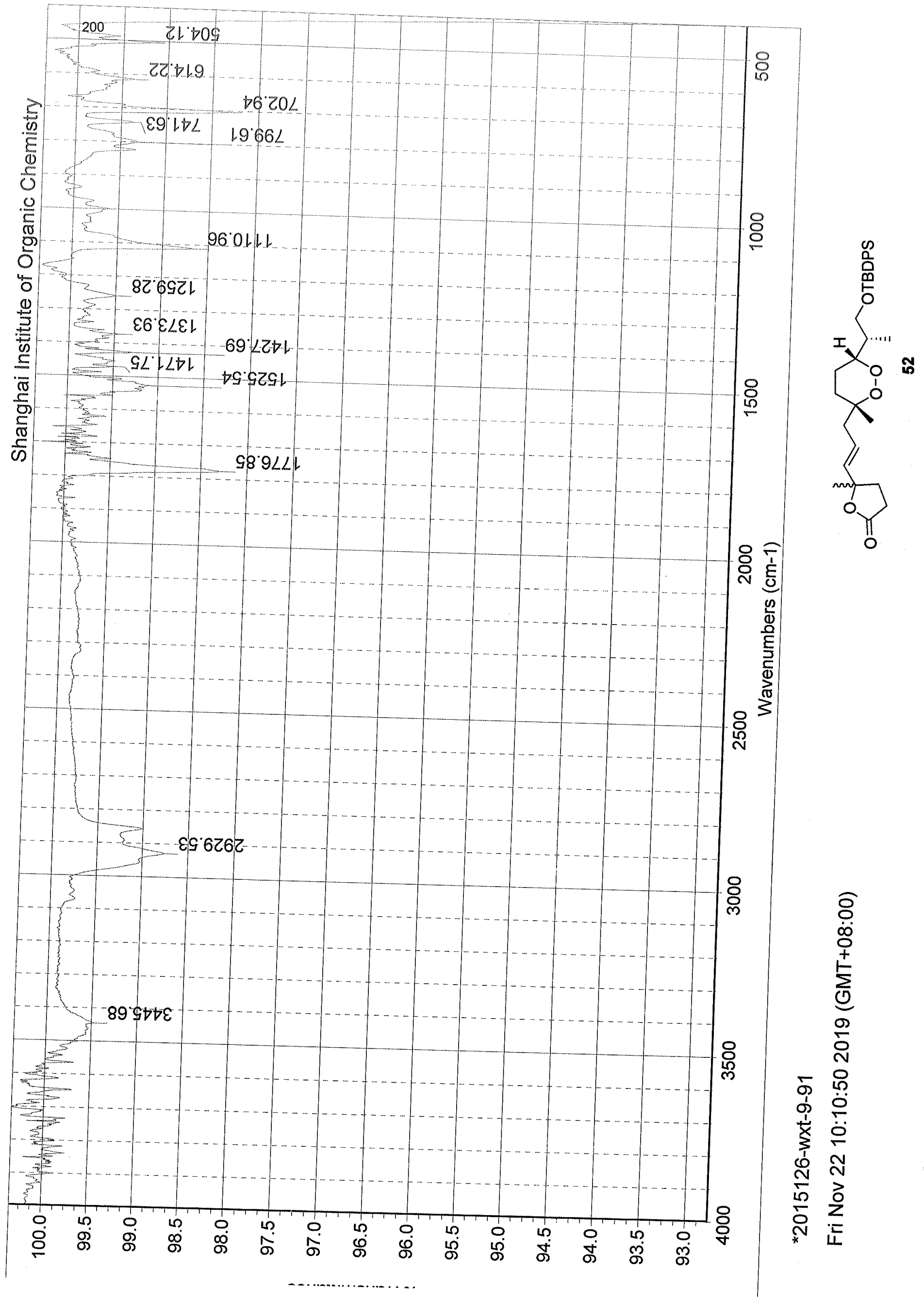



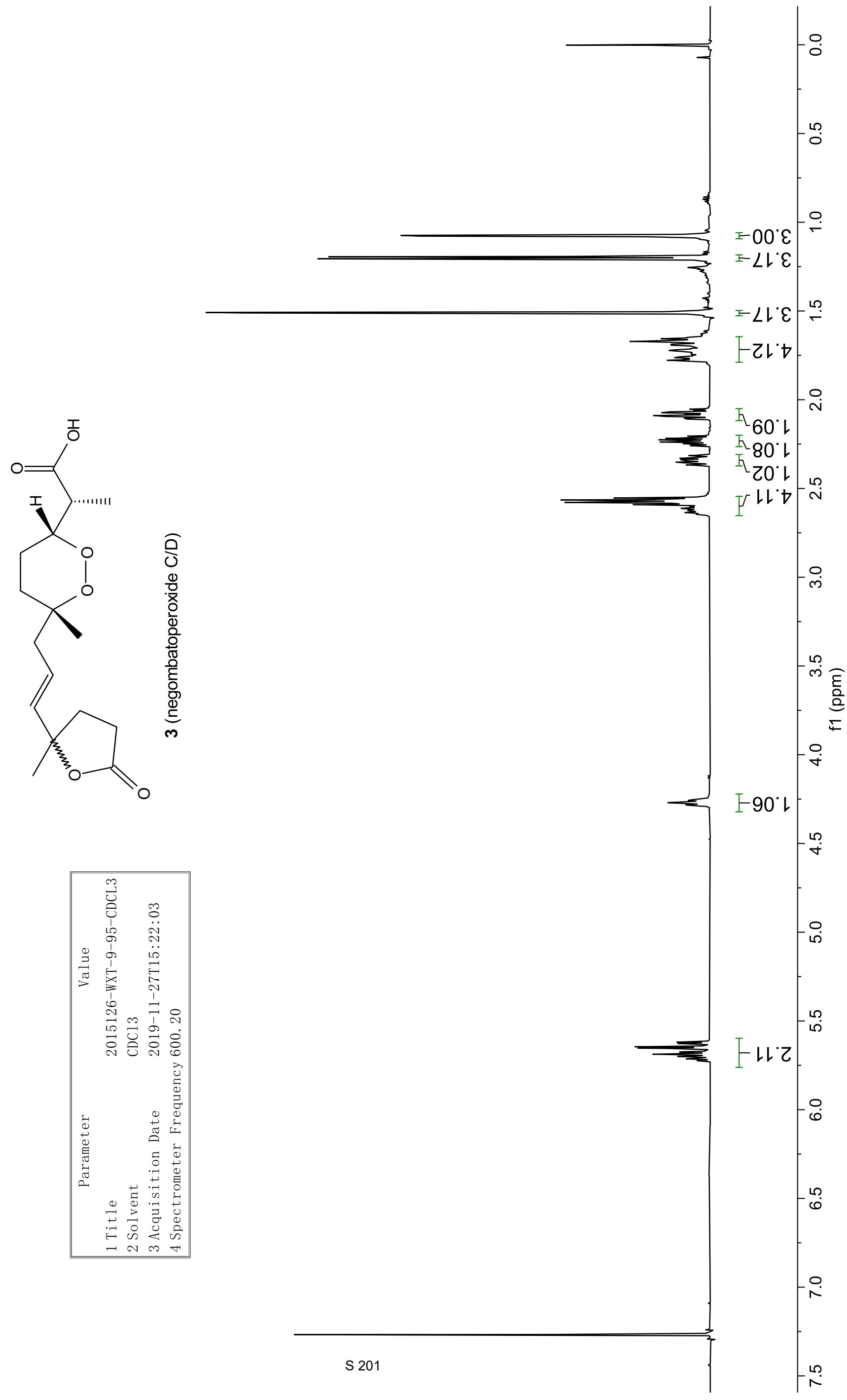
†6 $19^{\circ} \mathrm{S}-$

ZLZ9'9-

99+9:9-

$\forall \varepsilon 99$.

$\left\llcorner\nabla \angle 9^{\circ} G\right.$

$\varepsilon \angle \angle 9^{\circ} G$

$1789^{\circ} 9$

$\nabla \angle 89^{\circ}$ ᄂ

$7069^{\circ} \mathrm{C}-$

9L69 9 -

SOOL 9 '

$\mathrm{S} Q L^{\circ} \mathrm{G} /$

LOLLG

$\varepsilon \varepsilon L L^{\prime}$

$\varepsilon 9 L L^{\prime} G$

$\angle E Z L ' G$

$\angle 9 Z L G$
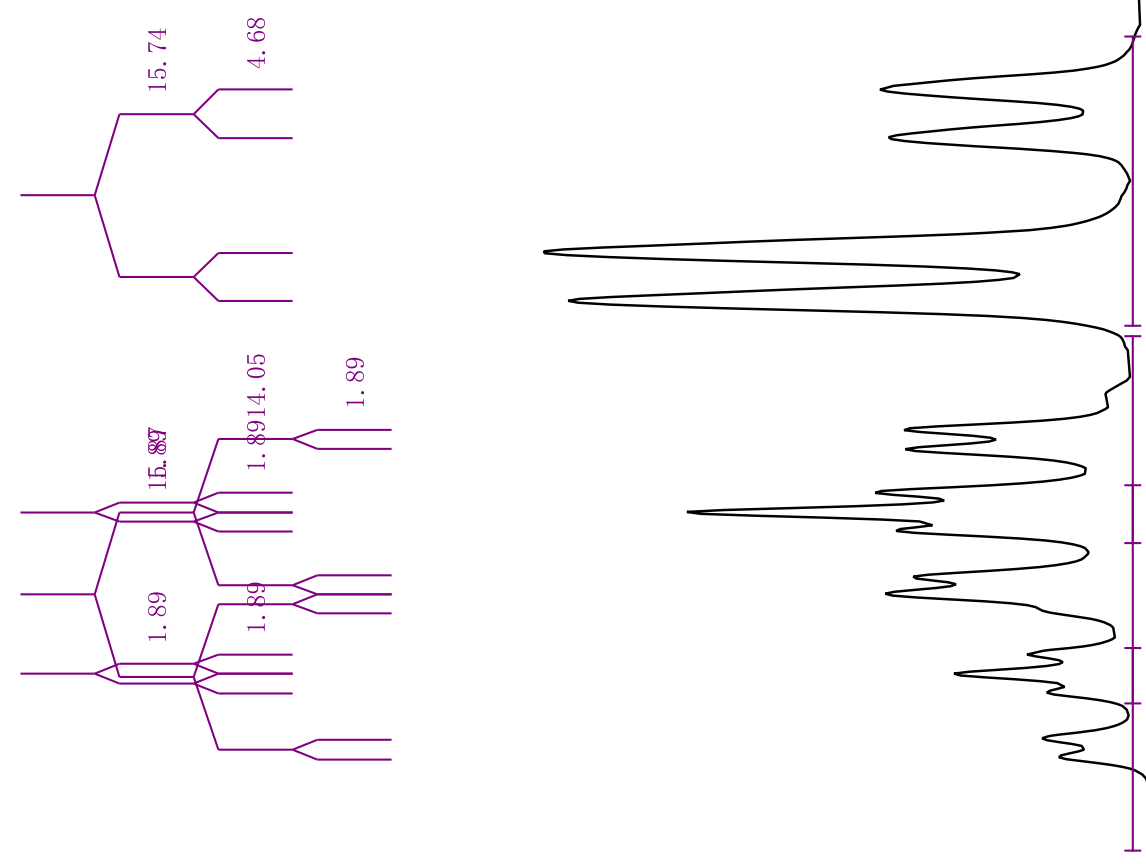

oิ

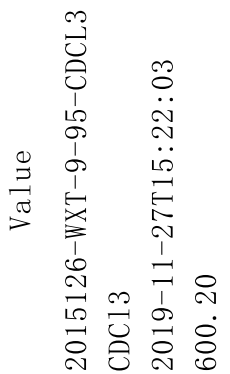

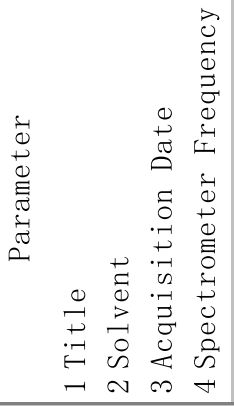<smiles>[CH2]C(=O)[C@H](C)[C@]1(C)CC[C@@](C)(CC)OO1</smiles> 

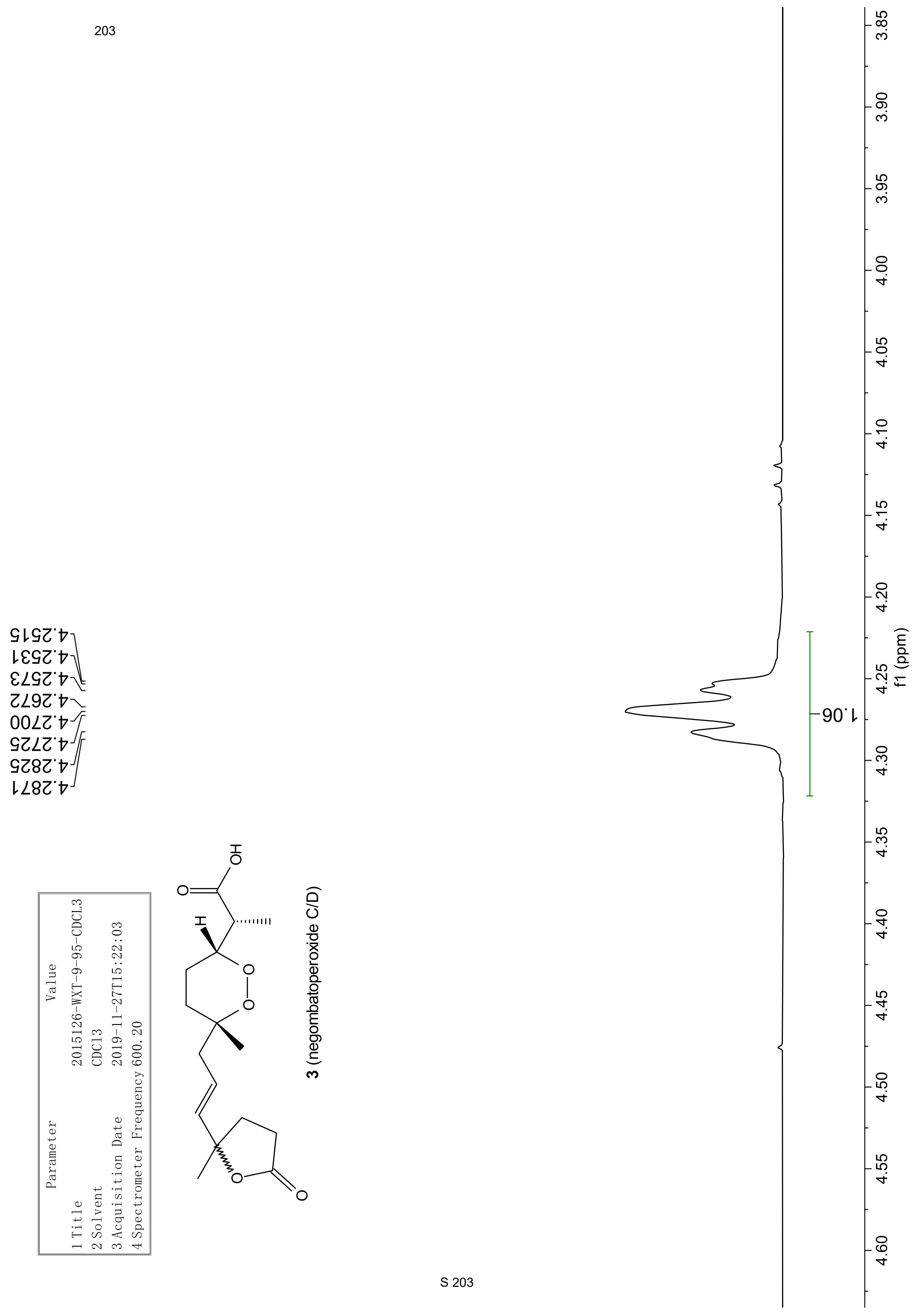
0E90 乙 $\angle 890^{\circ} \mathrm{Z}$ $8 \angle 90^{\circ} Z$ $9 \varepsilon \angle 0^{\circ} Z$ 66L0乙$9280^{\circ} \mathrm{C}$ $8880^{\circ} 乙$ 9†60乙] LEOL $ح$ Z60l' $ح$

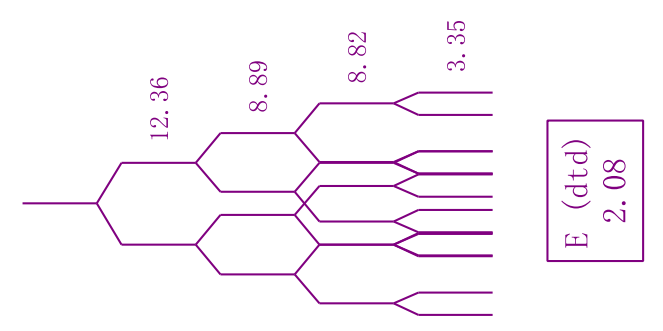

$6 \mapsto 0 乙 乙$ $\angle \varepsilon L 乙 Z$ \&LIZZ 09टZ乙 †9Z乙乙 ง6乙てて 8†ย乙て เ8EZ $Z^{-}$ 69t乙て8Lt乙 LOSZ $Z$ 689乙 乙 8†เE'乙 ๑८८ 乙 乙เยะ乙 乙8E६乙 งฤヤยてงIGE'て งเG\&'乙]
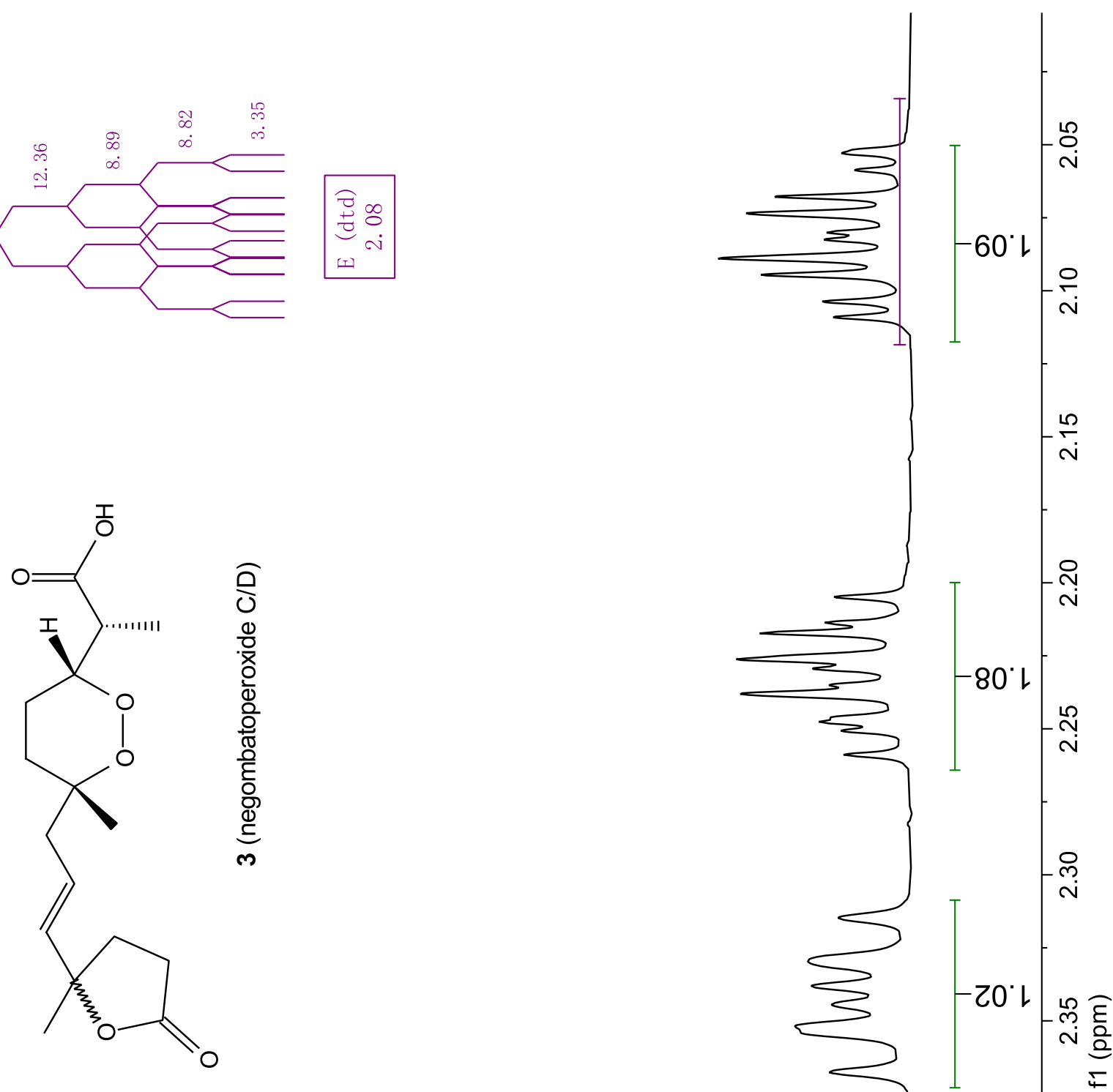

9L9E乙
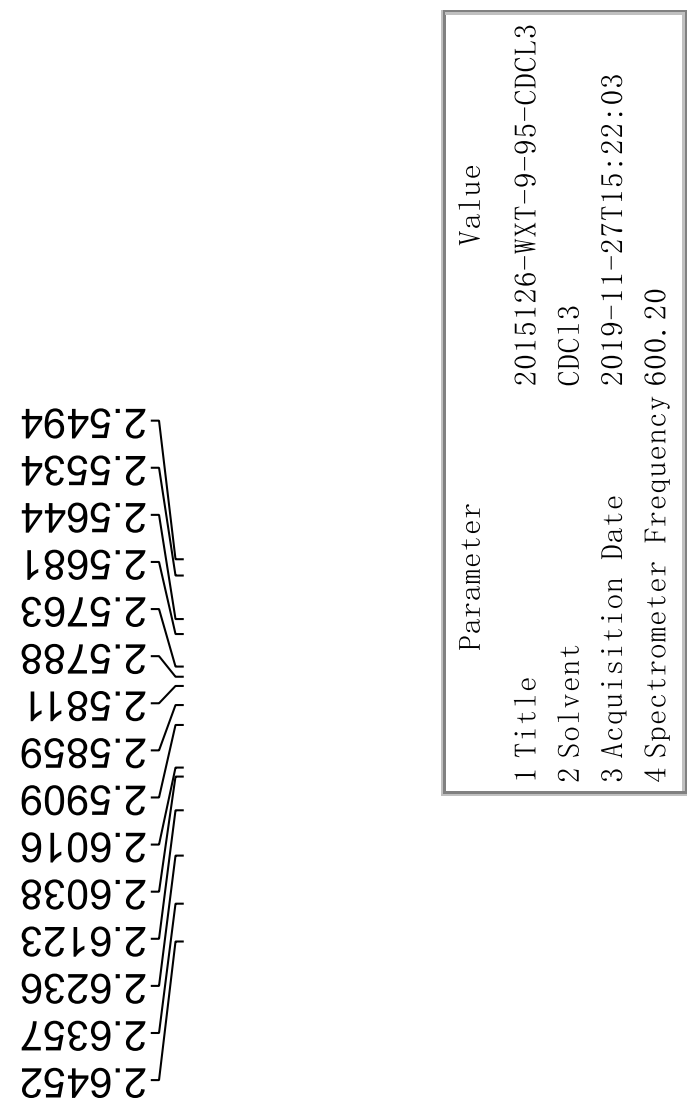
$\angle E \angle 0^{\circ} \mathrm{C}$

$28 \angle 0^{\circ} \mathrm{L}^{-}$

ยャ6เレ

ย90Zレ-
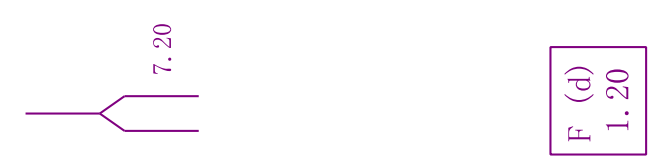

O
0
0
$\frac{0}{x}$
0
$\frac{0}{0}$
$\frac{0}{0}$
$\frac{0}{\pi}$
$\frac{0}{E}$
0
0
0
0
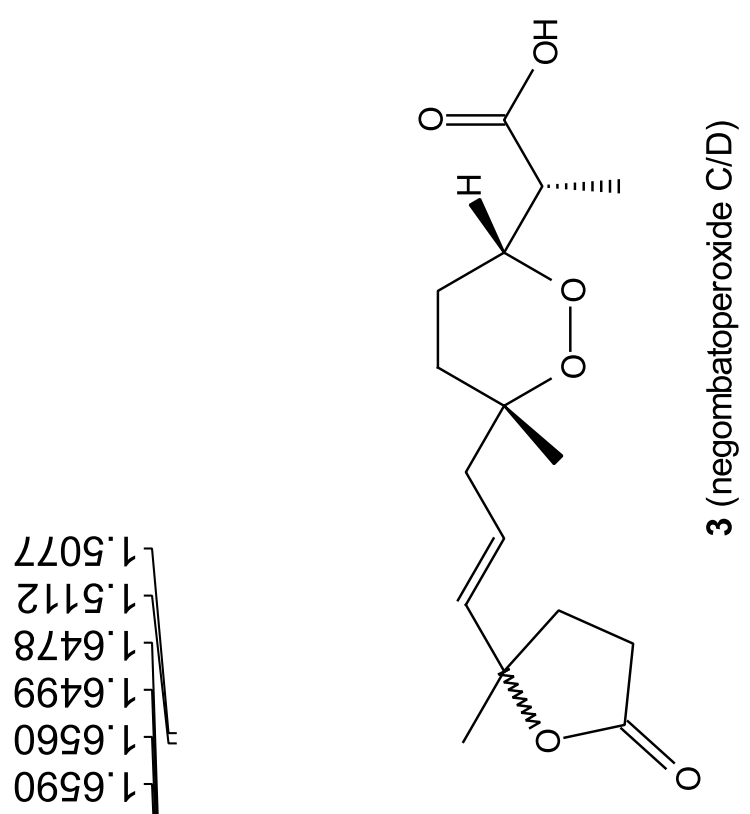

$7999^{\circ}$

EL $\angle 9^{\circ}$

$89 \angle 9$.

$6989^{\circ}$

LE69.

OLOL

$\angle \supset O L$

6ILL

†6LL'

6ZZL'L]

09ع L $L]$

9Z७LL]

OLGL $]$

ISGL'L

L8GL' $-F$

乙E9L $L$

Z89L' $\mathrm{L}$

$6 L \angle L L$

$\varepsilon \angle L L L$

†C8L'

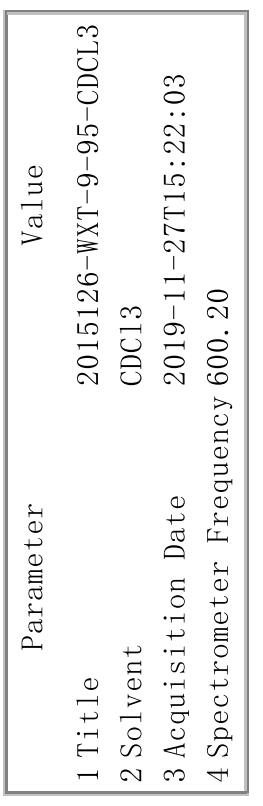

토ำ

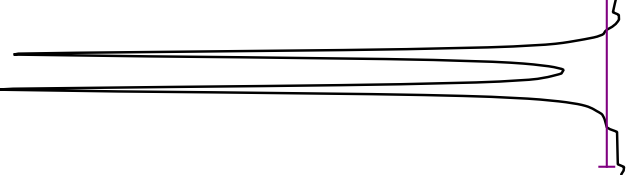

TLLE

$\stackrel{\leftrightarrow}{\stackrel{n}{\longrightarrow}}$

- 음

永.

ㅇ.

$\widehat{\overline{\mathrm{a}}}$

오

-

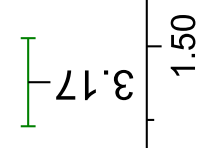

员

$-\stackrel{8}{-}$

-

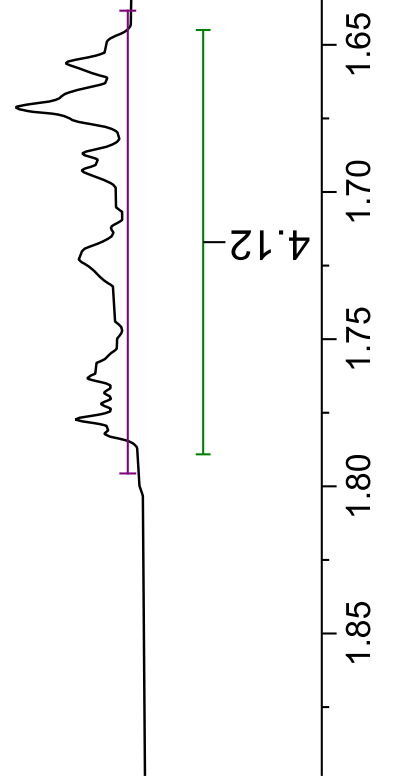


LE8'Zレー

GZLZZ

$\nabla 6 L^{\circ} \circ 2$

699. 92

$8 S L 9 Z$

จเL'6Z

SO0'टह 5

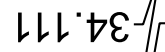

$\varepsilon 0 Z^{\circ} \downarrow \varepsilon$

$089^{\circ} \angle \varepsilon^{\top}$

$\downarrow 89)^{\circ} \mathrm{\nabla}$

188.94

$\varepsilon 60^{\circ} L L$

$\left.\neg 0 \varepsilon^{\prime} \angle L\right]$

910 02

IS6 62

89E. 18

$\neg 8 \varepsilon^{\prime} 18$

98+' 98

ILG'S8

ع00.9ZL

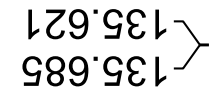

$\varepsilon 68^{\circ} 9 L \mathrm{~L}$

Z28 8LL-
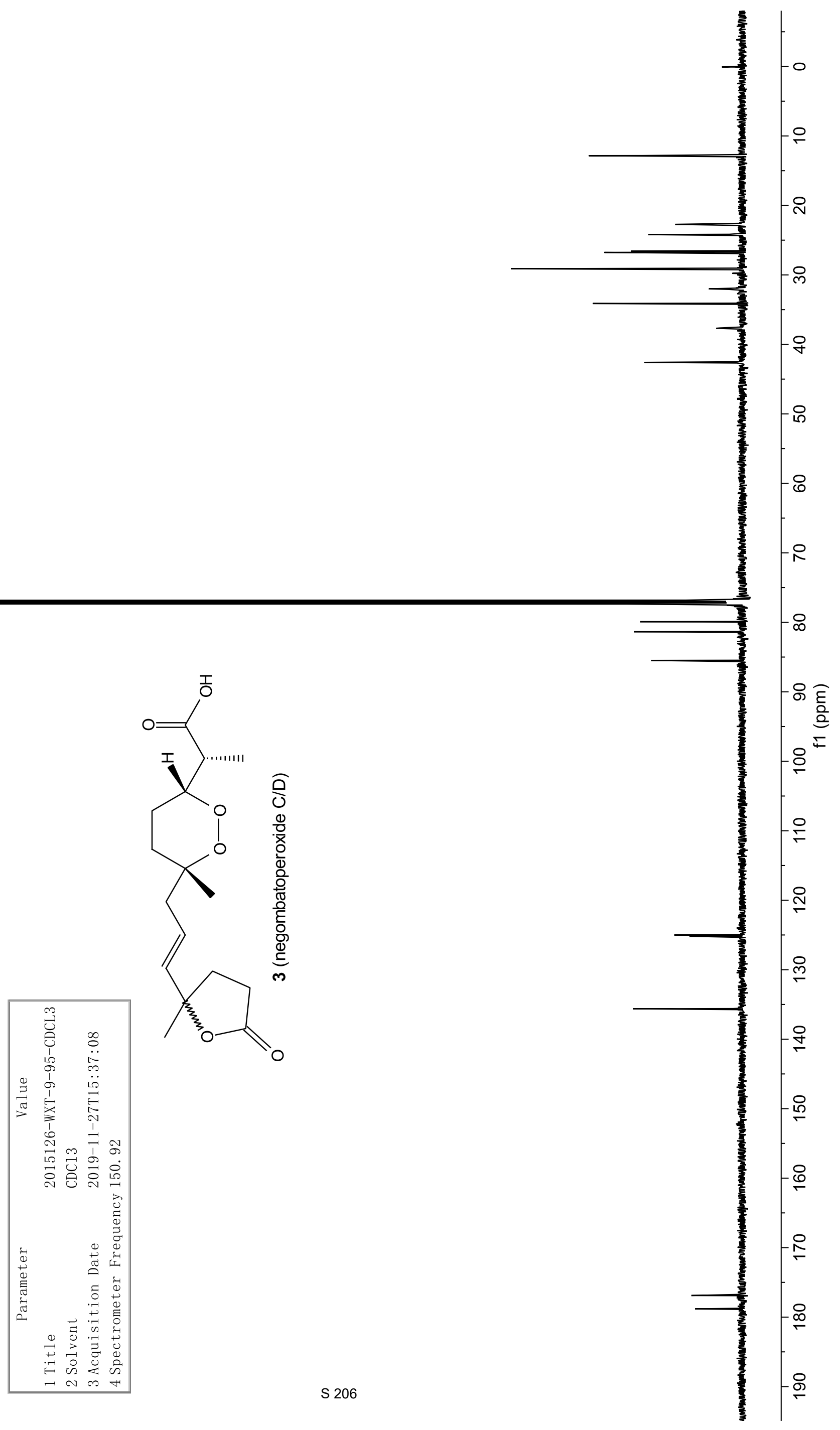


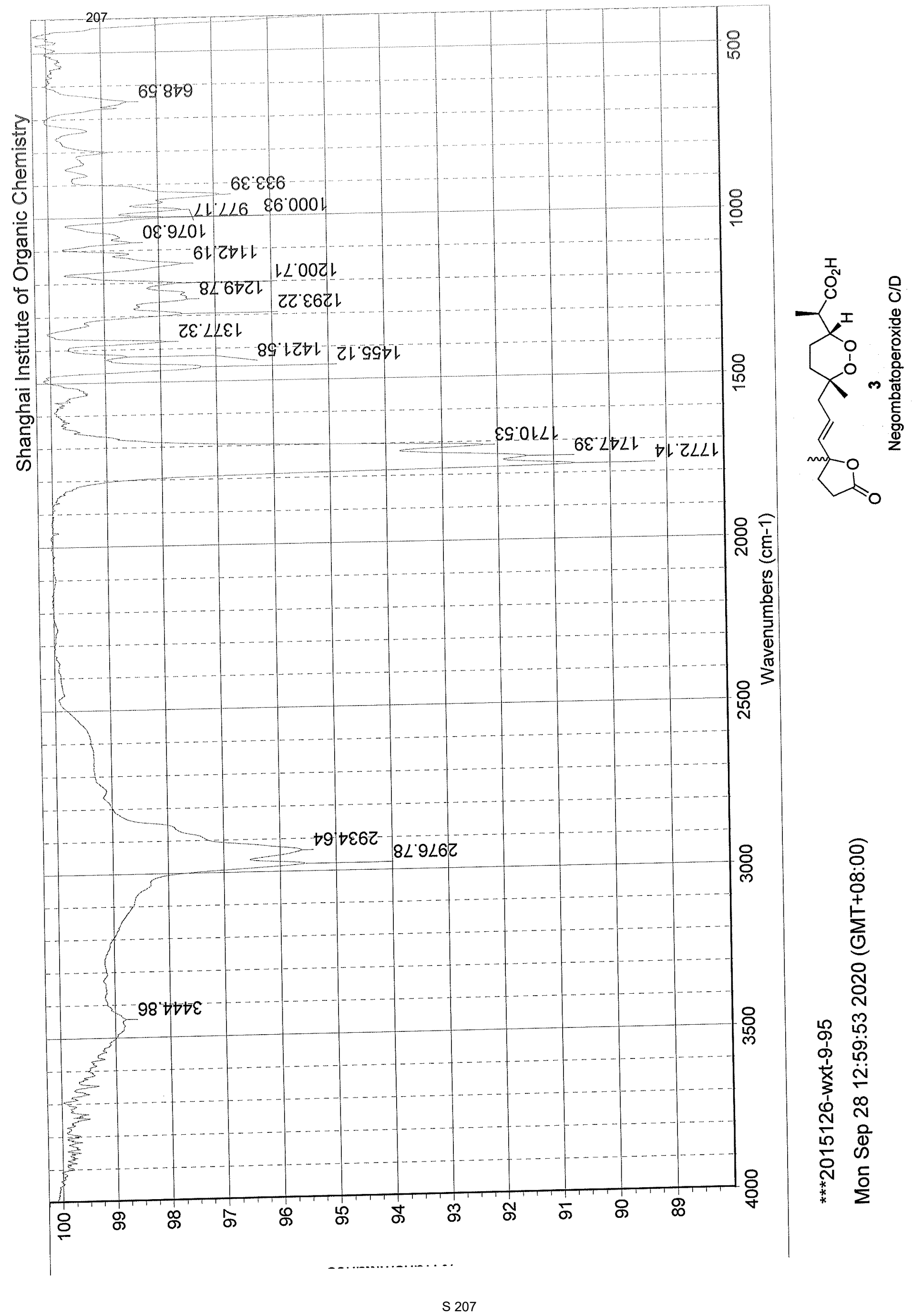


$\left.\begin{array}{l}\text { レ゙0 } \\ \text { Zレ゙ }\end{array}\right\}$

$26^{\circ} 0$

90*

$62^{\circ}$

OE'

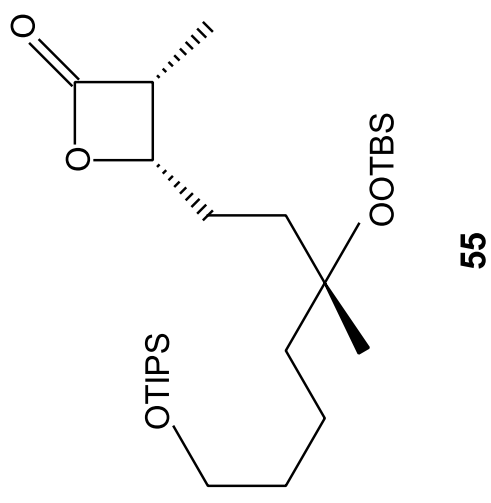

ผึ

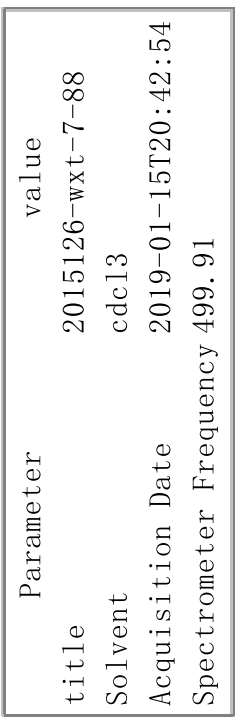


ZᄂL9'Eเ†89' $0 \angle 69^{\circ} \varepsilon_{\supset}$ $\angle O L L^{\prime} \varepsilon$ 9EL $\angle \mathcal{E} L$ Z9ZL $\varepsilon$ 062L $\varepsilon-$ $81 \nabla L L^{\circ} \varepsilon$ $\angle \nabla \nabla L^{\circ} \varepsilon^{\prime}$ $\varepsilon \angle S L L^{\circ} \varepsilon-$
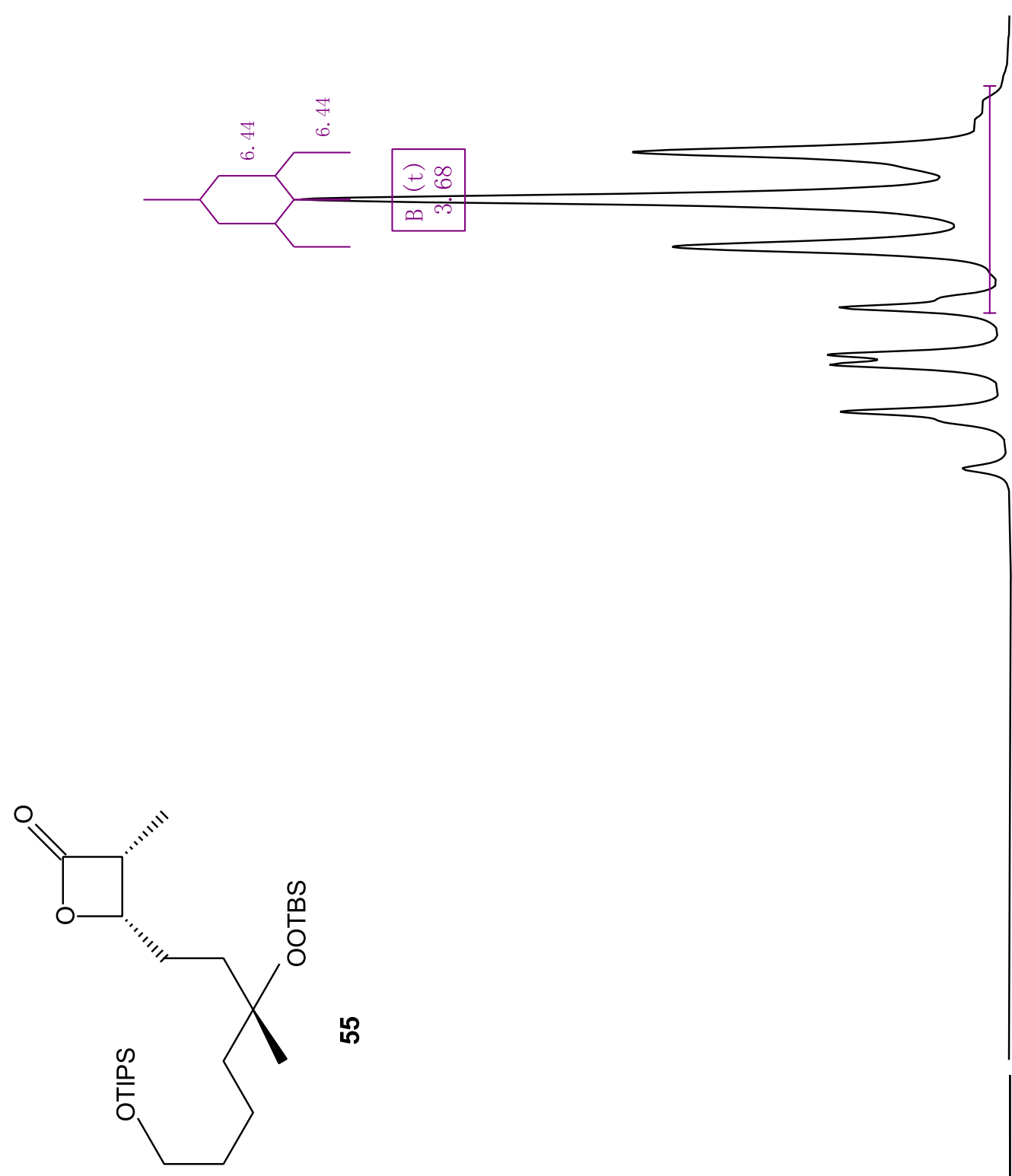

$\nabla \nabla 0 G^{\circ} \nabla>$ 89L't EOZ9" $\nabla-$ 98ZG" $\angle \nabla \nabla G^{\circ} \nabla^{\top}$

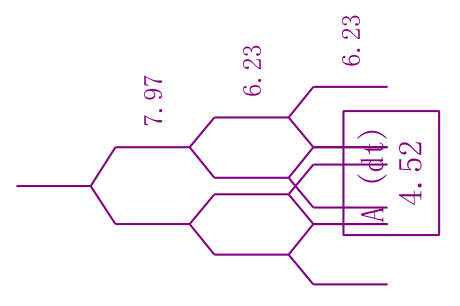




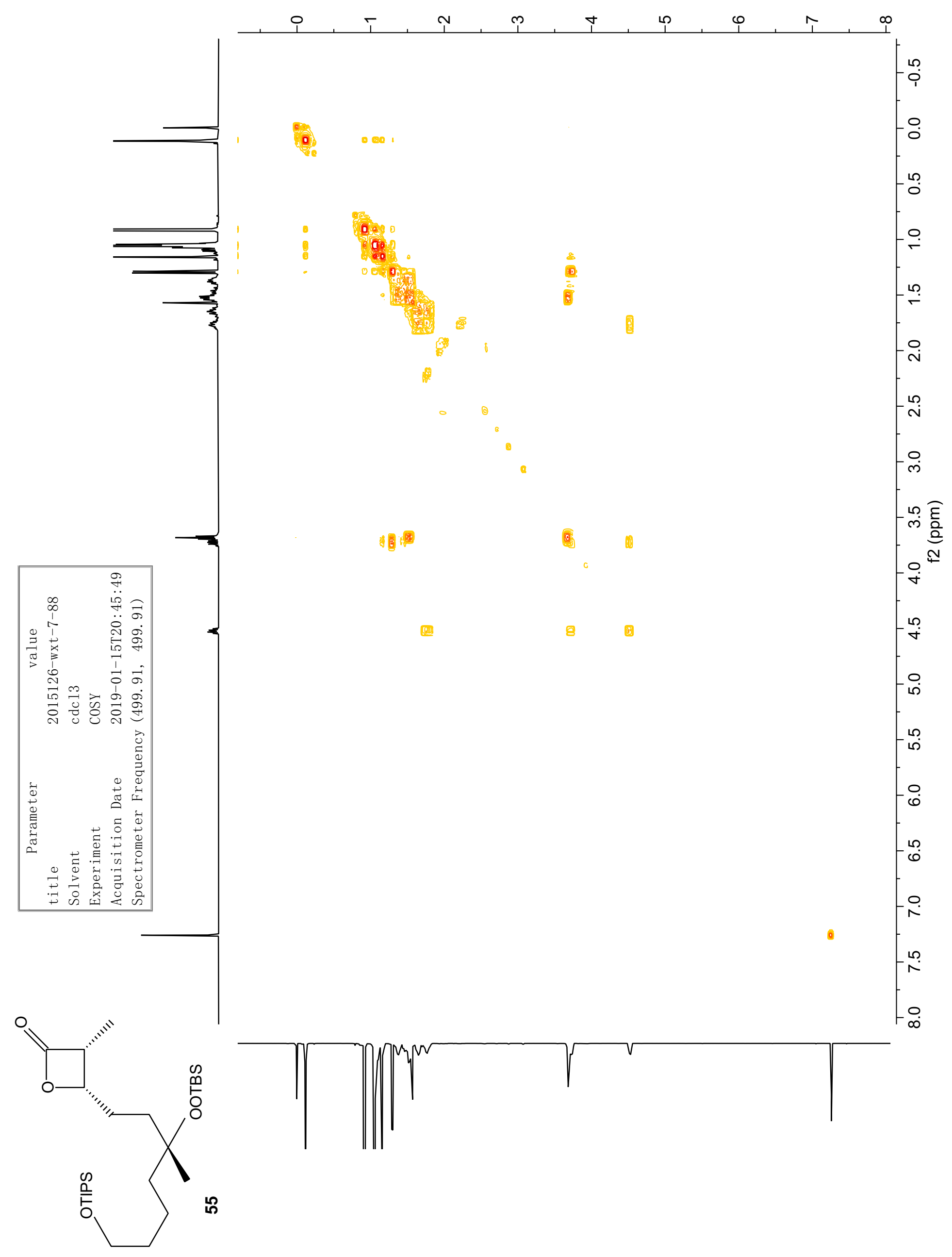


09: G--

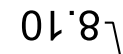

66เレ

$20.8 \mathrm{~L}$

$7 \mathrm{Z}^{\circ} 8 \mathrm{~L}$

ZLOZ

86.て-

$9 Z^{\prime} \sqcup \tau^{5}$

$6 เ 92$

เE' $ح \varepsilon^{\varsigma}$

6ฤ $\varepsilon \varepsilon$

ง $9 \varepsilon$

L $\angle \nabla-$

8เะ9-

$\angle 0^{\circ} 9 L-$

$69^{\circ} \varepsilon 8-$
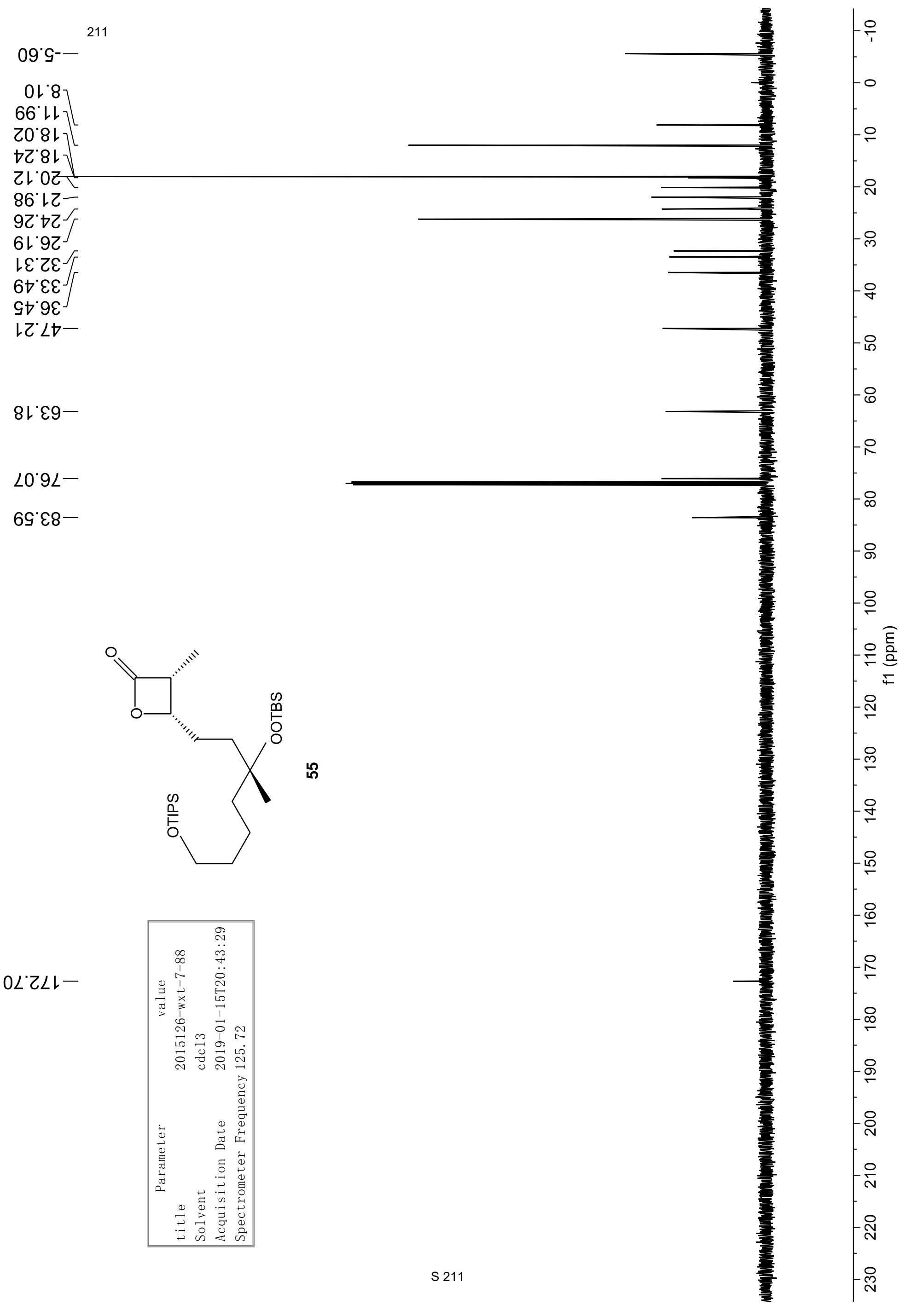


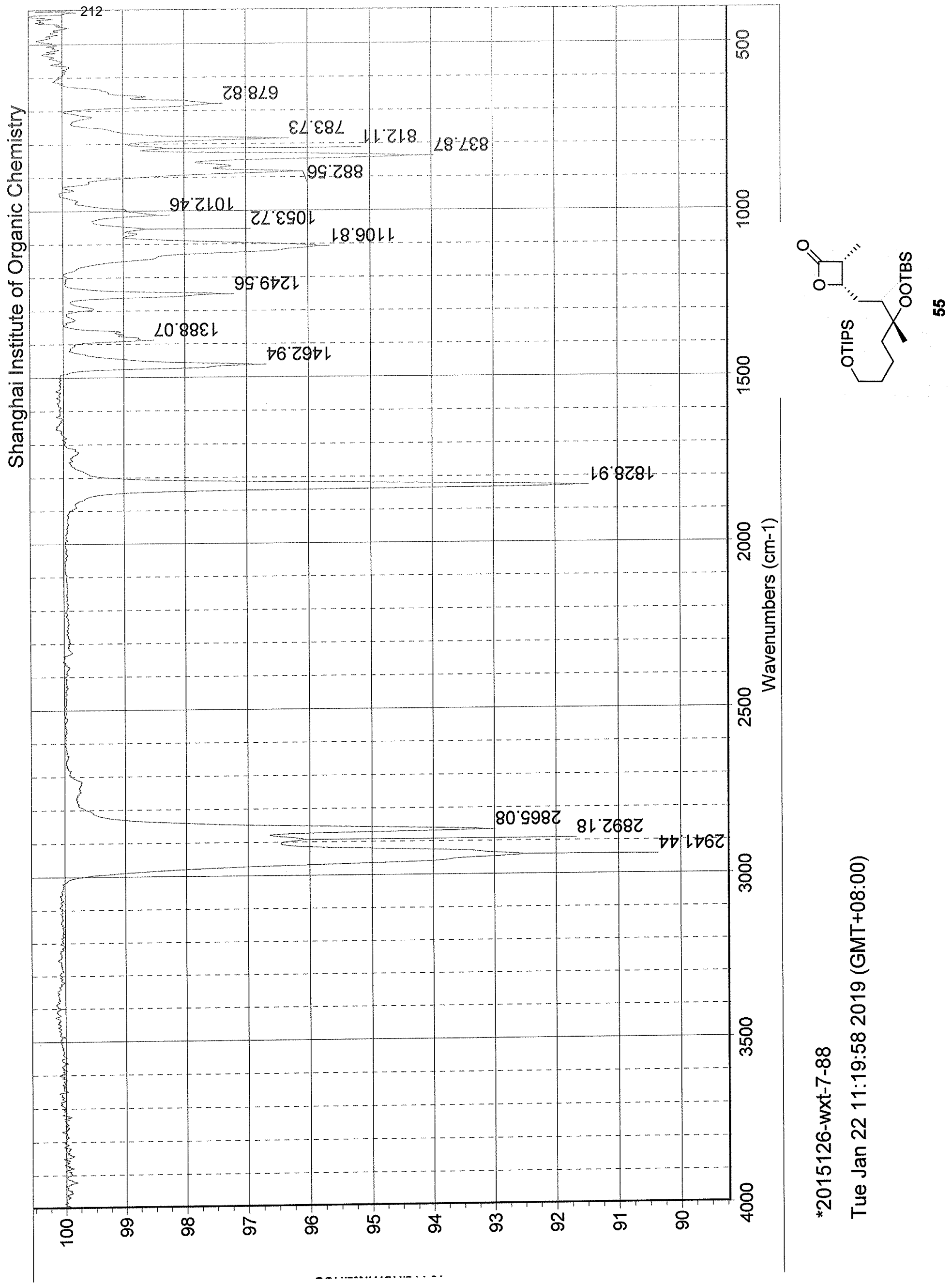


$\frac{\angle L^{\circ} L}{6 Z^{2} L}$ $0 \varepsilon^{\circ} \vdash$
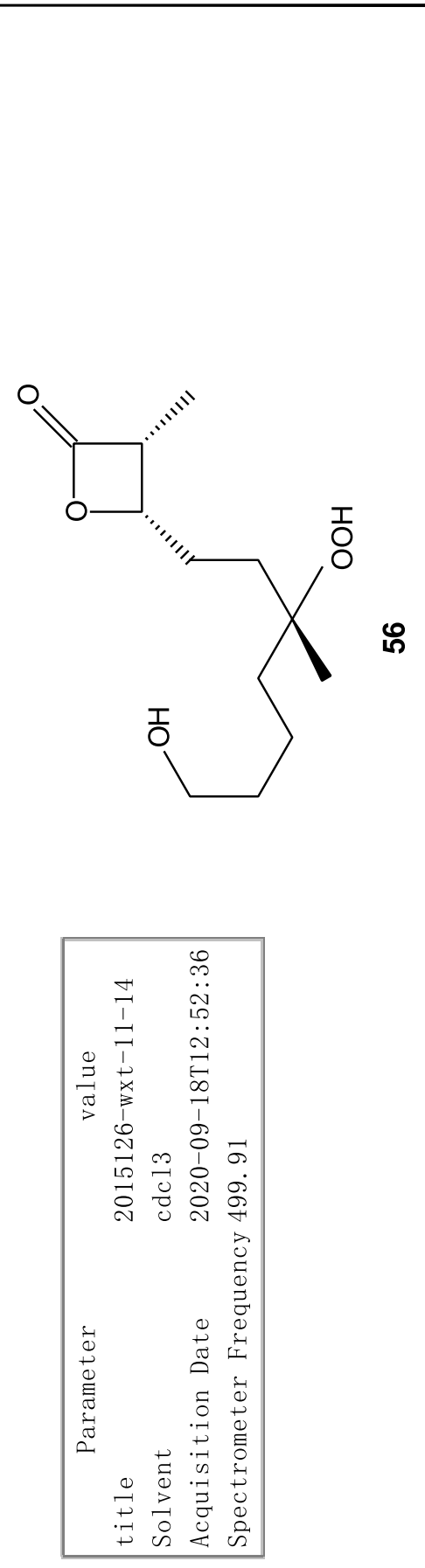

凡 $9 L^{\prime} \varepsilon$.

凡 $80^{\circ} \varepsilon$ $\checkmark t L L L \stackrel{\circ}{\sim}$ ${ }^{-} \varepsilon \mathcal{L}^{-} \varepsilon$ 


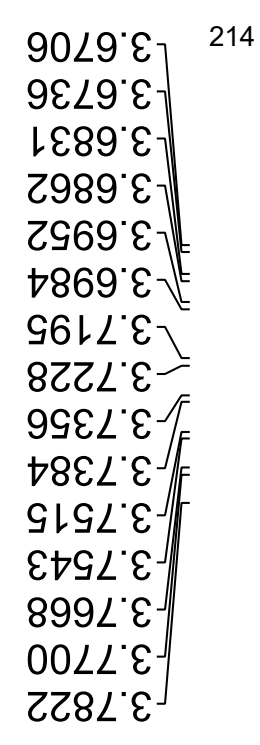

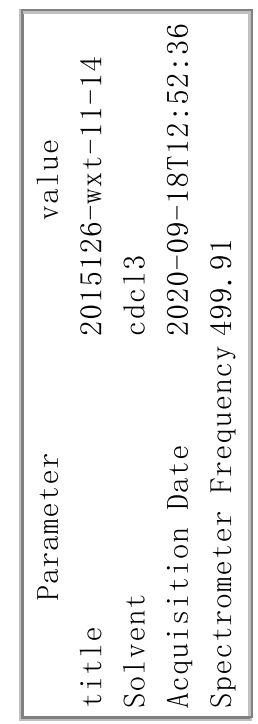

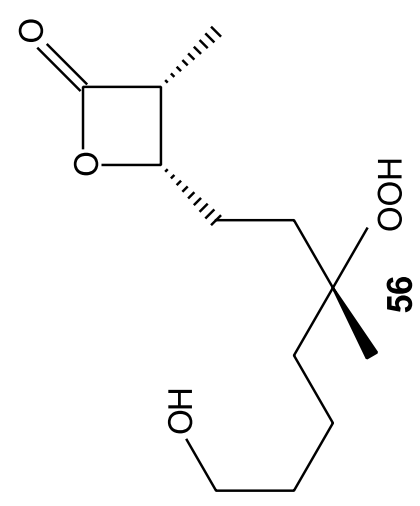

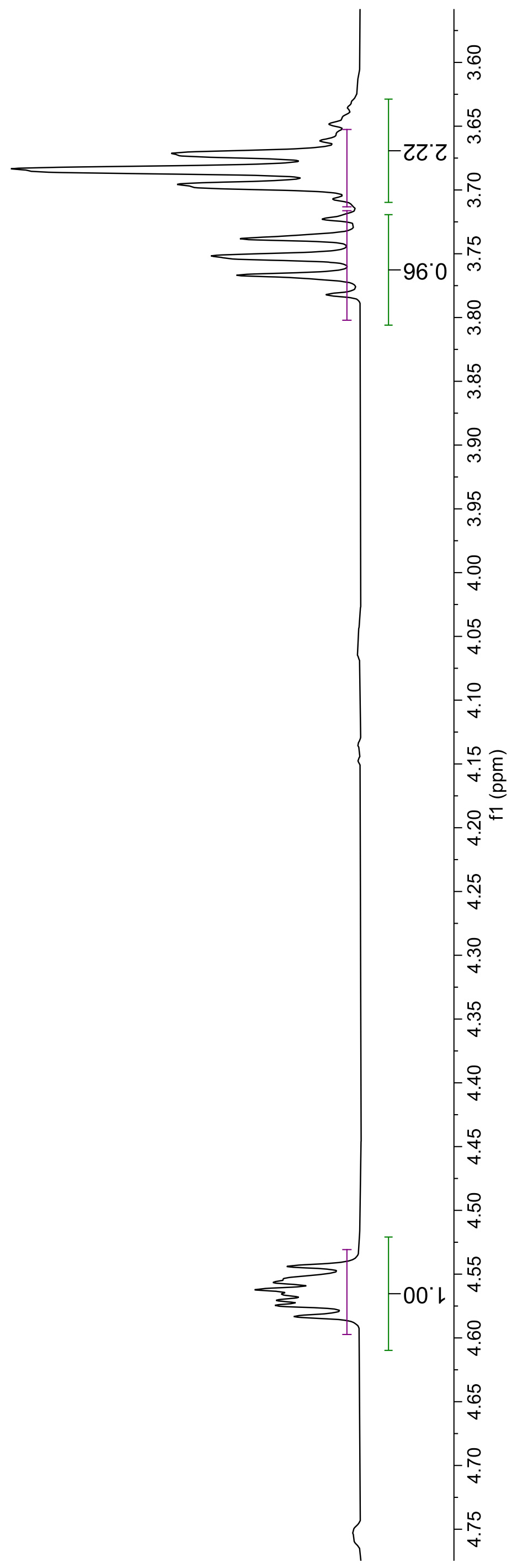


$80 \cdot 8-$

$\varepsilon \varepsilon^{\prime} 6$ -

91.レ乙-

$0 Z^{\prime} \downarrow Z^{-}$

$\varepsilon 乙 ' \measuredangle \varepsilon$

$0 \varepsilon^{\prime} 乙 \varepsilon$

$69{ }^{\circ} \varepsilon^{-}$

$\nabla Z \angle \nabla-$

оะ'Z9-

$600^{\circ} 9 L-$

เ9'ย8-

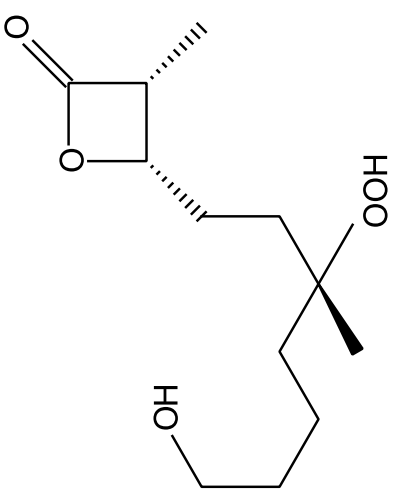

in

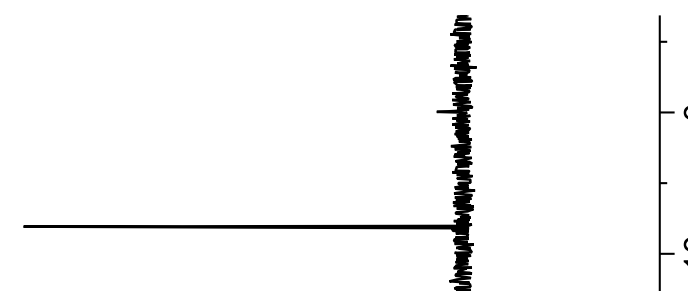

으

ำ

요

우

요

$-8$

웅

$\infty$

응

E

음

음

온

요

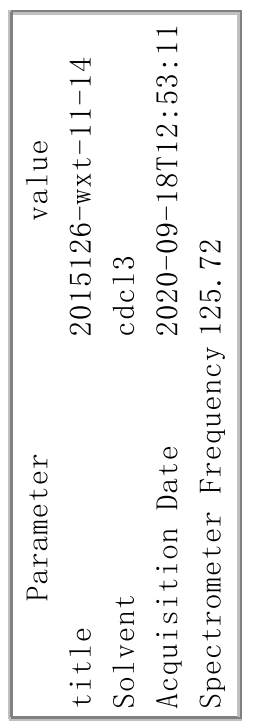




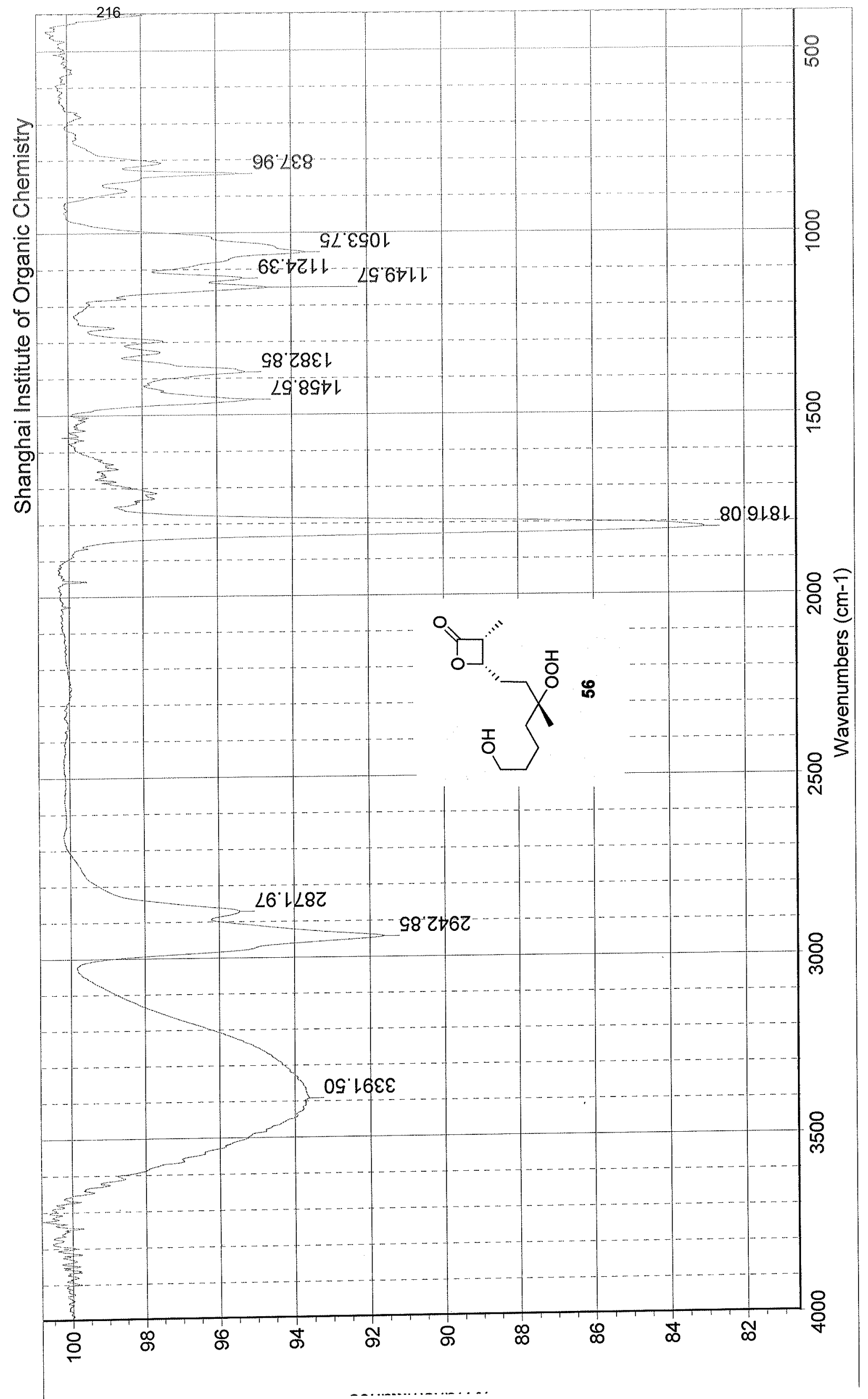

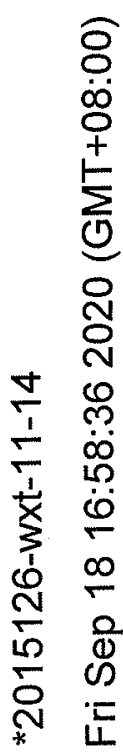


$80^{\circ} \mathrm{L}$ टLレテ

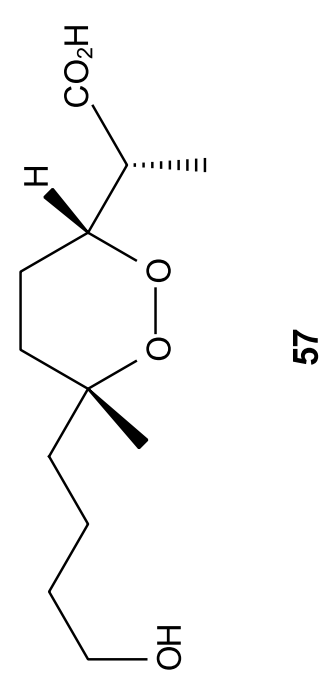

$\mathrm{GG}^{\circ} \varepsilon$

$99^{\circ} \varepsilon$

$\angle \mathcal{G}^{\circ} \varepsilon$

$\angle 8^{\circ} \circ-$ in

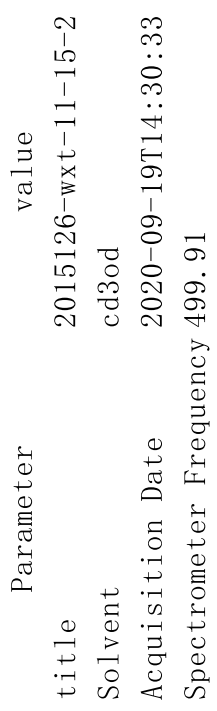

$\left\{\begin{array}{c}0 \\ 0 \\ 0 \\ 0 \\ 1 \\ 1 \\ 0 \\ 0\end{array}\right.$

t

$\stackrel{0}{0}$

$\infty$

$-\stackrel{\circ}{\circ}$

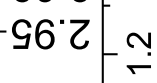

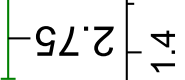

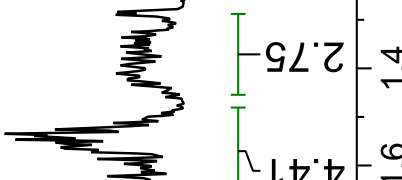

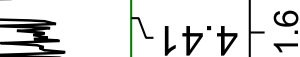

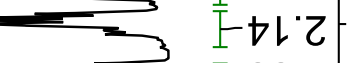

โ88.
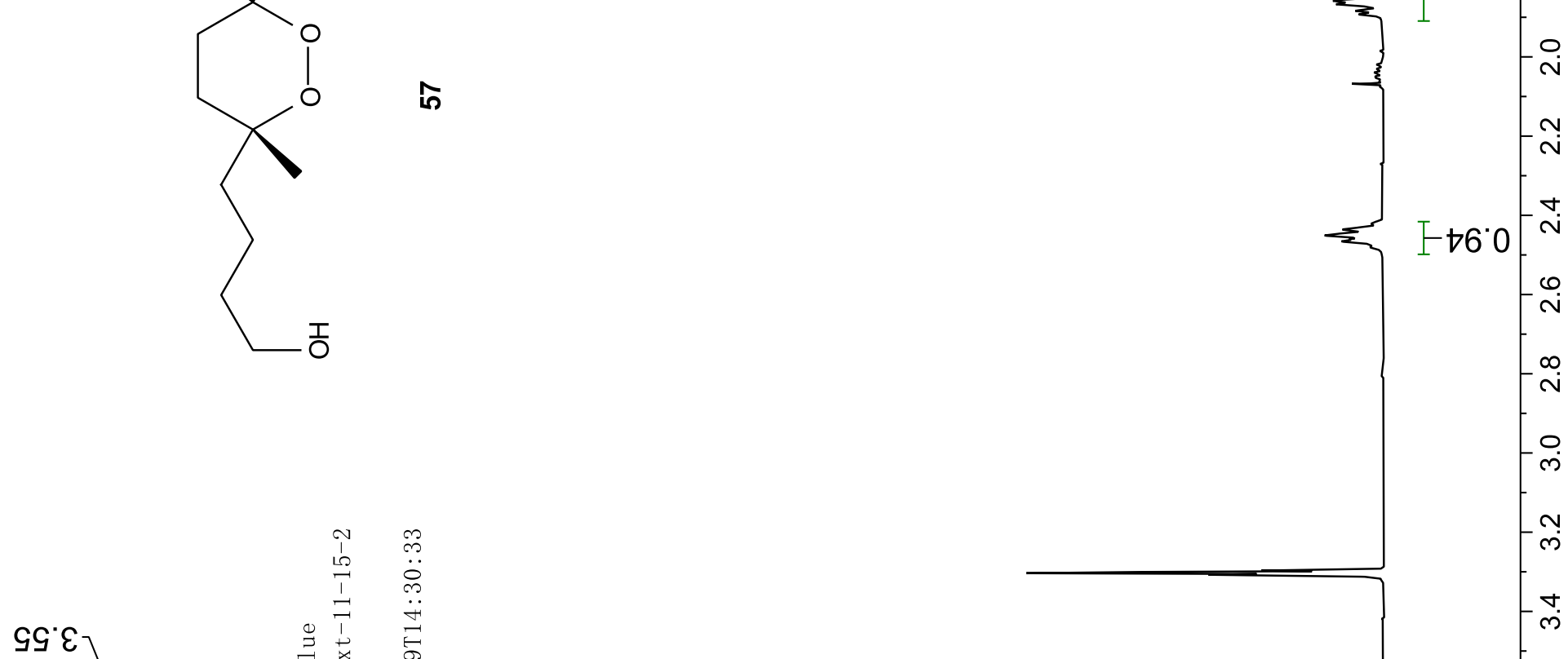
${ }^{\circ} \nabla \mathcal{G}^{\prime} \varepsilon-$ 9199. $\varepsilon-$ $8 \nabla \angle G^{\prime} \varepsilon-$

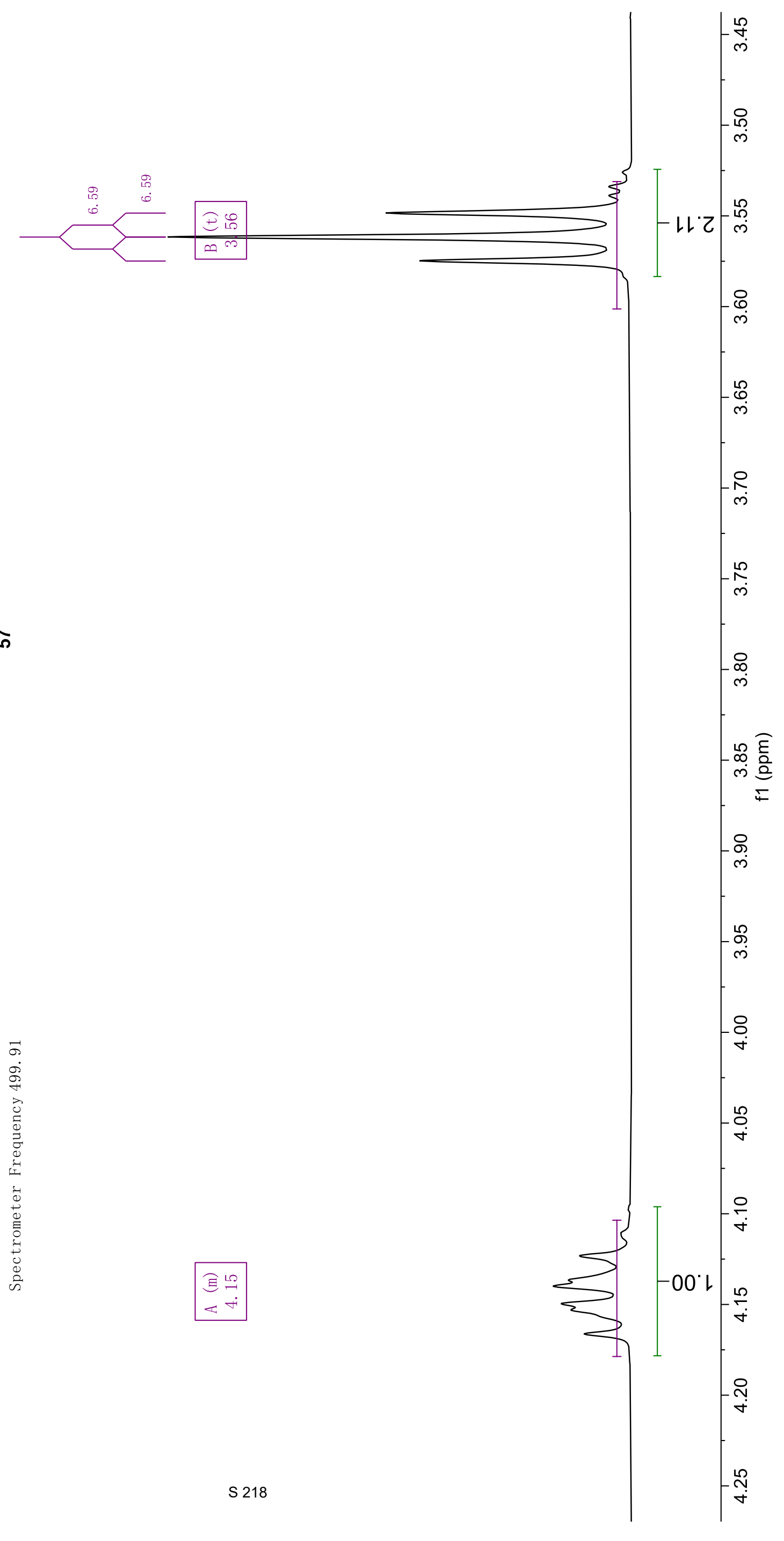




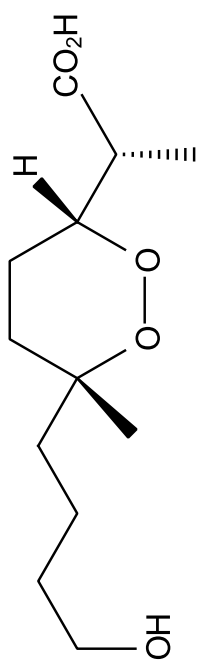

is

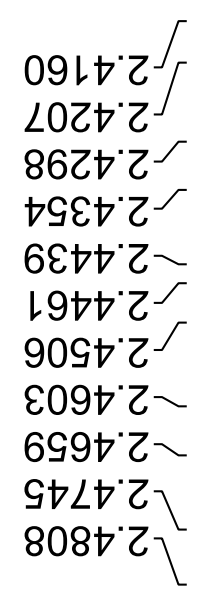

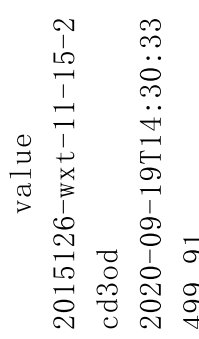

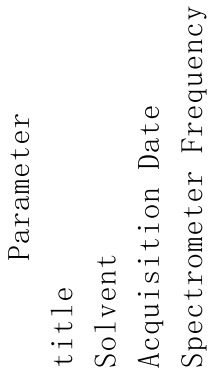

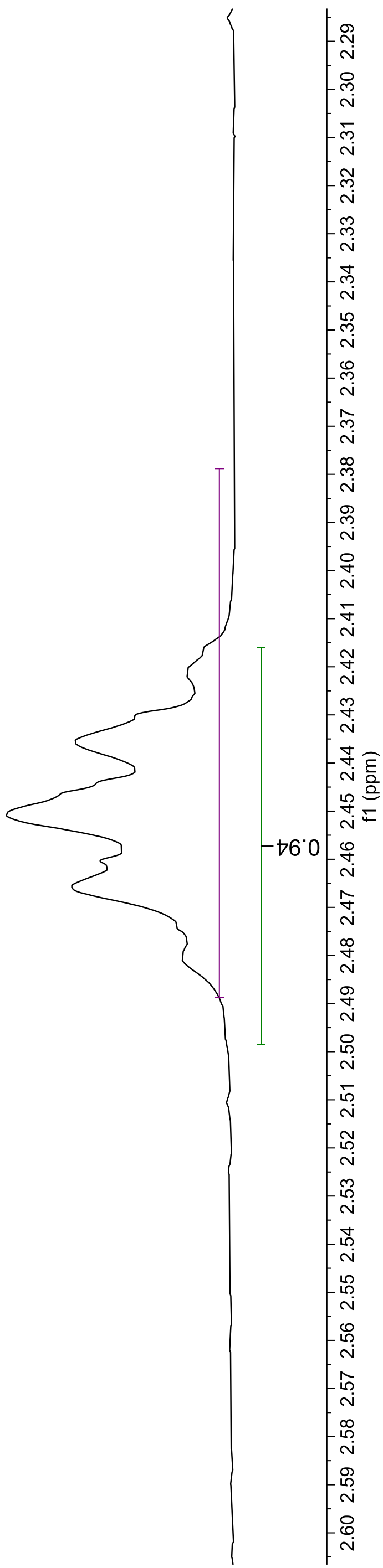


0ฤะ์ -

$76^{\circ} 0 乙 \backslash$

$\varepsilon L{ }^{\prime} \varepsilon 乙 \backslash$

$\left\llcorner 乙^{*} \nabla 乙^{\nearrow}\right.$

$0\llcorner\varepsilon \varepsilon]$

$\varepsilon L \nabla \varepsilon$

$89.9 \varepsilon^{\top}$

$\varepsilon 0^{\circ} \nabla \nabla^{\circ}$

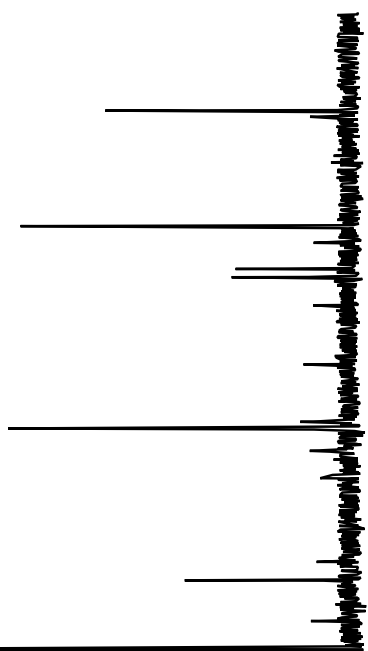

26 २9-

カレはレ

96 28
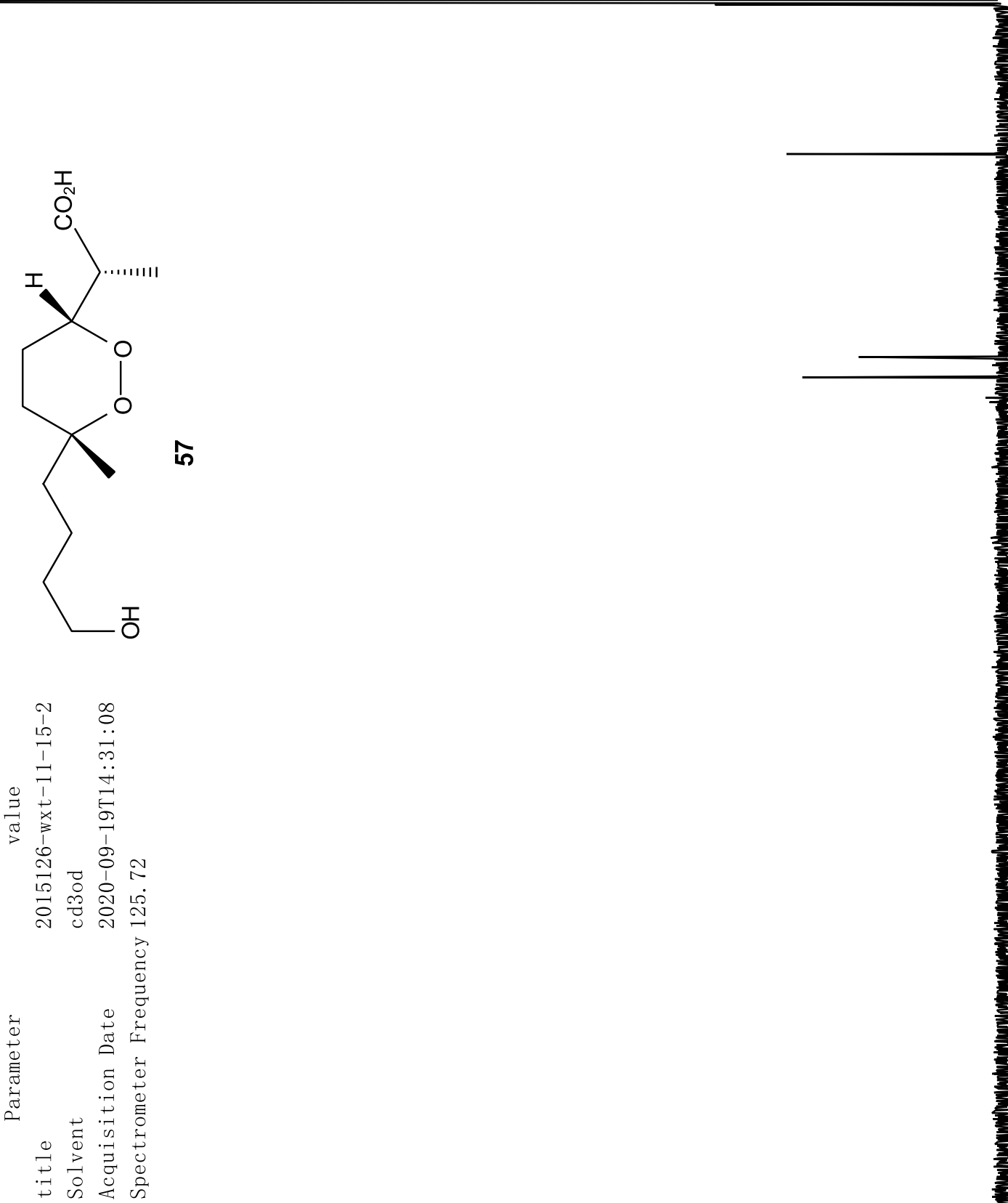

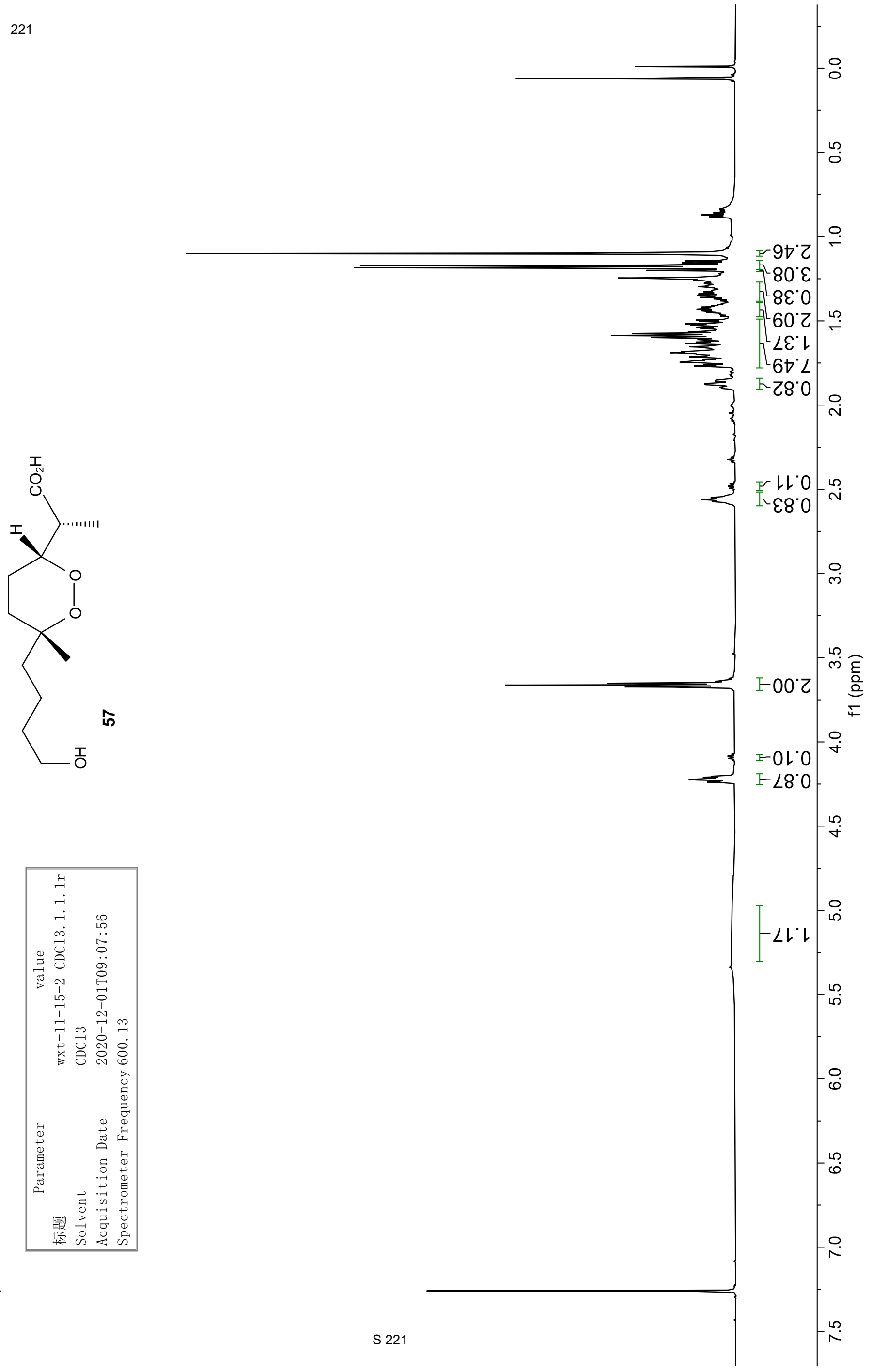
†เS9

\&乙99 $\varepsilon-$

乙E $\angle 9^{\circ} \varepsilon-$
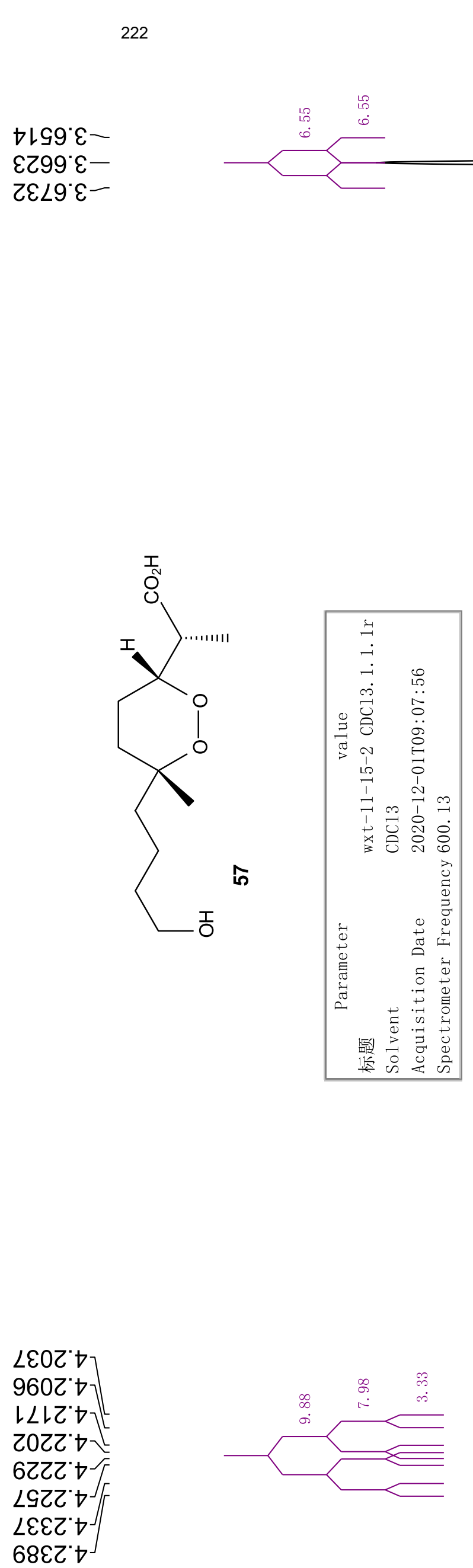


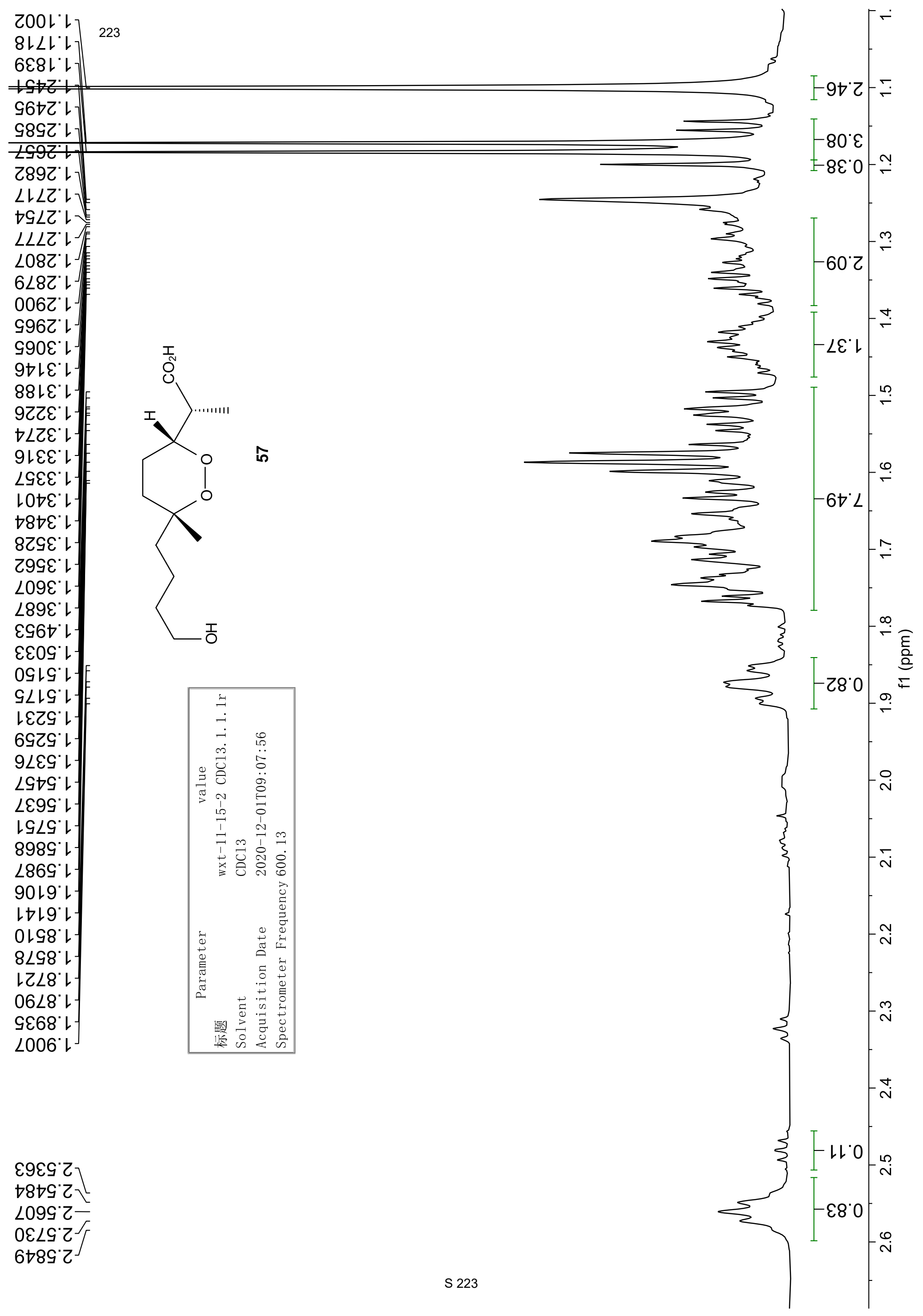


96てレ

99.6L

$\angle 9: 2 Z$

98. $\varepsilon Z^{-}$

IS'ZE

$18 . Z \varepsilon$

$6 Z^{\circ} \downarrow \varepsilon$

09'Z๐'

L9'29-

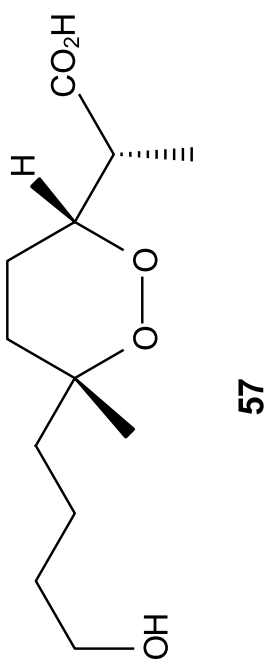

SI. 08

IE'L8

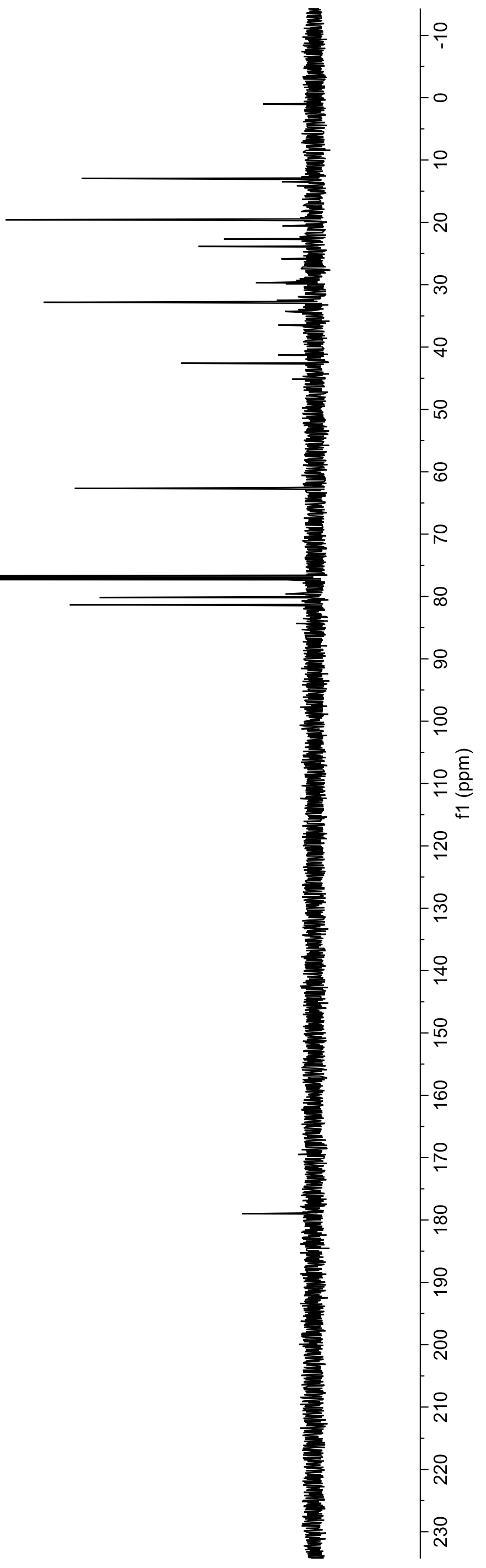




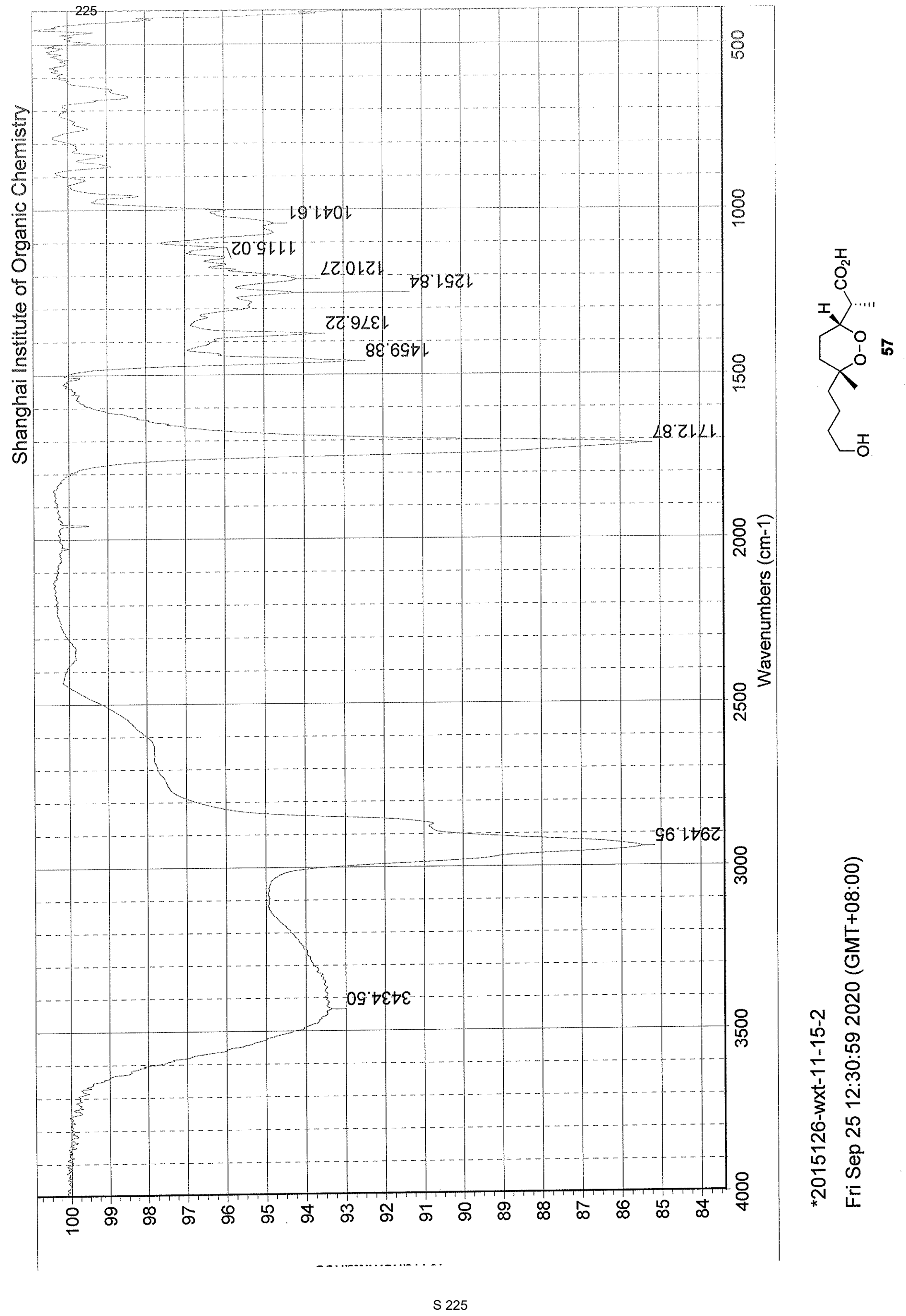




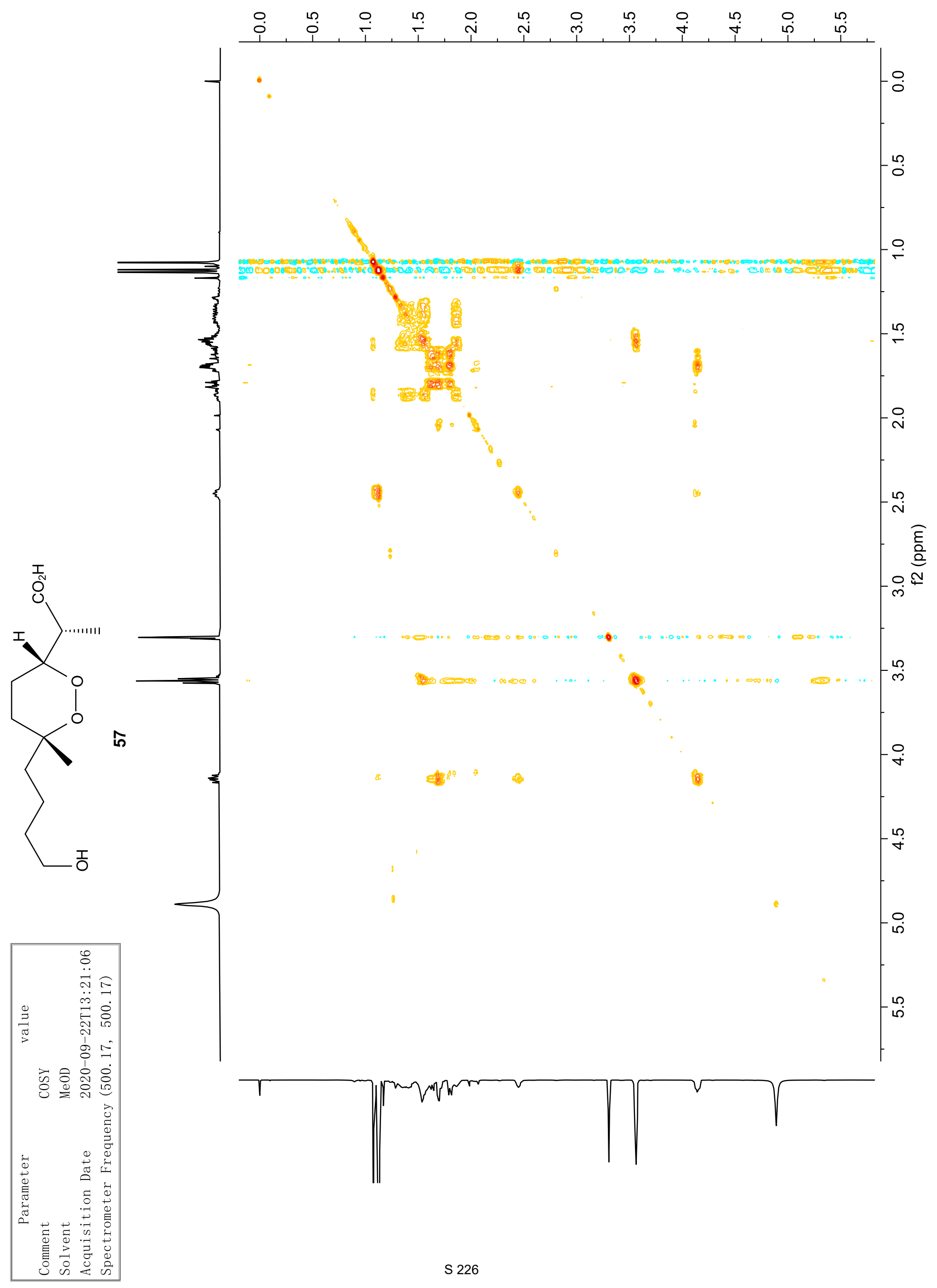




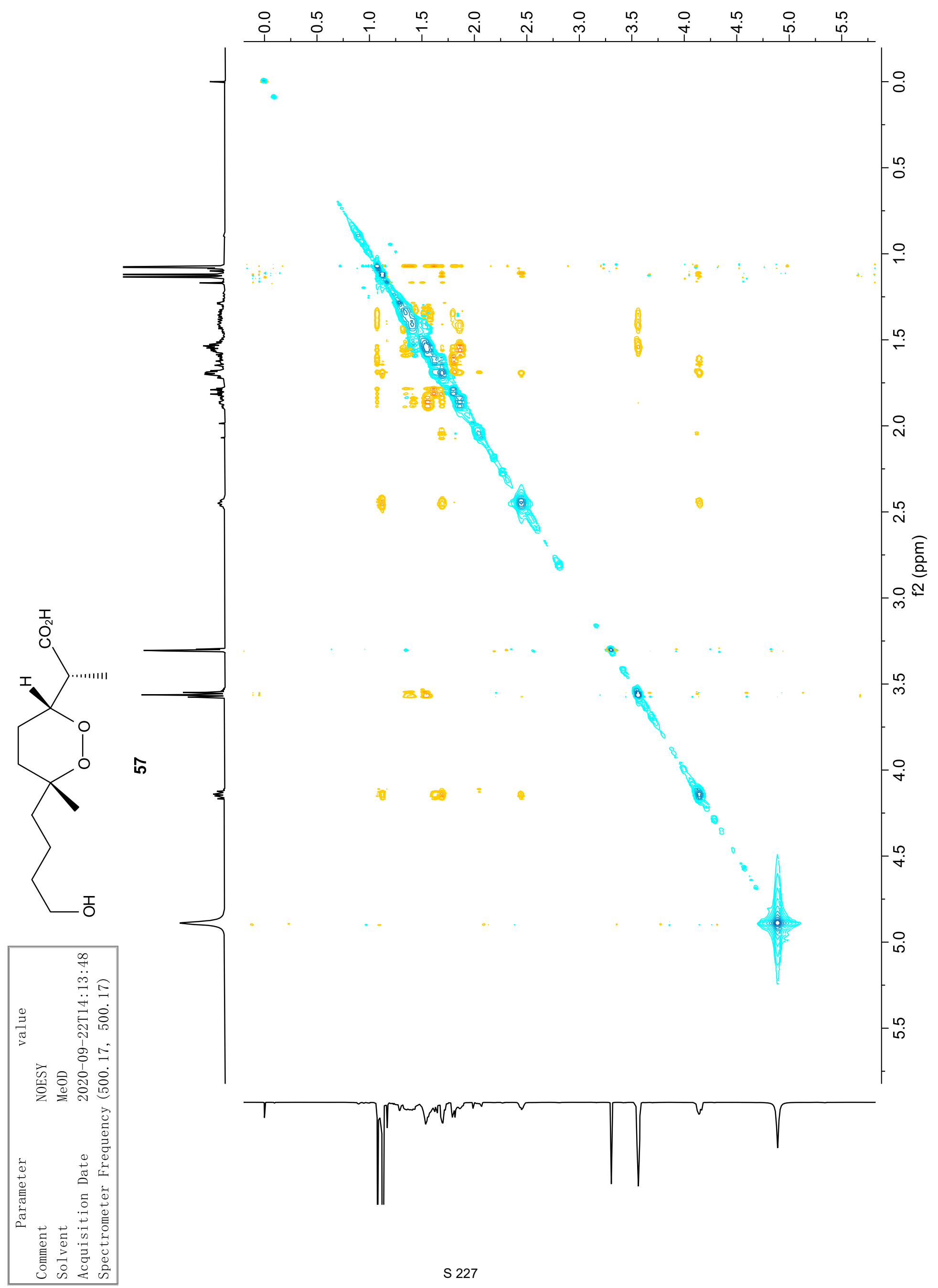




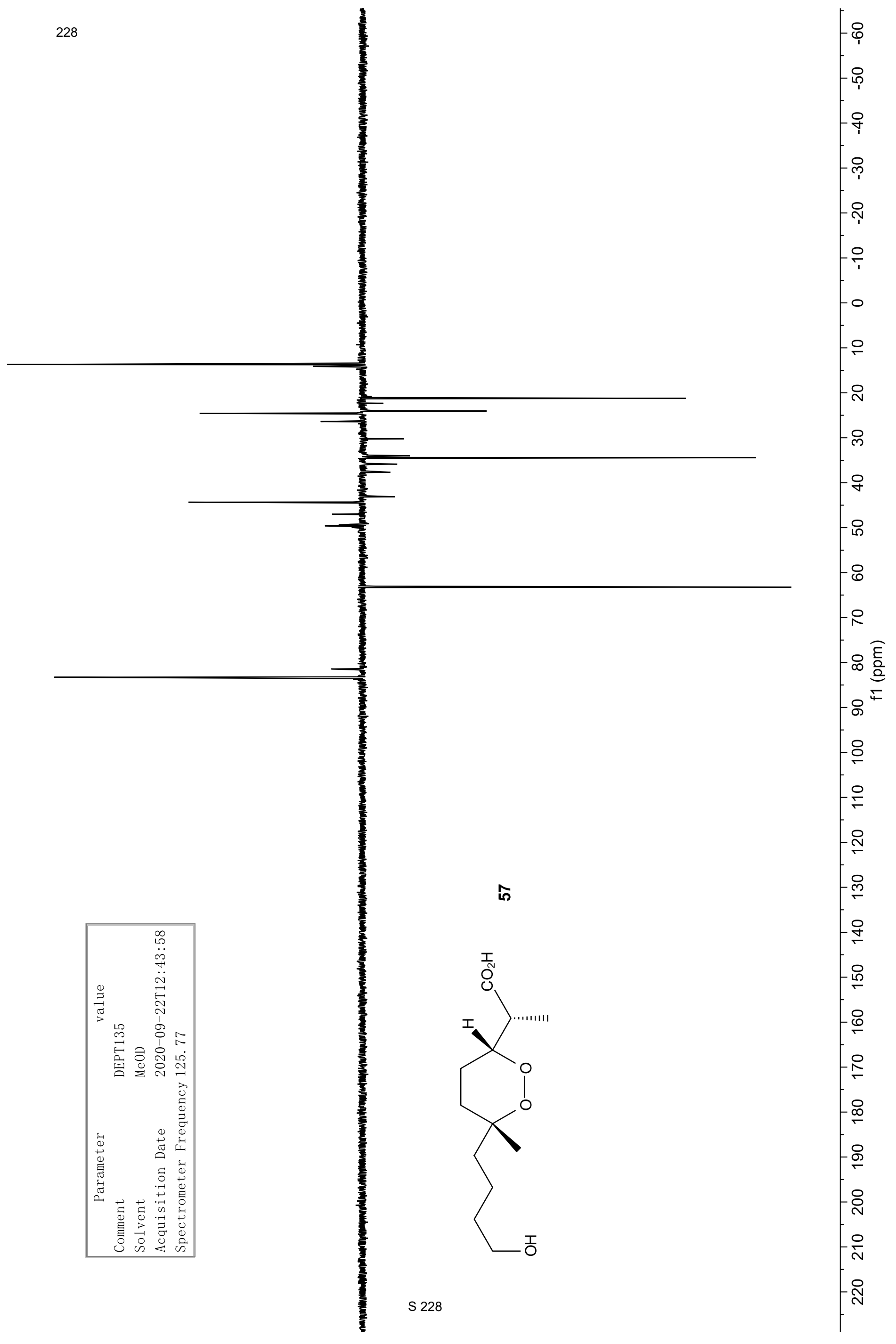




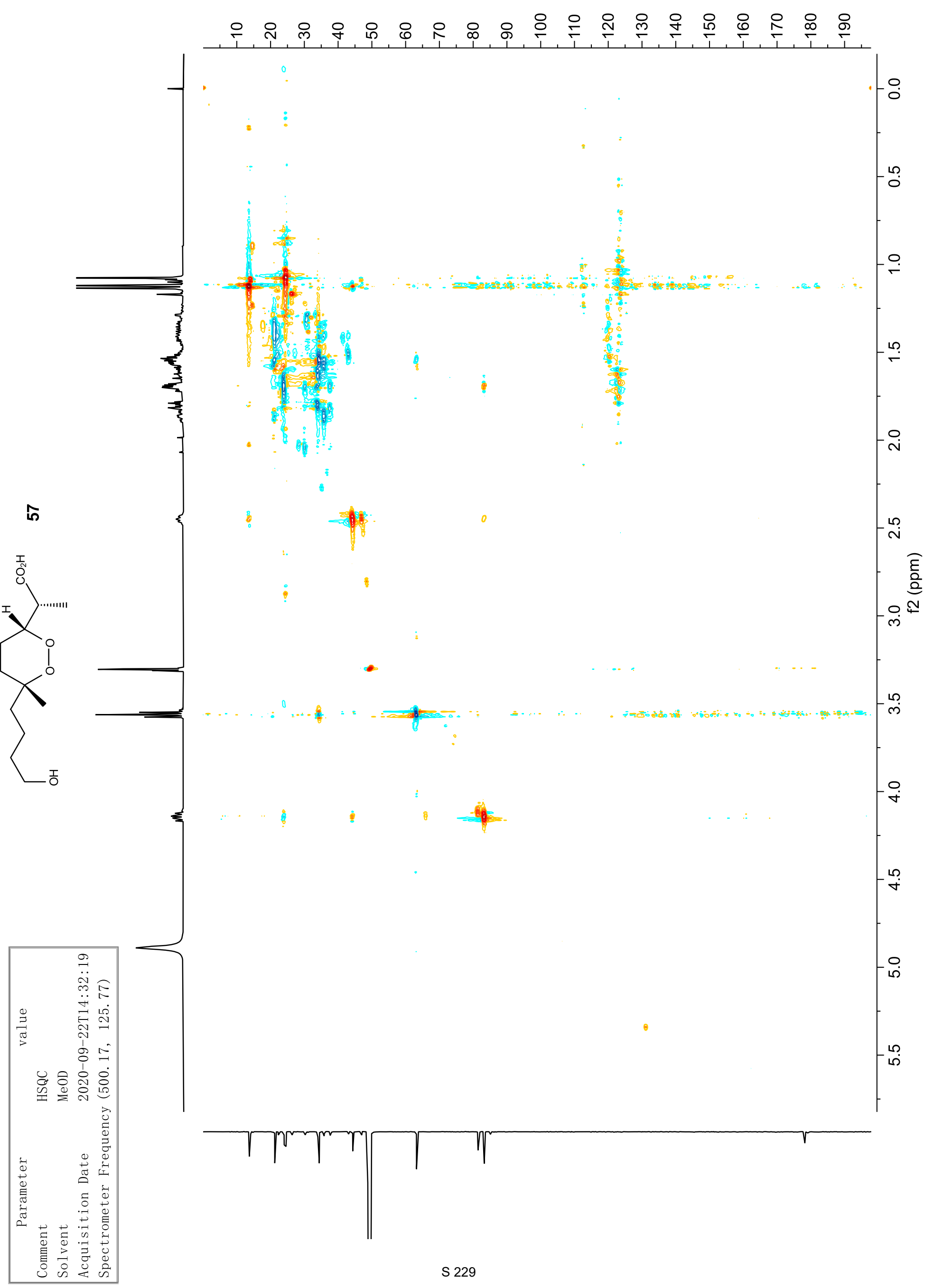




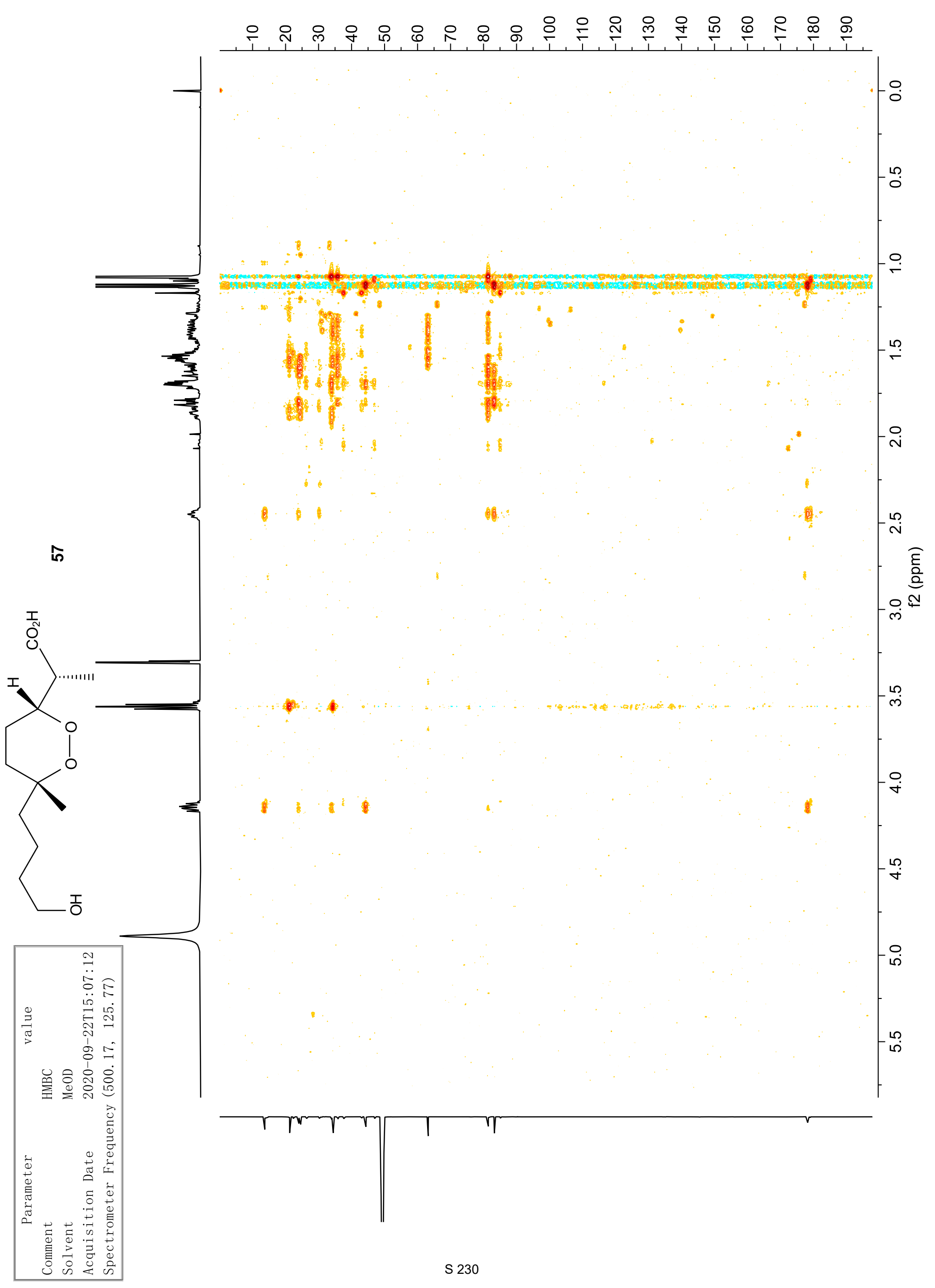




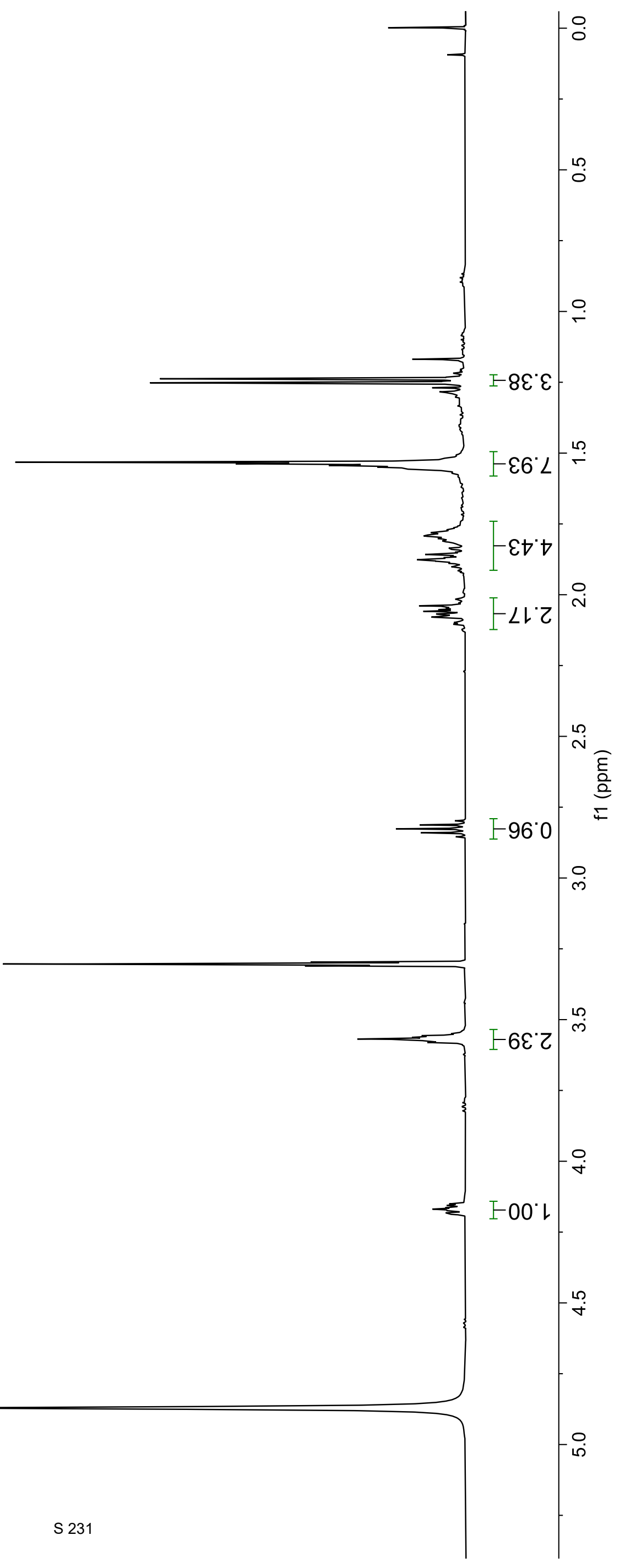

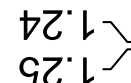

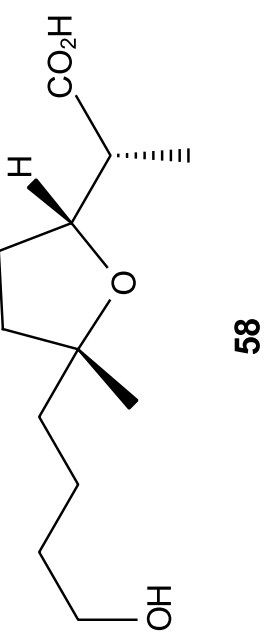

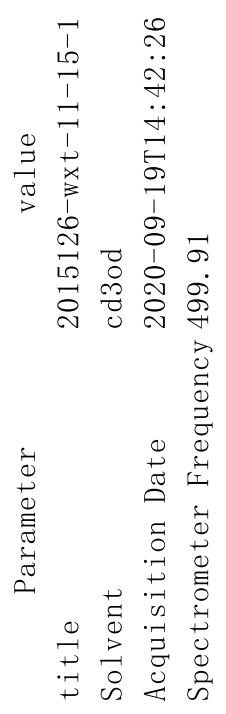

$\stackrel{\circ}{n}$ 


\section{$0 \angle t \mathcal{G}^{\circ} \varepsilon$ \\ 96tG $\varepsilon$ \\ 86tG $\varepsilon$ \\ Z999 $\varepsilon$ \\ \&699. $\varepsilon-$ \\ 9799' $\varepsilon^{\prime}$ \\ ${ }^{\circ} 899^{\circ} \varepsilon^{]}$ \\ $\angle Z \angle G^{\circ} \varepsilon$ \\ $\nabla G \angle G^{\circ} \varepsilon$ \\ 8089 ${ }^{\circ}$}
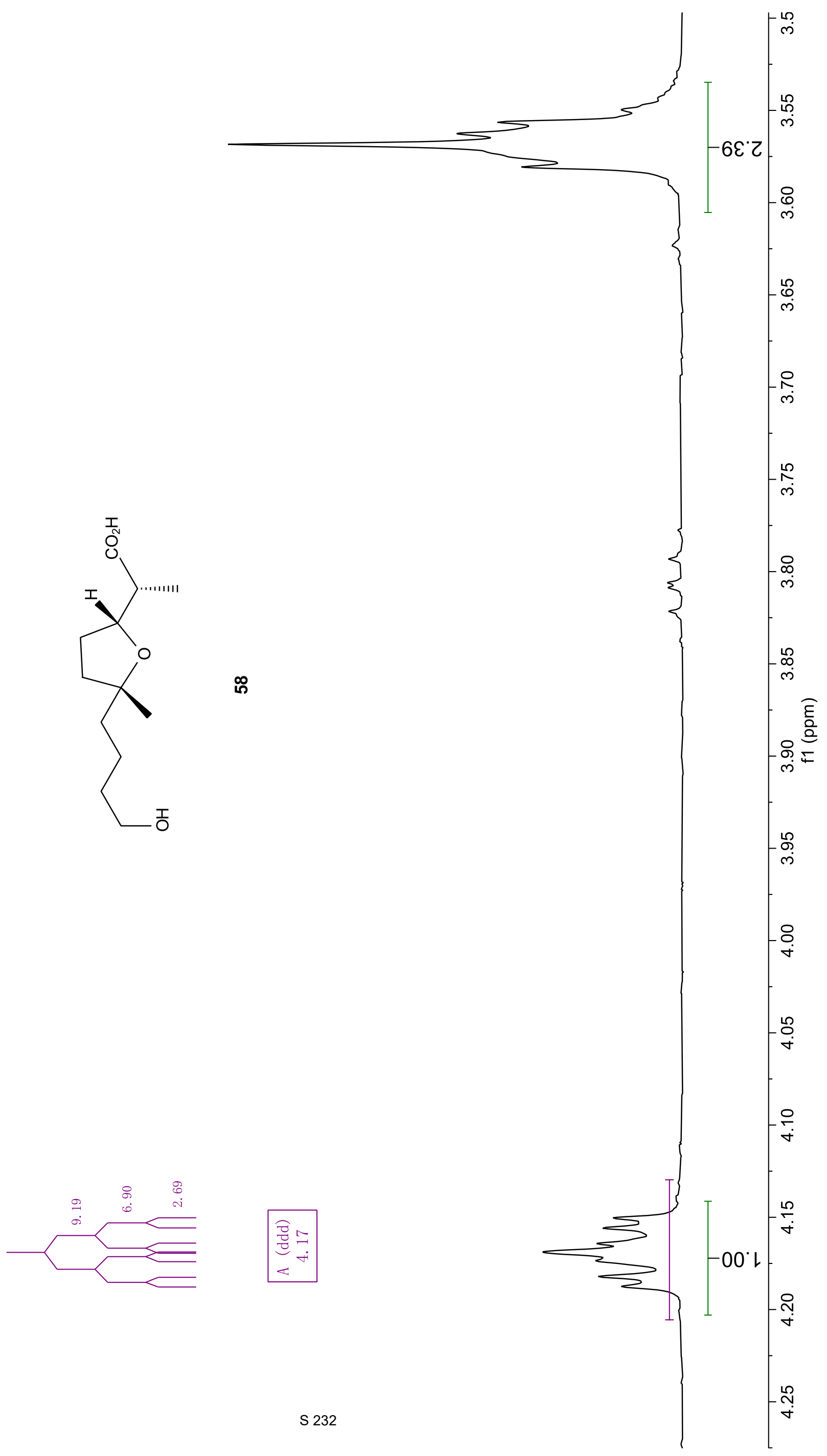

$889 L^{\circ} t$

$8 \varepsilon L L^{\circ} \nabla^{\top}$

乙८8 $\nabla$

$9 \angle 8 L^{\circ}$ 


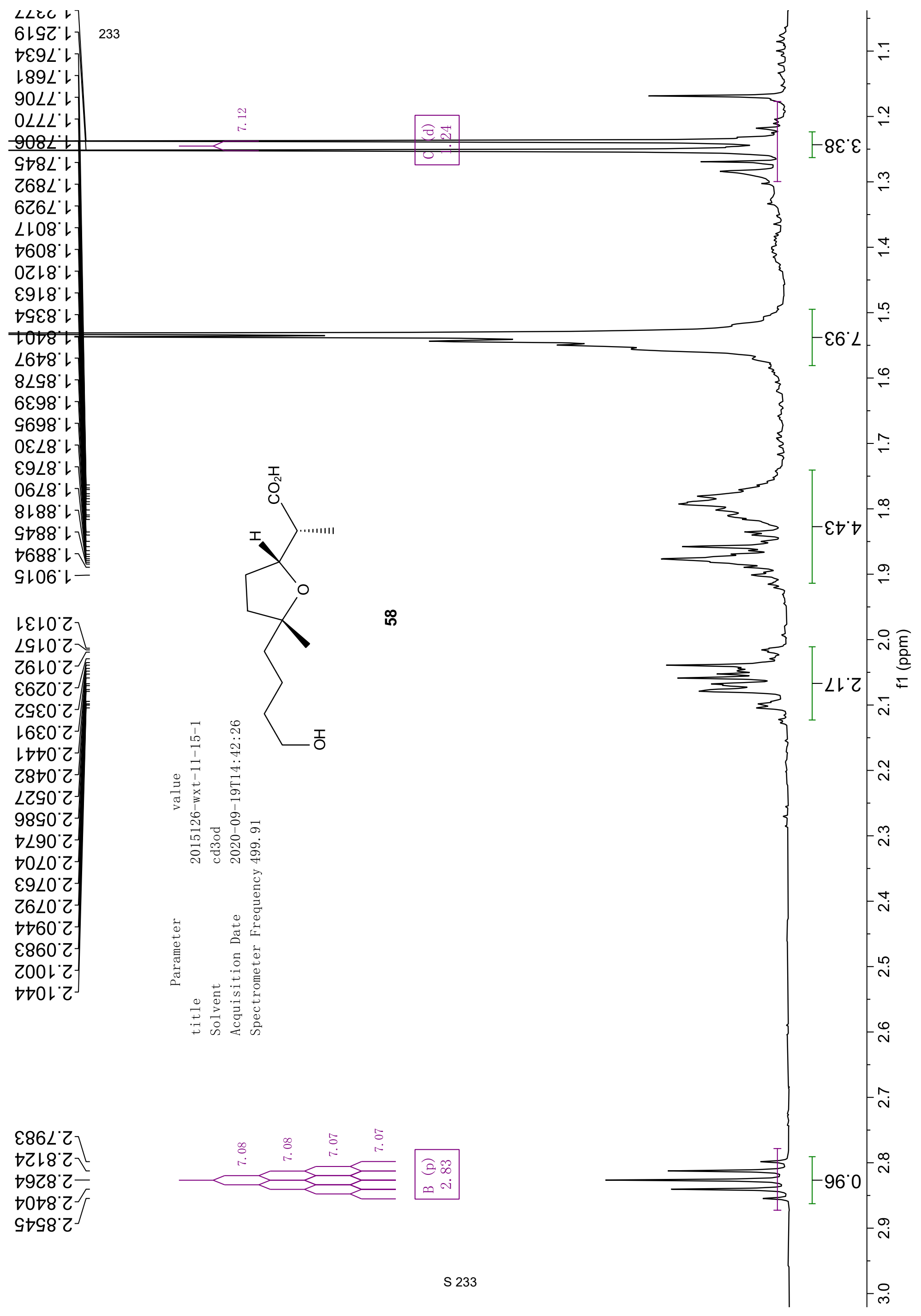


งでャレー

OZ'ZZ-

$\varepsilon L^{\circ} 0 \varepsilon>$

$80^{\circ} L \varepsilon^{-}$

L' $\varepsilon^{\prime} \varepsilon^{\prime}$

SLIVD

$\nabla Z \cdot S b-$

$\varepsilon L ' 79-$

90.99-

$\downarrow 8 \cdot \nabla L-$

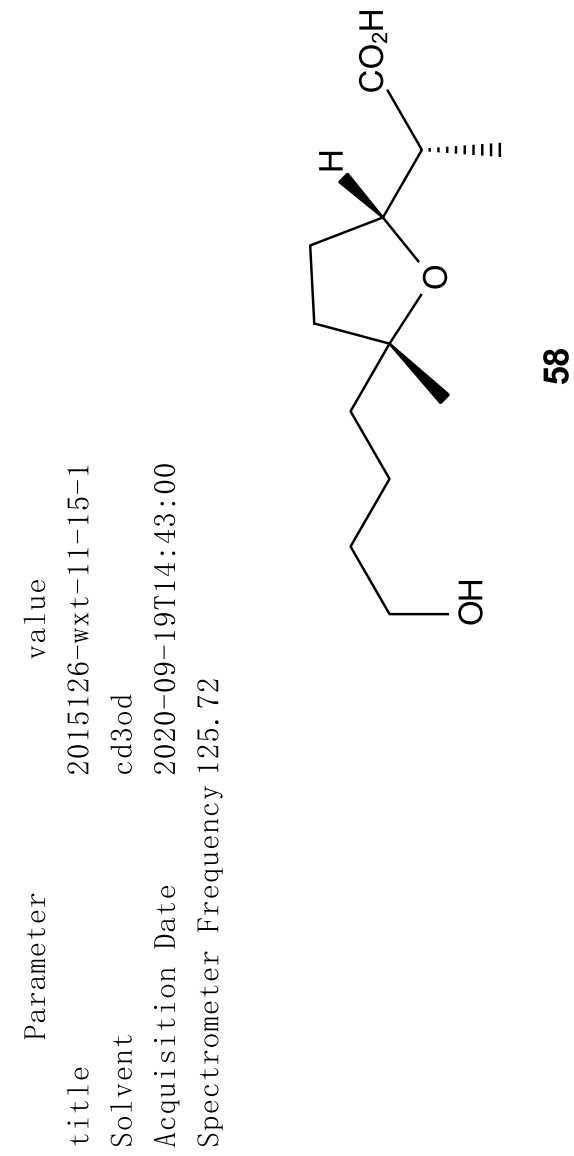

$18^{\circ} 9 L 1-$

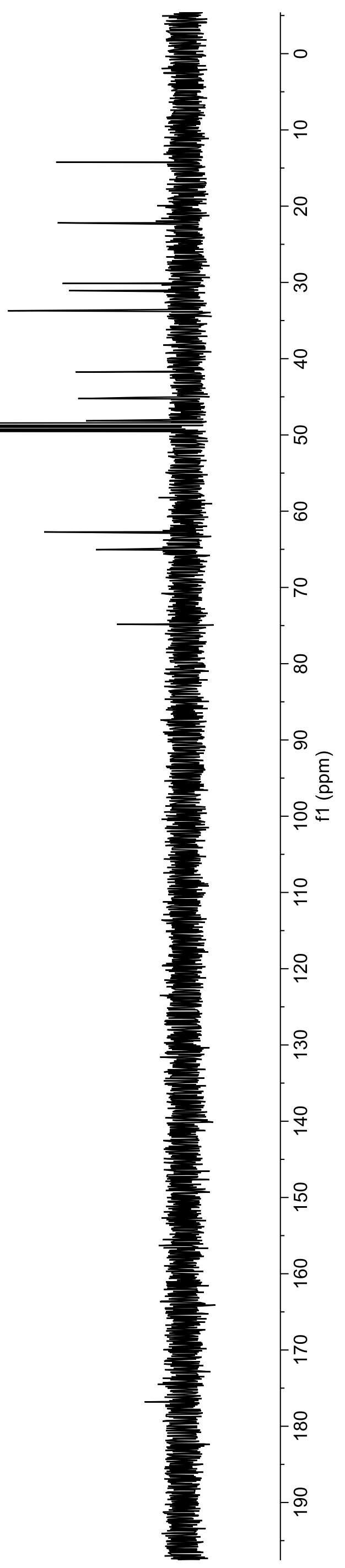




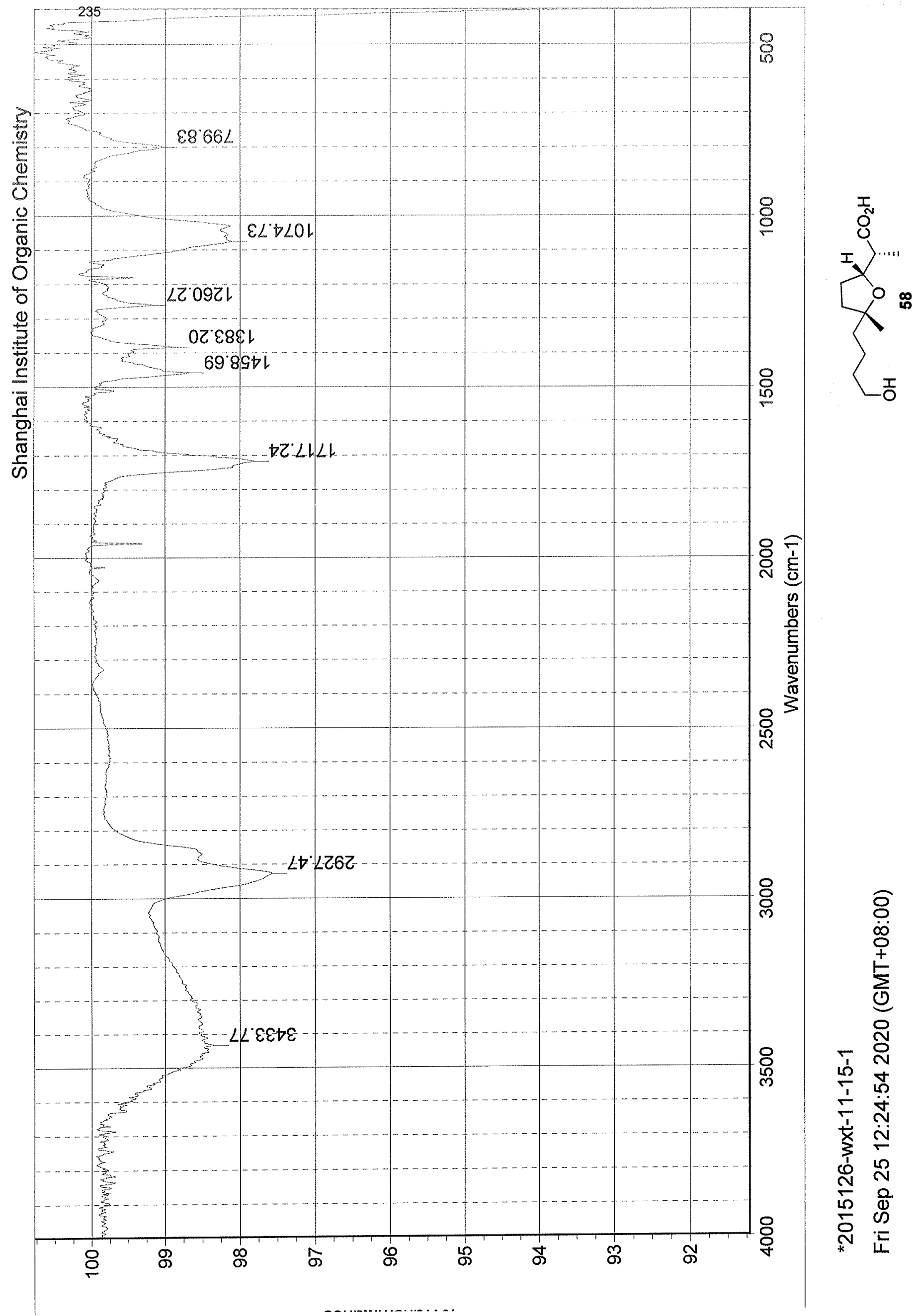


(The numbering system used in $J$ Nat Prod 2013, 76, 1541 is adopted)

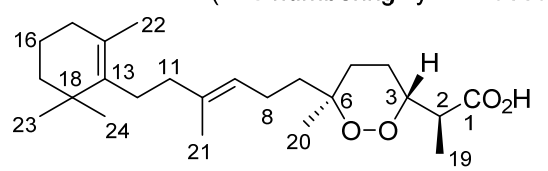

(+)-muqubilin A

$T L$ 1984, 931, $[\alpha]_{\mathrm{D}}+31.6\left(c 0.18 \mathrm{M}, \mathrm{CHCl}_{3}\right)$

This work, $[\alpha]_{D}+30.4\left(c 0.18, \mathrm{CHCl}_{3}\right)$

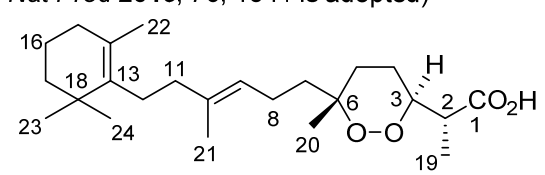

(-)-muqubilin A

J Nat Prod 1998, 61, 241, $[\alpha]_{D}-35.6\left(c 9.8, \mathrm{CHCl}_{3}\right)$

J Nat Prod 2013, 76, 1541, $[\alpha]_{D}-32.7\left(c 0.34, \mathrm{CHCl}_{3}\right)$

Table S1. Comparison of ${ }^{13} \mathrm{C}$ NMR (in $\mathrm{CDCl}_{3}$ ) of muqubilin 1.

\begin{tabular}{|c|c|c|c|c|}
\hline $\begin{array}{l}(+) \text {-natural } \\
(25 \mathrm{MHz})^{a}\end{array}$ & $\begin{array}{l}\text { (-)-natural } \\
(62.5 \mathrm{MHz})^{b}\end{array}$ & $\begin{array}{l}(-) \text {-natural } \\
(75 \mathrm{MHz})^{c}\end{array}$ & $\begin{array}{l}\text { (+)-synthetic } \\
(125 \mathrm{MHz})^{d}\end{array}$ & $\begin{array}{l}\text { (+)-synthetic } \\
(125 \mathrm{MHz})^{e}\end{array}$ \\
\hline $180.4(\mathrm{C}-1)$ & $180.0(\mathrm{C}-1)$ & $178.25(\mathrm{C}-1)$ & 177.92 & 178.49 \\
\hline $137.1(\mathrm{C}-13)$ & $137.2(\mathrm{C}-13)$ & $137.10(\mathrm{C}-13)$ & 137.11 & 137.10 \\
\hline $136.5(\mathrm{C}-10)$ & $136.6(\mathrm{C}-10)$ & $136.50(\mathrm{C}-10)$ & 136.55 & 136.51 \\
\hline $126.9(\mathrm{C}-14)$ & $127.0(\mathrm{C}-14)$ & $126.93(\mathrm{C}-14)$ & 126.99 & 126.95 \\
\hline $123.3(\mathrm{C}-9)$ & $123.3(\mathrm{C}-9)$ & $123.21(\mathrm{C}-9)$ & 123.23 & 123.22 \\
\hline $81.0(\mathrm{C}-3)$ & $81.1(\mathrm{C}-3)$ & $81.24(\mathrm{C}-3)$ & 81.80 & 80.98 \\
\hline $80.2(\mathrm{C}-6)$ & $80.3(\mathrm{C}-6)$ & $80.29(\mathrm{C}-6)$ & 80.30 & 80.26 \\
\hline $43.0(\mathrm{C}-2)$ & $43.0(\mathrm{C}-2)$ & $43.03(\mathrm{C}-2)$ & 42.64 & 42.71 \\
\hline $40.3(\mathrm{C}-17)$ & $40.3(\mathrm{C}-16)$ & $40.22(\mathrm{C}-17)$ & 40.19 & 40.20 \\
\hline $39.9(\mathrm{C}-11)$ & $39.9(\mathrm{C}-11)$ & $39.86(\mathrm{C}-11, \mathrm{C}-7)$ & 39.81 & 39.83 \\
\hline 39.7 (C-5) & $39.7(\mathrm{C}-5)$ & & 39.80 & 39.81 \\
\hline $35.0(\mathrm{C}-18)$ & $35.0(\mathrm{C}-18)$ & $34.97(\mathrm{C}-18)$ & 34.96 & 34.96 \\
\hline $32.8(\mathrm{C}-15)$ & $32.8(\mathrm{C}-15)$ & $32.76(\mathrm{C}-15)$ & 32.73 & 32.75 \\
\hline $32.0(\mathrm{C}-4)$ & $32.0(\mathrm{C}-7)$ & $32.03(\mathrm{C}-5)$ & 32.17 & 31.91 \\
\hline $28.7(\mathrm{C}-23, \mathrm{C}-24)$ & $28.7(\mathrm{C}-23, \mathrm{C}-24)$ & $28.62(\mathrm{C}-23, \mathrm{C}-24)$ & 28.59 (2C here) & 28.60 (2 C here) \\
\hline $27.9(\mathrm{C}-12)$ & $27.9(\mathrm{C}-12)$ & $27.83(\mathrm{C}-12)$ & 27.80 & 27.82 \\
\hline $23.5(\mathrm{C}-7)$ & $23.5(\mathrm{C}-4)$ & $23.41(\mathrm{C}-4)$ & 23.42 & 23.39 \\
\hline $21.7(\mathrm{C}-8)$ & $21.7(\mathrm{C}-8)$ & $21.65(\mathrm{C}-8)$ & 21.62 & 21.63 \\
\hline $20.8(\mathrm{C}-20)$ & $20.8(\mathrm{C}-20)$ & $20.66(\mathrm{C}-20)$ & 20.65 & 20.67 \\
\hline $19.9(\mathrm{C}-22)$ & $19.9(\mathrm{C}-22)$ & $19.81(\mathrm{C}-22$ or $\mathrm{C}-16)$ & 19.80 & 19.80 \\
\hline $19.6(\mathrm{C}-16)$ & $19.6(\mathrm{C}-17)$ & $19.55(\mathrm{C}-16$ or $\mathrm{C}-22)$ & 19.52 & 19.54 \\
\hline $16.1(\mathrm{C}-21)$ & $16.1(\mathrm{C}-21)$ & $15.99(\mathrm{C}-21)$ & 15.99 & 16.00 \\
\hline $13.2(\mathrm{C}-19)$ & $13.3(\mathrm{C}-19)$ & $13.21(\mathrm{C}-19)$ & 13.20 & 13.19 \\
\hline
\end{tabular}

${ }^{a}$ Data along with the assignments (converted to the present numbering system for direct comparison) taken from Manes, L. V.; Bakus, G. J.; Crews, P. Tetrahedron Lett. 1984, 931-934 (the sample was obtained from a MeOH solution without specifying chromatographic conditions). ${ }^{b}$ Data along with the assignments (converted to the present numbering system for direct comparison) taken from Sperry, S.; Valeriote, F. A.; Corbett, T. H.; Crews, P. J. Nat. Prod. 1998, 61, 931-934 (the sample was obtained by reverse phase chromatography eluting with $90 \%$ MeCN). ${ }^{c}$ Data taken from Lefranc, F.; Nuzzo, G.; Hamdy, N. A.; Fakhr, I. Banuls, L. M. Y.; van Goietenoven, G.; Villani, G.; Mathieu, V.; van Soest, R.; Kiss, R.; Ciavatta, M. L. J. Nat. Prod. 2013, 76, 1541-1547 (the sample was obtained by normal phase chromatography eluting with petroleum ether/ $\mathrm{Et}_{2} \mathrm{O}$ ). The chemical shifts were directly read from the scanned spectrum in the Supporting Information (p. 17) without including the signal of grease/high boiling residues (cf. Gottlib, H. E.; Kotlyar, V.; Nudelman, A. J. Org. Chem. 1997, 62, 7512-7515) at $\delta 29.652$ ppm. The assignments were taken from the Table A on p. 19 of the Supporting Information, where the shift of C-12 was erroneously typed as 28.6 (which was already assigned to C-23 and C-24) instead of 27.83 (clearly seen the scanned spectrum on p. 17 of the Supporting Information). The assignments of C-16 and C-22 might be switchable because the two peaks at 19.81 and $19.55 \mathrm{ppm}$ in the scanned spectrum were both listed as 19.8 in the Table A and assigned to $\mathrm{C}-16$ and $\mathrm{C}-22$, respectively. ${ }^{d}$ This work (the sample was obtained by normal phase chromatography). ${ }^{e}$ This work, the sample was dissolved in $\mathrm{MeCN}$ (containing $\mathrm{H}_{2} \mathrm{O}$ ) and concentrated before recording NMR (note that the change of the signal for $\mathrm{C}-1$ was much more significant than all other signals). 


\section{Additional experimental procedures}
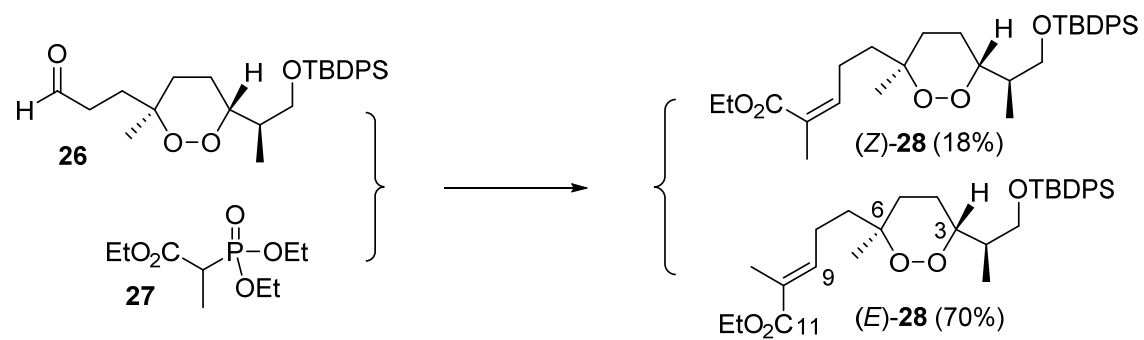

(E)-5-((3S,6R)-6-((R)-1-((tert-Butyldiphenylsilyl)oxy)propan-2-yl)-3-methyl-1,2-dioxan-3-yl)-2-me thylpent-2-enoate ((E)-28). Ethyl 2-(diethoxyphosphoryl)propanoate $27(22.6 \mathrm{mg}, 0.095 \mathrm{mmol})$ and DBU $(13 \mu \mathrm{L}, 0.083 \mathrm{mmol})$ were added in turn to a solution of $\mathrm{LiCl}(4 \mathrm{mg}, 0.095 \mathrm{mmol})$ in THF $(1 \mathrm{~mL})$ stirred in an ice-water bath. After completion of the addition, stirring was continued at the same temperature for $30 \mathrm{~min}$. A solution of aldehyde $26(29 \mathrm{mg}, 0.064 \mathrm{mmol})$ in THF $(0.2$ $\mathrm{mL}$ ) was then introduced slowly via a syringe. The mixture was stirred in the ice-water bath for another $30 \mathrm{~min}$. Water $(1 \mathrm{~mL})$ was added. The mixture was extracted with EtOAc $(5 \mathrm{~mL} \times 2)$. The combined organic layers were washed with brine $(5 \mathrm{~mL})$ and dried over anhydrous $\mathrm{Na}_{2} \mathrm{SO}_{4}$. Removal of the drying agent by filtration and rotary evaporation of the filtrate gave a crude oil, which was purified by column chromatography $(20: 1 \mathrm{PE} / \mathrm{EtOAc})$ on silica gel to afford first (Z)-28 (a colorless oil, $6.3 \mathrm{mg}, 0.012 \mathrm{mmol}, 18 \%$ from 26) and then (E)-28 as (a colorless oil, 24 $\mathrm{mg}, 0.045 \mathrm{mmol}, 70 \%$ ). Data for (E)-28 (more polar than (Z)-28): $[\alpha]_{\mathrm{D}}{ }^{25}+34.5\left(c 1.0, \mathrm{CHCl}_{3}\right) .{ }^{1} \mathrm{H}$ $\operatorname{NMR}\left(500 \mathrm{MHz}, \mathrm{CDCl}_{3}\right) \delta$ 7.71-7.62 (m, 4H), 7.47-7.33 (m, 6H), $6.71(\mathrm{t}, J=7.4 \mathrm{~Hz}, 1 \mathrm{H}), 4.18(\mathrm{q}$ $J=7.1 \mathrm{~Hz}, 2 \mathrm{H}), 4.10-4.01(\mathrm{~m}, 1 \mathrm{H}), 3.65(\mathrm{dd}, J=10.1,5.2 \mathrm{~Hz}, 1 \mathrm{H}), 3.59(\mathrm{dd}, J=10.1,5.6 \mathrm{~Hz}$, $1 \mathrm{H}), 2.31-2.17(\mathrm{~m}, 2 \mathrm{H}), 1.83(\mathrm{~s}, 3 \mathrm{H}), 1.78-1.68(\mathrm{~m}, 2 \mathrm{H}), 1.66-1.49(\mathrm{~m}, 5 \mathrm{H}), 1.32(\mathrm{~s}, 3 \mathrm{H}), 1.29(\mathrm{t}, J$ $=7.1 \mathrm{~Hz}, 3 \mathrm{H}), 1.06(\mathrm{~s}, 9 \mathrm{H}), 1.04(\mathrm{~d}, J=6.9 \mathrm{~Hz}, 3 \mathrm{H}) ;{ }^{13} \mathrm{C}\left\{{ }^{1} \mathrm{H}\right\} \mathrm{NMR}\left(126 \mathrm{MHz}, \mathrm{CDCl}_{3}\right) \delta 168.1$, 141.5, 135.6, 135.6, 133.7, 133.6, 129.6, 128.1, 127.6, 82.1, 79.4, 65.5, 60.4, 39.3, 39.0, 32.7, 26.8, 23.6, 22.4, 20.3, 19.3, 14.3, 12.7, 12.3. FT-IR (film of a concd solution in $\mathrm{CHCl}_{3}$ ) 2931, 2858, 1709, 1428, 1279, 1112, 823, $702 \mathrm{~cm}^{-1}$. MS (ESI) $\mathrm{m} / \mathrm{z}: 561.55\left([\mathrm{M}+\mathrm{Na}]^{+}\right)$; HRMS (ESI) $\mathrm{m} / z$ : $[\mathrm{M}$ $+\mathrm{Na}]^{+}$calcd. for $\mathrm{C}_{32} \mathrm{H}_{46} \mathrm{O}_{5} \mathrm{NaSi}$ 561.3007, found 561.3015. Data for (Z)-28 (less polar than (E)-28): $[\alpha]_{\mathrm{D}}{ }^{25}+43.0\left(c \mathrm{c} 0.5, \mathrm{CHCl}_{3}\right) .{ }^{1} \mathrm{H}$ NMR $\left(500 \mathrm{MHz}, \mathrm{CDCl}_{3}\right) \delta$ 7.68-7.62 (m, 4H), 7.47-7.34 (m, 6H), 5.90 (t, $J=7.6 \mathrm{~Hz}, 1 \mathrm{H}), 4.19(\mathrm{q}, J=7.1 \mathrm{~Hz}, 2 \mathrm{H}), 4.07-4.01(\mathrm{~m}, 1 \mathrm{H}), 3.64(\mathrm{dd}, J=10.1$, $5.1 \mathrm{~Hz}, 1 \mathrm{H}), 3.58(\mathrm{dd}, J=10.1,5.7 \mathrm{~Hz}, 1 \mathrm{H}), 2.61-2.50(\mathrm{~m}, 1 \mathrm{H}), 2.50-2.37(\mathrm{~m}, 1 \mathrm{H}), 1.88(\mathrm{~s}, 3 \mathrm{H})$, 1.77-1.68 (m, 2H), 1.66-1.47 (m, 6H), 1.31 (s, 3H), 1.30 (t, J=7.1 Hz, 3H), 1.05 (s, 9H), 1.04 (d, $J=7.0 \mathrm{~Hz}, 3 \mathrm{H}) ;{ }^{13} \mathrm{C}\left\{{ }^{1} \mathrm{H}\right\} \mathrm{NMR}\left(126 \mathrm{MHz}, \mathrm{CDCl}_{3}\right) \delta 168.0,142.2,135.6,135.6,133.7,133.6$, 129.6, 127.6, 127.4, 82.1, 79.7, 65.5, 60.1, 39.3, 32.6, 26.8, 24.1, 23.6, 23.5, 20.7, 20.2, 19.3, 14.3, 12.7. FT-IR (film of a concd solution in $\mathrm{CHCl}_{3}$ ) 2929, 2857, 1714, 1472, 1374, 1261, 1230, 1112, 1026, 802, $702 \mathrm{~cm}^{-1}$. MS (ESI) m/z: $561.45\left([\mathrm{M}+\mathrm{Na}]^{+}\right)$; HRMS (ESI) $\mathrm{m} / \mathrm{z}:[\mathrm{M}+\mathrm{Na}]^{+}$calcd. for $\mathrm{C}_{32} \mathrm{H}_{46} \mathrm{O}_{5} \mathrm{NaSi}$ 561.3007, found 561.3009 .

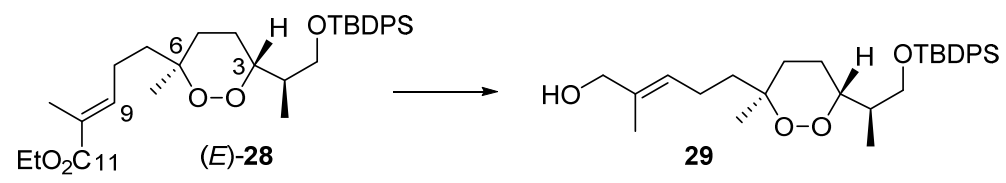

(E)-5-((3S,6R)-6-((R)-1-((tert-Butyldiphenylsilyl)oxy)propan-2-yl)-3-methyl-1,2-dioxan-3-yl)-2-me thylpent-2-en-1-ol (29). DIABL-H $(1.0 \mathrm{M}$, in cyclohexane, $0.11 \mathrm{~mL}, 0.11 \mathrm{mmol})$ was added 
dropwise via a syringe to a solution of $(E)-28(8.05 \mathrm{~g}, 16.4 \mathrm{mmol})$ in dry $\mathrm{CH}_{2} \mathrm{Cl}_{2}(0.5 \mathrm{~mL})$ stirred in a dry ice-acetone bath $\left(-78{ }^{\circ} \mathrm{C}\right)$ under argon (balloon). After completion of the addition, the mixture was stirred at the same temperature for ca. $30 \mathrm{~min}$ (when TLC showed completion of the reduction). A saturated solution $(3 \mathrm{~mL})$ of sodium potassium tartrate in water was then added. The mixture was stirred violently for $2 \mathrm{~h}$, when both organic and the aqueous phases became clear. The mixture was extracted with EtOAc $(5 \mathrm{~mL} \times 3)$. The combined organic layers were washed with brine and dried over anhydrous $\mathrm{Na}_{2} \mathrm{SO}_{4}$. Removal of the drying agent by filtration and rotary evaporation of the filtrate gave a crude oil, which was purified by column chromatography (3:1 $\mathrm{PE} / \mathrm{EtOAc})$ on silica gel to afford 29 as a colorless oil $(18.5 \mathrm{mg}, 0.037 \mathrm{mmol}, 85 \%)$. $[\alpha]_{\mathrm{D}}{ }^{25}+51.1$ (c 1.0, $\left.\mathrm{CHCl}_{3}\right) .{ }^{1} \mathrm{H}$ NMR $\left(500 \mathrm{MHz}, \mathrm{CDCl}_{3}\right) \delta$ 7.73-7.59 (m, 4H), 7.51-7.34 (m, 6H), 5.38 (t, $J=$ $7.3 \mathrm{~Hz}, 1 \mathrm{H}), 4.08-4.02(\mathrm{~m}, 1 \mathrm{H}), 3.99$ (s, 2H), 3.65 (dd, $J=10.2,5.1 \mathrm{~Hz}, 1 \mathrm{H}), 3.59$ (dd, $J=10.2$, $5.6 \mathrm{~Hz}, 1 \mathrm{H}), 2.14-2.03(\mathrm{~m}, 2 \mathrm{H}), 1.81-1.68(\mathrm{~m}, 2 \mathrm{H}), 1.67(\mathrm{~s}, 3 \mathrm{H}), 1.64-1.41(\mathrm{~m}, 6 \mathrm{H}), 1.32(\mathrm{~s}, 3 \mathrm{H})$, $1.06(\mathrm{~s}, 9 \mathrm{H}), 1.04(\mathrm{~d}, J=6.9 \mathrm{~Hz}, 3 \mathrm{H}) ;{ }^{13} \mathrm{C}\left\{{ }^{1} \mathrm{H}\right\} \mathrm{NMR}\left(126 \mathrm{MHz}, \mathrm{CDCl}_{3}\right) \delta$ 135.6, 135.6, 135.0, 133.7, 133.6, 129.6, 127.6, 125.7, 82.1, 79.6, 68.8, 65.5, 40.1, 39.3, 32.8, 26.8, 23.6, 21.3, 20.2, 19.3, 13.6, 12.7. FT-IR (film of a concd solution in $\mathrm{CHCl}_{3}$ ) 3399, 2931, 2857, 1428, 1112, 1008, 823, 740, $702 \mathrm{~cm}^{-1}$. MS (ESI) $\mathrm{m} / \mathrm{z}: 519.45\left([\mathrm{M}+\mathrm{Na}]^{+}\right.$); HRMS (ESI) $\mathrm{m} / \mathrm{z}:[\mathrm{M}+\mathrm{Na}]^{+}$calcd. for $\mathrm{C}_{30} \mathrm{H}_{44} \mathrm{O}_{4} \mathrm{NaSi}$ 519.2901; found 519.2908.

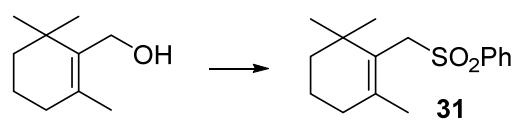

(((2,6,6-Trimethylcyclohex-1-en-1-yl)methyl)sulfonyl)benzene (31). To a solution of (2,6,6-trimethylcyclohex-1-en-1-yl)methanol $(1.0 \mathrm{~g}, 6.5 \mathrm{mmol})$ in THF $(40 \mathrm{~mL})$ stirred in a -30 ${ }^{\circ} \mathrm{C}$ bath (controlled by a cooling pump system) were added in turn $\mathrm{Et}_{3} \mathrm{~N}(1.8 \mathrm{~mL}, 13.0 \mathrm{mmol})$ and $\mathrm{MsCl}(0.6 \mathrm{~mL}, 7.8 \mathrm{mmol})$. The mixture was stirred at the same temperature for $30 \mathrm{~min}$. A solution of $\operatorname{LiBr}(2.25 \mathrm{~g}, 26 \mathrm{mmol})$ in THF $(20 \mathrm{~mL})$ was then introduced (via syringe) dropwise. After completion of the addition, the bath temperature was allowed to warm to $0{ }^{\circ} \mathrm{C}$. The mixture was stirred for another $30 \mathrm{~min}$. Aq. sat. $\mathrm{NH}_{4} \mathrm{Cl}(10 \mathrm{~mL})$ was added. The mixture was extracted with EtOAc $(5 \mathrm{~mL} \times 2)$. The combined organic layers were washed with brine and dried over anhydrous $\mathrm{Na}_{2} \mathrm{SO}_{4}$. Removal of the drying agent by filtration and rotary evaporation of the filtrate gave the oily crude bromide ( $1.3 \mathrm{~g}, 6.0 \mathrm{mmol}, 92 \%)$, a portion $(1.09 \mathrm{~g}, 5.0 \mathrm{mmol})$ of which was dissolved in DMF $(20 \mathrm{~mL})$. To the solution was added sodium benzenesulphinate $\left(\mathrm{PhSO}_{2} \mathrm{Na}, 1.9 \mathrm{~g}\right.$, $11.5 \mathrm{mmol})$. The mixture was stirred at ambient temperature overnight. Water $(40 \mathrm{~mL})$ was added. The mixture was extracted with $\mathrm{Et}_{2} \mathrm{O}(80 \mathrm{~mL})$. The aqueous layer was back-extracted with $\mathrm{Et}_{2} \mathrm{O}$ $(30 \mathrm{~mL})$. The combined organic layers were washed with brine and dried over anhydrous $\mathrm{Na}_{2} \mathrm{SO}_{4}$. Removal of the drying agent by filtration and rotary evaporation of the filtrate gave a crude oil, which was purified by column chromatography $(10: 1 \mathrm{PE} / \mathrm{EtOAc})$ on silica gel to afford known ${ }^{1}$ 31 as a white solid (1.18 g, $4.2 \mathrm{mmol}, 84 \%$ ). M. p. 92-94 ${ }^{\circ} \mathrm{C}$ (lit. ${ }^{1}$ m. p. 89-91 $\left.{ }^{\circ} \mathrm{C}\right) .{ }^{1} \mathrm{H}$ NMR (500 $\left.\mathrm{MHz}, \mathrm{CDCl}_{3}\right) \delta$ 7.96-7.89 (m, 2H), 7.68-7.62 (m, 1H), 7.58-7.53 (m, 2H), $3.96(\mathrm{~s}, 2 \mathrm{H}), 2.05(\mathrm{t}, J=$ $6.4 \mathrm{~Hz}, 2 \mathrm{H}), 1.67$ (s, 3H), 1.67-1.59 (m, 2H), 1.52-1.45 (m, 2H), $1.04(\mathrm{~s}, 6 \mathrm{H})$.

(1) Proszenyák, Á.; Charnock, C.; Hedner, E.; Larsson, R.; Bolin, L.; Gunersen, L.-L. Synthesis, Antimicrobial and Anti neoplastic Activities for Agelastine and Agelasimine Analogs with a $\beta$-Cyclocitral Derived Substituent. Arch. Pharm. Chem. Life Sci. 2007, 340, 625-634 (DOI: 10.1002/ardp.200700137). 


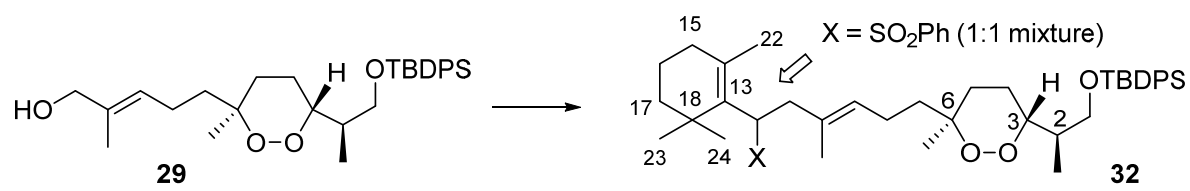

tert-Butyl((2R)-2-((3R,6S)-6-methyl-6-((E)-4-methyl-6-(phenylsulfonyl)-6-(2,6,6-trimethylcyclohex -1-en-1-yl)hex-3-en-1-yl)-1,2-dioxan-3-yl)propoxy)diphenylsilane (32). Synthesis of 32. To a solution of alcohol $29(17 \mathrm{mg}, 0.034 \mathrm{mmol})$ in THF $(0.4 \mathrm{~mL})$ stirred at $-30{ }^{\circ} \mathrm{C}$ under argon (balloon) were added in turn $\mathrm{Et}_{3} \mathrm{~N}(14 \mu \mathrm{L}, 0.102 \mathrm{mmol})$ and $\mathrm{MsCl}(5.3 \mu \mathrm{L}, 0.068 \mathrm{mmol})$. After completion of the addition, the mixture was stirred at the same temperature for $1 \mathrm{~h}$. A solution of $\mathrm{LiBr}(12 \mathrm{mg}, 0.137 \mathrm{mmol})$ in THF $(0.2 \mathrm{~mL})$ was introduced via a syringe. Stirring was continued at $0{ }^{\circ} \mathrm{C}$ for $30 \mathrm{~min}$. The mixture was filtered through a short pad of silica gel (washing with petroleum ether). The combined filtrate and washings were concentrated on a rotary evaporator to give crude bromide $\mathbf{3 0}(19 \mathrm{mg})$.

$n$-BuLi (2.0 M, in cyclohexane, $25.5 \mu \mathrm{L}, 0.051 \mathrm{mmol})$ was added to a solution of sulfone 31 (18.9 $\mathrm{mg}, 0.068 \mathrm{mmol})$ in THF $(0.3 \mathrm{~mL})$ stirred in $-78{ }^{\circ} \mathrm{C}$ (dry ice-EtOH bath) under argon (balloon). After completion of the addition, the mixture was stirred at the same temperature for $1 \mathrm{~h}$. Then the solution of the above prepared crude bromide $30(19 \mathrm{mg}, 0.034 \mathrm{mmol})$ in THF $(0.2 \mathrm{~mL})$ was introduced dropwise. The mixture was then stirred at $-78{ }^{\circ} \mathrm{C}$ overnight. Aq. sat. $\mathrm{NH}_{4} \mathrm{Cl}(1 \mathrm{~mL})$ was added. The mixture was extracted with EtOAc $(5 \mathrm{~mL} \times 2)$. The combined organic layers were dried over anhydrous $\mathrm{Na}_{2} \mathrm{SO}_{4}$. Removal of the drying agent by filtration and rotary evaporation of the filtrate gave a crude oil, which was purified by column chromatography (30:1 PE/EtOAc) on silica gel to afford $32(23 \mathrm{mg}, 0.030 \mathrm{mmol}, 88 \%$ from 29) as a colorless oil (1:1 inseparable mixture of the sulfonyl epimers). Data for 32: $[\alpha]_{\mathrm{D}}{ }^{25}+19.9\left(\right.$ c $\left.1.0, \mathrm{CHCl}_{3}\right) .{ }^{1} \mathrm{H}$ NMR $(500 \mathrm{MHz}$, $\left.\mathrm{CDCl}_{3}\right) \delta$ 7.90-7.86 (m, 2H), 7.68-7.62 (m, 4H), 7.61-7.48 (m, 3H), 7.45-7.33 (m, 6H), $5.12(\mathrm{t}, J=$ $7.0 \mathrm{~Hz}, 1 \mathrm{H}), 4.07-4.00(\mathrm{~m}, 1 \mathrm{H}), 3.91(\mathrm{t}, J=6.6 \mathrm{~Hz}, 1 \mathrm{H}), 3.64(\mathrm{dd}, J=10.1,5.2 \mathrm{~Hz}, 1 \mathrm{H}), 3.58(\mathrm{dd}$, $J=10.1,5.7 \mathrm{~Hz}, 1 \mathrm{H}), 2.97$ (dd, $J=14.5,6.8 \mathrm{~Hz}, 1 \mathrm{H}), 2.56(\mathrm{dd}, J=14.5,6.5 \mathrm{~Hz}, 1 \mathrm{H}), 2.18-2.10$ (m, 1H), $2.01(\mathrm{~s}, 3 \mathrm{H}), 2.05-1.97(\mathrm{~m}, 1 \mathrm{H}), 1.91-1.84(\mathrm{~m}, 2 \mathrm{H}), 1.79-1.68(\mathrm{~m}, 2 \mathrm{H}), 1.63-1.48(\mathrm{~m}, 8 \mathrm{H})$, 1.46-1.34 (m, 3H), 1.28 and $1.27(\mathrm{~s}, 3 \mathrm{H}), 1.18(\mathrm{~s}, 3 \mathrm{H}), 1.09$ and $1.08(\mathrm{~s}, 3 \mathrm{H}), 1.05(\mathrm{~s}, 9 \mathrm{H}), 1.03(\mathrm{~d}$, $J=7.3 \mathrm{~Hz}, 3 \mathrm{H}), 0.81(\mathrm{~s}, 3 \mathrm{H}) ;{ }^{13} \mathrm{C}\left\{{ }^{1} \mathrm{H}\right\} \operatorname{NMR}\left(126 \mathrm{MHz}, \mathrm{CDCl}_{3}\right) \delta 142.2,137.7,135.6,135.6$, 133.7, 133.6, 133.0, 130.8, 130.7, 129.6, 128.9, 128.8, 128.7, 128.5, 127.6, 82.1, 82.1, 79.5, 65.6, 65.5, 41.2, 39.7, 39.4, 36.0, 34.5, 29.2, 28.3, 26.9, 23.7, 23.3, 21.7, 20.2, 19.3, 19.0, 15.3, 12.7. FT-IR (film of a concd solution in $\mathrm{CHCl}_{3}$ ) 3442, 2930, 2858, 1446, 1304, 1143, 1112, 1084, 823, $702 \mathrm{~cm}^{-1}$. MS (ESI) m/z: $779.70\left([\mathrm{M}+\mathrm{Na}]^{+}\right)$; HRMS (ESI) $\mathrm{m} / \mathrm{z}:[\mathrm{M}+\mathrm{Na}]^{+}$calcd. for $\mathrm{C}_{46} \mathrm{H}_{64} \mathrm{O}_{5} \mathrm{SNaSi}$ 779.4136; found 779.4141.
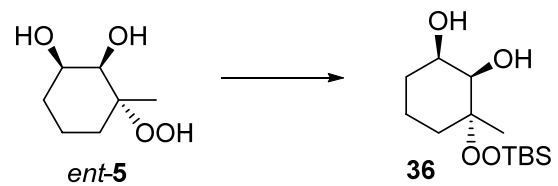

(1R,2R,3R)-3-((tert-Butyldimethylsilyl)peroxy)-3-methylcyclohexane-1,2-diol (36). Imidazole (5.2 $\mathrm{g}, 77.0 \mathrm{mmol})$ was added to a solution of ent-5 $(7.2 \mathrm{~g}, 44.3 \mathrm{mmol})$ in $\mathrm{CH}_{2} \mathrm{Cl}_{2}(240 \mathrm{~mL})$ and acetone $(60 \mathrm{~mL})$ stirred in an ice-water bath. After stirring at the same temperature for $10 \mathrm{~min}$, a solution of TBSCl $(9.8 \mathrm{~g}, 65.1 \mathrm{mmol})$ in $\mathrm{CH}_{2} \mathrm{Cl}_{2}(50 \mathrm{~mL})$ was the introduced slowly over $1 \mathrm{~h}$. 
Stirring was then continued at the same temperature for $4 \mathrm{~h}$. Water $(50 \mathrm{~mL})$ was added to quench the reaction. The mixture was extracted with $\mathrm{CH}_{2} \mathrm{Cl}_{2}(50 \mathrm{~mL} \times 3)$. The combined organic layers were dried over anhydrous $\mathrm{Na}_{2} \mathrm{SO}_{4}$. Removal of the drying agent by filtration and the solvent by rotary evaporation left an oily residue, which was purified by column chromatography (4:1 $\mathrm{PE} / \mathrm{EtOAc})$ on silica gel to afford $36(10.3 \mathrm{~g}, 37.3 \mathrm{mmol}, 63 \%)$ as a colorless oil, along with recovered ent-5 (2.0 g, 21\%). Data for 36: $[\alpha]_{\mathrm{D}}{ }^{25}-6.2\left(c 1.0, \mathrm{CHCl}_{3}\right) .{ }^{1} \mathrm{H} \mathrm{NMR}\left(500 \mathrm{MHz}, \mathrm{CDCl}_{3}\right)$ $\delta 4.01(\mathrm{dt}, J=6.3,3.2 \mathrm{~Hz}, 1 \mathrm{H}), 3.88(\mathrm{dd}, J=3.3,1.8 \mathrm{~Hz}, 1 \mathrm{H}), 3.08(\mathrm{br} \mathrm{s}, 1 \mathrm{H}), 2.28(\mathrm{br} \mathrm{s}, 1 \mathrm{H})$, 1.74-1.68 (m, 1H), 1.64-1.46 (m, 4H), 1.45-1.40 (m, 1H), 1.36 (s, 3H), $0.92(\mathrm{~s}, 9 \mathrm{H}), 0.14(\mathrm{~s}, 3 \mathrm{H})$, $0.13(\mathrm{~s}, 3 \mathrm{H}) ;{ }^{13} \mathrm{C}\left\{{ }^{1} \mathrm{H}\right\}$ NMR $\left(125 \mathrm{MHz}, \mathrm{CDCl}_{3}\right) \delta 84.9,75.0,69.3,31.8,28.8,26.2,19.8,18.2$, 18.2, -5.6, -5.6; FT-IR (film of a concd solution in $\mathrm{CHCl}_{3}$ ) 3418, 2932, 1462, 1250, 1062, 834, $785 \mathrm{~cm}^{-1}$. MS (ESI) m/z: $299.10\left([\mathrm{M}+\mathrm{Na}]^{+}\right)$; HRMS (ESI) $\mathrm{m} / \mathrm{z}:[\mathrm{M}+\mathrm{Na}]^{+}$calcd. for $\mathrm{C}_{13} \mathrm{H}_{28} \mathrm{O}_{4} \mathrm{NaSi} 299.1649$; found 299.1655.

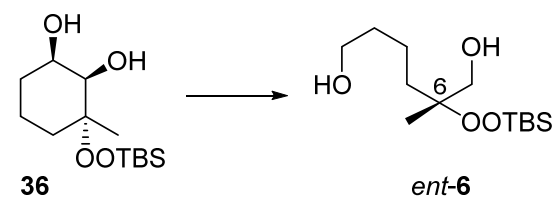

(R)-2-((tert-Butyldimethylsilyl)peroxy)-2-methylhexane-1,6-diol (ent-6). Powdered $\mathrm{NaIO}_{4}(13.7 \mathrm{~g}$, $64.0 \mathrm{mmol})$ was added in three portions to a solution of $\mathbf{3 6}(8.86 \mathrm{~g}, 32.0 \mathrm{mmol})$ in a mixture of THF $(150 \mathrm{~mL})$ and $\mathrm{H}_{2} \mathrm{O}(50 \mathrm{~mL})$ stirred at ambient temperature. The mixture was stirred at ambient temperature (the clear solution became turbid with the addition). After completion of the addition, the mixture was stirred at ambient temperature for $30 \mathrm{~min}$ (TLC showed completion of the reaction). Solids were filtered off through Celite (with suction). The filter cake was washed with $\mathrm{Et}_{2} \mathrm{O}(30 \mathrm{~mL})$. The combined filtrate and washing were partially concentrated on a rotary evaporator to remove most of the volatiles. The concentrated mixture was extracted with $\mathrm{Et}_{2} \mathrm{O}$ $(200 \mathrm{~mL})$. The combined organic layers were washed with water $(50 \mathrm{~mL} \times 2)$ and brine $(50 \mathrm{~mL})$ and dried over anhydrous $\mathrm{Na}_{2} \mathrm{SO}_{4}$. Filtration and rotary evaporation gave crude intermediate dialdehyde $(8.9 \mathrm{~g})$, which was dissolved in EtOH $(100 \mathrm{~mL})$ and cooled in a $-78{ }^{\circ} \mathrm{C}$ bath (dry ice-EtOH). $\mathrm{NaBH}_{4}(2.42 \mathrm{~g}, 64.0 \mathrm{mmol})$ was added. The mixture was stirred at $-78{ }^{\circ} \mathrm{C}$ for another $30 \mathrm{~min}$. Most of the EtOH was removed by rotary evaporation. The residue was extracted with EtOAc $(150 \mathrm{~mL} \times 2)$. The combined organic layers were washed with brine $(30 \mathrm{~mL})$ and dried over anhydrous $\mathrm{Na}_{2} \mathrm{SO}_{4}$. Removal of the drying agent by filtration and the solvent by rotary evaporation left an oily residue, which was purified by column chromatography (2:1 PE/EtOAc) on silica gel to afford ent-6 as a colorless oil $(7.4 \mathrm{~g}, 26.6 \mathrm{mmol}, 83 \%$ from 36). Data for ent-6: $[\alpha]_{\mathrm{D}}{ }^{25}+22.9\left(c_{1} 1.0, \mathrm{CHCl}_{3}\right) .{ }^{1} \mathrm{H}$ NMR $\left(500 \mathrm{MHz}, \mathrm{CDCl}_{3}\right) \delta 3.66-3.61(\mathrm{~m}, 3 \mathrm{H}), 3.56(\mathrm{dd}, J=11.7$, $7.0 \mathrm{~Hz}, 1 \mathrm{H}), 2.44(\mathrm{t}, J=6.5 \mathrm{~Hz}, 1 \mathrm{H}), 1.80(\mathrm{br} \mathrm{s}, 1 \mathrm{H}), 1.63-1.47$ (m, 4H), 1.43-1.33 (m, 2H), 1.11 (s, 3H), $0.91(\mathrm{~s}, 9 \mathrm{H}), 0.14(\mathrm{~s}, 3 \mathrm{H}), 0.14(\mathrm{~s}, 3 \mathrm{H}) ;{ }^{13} \mathrm{C}\left\{{ }^{1} \mathrm{H}\right\} \mathrm{NMR}\left(126 \mathrm{MHz}, \mathrm{CDCl}_{3}\right) \delta 84.9,67.5$, 62.5, 33.6, 33.1, 26.1, 19.6, 18.5, 18.2, -5.7. FT-IR (film of a concd solution in $\mathrm{CHCl}_{3}$ ) 3360, 2931, 2859, 1463, 1250, 1054, 838, $785 \mathrm{~cm}^{-1}$. MS (ESI) $\mathrm{m} / \mathrm{z}: 301.10\left([\mathrm{M}+\mathrm{Na}]^{+}\right)$; HRMS (ESI) $\mathrm{m} / \mathrm{z}:[\mathrm{M}+\mathrm{Na}]^{+}$calcd. for $\mathrm{C}_{13} \mathrm{H}_{30} \mathrm{O}_{4} \mathrm{NaSi} 301.1806$; found 301.1813 . 


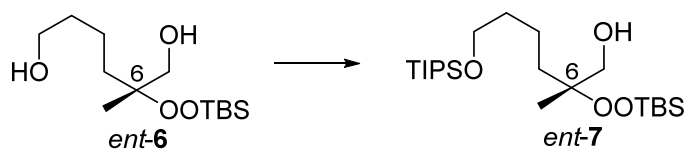

(R)-2-((tert-Butyldimethylsilyl)peroxy)-2-methyl-6-((triisopropylsilyl)oxy)hexan-1-ol (ent-7). This compound was obtained using the same procedure given above for 7 (i.e., conversion of 6 into 7 ) except that using ent-6 to replace 6. Data for ent-7: $[\alpha]_{\mathrm{D}}{ }^{25}+13.4\left(\right.$ c $\left.1.0, \mathrm{CHCl}_{3}\right) .{ }^{1} \mathrm{H}$ NMR (500 $\left.\mathrm{MHz}, \mathrm{CDCl}_{3}\right) \delta 3.68(\mathrm{t}, J=6.4 \mathrm{~Hz}, 1 \mathrm{H}), 3.62(\mathrm{dd}, J=11.8,6.5 \mathrm{~Hz}, 1 \mathrm{H}), 3.59(\mathrm{dd}, J=11.8,6.6 \mathrm{~Hz}$, $1 \mathrm{H}), 2.36(\mathrm{t}, J=6.5 \mathrm{~Hz}, 1 \mathrm{H}), 1.63-1.45(\mathrm{~m}, 4 \mathrm{H}), 1.43-1.32(\mathrm{~m}, 2 \mathrm{H}), 1.13(\mathrm{~s}, 3 \mathrm{H}), 1.11-1.02(\mathrm{~m}$, 21H), $0.92(\mathrm{~s}, 9 \mathrm{H}), 0.15(\mathrm{~s}, 3 \mathrm{H}), 0.15(\mathrm{~s}, 3 \mathrm{H}) ;{ }^{13} \mathrm{C}\left\{{ }^{1} \mathrm{H}\right\} \mathrm{NMR}\left(125 \mathrm{MHz}, \mathrm{CDCl}_{3}\right) \delta$ 84.9, 67.8, 63.2, 34.0, 33.5, 26.2, 19.8, 18.4, 18.2, 18.0, 12.0, -5.7, -5.7. FT-IR (film of a concd solution in $\mathrm{CHCl}_{3}$ ) 2942, 2866, 1463, 1258, 1106, 1057, 838, $786 \mathrm{~cm}^{-1}$. MS (ESI) m/z: $457.35\left([\mathrm{M}+\mathrm{Na}]^{+}\right)$; HRMS (ESI) $\mathrm{m} / \mathrm{z}$ : $[\mathrm{M}+\mathrm{Na}]^{+}$calcd. for $\mathrm{C}_{22} \mathrm{H}_{50} \mathrm{O}_{4} \mathrm{NaSi}_{2}$ 457.3140; found 457.3147 .

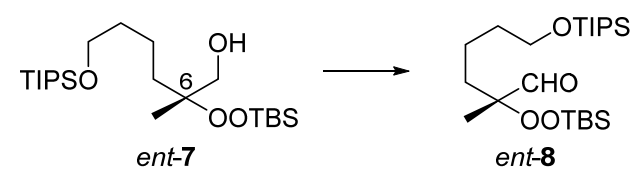

(R)-2-((tert-Butyldimethylsilyl)peroxy)-2-methyl-6-((triisopropylsilyl)oxy)hexanal (ent-8). A solution of DMSO $(3.6 \mathrm{~mL}, 51.4 \mathrm{mmol})$ in dry $\mathrm{CH}_{2} \mathrm{Cl}_{2}(10 \mathrm{~mL})$ was added very slowly to a solution of $(\mathrm{COCl})_{2}(3.3 \mathrm{~mL}, 38.5 \mathrm{mmol})$ in dry $\mathrm{CH}_{2} \mathrm{Cl}_{2}(60 \mathrm{~mL})$ stirred at $-78{ }^{\circ} \mathrm{C}$ under argon (balloon). After completion of the addition, the mixture was stirred at the same temperature for 30 min. A solution of alcohol ent-7 $(10.1 \mathrm{~g}, 23.4 \mathrm{mmol})$ in dry $\mathrm{CH}_{2} \mathrm{Cl}_{2}(30 \mathrm{~mL})$ was introduced dropwise. Stirring was then continued at the same temperature for another $30 \mathrm{~min}$. Finally, $\mathrm{Et}_{3} \mathrm{~N}$ $(14.3 \mathrm{~mL}, 102.8 \mathrm{mmol})$ was added very slowly. The mixture was stirred at $-78{ }^{\circ} \mathrm{C}$ for $30 \mathrm{~min}$. The cooling bath was allowed to warm to ambient temperature, at which the mixture was stirred for another $30 \mathrm{~min}$. Water $(50 \mathrm{~mL})$ was added. The phases were separated. The aqueous layer was back-extracted with $\mathrm{CH}_{2} \mathrm{Cl}_{2}(30 \mathrm{~mL})$. The combined organic layers were washed with brine (30 $\mathrm{mL}$ ) and dried over anhydrous $\mathrm{Na}_{2} \mathrm{SO}_{4}$. Removal of the drying agent by filtration and rotary evaporation of the filtrate gave a crude oil, which was purified by column chromatography (30:1 $\mathrm{PE} / \mathrm{EtOAc})$ on silica gel to afford aldehyde ent-8 as a colorless oil $(9.20 \mathrm{~g}, 21.2 \mathrm{mmol}, 91 \%$ from ent-7). Data for ent-8: $[\alpha]_{\mathrm{D}}{ }^{25}+55.8\left(c 1.0, \mathrm{CHCl}_{3}\right) .{ }^{1} \mathrm{H} \mathrm{NMR}\left(500 \mathrm{MHz}, \mathrm{CDCl}_{3}\right) \delta 9.68(\mathrm{~s}, 1 \mathrm{H})$, $3.66(\mathrm{t}, J=6.3 \mathrm{~Hz}, 2 \mathrm{H}), 1.71-1.63(\mathrm{~m}, 1 \mathrm{H}), 1.58-1.47(\mathrm{~m}, 3 \mathrm{H}), 1.44-1.36(\mathrm{~m}, 2 \mathrm{H}), 1.26(\mathrm{~s}, 3 \mathrm{H})$, 1.13-1.01 (m, 21H), $0.91(\mathrm{~s}, 9 \mathrm{H}), 0.16(\mathrm{~s}, 3 \mathrm{H}), 0.15(\mathrm{~s}, 3 \mathrm{H}) ;{ }^{13} \mathrm{C}\left\{{ }^{1} \mathrm{H}\right\}$ NMR $\left(126 \mathrm{MHz}, \mathrm{CDCl}_{3}\right) \delta$ 204.2, 88.7, 62.9, 33.2, 33.0, 26.1, 19.2, 18.2, 18.0, 16.6, 11.9, -5.7, -5.7. FT-IR (film of a concd solution in $\mathrm{CHCl}_{3}$ ) 2942, 2866, 1743, 1463, 1251, 1108, 882, 840, 786, $679 \mathrm{~cm}^{-1}$. MS (ESI) $\mathrm{m} / \mathrm{z}$ : $487.35\left([\mathrm{M}+\mathrm{Na}+\mathrm{MeOH}]^{+}\right)$; HRMS (ESI) $m / z:[\mathrm{M}+\mathrm{Na}+\mathrm{MeOH}]^{+}$calcd. for $\mathrm{C}_{23} \mathrm{H}_{52} \mathrm{O}_{5} \mathrm{NaSi}_{2}$ 487.3245; found 487.3248.

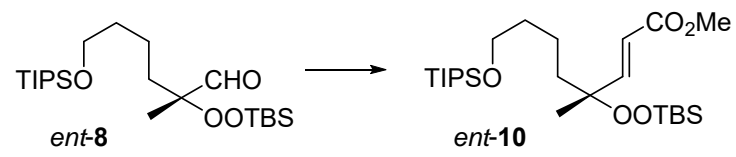

Methyl-(R,E)-4-((tert-butyldimethylsilyl)peroxy)-4-methyl-8-((triisopropylsilyl)oxy)oct-2-enoate (ent-10). NaHMDS $(9.8 \mathrm{~mL}, 19.7 \mathrm{mmol})$ was added to a solution of trimethyl phosphate $(4.0 \mathrm{~mL}$, $24.6 \mathrm{mmol})$ in THF $(150 \mathrm{~mL})$ stirred in an ice-water bath under argon (balloon). After completion 
of the addition, stirring was continued for $30 \mathrm{~min}$. The ice-water bath was then replaced by a dry ice-EtOH bath $\left(-78^{\circ} \mathrm{C}\right)$. With stirring, a solution of the above obtained aldehyde ent-8 $(7.10 \mathrm{~g}$, $16.4 \mathrm{mmol})$ in THF $(50 \mathrm{~mL})$ was introduce dropwise via a syringe. After completion of the addition, stirring was continued for ca. $3 \mathrm{~h}$, when TLC showed completion of the reaction. Water $(50 \mathrm{~mL})$ was added. The mixture was concentrated by rotary evaporation (to remove THF). The residue was extracted by EtOAc $(100 \mathrm{~mL})$. The aqueous layer was back-extracted with EtOAc (50 $\mathrm{mL})$. The combined organic layers were washed with brine $(30 \mathrm{~mL})$ and dried over anhydrous $\mathrm{Na}_{2} \mathrm{SO}_{4}$. Removal of the drying agent by filtration and rotary evaporation of the filtrate gave a crude oil $(9.0 \mathrm{~g})$, the majority ( $8.8 \mathrm{~g}$ ) of which was used directly in the next step. A small portion $(50 \mathrm{mg})$ of the crude ent-10 was purified by column chromatography (20:1 PE/EtOAc) on silica gel to afford the analytic sample $(32 \mathrm{mg})$ of ent-10 as a colorless oil. Data for ent-10: $[\alpha]_{\mathrm{D}}{ }^{25}+14.3$ $\left(c 1.0, \mathrm{CHCl}_{3}\right) .{ }^{1} \mathrm{H}$ NMR $\left(500 \mathrm{MHz}, \mathrm{CDCl}_{3}\right) \delta 6.98(\mathrm{~d}, J=16.1 \mathrm{~Hz}, 1 \mathrm{H}), 5.88(\mathrm{~d}, J=16.1 \mathrm{~Hz}, 1 \mathrm{H})$, $3.74(\mathrm{~s}, 3 \mathrm{H}), 3.65(\mathrm{t}, J=6.4 \mathrm{~Hz}, 2 \mathrm{H}), 1.69-1.56(\mathrm{~m}, 2 \mathrm{H}), 1.50$ (quint, $J=6.9 \mathrm{~Hz}, 2 \mathrm{H}), 1.43-1.32$ (m, 2H), 1.33 (s, 3H), 1.10-1.02 (m, 21H), $0.92(\mathrm{~s}, 9 \mathrm{H}), 0.13(\mathrm{~s}, 3 \mathrm{H}), 0.13(\mathrm{~s}, 3 \mathrm{H}),{ }^{13} \mathrm{C}\left\{{ }^{1} \mathrm{H}\right\}$ NMR $\left(126 \mathrm{MHz}, \mathrm{CDCl}_{3}\right) \delta 167.1,151.7,119.8,83.9,63.1,51.6,37.7,33.2,26.2,21.8,20.0,18.3,18.0$, 12.0, -5.6, -5.6. FT-IR (film of a concd solution in $\mathrm{CHCl}_{3}$ ) 2942, 2865, 1732, 1463, 1311, 1107, 883, 838, $785 \mathrm{~cm}^{-1}$. MS (ESI) $\mathrm{m} / \mathrm{z}: 511.40\left([\mathrm{M}+\mathrm{Na}]^{+}\right)$; HRMS (ESI) $\mathrm{m} / \mathrm{z}:[\mathrm{M}+\mathrm{Na}]^{+}$calcd. for $\mathrm{C}_{25} \mathrm{H}_{52} \mathrm{O}_{5} \mathrm{Si}_{2} \mathrm{Na}$ 511.3245; found 511.3251; HRMS (ESI) m/z: $[\mathrm{M}+\mathrm{H}]^{+}$calcd. for $\mathrm{C}_{25} \mathrm{H}_{53} \mathrm{O}_{5} \mathrm{Si}_{2}$ 489.3426; found 489.3426 .

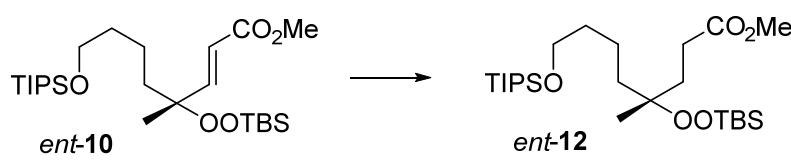

Methyl-(R)-4-((tert-butyldimethylsilyl)peroxy)-4-methyl-8-((triisopropylsilyl)oxy)-octanoate

(ent-12). This compound $(6.90 \mathrm{~g}, 14.1 \mathrm{mmol}, 86 \%$ overall from ent-8) was obtained using the same procedure given above for $\mathbf{1 2}$ (i.e., conversion of $\mathbf{1 0}$ into 12) except that using (crude) ent-10 to replace 10. Data for ent-12: $[\alpha]_{\mathrm{D}}{ }^{25}+5.7\left(c 1.0, \mathrm{CHCl}_{3}\right) .{ }^{1} \mathrm{H} \mathrm{NMR}\left(500 \mathrm{MHz}, \mathrm{CDCl}_{3}\right) \delta 3.67(\mathrm{t}, J$ $=6.5 \mathrm{~Hz}, 2 \mathrm{H}), 3.66(\mathrm{~s}, 3 \mathrm{H}), 2.41-2.31(\mathrm{~m}, 2 \mathrm{H}), 1.93-1.86(\mathrm{~m}, 2 \mathrm{H}), 1.57-1.43(\mathrm{~m}, 4 \mathrm{H}), 1.40-1.31$ $(\mathrm{m}, 2 \mathrm{H}), 1.11(\mathrm{~s}, 3 \mathrm{H}), 1.10-1.02(\mathrm{~m}, 21 \mathrm{H}), 0.91(\mathrm{~s}, 9 \mathrm{H}), 0.11(\mathrm{~s}, 6 \mathrm{H}) ;{ }^{13} \mathrm{C}\left\{{ }^{1} \mathrm{H}\right\}$ NMR $(126 \mathrm{MHz}$, $\left.\mathrm{CDCl}_{3}\right) \delta 174.6,83.4,63.2,51.6,36.7,33.5,31.7,28.6,26.2,21.5,20.0,18.3,18.0,12.0,-5.6$. FT-IR (film of a concd solution in $\mathrm{CHCl}_{3}$ ) 2943, 2865, 1745, 1463, 1250, 1108, 882, 838, 784, 679 $\mathrm{cm}^{-1}$. MS (ESI) $\mathrm{m} / \mathrm{z}: 513.45\left([\mathrm{M}+\mathrm{Na}]^{+}\right)$; HRMS (ESI) $\mathrm{m} / \mathrm{z}:[\mathrm{M}+\mathrm{Na}]^{+}$calcd. for $\mathrm{C}_{25} \mathrm{H}_{54} \mathrm{O}_{5} \mathrm{Si}_{2} \mathrm{Na}$ 513.3402; found 513.3407.

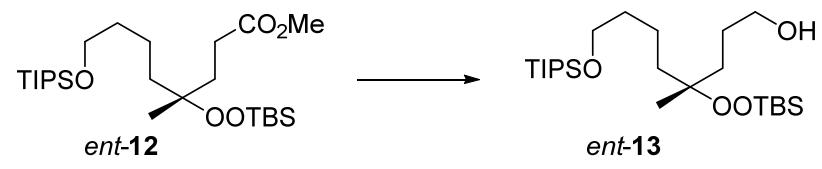

(R)-4-((tert-Butyldimethylsilyl)peroxy)-4-methyl-8-((triisopropylsilyl)oxy)octan-1-ol (ent-13). This compound $(6.03 \mathrm{~g}, 13.0 \mathrm{mmol}, 95 \%$ from ent-12) was obtained using the same procedure given above for $\mathbf{1 3}$ (i.e., conversion of $\mathbf{1 2}$ into $\mathbf{1 3}$ ) except that using ent-12 to replace $\mathbf{1 2}$. Data for ent-13 $[\alpha]_{\mathrm{D}}{ }^{25}+7.9\left(c 1.0, \mathrm{CHCl}_{3}\right) .{ }^{1} \mathrm{H}$ NMR $\left(500 \mathrm{MHz}, \mathrm{CDCl}_{3}\right) \delta 3.70-3.59(\mathrm{~m}, 4 \mathrm{H}), 1.90(\mathrm{t}, J=5.3 \mathrm{~Hz}$, $1 \mathrm{H}), 1.71-1.47(\mathrm{~m}, 9 \mathrm{H}), 1.40-1.30(\mathrm{~m}, 2 \mathrm{H}), 1.14(\mathrm{~s}, 3 \mathrm{H}), 1.11-1.00(\mathrm{~m}, 21 \mathrm{H}), 0.91(\mathrm{~s}, 9 \mathrm{H}), 0.12(\mathrm{~s}$, 3H), $0.12(\mathrm{~s}, 3 \mathrm{H}) ;{ }^{13} \mathrm{C}\left\{{ }^{1} \mathrm{H}\right\}$ NMR $\left(126 \mathrm{MHz}, \mathrm{CDCl}_{3}\right) \delta$ 84.6, 63.3, 63.2, 36.6, 33.6, 32.2, 27.0, 
26.2, 22.1, 20.2, 18.3, 18.0, 12.0, -5.5. FT-IR (film of a concd solution in $\mathrm{CHCl}_{3}$ ) 3321, 2942, 2865, 1463, 1249, 1107, 882, 838, $784 \mathrm{~cm}^{-1}$. MS (ESI) $\mathrm{m} / \mathrm{z}: 485.40\left([\mathrm{M}+\mathrm{Na}]^{+}\right)$; HRMS (ESI) $\mathrm{m} / \mathrm{z}$ : $[\mathrm{M}+\mathrm{Na}]^{+}$calcd. for $\mathrm{C}_{24} \mathrm{H}_{54} \mathrm{O}_{4} \mathrm{Si}_{2} \mathrm{Na}$ 485.3453; found 485.3453.

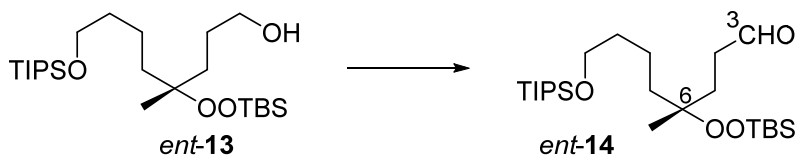

(R)-4-((tert-Butyldimethylsilyl)peroxy)-4-methyl-8-((triisopropylsilyl)oxy)octanal (ent-14). This compound $(5.63 \mathrm{~g}, 12.2 \mathrm{mmol}, 95 \%$ from ent-13) was obtained using the same procedure given above for 14 (i.e., conversion of $\mathbf{1 3}$ into 14) except that using ent-13 to replace 13. Data for ent-14: $[\alpha]_{\mathrm{D}}{ }^{25}+8.5\left(c 1.0, \mathrm{CHCl}_{3}\right) .{ }^{1} \mathrm{H} \mathrm{NMR}\left(500 \mathrm{MHz}, \mathrm{CDCl}_{3}\right) \delta 9.76(\mathrm{t}, J=1.8 \mathrm{~Hz}, 1 \mathrm{H}), 3.67$ (t, $J=6.4$ $\mathrm{Hz}, 2 \mathrm{H}), 2.53-2.37(\mathrm{~m}, 2 \mathrm{H}), 1.96-1.81(\mathrm{~m}, 2 \mathrm{H}), 1.58-1.42(\mathrm{~m}, 4 \mathrm{H}), 1.39-1.30(\mathrm{~m}, 2 \mathrm{H}), 1.13(\mathrm{~s}, 3 \mathrm{H})$, 1.12-1.00 (m, 21H), $0.91(\mathrm{~s}, 9 \mathrm{H}), 0.11(\mathrm{~s}, 3 \mathrm{H}), 0.10(\mathrm{~s}, 3 \mathrm{H}) ;{ }^{13} \mathrm{C}\left\{{ }^{1} \mathrm{H}\right\}$ NMR $\left(126 \mathrm{MHz}, \mathrm{CDCl}_{3}\right) \delta$ 203.1, 83.4, 63.2, 38.7, 36.7, 33.5, 28.9, 26.2, 21.6, 20.1, 18.2, 18.0, 12.0, -5.6, -5.6. FT-IR (film of a concd solution in $\left.\mathrm{CHCl}_{3}\right) 2942,2865,1729,1463,1250,1107,882,838,784,679 \mathrm{~cm}^{-1}$. MS (ESI) $\mathrm{m} / \mathrm{z} 483.35\left([\mathrm{M}+\mathrm{Na}]^{+}\right)$; MS (ESI) $m / z: 515.40\left([\mathrm{M}+\mathrm{Na}+\mathrm{MeOH}]^{\dagger}\right)$; HRMS (ESI) $m / z:[\mathrm{M}$ $+\mathrm{Na}]^{+}$calcd. for $\mathrm{C}_{24} \mathrm{H}_{52} \mathrm{O}_{4} \mathrm{Si}_{2} \mathrm{Na}$ 483.3296; found 483.3290 . 